\title{
Mass Spectrometric Study of the Conversion of Rhenium Diolates to Metallaoxetanes and Carbenes. Coordination Number, Polar, and Steric Effects
}

Xiangyang Zhang, Xueyi Chen, and Peter Chen*

Laboratorium für Organische Chemie

ETH Zürich

Zürich, Switzerland

\section{Supporting Material}

Part I: Electrospray and CID experiments on Bipyridine Rhenium Oxo Diolates

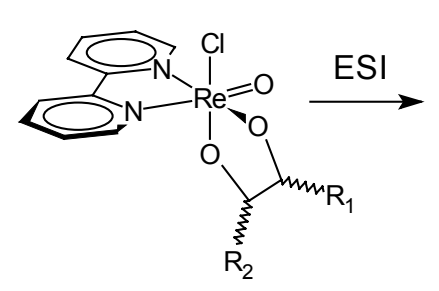

1

(a) $\mathrm{R}_{1}=\mathrm{R}_{2}=\mathrm{CO}_{2} \mathrm{Me}$

(b) $\mathrm{R}_{1}=\mathrm{CO}_{2} \mathrm{Et}, \mathrm{R}_{2}=\mathrm{Ph}$

(c) $R_{1}=M e, R_{2}=H$

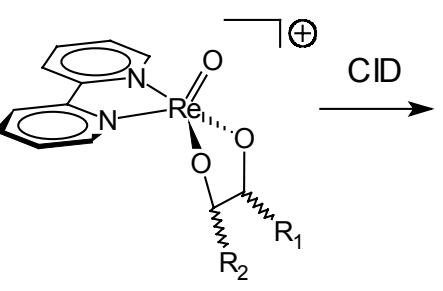

2
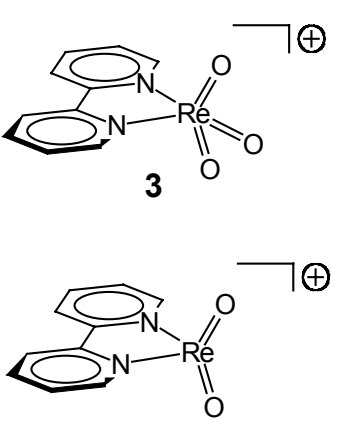

4

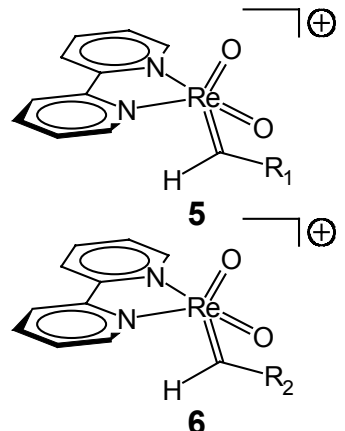

6

1. CID spectrum of $2 \mathrm{a}\left(\mathrm{R}_{1}=\mathrm{R}_{2}=\mathrm{CO}_{2} \mathrm{Me}\right)$

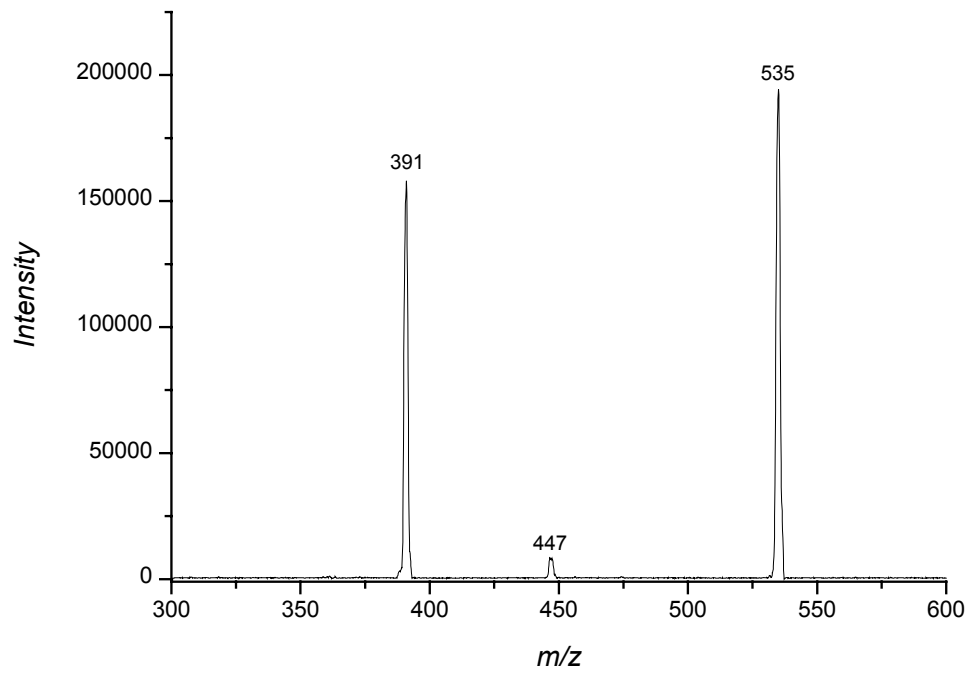

2. CID spectrum of $2 b\left(R_{1}=C O_{2} E t, R_{2}=P h\right)$ 


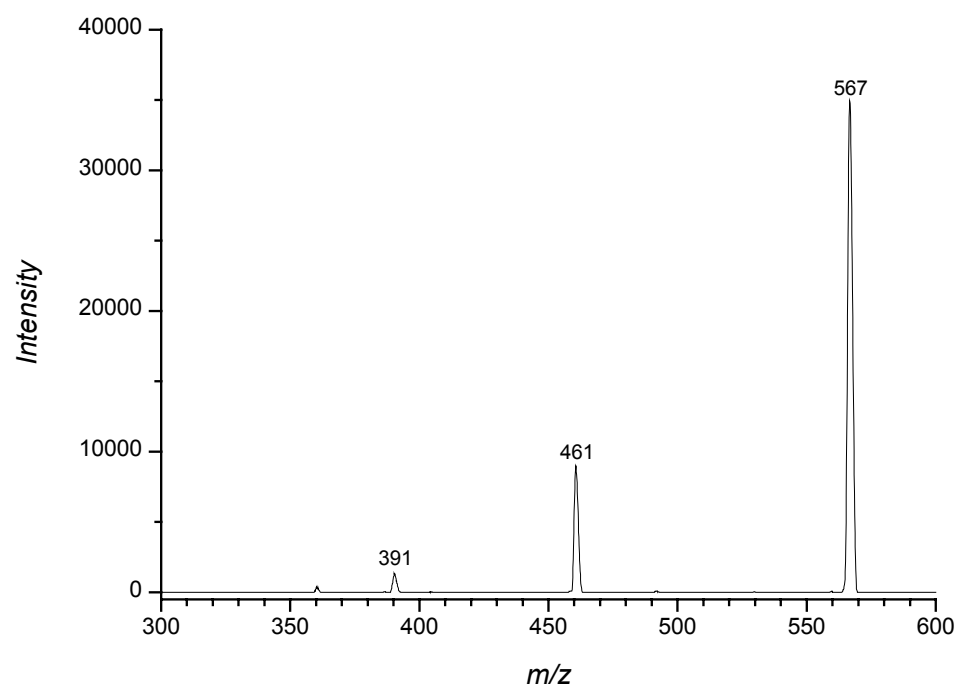

3. CID spectrum of $2 \mathrm{c}\left(\mathrm{R}_{1}=\mathrm{Me}, \mathrm{R}_{2}=\mathrm{H}\right)$

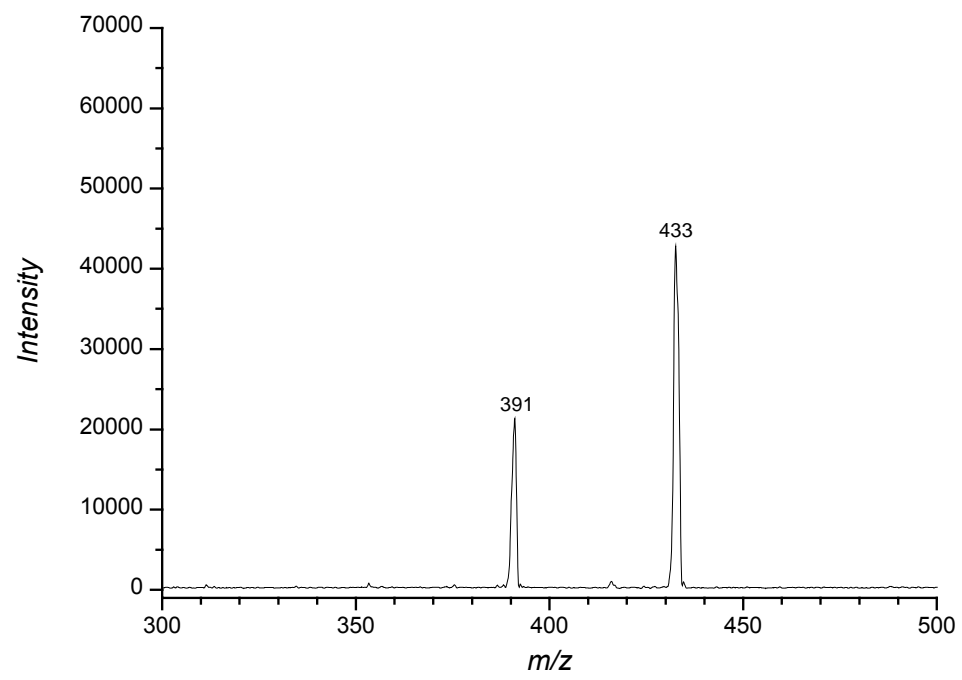


Part II. Independent preparation of benzylidene complex from (pyr) $)_{2}$ (bipy) $\mathrm{ReClO}_{2}$ and phenyldiazomethane in solution, followed by ESI-MS. Besides several species [(bipy)ReO $\left.\mathrm{R}_{2}(\mathrm{pyr})_{\mathrm{n}}\right]^{+}$ $(\mathrm{m} / \mathrm{z}=375,454,533)$ and the adduct ion $\left[(\text { bipy }) \mathrm{ReO}_{2}\left(\mathrm{PhHC}=\mathrm{N}_{2}\right)\right]^{+}(\mathrm{m} / \mathrm{z}=493)$, there is a small but definite peak at $\mathrm{m} / \mathrm{z}=465$ which corresponds to $\left[(\text { bipy }) \mathrm{ReO}_{2}(=\mathrm{CHPh})\right]^{+}$, a benzylidene complex.

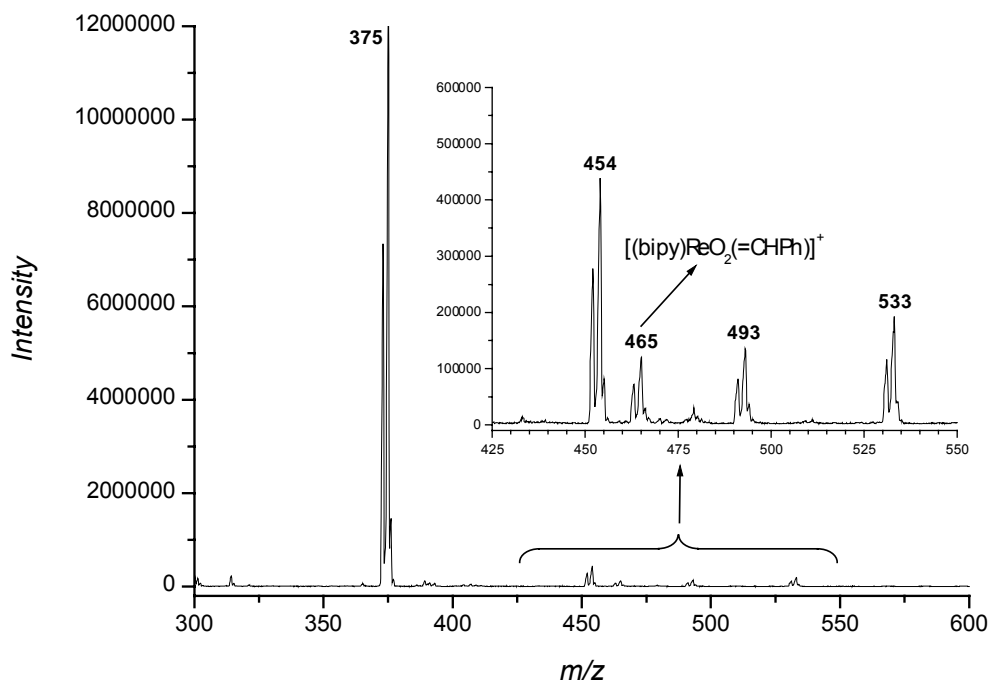


Part III: Electrospray and CID experiments of Bispyridine Rhenium Oxo Diolates
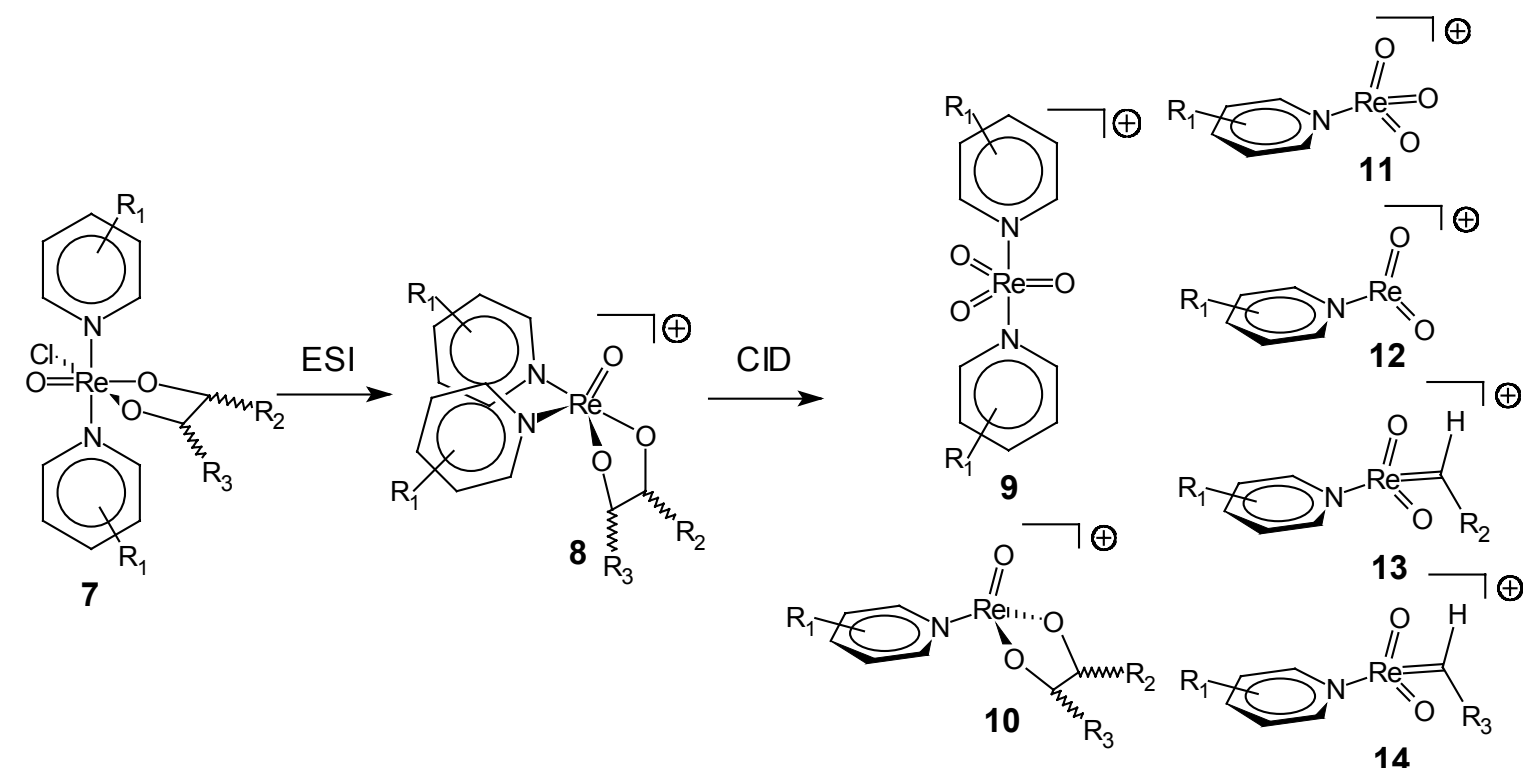
(a) $\mathrm{R}_{1}=\mathrm{H}, \mathrm{R}_{2}=\mathrm{H}, \mathrm{R}_{3}=\mathrm{H}$
(g) $\mathrm{R}_{1}=\mathrm{H}, \mathrm{R}_{2}=\mathrm{Ph}, \mathrm{R}_{3}=\mathrm{H}$
(m) $\mathrm{R}_{1}=\mathrm{H}, \mathrm{R}_{2}=\mathrm{Ph}, \mathrm{R}_{3}=\mathrm{Ph}$ (trans)
(b) $\mathrm{R}_{1}=\mathrm{H}, \mathrm{R}_{2}=\mathrm{Me}, \mathrm{R}_{3}=\mathrm{H}$
(h) $\mathrm{R}_{1}=\mathrm{H}, \mathrm{R}_{2}=(p-\mathrm{Me}) \mathrm{C}_{6} \mathrm{H}_{4}, \mathrm{R}_{3}=\mathrm{H}$
(n) $\mathrm{R}_{1}=\mathrm{H}, \mathrm{R}_{2}=(p-\mathrm{MeO}) \mathrm{C}_{6} \mathrm{H}_{4}, \mathrm{R}_{3}=\mathrm{Me}(c i s)$
(c) $R_{1}=H, R_{2}=E t, R_{3}=H$
(i) $\mathrm{R}_{1}=\mathrm{H}, \mathrm{R}_{2}=\left(p-\mathrm{CF}_{3}\right) \mathrm{C}_{6} \mathrm{H}_{4}, \mathrm{R}_{3}=\mathrm{H}$
(o) $\mathrm{R}_{1}=\mathrm{H}, \mathrm{R}_{2}=\mathrm{CO}_{2} \mathrm{Et}, \mathrm{R}_{3}=\mathrm{CO}_{2} \mathrm{Et}$ (trans)
(d) $\mathrm{R}_{1}=\mathrm{H}, \mathrm{R}_{2}=n-\mathrm{Pr}, \mathrm{R}_{3}=\mathrm{H}$
(j) $\mathrm{R}_{1}=\mathrm{H}, \mathrm{R}_{2}=\mathrm{CF}_{3}, \mathrm{R}_{3}=\mathrm{H}$
(p) $\mathrm{R}_{1}=p-\mathrm{Me}, \mathrm{R}_{2}=\mathrm{Me}, \mathrm{R}_{3}=\mathrm{H}$
(e) $\mathrm{R}_{1}=\mathrm{H}, \mathrm{R}_{2}=n-\mathrm{Bu}, \mathrm{R}_{3}=\mathrm{H}$
(k) $\mathrm{R}_{1}=\mathrm{H}, \mathrm{R}_{2}=\mathrm{Me}, \mathrm{R}_{3}=\mathrm{Me}$ (cis)
(q) $\mathrm{R}_{1}=p-\mathrm{NMe}_{2}, \mathrm{R}_{2}=\mathrm{Me}, \mathrm{R}_{3}=\mathrm{H}$
(f) $\mathrm{R}_{1}=\mathrm{H}, \mathrm{R}_{2}=t-\mathrm{Bu}, \mathrm{R}_{3}=\mathrm{H}$
(l) $\mathrm{R}_{1}=\mathrm{H}, \mathrm{R}_{2}=\mathrm{Me}, \mathrm{R}_{3}=\mathrm{Me}$ (trans)
(r) $\mathrm{R}_{1}=p-\mathrm{F}, \mathrm{R}_{2}=\mathrm{Me}, \mathrm{R}_{3}=\mathrm{H}$

4. CID spectrum of $8 a$ and $10 a\left(R_{1}=H, R_{2}=H, R_{3}=H\right)$
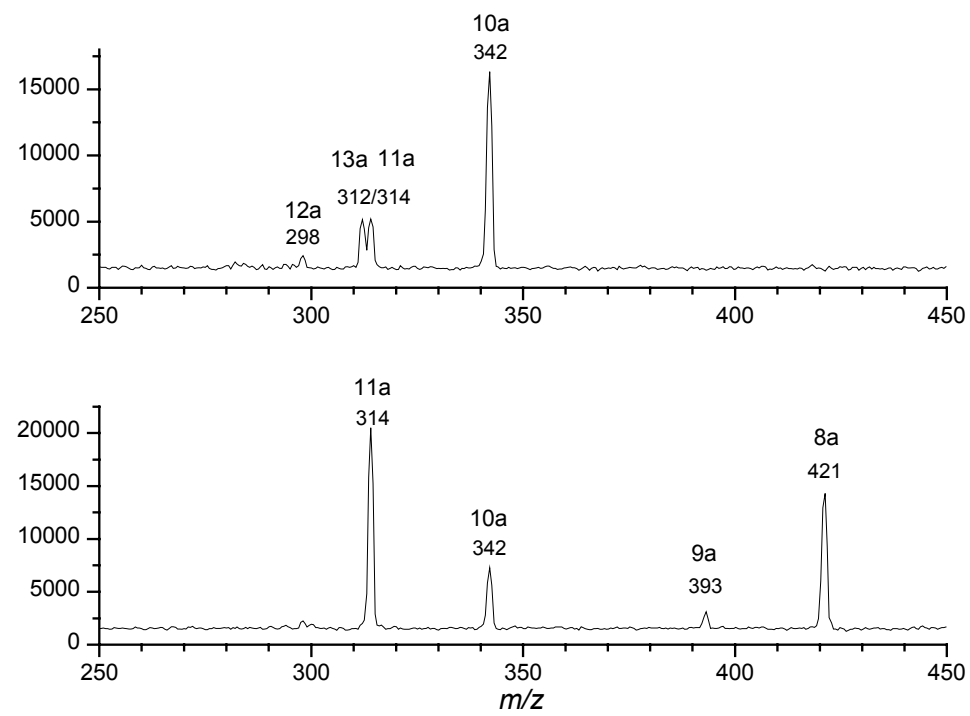
5. CID spectrum of $8 b$ and $10 b\left(R_{1}=H, R_{2}=M e, R_{3}=H\right)$
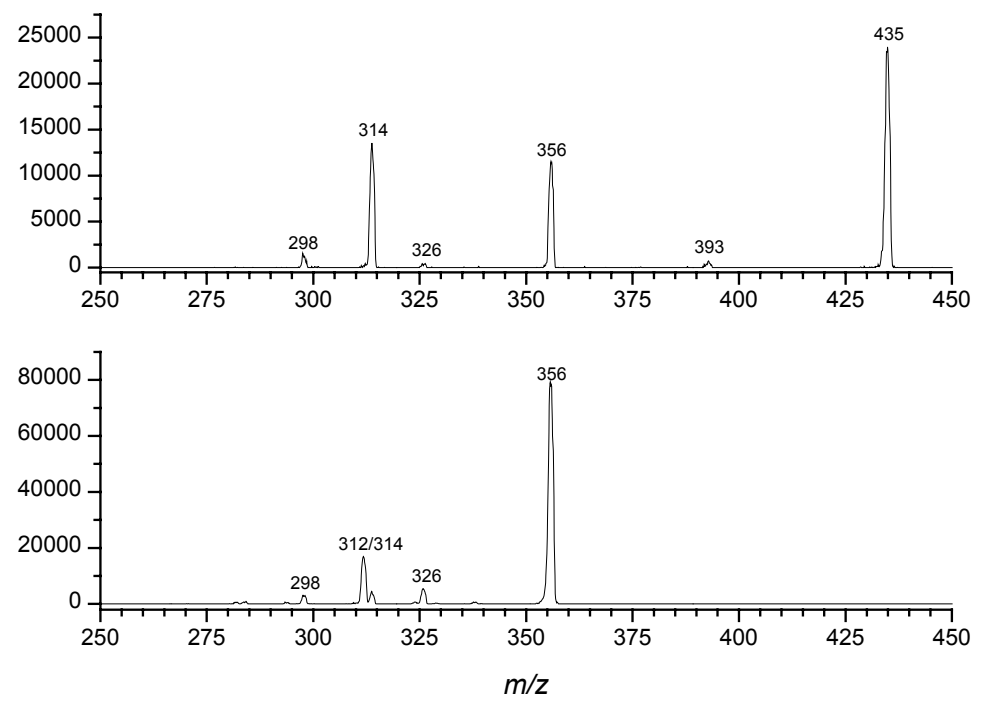

6. CID spectra of $8 c$ and $10 c\left(R_{1}=H, R_{2}=E t, R_{3}=H\right)$
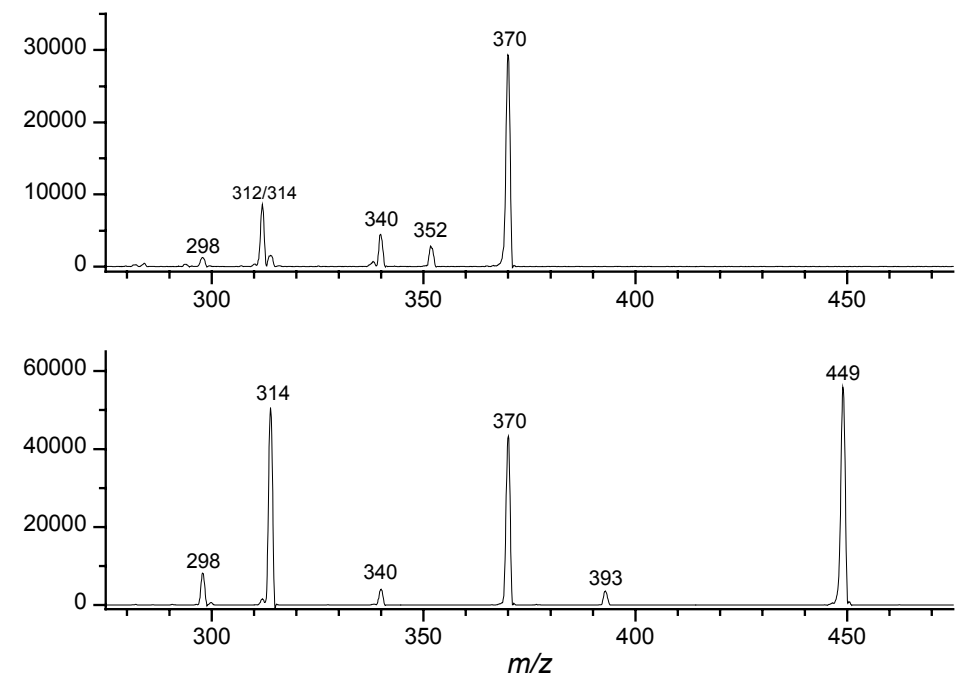
7. CID spectra of $8 \mathrm{~d}$ and $10 \mathrm{~d}\left(\mathrm{R}_{1}=\mathrm{H}, \mathrm{R}_{2}=n-\operatorname{Pr}, \mathrm{R}_{3}=\mathrm{H}\right)$
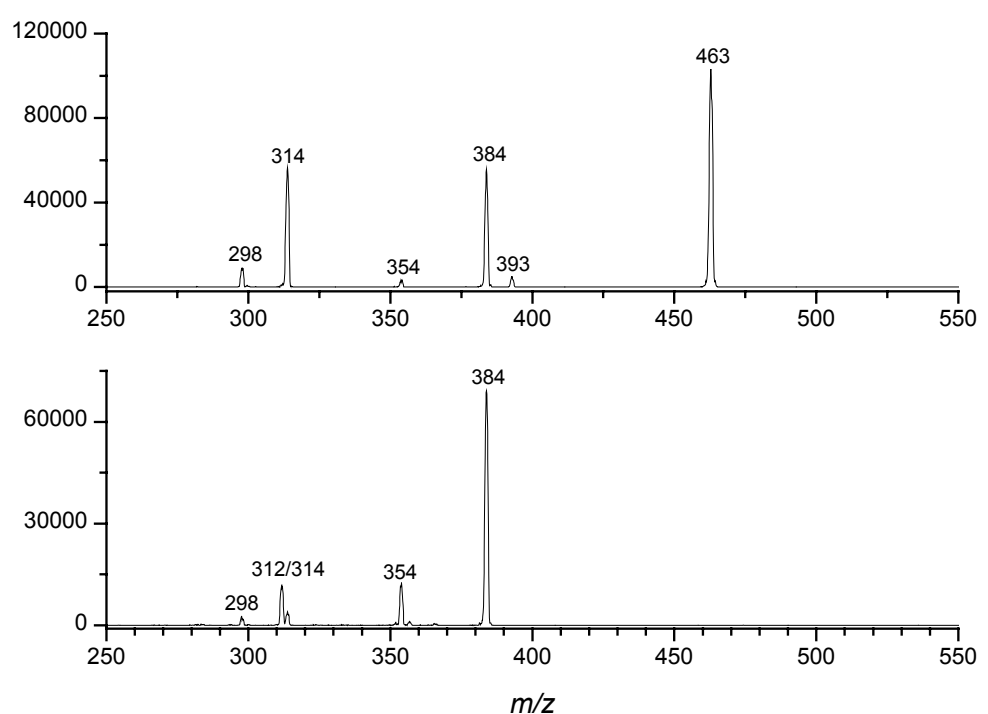

8. CID spectra of $8 \mathrm{e}$ and $10 \mathrm{e}\left(\mathrm{R}_{1}=\mathrm{H}, \mathrm{R}_{2}=n-\mathrm{Bu}, \mathrm{R}_{3}=\mathrm{H}\right)$
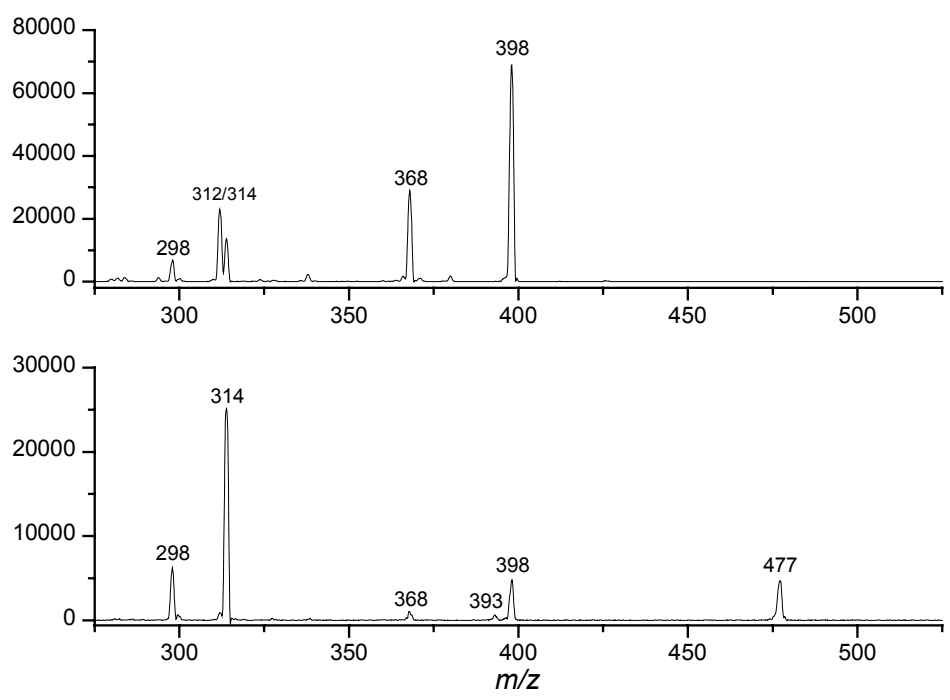
9. CID spectra of $8 \mathrm{f}$ and $10 \mathrm{f}\left(\mathrm{R}_{1}=\mathrm{H}, \mathrm{R}_{2}=t-\mathrm{Bu}, \mathrm{R}_{3}=\mathrm{H}\right)$
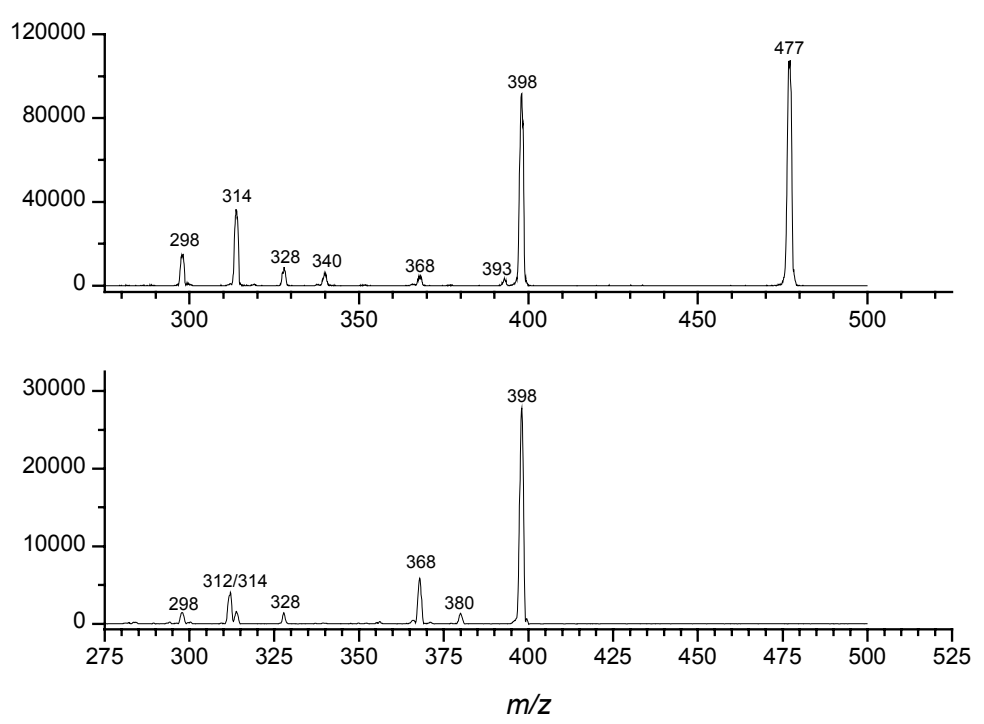

10. CID spectra of $8 \mathrm{~g}$ and $10 \mathrm{~g}\left(\mathrm{R}_{1}=\mathrm{H}, \mathrm{R}_{2}=\mathrm{Ph}, \mathrm{R}_{3}=\mathrm{H}\right)$
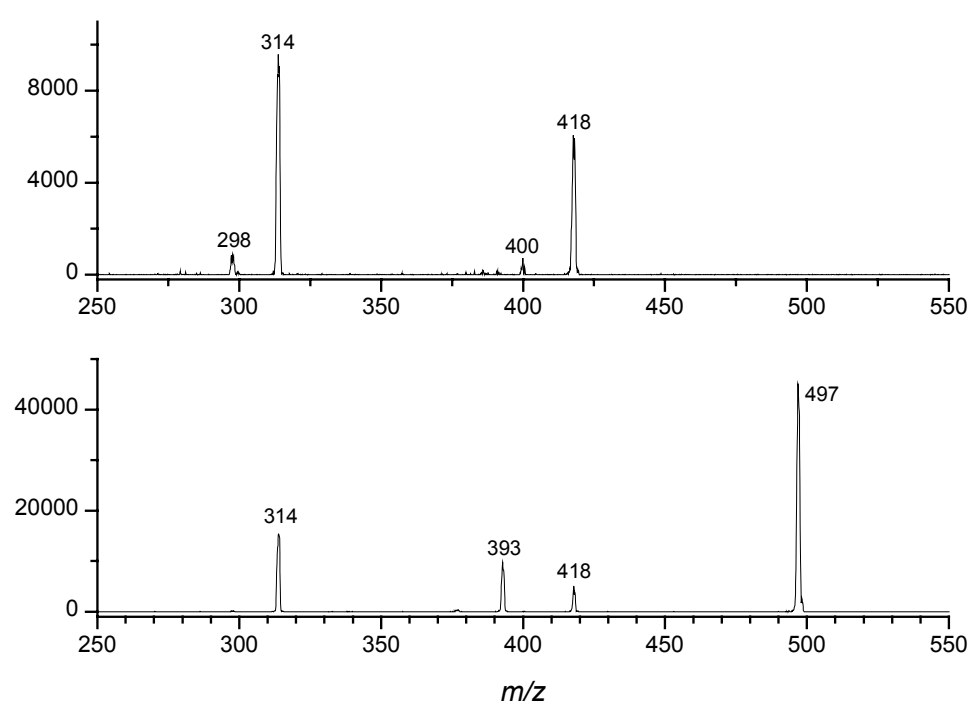
11. CID spectra of $8 \mathrm{~h}$ and $10 \mathrm{~h}\left(\mathrm{R}_{1}=\mathrm{H}, \mathrm{R}_{2}=(p-\mathrm{Me}) \mathrm{C}_{6} \mathrm{H}_{4}, \mathrm{R}_{3}=\mathrm{H}\right)$
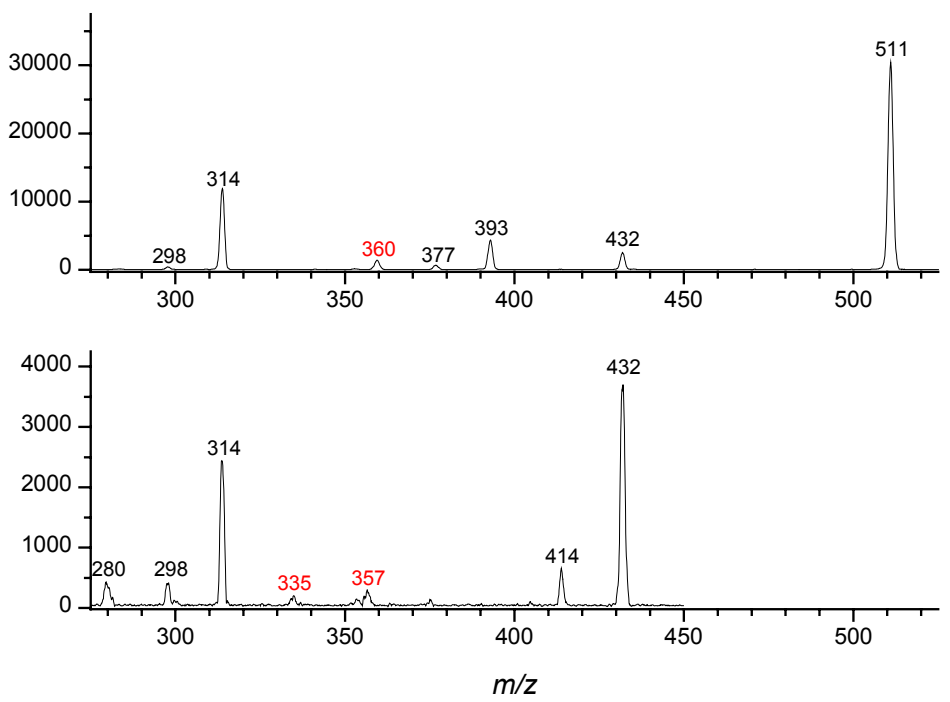

12. CID spectra of $8 \mathrm{i}$ and $10 \mathrm{i}\left(\mathrm{R}_{1}=\mathrm{H}, \mathrm{R}_{2}=\left(p-\mathrm{CF}_{3}\right) \mathrm{C}_{6} \mathrm{H}_{4}, \mathrm{R}_{3}=\mathrm{H}\right)$
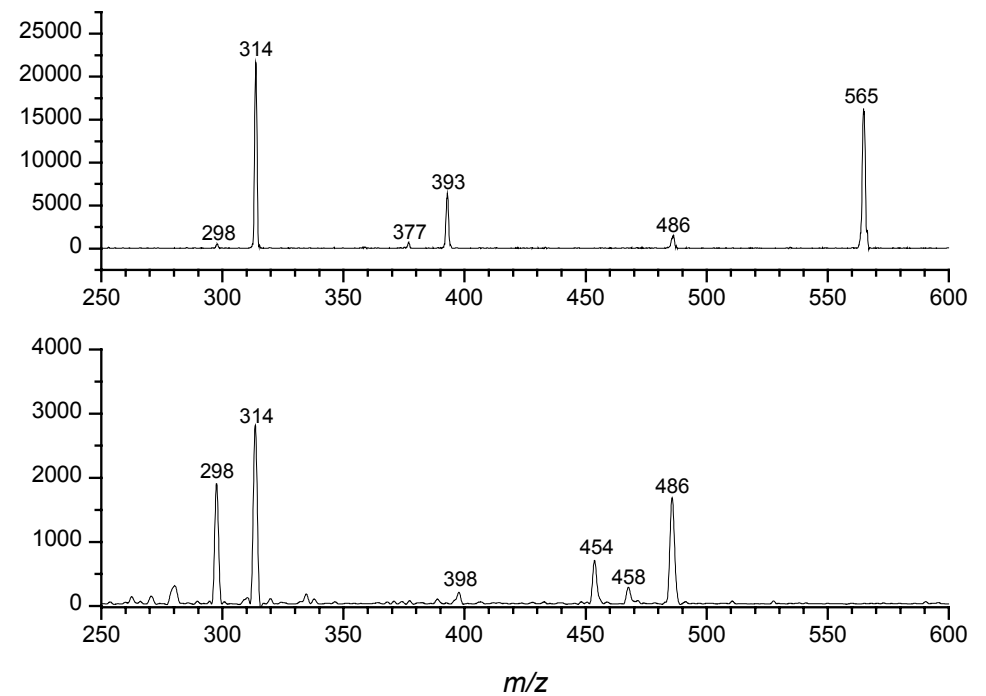
13. CID spectra of $8 j$ and $10 j\left(R_{1}=H, R_{2}=C F_{3}, R_{3}=H\right)$
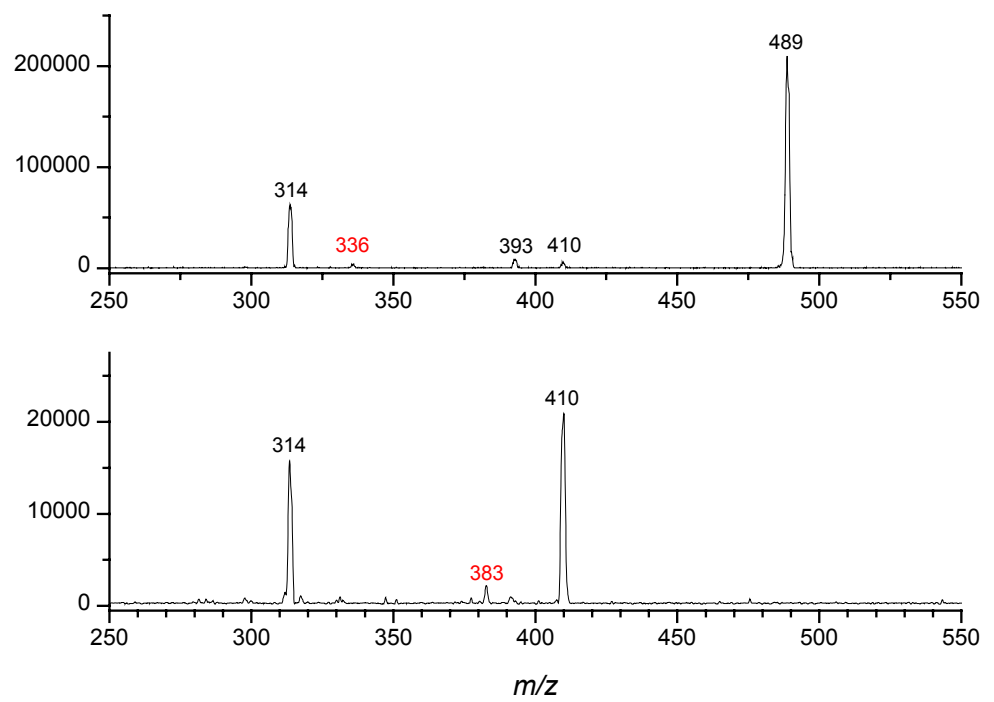
14. CID spectra of $8 \mathrm{k}$ and $10 \mathrm{k}\left(\mathrm{R}_{1}=\mathrm{H}, \mathrm{R}_{2}=\mathrm{Me}, \mathrm{R}_{3}=\mathrm{Me}\right.$, cis $)$
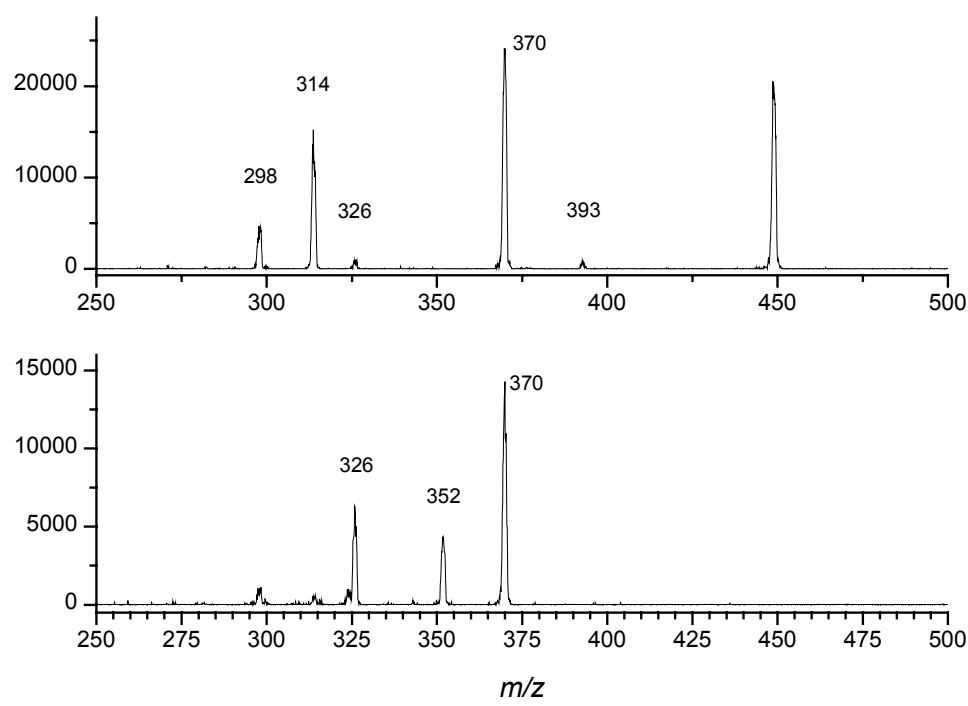

15. CID spectra of 81 and $101\left(\mathrm{R}_{1}=\mathrm{H}, \mathrm{R}_{2}=\mathrm{Me}, \mathrm{R}_{3}=\mathrm{Me}\right.$, trans $)$
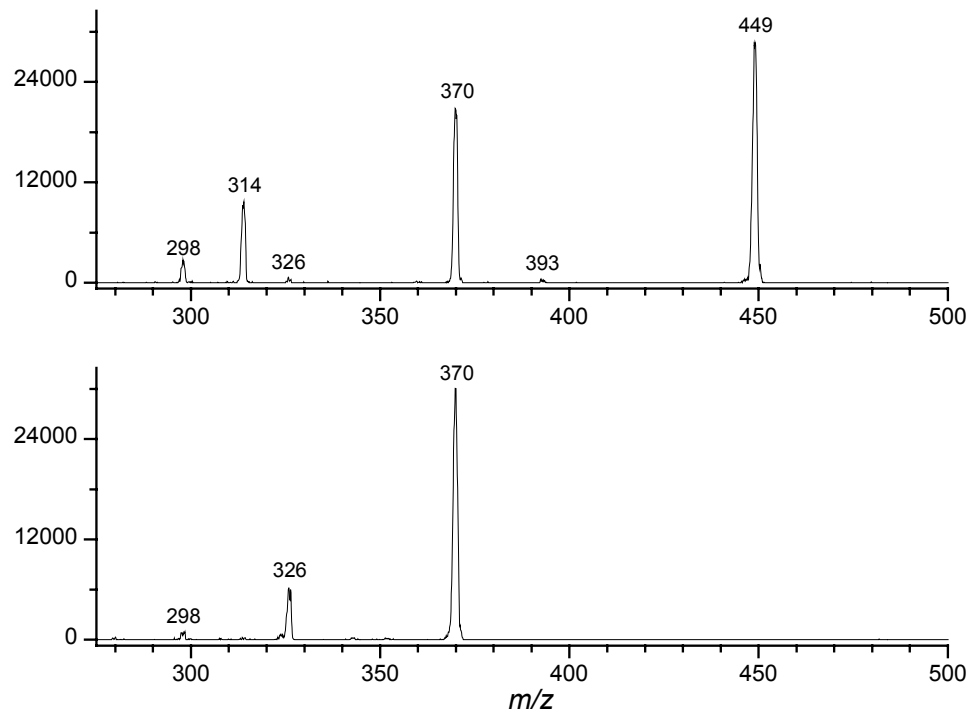
16. CID spectra of $8 \mathrm{~m}\left(\mathrm{R}_{1}=\mathrm{H}, \mathrm{R}_{2}=\mathrm{Ph}, \mathrm{R}_{3}=\mathrm{Ph}\right.$, trans $)$

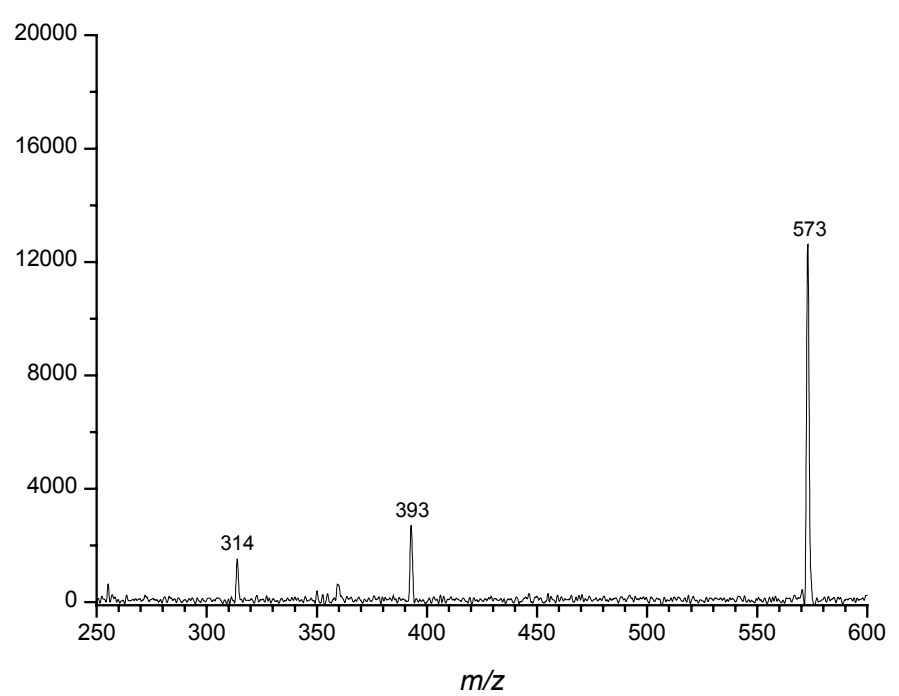

17. CID spectra of $8 \mathrm{n}$ and $10 \mathrm{n}\left(\mathrm{R}_{1}=\mathrm{H}, \mathrm{R}_{2}=p-\mathrm{OMeC}_{6} \mathrm{H}_{4}, \mathrm{R}_{3}=\mathrm{Me}\right)$
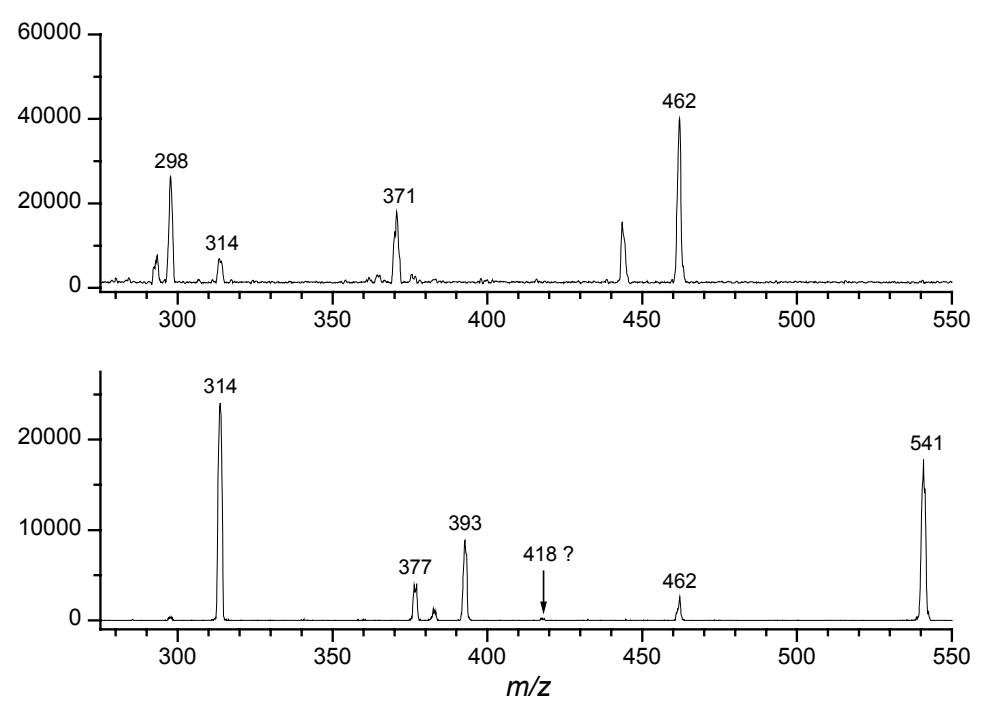
18. CID spectra of $8 \mathrm{o}$ and $10 \mathrm{o}\left(\mathrm{R}_{1}=\mathrm{H}, \mathrm{R}_{2}=\mathrm{CO}_{2} \mathrm{Et}, \mathrm{R}_{3}=\mathrm{CO}_{2} \mathrm{Et}\right)$
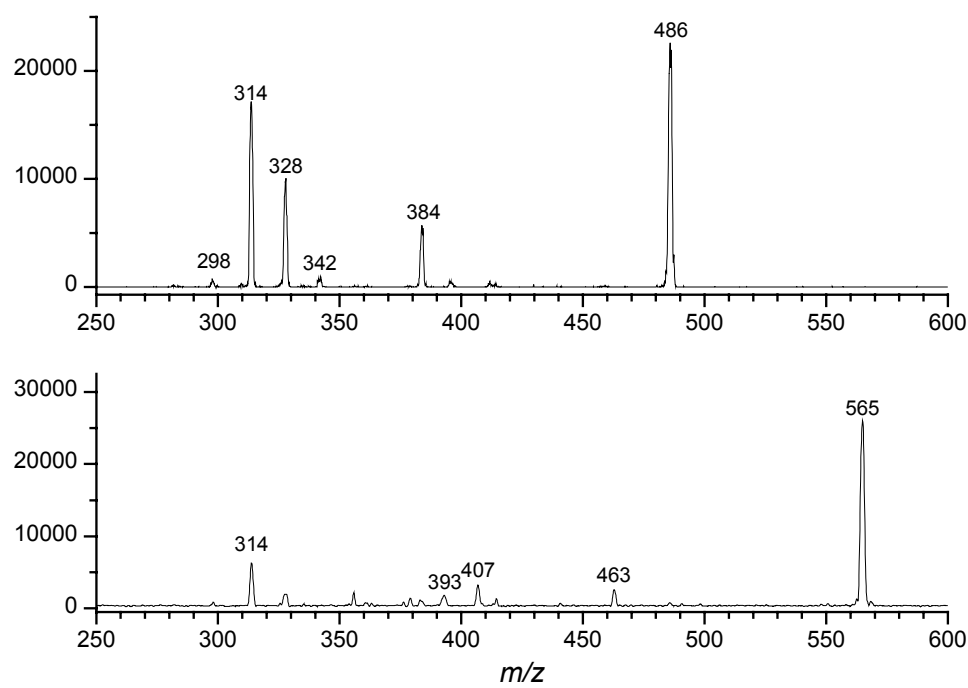
19. CID spectra of $8 \mathrm{q}, 8 \mathrm{p}, 8 \mathrm{~b}$ and $8 \mathrm{r}\left(\mathrm{R}_{1}=p-\mathrm{NMe}_{2}, p-\mathrm{Me}, \mathrm{H}, p-\mathrm{F} ; \mathrm{R}_{2}=\mathrm{Me}, \mathrm{R}_{3}=\mathrm{H}\right)$

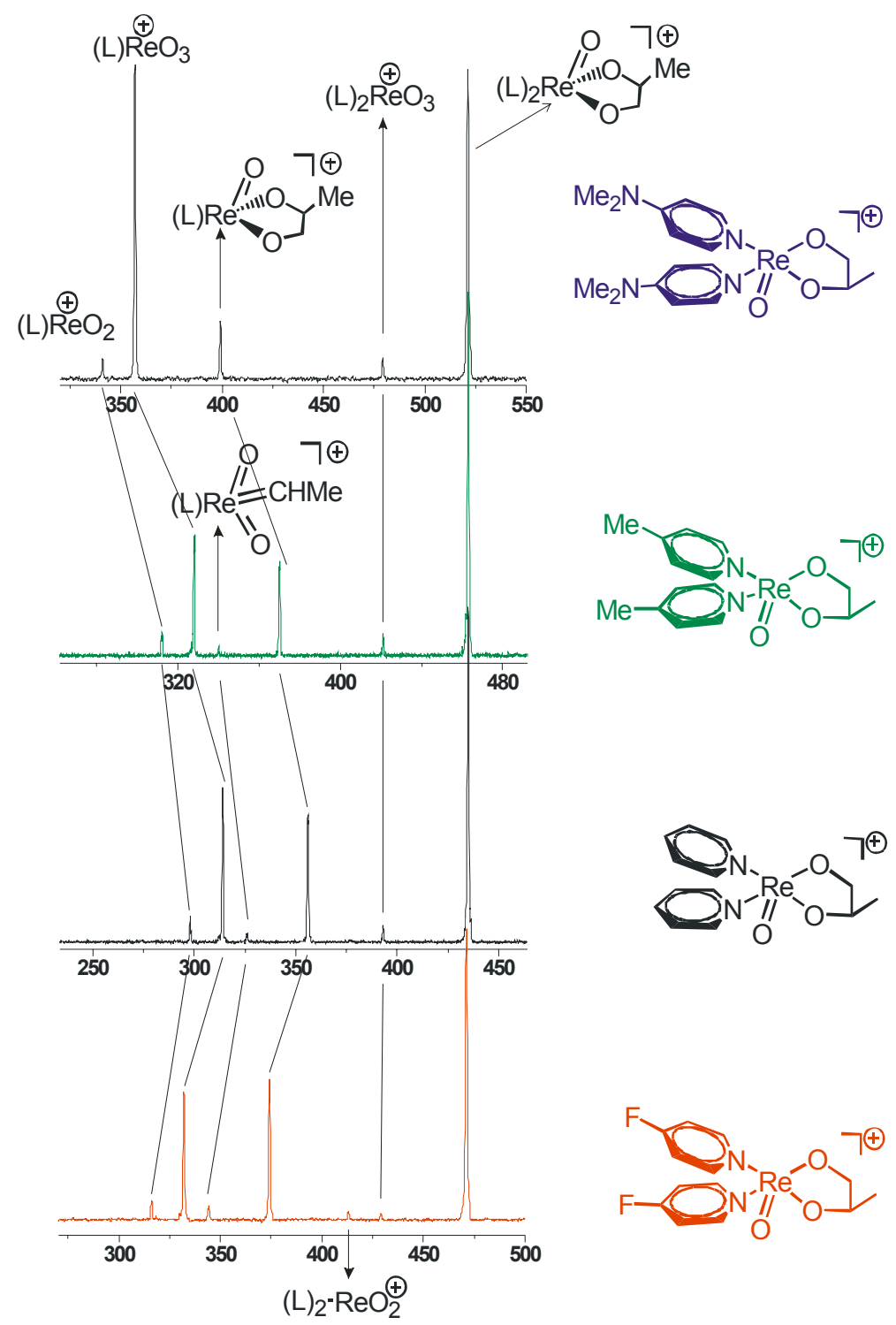


Part IV. NMR evidences for the clean formation of diolates in solution.

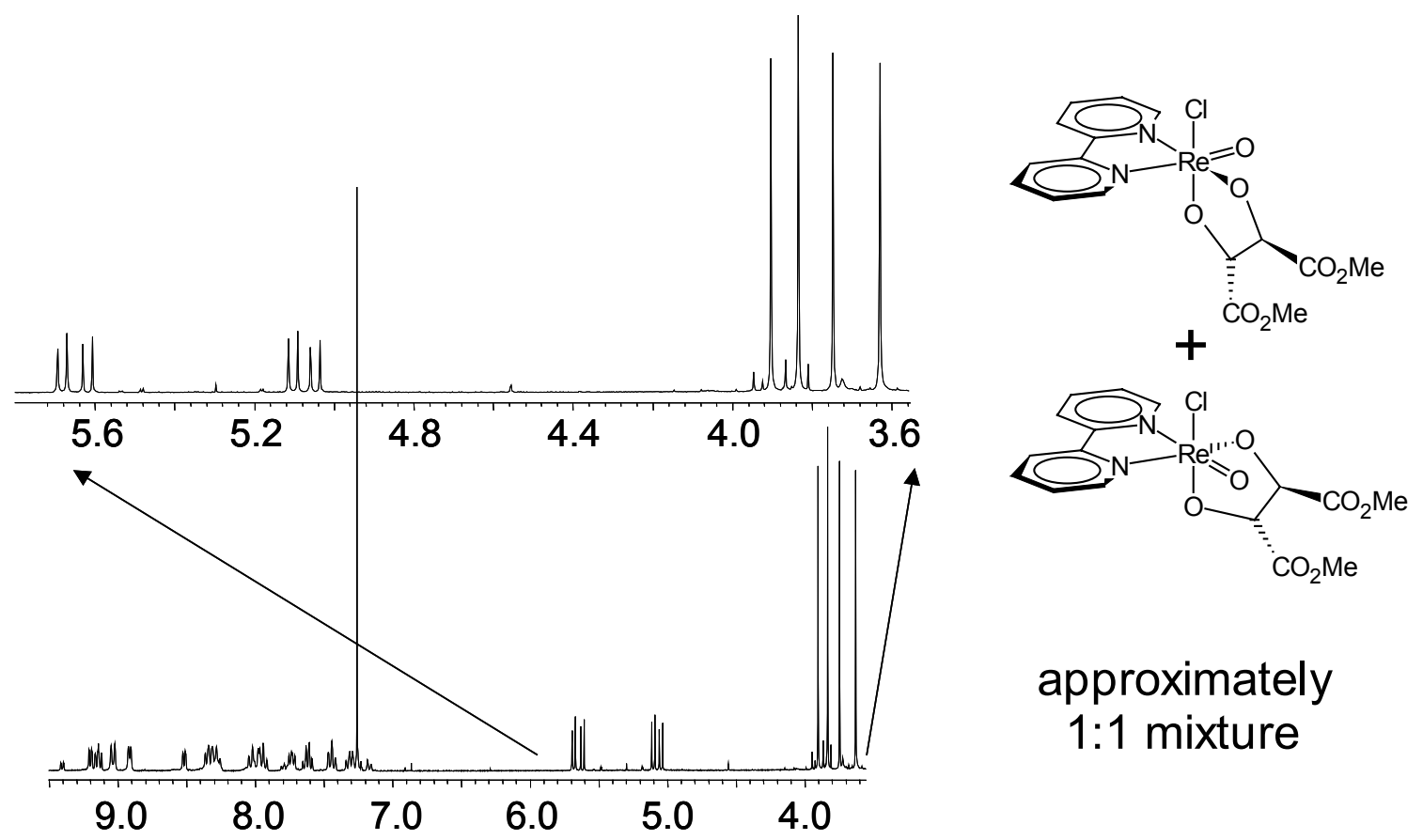

This is the ${ }^{1} \mathrm{H}$ nmr spectrum (Varian Gemini 300) of the solution made from

$\mathrm{ReOCl}_{3}\left(\mathrm{O}=\mathrm{PPh}_{3}\right)\left(\mathrm{Me}_{2} \mathrm{~S}\right), 2$,2'-bipyridine, and dimethyl tartrate in equimolar ratio in $\mathrm{CDCl}_{3}$. From the four methyl resonances and the four $\mathrm{C}-\mathrm{H} \alpha$ to the carboxyl groups, one sees that conversion was clean and quantitative.

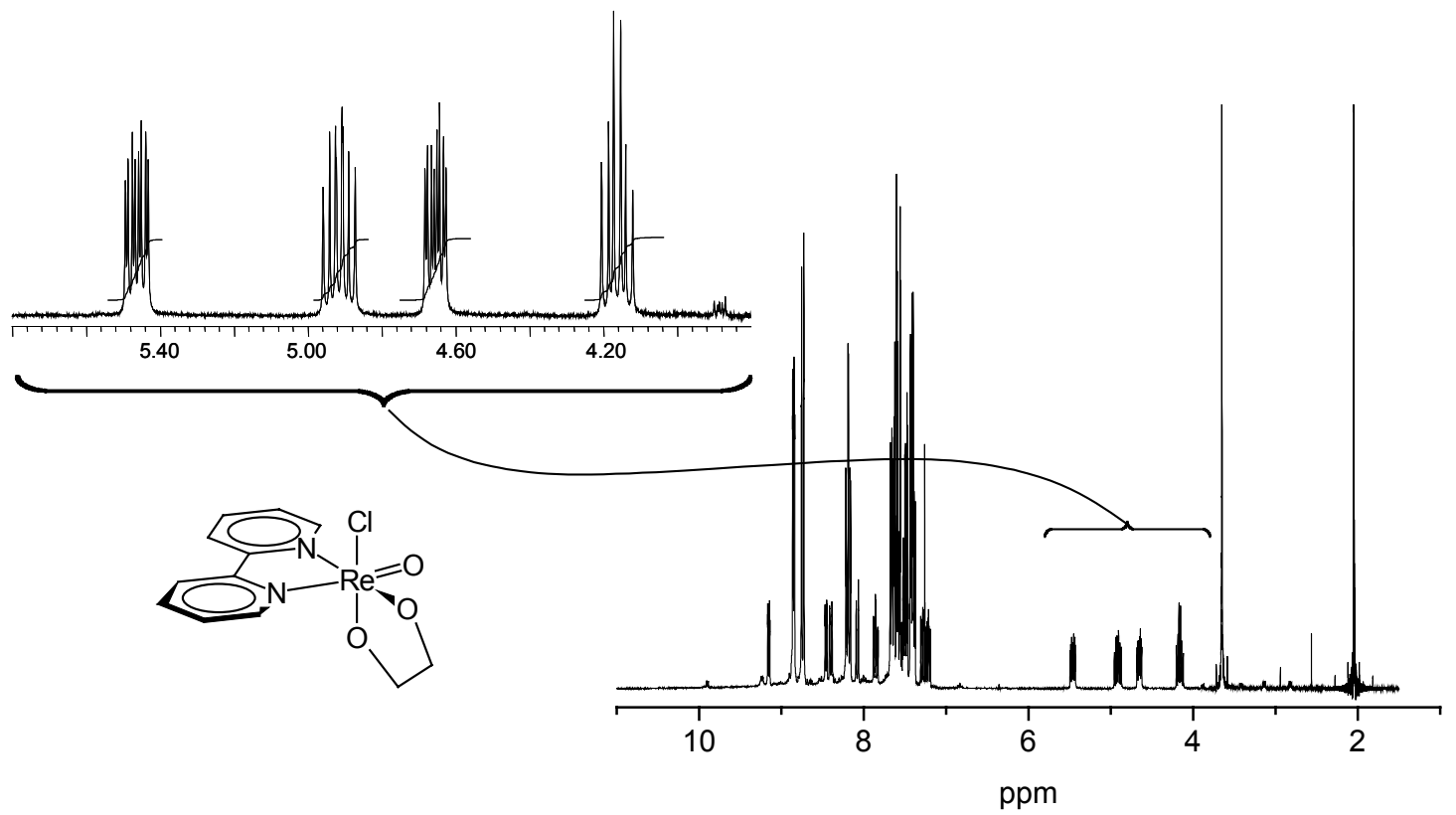

This is the ${ }^{1} \mathrm{H}$ nmr spectrum (Varian Gemini 300) of the material made by dissolving $\mathrm{ReOCl}_{3}\left(\mathrm{O}=\mathrm{PPh}_{3}\right)\left(\mathrm{Me}_{2} \mathrm{~S}\right), 2,2^{\prime}$ '-bipyridine, and ethylene glycol in near equimolar ratio in $\mathrm{CH}_{2} \mathrm{Cl}_{2}$, precipitation with hexane, and redissolution in $\mathrm{CDCl}_{3}$. In the structure analogous to Davison's complexes, all four methylene protons are magnetically inequivalent. 
Part V. (Abridged) Output files for the quantum chemical calculations

\section{Scheme 5}

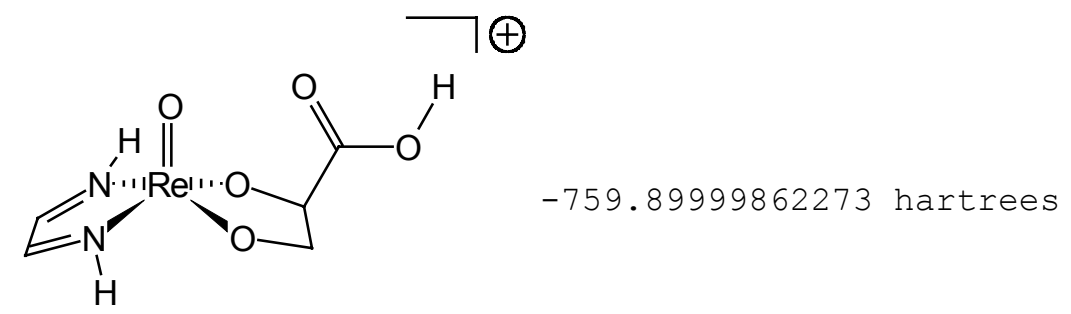

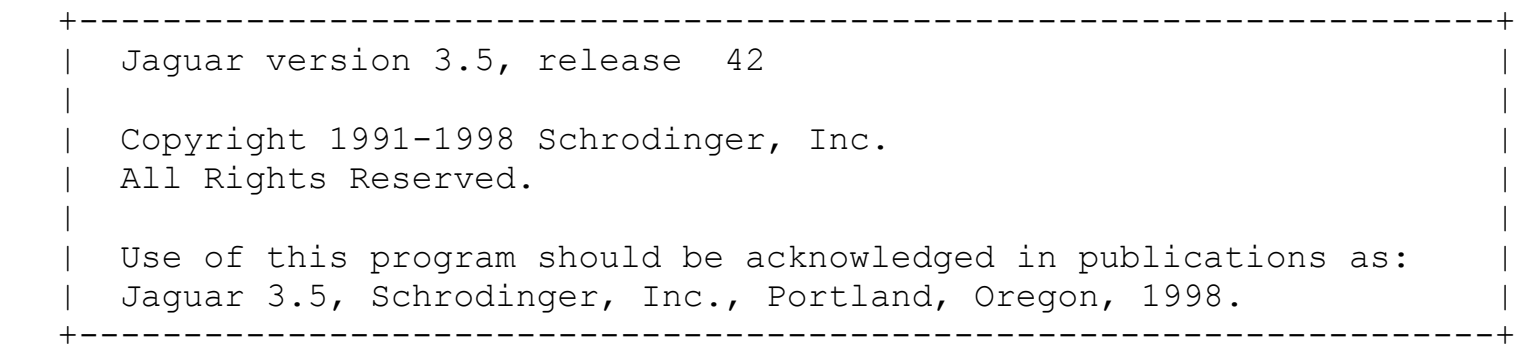

Job WF31322 started on CHENB091 at Sat Jan 10 10:18:38 2004

Jaguar version 3.5, release 42

Job name:

Task:

Stoichiometry: Molecular weight: Molecular charge: Spin multiplicity: Point Group:

SCF method:

Basis set:

Basis functions:

Total electrons:

Alpha electrons:

Beta electrons:

Total orbitals:

Core orbitals:

Open-shell orbitals:

occupied orbitals:

Shells:
WF31322

Geometry optimization

ReC5N2H 805

$363.00 \mathrm{amu}$

1

1

C1

B3LYP

$\mathrm{LACVP} * *$

230

106

53

53

245

53

0

53

Non-default options chosen:

SCF calculation type: DFT

$\mathrm{DFT}=$ Becke_3_Parameter/HF+Slater+Becke88+VWN+LYP (B3LYP)

Geometry will be optimized in redundant internal coordinates

Numerical 2nd derivatives will be computed

Vibrational frequencies and related properties will be computed

Molecular symmetry not used 


\begin{tabular}{|c|c|c|c|c|c|c|c|c|}
\hline ter & Energy & & Gmax & & Grms & & Dmax & Drms \\
\hline 1 & -759.877458 & * & 0.06393 & & 0.01617 & & 0.12590 & 0.03561 \\
\hline 2 & -759.894736 & & 0.03046 & - & 0.00638 & & 0.10417 & 0.03508 \\
\hline 3 & -759.897259 & . & 0.03166 & . & 0.00428 & & 0.11538 & 3073 \\
\hline 4 & -759.897174 & & 0.02032 & & 0.00370 & & 07088 & 02558 \\
\hline 5 & -759.896993 & - & 0.01504 & . & 0.00392 & & 6544 & 0.02495 \\
\hline 6 & -759.898092 & . & 0.00570 & . & 0.00130 & & 3778 & 436 \\
\hline 7 & -759.898072 & * & 0.00833 & & 0.00170 & & 2663 & 925 \\
\hline 8 & -759.898240 & . & 0.00172 & . & 0.00040 & & 0.01918 & 0.00476 \\
\hline 9 & -759.898278 & * & 0.00115 & . & 0.00033 & & & 466 \\
\hline 10 & -759.898242 & * & 0.00062 & . & 0.00023 & * & 2972 & 562 \\
\hline 11 & -759.898392 & . & 0.00238 & . & 0.00077 & & 0.12581 & 3563 \\
\hline 12 & -759.898743 & . & 0.00359 & . & 0.00101 & & & 560 \\
\hline$\star \star \quad r \in$ & estarting opt & in & ation fr & & step 1 & 2 & $\star \star$ & \\
\hline 12 & -759.898473 & . & 0.00359 & . & 0.00101 & & 0.12311 & 563 \\
\hline 14 & -759.8 & . & 0.00774 & . & 0.00188 & & 49 & 71 \\
\hline$x \in$ & estarting opt & in & ation $\mathrm{fr}$ & om & step 1 & 4 & $\star \star$ & \\
\hline 14 & -759.896991 & . & 0.00774 & . & 0.00188 & & 0.05 & 81 \\
\hline 16 & -759. & . & 0.00517 & . & 0.00 & & 19 & 91 \\
\hline 17 & -759. & - & 0.00532 & . & 145 & & 1331 & 47 \\
\hline 18 & -759.899 & & 0.00145 & . & 0.00044 & & 3299 & 398 \\
\hline 19 & -759.89 & . & 0.00142 & . & 0.00048 & & 563 & 85 \\
\hline 20 & -759.899877 & ${ }^{\circ}$ & 0.00363 & . & 0.00069 & & 0.09789 & 518 \\
\hline$\star \star x \in$ & estarting opt & & ation fr & & step 2 & 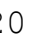 & $\star \star$ & \\
\hline 20 & -759.8 & . & 0.00363 & . & 0.00069 & & 0.05021 & 60 \\
\hline 22 & -759.89 & * & 0.00447 & . & 0.00121 & & 1557 & $\$ 30$ \\
\hline 23 & -759.89 & $\cdot$ & 0.00369 & . & 0.00090 & & 0772 & 322 \\
\hline 24 & -759.9 & . & 0.00080 & . & 0.00025 & * & 0.01765 & 446 \\
\hline 25 & -759.899985 & * & 0.00105 & - & 0.00029 & * & 0.01209 & 233 \\
\hline 26 & -759.90 & * & 0.00045 & . & 0.00015 & * & 0867 & 192 \\
\hline 27 & -759.900001 & * & 0.00051 & . & 0.00016 & * & 0.00781 & 165 \\
\hline 28 & -759.899990 & 夫 & 0.00030 & 夫 & 0.00009 & * & 0.00292 & 0.00082 \\
\hline 29 & -759.899996 & * & 0.00034 & * & 0.00008 & * & 0.00201 & 067 \\
\hline 30 & -759.899994 & $\#$ & 0.00020 & $\star$ & 0.00005 & $\#$ & 0.00530 & 131 \\
\hline
\end{tabular}

energy change:

gradient maximum:

gradient rms:

step size:

0.01106

displacement maximum:

displacement rms:

predicted energy change:

step: $\quad 1.1059 \mathrm{E}-02$
$1.8543 \mathrm{E}-06 \#(5.0000 \mathrm{E}-05)$
$2.0386 \mathrm{E}-04$ *
$5.1654 \mathrm{E}-05 \#(4.5000 \mathrm{E}-04)$

trust radius:

$5.2964 \mathrm{E}-03 \cdot(1.8000 \mathrm{E}-03)$

$1.3125 \mathrm{E}-03$. (1.2000E-03)

$-7.2971 \mathrm{E}-07$ geom step:

1.1059E-02 full

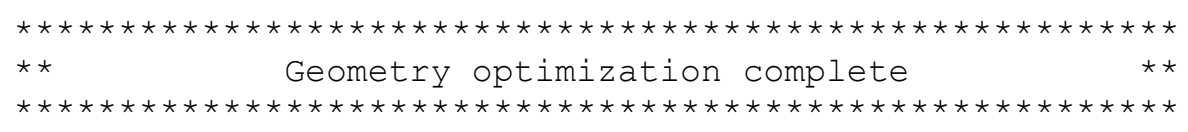

center of mass moved by:
$\mathrm{x}$ :
$0.0000 \mathrm{E}+00$
$\mathrm{Y}:$
$0.0000 \mathrm{E}+00$
$\mathrm{z}:$
$0.0000 \mathrm{E}+00$

final geometry:

\begin{tabular}{lcrr} 
atom & $x$ & $y$ & \multicolumn{1}{c}{$z$} \\
Re1 & 0.9516914368 & -0.4070752204 & 0.8260039884 \\
N2 & 1.5169370088 & 1.2579907525 & -0.2577820310
\end{tabular}




$\begin{array}{lr}\text { C10 } & 1.0912037492 \\ \text { C1 } & 0.6731100244 \\ \text { N1 } & 0.8040711610 \\ \text { O1 } & 2.2481931931 \\ \text { O2 } & -0.6629781398 \\ \text { O3 } & 0.0486238961 \\ \text { C4 } & -1.3421679152 \\ \text { C7 } & -1.1650260940 \\ \text { H21 } & -1.0805537109 \\ \text { H4 } & -1.9888767458 \\ \text { H12 } & 0.6745118218 \\ \text { H20 } & 0.3687470800 \\ \text { H22 } & 1.1381828269 \\ \text { H26 } & 1.9702456528 \\ \text { H2 } & -2.3965118918 \\ \text { C2 } & -0.7529424426 \\ \text { O4 } & 0.3105105765 \\ \text { O5 } & -1.5711754706 \\ \text { H3 } & -1.1558934422\end{array}$

1.2585981240

$-0.0367974721$

$-1.0188123939$

$-1.0956910916$

$-1.3948495800$

0.8212328491

$-1.1185255909$

0.3827914215

0.5975794069

0.9696851787

$-1.9759961937$

$-0.1939732079$

2. 1374578877

2.0979369320

$-1.3795412134$

$-1.9906984533$

$-2.5568636562$

$-1.9956734025$

$-2.5321584947$
-1.4963155065
-1.9931804751
-1.1340740999
1.6214765501
1.0896520728
1.9636020140
2.3220362015
2.6118338417
3.6808259464
2.1946856876
-1.4641692539
-3.0244139931
-2.1337760887
0.1033696838
2.1897630991
3.4347151058
3.3668655542
4.4977906598
5.1960702436 calculating forces at perturbed geometry calculating forces at perturbed geometry calculating forces at perturbed geometry calculating forces at perturbed geometry calculating forces at perturbed geometry calculating forces at perturbed geometry calculating forces at perturbed geometry calculating forces at perturbed geometry calculating forces at perturbed geometry calculating forces at perturbed geometry calculating forces at perturbed geometry calculating forces at perturbed geometry calculating forces at perturbed geometry calculating forces at perturbed geometry calculating forces at perturbed geometry calculating forces at perturbed geometry calculating forces at perturbed geometry calculating forces at perturbed geometry calculating forces at perturbed geometry calculating forces at perturbed geometry calculating forces at perturbed geometry calculating forces at perturbed geometry calculating forces at perturbed geometry calculating forces at perturbed geometry calculating forces at perturbed geometry calculating forces at perturbed geometry calculating forces at perturbed geometry calculating forces at perturbed geometry calculating forces at perturbed geometry calculating forces at perturbed geometry calculating forces at perturbed geometry calculating forces at perturbed geometry calculating forces at perturbed geometry calculating forces at perturbed geometry calculating forces at perturbed geometry calculating forces at perturbed geometry calculating forces at perturbed geometry calculating forces at perturbed geometry calculating forces at perturbed geometry calculating forces at perturbed geometry
1 of $126 \ldots$

2 of $126 \ldots$

3 of $126 \ldots$

4 of $126 \ldots$

5 of $126 \ldots$

6 of $126 \ldots$

7 of $126 \ldots$

8 of $126 \ldots$

9 of $126 \ldots$ 10 of $126 \ldots$ 11 of $126 \ldots$ 12 of $126 \ldots$ 13 of $126 \ldots$ 14 of $126 \ldots$ 15 of $126 \ldots$ 16 of $126 \ldots$ 17 of $126 \ldots$ 18 of $126 \ldots$ 19 of $126 \ldots$ 20 of $126 \ldots$ 21 of $126 \ldots$ 22 of $126 \ldots$ 23 of $126 \ldots$ 24 of $126 \ldots$ 25 of $126 \ldots$ 26 of $126 \ldots$ 27 of $126 \ldots$ 28 of $126 \ldots$ 29 of $126 \ldots$ 30 of $126 \ldots$ 31 of $126 \ldots$ 32 of $126 \ldots$ 33 of $126 \ldots$ 34 of $126 \ldots$ 35 of $126 \ldots$ 36 of $126 \ldots$ 37 of $126 \ldots$ 38 of $126 \ldots$ 39 of $126 \ldots$ 40 of $126 \ldots$ 
calculating forces at perturbed geometry calculating forces at perturbed geometry calculating forces at perturbed geometry calculating forces at perturbed geometry calculating forces at perturbed geometry calculating forces at perturbed geometry calculating forces at perturbed geometry calculating forces at perturbed geometry calculating forces at perturbed geometry calculating forces at perturbed geometry calculating forces at perturbed geometry calculating forces at perturbed geometry calculating forces at perturbed geometry calculating forces at perturbed geometry calculating forces at perturbed geometry calculating forces at perturbed geometry calculating forces at perturbed geometry calculating forces at perturbed geometry calculating forces at perturbed geometry calculating forces at perturbed geometry calculating forces at perturbed geometry calculating forces at perturbed geometry calculating forces at perturbed geometry calculating forces at perturbed geometry calculating forces at perturbed geometry calculating forces at perturbed geometry calculating forces at perturbed geometry calculating forces at perturbed geometry calculating forces at perturbed geometry calculating forces at perturbed geometry calculating forces at perturbed geometry calculating forces at perturbed geometry calculating forces at perturbed geometry calculating forces at perturbed geometry calculating forces at perturbed geometry calculating forces at perturbed geometry calculating forces at perturbed geometry calculating forces at perturbed geometry calculating forces at perturbed geometry calculating forces at perturbed geometry calculating forces at perturbed geometry calculating forces at perturbed geometry calculating forces at perturbed geometry calculating forces at perturbed geometry calculating forces at perturbed geometry calculating forces at perturbed geometry calculating forces at perturbed geometry calculating forces at perturbed geometry calculating forces at perturbed geometry calculating forces at perturbed geometry calculating forces at perturbed geometry calculating forces at perturbed geometry calculating forces at perturbed geometry calculating forces at perturbed geometry calculating forces at perturbed geometry calculating forces at perturbed geometry calculating forces at perturbed geometry calculating forces at perturbed geometry calculating forces at perturbed geometry calculating forces at perturbed geometry calculating forces at perturbed geometry
41 of $126 \ldots$ 42 of $126 \ldots$ 43 of $126 \ldots$ 44 of $126 \ldots$ 45 of $126 \ldots$ 46 of $126 \ldots$ 47 of $126 \ldots$ 48 of $126 \ldots$ 49 of $126 \ldots$ 50 of $126 \ldots$ 51 of $126 \ldots$ 52 of $126 \ldots$ 53 of $126 \ldots$ 54 of $126 \ldots$ 55 of $126 \ldots$ 56 of $126 \ldots$ 57 of $126 \ldots$ 58 of $126 \ldots$ 59 of $126 \ldots$ 60 of $126 \ldots$ 61 of $126 \ldots$ 62 of $126 \ldots$ 63 of $126 \ldots$ 64 of $126 \ldots$ 65 of $126 \ldots$ 66 of $126 \ldots$ 67 of $126 \ldots$ 68 of $126 \ldots$ 69 of $126 \ldots$ 70 of $126 \ldots$ 71 of $126 \ldots$ 72 of $126 \ldots$ 73 of $126 \ldots$ 74 of $126 \ldots$ 75 of $126 \ldots$ 76 of $126 \ldots$ 77 of $126 \ldots$ 78 of $126 \ldots$ 79 of $126 \ldots$ 80 of $126 \ldots$ 81 of $126 \ldots$ 82 of $126 \ldots$ 83 of $126 \ldots$ 84 of $126 \ldots$ 85 of $126 \ldots$ 86 of $126 \ldots$ 87 of $126 \ldots$ 88 of $126 \ldots$ 89 of $126 \ldots$ 90 of $126 \ldots$ 91 of $126 \ldots$ 92 of $126 \ldots$ 93 of $126 \ldots$ 94 of $126 \ldots$ 95 of $126 \ldots$ 96 of $126 \ldots$ 97 of $126 \ldots$ 98 of $126 \ldots$ 99 of $126 \ldots$ 100 of $126 \ldots$ 101 of $126 \ldots$ 
calculating forces at perturbed geometry 102 of $126 \ldots$ calculating forces at perturbed geometry 103 of $126 \ldots$ calculating forces at perturbed geometry 104 of 126... calculating forces at perturbed geometry 105 of $126 \ldots$ calculating forces at perturbed geometry 106 of $126 \ldots$ calculating forces at perturbed geometry 107 of $126 \ldots$ calculating forces at perturbed geometry 108 of $126 \ldots$ calculating forces at perturbed geometry 109 of $126 \ldots$ calculating forces at perturbed geometry 110 of $126 \ldots$ calculating forces at perturbed geometry 111 of $126 \ldots$ calculating forces at perturbed geometry 112 of $126 \ldots$ calculating forces at perturbed geometry 113 of $126 \ldots$ calculating forces at perturbed geometry 114 of $126 \ldots$ calculating forces at perturbed geometry 115 of $126 \ldots$ calculating forces at perturbed geometry 116 of $126 \ldots$ calculating forces at perturbed geometry 117 of $126 \ldots$ calculating forces at perturbed geometry 118 of $126 \ldots$ calculating forces at perturbed geometry 119 of $126 \ldots$ calculating forces at perturbed geometry 120 of $126 \ldots$ calculating forces at perturbed geometry 121 of $126 \ldots$ calculating forces at perturbed geometry 122 of $126 \ldots$ calculating forces at perturbed geometry 123 of $126 \ldots$ calculating forces at perturbed geometry 124 of $126 \ldots$ calculating forces at perturbed geometry 125 of $126 \ldots$ calculating forces at perturbed geometry 126 of $126 \ldots$

numerical derivatives complete;

calculation at normal geometry follows...

start of program freq

harmonic frequencies in $\mathrm{cm}^{\wedge}-1$, IR intensities in $\mathrm{km} / \mathrm{mol}$, and normal modes:

\begin{tabular}{|c|c|c|c|c|c|c|c|c|}
\hline frequ & cies & 49.74 & 59.56 & 91.49 & 101.24 & 133.75 & 179.38 & 220.8 \\
\hline inten & ties & 3.54 & 1.37 & 7.83 & 4.44 & 2.49 & 3.39 & \\
\hline Re1 & $\mathrm{X}$ & -0.01037 & -0.01102 & -0.01621 & -0.01600 & 0.01463 & 0.00130 & -0.0023 \\
\hline $\operatorname{Re} 1$ & Y & -0.00066 & -0.01716 & 0.00028 & 0.01458 & 0.00053 & -0.02176 & \\
\hline Re1 & Z & 0.00103 & -0.00758 & 0.01252 & -0.00869 & 0.00959 & -0.00680 & \\
\hline N2 & X & 0.01475 & 0.05293 & 0.01640 & 0.07024 & 0.07644 & -0.03055 & -0 . \\
\hline N2 & $Y$ & 0.00609 & -0.00230 & -0.02229 & -0.02277 & -0.04863 & 0.00821 & -0 . \\
\hline N2 & Z & 0.02125 & 0.04698 & -0.00014 & -0.02872 & -0.007 & 0.04100 & \\
\hline $\mathrm{C} 10$ & $\mathrm{X}$ & 0.04105 & 0.05967 & 0.05204 & 0.11609 & -0.00495 & -0.06375 & \\
\hline $\mathrm{C} 10$ & Y & 0.01358 & 0.05362 & -0.04274 & -0.02184 & -0.01465 & 0.03712 & \\
\hline $\mathrm{C} 10$ & Z & 0.012 & 0.04589 & -0.01299 & -0.04404 & & & -0 \\
\hline $\mathrm{C} 1$ & $\mathrm{X}$ & 0.04742 & 0.02528 & 0.04275 & 0.05162 & -0.123 & 0. & \\
\hline $\mathrm{C} 1$ & Y & 0.01735 & 0.08140 & -0.04496 & -0.00634 & 0.014 & 0.026 & \\
\hline $\mathrm{C} 1$ & Z & -0.00319 & 0 & 0.001 & -0.03100 & & & -0 . \\
\hline N1 & X & 0.02145 & -0.02467 & 0.01701 & -0.03241 & -0.102 & 0 . & -0 . \\
\hline N1 & $Y$ & 0.0 & 0.04851 & -0.03062 & 0.00093 & -0.003 & 0. & \\
\hline N1 & Z & -0.00558 & -0.02877 & 0.02143 & -0.01028 & 0 & -0.0 & -0 \\
\hline 01 & X & -0.0 & -0 & -0.028 & -0.02046 & 0.01 & -0.0 & \\
\hline 01 & $Y$ & 0.0 & -0.02617 & -0.01508 & 0.03822 & 0.04 & 0 & \\
\hline 01 & Z & 0.02559 & -0.0 & 0.01859 & 0.01926 & 0.05 & 0 & -0 . \\
\hline 02 & $\mathrm{X}$ & -0.0 & -0.0 & -0.05817 & 0.01471 & 0.01 & -0.0 & -0 . \\
\hline 02 & $Y$ & -0.01 & -0.03484 & 0.05313 & -0.01801 & -0.01 & 0.0 & -0 . \\
\hline 02 & Z & -0.01220 & 0.0 & -0.0292 & 0.04214 & -0.00 & 0.0 & \\
\hline 03 & $\mathrm{X}$ & -0.03420 & -0.02613 & 0.0047 & -0.02889 & -0.04276 & -0.03015 & -0 . \\
\hline 03 & $Y$ & -0.00473 & -0.0 & 0.0129 & 0.03207 & & 0.05572 & -0 . \\
\hline 03 & Z & -0.01379 & -0.02147 & 0.0189 & -0.04827 & -0.065 & -0.11 & 0 \\
\hline $\mathrm{C} 4$ & $\mathrm{X}$ & 0.00135 & -0.0 & -0.02108 & 0.02593 & -0.00371 & 0.0 & -0 . \\
\hline C4 & $\mathrm{Y}$ & -0.02430 & -0.0 & 0.03273 & -0.01240 & -0.00285 & 0.0 & 0 \\
\hline
\end{tabular}




\begin{tabular}{|c|c|c|c|c|c|c|c|c|}
\hline 4 & Z & -0.00858 & 0.00153 & -0.00563 & 0.04724 & -0.02577 & 0.01969 & -0.00175 \\
\hline C7 & $\mathrm{x}$ & -0.03958 & -0.03947 & -0.00565 & 0.03925 & -0.03253 & 0.05986 & -0.00134 \\
\hline C7 & $\mathrm{Y}$ & -0.01785 & -0.01172 & 0.02795 & -0.00929 & 0.00663 & 0.04311 & 0.00369 \\
\hline C7 & z & -0.02994 & -0.04253 & 0.00802 & 0.05829 & -0.05222 & 0.03474 & 0.02031 \\
\hline H21 & $\mathrm{x}$ & -0.06502 & -0.07378 & -0.01102 & 0.13897 & -0.03334 & 0.20645 & 0.02078 \\
\hline H21 & $\mathrm{Y}$ & 0.00028 & 0.02655 & 0.01827 & -0.02680 & 0.03002 & 0.01103 & -0.01549 \\
\hline H21 & z & -0.03188 & -0.04743 & 0.01067 & 0.05405 & -0.05696 & 0.03040 & 0.02243 \\
\hline H4 & $\mathrm{x}$ & -0.04280 & -0.03677 & 0.00299 & -0.00748 & -0.04455 & 0.00895 & 0.00584 \\
\hline H4 & $\mathrm{Y}$ & -0.04238 & -0.03972 & 0.03903 & -0.01164 & -0.01846 & 0.06425 & 0.03030 \\
\hline H4 & z & -0.05791 & -0.08732 & 0.00613 & 0.14894 & -0.06263 & 0.16671 & 0.04374 \\
\hline H12 & $\mathrm{x}$ & 0.02419 & -0.04636 & 0.01327 & -0.07944 & -0.19965 & 0.11677 & \\
\hline $\mathrm{H} 12$ & $\mathrm{Y}$ & 0.01456 & 0.06451 & -0.03622 & 0.00250 & 0.00732 & 0.00964 & 0.01481 \\
\hline $\mathrm{H} 12$ & Z & -0.01494 & -0.06693 & 0.03970 & 0.00383 & 0.02518 & -0.06226 & -0.03096 \\
\hline H2O & $\mathrm{x}$ & 0.07015 & 0.03644 & 0.06052 & 0.07145 & -0.22651 & 0.06846 & \\
\hline H2O & $\mathrm{Y}$ & 0.02427 & 0.12401 & -0.05917 & -0.00792 & 0.04363 & 0.04162 & \\
\hline H2O & z & -0.01089 & -0.00916 & -0.00149 & -0.03692 & 0.06984 & -0.01026 & -0.04673 \\
\hline H22 & $\mathrm{x}$ & 0.05618 & 8906 & 8365 & 0.19041 & 0.01133 & -0.14622 & \\
\hline H22 & $\mathrm{Y}$ & & 0.0 & -0.0 & -0.03771 & -0.0 & & \\
\hline H22 & Z & .788 & 7464 & -0.02931 & -0.06041 & 0.02027 & 0.09388 & -0.00917 \\
\hline H 26 & $\mathrm{x}$ & 0.00855 & 0.0 & 2633 & 0.11204 & 0.16475 & 10963 & -0 \\
\hline H26 & $\mathrm{Y}$ & 343 & -0.0 & -0. & 4296 & -0 . & & -0 \\
\hline H 26 & Z & 3526 & 0.0 & -0.0 & -0.03416 & -0.03692 & 8572 & 3796 \\
\hline H2 & $\mathrm{x}$ & 0.00640 & 0.0 & -0.02505 & 0.02096 & 0.00021 & 0142 & -0 . \\
\hline $\mathrm{H} 2$ & Y & -0.0 & -0.0 & 0 & & -0 . & & \\
\hline H2 & Z & 439 & 0. & 0. & 4772 & -0 . & 3147 & -0 \\
\hline $\mathrm{C} 2$ & $\mathrm{x}$ & .708 & 0. & 0. & 0.00506 & 179 & -0 . & -0 \\
\hline $\mathrm{C} 2$ & $\mathrm{Y}$ & -0.0 & 0.0 & 0.0 & -0.0 & -0 . & & \\
\hline $\mathrm{C} 2$ & Z & -0. & 0. & -0.0 & 3683 & 2685 & -0 & -0 \\
\hline 04 & $\mathrm{x}$ & -0.0 & 0.0 & 0. & 0152 & -0 . & -0 & -0 \\
\hline 04 & $\mathrm{Y}$ & -0 . & 0. & 0 . & -0 . & -0 & & \\
\hline 04 & Z & -0.06049 & 0.0 & -0.10341 & 0.04074 & -0.04604 & -0.02354 & -0 \\
\hline 05 & $\mathrm{X}$ & 0.11179 & 0.0 & 0.07589 & -0.01477 & 0.01216 & 0.00686 & 0040 \\
\hline 05 & $\mathrm{Y}$ & & 0. & -0 & -0.0 & -0 . & -0 . & \\
\hline 05 & Z & 152 & 0. & -0.0 & 0.02261 & -0.02109 & 330 & 474 \\
\hline H3 & $\mathrm{x}$ & 0.11738 & 0.0 & 0.12643 & -0.03022 & 0.01595 & 0.01013 & 0.02331 \\
\hline H3 & Y & & 0 . & -0 & -0.0 & -0 . & -0 & \\
\hline H3 & z & 0.05354 & 0.0 & -0.0 & 0.01549 & -0.0 & -0 & \\
\hline & & & & & & & & \\
\hline & & & & & & & .19 & 2 \\
\hline Re1 & $\mathrm{x}$ & -0.00727 & 0.00864 & 0.00183 & -0.01449 & 0.01835 & -0.01148 & -0.00616 \\
\hline Re1 & $\mathrm{Y}$ & & -0.0 & & -0.00419 & -0.00241 & -0 & -0 \\
\hline Re1 & z & -0 & & & & -0 . & & -0 \\
\hline N2 & $\mathrm{x}$ & 0.08403 & -0.05357 & 0.00927 & -0.02315 & 0.00414 & 0.08430 & -0.02092 \\
\hline N2 & $\mathrm{Y}$ & -0.02550 & & & -0.01149 & -0.00638 & -0 & 0.11856 \\
\hline N2 & Z & & -0.0 & & & -0 . & & -0 \\
\hline C10 & $\mathrm{x}$ & -0.02604 & 0.00189 & 0.00004 & 0.00838 & -0.00029 & -0.04862 & 0.07805 \\
\hline C10 & $\mathrm{Y}$ & -0.01756 & 0.01995 & -0.0 & -0.01470 & 0.00379 & 3628 & 0.03599 \\
\hline & Z & & -0.0 & & & -0.0 & -0 . & \\
\hline $\mathrm{C} 1$ & $\mathrm{x}$ & -0.00774 & 0.0 & 0.0 & 0.01470 & -0.01580 & -0.04036 & -0.06396 \\
\hline $\mathrm{C} 1$ & $\mathrm{Y}$ & -0.02048 & 0.00554 & -0.02641 & -0.01289 & 0.00901 & 0.03326 & 0.02347 \\
\hline $\mathrm{C} 1$ & Z & & -0.0 & & & -0.0 & & \\
\hline N1 & $\mathrm{x}$ & 000 & -0.0 & 0. & -0.02400 & 1939 & 542 & 0.07047 \\
\hline N1 & $\mathrm{Y}$ & -0.05018 & 0.03514 & -0.01500 & -0.01642 & 0.00503 & 0.03335 & 0.02977 \\
\hline N1 & Z & & -0.0 & & & -0.0 & -0.06721 & \\
\hline 01 & $\mathrm{x}$ & & & & & & 1663 & \\
\hline 01 & $\mathrm{Y}$ & 0.02523 & -0.08017 & -0.09443 & 0.03392 & 0.00683 & 0.00134 & 0.02607 \\
\hline 01 & Z & -0.07155 & -0.04299 & -0.11860 & -0.03174 & 0.00511 & -0.01776 & \\
\hline $\mathrm{O} 2$ & $\mathrm{x}$ & -0.00026 & & & -0.03115 & -0.11845 & 0.01429 & -0.0223 \\
\hline $\mathrm{O} 2$ & $\mathrm{Y}$ & 0.00610 & -0.00170 & -0.00371 & 0.03373 & 0.12971 & 0.00207 & -0.01713 \\
\hline 02 & Z & 0.04630 & 0.04508 & -0.01600 & -0.03932 & -0.06681 & 0.03992 & -0.01300 \\
\hline
\end{tabular}




\begin{tabular}{|c|c|c|c|c|c|c|c|c|}
\hline 03 & $\mathrm{x}$ & -0.00107 & -0.01888 & -0.09007 & 0.02977 & -0.06111 & -0.02282 & 0.01150 \\
\hline 03 & $\mathrm{Y}$ & -0.00376 & 0.04845 & 0.03500 & 0.04260 & -0.02594 & -0.00372 & -0.02135 \\
\hline 03 & Z & 0.00633 & -0.05686 & -0.08566 & -0.00997 & -0.01400 & 0.00732 & -0.0171 \\
\hline C4 & $\mathrm{X}$ & -0.01409 & 0.00815 & -0.00361 & 0.07381 & -0.03831 & 0.01164 & 0.00384 \\
\hline C4 & $\mathrm{Y}$ & 0.02403 & 0.03901 & -0.02369 & 0.02123 & -0.00800 & 0.01471 & \\
\hline C4 & Z & 0.03869 & 0.03876 & -0.02588 & 0.00540 & 0.01708 & 0.02349 & \\
\hline C7 & $\mathrm{x}$ & -0.03520 & -0.04677 & 0.01078 & 0.01687 & -0.05756 & -0.02101 & \\
\hline C7 & Y & 0.03584 & 0.06670 & -0.03090 & 0.03738 & -0.01621 & 0.01294 & 0.01297 \\
\hline C7 & Z & -0.03306 & -0.08732 & 0.05632 & -0.04725 & 0.02005 & 0.02336 & -0.00716 \\
\hline H21 & $\mathrm{x}$ & -0.09396 & -0.12870 & & -0.05540 & -0.03341 & -0.01338 & \\
\hline H21 & $\mathrm{Y}$ & 0.10771 & 0.18261 & -0.11897 & 0.09428 & 0.00513 & 0.01987 & 0.01663 \\
\hline H21 & Z & -0.04247 & -0.10390 & 0.06181 & -0.05346 & 0.01361 & 0.02127 & \\
\hline H4 & $\mathrm{x}$ & -0.01792 & -0.03987 & -0.05633 & 0.01415 & -0.06403 & -0.02796 & \\
\hline H4 & $\mathrm{Y}$ & 0.00082 & -0.00934 & -0.00197 & -0.02294 & -0.02568 & 0.00393 & \\
\hline H4 & Z & -0.11709 & -0.2 & & & 0.01985 & & \\
\hline H12 & $\mathrm{x}$ & 0.16183 & -0.0 & 0.0 & -0.02244 & 0.00720 & 8651 & \\
\hline H12 & $\mathrm{Y}$ & -0.05251 & 0.02750 & -0.02385 & -0.01641 & 0.00889 & 3325 & \\
\hline $\mathrm{H} 12$ & Z & 313 & 729 & 0. & 0.0 & 3561 & -0 & \\
\hline H 20 & $\mathrm{x}$ & -0. & 0.0 & -0 & & -0.0 & -0 . & \\
\hline H2O & $\mathrm{Y}$ & 0.00207 & -0.02661 & -0.03981 & -0.00599 & 0.01626 & 0.02264 & -0.02835 \\
\hline H2O & Z & 0.08667 & -0.0 & 0. & 0.01234 & -0.01225 & -0 . & \\
\hline H22 & $\mathrm{x}$ & -0.1 & 0.0 & -0 & & & -0 . & \\
\hline H22 & Y & -0.0 & 998 & -0.0 & -0.01452 & 0.00632 & 4968 & -0 . \\
\hline H22 & Z & 0.08964 & -0.0 & -0.0 & 3685 & -0.01329 & -0 & -0 . \\
\hline H 26 & $\mathrm{x}$ & & -0.1 & 0 & -0 . & & & \\
\hline H26 & Y & -0.03741 & 0.06300 & 0. & -0.01335 & -0.01541 & -0.04791 & \\
\hline H26 & z & 0.02746 & -0.02192 & -0.0 & 0.06280 & -0.02235 & -0.13550 & -0 . \\
\hline H2 & $\mathrm{x}$ & -0.0 & 0 & -0 . & & -0 . & & \\
\hline H2 & $\mathrm{Y}$ & 427 & 0. & -0. & -0.0 & -0.03892 & -0.01580 & 0 . \\
\hline H2 & Z & 0. & 0.0 & -0 & & & & \\
\hline $\mathrm{C} 2$ & $\mathrm{X}$ & -0.0 & 0. & -0 . & & & & \\
\hline C2 & $\mathrm{Y}$ & 344 & 0.0 & -0.00792 & -0.00471 & -0.02475 & 0.01070 & 827 \\
\hline $\mathrm{C} 2$ & Z & 3216 & 0.0 & -0.0 & -0.01366 & 0.02915 & -0 & \\
\hline O4 & $\mathrm{X}$ & -0.0 & -0.0 & & & & & \\
\hline 04 & $\mathrm{Y}$ & -0.01810 & -0.03267 & 0.01440 & -0.08717 & -0.02810 & -0.01692 & -0.00196 \\
\hline 04 & Z & -0.01487 & -0.0 & -0.0 & & & -0 & \\
\hline 05 & $\mathrm{X}$ & & 0 & -0 . & -0. & & -0 & \\
\hline 05 & $\mathrm{Y}$ & -0.04921 & -0.07058 & 0.03867 & 0.02960 & -0.02276 & 2807 & -0.0004 \\
\hline 05 & Z & 0.04598 & 0.03513 & -0.02 & -0.09982 & 0.04483 & -0 & \\
\hline H3 & $\mathrm{X}$ & & & -0 . & -0 . & & -0 & \\
\hline H3 & $\mathrm{Y}$ & -0.09927 & -0.1 & 0 & -0.0 & -0.00797 & 621 & -0 . \\
\hline H3 & z & 0.00227 & -0.02403 & 0.00617 & -0.05207 & 0.03760 & -0.02672 & -0.00971 \\
\hline & & & & & & & & \\
\hline ter & itie & 7.63 & 1.80 & & .81 & .44 & 5.08 & 63.03 \\
\hline Re1 & $\mathrm{x}$ & & 0.00086 & -0.0 & -0.0 & -0.0 & -0.0 & \\
\hline & Y & -0.0 & 0.0 & -0. & -0.0 & -0.0 & -0 & -0 \\
\hline Re1 & $\mathrm{z}$ & -0.01788 & 0459 & 0. & -0.00377 & -0.00588 & -0.00428 & 0.00539 \\
\hline & $\mathrm{x}$ & -0.02809 & -0.1 & & & & & \\
\hline & Y & & -0.0 & & & -0. & & \\
\hline N2 & $\mathrm{z}$ & 0.02462 & 0.06285 & -0.00068 & -0.00984 & -0.00045 & -0.01065 & 0818 \\
\hline C10 & $\mathrm{x}$ & & & & -0.00586 & & & \\
\hline & $\mathrm{Y}$ & -0.0 & -0.0 & & & & & \\
\hline C10 & z & 0513 & 657 & 0.0 & -0.00260 & -0.00811 & -0.00655 & 0 . \\
\hline $\mathrm{C} 1$ & $\mathrm{x}$ & 0.01405 & -0.08742 & & 0.00917 & -0.00867 & -0.00479 & -0. \\
\hline & Y & -0.00773 & & -0.0 & -0.00 & & & \\
\hline $\mathrm{C} 1$ & Z & & -0.0 & & & 0.01 & 0.01263 & \\
\hline & $\mathrm{X}$ & -0.03527 & 0.08265 & -0.0 & -0.011 & 0.007 & 0.00803 & -0 \\
\hline & Y & -0.01911 & -0.03937 & & & 0.00376 & -0.00483 & \\
\hline N1 & Z & & -0.10248 & -0.11412 & & & & \\
\hline 01 & $\mathrm{X}$ & -0.01375 & -0.00475 & -0.00264 & 0.00187 & -0.00042 & 0.00433 & -0.00 \\
\hline
\end{tabular}




\begin{tabular}{|c|c|c|c|c|c|c|c|c|}
\hline 01 & $\mathrm{Y}$ & -0.00501 & -0.02049 & 0.00302 & 0.00672 & 0.00218 & 0.00736 & 0.00133 \\
\hline 01 & Z & 0.02427 & -0.00977 & -0.00368 & 0.00007 & 0.00303 & -0.00082 & 0.00251 \\
\hline $\mathrm{O} 2$ & $\mathrm{x}$ & 0.02351 & 0.02647 & 0.00437 & 0.07274 & 0.05675 & 0.03865 & 113 \\
\hline $\mathrm{O} 2$ & $\mathrm{Y}$ & .03923 & -0.01544 & 0.00553 & -0.00099 & 0.04139 & 0.04923 & 0.15097 \\
\hline $\mathrm{O} 2$ & Z & 0.09165 & -0.00557 & -0.00116 & -0.03981 & 0.04571 & -0.05982 & 0.01795 \\
\hline 03 & $\mathrm{x}$ & -0.07573 & -0.01330 & 0.00876 & & & & \\
\hline 03 & $\mathrm{Y}$ & -0.04983 & 0.01056 & 0.00275 & 0.02910 & 0.06128 & 0.14211 & \\
\hline 03 & Z & 0.03861 & -0.00643 & -0.00117 & 0.05691 & 0.03311 & 0.05945 & -0.05571 \\
\hline C4 & $\mathrm{x}$ & 0.00375 & 0.01620 & -0.00223 & 0.00011 & -0.02273 & 0.07754 & \\
\hline C4 & $\mathrm{Y}$ & 0.02514 & 0.01669 & 0.00065 & 0.05591 & 0.01828 & -0.04355 & \\
\hline C4 & Z & 0.07266 & -0.00421 & -0.00816 & -0.08047 & -0.01091 & 0.00079 & -0.02080 \\
\hline C7 & $\mathrm{x}$ & -0.08425 & 0.00017 & 0.00714 & -0.05193 & -0.03400 & -0.01508 & 0.03604 \\
\hline C7 & $\mathrm{Y}$ & 0.00820 & & & & & -0.0 & \\
\hline C7 & Z & 0.06977 & 0.00342 & -0.00784 & & & & \\
\hline H21 & $\mathrm{x}$ & -0.07643 & 456 & -0.00518 & -0.01824 & -0.07734 & -0.11026 & 6181 \\
\hline H21 & $\mathrm{Y}$ & & & & -0.1 & & -0 & \\
\hline H21 & Z & 5553 & 595 & -0.0 & & & & \\
\hline H4 & $\mathrm{x}$ & -0.09224 & -0.0 & 0.0 & -0.0 & -0.03964 & -0.0 & \\
\hline H4 & $\mathrm{Y}$ & -0.0 & & -0 & & & & \\
\hline H4 & Z & 0.05944 & 0.02592 & -0.01760 & 0.15310 & -0.00840 & -0.011 & -0 \\
\hline $\mathrm{H} 12$ & $\mathrm{x}$ & -0.07979 & -0.02427 & -0.19009 & -0.01675 & & -0.00356 & \\
\hline 12 & $\mathrm{Y}$ & -0.0 & -0 & -0 . & & & & \\
\hline 12 & Z & 0.0 & -0.1 & -0.1 & & -0.000 & -0 & -0 \\
\hline H2O & $\mathrm{x}$ & -0.00630 & -0.27836 & -0.1 & & -0.02537 & -0.01863 & \\
\hline $\mathrm{H} 20$ & Y & & & & -0 . & -0 & -0 . & \\
\hline 20 & Z & & 0. & & & & & -0 \\
\hline H22 & $\mathrm{x}$ & 0.0 & 915 & -0.16199 & -0.0 & 4227 & -0 . & \\
\hline H22 & $\mathrm{Y}$ & 02 & -0 & -0 & & -0 & & \\
\hline H22 & $\mathrm{z}$ & & & -0 & -0 . & -0 & -0 . & -0 \\
\hline H 26 & $\mathrm{x}$ & -0.06534 & -0.0 & -0.1 & -0 & -0.00942 & -0 & 963 \\
\hline $\mathrm{H} 2$ & $\mathrm{Y}$ & & -0 & & & -0 & & \\
\hline H 26 & $\mathrm{z}$ & & & -0 . & -0 & & & -0 \\
\hline H2 & $\mathrm{x}$ & 693 & 0. & -0.0 & & -0.0 & 070 & 631 \\
\hline H2 & $\mathrm{Y}$ & & & & -0 . & & & \\
\hline H2 & Z & & -0. & -0 . & -0 . & -0 & -0 & \\
\hline C2 & $\mathrm{x}$ & & 0 & -0 & & -0. & -0 . & -0 \\
\hline C2 & $\mathrm{Y}$ & & & -0 . & & -0 & -0 & \\
\hline $\mathrm{C} 2$ & $\mathrm{Z}$ & & 0. & -0 . & -0 . & -0 & & \\
\hline 04 & $\mathrm{x}$ & 033 & -0.0 & -0 . & -0 . & & & -0 \\
\hline 04 & $\mathrm{Y}$ & & & -0 & -0 & & & \\
\hline 0 & Z & 59 & -0 & & & & -0 & -0 \\
\hline 05 & $\mathrm{x}$ & 177 & 0.0 & & & & -0 . & \\
\hline 05 & Y & & -0.0 & -0 & -0 . & & & \\
\hline 0 & Z & & & -0 . & & -0 & & \\
\hline H3 & $\mathrm{x}$ & -0 . & -0 & & & & -0 & -0 \\
\hline H3 & $\mathrm{Y}$ & & -0. & & -0 . & & -0 . & -0 \\
\hline $\mathrm{H} 3$ & z & -0.03603 & -0.02875 & & & & 8816 & \\
\hline \multirow{2}{*}{\multicolumn{2}{|c|}{$\begin{array}{l}\text { Erequencies } \\
\text { intensities }\end{array}$}} & & & & & & & \\
\hline & & & & & & & & \\
\hline & $\mathrm{x}$ & 269 & -0.0 & -0.0 & & -0.0 & -0 & \\
\hline e1 & $\mathrm{Y}$ & -0.00140 & 0041 & 0.00109 & -0.00081 & 0.00006 & -0.00525 & -0.00135 \\
\hline e1 & Z & & & & -0.0 & -0.0 & -0.0 & \\
\hline & $\mathrm{x}$ & & & -0.0 & & & & \\
\hline & $\mathrm{Y}$ & -0.00327 & -0.06641 & -0.01068 & -0.01248 & -0.00838 & 0.07249 & 0 \\
\hline & Z & -0.00058 & -0.02021 & -0.02023 & -0.018 & -0.00847 & 0.06376 & \\
\hline $\mathrm{C} 1 \mathrm{C}$ & $\mathrm{x}$ & & & -0. & -0.0 & & & \\
\hline C & $\mathrm{Y}$ & & 0.00076 & & & 811 & -0.09417 & 0. \\
\hline & Z & -0.00 & -0.02669 & -0.0 & -0.01392 & -0.00 & 0.06395 & \\
\hline & $\mathrm{x}$ & -0.0 & & & & & & \\
\hline $\mathrm{C} 1$ & $\mathrm{Y}$ & 0.00488 & 0.00006 & 0.00623 & 0.00863 & 0.00772 & -0.04006 & -0.0051 \\
\hline
\end{tabular}




\begin{tabular}{|c|c|c|c|c|c|c|c|c|}
\hline $\mathrm{C} 1$ & Z & 0.00536 & -0.02071 & 0.04221 & 0.03662 & 0.02210 & -0.11699 & -0.00709 \\
\hline N1 & $\mathrm{X}$ & 0.00849 & 0.05432 & 0.02110 & 0.01900 & -0.00086 & 0.04285 & -0.00340 \\
\hline N1 & $\mathrm{Y}$ & -0.00127 & 0.03578 & -0.05082 & -0.03521 & -0.01691 & 0.08861 & 0.00092 \\
\hline N1 & Z & -0.00033 & 0.02243 & -0.00408 & -0.00194 & 0.00066 & 0.00013 & -0.00073 \\
\hline 01 & $\mathrm{x}$ & -0.00159 & 0.00671 & 0.00059 & -0.00352 & 0.00105 & 0.00330 & -0.00526 \\
\hline 01 & $\mathrm{Y}$ & -0.00059 & 0.00215 & -0.00358 & -0.00053 & -0.00125 & 0.00700 & 0.00141 \\
\hline 01 & Z & 0.00169 & -0.01010 & -0.00034 & -0.00196 & 0.00052 & 0.00434 & -0.00207 \\
\hline $\mathrm{O} 2$ & $\mathrm{x}$ & -0.01797 & 0.02146 & 0.01581 & -0.03241 & 0.00815 & 0.00388 & -0.02356 \\
\hline $\mathrm{O} 2$ & $\mathrm{Y}$ & 0.01726 & 0.02670 & -0.01847 & 0.02715 & 0.03527 & 0.01404 & 0.00169 \\
\hline O2 & Z & 0.07770 & 0.00162 & -0.03129 & 0.05970 & 0.05082 & 0.01777 & \\
\hline 03 & $\mathrm{x}$ & 0.02881 & 0.00232 & -0.00982 & 0.00772 & 0.06307 & 0.00999 & -0.09848 \\
\hline 03 & $\mathrm{Y}$ & 0.06659 & -0.02360 & -0.02701 & 0.04874 & 0.03892 & & \\
\hline 03 & Z & 0.00042 & -0.01249 & -0.00038 & 0.00109 & -0.01404 & 0.00053 & 0.06728 \\
\hline C4 & $\mathrm{x}$ & -0.08625 & 0.00836 & -0.01826 & 0.03084 & -0.13231 & -0.02002 & 0.01393 \\
\hline C4 & $\mathrm{Y}$ & -0.04199 & 0.00221 & 0.02078 & -0.03727 & & & \\
\hline C4 & Z & 0.00441 & -0.00235 & -0.00813 & 0.03337 & -0.06837 & 0082 & -0 . \\
\hline C7 & $\mathrm{x}$ & 0.00189 & 0.00630 & -0.00864 & 0.03197 & -0.03054 & 0.00039 & 8772 \\
\hline C7 & $\mathrm{Y}$ & -0.00908 & -0.0 & 0 & -0. & $-0 . c$ & 153 & \\
\hline C7 & $\mathrm{z}$ & -0.00944 & -0.0 & & -0 & -0.0 & -0 & -0 . \\
\hline H21 & $\mathrm{X}$ & -0.03547 & 0.02359 & 0.02102 & -0.04567 & 0.13733 & 0.01917 & 0.01371 \\
\hline H21 & $\mathrm{Y}$ & 480 & 0.0 & 718 & -0 & -0.2 & -0 . & \\
\hline H21 & Z & -0. & -0. & & -0 . & -0.0 & -0 & \\
\hline H4 & $\mathrm{x}$ & 0.00963 & 441 & 775 & -0.01096 & 0.03279 & -0.00384 & 1852 \\
\hline H4 & $\mathrm{Y}$ & -0.0 & -0.0 & 934 & -0.2 & & 0295 & -0 . \\
\hline H4 & $\mathrm{Z}$ & -0 & -0 & & -0 & & & \\
\hline H12 & $\mathrm{x}$ & 0.02119 & -0.46502 & -0.4 & -0.22227 & 0.01987 & -0.27309 & \\
\hline $\mathrm{H} 12$ & $\mathrm{Y}$ & 0.00170 & 0.06134 & & & -0.00403 & 0.04627 & -0 . \\
\hline H12 & Z & -0 & & & -0 & -0 & & \\
\hline H2O & $\mathrm{x}$ & -0 & -0.3 & -0 & -0 . & & -0 & \\
\hline $\mathrm{H} 2 \mathrm{C}$ & $\mathrm{Y}$ & & -0. & & & & -0 & \\
\hline H2O & Z & & & & & & -0 & \\
\hline H22 & $\mathrm{x}$ & 0.00911 & -0.31012 & & & 0.0 & 704 & -0. \\
\hline H22 & $\mathrm{Y}$ & & 0.1 & & & & -0 & \\
\hline 22 & Z & & & -0 . & -0 . & -0 . & & \\
\hline H26 & $\mathrm{x}$ & -0.06093 & -0.44963 & 0 & 0.1 & -0.01959 & 0005 & 2951 \\
\hline H26 & $\mathrm{Y}$ & 0.00978 & 0.10588 & -0 & -0. & -0.02 & 3738 & 1410 \\
\hline H26 & Z & & & -0 & -0 & & -0 & \\
\hline $\mathrm{H} 2$ & $\mathrm{x}$ & -0.10228 & 0.01368 & -0.01304 & 0.01542 & -0.09244 & -0.01396 & -0.05001 \\
\hline $\mathrm{H} 2$ & $\mathrm{Y}$ & 0.03733 & -0.00702 & 0.03129 & -0.02901 & -0.20322 & -0.03644 & 3458 \\
\hline H2 & Z & -0 & -0 & -0 & & -0 & -0 & \\
\hline $\mathrm{C} 2$ & $\mathrm{x}$ & -0. & & & -0 . & & 577 & \\
\hline $\mathrm{C} 2$ & $\mathrm{Y}$ & -0.1 & 0013 & -0.0 & & 0.0 & 1039 & -0.02125 \\
\hline C2 & Z & & & & -0 & & & \\
\hline 04 & $\mathrm{x}$ & & 0. & & -0 . & 27 & -0 . & \\
\hline 04 & $\mathrm{Y}$ & 0.02475 & 0.0 & -0.00392 & 0.0 & -0.04564 & -0.00267 & -0.01305 \\
\hline 04 & Z & & -0.0 & -0 . & & & & \\
\hline 05 & $\mathrm{x}$ & & -0 & -0 & & -0 & & -0 \\
\hline 05 & $\mathrm{Y}$ & 0.06281 & -0.0 & 0.0 & -0.01436 & -0.02090 & -0.00117 & -0.00157 \\
\hline 05 & Z & & & & -0. & & -0 & \\
\hline H3 & $\mathrm{x}$ & & & -0 . & & -0 . & & -0 . \\
\hline H3 & $\mathrm{Y}$ & -0.36721 & 0.00576 & -0.0 & -0.00893 & 0.06898 & 0.00376 & 0.01305 \\
\hline H3 & z & -0.17382 & 0.00617 & 0.06863 & -0.15065 & 0.10734 & -0.00138 & 0.06844 \\
\hline \multirow{2}{*}{\multicolumn{2}{|c|}{ Erequencies }} & & & & & & & \\
\hline & & & & & & & & \\
\hline Re1 & $\mathrm{X}$ & & & & -0.00 & -0.00315 & & \\
\hline Re1 & $\mathrm{Y}$ & -0.00042 & & & & & -0.0 & -0 \\
\hline & Z & & 0032 & & -0.00 & -0.00028 & 0.00582 & \\
\hline & $\mathrm{x}$ & -0.04472 & 0.03221 & -0.01670 & & -0.00612 & -0.00305 & \\
\hline N2 & $\mathrm{Y}$ & & -0.01312 & & & & -0.00096 & -0.00315 \\
\hline N2 & Z & 0.02091 & -0.01640 & 0.00779 & 0.02 & -0.00231 & 0.00248 & 0.00167 \\
\hline
\end{tabular}




\begin{tabular}{|c|c|c|c|c|c|c|c|c|}
\hline $\mathrm{C} 10$ & $\mathrm{X}$ & 0.06231 & -0.08782 & 0.03533 & 0.01959 & -0.00092 & 0.00204 & \\
\hline $\mathrm{C} 10$ & $\mathrm{Y}$ & 0.00081 & 0.02838 & -0.00763 & 0.14828 & -0.02021 & 0.00636 & 0.01008 \\
\hline $\mathrm{C} 10$ & Z & -0.03732 & 0.03663 & -0.01314 & 0.00406 & 0.00024 & 0.00112 & 0.00174 \\
\hline 1 & $\mathrm{x}$ & 0.06598 & 0.08453 & -0.03636 & -0.06792 & 0.01354 & -0.00418 & 0.00140 \\
\hline $\mathrm{C} 1$ & $\mathrm{Y}$ & -0.00783 & -0.01316 & 0.00154 & -0.10057 & 0.01098 & -0.00677 & -0.00727 \\
\hline $\mathrm{C} 1$ & Z & -0.04039 & -0.03294 & 0.00939 & -0.08771 & 0.01279 & -0.00638 & -0.00533 \\
\hline & $\mathrm{x}$ & -0.05559 & -0.03389 & 0.01945 & 0.00394 & -0.00283 & 0.00269 & \\
\hline N1 & $\mathrm{Y}$ & -0.00624 & -0.00565 & 0.00117 & -0.04057 & 0.00657 & 0.00407 & 0.00047 \\
\hline & Z & 0.01573 & 0.00982 & -0.00352 & 0.00522 & 0.00093 & 0.00422 & 0.00333 \\
\hline & $\mathrm{x}$ & & -0. & -0.00947 & & & -0.11413 & -0.14063 \\
\hline $\mathrm{O} 1$ & $\mathrm{Y}$ & -0.00190 & -0.00066 & 0.00540 & -0.00972 & -0.01088 & 0.06055 & 0.07790 \\
\hline $\mathrm{O} 1$ & Z & 0.00200 & 0.00054 & -0.00579 & 0.01292 & 0.01184 & -0.07068 & -0.09087 \\
\hline 02 & $\mathrm{x}$ & -0.00334 & -0.02908 & -0.06188 & & & -0.02412 & \\
\hline $\mathrm{O} 2$ & $\mathrm{Y}$ & -0.00140 & -0.00234 & -0.00539 & -0.00046 & -0.01734 & 0.00228 & 0.00255 \\
\hline $\mathrm{O} 2$ & Z & -0.00296 & 0.03001 & 0.06308 & -0.00934 & -0.04394 & 0.03820 & -0.04544 \\
\hline & $\mathrm{x}$ & & & & & & -0 & \\
\hline & $\mathrm{Y}$ & & -0.00639 & -0. & -0. & & -0.00428 & \\
\hline 03 & Z & -0.00289 & -0.01349 & -0.03995 & -0.00871 & -0.05598 & -0.02087 & 00019 \\
\hline C4 & $\mathrm{x}$ & & & & -0 & & & -0 . \\
\hline C4 & $\mathrm{Y}$ & -0.0 & 0.01846 & & & & -0 & \\
\hline C4 & Z & -0.00247 & -0.03968 & -0.07327 & 0. & 0.03132 & -0.11197 & 9949 \\
\hline C7 & $\mathrm{x}$ & -0 . & -0 & -0 . & -0 . & -0 . & & \\
\hline & & & -0 & -0 . & -0 . & & & \\
\hline C7 & Z & & 296 & & & & & -0 . \\
\hline 21 & $\mathrm{x}$ & & -0 & -0 . & -0 . & -0 . & -0 & \\
\hline H21 & & & -0 & -0 . & 0 & -0 . & & \\
\hline H21 & Z & & 674 & & & & & -0 . \\
\hline H4 & $\mathrm{X}$ & & -0 & -0 . & -0 . & & & -0 . \\
\hline $\mathrm{H}$ & $\mathrm{Y}$ & & & & -0 . & -0 . & -0 & \\
\hline $\mathrm{H}$ & $\mathrm{z}$ & -0 . & 97 & & -0 & -0 & -0 & \\
\hline & $\mathrm{X}$ & & & -0 . & & & -0 & \\
\hline $\mathrm{H}$ & & -0 & -0 & & -0 . & & & -0 . \\
\hline H12 & Z & & -0.006 & 0 . & 0 & -0 . & -0 . & 881 \\
\hline & $\mathrm{x}$ & & -0.447 & & -0 & & -0 . & -0 . \\
\hline 20 & $\mathrm{Y}$ & & & -0 . & -0 . & & -0 & -0 \\
\hline 20 & $\mathrm{z}$ & & 0.1 & -0 . & -0 & & -0 . & 537 \\
\hline 22 & $\mathrm{x}$ & & 0.4 & -0 & & & & .782 \\
\hline & $\mathrm{Y}$ & & -0 . & & & & & \\
\hline 22 & Z & & -0 & & & -0 & & \\
\hline H26 & $\mathrm{X}$ & & -0.7 & & 0 . & & 671 & -0 . \\
\hline & $\mathrm{Y}$ & & & -0 . & -0 . & & & -0 \\
\hline H26 & $\mathrm{z}$ & -0 . & & -0 . & & -0 & -0 & \\
\hline H2 & $\mathrm{x}$ & & 0.0 & 168 & -0 & -0.10481 & -0.02678 & -0 \\
\hline & $\mathrm{Y}$ & & & -0 . & & & & -0 . \\
\hline H2 & $\mathrm{z}$ & -0 . & -0 & -0 & & -0 . & -0 . & \\
\hline $\mathrm{C} 2$ & $\mathrm{x}$ & -0 & -0.0 & -0.0 & & & 17 & -0. \\
\hline $\mathrm{C} 2$ & $\mathrm{Y}$ & & -0.0 & -0. & & & & \\
\hline $\mathrm{C} 2$ & Z & & -0 . & -0 . & & & & \\
\hline 04 & $\mathrm{x}$ & -0.00370 & 0.0 & & 0.0 & 34 & 93 & -0.0 \\
\hline 04 & $\mathrm{Y}$ & & & & -0. & -0 & -0 & \\
\hline 04 & Z & -0.0 & & -0 & & & & \\
\hline 05 & $\mathrm{x}$ & & -0.00697 & -0.0 & -0.0 & & -0 & \\
\hline 05 & $\mathrm{Y}$ & 12 & & & & -0. & -0 & \\
\hline 05 & z & & & & & & & \\
\hline H3 & $\mathrm{x}$ & & & & -0. & -0.0 & -0 & -0 . \\
\hline H3 & $\mathrm{Y}$ & 72 & -0.00457 & -0.0 & & & -0.0 & \\
\hline H3 & z & -0.00641 & -0.00173 & -0.01038 & & & & \\
\hline \multirow{2}{*}{\multicolumn{2}{|c|}{ ntensities }} & & & & & & & \\
\hline & & & & & & & & \\
\hline & $\mathrm{x}$ & & -0.0 & & & -0.00029 & -0.00071 & 0 \\
\hline
\end{tabular}




\begin{tabular}{|c|c|c|c|c|c|c|c|c|}
\hline Re1 & Y & 0.00017 & 0.00077 & -0.00082 & 0.00028 & -0.00057 & -0.00040 & -0.00138 \\
\hline Re1 & Z & 0.00003 & -0.00152 & 0.00016 & 0.00035 & 0.00001 & -0.00019 & -0.00064 \\
\hline N2 & $\mathrm{X}$ & 0.00149 & -0.02121 & -0.02145 & 0.01134 & -0.00071 & -0.00285 & -0.00100 \\
\hline N2 & $\mathrm{Y}$ & 0.00048 & -0.01832 & -0.01400 & 0.00967 & 0.00125 & 0.00109 & -0.00266 \\
\hline N2 & Z & 0.00165 & -0.01517 & -0.03927 & 0.02149 & 0.00081 & -0.00364 & \\
\hline C10 & $\mathrm{X}$ & -0.00080 & 0.01521 & 0.01419 & -0.00835 & -0.00063 & 0.00127 & \\
\hline C10 & & 0.00150 & -0.00912 & 0.00818 & -0.00468 & -0.00090 & -0.00097 & \\
\hline $\mathrm{C} 10$ & Z & -0.00189 & 0.02975 & 0.01749 & -0.00932 & -0.00284 & 0.00271 & \\
\hline $\mathrm{C} 1$ & $\mathrm{X}$ & -0.00171 & 0.01230 & -0.01002 & 0.00555 & -0.00097 & -0.00020 & \\
\hline C1 & Y & 0.00122 & -0.01213 & 0.02909 & -0.01630 & 0.00231 & -0.00210 & -0.00 \\
\hline $\mathrm{C} 1$ & Z & -0.00305 & 0.02493 & -0.01227 & & -0.00260 & & \\
\hline N1 & $\mathrm{X}$ & 0.00082 & -0.00555 & 0.00464 & -0.00440 & 0.00103 & -0.00011 & -0.00 \\
\hline & Y & -0.00308 & 0.01300 & -0.05042 & 0.02777 & -0.00258 & & \\
\hline N1 & Z & 0.00129 & 0.00785 & 0.01479 & -0.00961 & & & -0 . \\
\hline 01 & $\mathrm{X}$ & -0.002 & 00730 & 0222 & -0.00243 & -0.001 & & -0 \\
\hline b1 & Y & 0.00105 & -0.00376 & 0.00249 & 0.00062 & 0.00073 & -0 . & \\
\hline O1 & Z & -0.00126 & 0.00505 & -0.00123 & -0.00179 & -0.00046 & & -0 \\
\hline 02 & $\mathrm{X}$ & -0.02 & -0.0 & -0.0 & -0.010 & & & \\
\hline $\mathrm{O} 2$ & $Y$ & -0.00100 & -0.00105 & -0.00287 & -0.00641 & 0.02092 & & -0 . \\
\hline $\mathrm{O} 2$ & Z & 0.00354 & -0.00053 & 0.00403 & 0.00547 & -0.00427 & & \\
\hline 3 & $\mathrm{X}$ & -0 & & 0 & & & & -0 \\
\hline 03 & Y & -0 & -0.00023 & -0.00047 & -0.00248 & -0 & -0 . & \\
\hline 03 & Z & 00162 & 0.00209 & 0.01593 & 0.02871 & -0 . & & \\
\hline C 4 & $\mathrm{X}$ & & -0 . & -0 . & -0 . & & -0 & \\
\hline C4 & Y & -0 & 00224 & 0.00322 & 0 & -0 & -0 . & \\
\hline C4 & Z & -0 & 00009 & -0.01019 & -0 . & -0 . & -0 . & -0 \\
\hline C7 & $\mathrm{X}$ & -0 & -0 & -0 . & -0 . & -0 . & -0 & \\
\hline C7 & $\mathrm{Y}$ & 97 & -0 . & 00720 & -0.01125 & & & -0 \\
\hline C7 & Z & 0.0 & -0 & -0 & -0.02031 & -0 . & & \\
\hline $\mathrm{H} 21$ & $\mathrm{X}$ & & 0 & & & -0 . & & \\
\hline $\mathrm{H} 21$ & Y & 2089 & 1791 & 0330 & 0 . & -0 . & & \\
\hline $\mathrm{H} 21$ & Z & -0.0 & -0 & -0.07809 & -0 & 0.030 & -0 . & -0 . \\
\hline $\mathrm{H} 4$ & $\mathrm{X}$ & -0 . & -0 & -0 . & -0 . & -0 . & -0 & \\
\hline $\mathrm{H} 4$ & $Y$ & 85 & 1726 & 9608 & -0 . & 0 & -0 . & \\
\hline H4 & Z & 1081 & 1312 & 0.09413 & 0 & 0 & 0 & -0 \\
\hline $\mathrm{H} 12$ & $\mathrm{X}$ & -0 . & -0 & 0 & -0 . & -0 & & \\
\hline & Y & -0 & 0.15267 & -0.19772 & 0686 & & & -0 \\
\hline $\mathrm{H} 12$ & Z & 00566 & -0.38862 & 0.41615 & -0.22820 & -0.003 & & \\
\hline $\mathrm{H} 2 \mathrm{O}$ & $\mathrm{x}$ & 0 . & -0 . & & -0 . & & & -0 \\
\hline & Y & 0147 & 38019 & 0 & -0.24253 & & & -0 \\
\hline $\mathrm{H} 20$ & Z & -0.00543 & 10719 & -0.10911 & 0.0543 & -0.004 & -0 . & \\
\hline $\mathrm{H} 22$ & $\mathrm{x}$ & 0 & 0 . & & -0 . & & & -0 \\
\hline & $\mathrm{Y}$ & 0375 & 0 . & .7518 & -0 . & & -0 & -0 \\
\hline H22 & Z & 0109 & 0 & 25618 & -0 & & & -0 \\
\hline H26 & $\mathrm{X}$ & -0 . & -0 . & -0 . & & -0 . & & -0 \\
\hline & $\mathrm{Y}$ & & 0 & 0 . & -0 . & & -0 & \\
\hline $\mathrm{H} 2$ & Z & 0 . & -0 & -0 & 0 & & & -0 \\
\hline $\mathrm{H} 2$ & $\mathrm{X}$ & & -0 . & -0 . & -0 . & -0 & -0 . & -0 \\
\hline $\mathrm{H} 2$ & $\mathrm{Y}$ & & 0 & 0.09615 & 0 & & -0 . & \\
\hline $\mathrm{H} 2$ & Z & -0 & -0 . & -0.13362 & -0 & 0.211 & & \\
\hline $\mathrm{C} 2$ & $\mathrm{x}$ & -0 & 0 & & & & & -0 \\
\hline $\mathrm{C} 2$ & $\mathrm{Y}$ & -0.01916 & -0.00010 & 0.00873 & 0.014 & 0 . & & -0 \\
\hline $\mathrm{C} 2$ & Z & 0 & 0 & 0 . & 0 . & & -0 & \\
\hline 04 & $\mathrm{X}$ & -0.06344 & 0.00004 & & 0.013 & & -0 . & \\
\hline 04 & $\mathrm{Y}$ & 0.03154 & -0.00012 & -0.00682 & -0.01100 & -0.00388 & 0.00 & \\
\hline 04 & Z & 0. & -0 . & -0.00105 & -0 . & -0 . & 0.01 & -0 \\
\hline 05 & $\mathrm{X}$ & 0.11369 & 0.00092 & -0.00779 & -0.01117 & -0.00623 & 0.02171 & \\
\hline 05 & Y & -0.02798 & -0.00051 & 0.00171 & 0.00166 & 0.00743 & -0.02634 & 0.00465 \\
\hline 05 & Z & -0.08504 & -0.00032 & & 0.005 & -0.01877 & & -0 . \\
\hline H3 & $\mathrm{X}$ & -0.449 & -0.00 & 0.03381 & 0.04562 & 0.12141 & -0.3 & 0 \\
\hline H3 & Y & 0.10996 & -0.00020 & -0.00568 & -0.00958 & -0.03162 & 0.05639 & -0.00 \\
\hline
\end{tabular}




\begin{tabular}{|c|c|c|c|c|c|c|c|c|}
\hline H3 & Z & 0.33308 & 0.00389 & -0.02495 & -0.03463 & -0.11743 & 0.28656 & -0.0443 \\
\hline \multicolumn{2}{|c|}{ frequencies } & 1385.80 & 1416.49 & 1438.34 & 1505.44 & 1515.28 & 1598.66 & 1866. \\
\hline \multicolumn{2}{|c|}{ intensities } & 5.16 & 71.86 & 21.70 & 278.55 & 3.56 & 2.45 & 200 \\
\hline Re1 & $\mathrm{X}$ & 0.00001 & -0.00016 & -0.00018 & 0.00005 & 0.00022 & -0.00022 & \\
\hline Re1 & $\mathrm{Y}$ & -0.00021 & 0.00020 & 0.00013 & -0.00066 & -0.00011 & -0.00070 & \\
\hline Re1 & Z & -0.00004 & 0.00007 & -0.00015 & 0.00150 & -0.00036 & -0.00024 & -0.000 \\
\hline N2 & $\mathrm{X}$ & 0.00314 & -0.00103 & -0.02814 & 0.02624 & -0.00478 & 0.03289 & \\
\hline N2 & $\mathrm{Y}$ & 0.05885 & 0.00227 & 0.02857 & 0.04245 & -0.00596 & 0.00062 & \\
\hline N2 & Z & -0.06355 & -0.00387 & -0.11956 & 0.02559 & -0.00681 & 0.10019 & -0.00 \\
\hline C10 & $\mathrm{X}$ & -0.01938 & -0.00006 & 0.02072 & -0.05738 & 0.00959 & -0.05309 & \\
\hline 10 & $\mathrm{Y}$ & -0.06207 & -0.00319 & -0.04252 & -0.09728 & 0.01449 & -0.01005 & \\
\hline C10 & Z & 0.00020 & 0.00190 & 0.09110 & -0.09167 & 0.01642 & -0.15279 & \\
\hline $\mathrm{C} 1$ & $\mathrm{X}$ & -0.01424 & 0.00105 & 0.02317 & 0.01850 & -0.00239 & 0.01056 & \\
\hline $\mathrm{C} 1$ & $\mathrm{Y}$ & -0.03941 & -0.00006 & -0.04726 & 0.15686 & -0.02655 & -0.11034 & \\
\hline $\mathrm{C} 1$ & $\mathrm{Z}$ & -0.04086 & 0.00233 & 0.09671 & -0.00669 & 0.00404 & 0.09456 & -0.0 \\
\hline N1 & $\mathrm{X}$ & 0.01521 & -0.001 & -0.01 & -0.00382 & & -0.00817 & \\
\hline N1 & $\mathrm{Y}$ & -0.00531 & & 0.07443 & -0.05941 & & & \\
\hline N1 & Z & 0.08384 & -0.002 & -0.10636 & -0.01040 & -0.00161 & -0.06425 & \\
\hline 01 & $\mathrm{X}$ & -0 & -0.000 & -0.00173 & & -0.001 & & \\
\hline 01 & Y & -0.00 & -0.00 & -0.00005 & 60 & & & -0 \\
\hline 01 & Z & -0.00074 & 0.00 & 0.00197 & -0.002 & -0.000 & 0.00016 & \\
\hline 02 & $\mathrm{X}$ & 0.001 & 0. & -0.00 & -0 & & -0.00 & \\
\hline 02 & Y & -0.00 & & & -0. & -0 & 0.00 & \\
\hline $\mathrm{O} 2$ & Z & -0.00 & 0.00 & 0.00 & 0.0 & -0 & 0.002 & -0 \\
\hline 03 & $\mathrm{X}$ & -0 & 0 & 0.00 & -0 & -0 & 0.00 & \\
\hline 03 & Y & 0 . & -0.00 & -0 . & 0 & & 0.00 & \\
\hline 03 & Z & 0 . & -0.00 & 0 & 0.0 & 75 & -0.00101 & -0 \\
\hline C4 & $\mathrm{X}$ & 53 & -0.0 & 0 & -0 & -0 & 0.00 & \\
\hline $\mathrm{C} 4$ & $\mathrm{Y}$ & 0 . & & -0 . & 0 & & -0.00 & -0 \\
\hline $\mathrm{C} 4$ & Z & 0 . & -0.08 & 0 & -0.0 & -0 & -0.00064 & \\
\hline C7 & $\mathrm{X}$ & -0 & 0.02 & 0 . & -0 & -0 & -0.00 & \\
\hline C7 & Y & -0 & 0.01 & 0 & 0 & & 100 & -0 \\
\hline C7 & Z & -0 . & 0.01 & -0 . & 0 . & 76 & -0.00046 & \\
\hline H21 & $\mathrm{X}$ & 0 & -0.07 & -0.02173 & 0 & & -0.00 & -0 \\
\hline H21 & Y & 0.02 & 0.00 & 0 & -0 & -0 . & 0.00 & -0 \\
\hline H21 & Z & -0 & 0.023 & 0 & 0.0 & 0 & -0.00 & \\
\hline $\mathrm{H} 4$ & $\mathrm{X}$ & 0 & -0.15499 & -0.00156 & 0 & & -0.00 & \\
\hline $\mathrm{H} 4$ & $\mathrm{Y}$ & 0.03 & -0.20 & 186 & -0 . & -0.2 & -0.00561 & \\
\hline H4 & $\mathrm{Z}$ & -0.01121 & 0.04484 & 0 . & -0.09 & -0.6 & 0.00438 & \\
\hline H12 & $\mathrm{X}$ & -0 . & 0.00 & 0 & 0.00 & -0 . & 0.02 & -0 \\
\hline $\mathrm{H} 12$ & $\mathrm{Y}$ & 0.16684 & -0.00026 & -0.06047 & -0.1 & 1884 & 0.03637 & \\
\hline H12 & Z & -0.39566 & 0.00257 & 0.28469 & 0.19508 & -0.021 & 0.05677 & -0 \\
\hline $\mathrm{H} 20$ & $\mathrm{X}$ & 0.03171 & 0.00210 & -0.00498 & -0.00279 & 0 & 0.03 & -0 \\
\hline $\mathrm{H} 20$ & $\mathrm{Y}$ & 0.35878 & -0.00507 & -0.14385 & -0.341 & & 0.24615 & \\
\hline $\mathrm{H} 20$ & Z & -0.12163 & 0.00311 & 0.13654 & 0.08580 & -0.00978 & 0.04786 & -0 . \\
\hline $\mathrm{H} 22$ & $\mathrm{X}$ & 0.142 & 0.002 & 0 . & 0. & -0 & 0.122 & -0 \\
\hline H22 & $\mathrm{Y}$ & 0.16531 & 0.00297 & 0.01923 & 0.12 & -0.01794 & 0.20095 & -0 . \\
\hline H22 & Z & 0.33801 & 0.01141 & 0.20485 & 0.24755 & -0.03285 & 0.12877 & -0 . \\
\hline $\mathrm{H} 26$ & $\mathrm{X}$ & 0.06504 & 0.00383 & 0.03572 & 0.02707 & -0 & 0.016 & -0 . \\
\hline H26 & $\mathrm{Y}$ & -0.17919 & -0.00927 & -0.17207 & -0.03619 & 0.00226 & 0.07088 & -0.00 \\
\hline H26 & Z & 0.39596 & 0.01413 & 0.23461 & 0.21118 & -0.02811 & -0.00525 & \\
\hline $\mathrm{H} 2$ & $\mathrm{X}$ & 0.00348 & -0.02376 & 0.00075 & -0.00173 & -0.00978 & 0.00084 & 0.0 \\
\hline $\mathrm{H} 2$ & $\mathrm{Y}$ & -0.00560 & -0.20614 & 0.01240 & 0.00026 & 0.01491 & 0.00002 & -0.03 \\
\hline H2 & $\mathrm{Z}$ & -0.00135 & 0.35188 & -0.02461 & 0.00870 & 0.00724 & -0.00154 & 0.04 \\
\hline $\mathrm{C} 2$ & $\mathrm{X}$ & -0.00001 & -0.03729 & 0.00071 & -0.00019 & -0.00253 & -0.00028 & -0.2 \\
\hline $\mathrm{C} 2$ & $\mathrm{Y}$ & -0.00146 & -0.06901 & 0.00199 & 0.00151 & -0.00160 & 0.00043 & 0.108 \\
\hline $\mathrm{C} 2$ & Z & 0.00016 & 0.16689 & -0.00189 & -0.00253 & 0.01335 & -0.00004 & 0.009 \\
\hline 04 & $\mathrm{X}$ & 0.00014 & 0.00679 & -0.00060 & 0.00086 & 0.00145 & 0.00017 & \\
\hline 04 & $\mathrm{Y}$ & 0.00003 & 0.00804 & 0.00034 & -0.00075 & -0.00046 & -0.00013 & -0.06 \\
\hline 04 & Z & -0.00015 & -0.02219 & 0.00016 & 0.00046 & -0.00184 & 0.00007 & -0.006 \\
\hline
\end{tabular}




\begin{tabular}{|c|c|c|c|c|c|c|c|c|}
\hline 05 & $\mathrm{x}$ & -0.00035 & 0.00433 & 0.00061 & -0.00062 & 0.00056 & 0.00009 & 0.01974 \\
\hline 05 & $\mathrm{Y}$ & 0.00020 & 0.02399 & -0.00043 & -0.00028 & 0.00084 & -0.00010 & -0.00698 \\
\hline 05 & Z & -0.00004 & -0.05611 & 0.00001 & 0.00122 & -0.00482 & -0.00001 & -0.00896 \\
\hline H3 & $\mathrm{x}$ & 0.00416 & 0.26570 & -0.00863 & -0.00041 & 0.01474 & -0.00044 & -0.08560 \\
\hline H3 & $\mathrm{Y}$ & -0.00172 & -0.04028 & 0.00218 & 0.00099 & -0.00026 & 0.00051 & 0.01976 \\
\hline H3 & Z & -0.00378 & -0.24020 & 0.00688 & 0.00262 & -0.01194 & 0.00064 & 0.06867 \\
\hline \multicolumn{2}{|c|}{ Erequencies } & 3076.50 & 3104.76 & 3135.60 & 3213.71 & 3224.77 & 3537.76 & 3541.69 \\
\hline & ities & 13.84 & 4.35 & 2.68 & 3.50 & 29.68 & 200.59 & 61.98 \\
\hline $\operatorname{Re} 1$ & $\mathrm{x}$ & -0.00004 & -0.00008 & -0.00001 & -0.00002 & -0.00001 & 0.00000 & 0.00001 \\
\hline Re1 & $\mathrm{Y}$ & 0.00003 & -0.00003 & 0.00002 & -0.00003 & -0.00001 & -0.00002 & -0.00001 \\
\hline $\operatorname{Re} 1$ & z & 0.00005 & 0.00004 & 0.00002 & -0.00001 & 0.00006 & 0.00000 & \\
\hline N2 & $\mathrm{x}$ & 0.00007 & 0.00016 & -0.00006 & 0.00013 & 0.00062 & 0.02895 & 0.01326 \\
\hline N2 & $\mathrm{Y}$ & 0.00005 & 0.00012 & -0.00001 & -0.00132 & -0.00046 & 0.05182 & 0.02408 \\
\hline N2 & Z & 0005 & 0.00016 & -0.00011 & 0.00180 & 0.00217 & 0.02555 & \\
\hline C10 & $\mathrm{x}$ & 0.00004 & -0.00007 & 0.00004 & 0.00227 & 0.00281 & -0.00096 & \\
\hline $\mathrm{C} 10$ & $\mathrm{Y}$ & 0.00034 & 0.00031 & -0.00011 & 0.05407 & 0.04345 & 0.00152 & \\
\hline $\mathrm{C} 10$ & Z & 0036 & -0.0 & 015 & -0.04104 & 2869 & 0365 & \\
\hline & $\mathrm{x}$ & -0.0 & -0.00013 & 0.00005 & 0.01387 & 1978 & & -0 \\
\hline $\mathrm{C} 1$ & $\mathrm{Y}$ & 0.00014 & -0.00007 & -0.00003 & 0.00508 & -0.01175 & -0.00170 & 0.00093 \\
\hline $\mathrm{C} 1$ & Z & -0.00037 & -0.00063 & 0.00025 & 0.04833 & -0.06515 & 00152 & -0 \\
\hline N1 & $\mathrm{x}$ & & -0.0 & -0.0 & -0.00040 & & & -0 \\
\hline N1 & Y & 0002 & -0.00008 & -0.0 & -0.00013 & -0.00146 & 2799 & -0.06150 \\
\hline N1 & Z & 011 & -0.00002 & -0.00 & -0.00155 & 0.00220 & 0795 & -0.0 \\
\hline 01 & $\mathrm{x}$ & & 0.0 & 0 & & & & \\
\hline 01 & $\mathrm{Y}$ & 0.00002 & 0.00001 & 0.00000 & -0.00010 & -0.00008 & 0.00004 & -0 . \\
\hline 01 & Z & -0.00003 & 0.00000 & -0.00003 & -0.00003 & 0.00004 & 0.00002 & \\
\hline 02 & $\mathrm{x}$ & -0 & 0. & -0 . & -0 & & -0 & -0 \\
\hline $\mathrm{O} 2$ & $\mathrm{Y}$ & 031 & -0.0 & 0 & 0002 & -0 . & -0 . & -0 \\
\hline 02 & Z & 0 . & -0.0 & 0 . & & -0 . & -0 . & -0 \\
\hline 03 & $\mathrm{x}$ & 90 & -0 . & 0 . & & -0 . & -0 & -0 \\
\hline 03 & $\mathrm{Y}$ & 0079 & -0.0 & 0.0 & -0.00004 & 07 & 0011 & 0 \\
\hline 03 & Z & -0. & & 0 . & -0.0 & -0 . & & \\
\hline C4 & $\mathrm{x}$ & -0 & 0. & -0 & & -0 . & & -0 \\
\hline C4 & Y & -0.00157 & 0.0 & -0.00243 & 0.00000 & -0.00017 & 0005 & -0.00010 \\
\hline $\mathrm{C} 4$ & Z & -0.00064 & & -0.00214 & & -0.00013 & -0 . & -0.0 \\
\hline C7 & $\mathrm{x}$ & & -0.0 & -0 & -0.00011 & -0 & -0 & \\
\hline C7 & $\mathrm{Y}$ & -0.04587 & -0.004 & 0. & 0.00012 & 0.00043 & 0.00005 & -0 \\
\hline C7 & z & -0.01837 & -0.012 & -0 & 0.0 & & & \\
\hline H & $\mathrm{x}$ & & & & -0 . & -0 . & -0 & \\
\hline 21 & $\mathrm{Y}$ & 700 & & & -0.00027 & -0.00044 & -0 & -0.0 \\
\hline H21 & $\mathrm{z}$ & 4093 & 0. & 0. & -0.00174 & -0.00237 & -0.00062 & -0.00131 \\
\hline H4 & $\mathrm{x}$ & -0. & -0 . & & & & & \\
\hline H4 & $\mathrm{Y}$ & 757 & 0.0 & -0.2 & -0.00088 & -0.00396 & -0 & -0. \\
\hline H4 & $\mathrm{z}$ & -0.31706 & -0.00699 & 0.2 & 0.00074 & 0.00291 & 0.00050 & -0.00029 \\
\hline & $\mathrm{x}$ & & & & & -0 . & & \\
\hline & $\mathrm{Y}$ & -0 . & & & 0 . & -0 & -0 & \\
\hline $\mathrm{H} 12$ & z & 0041 & 0.0 & 0.0 & 0.00509 & -0.00533 & -0.12801 & 961 \\
\hline & $\mathrm{x}$ & & & -0. & & & & \\
\hline $\mathrm{H}$ & $\mathrm{Y}$ & & & -0 & -0.0 & & -0 . & \\
\hline H2O & z & 0.00352 & 0.007 & -0.00297 & -0.54192 & 0.72023 & -0.00642 & 0793 \\
\hline & $\mathrm{x}$ & -0.0 & -0.0 & & -0.03 & -0.02785 & & \\
\hline H22 & $\mathrm{Y}$ & -0.0 & -0. & & -0.617 & -0.46472 & -0.0 & -0 . \\
\hline H 22 & Z & & & -0.0 & & 2784 & 0.0 & \\
\hline & $\mathrm{x}$ & -0.0 & -0.0 & -0 & & & -0.38 & -0 \\
\hline H26 & $\mathrm{Y}$ & -0.0 & -0.0 & -0. & & & & \\
\hline H26 & $\mathrm{z}$ & -0.0 & -0.0 & -0.0 & 0.0 & & -0.31242 & -0.1 \\
\hline & $\mathrm{x}$ & 207 & -0.907 & 0.1 & -0.0 & & 0.00082 & \\
\hline $\mathrm{H} 2$ & $\mathrm{Y}$ & & -0.22061 & & -0.00035 & & -0.00005 & \\
\hline $\mathrm{H} 2$ & Z & & -0.12475 & 0.01699 & -0.00021 & & -0.00014 & \\
\hline $\mathrm{C} 2$ & $\mathrm{x}$ & -0.00041 & 0.00111 & -0.00004 & 0.00003 & 0.00010 & 0.00012 & -0.00 \\
\hline
\end{tabular}




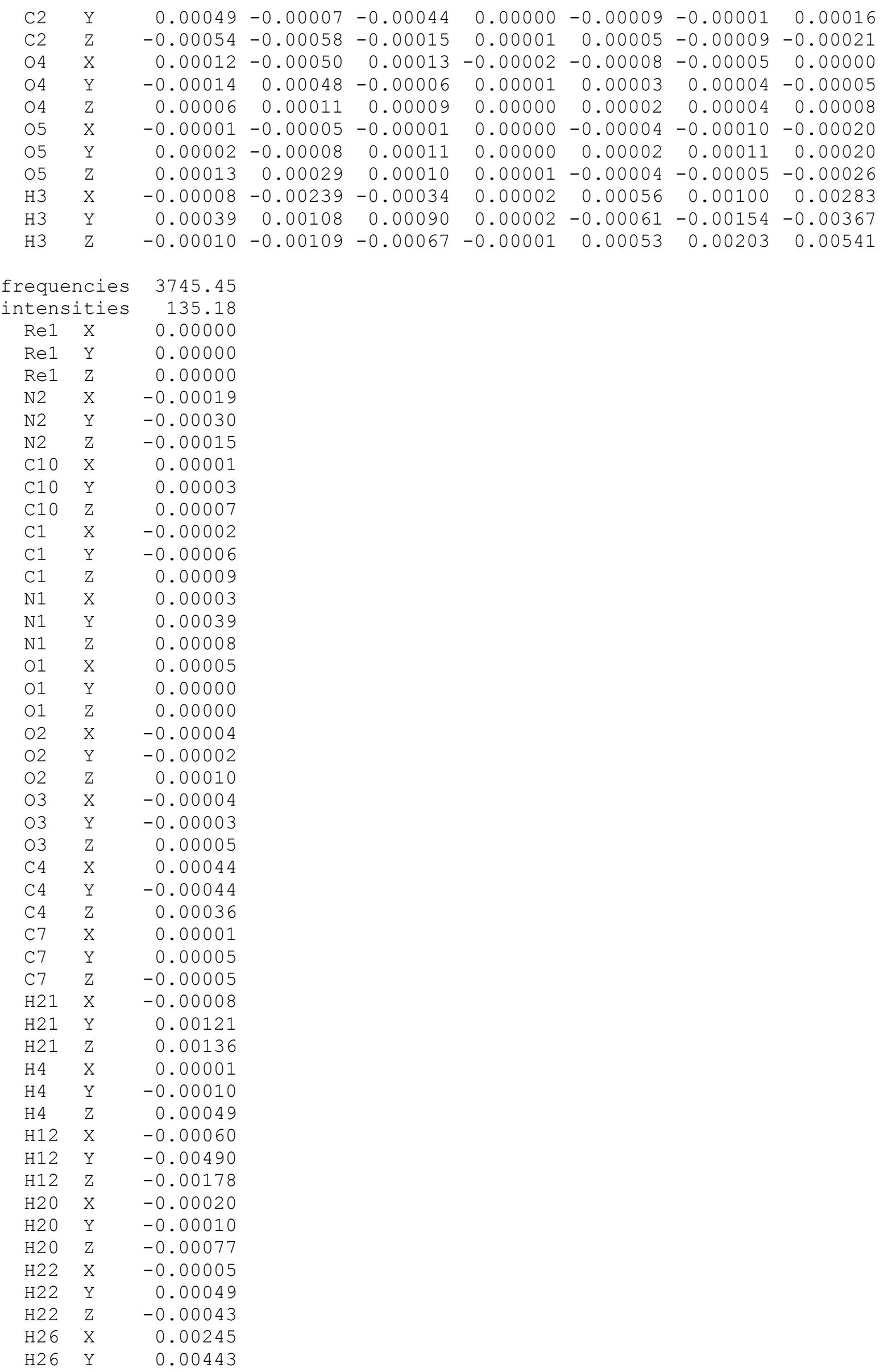




$\begin{array}{llr}\text { H26 } & \text { Z } & 0.00179 \\ \text { H2 } & X & -0.00196 \\ \text { H2 } & \text { Y } & -0.00091 \\ \text { H2 } & Z & 0.00056 \\ \text { C2 } & X & -0.00028 \\ \text { C2 } & \text { Y } & 0.00116 \\ \text { C2 } & Z & -0.00218 \\ \text { O4 } & X & -0.00072 \\ \text { O4 } & \text { Y } & 0.00016 \\ \text { O4 } & \text { Z } & 0.00047 \\ \text { O5 } & X & -0.02402 \\ \text { O5 } & \text { Y } & 0.03260 \\ \text { O5 } & Z & -0.04394 \\ \text { H3 } & X & 0.39349 \\ \text { H3 } & \text { Y } & -0.52850 \\ \text { H3 } & Z & 0.70800\end{array}$

Thermochemical Properties:

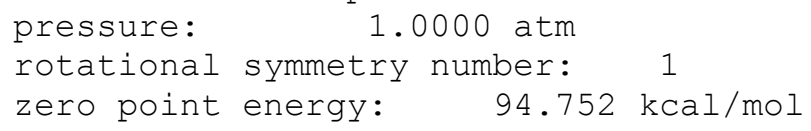




\section{Scheme 5}

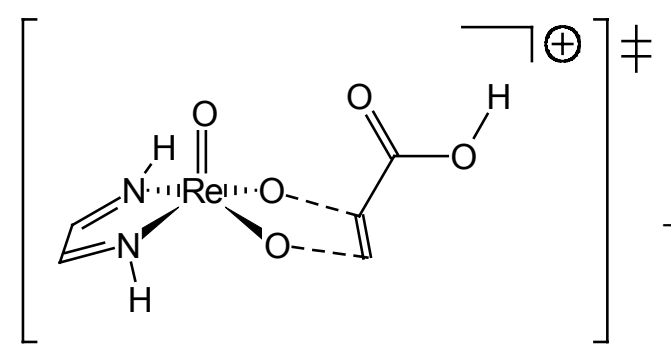

-759.83704278810 hartrees

Jaguar version 3.5, release 42

| Copyright 1991-1998 Schrodinger, Inc.

| All Rights Reserved.

Use of this program should be acknowledged in publications as:

| Jaguar 3.5, Schrodinger, Inc., Portland, Oregon, 1998.

Job WF3681 started on CHENB071 at Wed Jan 21 08:45:18 2004

Jaguar version 3.5, release 42

Job name:

Task:

Stoichiometry:

Molecular weight:

Molecular charge:

Spin multiplicity:

Point Group:

SCF method:

Basis set:

Basis functions:

Total electrons:

Alpha electrons:

Beta electrons:

Total orbitals:

Core orbitals:

Open-shell orbitals:

occupied orbitals:

Shells:

\section{WF3681}

Calculate energy ("single-point job")

ReC5N2H8O5

$363.00 \mathrm{amu}$

1

1

B3LYP

$\mathrm{LACVP} * *$

230

106

53

53

245

53

0

53

1

Non-default options chosen:

SCF calculation type: DFT

$\mathrm{DFT}=$ Becke 3 Parameter/HF+Slater+Becke88+VWN+LYP (B3LYP)

Numerical $2 \bar{n}$ d derivatives will be computed

Vibrational frequencies and related properties will be computed

Molecular symmetry not used

Input geometry:
atom
$\mathrm{x}$
angstroms
Y
$\operatorname{Re} 1$
0.6948360000
$-0.3546770000$
0.8067280000 


$\begin{array}{lrrr}\text { N2 } & 1.4301100000 & 1.2603360000 & -0.5540900000 \\ \text { C10 } & 1.2353740000 & 1.1538370000 & -1.8158510000 \\ \text { C1 } & 0.6385730000 & -0.1371560000 & -2.2396310000 \\ \text { N1 } & 0.3725730000 & -0.9577320000 & -1.2893170000 \\ \text { O1 } & 2.1307920000 & -0.9163850000 & 1.5022650000 \\ \text { O2 } & -0.5896230000 & -1.4801820000 & 1.2033420000 \\ \text { O3 } & 0.0284510000 & 0.9547780000 & 1.7813530000 \\ \text { C4 } & -1.6981520000 & -0.8795110000 & 2.9217670000 \\ \text { C7 } & -1.3604350000 & 0.4595890000 & 3.1181550000 \\ \text { H21 } & -0.6703820000 & 0.7025370000 & 3.9183120000 \\ \text { H4 } & -2.0328600000 & 1.2400430000 & 2.7842060000 \\ \text { H12 } & -0.0036080000 & -1.8743840000 & -1.5364160000 \\ \text { H20 } & 0.4762220000 & -0.3698790000 & -3.2909750000 \\ \text { H22 } & 1.5092740000 & 1.9166610000 & -2.5426110000 \\ \text { H26 } & 1.8720530000 & 2.1145800000 & -0.2101630000 \\ \text { H2 } & -2.6147220000 & -1.1647120000 & 2.4210460000 \\ \text { C2 } & -0.9954640000 & -1.9036370000 & 3.7462340000 \\ \text { 04 } & 0.0773320000 & -1.7053880000 & 4.2795100000 \\ \text { O5 } & -1.6816210000 & -3.0544870000 & 3.8147960000 \\ \text { H3 } & -1.1684710000 & -3.6646350000 & 4.3726220000\end{array}$

calculating forces at perturbed geometry calculating forces at perturbed geometry calculating forces at perturbed geometry calculating forces at perturbed geometry calculating forces at perturbed geometry calculating forces at perturbed geometry calculating forces at perturbed geometry calculating forces at perturbed geometry calculating forces at perturbed geometry calculating forces at perturbed geometry calculating forces at perturbed geometry calculating forces at perturbed geometry calculating forces at perturbed geometry calculating forces at perturbed geometry calculating forces at perturbed geometry calculating forces at perturbed geometry calculating forces at perturbed geometry calculating forces at perturbed geometry calculating forces at perturbed geometry calculating forces at perturbed geometry calculating forces at perturbed geometry calculating forces at perturbed geometry calculating forces at perturbed geometry calculating forces at perturbed geometry calculating forces at perturbed geometry calculating forces at perturbed geometry calculating forces at perturbed geometry calculating forces at perturbed geometry calculating forces at perturbed geometry calculating forces at perturbed geometry calculating forces at perturbed geometry calculating forces at perturbed geometry calculating forces at perturbed geometry calculating forces at perturbed geometry calculating forces at perturbed geometry calculating forces at perturbed geometry calculating forces at perturbed geometry calculating forces at perturbed geometry calculating forces at perturbed geometry
1 of $126 \ldots$

2 of $126 \ldots$

3 of $126 \ldots$

4 of $126 \ldots$

5 of $126 \ldots$

6 of $126 \ldots$

7 of $126 \ldots$

8 of $126 \ldots$

9 of $126 \ldots$

10 of $126 \ldots$

11 of $126 \ldots$

12 of $126 \ldots$

13 of $126 \ldots$

14 of $126 \ldots$

15 of $126 \ldots$

16 of $126 \ldots$

17 of $126 \ldots$

18 of $126 \ldots$

19 of $126 \ldots$

20 of $126 \ldots$

21 of $126 \ldots$

22 of $126 \ldots$

23 of $126 \ldots$

24 of $126 \ldots$

25 of $126 \ldots$

26 of $126 \ldots$

27 of $126 \ldots$

28 of $126 \ldots$

29 of $126 \ldots$

30 of $126 \ldots$

31 of $126 \ldots$

32 of $126 \ldots$

33 of $126 \ldots$

34 of $126 \ldots$

35 of $126 \ldots$

36 of $126 \ldots$

37 of $126 \ldots$

38 of $126 \ldots$

39 of $126 \ldots$ 
calculating forces at perturbed geometry calculating forces at perturbed geometry calculating forces at perturbed geometry calculating forces at perturbed geometry calculating forces at perturbed geometry calculating forces at perturbed geometry calculating forces at perturbed geometry calculating forces at perturbed geometry calculating forces at perturbed geometry calculating forces at perturbed geometry calculating forces at perturbed geometry calculating forces at perturbed geometry calculating forces at perturbed geometry calculating forces at perturbed geometry calculating forces at perturbed geometry calculating forces at perturbed geometry calculating forces at perturbed geometry calculating forces at perturbed geometry calculating forces at perturbed geometry calculating forces at perturbed geometry calculating forces at perturbed geometry calculating forces at perturbed geometry calculating forces at perturbed geometry calculating forces at perturbed geometry calculating forces at perturbed geometry calculating forces at perturbed geometry calculating forces at perturbed geometry calculating forces at perturbed geometry calculating forces at perturbed geometry calculating forces at perturbed geometry calculating forces at perturbed geometry calculating forces at perturbed geometry calculating forces at perturbed geometry calculating forces at perturbed geometry calculating forces at perturbed geometry calculating forces at perturbed geometry calculating forces at perturbed geometry calculating forces at perturbed geometry calculating forces at perturbed geometry calculating forces at perturbed geometry calculating forces at perturbed geometry calculating forces at perturbed geometry calculating forces at perturbed geometry calculating forces at perturbed geometry calculating forces at perturbed geometry calculating forces at perturbed geometry calculating forces at perturbed geometry calculating forces at perturbed geometry calculating forces at perturbed geometry calculating forces at perturbed geometry calculating forces at perturbed geometry calculating forces at perturbed geometry calculating forces at perturbed geometry calculating forces at perturbed geometry calculating forces at perturbed geometry calculating forces at perturbed geometry calculating forces at perturbed geometry calculating forces at perturbed geometry calculating forces at perturbed geometry calculating forces at perturbed geometry calculating forces at perturbed geometry
40 of $126 \ldots$ 41 of $126 \ldots$ 42 of $126 \ldots$ 43 of $126 \ldots$ 44 of $126 \ldots$ 45 of $126 \ldots$ 46 of $126 \ldots$ 47 of $126 \ldots$ 48 of $126 \ldots$ 49 of $126 \ldots$ 50 of $126 \ldots$ 51 of $126 \ldots$ 52 of $126 \ldots$ 53 of $126 \ldots$ 54 of $126 \ldots$ 55 of $126 \ldots$ 56 of $126 \ldots$ 57 of $126 \ldots$ 58 of $126 \ldots$ 59 of $126 \ldots$ 60 of $126 \ldots$ 61 of $126 \ldots$ 62 of $126 \ldots$ 63 of $126 \ldots$ 64 of $126 \ldots$ 65 of $126 \ldots$ 66 of $126 \ldots$ 67 of $126 \ldots$ 68 of $126 \ldots$ 69 of $126 \ldots$ 70 of $126 \ldots$ 71 of $126 \ldots$ 72 of $126 \ldots$ 73 of $126 \ldots$ 74 of $126 \ldots$ 75 of $126 \ldots$ 76 of $126 \ldots$ 77 of $126 \ldots$ 78 of $126 \ldots$ 79 of $126 \ldots$ 80 of $126 \ldots$ 81 of $126 \ldots$ 82 of $126 \ldots$ 83 of $126 \ldots$ 84 of $126 \ldots$ 85 of $126 \ldots$ 86 of $126 \ldots$ 87 of $126 \ldots$ 88 of $126 \ldots$ 89 of $126 \ldots$ 90 of $126 \ldots$ 91 of $126 \ldots$ 92 of $126 \ldots$ 93 of $126 \ldots$ 94 of $126 \ldots$ 95 of $126 \ldots$ 96 of $126 \ldots$ 97 of $126 \ldots$ 98 of $126 \ldots$ 99 of $126 \ldots$ 100 of $126 \ldots$ 
calculating forces at perturbed geometry 101 of $126 \ldots$ calculating forces at perturbed geometry 102 of $126 \ldots$ calculating forces at perturbed geometry 103 of $126 \ldots$ calculating forces at perturbed geometry 104 of $126 \ldots$ calculating forces at perturbed geometry 105 of $126 \ldots$ calculating forces at perturbed geometry 106 of $126 \ldots$ calculating forces at perturbed geometry 107 of $126 \ldots$ calculating forces at perturbed geometry 108 of $126 \ldots$ calculating forces at perturbed geometry 109 of $126 \ldots$ calculating forces at perturbed geometry 110 of $126 \ldots$ calculating forces at perturbed geometry 111 of $126 \ldots$ calculating forces at perturbed geometry 112 of $126 \ldots$ calculating forces at perturbed geometry 113 of $126 \ldots$ calculating forces at perturbed geometry 114 of $126 \ldots$ calculating forces at perturbed geometry 115 of $126 \ldots$ calculating forces at perturbed geometry 116 of $126 \ldots$ calculating forces at perturbed geometry 117 of $126 \ldots$ calculating forces at perturbed geometry 118 of $126 \ldots$ calculating forces at perturbed geometry 119 of $126 \ldots$ calculating forces at perturbed geometry 120 of $126 \ldots$ calculating forces at perturbed geometry 121 of $126 \ldots$ calculating forces at perturbed geometry 122 of $126 \ldots$ calculating forces at perturbed geometry 123 of $126 \ldots$ calculating forces at perturbed geometry 124 of $126 \ldots$ calculating forces at perturbed geometry 125 of $126 \ldots$ calculating forces at perturbed geometry 126 of $126 \ldots$

numerical derivatives complete;

calculation at normal geometry follows...

start of program freq

harmonic frequencies in $\mathrm{cm}^{\wedge}-1$, IR intensities in $\mathrm{km} / \mathrm{mol}$, and normal modes:

\begin{tabular}{|c|c|c|c|c|c|c|c|c|}
\hline \multicolumn{2}{|c|}{ frequencies } & $-342 \cdot 30$ & 36.81 & 57.55 & 73.85 & 83.17 & 97.66 & 135. \\
\hline into & ities & 152.22 & 2.57 & 8.28 & 3.07 & 4.64 & 4.41 & \\
\hline Re1 & $\mathrm{X}$ & -0.00055 & -0.00980 & 0.02449 & -0.00521 & -0.01051 & 0.01436 & 0.0 \\
\hline Re1 & Y & -0.00135 & -0.01675 & -0.00556 & -0.00091 & 0.01931 & -0.00268 & \\
\hline $\operatorname{Re1}$ & Z & 0.00117 & -0.00583 & -0.00512 & 0.00367 & -0.00065 & 0.01969 & \\
\hline N2 & $\mathrm{X}$ & 0.01336 & 0.08940 & -0.00036 & 0.01800 & 0.08011 & 0.01773 & \\
\hline N2 & $\mathrm{Y}$ & 0.00568 & -0.01697 & 0.00162 & -0.02037 & -0.04460 & -0.03319 & -0 \\
\hline N2 & Z & -0.02279 & 0.04299 & -0.01263 & -0.00795 & -0.03562 & -0.008 & -0 . \\
\hline $\mathrm{C} 10$ & $\mathrm{x}$ & 0.01249 & 0.07615 & -0.06515 & 0.01389 & 0.09950 & -0.04918 & \\
\hline $\mathrm{C} 10$ & $\mathrm{Y}$ & 0.00 & 0.035 & 0.02825 & -0.02559 & -0.07271 & -0.023 & -0 \\
\hline $\mathrm{C} 10$ & Z & -0.01700 & 0.040 & -0.00504 & -0.00679 & -0.03616 & 0.00 & -0 \\
\hline $\mathrm{C} 1$ & $\mathrm{X}$ & 0.00770 & -0.00138 & -0.09503 & -0.01501 & 0.00429 & -0.09902 & -0 . \\
\hline $\mathrm{C} 1$ & $\mathrm{Y}$ & 0.00 & 0.083 & 0.04044 & -0.015 & -0.038 & -0.00 & -0 \\
\hline C1 & Z & -0.01 & 0.007 & 0 & 0 & -0 . & 0.02 & -0 \\
\hline $\mathrm{N} 1$ & $\mathrm{x}$ & -0.000 & -0.05589 & -0.06012 & -0.02849 & -0.06777 & -0.054 & -0 \\
\hline N1 & Y & 0.01 & 0.07 & 0.027 & -0.00 & 0.006 & -0.00 & -0 \\
\hline N1 & Z & -0 & -0 & 0 . & & & 0.0 & \\
\hline 01 & $\mathrm{x}$ & 0.012 & -0.011 & 0.049 & -0.01138 & 0.00832 & 0.00374 & -0 \\
\hline 01 & $\mathrm{Y}$ & 0.00 & -0.0 & 0.049 & -0.02 & 0.07 & 0.01 & -0 \\
\hline 01 & Z & -0 . & 0 & -0.010 & -0.0001 & 0. & 0.05 & -0 \\
\hline 02 & $\mathrm{X}$ & 0 & -0.00002 & 0.061 & -0.01696 & 0.024 & 0.00 & -0 \\
\hline 02 & $\mathrm{Y}$ & -0.02568 & -0.027 & -0.036 & 0.015 & -0.007 & 0.003 & \\
\hline 02 & Z & -0.06239 & -0.00357 & 0.0253 & 0.01167 & 0.04187 & 0.00174 & -0 . \\
\hline 03 & $\mathrm{X}$ & 0.08168 & -0.03614 & -0.009 & 0.01656 & -0.04013 & -0.01166 & \\
\hline 03 & $\mathrm{Y}$ & 0.02 & -0.011 & -0.0081 & 0.00803 & 0.02297 & 0.01667 & -0 . \\
\hline 03 & Z & -0.06956 & -0.02937 & -0.02445 & 0.00645 & -0.02499 & -0.02592 & 0 \\
\hline $\mathrm{C} 4$ & $\mathrm{X}$ & -0.08957 & -0.00605 & -0.00352 & 0.00362 & 0.02404 & -0.03013 & -0 \\
\hline
\end{tabular}




\begin{tabular}{|c|c|c|c|c|c|c|c|c|}
\hline $\mathrm{C4}$ & Y & 0.02733 & -0.01988 & -0.01221 & 0.02632 & -0.02462 & 0.01844 & -0.06969 \\
\hline C4 & Z & .11529 & -0.01160 & -0.01890 & 0.01163 & 01187 & -0.03527 & \\
\hline C7 & $\mathrm{x}$ & -0.12551 & -0.04321 & -0.00669 & 0.00797 & 0.02907 & -0.04039 & -0.08111 \\
\hline C7 & $\mathrm{Y}$ & -0.01836 & -0.00367 & -0.00982 & 0.02433 & -0.02665 & 0.01877 & -0.05449 \\
\hline C7 & Z & 0.12060 & -0.04859 & -0.02787 & 0.01489 & 0.02991 & -0.02984 & -0.03808 \\
\hline H21 & $\mathrm{x}$ & -0.01753 & -0.06206 & -0.02380 & 0.01222 & 0.05311 & -0.03881 & -0.14338 \\
\hline H21 & $\mathrm{Y}$ & 0.01497 & 0.03779 & -0.00190 & 0.01749 & -0.04282 & 0.02455 & -0.01634 \\
\hline H21 & $\mathrm{z}$ & 0.01604 & -0.04519 & -0.01534 & 0.01337 & 0.01468 & -0.03333 & \\
\hline H4 & $\mathrm{x}$ & -0.04155 & -0.06283 & -0.00199 & 0.01462 & 0.01396 & -0.04095 & -0.08031 \\
\hline H4 & $\mathrm{Y}$ & 0.00969 & -0.03228 & -0.01417 & 0.02927 & -0.02207 & 0.01632 & -0.08208 \\
\hline H4 & Z & 0.02191 & -0.07656 & -0.04756 & 0.01299 & 0.07174 & -0.03479 & \\
\hline $\mathrm{H} 12$ & $\mathrm{x}$ & -0.02432 & -0.10726 & -0.07769 & -0.04936 & -0.13546 & -0.08634 & -0.07551 \\
\hline $\mathrm{H} 12$ & $\mathrm{Y}$ & 0.01618 & 0.10140 & 0.03371 & 0.00230 & 0.02860 & -0.00155 & 0.00494 \\
\hline $\mathrm{H} 12$ & Z & -0.00295 & -0.04378 & 0.00529 & 0.01561 & 0.03363 & 0.05622 & 0.04196 \\
\hline H2O & $\mathrm{x}$ & 0240 & -0.01287 & -0.14269 & -0.02436 & -0.00552 & -0.16484 & -0.07152 \\
\hline $\mathrm{H} 2 \mathrm{O}$ & $\mathrm{Y}$ & 00119 & 0.11866 & 0.05547 & -0.01777 & -0.05359 & 0.00231 & -0.03236 \\
\hline H 20 & Z & -0.0 & 149 & 0686 & 0.00446 & -0.00140 & 0.03269 & \\
\hline H22 & $\mathrm{x}$ & -0.0 & & -0.0 & & & -0 . & \\
\hline H22 & $\mathrm{Y}$ & 0854 & 059 & 0.03950 & -0.03643 & -0.11920 & -0.02646 & -0.07088 \\
\hline H22 & z & -0.01960 & 136 & -0.00485 & -0.01259 & -0.05693 & -0 & -0 . \\
\hline H26 & $\mathrm{x}$ & -0. & & & & & & \\
\hline H 26 & $\mathrm{Y}$ & 1389 & -0.0 & -0.00826 & -0.02702 & -0.06780 & 04417 & -0 . \\
\hline H26 & $\mathrm{z}$ & -0.01371 & 5751 & -0.01858 & -0.01512 & -0.05892 & -0 . & -0 . \\
\hline H2 & $\mathrm{x}$ & -0. & & & & & -0 & \\
\hline H2 & $\mathrm{Y}$ & -0. & -0. & -0 & 0.02738 & 0751 & 1264 & -0 \\
\hline H2 & Z & 1791 & -0. & -0. & 0.01135 & 1851 & -0 & -0 \\
\hline $\mathrm{C} 2$ & $\mathrm{x}$ & -0.0 & & -0 . & & & & \\
\hline C2 & $\mathrm{Y}$ & 0.0 & & -0. & 01832 & -0 . & & -0 \\
\hline $\mathrm{C} 2$ & Z & 0.01905 & & & -0.00606 & 0371 & -0 & \\
\hline 04 & $\mathrm{x}$ & 132 & & -0 & -0 . & 1080 & & \\
\hline 04 & $\mathrm{Y}$ & -0. & & -0 & 5688 & $-0 . c$ & 0845 & 291 \\
\hline 04 & Z & -0.0 & 397 & 620 & 9681 & 0307 & -0 . & 343 \\
\hline 05 & $\mathrm{x}$ & & & & 123 & -0 & & \\
\hline 05 & $\mathrm{Y}$ & -0.0 & 340 & -0. & -0.04376 & -0.02856 & 1168 & -0 . \\
\hline 05 & Z & 0.00307 & 782 & 0.0 & -0.16140 & -0.00214 & -0.05229 & 0.05563 \\
\hline H3 & $\mathrm{x}$ & & & -0 . & & & & \\
\hline H3 & $\mathrm{Y}$ & -0.0 & & & -0.04757 & -0.0 & 399 & 0482 \\
\hline H3 & z & -0.02515 & 0.07357 & 0.03843 & -0.16814 & -0.00679 & -0.08925 & 0.09738 \\
\hline \multicolumn{2}{|c|}{ requencies } & 180 & & & & & & \\
\hline & & 4.66 & & & 3.05 & 2.94 & 5.43 & 2.55 \\
\hline e1 & $\mathrm{x}$ & -0.00633 & -0.00544 & -0.002 & 0.00847 & -0.01046 & & \\
\hline$e 1$ & $\mathrm{Y}$ & -0. & & -0 & & & -0 & -0 \\
\hline Re1 & z & 0.01179 & -0.01545 & 0.00975 & -0.00664 & 0.00486 & -0.00113 & 0.01127 \\
\hline N2 & $\mathrm{x}$ & -0.04265 & & -0.01 & -0.10 & 5124 & -0.04208 & -0 \\
\hline $\mathrm{N}$ & $\mathrm{Y}$ & 0.0 & -0. & & -0.0 & -0 & -0 & \\
\hline N2 & z & 0.00880 & 0.07695 & -0.03929 & 0.08076 & -0.01231 & 0.05177 & -0.01397 \\
\hline C10 & $\mathrm{x}$ & 0.03895 & -0.04024 & -0.00824 & $0.03 \xi$ & -0.01155 & -0.01740 & 0.00327 \\
\hline & $\mathrm{Y}$ & -0.0 & -0. & & -0.0 & & -0 & \\
\hline $\mathrm{C} 10$ & Z & 0 . & & -0.03533 & 0.05982 & -0.00900 & 0.04392 & -0.01614 \\
\hline $\mathrm{C} 1$ & $\mathrm{x}$ & 0.04770 & -0.01805 & 0.05092 & 0.00363 & -0.05174 & 0.03653 & -0.00298 \\
\hline $\mathrm{C} 1$ & $\mathrm{Y}$ & -0.01988 & -0.0 & -0.0 & -0.02 & & & \\
\hline $\mathrm{C} 1$ & Z & & & -0.01528 & 0.01325 & -0.05557 & -0.0 & -0.0 \\
\hline N1 & $\mathrm{x}$ & -0.04395 & 0.05559 & -0.03015 & -0.07418 & 0.08021 & -0.05015 & 0.00603 \\
\hline & $\mathrm{Y}$ & & -0.0 & & -0.00734 & -0.04763 & & \\
\hline N1 & $\mathrm{z}$ & & & -0.00086 & & -0.09138 & -0 & -0 \\
\hline 01 & $\mathrm{x}$ & 0.08992 & 0.03107 & -0.01302 & 0.00358 & 0.01228 & 0.02056 & 0.05013 \\
\hline 01 & Y & & -0.00431 & -0.04917 & & & & \\
\hline 01 & Z & -0.1 & -0.0 & -0.0 & & & & -0. \\
\hline $\mathrm{O} 2$ & $\mathrm{x}$ & 0.03957 & -0.01253 & -0.00255 & -0.00699 & -0.00018 & -0.04777 & -0.08576 \\
\hline $\mathrm{O} 2$ & $\mathrm{Y}$ & -0.04186 & 0.02392 & 0.02231 & -0.00468 & -0.00613 & 0.06562 & 0.08670 \\
\hline
\end{tabular}




\begin{tabular}{|c|c|c|c|c|c|c|c|c|}
\hline $\mathrm{O} 2$ & z & 0.05014 & 0.02824 & 0.09629 & -0.10355 & 0.01639 & 0.06793 & -0.01697 \\
\hline 03 & $\mathrm{x}$ & 0.00361 & 0.00352 & 0.02531 & 0.00018 & 0.03955 & 0.08664 & -0.12380 \\
\hline 03 & $\mathrm{Y}$ & -0.03690 & 0.04088 & & -0.01076 & -0.03877 & 0.05300 & \\
\hline 03 & Z & 0.06240 & -0.05361 & -0.06563 & 0.00857 & 0.08832 & -0.03405 & -0.03878 \\
\hline C4 & $\mathrm{x}$ & -0.02530 & 0.00221 & 0.04781 & 0.02936 & 0.01747 & -0.00288 & -0.05489 \\
\hline C4 & $\mathrm{Y}$ & 0.02149 & -0.00488 & -0.00733 & 0.00845 & 0.00763 & -0.01922 & 0.01601 \\
\hline C4 & Z & -0.01172 & 0.00715 & -0.01012 & 0.02069 & 0.01463 & -0.04402 & \\
\hline C7 & $\mathrm{x}$ & -0.01577 & -0.03762 & -0.07544 & -0.03499 & -0.05791 & 0.00216 & -0.00561 \\
\hline C7 & $\mathrm{Y}$ & 0.01943 & 0.00490 & 0.02828 & 0.02747 & 0.03104 & -0.03095 & 0.02858 \\
\hline C7 & Z & -0.00816 & -0.00679 & -0.06297 & -0.03704 & -0.02576 & 0.02671 & \\
\hline H21 & $\mathrm{x}$ & -0.04749 & -0.03006 & -0.11599 & -0.08104 & -0.13849 & 0.03337 & \\
\hline H21 & $\mathrm{Y}$ & 0.01293 & 0.04555 & 0.15987 & & 0.12134 & -0.07771 & \\
\hline H21 & Z & 0.02097 & -0.02601 & -0.06827 & -0.02371 & 0.01656 & 0.01276 & -0.04478 \\
\hline H4 & $\mathrm{x}$ & -0.00483 & -0.05045 & -0.12214 & -0.05850 & -0.07503 & 0.03398 & -0.04114 \\
\hline H 4 & $\mathrm{Y}$ & 0.02383 & -0.0 & -0.0 & -0.0 & -0.02373 & 0118 & \\
\hline H4 & Z & -0.02180 & -0.0 & -0. & -0.1 & -0.12274 & 0.04041 & \\
\hline H12 & $\mathrm{x}$ & -0.05510 & 0.10645 & -0.00305 & -0.13856 & 0.09357 & -0.02613 & -0.00481 \\
\hline H12 & $\mathrm{Y}$ & 207 & -0.0 & 0.0 & & -0.04286 & & \\
\hline $\mathrm{H} 12$ & z & 0.0 & 0.0 & -0.0 & & -0.1 & -0 . & \\
\hline H2O & $\mathrm{X}$ & 0.11475 & -0.03591 & 0.12926 & 0.01831 & -0.13453 & 0.12898 & -0.00995 \\
\hline H2O & $\mathrm{Y}$ & -0.0 & -0.0 & -0. & & & & \\
\hline H2O & Z & -0.0 & & -0 & & -0 . & -0 & \\
\hline H22 & $\mathrm{x}$ & 0.08491 & -0.7 & -0.0 & & 0.00261 & -0.03569 & 1384 \\
\hline H22 & $\mathrm{Y}$ & -0.0 & 0. & 0.0 & -0. & & & \\
\hline H 22 & z & -0 & 0. & -0 . & & & & \\
\hline H26 & $\mathrm{x}$ & -0.0 & 3395 & -0.08200 & -0.11 & 0.12373 & -0.09891 & 0. \\
\hline H26 & $\mathrm{Y}$ & 0.02990 & -0.06181 & 0.08616 & -0.03775 & -0.09922 & -0.06103 & \\
\hline H26 & Z & & & -0 . & & -0 & & \\
\hline H2 & $\mathrm{x}$ & -0 & 0.0 & 0 & & 3436 & -0.017 & -0 . \\
\hline $\mathrm{H} 2$ & $\mathrm{Y}$ & & -0. & -0 & -0 & -0 . & & \\
\hline H2 & Z & -0.0 & & & & & -0 . & \\
\hline C2 & $\mathrm{x}$ & -0.0 & 0.0 & 0. & & 579 & -0.01786 & -0 \\
\hline $\mathrm{C} 2$ & $\mathrm{Y}$ & & -0.0 & -0. & & & -0 & \\
\hline $\mathrm{C} 2$ & Z & -0. & 0 & & & & -0 & \\
\hline 04 & $\mathrm{x}$ & -0.00414 & 0.00840 & 0.0 & 0.0 & 0.03398 & -0.02792 & -0 \\
\hline 04 & $\mathrm{Y}$ & 0.03316 & -0.02482 & -0.0 & -0.0 & -0.03728 & 0.02702 & -0 \\
\hline 04 & Z & -0 . & & & & & -0 . & \\
\hline 05 & $\mathrm{x}$ & -0.00304 & -0.00336 & -0.01711 & -0.01538 & -0.01244 & 0.01380 & \\
\hline 05 & $\mathrm{Y}$ & 0.01842 & -0.00387 & 0.02455 & & 0.03261 & -0.03410 & -0. \\
\hline 05 & Z & -0 . & -0 & -0 . & -0 & & & \\
\hline H3 & $\mathrm{x}$ & 587 & -0.0 & -0.0 & -0 . & -0 . & 2954 & \\
\hline H3 & $\mathrm{Y}$ & 503 & -0.0 & -0.0 & -0 . & -0.0 & 815 & -0 \\
\hline H3 & Z & -0.04663 & -0.01255 & -0.04007 & -0.02818 & -0.05026 & 0.05531 & \\
\hline \multicolumn{2}{|c|}{ Erequencies } & & & & & & & \\
\hline & itioc & & & & & & & \\
\hline & $x$ & -0.004 & & -0.00 & & -0.0 & & \\
\hline$e 1$ & $\mathrm{Y}$ & -0.02124 & 0.00572 & -0.00217 & -0.00400 & 0.00242 & -0.00041 & -0.00150 \\
\hline & Z & -0.00330 & -0.0 & & & & & \\
\hline & $\mathrm{x}$ & & & -0 & -0 & & -0 . & \\
\hline $\mathrm{N}$ & $\mathrm{Y}$ & 0.07601 & 0.00461 & 0. & 0 & 5302 & -0.13718 & 0743 \\
\hline & Z & -0.0 & & & & & -0 & \\
\hline C & $\mathrm{x}$ & & -0.0 & & & & -0 . & \\
\hline C & $\mathrm{Y}$ & & -0.0 & -0.0 & -0.0 & -0.0 & 670 & -0 . \\
\hline & Z & 0.0 & & -0.0 & 0.0 & & -0 & \\
\hline & $\mathrm{x}$ & -0.0 & & -0.0 & -0.0 & & & \\
\hline $\mathrm{C} 1$ & $\mathrm{Y}$ & -0.0 & -0.01435 & & & -0.0 & 2944 & 009 \\
\hline & Z & & 0.0 & & & 84 & -0.02987 & \\
\hline & $\mathrm{x}$ & & -0.02661 & & & -0.01887 & 0.05850 & \\
\hline & $\mathrm{Y}$ & & & -0.02272 & -0.01630 & -0.04876 & 0.12591 & \\
\hline $\mathrm{N}$ & Z & 0.08099 & 0.02140 & -0.00709 & 0.01 & -0.02818 & 0.07882 & -0 . \\
\hline
\end{tabular}




\begin{tabular}{|c|c|c|c|c|c|c|c|c|}
\hline 01 & $\mathrm{X}$ & 0.04321 & -0.03460 & 0.00199 & -0.02036 & -0.00157 & 0.00357 & 0.00316 \\
\hline 01 & $\mathrm{Y}$ & 0.12207 & 0.00318 & -0.01497 & -0.00658 & 0.00199 & 0.00198 & \\
\hline 01 & Z & 0.01685 & 0.04793 & -0.01789 & 0.03913 & 0.00212 & 0.00459 & -0.00257 \\
\hline $\mathrm{O} 2$ & $\mathrm{x}$ & -0.09803 & 0.03250 & 0.01267 & -0.01774 & -0.00389 & -0.01010 & -0.00745 \\
\hline $\mathrm{O} 2$ & $\mathrm{Y}$ & & -0.00751 & -0.01510 & 0.04565 & 0.01373 & 0.00648 & \\
\hline $\mathrm{O} 2$ & Z & -0.05124 & 0.06063 & 0.03411 & 0.10929 & 0.01672 & 0.02102 & -0.0247 \\
\hline 03 & $\mathrm{x}$ & 0.06544 & 0.03915 & 0.03652 & -0.04542 & 0.01774 & 0.00135 & -0.00532 \\
\hline 03 & $\mathrm{Y}$ & -0.01204 & -0.01788 & 0.05201 & -0.05728 & -0.00246 & 0.00380 & \\
\hline 03 & Z & 1511 & 0.05955 & -0.05963 & 0.03686 & -0.00779 & 0.01206 & -0.012 \\
\hline C4 & $\mathrm{x}$ & 0.00107 & -0.07527 & -0.00317 & -0.00266 & -0.06417 & -0.02366 & 0.10994 \\
\hline C4 & $\mathrm{Y}$ & -0.00171 & 0.02800 & 0.00536 & -0.00397 & -0.08217 & -0.03473 & -0.01835 \\
\hline C4 & Z & -0.00268 & 0.09731 & -0.00143 & -0.00374 & 0.06210 & 0.02816 & \\
\hline C7 & $\mathrm{x}$ & -0.00122 & 0.02728 & 0.03327 & -0.08863 & 0.03908 & 0.00149 & \\
\hline C7 & $\mathrm{Y}$ & 0.00094 & 0.04715 & 0.00534 & 0.00094 & -0.11446 & -0.03843 & 0.01202 \\
\hline C7 & $\mathrm{Z}$ & -0.01596 & -0.09894 & -0.04505 & & -0.05732 & -0.0 & \\
\hline H21 & $\mathrm{x}$ & -0.01298 & -0.0 & 0.0 & -0.09635 & 0.12122 & 0.01296 & -0 \\
\hline H21 & $\mathrm{Y}$ & 0.01971 & 2818 & 393 & -0.01628 & -0.17284 & -0.05565 & 0 \\
\hline H21 & $\mathrm{z}$ & -0.0 & -0.0 & -0.0 & & -0.1 & -0 & \\
\hline H4 & $\mathrm{x}$ & 0.0 & 0.0 & 0.0 & -0.10172 & 0.06046 & 0.00328 & -0 . \\
\hline H4 & $\mathrm{Y}$ & -0.00494 & 1482 & 0.0 & -0.00082 & -0.07087 & -0.03043 & -0 . \\
\hline H4 & $\mathrm{z}$ & -0.0 & -0.1 & -0.0 & 3064 & 0874 & 27 & -0 \\
\hline $\mathrm{H} 12$ & $\mathrm{X}$ & -0. & -0.0 & -0. & -0.04304 & -0. & 1182 & \\
\hline $\mathrm{H} 12$ & $\mathrm{Y}$ & 0.03036 & -0.00241 & 0.0 & 0.00736 & -0.02897 & 8388 & -0 \\
\hline H12 & Z & 577 & 0. & -0 & & -0 & & -0 . \\
\hline H2O & $\mathrm{X}$ & -0.0 & 0.0 & -0 & -0.1 & -0.0 & & \\
\hline H2O & $\mathrm{Y}$ & -0.04810 & -0.03420 & 0.1 & 0.04408 & 0.04610 & -0.7 & \\
\hline H2O & Z & 390 & 0. & 0. & & & & -0 \\
\hline H 22 & $\mathrm{x}$ & 1799 & -0.0 & 0.3 & & -0.0 & & \\
\hline H22 & $\mathrm{Y}$ & -0.03174 & 0373 & -0.12408 & -0.06445 & -0.02180 & 5240 & -0.00944 \\
\hline H22 & Z & -0. & 0.0 & -0 & -0 . & -0. & & -0 . \\
\hline H 26 & $\mathrm{x}$ & 101 & -0.0 & -0 & -0.0 & -0.0 & -0 . & \\
\hline H26 & Y & 0.08474 & 0.01479 & $-0 . c$ & 0.04104 & 0.07691 & -0.20119 & 0.00362 \\
\hline H 26 & z & -0. & 0.0 & 0. & & -0.0 & & -0 . \\
\hline H2 & $\mathrm{x}$ & -0. & -0.0 & -0 & & -0 . & -0 & \\
\hline H2 & $\mathrm{Y}$ & -0.00068 & 4125 & 0.02354 & -0.02271 & -0.13881 & -0.05843 & 0.00757 \\
\hline H2 & $\mathrm{z}$ & 0.0 & 0.0 & -0.0 & -0.013 & & & -0 . \\
\hline C2 & $\mathrm{X}$ & & -0. & -0 & & & & -0 \\
\hline C2 & $\mathrm{Y}$ & -0.00539 & -0.01252 & -0.01033 & 0.00905 & 0.01663 & 0311 & -0.02518 \\
\hline $\mathrm{C} 2$ & Z & & & & -0. & & & \\
\hline 04 & $\mathrm{x}$ & -0 & -0. & -0 . & & & & \\
\hline 04 & $\mathrm{Y}$ & -0.01083 & -0.02403 & -0.00425 & 0.01575 & 0.06213 & 2884 & 0. \\
\hline 04 & Z & & -0.03921 & -0.01013 & -0.00879 & -0.0 & -0 & -0 \\
\hline 05 & $\mathrm{x}$ & & & & -0 . & -0 . & -0 & -0 \\
\hline 05 & $\mathrm{Y}$ & -0.0 & -0.0 & -0.0 & 0 & 278 & 2247 & -0 \\
\hline 05 & z & 0598 & 0.0 & 0. & -0.01169 & -0.05658 & -0.02308 & 0. \\
\hline H3 & $\mathrm{x}$ & & & & & & & \\
\hline H3 & $\mathrm{Y}$ & 124 & -0.0 & -0.0 & -0.00250 & -0.09815 & -0 & -0.32 \\
\hline H3 & Z & 0.01724 & -0.10087 & 0.00345 & -0.02481 & -0.13798 & -0.07279 & -0.50035 \\
\hline & & & & & & & & \\
\hline & ities & & 1.13 & & 91 & 6.35 & 1.95 & 62.71 \\
\hline Re1 & $\mathrm{X}$ & & & -0.0 & -0.0 & & & \\
\hline $\mathrm{Re} 1$ & $\mathrm{Y}$ & & -0.0 & 0.0 & & -0. & & \\
\hline Re1 & Z & 0.00018 & -0.00019 & 0.0 & 546 & -0.00010 & 0.00282 & -0. \\
\hline N2 & $\mathrm{x}$ & -0.0 & -0.02787 & & -0.0 & -0.03740 & -0.00688 & \\
\hline N2 & $\mathrm{Y}$ & -0.0 & & -0.0 & -0.0 & -0.06109 & & \\
\hline N2 & Z & -0.00028 & 0.00105 & 0.0 & 0.003 & -0.06301 & -0.00368 & 0.0072 \\
\hline 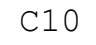 & $\mathrm{x}$ & 050 & -0.05011 & 0.0 & -0.00342 & 0.02663 & -0.00335 & \\
\hline $\mathrm{C} 10$ & Y & & & -0.00250 & & & & \\
\hline $\mathrm{C} 10$ & Z & 0.00002 & 0.00317 & & 0.00711 & -0.07385 & -0.00304 & \\
\hline $\mathrm{C} 1$ & $\mathrm{x}$ & 0.00026 & -0.03659 & 261 & -0.00605 & 0.05331 & 0.00831 & -0.0241 \\
\hline
\end{tabular}




\begin{tabular}{|c|c|c|c|c|c|c|c|c|}
\hline $\mathrm{C} 1$ & $\mathrm{Y}$ & -0.00109 & 0.02457 & -0.00142 & -0.00310 & 0.03761 & -0.00214 & 0.01357 \\
\hline $\mathrm{C} 1$ & Z & -0.00072 & 0.01752 & -0.00092 & 0.00208 & 0.12625 & 0.01097 & \\
\hline N1 & $\mathrm{x}$ & 0.00090 & -0.04254 & 0.00426 & 0.00352 & -0.03195 & -0.00070 & -0.00315 \\
\hline N1 & $\mathrm{Y}$ & 0.00262 & -0.00843 & 0.00145 & 0.00611 & -0.09804 & -0.00714 & 0.01388 \\
\hline N1 & & 0.00176 & -0.00717 & 0.00149 & 0.00642 & 0.00716 & 0.00290 & \\
\hline 01 & $\mathrm{x}$ & -0.00071 & 0.00039 & 0.00205 & 0.01163 & -0.00017 & 0.00832 & -0.00354 \\
\hline 01 & $\mathrm{Y}$ & 0.00045 & -0.00202 & -0.00104 & -0.00862 & -0.00302 & -0.00513 & 0.00593 \\
\hline $\mathrm{O} 1$ & & -0.00014 & 0.00561 & 0.00078 & 0.01058 & 0.00075 & 0.00569 & \\
\hline O2 & $\mathrm{x}$ & -0.00268 & -0.00269 & 0.00640 & 0.05004 & 0.02174 & 0.01859 & 0.034 \\
\hline 02 & $\mathrm{Y}$ & -0.00299 & -0.00093 & 0.02565 & 0.09177 & 0.01979 & 0.02184 & 0.04171 \\
\hline 02 & Z & 0.00216 & -0.00334 & -0.00439 & 0.01208 & -0.01403 & -0.00084 & \\
\hline 03 & & 0.00404 & 0.00154 & -0.00909 & 0.01169 & -0.01523 & 0.01905 & -0.04272 \\
\hline 03 & $\mathrm{Y}$ & -0.00177 & 0.00984 & -0.01767 & -0.13311 & 0.02812 & -0.07284 & 0.10555 \\
\hline 03 & Z & -0.00184 & -0.00363 & -0.01823 & -0.06563 & 0.02194 & -0.02806 & 0.05085 \\
\hline C4 & & -0.05652 & -0.01168 & 0.01237 & 0.01017 & -0.00722 & -0.05321 & -0.08940 \\
\hline C4 & $\mathrm{Y}$ & 0.00137 & -0.00329 & -0.01049 & 0.00994 & -0.00348 & 0.05498 & \\
\hline C4 & Z & -0.07884 & -0.00131 & -0.02339 & 0.04529 & 0.01086 & -0.07907 & 0.02406 \\
\hline C7 & & -0.01 & -0.0 & 0.01900 & 3267 & & -0.0 & \\
\hline C7 & Y & -0.03338 & -0.00119 & -0.03826 & -0.04535 & -0.00603 & 0.06662 & \\
\hline C7 & Z & -0.00661 & 0.00441 & 0.01411 & 0.00049 & 0.00044 & -0.02987 & -0.00 \\
\hline H21 & $\mathrm{x}$ & & -0.0 & -0.10843 & -0 & -0 & & -0 . \\
\hline H 21 & $\mathrm{Y}$ & -0.21899 & -0.02257 & -0.07229 & 0.09097 & 0020 & -0.20274 & -0.1 \\
\hline H21 & Z & -0.00188 & 0.02107 & 0.13647 & -0.00836 & 5015 & -0.11546 & \\
\hline H4 & $\mathrm{x}$ & 595 & 0.0 & 0.1 & 401 & 1162 & & \\
\hline H 4 & & 850 & 0.0 & 0.00757 & -0.06696 & -0 & 030 & \\
\hline H4 & Z & 838 & 0.0 & -0.15566 & -0.32102 & -0.02908 & 6189 & -0.14546 \\
\hline H12 & $\mathrm{x}$ & -0.0 & 0.4 & -0.0 & 2282 & -0.1 & -0. & \\
\hline H12 & $\mathrm{Y}$ & 0.00433 & -0.18547 & 0.01565 & -0.00425 & 0.01817 & 0 & -0 \\
\hline H12 & Z & 0.00458 & -0.07358 & 0.00644 & 0.01619 & -0.21288 & -0.00621 & -0.00589 \\
\hline H2O & $\mathrm{x}$ & -0. & 0.2 & -0.03325 & 107 & -0.0 & -0 & \\
\hline H2O & $\mathrm{Y}$ & -0.0 & -0.1 & 059 & -0.02027 & 799 & 0 & \\
\hline H2O & z & 0.00019 & -0.01664 & 0.00188 & 0.00154 & 0.13126 & 0.01177 & -0.01474 \\
\hline H22 & $\mathrm{x}$ & -0.00 & 0.3 & -0.03424 & 003 & 576 & & -0 . \\
\hline H22 & $\mathrm{Y}$ & & -0 & 0 & -0. & 964 & & -0 \\
\hline H22 & z & 0.00265 & -0.06208 & 0.00659 & 0.00956 & -0.07558 & -0.00123 & -0.00081 \\
\hline H26 & $\mathrm{x}$ & -0.00 & 0.4 & -0.04058 & 0.02235 & 0.02577 & & -0 \\
\hline H26 & $\mathrm{Y}$ & & -0 & & -0. & -0 & -0 & \\
\hline H26 & Z & 0.00377 & -0.06324 & 0.00553 & 0.00754 & 0.16029 & 0.01527 & -0.02582 \\
\hline H2 & $\mathrm{x}$ & -0.0 & & & & & & \\
\hline H2 & $\mathrm{Y}$ & -0. & -0.0 & -0.0 & 0 & -0. & -0. & -0 . \\
\hline H2 & Z & -0.07947 & -0.02003 & -0.27367 & -0.19729 & -0.03697 & -0.17243 & -0.34946 \\
\hline C2 & $\mathrm{x}$ & -0.02633 & -0.006 & -0.09921 & 0.02619 & 0.00379 & 0.0 & \\
\hline C2 & $\mathrm{Y}$ & & & & -0.0 & -0 & -0 . & -0 . \\
\hline C2 & Z & 960 & 0.0 & 621 & -0.08847 & -0.01294 & 5836 & -0.04112 \\
\hline 04 & $\mathrm{X}$ & -0.00249 & 0.0 & 554 & -0.03024 & -0.00362 & 7380 & 2399 \\
\hline O4 & $\mathrm{Y}$ & -0.0 & -0.0 & -0. & & -0 & & \\
\hline 04 & Z & 306 & -0.0 & -0.04939 & 1131 & 237 & 0. & \\
\hline 05 & $\mathrm{X}$ & 0.03874 & 0.00703 & 0.04648 & 0.00151 & 0.00204 & -0.03960 & -0.01126 \\
\hline 05 & $\mathrm{Y}$ & & & & & & -0 & \\
\hline 05 & Z & 12 & -0.0 & -0.05 & 0.01830 & 191 & 0 & 0.0 \\
\hline H3 & $\mathrm{X}$ & 0.44591 & -0.01722 & -0.12276 & 0.09077 & 0.01031 & -0.18108 & -0.06432 \\
\hline H3 & $\mathrm{Y}$ & $-0.0^{-}$ & & & & & & \\
\hline H3 & Z & -0.4 & & & -0.0 & -0.0 & -0.01519 & -0.0154 \\
\hline & & & & & & & & \\
\hline & & & & & & & & \\
\hline $\operatorname{Re} 1$ & $\mathrm{x}$ & 0.00122 & -0.01021 & -0.00015 & 0.00440 & -0.01558 & 0.00033 & 0.00093 \\
\hline $\operatorname{Re} 1$ & $\mathrm{Y}$ & -0.00247 & -0.01504 & -0.00152 & 0.00243 & 0.00700 & -0.00023 & -0.00034 \\
\hline $\operatorname{Re} 1$ & Z & -0.00139 & -0.00141 & -0.00052 & -0.00001 & -0.00960 & & \\
\hline N2 & $\mathrm{x}$ & -0.02239 & -0.00320 & & 0.00019 & 0.00178 & -0.06709 & -0.00916 \\
\hline N2 & Y & 0.03353 & 0.00921 & 0.02306 & -0.00292 & 0.00544 & 0.03366 & 0.00381 \\
\hline
\end{tabular}




\begin{tabular}{|c|c|c|c|c|c|c|c|c|}
\hline & Z & 0.01760 & 0.00886 & 0.02807 & -0.00202 & -0.00018 & 0.01181 & -0.00010 \\
\hline C10 & $\mathrm{X}$ & -0.09127 & -0.01375 & 0.05639 & 0.00087 & 0.00425 & 0.09863 & 0.04130 \\
\hline C10 & $\mathrm{Y}$ & 0.00908 & -0.01558 & 0.14577 & -0.00043 & -0.00473 & 04206 & \\
\hline $\mathrm{C} 10$ & Z & 0.02603 & 0.01299 & -0.00255 & -0.00283 & -0.00137 & -0.01521 & -0.00205 \\
\hline C1 & $\mathrm{x}$ & 0.07547 & 0.00248 & -0.06569 & 0.00148 & -0.00426 & 0.00819 & -0.11027 \\
\hline $\mathrm{C} 1$ & $\mathrm{Y}$ & -0.04431 & -0.00431 & -0.11124 & 0.00314 & 0.00446 & & \\
\hline $\mathrm{C} 1$ & $\mathrm{z}$ & -0.03817 & -0.01183 & -0.09394 & 0.00351 & -0.00124 & -0.00634 & 0. \\
\hline N1 & $\mathrm{x}$ & 0.04626 & 0.01120 & -0.01165 & -0.00349 & 0.00201 & -0.02121 & 0.06421 \\
\hline & $\mathrm{Y}$ & 0.00853 & 0.01252 & -0.03308 & -0.00239 & -0.00454 & 0.00763 & -0.02217 \\
\hline & Z & -0.00564 & 0.00137 & 0.01117 & -0.00113 & -0.00370 & 0.00274 & \\
\hline 01 & $\mathrm{x}$ & -0.00307 & 0.01055 & -0.00004 & -0.01351 & 0.19955 & -0.00543 & -0.01105 \\
\hline 01 & $\mathrm{Y}$ & 0.00133 & 0.00088 & -0.00008 & 0.00694 & -0.07910 & 0.00205 & 0.00414 \\
\hline $\mathrm{O} 1$ & Z & -0.00161 & 0.00735 & 0.00214 & -0.00692 & 0.10020 & -0 & \\
\hline 02 & $\mathrm{x}$ & -0.01032 & 0.13239 & 0.00367 & -0.05095 & -0.01687 & 0.00073 & -0.00037 \\
\hline $\mathrm{O} 2$ & $\mathrm{Y}$ & -0.00808 & 0.13472 & 0.00320 & -0.03256 & -0.02000 & 0.00122 & -0.00037 \\
\hline $\mathrm{O} 2$ & Z & & -0.0 & -0.0 & & & -0 & \\
\hline 03 & $\mathrm{x}$ & -0.00786 & -0.01577 & -0.00533 & 0.00761 & -0.00208 & 0.00192 & \\
\hline 03 & $\mathrm{Y}$ & 0.02997 & 0.03893 & 0.01269 & -0.00278 & 0.01632 & -0.00181 & -0 . \\
\hline 03 & Z & & & & & & -0 . & \\
\hline C4 & $\mathrm{x}$ & -0.02427 & 0.04607 & -0.00098 & 0.01140 & -0.00529 & 0.00254 & -0. \\
\hline C4 & $\mathrm{Y}$ & -0.00048 & -0.00421 & 0.00267 & -0.00458 & -0.00244 & -0.00268 & 0.00472 \\
\hline C4 & Z & 0. & -0 & 259 & & & & -0 \\
\hline C7 & $\mathrm{x}$ & 0.00990 & -0.0 & 0341 & -0.0 & 0.00642 & -0 & \\
\hline C7 & $\mathrm{Y}$ & -0.00223 & 0.0 & -0.00248 & 0.01253 & 0.00243 & 0.00234 & -0.00561 \\
\hline C7 & Z & & -0.0 & -0 . & -0 . & -0 & & -0 \\
\hline H21 & $\mathrm{x}$ & -0.08290 & 0.1 & -0. & & -0.02045 & 0524 & -0 \\
\hline H21 & $\mathrm{Y}$ & -0.01742 & -0.00632 & -0.0 & -0.05865 & 0.00273 & -0.00589 & 0.02635 \\
\hline H21 & Z & & -0 & & -0 . & & -0 & \\
\hline H4 & $\mathrm{x}$ & 462 & -0.2 & -0. & -0.2 & $-0.03 \varepsilon$ & & -0.0437 \\
\hline $\mathrm{H} 4$ & Y & 0.01272 & -0.05285 & -0.0 & 0.00744 & -0.01748 & 0.01286 & -0.04179 \\
\hline & Z & -0.0 & 0.2 & & & & -0 & \\
\hline $\mathrm{H} 12$ & $\mathrm{x}$ & -0 . & -0. & -0 . & & -0 . & & -0 . \\
\hline H12 & $\mathrm{Y}$ & 583 & 0. & -0.10373 & -0.00487 & 0069 & -0.07380 & 6744 \\
\hline $\mathrm{H} 12$ & Z & & 0. & & -0 & & -0 . & \\
\hline H2O & $\mathrm{x}$ & -0.7 & -0.0 & -0 . & & & -0 & \\
\hline H2O & $\mathrm{Y}$ & 986 & -0.0 & -0. & 0.0 & -0.00094 & 0.03236 & -0.25144 \\
\hline 20 & z & -0.0 & -0.0 & -0 & & -0 & & -0 . \\
\hline H22 & $\mathrm{x}$ & & 0 & & -0 . & -0. & -0 . & -0 . \\
\hline H22 & $\mathrm{Y}$ & -0.09024 & -0.0 & 0 . & & & 242 & 680 \\
\hline H22 & Z & & & & -0 & & & \\
\hline $\mathrm{H}$ & $\mathrm{x}$ & & 0. & & -0 . & & & \\
\hline H26 & $\mathrm{Y}$ & -0.1 & -0.0 & -0.0 & & 0044 & -0.2 & -0 \\
\hline H26 & Z & & -0.0 & & & & -0 & -0 \\
\hline & $\mathrm{x}$ & & & & & & -0 . & -0. \\
\hline H2 & $\mathrm{Y}$ & -0.01570 & -0.0 & -0.00 & -0.23 & 0161 & -0 & \\
\hline H2 & Z & -0.1 & 0.0 & -0.0 & -0.5 & -0.03599 & 1491 & -0.03135 \\
\hline & $\mathrm{x}$ & & -0.0 & & -0 . & & & \\
\hline $\mathrm{C} 2$ & $\mathrm{Y}$ & -0 & 0 & & -0 . & -0 & -0 . & -0 . \\
\hline C2 & Z & -0.01547 & 0.01934 & -0.0 & -0.0 & 0.00040 & -0.00025 & 0254 \\
\hline 04 & $\mathrm{X}$ & & -0.0 & & -0 . & & & \\
\hline 04 & $\mathrm{Y}$ & & -0.0 & & -0 . & -0. & -0 . & -0 . \\
\hline 04 & Z & 0.00650 & -0.01261 & 0.00085 & -0.01237 & -0.00136 & -0.00107 & 0.00247 \\
\hline 05 & $\mathrm{x}$ & -0.00025 & & -0.0 & & & & \\
\hline 05 & $\mathrm{Y}$ & & -0.0 & -0.0 & & & & -0 . \\
\hline 05 & Z & 0.00242 & -0.00239 & 0.00023 & -0.00079 & 0.00026 & -0.00008 & 0023 \\
\hline H3 & $\mathrm{x}$ & -0.01031 & 0.03842 & & & -0.00442 & 0.00128 & -0.0 \\
\hline H3 & $\mathrm{Y}$ & & & -0.0 & & -0.00080 & & \\
\hline H3 & z & -0.00151 & -0.00333 & -0.00047 & 0.01109 & 0.00065 & -0.00054 & -0.00169 \\
\hline & & & & & & & & \\
\hline & & & & & & .45 & 39.77 & \\
\hline
\end{tabular}




\begin{tabular}{|c|c|c|c|c|c|c|c|c|}
\hline Re1 & $\mathrm{X}$ & 0.00025 & -0.00219 & 0.00064 & -0.00012 & 0.00033 & 0.00120 & -0.00093 \\
\hline Re1 & $\mathrm{Y}$ & 0.00083 & 0.00277 & 0.00018 & 0.00025 & 0.00088 & -0.00036 & -0.00001 \\
\hline Re1 & Z & 0.00091 & 0.00301 & -0.00075 & -0.00082 & 0.00011 & -0.00101 & 0.00081 \\
\hline N2 & $\mathrm{X}$ & -0.00196 & -0.00023 & -0.00152 & -0.01343 & 0.01765 & -0.00199 & 0.00126 \\
\hline N2 & Y & -0.00101 & -0.00151 & -0.00055 & -0.02248 & 0.02729 & -0.00125 & \\
\hline N2 & Z & -0.00038 & 0.00031 & -0.00248 & 0.00232 & 0.02921 & -0.00382 & \\
\hline C10 & $\mathrm{X}$ & -0.00187 & 0.00020 & -0.00017 & 0.00663 & -0.01293 & 0.00002 & \\
\hline $\mathrm{C} 10$ & Y & 0.00031 & 0.00087 & -0.00218 & 0.00796 & -0.01729 & -0.00139 & \\
\hline $\mathrm{C} 10$ & Z & 0.00044 & -0.00014 & 0.00203 & 0.01580 & -0.00455 & 0.00353 & -0.00 \\
\hline $\mathrm{C} 1$ & $\mathrm{X}$ & 0.01282 & 0.00019 & 0.00109 & -0.00232 & -0.00869 & 0.00033 & \\
\hline $\mathrm{C} 1$ & $\mathrm{Y}$ & -0.00462 & -0.00046 & 0.00070 & -0.00608 & -0.02576 & & \\
\hline $\mathrm{C} 1$ & Z & 0.00046 & 0.00001 & 0.00292 & 0.01316 & 0.00070 & & -0 . \\
\hline & $\mathrm{X}$ & -0.00801 & 0.00024 & -0.00043 & 0.00025 & 0.01731 & -0.00 & -0 . \\
\hline N1 & $Y$ & 0.00361 & 0.00030 & 0.00192 & -0.00110 & 0.04587 & 0.0 & -0 . \\
\hline & Z & & 0.00051 & 0152 & 0.01544 & -0.00473 & -0 . & \\
\hline 01 & $\mathrm{X}$ & -0 & -0.00429 & 0.00385 & 0.00287 & 0.00048 & & -0 . \\
\hline O1 & $Y$ & 0 & 0.00095 & -0.00151 & -0.00079 & -0.00122 & -0.0 & \\
\hline 01 & Z & -0 & -0 & 0227 & & -0 . & & -0 \\
\hline 02 & $\mathrm{X}$ & -0.00008 & 01234 & -0.01030 & 0.00008 & -0.00071 & -0.0 & \\
\hline $\mathrm{O} 2$ & $\mathrm{Y}$ & 0.00015 & 0 & -0.00633 & -0.00079 & -0.00258 & -0.0 & \\
\hline 02 & Z & & -0 . & 0 & -0 & -0 . & & -0 \\
\hline 03 & $\mathrm{X}$ & 0 & 03460 & -0.00467 & 0.00141 & -0.000 & -0 . & \\
\hline 03 & $\mathrm{Y}$ & -0 & -0 & 0.00682 & 0.00134 & -0.001 & & -0 . \\
\hline & Z & -0 & -0 & 0 & -0 & & & -0 \\
\hline C4 & $\mathrm{X}$ & -0 & 0851 & -0.01725 & 0324 & 0 . & & -0 . \\
\hline C4 & $Y$ & 0 & -0 & 0.02388 & -0 & 0 . & 0 . & -0 . \\
\hline C4 & Z & -0 . & & -0 . & -0 . & & & -0 \\
\hline C7 & $\mathrm{X}$ & 6238 & 441 & 1668 & 0 & -0 . & -0 . & -0 \\
\hline C7 & $Y$ & -0 & 0 & -0.0 & 0.0 & -0 . & -0 . & \\
\hline C7 & Z & & -0 . & & & -0 . & -0 & -0 \\
\hline $\mathrm{H} 21$ & $\mathrm{X}$ & -0 . & -0 & 2862 & 0 . & & -0 . & -0 \\
\hline $\mathrm{H} 21$ & $Y$ & 0 & -0 & 5369 & 0.0 & -0.011 & -0.4 & \\
\hline $\mathrm{H} 21$ & Z & & & -0 . & -0 & -0 . & & -0 \\
\hline H 4 & $\mathrm{X}$ & 1715 & -0 & 5621 & -0 . & -0 . & & \\
\hline H 4 & $\mathrm{Y}$ & -0 & 0 & -0 & -0.000 & -0.000 & 0 & \\
\hline H4 & Z & & & -0 . & -0 . & & -0 . & \\
\hline & $\mathrm{X}$ & & 0 . & 0 & 0 & 0 . & & -0 \\
\hline $\mathrm{H} 1$ & $\mathrm{Y}$ & -0 & 0 & -0.00330 & 0.101 & 0.193 & -0.0 & \\
\hline & Z & -0 . & & & -0.3 & -0 . & & \\
\hline & $\mathrm{X}$ & -0 . & -0 & 00024 & -0.12242 & -0.190 & & -0 \\
\hline $\mathrm{H} 20$ & $\mathrm{Y}$ & 0 & -0 & 0.01017 & -0.33752 & -0.477 & & -0 \\
\hline & Z & 0 . & & 0 & 0.10 & & & -0 \\
\hline & $\mathrm{X}$ & & 0 . & -0 . & 0 . & -0 . & & \\
\hline H22 & $\mathrm{Y}$ & -0 . & 0404 & -0 . & 0.31396 & -0.208 & 0. & \\
\hline $\mathrm{H} 22$ & Z & 0 & 0 & & 0 . & -0 . & & -0 \\
\hline & $\mathrm{X}$ & & -0 & 0 & 0 . & -0 . & & -0 \\
\hline & $\mathrm{Y}$ & -0 . & 0 . & 00494 & 0 & -0 & & \\
\hline H26 & Z & -0 . & & -0 . & -0 . & & -0 . & \\
\hline $\mathrm{H} 2$ & $\mathrm{X}$ & -0 . & 0.00314 & 0 & -0 . & 0 . & -0.0 & -0 . \\
\hline $\mathrm{H} 2$ & $\mathrm{Y}$ & 0 & -0.17624 & -0 . & -0 . & & & \\
\hline $\mathrm{H} 2$ & Z & -0 . & & -0 & -0 & -0 . & -0.04 & -0 \\
\hline $\mathrm{C} 2$ & $\mathrm{X}$ & 0 & -0.00074 & 0.01532 & 0.00024 & -0.000 & -0.00059 & -0.01 \\
\hline $\mathrm{C} 2$ & $\mathrm{Y}$ & -0 . & 0 & 0.1124 & 0.000 & -0 . & -0.02 & -0 . \\
\hline $\mathrm{C} 2$ & Z & & -0.0 & -0.031 & 0.00002 & & & \\
\hline 04 & $\mathrm{X}$ & 0 & -0.00564 & 0.07053 & -0.00009 & 0.000 & 0.006 & 0 . \\
\hline 04 & $\mathrm{Y}$ & -0 . & 0 & 0 . & -0 . & 0.000 & & \\
\hline O4 & Z & 0 & -0.00317 & 0.03473 & -0.00001 & 0.00025 & 0.001 & \\
\hline 05 & $\mathrm{X}$ & 0.01454 & -0.00978 & -0.10343 & 0.00002 & -0.00076 & -0.00906 & -0.02375 \\
\hline 05 & $\mathrm{Y}$ & & -0.01009 & -0.10191 & -0.00001 & -0.00049 & 0.000 & \\
\hline 05 & Z & 0.00176 & -0.00073 & -0.01867 & -0.00011 & -0.00035 & -0.00467 & -0 . \\
\hline H3 & $\mathrm{X}$ & -0.11082 & 0.04854 & 0.40248 & -0.00085 & 0.00457 & 0.08272 & 0.35 \\
\hline
\end{tabular}




\begin{tabular}{|c|c|c|c|c|c|c|c|c|}
\hline H3 & $\mathrm{Y}$ & -0.11092 & 0.04675 & 0.38065 & 0.00001 & 0.00589 & 0.08918 & 0.40536 \\
\hline H3 & Z & -0.02051 & 0.01018 & 0.07502 & 0.00044 & 0.00210 & 0.01620 & 0.04273 \\
\hline \multicolumn{2}{|c|}{ frequencies } & 1386.94 & 1452.07 & 1454.42 & 1548.49 & 1611.75 & 1708.43 & 1818.88 \\
\hline \multicolumn{2}{|c|}{ intensities } & 2.34 & 59.31 & 160.79 & 80.61 & 172.10 & 2.27 & 167.14 \\
\hline Re1 & $\mathrm{X}$ & 0.00011 & 0.00003 & -0.00009 & 0.00071 & 0.00007 & 0.00030 & 0.00010 \\
\hline $\operatorname{Re1}$ & Y & 0.00013 & 0.00026 & -0.00016 & -0.00029 & 0.00018 & 0.00049 & 0.00008 \\
\hline $\operatorname{Re1}$ & Z & 0.00008 & -0.00048 & 0.00016 & -0.00073 & 0.00063 & 0.00014 & -0.00026 \\
\hline N2 & $\mathrm{x}$ & -0.01651 & -0.01082 & 0.00148 & -0.00440 & 0.02201 & -0.01522 & -0.00009 \\
\hline N2 & Y & -0.04816 & -0.03899 & 0.00398 & -0.00430 & 0.02050 & -0.00671 & 0.00003 \\
\hline N2 & $\mathrm{Z}$ & 0.06674 & 0.07384 & -0.00495 & -0.01406 & 0.10505 & -0.11184 & 0.00057 \\
\hline $\mathrm{C} 10$ & $\mathrm{X}$ & 0.02741 & 0.03563 & -0.00365 & 0.00619 & -0.03897 & 0.02470 & -0.00015 \\
\hline $\mathrm{C} 10$ & Y & 0.06177 & 0.08400 & -0.00808 & 0.00853 & -0.05144 & 0.01770 & 0.00000 \\
\hline $\mathrm{C} 10$ & Z & 0.00074 & -0.01535 & -0.00073 & 0.02082 & -0.13861 & 0.15484 & -0.00042 \\
\hline $\mathrm{C} 1$ & $\mathrm{X}$ & 0.02218 & -0.03120 & 0.00305 & -0.00713 & 0.04488 & 0.03371 & 0.00002 \\
\hline $\mathrm{C} 1$ & Y & 0.04782 & -0.06274 & 0.00676 & -0.01896 & 0.12472 & 0.10035 & 0.00035 \\
\hline $\mathrm{C} 1$ & Z & 0.03030 & -0.06581 & 0.00415 & 0.01231 & -0.08616 & -0.10875 & -0.00026 \\
\hline N1 & $\mathrm{X}$ & -0.00577 & 0.00156 & -0.00047 & 0.00362 & -0.02580 & -0.02186 & -0.00 \\
\hline $\mathrm{N} 1$ & Y & -0.00365 & -0.00986 & -0.00060 & 0.01231 & -0.07999 & -0.06621 & -0.00028 \\
\hline N1 & Z & -0.07775 & 0.09020 & -0.00628 & -0.00904 & 0.07526 & 0.08209 & 0.00 \\
\hline 01 & $\mathrm{X}$ & 0.00004 & 0.00130 & -0.00099 & -0.00038 & -0.00035 & -0.00075 & 082 \\
\hline 01 & Y & 0.00050 & -0.00025 & 0.00030 & -0.00021 & 0.00047 & -0.00015 & -0.00041 \\
\hline 01 & Z & 0.00003 & -0.00038 & -0.00072 & 0.00055 & -0.00208 & -0.00006 & 0.00 \\
\hline $\mathrm{O} 2$ & $\mathrm{X}$ & -0.00144 & 0.00030 & 0.00244 & -0.00609 & 0.00002 & -0.00075 & -0.0 \\
\hline 02 & $Y$ & 0.00026 & -0.00039 & 0.00063 & -0.00495 & 0.00118 & -0.00087 & -0.00012 \\
\hline $\mathrm{O} 2$ & Z & 0.00164 & -0.00171 & -0.00121 & 0.00306 & 0.00007 & -0.00176 & 0.0 \\
\hline 03 & $\mathrm{X}$ & 0.00145 & 0.00097 & -0.00117 & -0.00773 & 0.00157 & -0.00042 & 015 \\
\hline 03 & $\mathrm{Y}$ & 0.00043 & -0.00034 & -0.00100 & 0.00806 & -0.00394 & -0.00142 & 0.00032 \\
\hline 03 & Z & -0.00095 & -0.00132 & 0.00123 & 0.00903 & -0.00134 & 0.00104 & 037 \\
\hline $\mathrm{C} 4$ & $\mathrm{X}$ & 00074 & -0.00177 & -0.02421 & 0.00666 & 0.00223 & -0.00034 & 0.0 \\
\hline $\mathrm{C} 4$ & Y & 0.00059 & 0.00715 & 0.08856 & 0.15294 & 0.02735 & -0.00012 & 0.00414 \\
\hline $\mathrm{C} 4$ & $\mathrm{Z}$ & -0.00012 & -0.00119 & -0.03576 & -0.02502 & -0.00212 & 0.00012 & 0.0 \\
\hline $\mathrm{C} 7$ & $\mathrm{X}$ & 00062 & 0.00 & 0.02043 & -0.00807 & -0.00571 & 0.00036 & -0.0 \\
\hline $\mathrm{C} 7$ & $\mathrm{Y}$ & -0.00042 & 0.00422 & 0.04187 & -0.13636 & -0.02606 & 0.00043 & -0.00613 \\
\hline C7 & Z & 0.00035 & 0.00280 & 0.02663 & -0.02574 & -0.00332 & -0.00008 & -0.0 \\
\hline H21 & $\mathrm{X}$ & 0.00166 & 0.01699 & 0.15247 & -0.17454 & -0.02216 & -0.00048 & -0.0 \\
\hline $\mathrm{H} 21$ & $\mathrm{Y}$ & -0.00495 & -0.04591 & -0.36499 & 0.36883 & 0.04868 & -0.00004 & 0.02778 \\
\hline $\mathrm{H} 21$ & Z & -0.00035 & 0.00426 & 0.03684 & -0.04882 & -0.01516 & 0.00069 & -0.01144 \\
\hline H4 & $\mathrm{X}$ & -0.00112 & -0.02944 & -0.25629 & 0.18561 & 0.02549 & 0.00007 & 0.0 \\
\hline $\mathrm{H} 4$ & $\mathrm{Y}$ & -0.00276 & -0.03722 & -0.34372 & 0.13588 & 0.01450 & -0.00006 & 0.02433 \\
\hline $\mathrm{H} 4$ & Z & -0.00342 & -0.03303 & -0.30147 & 0.24283 & 0.03566 & -0.00036 & 0.02 \\
\hline $\mathrm{H} 12$ & $\mathrm{X}$ & -0.01563 & 0.00823 & -0.00290 & 0.01308 & -0.05116 & -0.02429 & 0.00 \\
\hline $\mathrm{H} 12$ & $\mathrm{Y}$ & -0.10821 & 0.09244 & -0.00795 & 0.01059 & -0.06936 & -0.05088 & -0.00010 \\
\hline $\mathrm{H} 12$ & Z & 0.34993 & -0.33704 & 0.02880 & -0.01127 & 0.02141 & -0.03567 & -0.00047 \\
\hline $\mathrm{H} 20$ & $\mathrm{X}$ & -0.15435 & 0.13152 & -0.01106 & 0.00981 & -0.04306 & -0.06781 & -0.00012 \\
\hline $\mathrm{H} 2 \mathrm{O}$ & $\mathrm{Y}$ & -0.38900 & 0.34769 & -0.03005 & 0.02584 & -0.12288 & -0.16890 & -0.00020 \\
\hline $\mathrm{H} 20$ & Z & 0.16048 & -0.20125 & 0.01598 & 0.00075 & -0.02734 & -0.05240 & -0.00012 \\
\hline H22 & $\mathrm{X}$ & -0.14534 & -0.10690 & 0.01166 & -0.01014 & 0.05686 & -0.08653 & 0.00003 \\
\hline $\mathrm{H} 22$ & $\mathrm{Y}$ & -0.24111 & -0.15828 & 0.01702 & -0.01962 & 0.09628 & -0.16559 & -0.00017 \\
\hline $\mathrm{H} 22$ & Z & -0.39328 & -0.35224 & 0.03370 & -0.01343 & 0.03960 & -0.05176 & -0.00038 \\
\hline H26 & $\mathrm{X}$ & 0.01263 & 0.01514 & -0.00263 & 0.00302 & 0.01278 & -0.02711 & 0.00073 \\
\hline H26 & $\mathrm{Y}$ & 0.11682 & 0.10712 & -0.00815 & -0.00691 & 0.05114 & -0.06135 & 0.00081 \\
\hline H26 & Z & -0.35784 & -0.29314 & 0.02786 & -0.02108 & 0.07937 & -0.01001 & -0.00165 \\
\hline $\mathrm{H} 2$ & $\mathrm{X}$ & 0.00062 & 0.00084 & 0.02029 & 0.06140 & 0.00820 & -0.00020 & 0.01999 \\
\hline $\mathrm{H} 2$ & $\mathrm{Y}$ & 0.00038 & -0.01740 & -0.29378 & -0.23903 & -0.02459 & -0.00023 & -0.05384 \\
\hline $\mathrm{H} 2$ & Z & 0.00035 & 0.00668 & 0.08473 & 0.11020 & 0.02024 & -0.00011 & 0.03336 \\
\hline $\mathrm{C} 2$ & $\mathrm{X}$ & 0.00014 & -0.00057 & -0.01011 & 0.00928 & 0.00249 & 0.00001 & -0.20240 \\
\hline $\mathrm{C} 2$ & $\mathrm{Y}$ & -0.00017 & -0.00709 & -0.12848 & -0.07430 & -0.00337 & -0.00014 & -0.03816 \\
\hline $\mathrm{C} 2$ & Z & 0.00013 & 0.00251 & 0.04593 & 0.03364 & 0.00255 & -0.00001 & -0.10279 \\
\hline 04 & $\mathrm{X}$ & -0.00016 & 0.00069 & 0.00893 & -0.00922 & -0.00150 & 0.00005 & 0.12565 \\
\hline 04 & Y & 0.00002 & 0.00089 & 0.01655 & 0.00919 & 0.00005 & 0.00001 & 0.02146 \\
\hline
\end{tabular}




\begin{tabular}{|c|c|c|c|c|c|c|c|c|}
\hline 04 & $\mathrm{z}$ & -0.00011 & 0.00000 & -0.00215 & -0.00801 & -0.00089 & 0.00000 & 0.06375 \\
\hline 05 & $\mathrm{x}$ & -0.00009 & -0.00039 & -0.00206 & 0.00140 & -0.00039 & 0.00002 & 0.02111 \\
\hline 05 & $\mathrm{Y}$ & 0.00004 & 0.00170 & 0.03563 & 0.01388 & -0.00105 & 0.00009 & 0.01360 \\
\hline 05 & Z & -0.00009 & -0.00099 & -0.01599 & -0.00479 & 0.00005 & -0.00002 & 0.00697 \\
\hline H3 & $\mathrm{x}$ & 0.00087 & 0.00983 & 0.15091 & 0.05731 & 0.00458 & -0.00044 & -0.08910 \\
\hline H3 & $\mathrm{Y}$ & 0.00009 & 0.01203 & 0.17922 & 0.06548 & 0.00335 & -0.00044 & \\
\hline H3 & $\mathrm{Z}$ & -0.00051 & 0.00215 & 0.02020 & 0.00874 & 0.00193 & -0.00002 & \\
\hline \multicolumn{2}{|c|}{ frequencies } & 3186.26 & 3194.54 & 3202.13 & 3240.94 & 3285.36 & 3535.77 & 3538.0 \\
\hline \multicolumn{2}{|c|}{ intensities } & 0.83 & 5.26 & 25.82 & 0.92 & 2.56 & & \\
\hline $\operatorname{Re} 1$ & $\mathrm{x}$ & -0.00004 & -0.00001 & 0.00001 & -0.00003 & 0.00000 & 0.00000 & \\
\hline Re1 & $\mathrm{Y}$ & 0.00001 & 0.00000 & 0.00001 & 0.00000 & 0.00000 & 0.00001 & \\
\hline $\operatorname{Re} 1$ & $\mathrm{z}$ & 0.00001 & 0.00002 & -0.00007 & 0.00001 & 0.00000 & -0.00001 & \\
\hline N2 & $\mathrm{x}$ & 0.00007 & -0.00035 & -0.00022 & -0.00003 & 0.00000 & -0.03052 & \\
\hline N2 & $\mathrm{Y}$ & 0.00016 & -0.00145 & 0.00026 & -0.00001 & -0.00004 & & \\
\hline N2 & $\mathrm{z}$ & -0.00040 & 0.00291 & -0.00271 & -0.00007 & 0.00000 & & \\
\hline C10 & $\mathrm{x}$ & -0.00191 & 0.01648 & -0.01309 & -0.00001 & 0.00000 & -0.00012 & \\
\hline C10 & $\mathrm{Y}$ & -0.00544 & 0.0 & -0.036 & -0.00008 & -0.00005 & -0 & \\
\hline $\mathrm{C} 10$ & Z & 0.00562 & -0.0 & 0.032 & 0.00018 & 0001 & & \\
\hline $\mathrm{C} 1$ & $\mathrm{x}$ & -0.00042 & 0.00645 & 0.01068 & -0.00016 & 0.00006 & 0.00032 & \\
\hline $\mathrm{C} 1$ & $\mathrm{Y}$ & -0.0 & 0.0 & 0. & -0.00 & & & \\
\hline $\mathrm{C} 1$ & Z & -0.0 & & & -0.00088 & & & \\
\hline N1 & $\mathrm{x}$ & 0.00009 & -0.00028 & 0.0 & 0.00003 & 0.00003 & -0 & -0 \\
\hline N1 & $\mathrm{Y}$ & 0.0 & -0.00026 & 0.0 & 0.00004 & -0.0 & & -0 \\
\hline N1 & Z & 0. & -0 & -0.0 & & -0. & & \\
\hline 01 & $\mathrm{x}$ & 0.00018 & 0.00006 & -0.00009 & 0.00012 & -0.00001 & -0.00007 & \\
\hline 01 & Y & -0.00003 & -0.00001 & 0.00000 & -0.00003 & 0.00003 & & -0 . \\
\hline 01 & Z & & -0 . & & & & & -0 \\
\hline 02 & $\mathrm{x}$ & 0. & -0 & & 0. & -0.0 & 0009 & \\
\hline $\mathrm{O} 2$ & $\mathrm{Y}$ & & -0 & -0. & 0. & -0.0 & & \\
\hline 02 & Z & -0 & -0 . & & -0. & -0 & & -0 \\
\hline 03 & $\mathrm{x}$ & 0.0 & 0. & -0.0 & 0.00032 & -0.00046 & -0 . & \\
\hline 03 & $\mathrm{Y}$ & -0.0 & -0. & & -0.0 & & & -0 \\
\hline 03 & Z & -0 & -0 & & -0 & -0 . & & \\
\hline C4 & $\mathrm{x}$ & 0.0 & -0.0 & -0.00 & -0.06959 & 0.00995 & -0.00001 & -0 \\
\hline C4 & $\mathrm{Y}$ & -0.0 & -0.0 & -0.0 & -0.02278 & & & \\
\hline C4 & Z & & -0 & -0 . & -0. & & -0 & \\
\hline C7 & $\mathrm{x}$ & 0.0 & 0.0 & -0.0 & 0.01050 & 0.06907 & 0009 & -0 . \\
\hline C7 & $\mathrm{Y}$ & 0.0 & & -0.0 & 0.00009 & -0.03101 & & -0 \\
\hline C7 & Z & & & -0 & & & & \\
\hline H 21 & $\mathrm{x}$ & -0.46098 & -0.0 & & -0.06526 & -0.39787 & -0 . & -0 \\
\hline H 21 & Y & -0.16 & -0.0 & & -0.02184 & -0.14899 & & -0 \\
\hline H21 & Z & -0 & -0 . & & -0 . & & & \\
\hline $\mathrm{H} 4$ & $\mathrm{x}$ & & & -0.0 & -0.05004 & -0.42500 & & \\
\hline H4 & Y & -0.46519 & -0.04577 & 0.01117 & 0.05653 & 0.50251 & -0.00042 & -0.0 \\
\hline H4 & Z & & & -0 & -0.0 & -0 . & & \\
\hline $\mathrm{H} 12$ & $\mathrm{x}$ & -0 . & & & -0 . & & & \\
\hline $\mathrm{H} 12$ & $\mathrm{Y}$ & -0.0 & 0.0 & & -0.00088 & 0.0 & 234 & 229 \\
\hline & Z & & & & & & & \\
\hline $\mathrm{H} 2 \mathrm{O}$ & $\mathrm{x}$ & & -0 & -0 & & -0 & & \\
\hline H2O & $\mathrm{Y}$ & & -0. & -0.1 & 221 & -0.00027 & & \\
\hline & Z & & -0.5 & -0 & & -0.0 & & \\
\hline H22 & $\mathrm{x}$ & & -0. & & & & & \\
\hline H22 & $\mathrm{Y}$ & 0. & -0.5 & & 0.0 & 0.0 & & \\
\hline H22 & Z & -0.0 & & -0.3 & -0.0 & -0.0 & -0 . & \\
\hline H26 & $\mathrm{x}$ & -0.0 & & & & & & \\
\hline H26 & $\mathrm{Y}$ & -0.0 & & -0.0 & & 0.0 & & -0 . \\
\hline & Z & -0.0 & & -0 & & 0.0 & & -0 . \\
\hline H2 & $\mathrm{x}$ & -0.0 & & & & -0.10827 & & \\
\hline $\mathrm{H} 2$ & Y & -0.0 & & & & -0.03634 & & \\
\hline $\mathrm{H} 2$ & Z & -0.01223 & 0.00252 & 0.00364 & 0.44443 & -0.05970 & -0.00033 & 0.0 \\
\hline
\end{tabular}




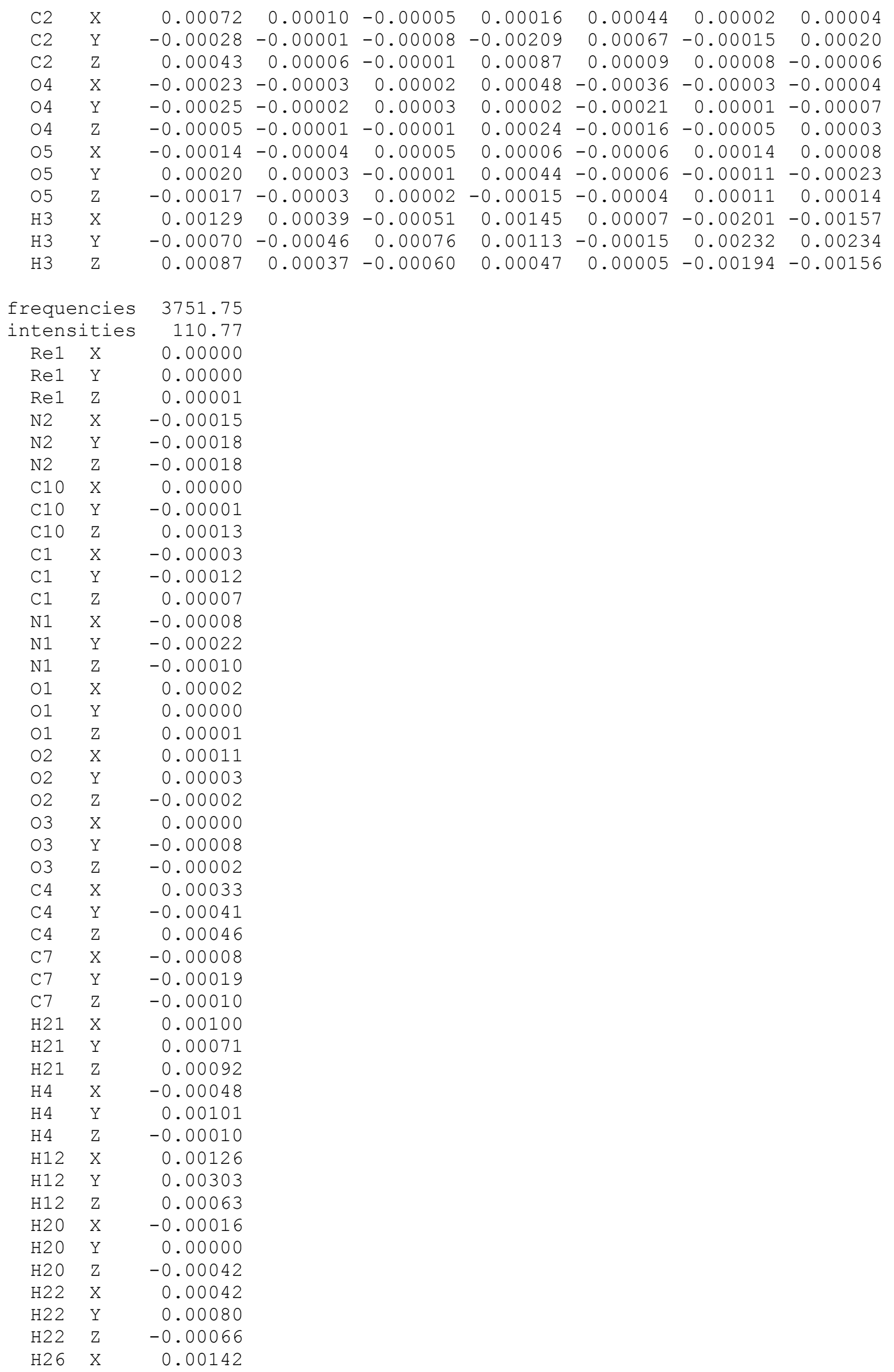




$\begin{array}{llr}\text { H26 } & \text { Y } & 0.00291 \\ \text { H26 } & \text { Z } & 0.00121 \\ \text { H2 } & \text { X } & -0.00013 \\ \text { H2 } & \text { Y } & -0.00076 \\ \text { H2 } & \text { Z } & 0.00052 \\ \text { C2 } & \text { X } & -0.00074 \\ \text { C2 } & \text { Y } & 0.00210 \\ \text { C2 } & \text { Z } & -0.00127 \\ \text { O4 } & \text { X } & -0.00068 \\ \text { O4 } & \text { Y } & -0.00057 \\ \text { O4 } & \text { Z } & -0.00017 \\ \text { O5 } & \text { X } & -0.02998 \\ \text { O5 } & \text { Y } & 0.03879 \\ \text { O5 } & \text { Z } & -0.03397 \\ \text { H3 } & \text { X } & 0.49177 \\ \text { H3 } & \text { Y } & -0.62463 \\ \text { H3 } & \text { Z } & 0.55104 \\ & & \end{array}$

Thermochemical Properties:
pressure:
$1.0000 \mathrm{~atm}$
rotational symmetry number:
vibrational frequencies below $10.0 \mathrm{~cm}-1$ excluded: 1
zero point energy:
$92.008 \mathrm{kcal} / \mathrm{mol}$
temp $(\mathrm{K})$
7.9489
$\mathrm{Cp}(\mathrm{cal} / \mathrm{mol} \mathrm{K})$
$\mathrm{S}(\mathrm{cal} / \mathrm{mol} \mathrm{K})$
H ( $\mathrm{kcal} / \mathrm{mol})$
30.0337
0.0000
G $(\mathrm{kcal} / \mathrm{mol})$
298.15
52.5681
120.9763
9.6773
0.0000
$-26.3918$

end of program freq

start of program ch

Moments from quantum mechanical wavefunction:

Dipole Moments (Debye)
$\mathrm{X}=$
$-0.7291$
$\mathrm{Y}=$
3.7057
$\mathrm{Z}=$
-11.3958 Tot $=$
12.0053

Einal energy .......... $\quad-759.83704278810$

Job WF3681 completed on CHENB071 at Wed Jan 21 18:06:13 2004

Reason for exit: Successful completion

Titan Program CPU Time : 008:51:56.0

Titan Program Wall Time: 009:20:55.5

TITAN Properties Program: (PC/x86)

Release 6.0 .6

Reason for exit: Successful completion

Properties Program CPU Time : 000:00:02.1

Properties Program Wall Time: 000:00:02.1 


\section{Scheme 5}

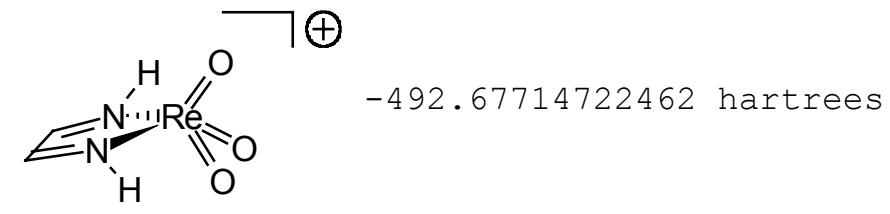

\begin{tabular}{l|} 
Jaguar version 3.5, release 42 \\
Copyright 1991-1998 Schrodinger, Inc. \\
All Rights Reserved. \\
Use of this program should be acknowledged in publications as: \\
Jaguar 3.5, Schrodinger, Inc., Portland, Oregon, 1998. \\
+
\end{tabular}

Job WF21072 started on CHENB091 at Wed Jan 07 17:19:50 2004

Jaguar version 3.5, release 42

Job name:

Task:

Stoichiometry:

Molecular weight:

Molecular charge:

Spin multiplicity:

Point Group:

SCF method:

Basis set:

Basis functions:

Total electrons:

Alpha electrons:

Beta electrons:

Total orbitals:

Core orbitals:

Open-shell orbitals:

Occupied orbitals:

Shells:
WF21072

Geometry optimization

$\mathrm{ReC} 2 \mathrm{~N} 2 \mathrm{H} 4 \mathrm{O} 3$

$290.98 \mathrm{amu}$

1

C1

B3LYP

$\mathrm{LACVP} * *$

140

68

34

34

150

34

0

34

1

Non-default options chosen:

SCF calculation type: DFT

$\mathrm{DFT}=$ Becke 3 Parameter/HF+Slater+Becke88+VWN+LYP (B3LYP)

Geometry will be optimized in redundant internal coordinates

Numerical 2nd derivatives will be computed

Vibrational frequencies and related properties will be computed

Molecular symmetry not used

Initial Hessian: from previous calculation

$\begin{array}{cccccc}\text { Iter } & \text { Energy } & \text { Gmax } & \text { Grms } & \text { Dmax } & \text { Drms } \\ 1 & -492.380958 & 0.15509 & 0.04719 & 0.17166 & 0.05003 .\end{array}$




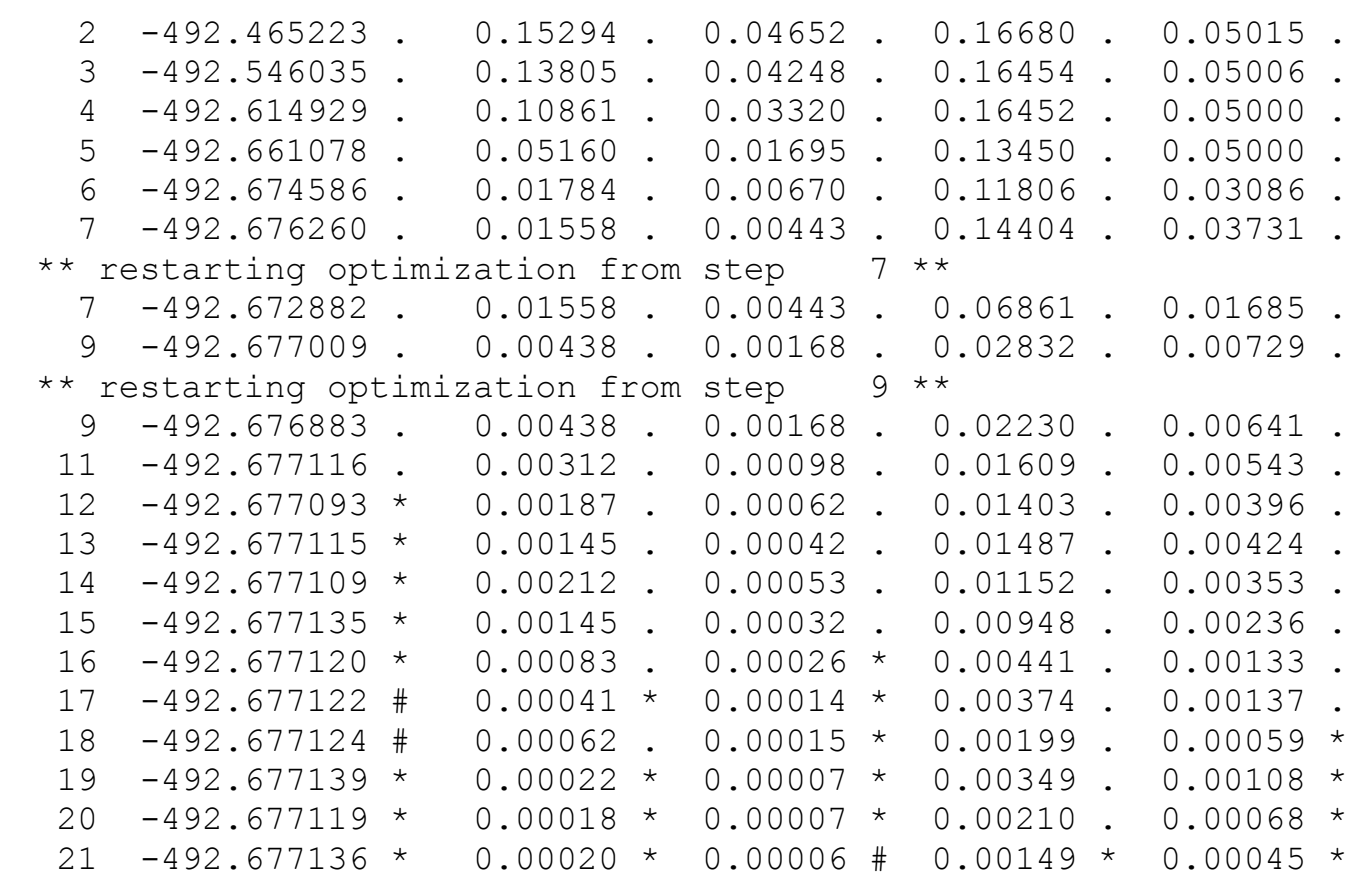

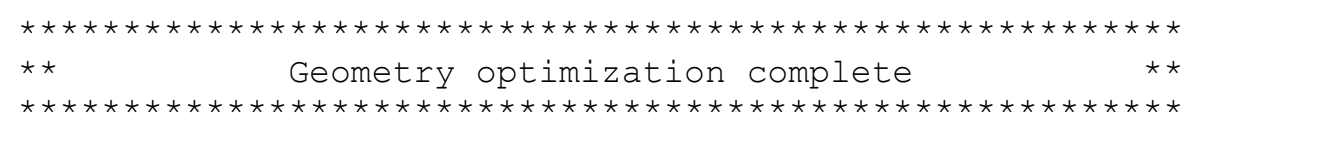

energy change:

gradient maximum:

gradient rms:

step size:

displacement maximum:

displacement rms:

predicted energy change:

step: $2.7270 \mathrm{E}-03$

$$
\begin{array}{r}
-1.7054 \mathrm{E}-05 *(5.0000 \mathrm{E}-05) \\
2.0181 \mathrm{E}-04 *(4.5000 \mathrm{E}-04) \\
5.8663 \mathrm{E}-05
\end{array} \text { \# }\left(\begin{array}{r}
(3.0000 \mathrm{E}-04) \\
\text { trust radius: }
\end{array}\right.
$$

$1.4888 \mathrm{E}-03$ * $(1.8000 \mathrm{E}-03)$

$4.5450 \mathrm{E}-04 *(1.2000 \mathrm{E}-03)$ $-3.5185 \mathrm{E}-07$ geom step:

center of mass moved by:
$\mathrm{x}$ :
$0.0000 \mathrm{E}+00$
$y:$
$0.0000 \mathrm{E}+00$
z :
$0.0000 E+00$

final geometry:

$\begin{array}{lr}\text { atom } & \mathrm{x} \\ \mathrm{N} 1 & 1.2556995468 \\ \mathrm{~N} 2 & -1.2921499042 \\ \mathrm{C} 4 & 0.8504049933 \\ \mathrm{C} 5 & -0.6298680718 \\ \mathrm{H} 5 & -2.3130201970 \\ \mathrm{H} 6 & -1.0645588400 \\ \mathrm{H} 4 & 1.5368637033 \\ \mathrm{H} 3 & 2.2618435377 \\ \mathrm{Re} 1 & -0.1312279966 \\ \mathrm{O} 1 & -1.0015445423 \\ \mathrm{O} 2 & -1.0326606704 \\ \mathrm{O} 3 & 1.2663123283\end{array}$

angstroms Y

1. 4245038259

1. 7475050338

2.6384210193

2.8330325911

1.8001689777

3. 8316457241

3.4843642890

1. 2457302129

$-0.2827812463$

$-0.6650365720$

$-0.6910574990$

$-1.2538774837$ z

$-0.0116535678$

$-0.0023478711$

$-0.0182675656$

$-0.0140559516$

0.0007887620

$-0.0207749626$

$-0.0263908920$

$-0.0141771401$

0.0085324531

1. 4199737985

$-1.3758284935$

0.0016026257

calculating forces at perturbed geometry calculating forces at perturbed geometry calculating forces at perturbed geometry calculating forces at perturbed geometry calculating forces at perturbed geometry

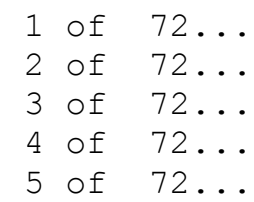


calculating forces at perturbed geometry calculating forces at perturbed geometry calculating forces at perturbed geometry calculating forces at perturbed geometry calculating forces at perturbed geometry calculating forces at perturbed geometry calculating forces at perturbed geometry calculating forces at perturbed geometry calculating forces at perturbed geometry calculating forces at perturbed geometry calculating forces at perturbed geometry calculating forces at perturbed geometry calculating forces at perturbed geometry calculating forces at perturbed geometry calculating forces at perturbed geometry calculating forces at perturbed geometry calculating forces at perturbed geometry calculating forces at perturbed geometry calculating forces at perturbed geometry calculating forces at perturbed geometry calculating forces at perturbed geometry calculating forces at perturbed geometry calculating forces at perturbed geometry calculating forces at perturbed geometry calculating forces at perturbed geometry calculating forces at perturbed geometry calculating forces at perturbed geometry calculating forces at perturbed geometry calculating forces at perturbed geometry calculating forces at perturbed geometry calculating forces at perturbed geometry calculating forces at perturbed geometry calculating forces at perturbed geometry calculating forces at perturbed geometry calculating forces at perturbed geometry calculating forces at perturbed geometry calculating forces at perturbed geometry calculating forces at perturbed geometry calculating forces at perturbed geometry calculating forces at perturbed geometry calculating forces at perturbed geometry calculating forces at perturbed geometry calculating forces at perturbed geometry calculating forces at perturbed geometry calculating forces at perturbed geometry calculating forces at perturbed geometry calculating forces at perturbed geometry calculating forces at perturbed geometry calculating forces at perturbed geometry calculating forces at perturbed geometry calculating forces at perturbed geometry calculating forces at perturbed geometry calculating forces at perturbed geometry calculating forces at perturbed geometry calculating forces at perturbed geometry calculating forces at perturbed geometry calculating forces at perturbed geometry calculating forces at perturbed geometry calculating forces at perturbed geometry calculating forces at perturbed geometry calculating forces at perturbed geometry
6 of $72 \ldots$

7 of $72 \ldots$

8 of $72 \ldots$

9 of $72 \ldots$

10 of $72 \ldots$

11 of $72 \ldots$

12 of $72 \ldots$

13 of $72 \ldots$

14 of $72 \ldots$

15 of $72 \ldots$

16 of $72 \ldots$

17 of $72 \ldots$

18 of $72 \ldots$

19 of $72 \ldots$

20 of $72 \ldots$

21 of $72 \ldots$

22 of $72 \ldots$

23 of $72 \ldots$

24 of $72 \ldots$

25 of $72 \ldots$

26 of $72 \ldots$

27 of $72 \ldots$

28 of $72 \ldots$

29 of $72 \ldots$

30 of $72 \ldots$

31 of $72 \ldots$

32 of $72 \ldots$

33 of $72 \ldots$

34 of $72 \ldots$

35 of $72 \ldots$

36 of $72 \ldots$

37 of $72 \ldots$

38 of $72 \ldots$

39 of $72 \ldots$

40 of $72 \ldots$

41 of $72 \ldots$

42 of $72 \ldots$

43 of $72 \ldots$

44 of $72 \ldots$

45 of $72 \ldots$

46 of $72 \ldots$

47 of $72 \ldots$

48 of $72 \ldots$

49 of $72 \ldots$

50 of $72 \ldots$

51 of $72 \ldots$

52 of $72 \ldots$

53 of $72 \ldots$

54 of $72 \ldots$

55 of $72 \ldots$

56 of $72 \ldots$

57 of $72 \ldots$

58 of $72 \ldots$

59 of $72 \ldots$

60 of $72 \ldots$

61 of $72 \ldots$

62 of $72 \ldots$

63 of $72 \ldots$

64 of $72 \ldots$

65 of $72 \ldots$

66 of $72 \ldots$ 
calculating forces at perturbed geometry calculating forces at perturbed geometry calculating forces at perturbed geometry calculating forces at perturbed geometry calculating forces at perturbed geometry calculating forces at perturbed geometry
67 of $72 \ldots$ 68 of $72 \ldots$ 69 of $72 \ldots$ 70 of $72 \ldots$ 71 of $72 \ldots$ 72 of $72 \ldots$

numerical derivatives complete;

calculation at normal geometry follows...

start of program freq

harmonic frequencies in $\mathrm{cm}^{\wedge}-1$, IR intensities in $\mathrm{km} / \mathrm{mol}$, and normal modes:

\begin{tabular}{|c|c|c|c|c|c|c|c|c|}
\hline \multirow{2}{*}{\multicolumn{2}{|c|}{$\begin{array}{l}\text { frequencies } \\
\text { intensities }\end{array}$}} & 83.66 & 122.52 & 170.64 & 251.80 & 287.09 & 290.48 & 347.8 \\
\hline & & 0.14 & 0.44 & 2.74 & 1.15 & 0.26 & 1.75 & \\
\hline N1 & $\mathrm{X}$ & 0.00066 & -0.00215 & 0.04491 & 0.03607 & 0.00407 & 0.07050 & -0.002 \\
\hline N1 & Y & 0.00129 & -0.00153 & 0.01009 & -0.05446 & 0.00527 & 0.10405 & \\
\hline N1 & Z & 0.10905 & -0.05769 & -0.00203 & 0.00138 & -0.15167 & 0.00863 & \\
\hline N2 & $\mathrm{X}$ & 0.00002 & -0.00344 & 0.05743 & 0.03432 & 0.00397 & 0.06105 & -0.00 \\
\hline N2 & $Y$ & -0.00071 & 0.00050 & -0.01009 & -0.15989 & -0.00642 & -0.06586 & \\
\hline N2 & Z & -0.10657 & -0.00396 & -0.00060 & 0.00203 & -0.13683 & & \\
\hline C4 & $\mathrm{X}$ & 0060 & -0.00265 & 0.06236 & 0.00029 & 0.00083 & 0.00489 & -0 . \\
\hline C4 & Y & 0.00106 & -0.00098 & 0.010 & -0.06883 & 0.005 & 0.082 & -0 \\
\hline $\mathrm{C} 4$ & Z & 0.09432 & 0.06267 & & & & -0.00 & \\
\hline C5 & $\mathrm{X}$ & 0002 & -0.002 & 0.05 & -0.00909 & -0.00 & -0.011 & -0 . \\
\hline C5 & Y & -0.00010 & 0.00128 & -0.007 & -0.13515 & -0.001 & -0.022 & -0 . \\
\hline $\mathrm{C} 5$ & Z & -0.04527 & 0.12 & & 0.000 & & -0.00 & -0 . \\
\hline H5 & $\mathrm{X}$ & -0.0 & -0.003 & 0.057 & 0.031 & 0.00 & 0.057 & -0 . \\
\hline H5 & Y & -0.0 & 0.001 & -0.004 & -0.220 & -0.012 & -0.13208 & \\
\hline H5 & Z & -0.2 & 0.03 & & & -0.24 & & -0 \\
\hline H 6 & $\mathrm{X}$ & -0.0 & -0.000 & 0.04 & -0.06015 & -0.00 & -0.092 & \\
\hline H 6 & Y & -0.0 & 0.003 & -0.01 & -0.15880 & -0.003 & -0.057 & -0 . \\
\hline H 6 & Z & -0.0 & 0.2 & & & & -0 . & -0 . \\
\hline H4 & $\mathrm{X}$ & 0 & -0.002 & 0.05 & -0.036 & -0.002 & $-0.05 \varepsilon$ & \\
\hline $\mathrm{H} 4$ & $Y$ & 0.0 & -0.000 & 0.01 & -0.040 & 0.009 & 0.13 & -0 . \\
\hline $\mathrm{H} 4$ & Z & 0.1 & 0.1 & -0.0 & & & -0.0 & \\
\hline H3 & $\mathrm{X}$ & 0 . & -0.002 & 0.03 & 0.036 & & 0.0 & -0 . \\
\hline H3 & Y & 0. & -0.000 & -0.02 & -0.053 & $0.00^{\circ}$ & 0.16 & -0 . \\
\hline $\mathrm{H} 3$ & Z & 0.2 & -0.1 & -0.0 & & -0 & & \\
\hline Re1 & $\mathrm{X}$ & -0.0 & 0.001 & -0.02 & -0.005 & 0 & 0.0 & \\
\hline Re1 & $Y$ & -0.00010 & -0.00031 & 0.005 & 0.019 & 0. & 0.0 & \\
\hline Re1 & Z & -0.0 & -0.0 & -0.00 & -0.00 & 0 & -0.0 & -0 . \\
\hline 01 & $\mathrm{X}$ & 0.0 & -0.003 & 0.00 & & -0 & -0.07 & \\
\hline 01 & Y & 0.03936 & 0.12533 & -0.095 & 0.055 & -0.059 & -0.034 & -0.0 \\
\hline 01 & $\mathrm{Z}$ & 0.0 & 0.0 & -0.00 & & -0 . & -0.0 & \\
\hline 02 & $\mathrm{X}$ & -0.07792 & 0.00290 & 0.008 & & 0 & -0.068 & -0 . \\
\hline $\mathrm{O} 2$ & Y & -0.03930 & -0.11482 & -0.108 & 0.054 & 0 . & -0.040 & \\
\hline 02 & Z & 0.0 & 0.0 & 0.01 & -0 & 0 & 0.06 & \\
\hline 03 & $\mathrm{X}$ & -0.00054 & -0.002 & 0.068 & 0.006 & -0 & -0.052 & -0.0 \\
\hline 03 & Y & -0.00020 & -0.006 & 0.136 & & -0 . & -0.077 & -0.0 \\
\hline 03 & Z & -0.06780 & 0.07 & 0.001 & -0.00077 & -0.02550 & 0.00208 & 0.15 \\
\hline Irequ & & & & & & & & \\
\hline & tie & & & & & & 13.66 & \\
\hline N1 & $\mathrm{X}$ & -0.04054 & -0.00198 & -0.001 & 0.17695 & -0.00157 & -0.10367 & 0.000 \\
\hline N1 & $Y$ & -0.09831 & 0.03339 & -0.002 & & -0.00023 & 0.05075 & \\
\hline N1 & Z & 0.00513 & -0.00568 & 0.07065 & -0.00053 & -0.03209 & -0.00011 & 0.043 \\
\hline N2 & $\mathrm{X}$ & -0.06065 & 0.03017 & -0.00184 & -0.15619 & 0.00059 & -0.08624 & -0.000 \\
\hline $\mathrm{N} 2$ & $\mathrm{Y}$ & 0.02108 & 0.00503 & -0.00058 & 0.03552 & -0.00070 & -0.03363 & -0.000 \\
\hline
\end{tabular}




\begin{tabular}{|c|c|c|c|c|c|c|c|c|}
\hline N2 & z & -0.00577 & 0.00567 & -0.10082 & -0.00086 & -0.03651 & 0.00062 & -0.03489 \\
\hline C4 & $\mathrm{x}$ & -0.01370 & 0.00385 & -0.00118 & 0.01827 & 0.00000 & 0.10207 & 0.00047 \\
\hline C4 & $\mathrm{Y}$ & -0.08954 & 0.03491 & -0.00330 & -0.02933 & 0.00105 & 0.10059 & 0.00056 \\
\hline C4 & Z & -0.00525 & 0.00659 & -0.09177 & -0.00126 & -0.04566 & -0.00077 & 0.09400 \\
\hline C5 & $\mathrm{x}$ & -0.00362 & 0.00404 & -0.00015 & -0.00935 & 0.00047 & 0.08789 & -0.00037 \\
\hline C5 & $\mathrm{Y}$ & -0.01418 & 0.01977 & 0.00015 & -0.05572 & -0.00048 & -0.12231 & -0.00117 \\
\hline C5 & Z & 0.00559 & -0.00631 & 0.09201 & 0.00085 & -0.06059 & 0.00108 & -0.09545 \\
\hline H5 & $\mathrm{x}$ & -0.05872 & 0.02918 & -0.00142 & -0.15144 & 0.00260 & -0.07324 & 0.00167 \\
\hline H5 & $\mathrm{Y}$ & 0.05916 & -0.02129 & 0.00053 & 0.16454 & 0.00462 & 0.25104 & 0.00473 \\
\hline H5 & Z & 0.00196 & -0.00290 & -0.01348 & 0.00157 & 0.52928 & -0.00413 & 0.52341 \\
\hline H6 & $\mathrm{x}$ & 0.06262 & -0.01838 & 0.00230 & 0.09886 & 0.00135 & 0.08950 & 0.00096 \\
\hline $\mathrm{H} 6$ & $\mathrm{Y}$ & 0.01491 & 0.00983 & 0.00287 & -0.00693 & 0.00328 & -0.12840 & 0.00106 \\
\hline H6 & Z & 0.02071 & -0.02307 & 0.32985 & 0.00430 & 0.45414 & -0.00217 & 0.14601 \\
\hline H4 & $\mathrm{x}$ & 0.02244 & 0.00372 & -0.00057 & -0.09753 & 0.00255 & 0.12227 & -0.00094 \\
\hline H 4 & $\mathrm{Y}$ & -0.11977 & 3531 & -0.00603 & & 0.00351 & 0.09079 & \\
\hline H4 & Z & -0.01810 & 0.02183 & -0.31809 & 0.00115 & 0.42907 & -0.00349 & -0 \\
\hline H3 & $\mathrm{x}$ & -0.04522 & -0.00308 & -0.00242 & 0.19435 & 0.00054 & -0.14654 & -0.00261 \\
\hline H3 & $\mathrm{Y}$ & -0.12 & 891 & -0 & & & & \\
\hline H3 & Z & -0.00 & 0.0 & -0 & & & & -0 \\
\hline Re1 & $\mathrm{X}$ & 0.02067 & 0324 & 0.00042 & -0.00290 & 0.00001 & 0.00158 & -0.00002 \\
\hline Re1 & $\mathrm{Y}$ & .01814 & -0.0 & 069 & -0.0 & -0.0 & & 0001 \\
\hline Re1 & z & & -0.0 & -0. & & & -0 . & -0 \\
\hline $\mathrm{O} 1$ & $\mathrm{x}$ & -0.09074 & -0.0 & 5437 & -0.0 & -0.00268 & 0.00078 & -0 . \\
\hline 01 & $\mathrm{Y}$ & -0.03083 & 0.08287 & -0.0 & & -0.00770 & 0496 & -0 . \\
\hline 01 & Z & -0.0 & -0. & & & & -0 & \\
\hline 02 & $\mathrm{x}$ & -0.07172 & -0.08919 & -0.0 & -0.00014 & 0.00266 & 0.00090 & 0108 \\
\hline 02 & $\mathrm{Y}$ & -0.04069 & 0.09439 & & & & & 0325 \\
\hline 02 & Z & & & & -0 . & & & \\
\hline 03 & $\mathrm{x}$ & & 0. & & & -0 & 110 & \\
\hline 03 & $\mathrm{Y}$ & & & -0 & & -0 & -0 . & -0 \\
\hline 03 & Z & -0.01936 & 0.0 & & -0. & & 007 & 0 . \\
\hline \multicolumn{2}{|c|}{ Erequencies } & & & & & & & \\
\hline & ities & & & & & & & \\
\hline N1 & $\mathrm{X}$ & -0.02564 & -0.00004 & 315 & -0.00 & 0.00003 & 0.00007 & 1228 \\
\hline & $\mathrm{Y}$ & 0.02608 & -0.00005 & & -0.0 & & 0113 & -0 \\
\hline & Z & -0.0 & 0 & -0 . & & -0 . & & 006 \\
\hline N2 & $\mathrm{x}$ & 0.04277 & -0.00027 & 969 & 0.0 & 0.00012 & 0039 & 0.03561 \\
\hline N2 & $\mathrm{Y}$ & 0.01315 & -0.00031 & 0.0 & -0.0 & 0.00071 & -0 & -0.01669 \\
\hline & Z & & -0 & & & & & -0 \\
\hline C4 & $\mathrm{x}$ & -0.1 & 0 & -0 & 0.0 & 024 & -0.00050 & -0 \\
\hline C4 & $\mathrm{Y}$ & -0.03958 & 0.00049 & -0 & -0.0 & 0010 & -0.00090 & -0.01082 \\
\hline C4 & Z & & & & -0 & & -0 & \\
\hline C5 & $\mathrm{x}$ & & -0.0 & & -0 . & -0.00045 & 031 & -0 \\
\hline C5 & $\mathrm{Y}$ & -0.06317 & 0.00013 & 0.0 & -0.0 & -0.00120 & 0.00010 & -0.00007 \\
\hline C5 & Z & & -0.0 & & & -0.1 & & \\
\hline & $\mathrm{x}$ & & -0 & & & -0 & -0 & \\
\hline H5 & $\mathrm{Y}$ & 0.26142 & 0030 & 0. & 0. & -0.00367 & -0.00008 & 10475 \\
\hline H5 & Z & & & & & -0.451 & -0 & \\
\hline & $\mathrm{x}$ & & -0 & & & & & -0 . \\
\hline H6 & $\mathrm{Y}$ & -0.00480 & 0.0 & 0. & -0.0 & 0.00555 & 170 & -0.23312 \\
\hline H6 & Z & & & & & & & \\
\hline & $\mathrm{x}$ & -0.3 & & -0.0 & & $-0 . c$ & & 6762 \\
\hline H4 & $\mathrm{Y}$ & 0.07660 & -0.00057 & & -0.0 & -0.0 & 454 & -0.15848 \\
\hline & Z & -0.00028 & 0.00117 & -0. & & $-0.2 \varepsilon$ & 212 & -0.0 \\
\hline & $\mathrm{x}$ & & -0.00026 & & -0.0 & & & \\
\hline H3 & $\mathrm{Y}$ & 0.26 & -0.00131 & & & -0.00120 & -0 & 0000 \\
\hline & Z & -0.00199 & -0.0 & 0.0 & & 0.0 & -0 & -0 \\
\hline Re1 & $\mathrm{x}$ & & & -0.02281 & -0.00162 & 0.00001 & -0.00005 & \\
\hline Re1 & $\mathrm{Y}$ & -0.00038 & & & -0.01253 & & & 0.00081 \\
\hline e1 & Z & 0.00004 & 0.02399 & 0.00092 & 0.000 & -0.00062 & -0.00008 & -0.00002 \\
\hline
\end{tabular}




01
01
01
02
02
02
03
03
03

$X$
$Y$
$Z$
$X$
$Y$
$Z$
$X$
$Y$
$Z$

frequencies

intensities

$\mathrm{N} 1 \mathrm{X}$

N1 Y

N1 Z

N2 X

N2 Y

N2 Z

C 4 X

C4 Y

C4 Z

C5 $X$

C5 Y

C5 Z

H5 X

H5 Y

H5 Z

H $6 \quad X$

H 6 Y

H6 Z

H4 X

H4 Y

H4 Z

H3 $\mathrm{X}$

H3 Y

H3 Z

Re1 X

Re1 Y

Re1 Z

O1 X

O1 Y

01 Z

O2 X

$02 \mathrm{Y}$

O2 Z

$03 \mathrm{X}$

$03 \mathrm{Y}$

O3 Z

frequencies

intensities

$\mathrm{N} 1 \mathrm{X}$

N1 Y

N1 Z

N2 X

N2 Y

N2 Z

C4 X

C4 Y

C4 Z

C5 $\mathrm{X}$ $\begin{array}{rr}-0.00281 & 0.08096\end{array}$

$-0.001430 .03864$

$0.00387-0.13546$

$-0.00320-0.08852$

$-0.00172-0.04314$

$-0.00433-0.13962$

$-0.01514-0.00352$

$0.01240 \quad 0.00242$

0.00002

1238.97

5.48

$-0.00473$

$-0.03788$

0.02057

1383.59

2.97

$0.03309-0$

0.00015

$0.05932-0$

$-0.03275$

$-0.00063$

$-0.01278-0.08652$

$0.00040 \quad 0.00045$

$0.01705-0.05135$

$-0.015140 .00011$

$-0.00002$

0.00031

0.01749

$-0.07180$

$-0.00981$

0.02871

$-0.00023$

$-0.04389$

0.00005

0.03692

0.36698

$-0.21262$

0.00014

$-0.00137$

0.16283

0.48157

0.05204

0.27973

0.00013

$-0.00467$

$0.48013 \quad 0.28087$

$-0.39694-0.27326$

$0.00180-0.00096$

$0.07736-0.04074$

$0.68301-0.33634$

$-0.00711$

0.00450

$-0.00135$

$-0.00030$

0.00043

0.00010

$0.00002-0.00001$

$0.00112-0.00060$

$0.00062 \quad 0.00001$

$-0.00070-0.00033$

$0.00103-0.00045$

$0.00057 \quad 0.00019$

0.00056

0.00073

0.00696

$-0.00228$

$-0.00149$

$-0.00003-0.00006$

0.05866

0.02547

$-0.08866$

0.05543

0.02451

0.07872

0.15029

$-0.10546$

$-0.00088$

1450.34

76.83

1668.35
16.76

$0.03443-0.04524$

$-0.08708 \quad 0.11973$

$0.00077-0.00074$

0.00885

$-0.06954$

0.05799

0.08179

0.00062

0.09942

$-0.00090$

$\begin{array}{rr}0.09942 & 0.08961 \\ 0.02431 & -0.15097\end{array}$

$-0.00046$

0.00048

$-0.10206$

$0.04160-0.08898$

$-0.00008$

0.01790

0.00119

0.07266

0.05477

$0.00088-0.00189$

$0.07183 \quad 0.18490$

$-0.02499-0.04795$

$\begin{array}{ll}-0.00038 & 0.00002\end{array}$

$\begin{array}{ll}-0.13265 & 0.15866\end{array}$

$0.00954-0.01687$

0.00154

$-0.07705$

0.00144

-0.07705
0.03856

0.05770

$0.00169-0.00191$

$0.00043-0.00049$

$0.00016 \quad 0.00012$

$0.00000 \quad 0.00000$

$\begin{array}{ll}-0.00142 & 0.00071\end{array}$

$-0.00057-0.00081$

$\begin{array}{rrr}0.00042 & 0.00253 & -0.00050 \\ -0.00031 & -0.00151 & 0.00067\end{array}$

$0.00071-0.00066-0.00100$

$-0.00261$

$0.00352 \quad 0.00022$

0.00186

$\begin{array}{lll}-0.00196 & 0.00082\end{array}$

0.00008

0.00005

$-0.00036-0.00239$

$\begin{array}{rr}-0.00036 & -0.00239 \\ -0.00028 & 0.00009\end{array}$

$0.00054 \quad 0.00223$

$0.00046-0.00228$

$0.00026 \quad 0.00012$

$0.00075-0.00195$

$\begin{array}{rr}0.00034 & 0.00577\end{array}$

$-0.00004-0.00148$

$0.00089-0.00009$

$3535.04 \quad 3538.02$

$173.64 \quad 130.49$

$0.00656-0.06952$

$-0.001490 .01628$

$\begin{array}{ll}-0.00001 & 0.00015\end{array}$

$0.07126 \quad 0.00742$

$0.00143 \quad 0.00012$

$-0.00028-0.00003$

$-0.00025-0.00016$

$0.00075-0.00561$

$0.00000 \quad 0.00004$

$-0.00136 \quad 0.00037$

3193.57

$3.12 \quad 12.90$

3199.43

$0.00122 \quad 0.00011$

$0.00320-0.00324$

$\begin{array}{lll}0.00002 & 0.00002\end{array}$

$\begin{array}{rr}-0.00002 & 0.00002 \\ 0.00062 & -0.00092\end{array}$

$-0.00277-0.00391$

0.000010 .00003

$\begin{array}{ll}-0.03920 & 0.03513\end{array}$

$\begin{array}{rrr}-0.05317 & 0.04274\end{array}$

$\begin{array}{rrr}-0.00050 & -0.00041\end{array}$

$-0.01899-0.02635$ 


\begin{tabular}{|c|c|c|c|}
\hline C5 & $Y$ & -0.00668 & -0.00060 \\
\hline $\mathrm{C} 5$ & Z & 0.00007 & 0.00000 \\
\hline H5 & X & -0.95336 & -0.09557 \\
\hline H5 & Y & 0.04335 & 0.00445 \\
\hline 5 & Z & 0.00306 & 0.00022 \\
\hline & $\mathrm{X}$ & -0.00945 & 0.00078 \\
\hline & Y & 0.01672 & -0.00140 \\
\hline 6 & Z & -0.00008 & 0.00003 \\
\hline & $\mathrm{X}$ & -0.00381 & 0.0120 \\
\hline & Y & -0.00411 & 0.0124 \\
\hline & Z & -0.00008 & -0.0000 \\
\hline & $\mathrm{X}$ & -0.09467 & 93917 \\
\hline $\mathrm{H}$ & Y & 0.01733 & -0.16954 \\
\hline H3 & Z & 0.00027 & -0.00 \\
\hline $\operatorname{Re} 1$ & $\mathrm{X}$ & 0.00000 & 0 \\
\hline $\operatorname{Re} 1$ & Y & -0.00001 & -0.00001 \\
\hline $\operatorname{Re} 1$ & Z & 0.00000 & 0.00 \\
\hline $\mathrm{O} 1$ & $\mathrm{X}$ & 0.00000 & 0.000 \\
\hline 01 & Y & -0.00003 & 0.0000 \\
\hline 01 & Z & -0.00003 & -0.000 \\
\hline $\mathrm{O} 2$ & $\mathrm{X}$ & 0.00002 & 0.000 \\
\hline $\mathrm{O} 2$ & Y & -0.00002 & 0.0000 \\
\hline 02 & Z & 0.00004 & 0.0 \\
\hline 03 & $\mathrm{X}$ & -0.00009 & -0.0000 \\
\hline 03 & Y & 0.00011 & -0.0000 \\
\hline & Z & 0.00000 & \\
\hline
\end{tabular}

Thermochemical Properties:

pressure: $\quad 1.0000$ atm

rotational symmetry number:

zero point energy: $\quad 48.609 \mathrm{kcal} / \mathrm{mol}$

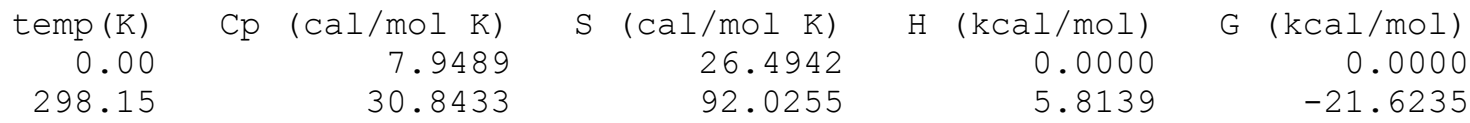

end of program freq

start of program ch

Moments from quantum mechanical wavefunction:

Dipole Moments (Debye)

$\mathrm{X}=1.5379 \quad \mathrm{Y}=12.3830 \quad \mathrm{Z}=\quad-0.1111$ Tot $=\quad 12.4786$

Final energy ........... -492.67714722462

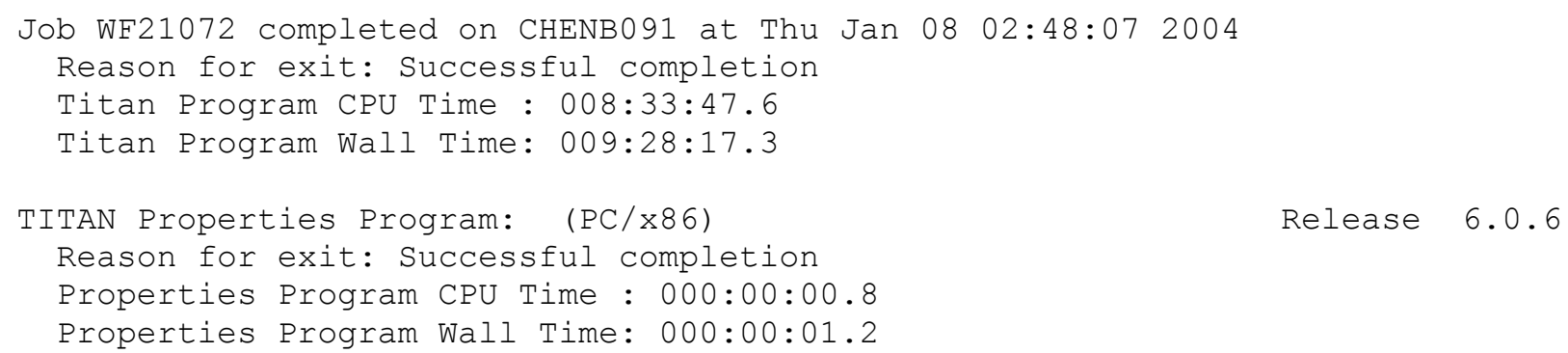




\section{Scheme 5}

$\overbrace{\mathrm{O}}^{\mathrm{H}} \quad-267.17007473960$ hartrees

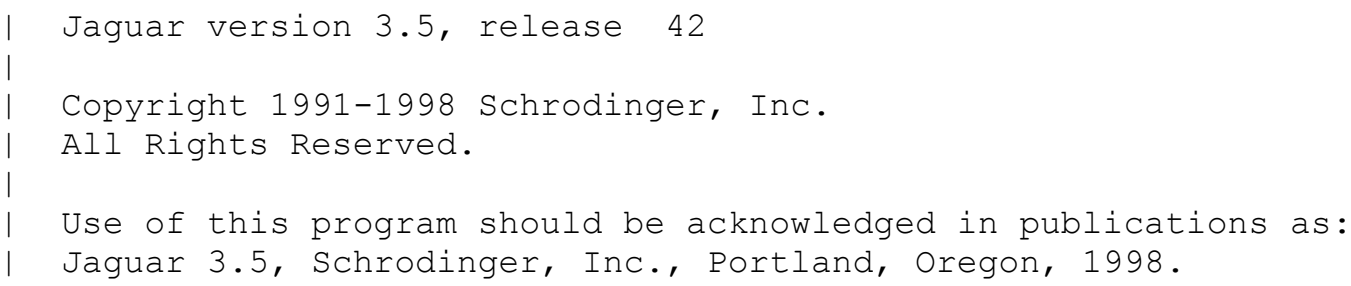

Job name:

Task:

Stoichiometry:

Molecular weight:

Molecular charge:

Spin multiplicity:

Point Group:

SCF method:

Basis set:

Basis functions:

Total electrons:

Alpha electrons:

Beta electrons:

Total orbitals:

Core orbitals:

Open-shell orbitals:

Occupied orbitals:

Shells:
WF11382

Geometry optimization

$\mathrm{C} 3 \mathrm{H} 4 \mathrm{O} 2$

$72.02 \mathrm{amu}$

0

1

Cs

B3LYP

LACVP **

95

38

19

19

95

19

0

19

1

Non-default options chosen:

SCF calculation type: DFT

$\mathrm{DFT}=$ Becke_3_Parameter/HF+Slater+Becke88+VWN+LYP (B3LYP)

Geometry $\overline{w i} \bar{l} l$ be optimized in redundant internal coordinates

Initial Hessian: from previous calculation

Powell Hessian-updating used

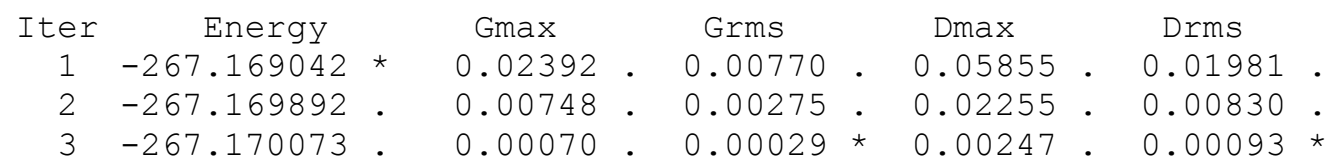




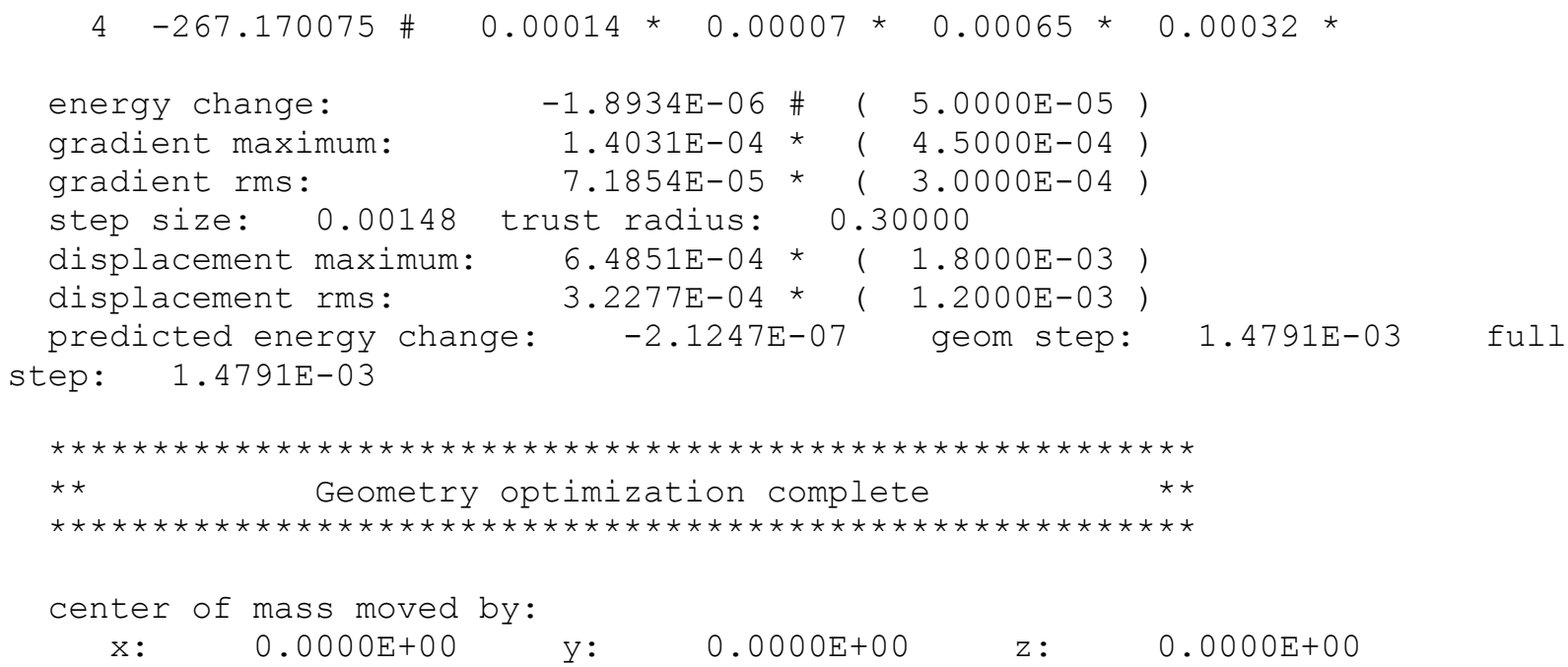

$\begin{array}{lrcc}\text { atom } & \mathrm{x} & \mathrm{y} & \mathrm{z} \\ \mathrm{H} 1 & 1.3089876396 & 1.8457575078 & 0.0000000000 \\ \mathrm{C} 1 & 0.2312585549 & 1.9721699010 & 0.0000000000 \\ \mathrm{H} 3 & -0.1538786480 & 2.9870842984 & 0.0000000000 \\ \text { C2 } & -0.5907374926 & 0.9203852274 & 0.0000000000 \\ \text { H6 } & -1.6702766412 & 1.0307772297 & 0.0000000000 \\ \text { C3 } & -0.1386955099 & -0.4891554774 & 0.0000000000 \\ \text { O1 } & -0.8923755807 & -1.4417312745 & 0.0000000000 \\ \text { O2 } & 1.2120147305 & -0.6307614689 & 0.0000000000 \\ \text { H4 } & 1.3739401854 & -1.5885231260 & 0.0000000000\end{array}$

Final energy ............. -267.17007473960

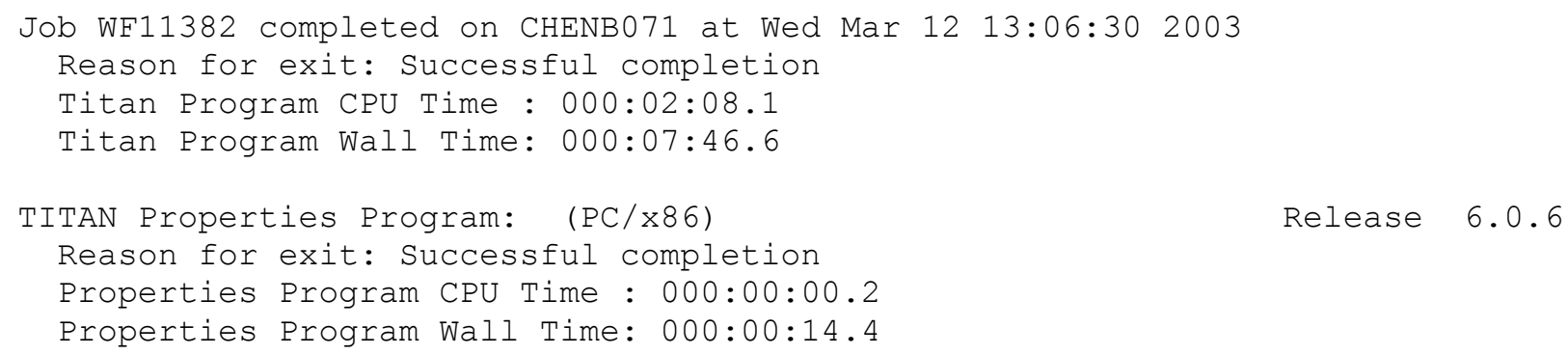




\section{Scheme 5}
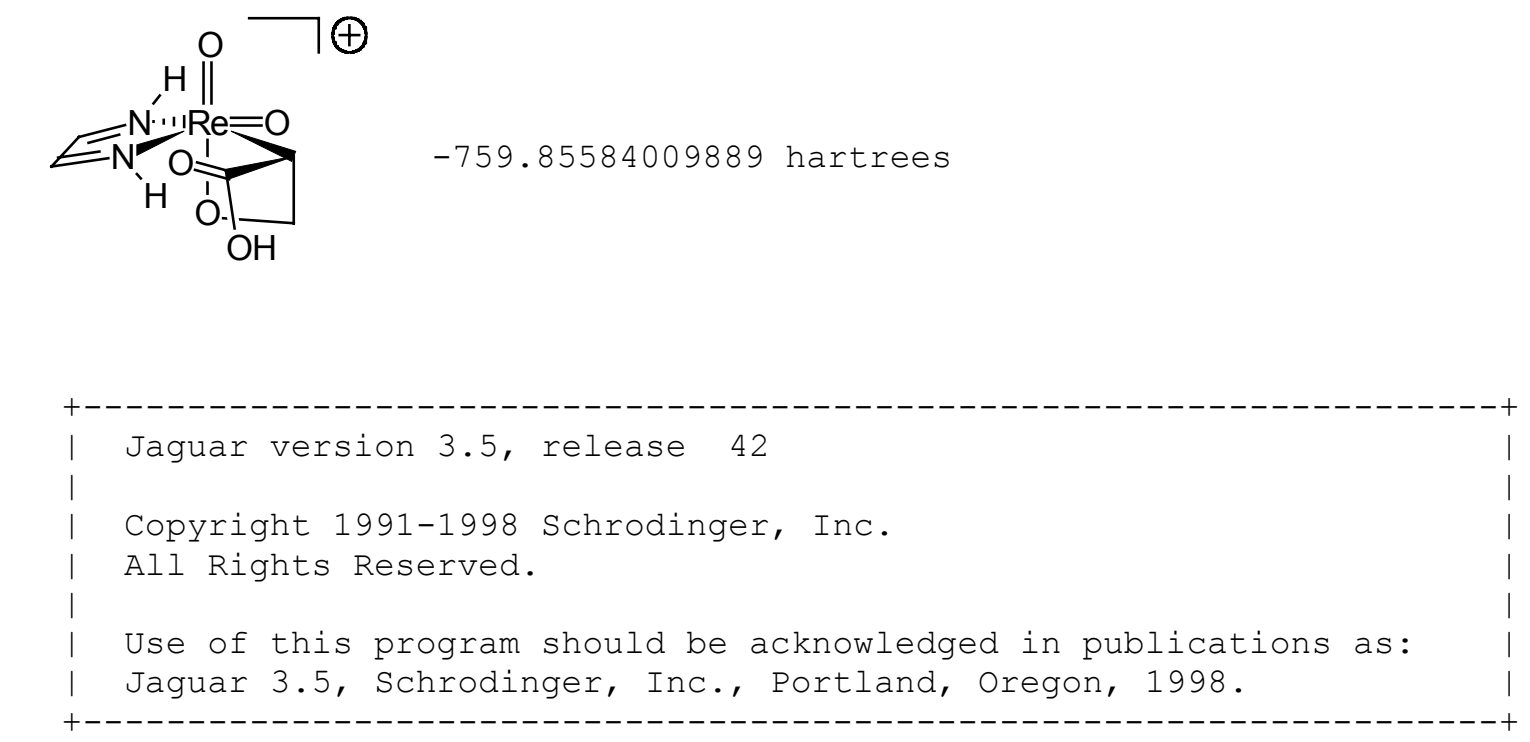

Job WF26685 started on CHENB071 at Fri Jan 23 07:18:33 2004 Jaguar version 3.5, release 42

Job name:

Task:

Stoichiometry:

Molecular weight:

Molecular charge:

Spin multiplicity:

Point Group:

SCF method:

Basis set:

Basis functions:

Total electrons:

Alpha electrons:

Beta electrons:

Total orbitals:

Core orbitals:

Open-shell orbitals:

Occupied orbitals:

Shells:

\section{WF2 6685}

Geometry optimization

ReC5N2H8O5

$363.00 \mathrm{amu}$

1

1

B3LYP

LACVP * *

230

106

53

53

245

53

0

53

1

Non-default options chosen:

SCF calculation type: DFT

DFT=Becke_3_Parameter/HF+Slater+Becke88+VWN+LYP (B3LYP)

Geometry will be optimized in redundant internal coordinates

Numerical 2nd derivatives will be computed

Vibrational frequencies and related properties will be computed Molecular symmetry not used

Initial Hessian: from previous calculation

Powell Hessian-updating used 


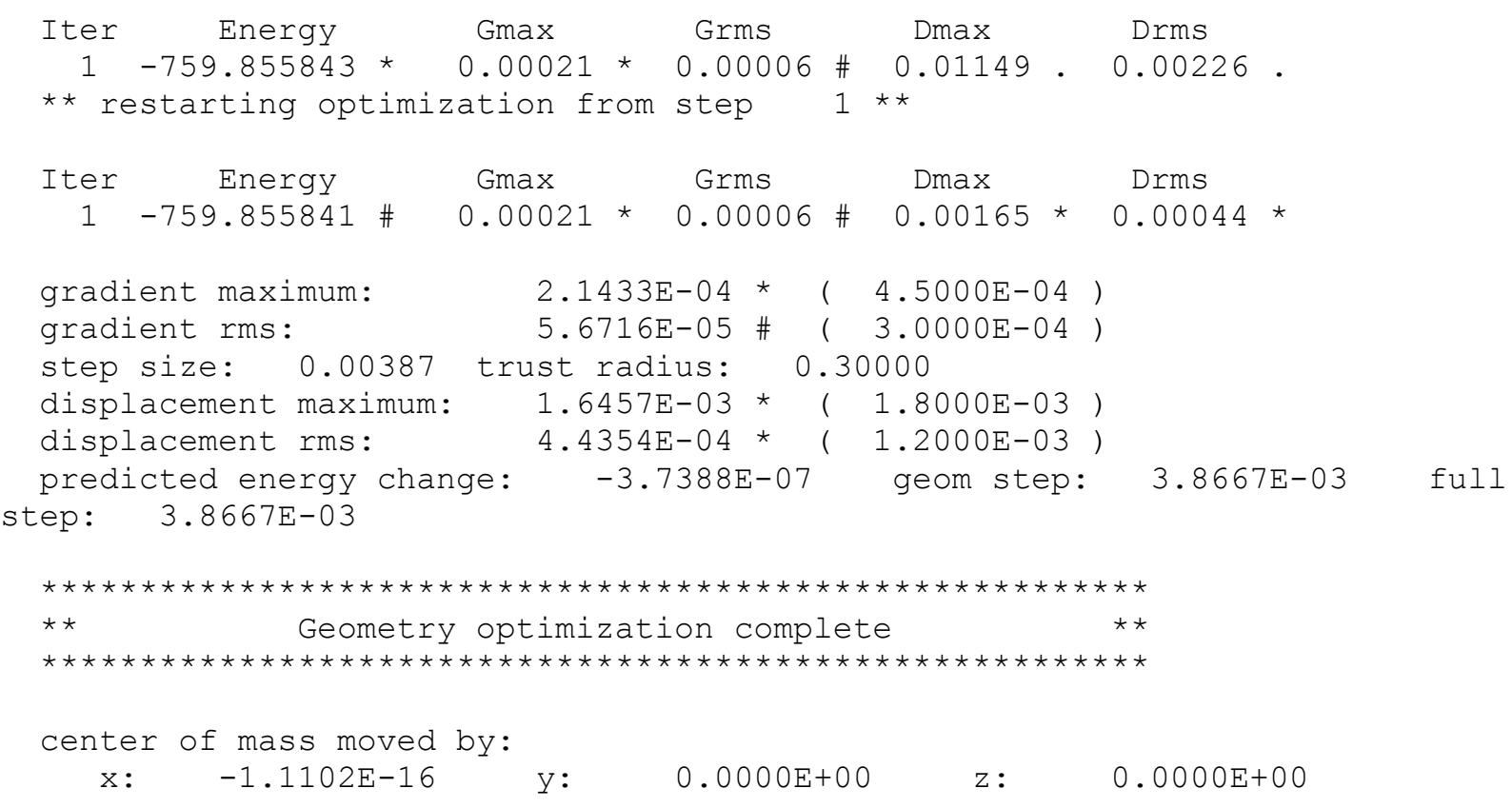

final geometry:

\begin{tabular}{lccc} 
atom & x & \multicolumn{1}{c}{ y } & \multicolumn{1}{c}{$z$} \\
Re1 & -0.0383500000 & -0.2364890000 & 1.2063340000 \\
N1 & -1.2731920000 & 0.1527510000 & -0.6720240000 \\
C4 & -2.5039540000 & 0.4622570000 & -0.6127200000 \\
C7 & -3.0753200000 & 0.4656570000 & 0.7655350000 \\
N2 & -2.2466550000 & 0.2163840000 & 1.7018940000 \\
H18 & -0.7700840000 & 0.1714100000 & -1.5634350000 \\
H21 & -3.1143880000 & 0.7219030000 & -1.4765880000 \\
H23 & -4.1308380000 & 0.6774170000 & 0.9344850000 \\
H26 & -2.5912260000 & 0.2193930000 & 2.6626510000 \\
O1 & 0.5097830000 & -1.5992440000 & 0.3289970000 \\
O2 & 0.0815210000 & -0.6447660000 & 2.8464130000 \\
O3 & 0.0163960000 & 1.7073770000 & 1.1521180000 \\
C1 & 1.4096640000 & 2.0130580000 & 1.0828470000 \\
H3 & 1.7374030000 & 2.4496140000 & 2.0319920000 \\
H4 & 1.6142740000 & 2.7097540000 & 0.2643560000 \\
C2 & 1.9648750000 & 0.6034830000 & 0.8449200000 \\
H1 & 2.7168950000 & 0.2156370000 & 1.5304300000 \\
C3 & 2.2634570000 & 0.3314230000 & -0.6090190000 \\
O4 & 3.4612710000 & -0.2155800000 & -0.7923290000 \\
H8 & 3.5904120000 & -0.3640380000 & -1.7467860000 \\
O5 & 1.4838760000 & 0.6055870000 & -1.5074700000 \\
& & &
\end{tabular}

calculating forces at perturbed geometry calculating forces at perturbed geometry calculating forces at perturbed geometry calculating forces at perturbed geometry calculating forces at perturbed geometry calculating forces at perturbed geometry calculating forces at perturbed geometry calculating forces at perturbed geometry calculating forces at perturbed geometry calculating forces at perturbed geometry calculating forces at perturbed geometry calculating forces at perturbed geometry calculating forces at perturbed geometry

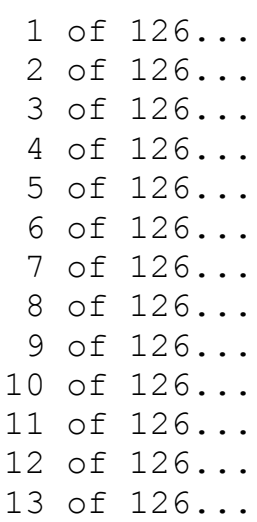


calculating forces at perturbed geometry calculating forces at perturbed geometry calculating forces at perturbed geometry calculating forces at perturbed geometry calculating forces at perturbed geometry calculating forces at perturbed geometry calculating forces at perturbed geometry calculating forces at perturbed geometry calculating forces at perturbed geometry calculating forces at perturbed geometry calculating forces at perturbed geometry calculating forces at perturbed geometry calculating forces at perturbed geometry calculating forces at perturbed geometry calculating forces at perturbed geometry calculating forces at perturbed geometry calculating forces at perturbed geometry calculating forces at perturbed geometry calculating forces at perturbed geometry calculating forces at perturbed geometry calculating forces at perturbed geometry calculating forces at perturbed geometry calculating forces at perturbed geometry calculating forces at perturbed geometry calculating forces at perturbed geometry calculating forces at perturbed geometry calculating forces at perturbed geometry calculating forces at perturbed geometry calculating forces at perturbed geometry calculating forces at perturbed geometry calculating forces at perturbed geometry calculating forces at perturbed geometry calculating forces at perturbed geometry calculating forces at perturbed geometry calculating forces at perturbed geometry calculating forces at perturbed geometry calculating forces at perturbed geometry calculating forces at perturbed geometry calculating forces at perturbed geometry calculating forces at perturbed geometry calculating forces at perturbed geometry calculating forces at perturbed geometry calculating forces at perturbed geometry calculating forces at perturbed geometry calculating forces at perturbed geometry calculating forces at perturbed geometry calculating forces at perturbed geometry calculating forces at perturbed geometry calculating forces at perturbed geometry calculating forces at perturbed geometry calculating forces at perturbed geometry calculating forces at perturbed geometry calculating forces at perturbed geometry calculating forces at perturbed geometry calculating forces at perturbed geometry calculating forces at perturbed geometry calculating forces at perturbed geometry calculating forces at perturbed geometry calculating forces at perturbed geometry calculating forces at perturbed geometry calculating forces at perturbed geometry
14 of $126 \ldots$ 15 of $126 \ldots$ 16 of $126 \ldots$ 17 of $126 \ldots$ 18 of $126 \ldots$ 19 of $126 \ldots$ 20 of $126 \ldots$ 21 of $126 \ldots$ 22 of $126 \ldots$ 23 of $126 \ldots$ 24 of $126 \ldots$ 25 of $126 \ldots$ 26 of $126 \ldots$ 27 of $126 \ldots$ 28 of $126 \ldots$ 29 of $126 \ldots$ 30 of $126 \ldots$ 31 of $126 \ldots$ 32 of $126 \ldots$ 33 of $126 \ldots$ 34 of $126 \ldots$ 35 of $126 \ldots$ 36 of $126 \ldots$ 37 of $126 \ldots$ 38 of $126 \ldots$ 39 of $126 \ldots$ 40 of $126 \ldots$ 41 of $126 \ldots$ 42 of $126 \ldots$ 43 of $126 \ldots$ 44 of $126 \ldots$ 45 of $126 \ldots$ 46 of $126 \ldots$ 47 of $126 \ldots$ 48 of $126 \ldots$ 49 of $126 \ldots$ 50 of $126 \ldots$ 51 of $126 \ldots$ 52 of $126 \ldots$ 53 of $126 \ldots$ 54 of $126 \ldots$ 55 of $126 \ldots$ 56 of $126 \ldots$ 57 of $126 \ldots$ 58 of $126 \ldots$ 59 of $126 \ldots$ 60 of $126 \ldots$ 61 of $126 \ldots$ 62 of $126 \ldots$ 63 of $126 \ldots$ 64 of $126 \ldots$ 65 of $126 \ldots$ 66 of $126 \ldots$ 67 of $126 \ldots$ 68 of $126 \ldots$ 69 of $126 \ldots$ 70 of $126 \ldots$ 71 of $126 \ldots$ 72 of $126 \ldots$ 73 of $126 \ldots$ 74 of $126 \ldots$ 
calculating forces at perturbed geometry calculating forces at perturbed geometry calculating forces at perturbed geometry calculating forces at perturbed geometry calculating forces at perturbed geometry calculating forces at perturbed geometry calculating forces at perturbed geometry calculating forces at perturbed geometry calculating forces at perturbed geometry calculating forces at perturbed geometry calculating forces at perturbed geometry calculating forces at perturbed geometry calculating forces at perturbed geometry calculating forces at perturbed geometry calculating forces at perturbed geometry calculating forces at perturbed geometry calculating forces at perturbed geometry calculating forces at perturbed geometry calculating forces at perturbed geometry calculating forces at perturbed geometry calculating forces at perturbed geometry calculating forces at perturbed geometry calculating forces at perturbed geometry calculating forces at perturbed geometry calculating forces at perturbed geometry calculating forces at perturbed geometry 100 of $126 \ldots$ calculating forces at perturbed geometry 101 of $126 \ldots$ calculating forces at perturbed geometry 102 of $126 \ldots$ calculating forces at perturbed geometry 103 of $126 \ldots$ calculating forces at perturbed geometry 104 of $126 \ldots$ calculating forces at perturbed geometry 105 of $126 \ldots$ calculating forces at perturbed geometry 106 of $126 \ldots$ calculating forces at perturbed geometry 107 of $126 \ldots$ calculating forces at perturbed geometry 108 of $126 \ldots$ calculating forces at perturbed geometry 109 of $126 \ldots$ calculating forces at perturbed geometry 110 of $126 \ldots$ calculating forces at perturbed geometry 111 of $126 \ldots$ calculating forces at perturbed geometry 112 of $126 \ldots$ calculating forces at perturbed geometry 113 of $126 \ldots$ calculating forces at perturbed geometry 114 of $126 \ldots$ calculating forces at perturbed geometry 115 of $126 \ldots$ calculating forces at perturbed geometry 116 of $126 \ldots$ calculating forces at perturbed geometry 117 of $126 \ldots$ calculating forces at perturbed geometry 118 of $126 \ldots$ calculating forces at perturbed geometry 119 of $126 \ldots$ calculating forces at perturbed geometry 120 of $126 \ldots$ calculating forces at perturbed geometry 121 of $126 \ldots$ calculating forces at perturbed geometry 122 of $126 \ldots$ calculating forces at perturbed geometry 123 of $126 \ldots$ calculating forces at perturbed geometry 124 of $126 \ldots$ calculating forces at perturbed geometry 125 of $126 \ldots$ calculating forces at perturbed geometry 126 of $126 \ldots$

numerical derivatives complete;

calculation at normal geometry follows...

start of program freq

harmonic frequencies in $\mathrm{cm}^{\wedge}-1$, IR intensities in $\mathrm{km} / \mathrm{mol}$, and normal modes:

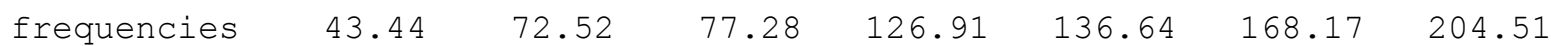




\begin{tabular}{|c|c|c|c|c|c|c|c|c|}
\hline ntens & ities & 1.02 & 7.92 & 7.49 & 1.52 & 3.85 & 2.49 & 4.34 \\
\hline Re1 & $\mathrm{X}$ & -0.00355 & 0.00117 & 0.00598 & -0.00648 & -0.00864 & 0.01366 & 0.00278 \\
\hline $\operatorname{Re} 1$ & $\mathrm{Y}$ & 0.00672 & -0.00162 & 0.01234 & 0.01226 & 0.00617 & 0.01385 & -0.00331 \\
\hline Re1 & Z & -0.01552 & 0.01876 & -0.01001 & 0.01357 & 0.00963 & -0.00633 & -0.00550 \\
\hline $\mathrm{N} 1$ & $\mathrm{x}$ & -0.03116 & 0.06440 & -0.01214 & 0.01928 & -0.00933 & 0.01153 & 0.00792 \\
\hline N1 & $\mathrm{Y}$ & -0.02848 & -0.00585 & -0.03804 & 0.04443 & 0.04867 & 0.08503 & 0.02204 \\
\hline N1 & Z & 0.02183 & -0.02134 & 0.00190 & 0.00421 & -0.00375 & 0.00197 & 0.06528 \\
\hline C4 & $\mathrm{X}$ & -0.04357 & 0.06128 & -0.02974 & -0.00611 & -0.02047 & 0.00243 & 0.00927 \\
\hline $\mathrm{C} 4$ & $\mathrm{Y}$ & -0.07502 & -0.01200 & -0.10389 & -0.05448 & 0.00165 & 0.04593 & 0.04044 \\
\hline C4 & Z & 0.01937 & -0.06776 & -0.00400 & -0.01594 & -0.00134 & -0.00301 & 0.04592 \\
\hline C7 & $\mathrm{X}$ & -0.02878 & 0.01883 & -0.01996 & -0.01579 & -0.03778 & -0.03430 & -0.05460 \\
\hline C7 & $\mathrm{Y}$ & -0.01955 & -0.00110 & -0.05876 & -0.06839 & -0.09107 & -0.08766 & -0.02053 \\
\hline $\mathrm{C} 7$ & Z & 0.02532 & -0.08604 & -0.00027 & -0.01965 & -0.00725 & -0.01749 & 0.02009 \\
\hline $\mathrm{N} 2$ & $\mathrm{X}$ & -0.00352 & -0.00860 & 0.00271 & -0.00619 & -0.03305 & -0.03451 & -0.06935 \\
\hline N2 & $\mathrm{Y}$ & 0.06428 & 0.01322 & 0.03373 & 0.00691 & -0.05679 & -0.06080 & -0.00303 \\
\hline $\mathrm{N} 2$ & Z & 0.02381 & -0.05709 & 0.00336 & -0.00720 & -0.00110 & -0.01006 & 0.03340 \\
\hline $\mathrm{H} 18$ & $\mathrm{x}$ & -0.04361 & 0.10564 & -0.02866 & 0.03478 & 0.00143 & 0.04537 & 0.03066 \\
\hline H18 & $\mathrm{Y}$ & -0.05884 & -0.00561 & -0.05528 & 0.05781 & 0.11650 & 0.17190 & 0.078 \\
\hline $\mathrm{H} 18$ & Z & 0.01485 & 0.00134 & -0.00709 & 0.01230 & 0.00314 & 0.02252 & 0.07810 \\
\hline H21 & $\mathrm{X}$ & -0.05862 & 0.08911 & -0.04744 & -0.01834 & -0.02278 & 0.01626 & 0.05 \\
\hline H21 & $\mathrm{Y}$ & -0.14163 & -0.02169 & -0.17834 & -0.11431 & 0.02535 & 0.09930 & 0.096 \\
\hline $\mathrm{H} 21$ & $\mathrm{Z}$ & 0.01023 & -0.09043 & -0.01386 & -0.02532 & 0.00727 & 0.00302 & 0.03042 \\
\hline $\mathrm{H} 23$ & $\mathrm{X}$ & -0.03339 & 0.01359 & -0.02773 & -0.02834 & -0.05610 & -0.05766 & -0.07 \\
\hline H23 & $\mathrm{Y}$ & -0.04325 & -0.00237 & -0.09479 & -0.12419 & -0.17538 & -0.19011 & -0.06869 \\
\hline $\mathrm{H} 23$ & Z & 0.02636 & -0.11871 & -0.00328 & -0.02909 & -0.01479 & -0.03346 & -0.02280 \\
\hline H26 & $\mathrm{X}$ & 0.01626 & -0.03926 & 0.01533 & -0.01334 & -0.05509 & -0.06526 & $-0.0 \varepsilon$ \\
\hline H26 & $\mathrm{Y}$ & 0.103 & 0.02350 & 0.06943 & 0.01054 & -0.11793 & -0.15389 & -0.058 \\
\hline H26 & $\mathrm{Z}$ & 0.03093 & -0.06833 & 0.00775 & -0.00974 & -0.00867 & -0.02083 & 0.02708 \\
\hline 01 & $\mathrm{X}$ & -0.01685 & -0.00050 & 0.02649 & -0.03816 & -0.07095 & 0.00716 & 0.1 \\
\hline O1 & $\mathrm{Y}$ & -0.00941 & -0.02135 & 0.02029 & 0.04070 & -0.02030 & 0.02064 & -0.00414 \\
\hline 01 & Z & 0.00128 & 0.04527 & -0.01352 & -0.05169 & 0.01408 & -0.01861 & 0.05676 \\
\hline 02 & $\mathrm{X}$ & -0.04138 & -0.03211 & -0.02289 & -0.00130 & 0.04827 & 0.01615 & -0.14 \\
\hline 02 & $Y$ & 0 & 0.01244 & -0.01045 & -0.07860 & 0.06533 & -0.01180 & -0.00370 \\
\hline $\mathrm{O} 2$ & Z & -0.01522 & 0.02414 & -0.01449 & -0.00984 & 0.02207 & -0.01207 & 0.004 \\
\hline 03 & $\mathrm{X}$ & 0.05750 & 0.02975 & 0.02684 & 0.01324 & 0.03234 & 0.00091 & 0.01 \\
\hline 03 & $Y$ & 0.019 & 0.00160 & 0.01632 & 0.01241 & -0.01749 & -0.00372 & -0.00750 \\
\hline 03 & Z & 0.00954 & 0.04767 & 0.02510 & 0.12089 & -0.07859 & -0.01375 & -0.00640 \\
\hline C1 & $\mathrm{X}$ & 0.06777 & 0.03281 & 0.03157 & 0.00396 & 0.04905 & 0.00281 & 0.01216 \\
\hline C1 & $Y$ & -0.028 & -0.02117 & 0.00137 & 0.01021 & -0.05014 & 0.00649 & 0.00206 \\
\hline $\mathrm{C} 1$ & Z & 0.05616 & 0.03146 & 0.05315 & -0.05460 & 0.01490 & 0.01004 & -0.04992 \\
\hline H3 & $\mathrm{X}$ & 0.06025 & 0.05782 & 0.02155 & 0.10846 & 0.00474 & -0.01878 & 0.02834 \\
\hline H3 & $Y$ & -0.074 & -0.05764 & -0.02718 & 0.07166 & -0.10619 & 0.01716 & 0.04749 \\
\hline H3 & Z & 0.07995 & 0.03980 & 0.06980 & -0.11909 & 0.05581 & 0.01215 & -0.07665 \\
\hline $\mathrm{H} 4$ & $\mathrm{X}$ & 0.11658 & 0.03186 & 0.05266 & -0.09345 & 0.11121 & 0.01314 & -0.01531 \\
\hline H 4 & $Y$ & -0.004 & 0.00278 & 0.01839 & -0.03919 & -0.01639 & 0.00124 & -0.03119 \\
\hline H4 & Z & 0.08794 & 0.05139 & 0.07298 & -0.12078 & 0.05999 & 0.00797 & -0.08495 \\
\hline $\mathrm{C} 2$ & $\mathrm{X}$ & 0.02405 & 0.00340 & 0.01742 & -0.00809 & 0.01629 & 0.01175 & 0.01 \\
\hline $\mathrm{C} 2$ & $Y$ & -0.04351 & -0.02468 & 0.00159 & 0.00087 & -0.05721 & 0.00638 & -0.00088 \\
\hline $\mathrm{C} 2$ & Z & 0.02411 & -0.02103 & 0.02612 & -0.04762 & -0.01044 & 0.03651 & -0.01792 \\
\hline $\mathrm{H} 1$ & $\mathrm{X}$ & 0.01173 & 0.02555 & 0.01174 & -0.01216 & 0.00974 & 0.01800 & 0.02241 \\
\hline $\mathrm{H} 1$ & $Y$ & -0.08114 & -0.05092 & -0.02763 & 0.01536 & -0.08916 & 0.02165 & 0.01381 \\
\hline $\mathrm{H} 1$ & Z & 0.01616 & -0.06006 & 0.01572 & -0.03451 & -0.02130 & 0.03795 & -0.01384 \\
\hline C3 & $\mathrm{X}$ & 0.01616 & -0.04689 & -0.01360 & 0.02467 & 0.03321 & -0.03053 & 0.02122 \\
\hline C3 & $\mathrm{Y}$ & -0.01382 & 0.01085 & 0.00543 & -0.02235 & 0.00467 & -0.03919 & -0.00161 \\
\hline $\mathrm{C} 3$ & Z & 0.01711 & -0.03960 & 0.02003 & -0.03859 & -0.02119 & 0.04110 & -0.02207 \\
\hline 04 & $\mathrm{X}$ & 0.06526 & -0.04385 & -0.07566 & 0.03674 & 0.03238 & -0.03400 & 0.01579 \\
\hline 04 & $\mathrm{Y}$ & 0.10401 & 0.04049 & -0.12183 & -0.01389 & -0.00100 & -0.02678 & -0.00861 \\
\hline 04 & Z & -0.01246 & -0.09901 & 0.00037 & 0.00374 & -0.02503 & 0.00607 & -0.03239 \\
\hline $\mathrm{H} 8$ & $\mathrm{X}$ & 0.06207 & -0.07577 & -0.10239 & 0.06579 & 0.05309 & -0.08212 & 0.01287 \\
\hline H8 & $\mathrm{Y}$ & 0.12734 & 0.06893 & -0.12521 & -0.02363 & 0.05858 & -0.07744 & 0.00801 \\
\hline H 8 & Z & -0.01679 & -0.10807 & -0.00261 & 0.00927 & -0.03157 & 0.00736 & -0.0356 \\
\hline
\end{tabular}




$\begin{array}{rrrr}05 & X & -0.02768 & -0.08106 \\ 05 & Y & -0.07805 & 0.01812 \\ 05 & Z & 0.03600 & -0.00918\end{array}$

frequencies intensities

Re1 X

Re1 Y

Re1 Z

N1 X

N1 Y

N1 Z

C4 X

C 4 Y

C4 Z

C7 $\mathrm{X}$

C7 Y

C7 Z

N2 $\mathrm{X}$

N2 Y

N2 Z

H18 X

H18 Y

H18 Z

H21 X

H21 Y

H21 Z

H23 X

H23 Y

H23 Z

H26 X

H26 Y

H26 Z

01

01

01

02

02

02

03

03

03

C1

C1

C1 1

H3

H3

H3

H 4

H 4

$\mathrm{H} 4$

C2

$\mathrm{C} 2$

C2

$\mathrm{H} 1$

$\mathrm{H} 1$

H1

C3

C3

C3

04

$0.00310-0.00354$

$0.00597-0.01984$

$-0.07362$

$-0.05093$

$-0.02287$

$-0.05651$

0.02495

$-0.02891$

$-0.07312$

0.06502

$-0.03489$

$-0.11135$

$-0.02955$

$-0.02484$

$-0.09961$

$-0.10367$

$-0.03747$

$-0.04009$

0.03726

$-0.03738$

$-0.06123$

0.14430

$-0.05488$

$-0.14736$

$-0.01830$

$-0.03750$

0.09299

0.04768

$-0.00462$

0.08905

0.01118

$-0.00022$

0.01194

$-0.02495$

0.05150

0.01521

$-0.02222$

0.02601

0.03043

$-0.03718$

0.02719

0.01989

0.03383

0.01771

0.01327

0.01252

0.02062

$-0.00168$

$-0.02868$

0.01539

0.00805

0.01085

0.01514

0.02717

0.07498

0.02075

0.03248

0.02178

$-0.01092$

$-0.07023$

0.00849

0.01477

$-0.00815$

0.01732

0.02733

$-0.04900$

0.06141

0.03220

0.09414

0.00099

$-0.01785$

0.01409

0.09536

$-0.01710$

$-0.00175$

0.09273

0.01033

$-0.10412$

$-0.00806-0.01474$

$-0.00930$

0.07911

0.03205

0.01407

0.16113

$-0.04193$

$-0.01810$

0.01239

$-0.00765$

0.00651

$$
-0.03167
$$

-0.03167
-0.04131

$-0.03267$

$-0.01202$

$-0.01089-0.03106$

$$
\begin{array}{rr}
228.65 & 243.71 \\
1.48 & 2.10 \\
0.00195 & -0.00102
\end{array}
$$

$0.02086-0$

$-0.03538-0$

0.00095

0.05205

$0.06297-0.08558$

0.02813

$0.09741-0.03909$

$0.07176-0.10340$

0.02604

$0.03484-0.06675$

247.66

4.79

272.01

7.47

0.01196

95.89
4.82

311.66

1.13

$0.02018-0.00593$

0.02928

$-0.00033$

$-0.00189-0.02922$

0.00183

$\begin{array}{lll}0.00721 & 0.00598 & 0.01674\end{array}$

$0.02894 \quad 0.04968-0.08660$

$-0.01543$

$-0.018670 .03781$

$0.08435-0.08816$

$0.00651-0.00310$

$0.00085-0.00991$

$0.11037-0.03364$

$-0.00826$

0.03604

0.00578

$0.04572-0.09003$

$0.00639-0.01339$

$578-0.02923 \quad 0.01956-0.02239-0.02720$

$0.06181-0.03447-0.01214-0.03507-0.00199$

$\begin{array}{llllll}-0.03013 & 0.03113-0.00466 & -0.01775 & -0.01746\end{array}$

$0.02123-0.01961-0.00201-0.02511-0.00302$

$\begin{array}{llllll}0.08240 & -0.09156 & 0.04160 & -0.00103 & 0.00139\end{array}$

$\begin{array}{rrrrr}-0.02175 & 0.02905 & 0.00772 & 0.13507 & 0.00613\end{array}$

$\begin{array}{llllll}0.00754 & 0.02962 & -0.04587 & -0.01088 & 0.00145\end{array}$

$\begin{array}{llllll}0.04097 & 0.10241 & -0.13512 & 0.02129 & -0.01168\end{array}$

$-0.00012-0.03910 \quad 0.05349$

$-0.00183$

0.11184

$-0.11407$

$0.16767-0.01039$

$\begin{array}{llllll}0.03689 & -0.06739 & 0.02739 & -0.07114 & 0.09034\end{array}$

$\begin{array}{lllll}0.02989 & -0.03711 & 0.00539 & -0.03322 & 0.01018\end{array}$

$0.05952-0.03859-0.00524-0.04944-0.01350$

$\begin{array}{llllll}-0.06792 & 0.07362 & -0.02553 & -0.08529 & -0.07055\end{array}$

$\begin{array}{lllllll}0.04893 & -0.09574 & 0.06794 & -0.02877 & -0.00788\end{array}$

$\begin{array}{llllll}0.09878 & -0.13943 & 0.08002 & 0.00080 & -0.01589\end{array}$

$\begin{array}{rrrrr}-0.05389 & 0.06972 & 0.00048 & 0.19961 & -0.03309\end{array}$

$\begin{array}{llllll}0.01327 & 0.01253 & -0.03222 & -0.01051 & -0.00436\end{array}$

$\begin{array}{lllll}0.12330 & -0.03594 & 0.00231 & 0.06646 & 0.02625\end{array}$

$\begin{array}{lllll}0.09560 & 0.02643 & -0.00300 & 0.01621 & -0.02744\end{array}$

$\begin{array}{llllll}-0.03729 & -0.06207 & 0.01388 & -0.01532 & 0.07039\end{array}$

$0.00642 \quad 0.00372-0.08768$

$-0.01478-0.05181-0.04260$

$0.00322-0.005590 .01165$

$0.00549-0.01552-0.00362$

$\begin{array}{lll}0.00367 & 0.00727 & 0.00029\end{array}$

$-0.05286-0.08863-0.04192$

$0.01909-0.00408$

0.00120

0.00896

0.05007

0.04065

0.00543

0.05439

$-0.02061$

$-0.07505$

$-0.04551$

$-0.07578$

0.10546

0.04762

0.08764

0.12869

0.08255

0.07594

0.01923

0.09143

0.09908

0.14686

$-0.00069-0.02152$

0.08246

0.12354

$-0.00135$

$0.01772 \quad 0.02496$

$-0.02751$

$-0$

0.01790

0.03561

0.06425

0.04431

$0.02675-0.08498$

$0.01309-0.03090$

0.017690 .03786

$0.02352 \quad 0.02563$

$-0.03605-0.01483$

$\begin{array}{ll}0.00451 & 0.05155\end{array}$

$0.02548-0.03097$

$-0.03668-0.05962$

0.008030 .09660

0.065630 .01993

$-0.05543-0.09945$

$\begin{array}{rr}0.01398 & 0.06522\end{array}$

0.01690

$-0.01057-0.10078$

$-0.06685-0.11515$

$0.00118-0.06614$

$0.01871-0.07042$

0.006360 .00016

$-0.00742-0.05907$

$-0.00763-0.05956-0.02498-0.05604-0.06218-0.01549-0.03065$

20.02507 $-0.00517-0.03268-0.02003$

$0.00695-0.01825$

0.01518

0.01560

$-0.01068-0.07656$

$0.00741-0.01325$

$-0.02024-0.02886-0.03313$

$0.00866 \quad 0.00556$

$0.00371-0.02600-0.01340-0.00829-0.04498$ 


O4
O4
H8
H8
H8
O5
O5
O5

frequencies intensities

$\operatorname{Re} 1 \mathrm{X}$

Re1 Y

Re1 Z

N1 $\mathrm{X}$

N1 Y

N1 Z

C4 X

C4 Y

C4 Z

C7 $\mathrm{X}$

C7 Y

C7 Z

N2 $\mathrm{X}$

N2 Y

N2 Z

H18 X

H18 Y

H18 Z

H21 X

H21 Y

H21 Z

H23 X

H23

H23

H26

H26 Y

H26 Z

$01 \mathrm{X}$

$01 Y$

01

02

02

02

03

03

03

C1

C1

C1

H3

H3

H3

H4

$\mathrm{H} 4$

$\mathrm{H} 4$

C2

C2

C2

$\mathrm{H} 1$

$\mathrm{H} 1$

$-0.00951-0.01323-0.00578$

$\begin{array}{llll}-0.01876 & 0.05469 & -0.01251\end{array}$

$\begin{array}{lll}0.01010 & 0.03088 & 0.00868\end{array}$

$-0.00901-0.06389-0.00308$

$\begin{array}{lll}-0.01996 & 0.06976-0.01226\end{array}$

$\begin{array}{llll}0.00959 & 0.01751 & 0.00436\end{array}$

$-0.01666-0.03883-0.03285$

$-0.00514-0.08220-0.03193$

$\begin{array}{rr}366.82 & 393.51 \\ 4.19 & 2.94\end{array}$

$-0.01490-0.00200$

$0.00417-0.01211$

$\begin{array}{rrr}-0.00140 & 0.00369\end{array}$

$-0.00132-0.03396$

$-0.01949-0.12375-0$

$0.00124-0.00114$

$0.00959 \quad 0.02218$

0.025790 .09588

$-0.00083 \quad 0.00415$

$-0.00765-0.01936-0$

$-0.02330-0.06705-0$

$\begin{array}{rr}-0.00822 & -0.01568 \\ -0.00004 & 0.01042\end{array}$

0.01042

0.03912

0.05411

$0.00427-0.00782$

$0.00970-0.00681$

$0.01329-0.07253$

$0.00755 \quad 0.01515$

0.02991

0.08977

$0.08787 \quad 0.31816$

0.00346

0.02348

$-0.02510-0.06431$

$-0.10077-0.28043$

$-0.01981-0.02928-0$

$-0.01996-0.01819$

$0.00487-0.07834$

$-0.00258-0.01756-0$.

$-0.00142-0.03566$

$\begin{array}{lll}-0.05504 & 0.02136\end{array}$

$0.10584-0.06685-0$

$0.06594-0.01075$

$-0.11560$

$-0.03974$

$-0.00509$

0.04424

0.01823

$0.04890-0$

441.58

0.33

0.01637

$0.00802-0.00118$

0.00192

$-0.01405$

$-0.03508$

0.00087

0.00497

0.03844

$-0.00171$

0.00065

0.03896

0.00338

$-0.16637$

0.04698

$-0.00854-0$

0.00574

0.04290

$-0.01967$

0.02748

01100

0.01417

$-0.07752$

0.09739

$-0.00054$

0.03224

0.14386

$0.00899-0.06002$

0.02475

0.01477

$0.01454-0.22097$

$0.04135-0$

0.15544

0.00758

$-0.06290-0$

0.01292

0.08690

0.01644

$-0.17914-0.03775$

$-0.02251-0.10220$

0.00761

0.08843

$-0.18472$

0.06066

0.10728

0.00278

0.00264

0.00802

$-0.00044$

0.00672

$-0.00413$

$0.00066-0.00895$

$-0.009440 .00414$

0.02770

$-0.02606$

0.02592

$0.00259-0$

$-0.00156$

$\begin{array}{ll}0.10593 & 0.00302\end{array}$

$0.00278-0.02027$

0.01987

0.00479

0.00058

$0.03770-0.08950-0.00275$

$-0.01040$

0.04528

$-0.08950$

0.00247

0.03917

$-0.00453$

0.01501

0.00200

$-0.05240$

$0.05642-0.08875$

$-0.00960$

$-0.00367$

$0.06014-0.08973$

0.07972

(1)

$\begin{array}{rr}-0.01709 & 0.02691\end{array}$

0.01057

0.00025

0.01448

$0.02267-0.05975$

$-0.01549$

0.11052

$0.03681-0.05846$

0.00096

$-0.00227$

0.00556

0.00488

0.00366

$0.02868-0.07432$

0.07252

$0.04936-0.08205$

$-0.00436$

$-0.00797$

0.01347

0.04668

$0.03197-0.11442$

0.08990

$0.03835-0.07945$

$0.01107-0.16074$
$0.00934-0.05905$

$0.01107-0.16074$
$0.00934-0.05905$

0.00287

0.02400

0.01545

$-0.03773$

0.03455

0.00718

$-0.02630$

$-0.06645$

582.24

25.61

0.00220

0.01555

0.04079

0.01380

0.00300

0.02151

.09952

0.10005

$0.01430 \quad 0.10943$

$-0.01176-0.02245$

$0.01672 \quad 0.04222$

$0.01418-0.02495$

$0.01061 \quad 0.00677-0.00118$

$0.00074-0.00271 \quad 0.00123$

$-0.00178-0.00290-0.00142$

$0.00184-0.00689-0.00238$

$-0.015720 .01155-0.00425$

$-0.00305-0.00181-0.00147$

$\begin{array}{llll}0.00237 & 0.00049 & -0.00325\end{array}$

$0.00031-0.00089-0.00139$

$0.00340-0.00416-0.00059$

$0.01204-0.00661-0.00257$

$\begin{array}{llll}0.00540 & -0.00090 & 0.00019\end{array}$

$\begin{array}{llll}-0.01683 & 0.01429 & -0.00351\end{array}$

$\begin{array}{llll}-0.04932 & 0.02649 & -0.00315\end{array}$

$\begin{array}{lll}0.00510 & -0.00702 & 0.00273\end{array}$

$\begin{array}{lll}-0.01418 & 0.01990 & 0.01067\end{array}$

$\begin{array}{lll}-0.01480 & 0.01642 & 0.03213\end{array}$

$\begin{array}{lll}-0.02368 & 0.02352 & 0.00268\end{array}$

$\begin{array}{lll}-0.02081 & 0.01299 & 0.00271\end{array}$

$\begin{array}{lll}-0.02648 & 0.02657 & 0.02429\end{array}$

$\begin{array}{llll}0.00547 & -0.00362 & 0.00381\end{array}$

$\begin{array}{llll}0.01222 & -0.00659 & 0.00498\end{array}$

$\begin{array}{lll}0.05593 & -0.02387 & 0.02481\end{array}$

$\begin{array}{lll}0.00519 & 0.00653 & 0.00070\end{array}$

$\begin{array}{lll}-0.02676 & 0.02749-0.00005\end{array}$

$\begin{array}{lll}-0.08815 & 0.06162 & 0.02378\end{array}$

$\begin{array}{lll}0.00189 & -0.00243 & 0.00389\end{array}$

$\begin{array}{llll}0.00026 & -0.00201 & 0.00232\end{array}$

$\begin{array}{lll}-0.00030 & -0.00246 & 0.00007\end{array}$

$\begin{array}{lll}-0.00860 & 0.00484 & -0.00251\end{array}$

$\begin{array}{lll}0.00339 & 0.01053-0.00235\end{array}$

$\begin{array}{llll}0.00698 & 0.00049 & -0.00079\end{array}$

$0.00397-0.00392 \quad 0.00160$

$\begin{array}{llll}0.00750 & 0.03017 & -0.01348\end{array}$

$\begin{array}{llll}0.13824 & -0.11576 & 0.03427\end{array}$

$\begin{array}{llll}-0.00438 & 0.00139 & -0.00340\end{array}$

$\begin{array}{llll}0.04153 & -0.01304 & 0.01441\end{array}$

$0.07560-0.00923-0.03662$

$\begin{array}{llll}0.01274 & 0.01016 & -0.01011\end{array}$

$\begin{array}{lll}0.07361 & -0.07856 & 0.04029\end{array}$

$\begin{array}{lll}0.09431 & 0.16773 & 0.00317\end{array}$

$-0.00542-0.05001-0.03880$

$\begin{array}{llll}0.11782 & -0.07046 & 0.04310\end{array}$

$0.01720-0.11402-0.09322$

$-0.01925-0.09374-0.05004$

$\begin{array}{llll}-0.09015 & 0.03845 & -0.04751\end{array}$

$0.00198-0.00446-0.03756$

$\begin{array}{lll}0.10820 & 0.00897\end{array}$

$0.10141-0.09933$

$0.02249-0.10349$ 


H1
C3
C3
C3
O4
O4
O4
H8
H8
H8
O5
O5
O5

$Z$
$X$
$Y$
$Z$
$X$
$Y$
$Z$
$X$
$Y$
$Z$
$X$
$Y$
$Z$

$$
\begin{array}{rr}
0.01078 & -0.01821 \\
0.05238 & -0.00196 \\
0.02373 & 0.02442 \\
-0.00606 & -0.00457 \\
0.02743 & -0.00781 \\
-0.02406 & 0.00393 \\
-0.05822 & 0.02375 \\
-0.06095 & 0.00028 \\
-0.08203 & -0.04961 \\
-0.06149 & 0.03331 \\
0.02040 & -0.00822 \\
-0.01858 & -0.00217 \\
0.00781 & -0.00706
\end{array}
$$

$\begin{array}{llll}0.06064 & 0.00001 & 0.10886 & 0.05512\end{array}$

$0.01010-0.00309-0.01374-0.03661$

$\begin{array}{lllll}-0.05528 & 0.00242 & -0.03002 & 0.02719\end{array}$

$\begin{array}{llll}0.01052 & -0.00142 & 0.04804 & 0.05670\end{array}$

$0.02489-0.00223-0.03029-0.08166$

$\begin{array}{llll}-0.00764 & 0.00396 & -0.00582 & 0.04046\end{array}$

0.02836

0.03006

0.06599

0.00434

0.01366

0.05319

frequencies intensities

$723.72 \quad 757.42$

$143.75 \quad 28.80$

$0.00053-0.00003$

$\operatorname{Re1} \mathrm{X}$

Re1 Y

Re1 Z

N1 X

N1 Y

N1 Z

C 4 X

C4 Y

C 4 Z

C7 $\mathrm{X}$

C7 Y

C7 Z

N2 $\mathrm{X}$

N2 Y

N2 Z

H18 X

H18 Y

H18 Z

H21 X

H21 Y

H21 Z

$\mathrm{H} 23 \mathrm{X}$

H23 Y

H23

H2 6

H26 Y

H26 Z

O1 X

01

01

02

02

02

03

03

$\mathrm{O} 3$

C1

C1

C1

H3

H3

H3

H4

H4

H4
$-0.00119-0.00421$

$0.00009-0.00062$

$0.00743 \quad 0.00149$

$0.03617-0.00082$

$0.01661-0.00637$

$0.00878 \quad 0.00069$

$0.04809-0.00215$

$0.00142 \quad 0.00335$

$0.01827-0.00405$

0.054420 .00302

$-0.002690 .00073$

$0.00817-0.00846$

$0.03673-0.00773$

$0.00320 \quad 0.00178$

$-0.14555-0.00649$

$-0.457150 .01231$

$-0.07391-0.01083$

$-0.10184-0.00096-0$

$-0.43672$

$-0.06471$

$0.00244-0$

$-0.08241-0.00192$

$-0.4164$

$-0.04435$

0.01574

$-0.11933$

$-0.00172-0$

$-0.48030-0.02397$

$-0.041050 .00263$

$0.00458-0.00571$

$-0.00503 \quad 0.00849$

0.00070

0.00322

0.00160

0.00152

0.00060

0.00512

0.00102

$-0.00331$

$-0.00087-0.08366$

$-0.00441$

0.11976

0.01164

$-0.00383$

$-0.00760$

$-0.00806-0.10199$

$0.00168-0.00823-0$

$-0.020150 .01879$

$0.00795-0.00640$

$-0.00418-0.06438$

$-0.01360 \quad 0.05836$

$-0.02134-0.20243$

$-0.00885-0.07654-0.04693$

0.00029

0.00355

0.00463

0.00475

$-0.00392$

0.00142

0.00436

0.00047

$-0.02558$

0.00557

$-0.00390$

$-0.00652$

0.001

0.00019

0.01019

0.03676

0.01257

$-0.04097$
$-0.05530-0.00068-0.06262$

$0.00752-0.00404-0.13064$

$-0.02697$

$\begin{array}{lll}0.12957 & 0.00181 & 0.22091\end{array}$

$-0.25043$

0.07652

$-0.07977-0.00054-0.11318-0.05646$

$\begin{array}{llll}0.02636 & -0.00218 & 0.07243 & 0.08275\end{array}$

$0.01108-0.00274-0.02905-0.03926$

$0.01824-0.00192-0.02225-0.07004$

786.22

27.42

0.00117

0.00045

$-0.00260-0$.

0.00253

$-0.00159-0$

$-0.00550$

$-0.00242-0$

0.00554

0.00756

810.30

11.51

0.00087

830.22

40.45

0.00010

0.00015

0.00047

$-0.00120$

$-0.00069-$

$0.07534-0.00676$

0.00074

0.06586

0.00898

$-0.06822$

$-0.00538$

0.00138

$-0.13100$

$-0.01018$

0.13552

0.01567

$-0.01459-0.00484$

$-0.04176-0.00607$

$\begin{array}{rr}0.00231 & 0.00159\end{array}$

$-0.00708-0.00494$

0.100190 .00715

0.192230 .00579

$0.04206 \quad 0.00400$

$0.21396 \quad 0.01717$

$-0.05809-0.00197$

0.050490 .01292

$-0.13288-0.00953$

$\begin{array}{lll}0.00214 & 0.13778 & 0.01983\end{array}$

$\begin{array}{lll}-0.01667 & -0.02551 & 0.01303\end{array}$

$-0.04255-0.00614$

$-0.00641-0.25276-0.02155$

0.02155

2509

$-0.00117$

$0.00474-0.00115$

$0.00630-0.00601-0.00338$

$\begin{array}{lll}0.00526 & 0.00116 & 0.00081\end{array}$

$-0.00265-0.00820$

0.00207

0.00074

0.00565

$0.00506-0.00991$

0.00079

$0.00928-0.00160-0.00264$

$0.00313-0.00531$

$0.00521-0.01091$

0.00094

$-0.00691$

$-0.00859$

0.07203

0.07188

$-0.00652$

$-0.09870$

0.00968

0.40770

75.78

6.30

0.00052

$-0.01134$

$-0.37966$

$-0.77480$

0.06429

0.01022

0.00023

$0.00422-0.05042-0.00277-0.00164$

$0.00183-0.03189-0.01414-0.00447$

$0.01646-0.16906-0.00360 \quad 0.00099$

$0.01372-0.14102-0.00328-0.00062$
$-0.00318$ 


C2
C2
C2
H1
H1
H1
C3
C3
C3
O4
O4
O4
H8
H8
H8
O5
O5
O5

$X$
$Y$
$Z$
$X$
$Y$
$Z$
$X$
$Y$
$Z$
$X$
$Y$
$Z$
$X$
$Y$
$Z$
$X$
$Y$
$Z$

$\begin{array}{llll}0.00079 & 0.15164 & -0.06283\end{array}$

$-0.00428-0.02133-0.01911$

$0.00526 \quad 0.04782-0.02138-0$

$\begin{array}{llll}0.00532 & 0.00617 & -0.00232 & 0.00038\end{array}$

$-0.01046 \quad 0.28250-0.15035$

$0.00389-0$.

$-0.01628$

0.06135

$-0.12651$

.00978

.04418

$-0.00370-0$.

0.00158

$0.01074-0.04586$

$-0.00301-0.00177$

0.01137

0.01840

0.00263

0.001310 .04242

$-0.21976$

$-0.01450$

0.00325

$-0.00213-0.00935$

0.21026

0.01225

0737

$0.00209-0.00150$

-0.00935
-0.03175

$-0.00394$

0.00601

$-0.00021$

$-0.03238-0$

$-0.00119$

0.01676

$-0.05775$

00786

0.00293

$0.00598 \quad 0.00083$

$-0.02974$

0.04631

0.00397

0.00957

0.00558

$0.00579-0.00076$

$-0.04642$

0.07157

0.06757

0.00542

$-0.08956$

$-0.00042-0.00032$

0.00291

0.01709

0.26755

$\begin{array}{ll}0.00032 & 0.00177\end{array}$

$0.00217-0.00274-0.01924$

$0.00196-0.01204-0.06145$

$-0.00370-0.03193$

0.01847

$-0.00242-0.00098$

$0.00053-0.00005$

$-0.01800 \quad 0.26718$

$0.01426 \quad 0.01141$

$0.00570-0.10802$

$0.00552-0.00595$

$-0.00070 \quad 0.03387$

0.001120 .00198

$0.00785-0.06742-0.00291-0.00260$

$\begin{array}{llll}0.00186 & 0.01879 & -0.00145 & 0.00240\end{array}$

frequencies

intensities

$\operatorname{Re} 1 \mathrm{X}$

Re1 Y

$$
992.36 \quad 1003.48 \quad 1037.99
$$

$0.00200-0.05023$

$0.00055-0.00115$

$\begin{array}{rrr}83.47 & 4.71 & 88.67\end{array}$

$-0.005540 .00133-0.00305$

$0.01233-0.00257 \quad 0.00926$

1053.27

23.44

1066.02

1075.07

1076.32

0.00046

0.00144

$-0.00009$

0.00010

$0.01677-0.00376-0.01428-0.00029$

$0.00458-0.00116-0.00519-0.00011$

0.00071

0.00094

0.00080

$-0.00338$

$-0.00166$

0.00062

0.00138

$-0.00654$

$0.00026-0.00245$

$0.00096-0.00051-0.00727$

$-0.00525$

$0.00303-0$

0.00344

.00331

$-0.00109-0.00007$

$0.00310 \quad 0.01712-0.01156$

$0.00624 \quad 0.05705-0.03762$

$-0.00343$

0.00094

$-0.00309$

0.00292

0.00371

$0.01856-0.01100$

$\begin{array}{lll}-0.00118 & -0.01961 & 0.01229\end{array}$

$-0.01006 \quad 0.00182$

0.00397

$0.00117-0$

0.05792

$0.00082-0$.

$-0.00724$

0.01711

$\begin{array}{llll}0.00597 & -0.00244 & 0.00974\end{array}$

$0.00661-0.00181-0.00768$

0.00483

0.00036

0.02966

0.021620 .01136

$0.00242-0.00040$

$0.00307 \quad 0.00409$

$0.01544-0.00722$

$-0.00042-0.00133-0.00387$

0.0035

0.00122

$0.00639-0.00470$

$0.00105-0.00108$

$-0.000350 .00060$

0.00029

$0.00105-0.00314$

$0.00194-0.00272$

0.013120 .00385

0.00363

$-0.00288-0.00018$

$0.00311-0.00366$

0.00853

$0.00411-0.02870$

$0.00839-0.00391$

$0.00508-0.00168$

0.01241

0.04272

-0.02870
-0.02278

$-0.19785$

0.11824

$-0.00215 \quad 0.00324$

0.00505

0.00685

0.36841

0.25092

$0.00584-0.00787-0$.

$-0.01079$

$-0.00315$

0.01314

$-0.10365$

0.06400

$0.02468-0.01375$

$\begin{array}{llll}0.01161 & -0.04307 & 0.06284\end{array}$

0.03342

$0.20588-0.12739$

$0.00218 \quad 0.00090$

$0.53141-0.33946$

$-0.01236$

0.00380

0.01023

$-0.00853$

$-0.00861$

$-0.00148-0.00383$

$-0.01090$

0.01100

$-0$

0.02379

-0.00799
0.00381

$-0.05854-0$

$-0.03177$

$-0.01386$

$-0.001$

0.00114

0.00937

0.00381
0.00778

.04385

$-0.00959$

$-0.06031$

0.01338

$-0.01899$

0.00983

0.01113

0.00658

0.02738

$-0.02172$

$0.00328 \quad 0.00234$

$0.01596-0.01242$

0.01461

$0.06730-0.01397$

$0.00871-0.00073$

$0.02786-0.07713$

0.06097

0.03430

0.02519

$0.00073 \quad 0.00468$

0.00438

0.00211

0.02333

$-0.06484$

0.04

$-0.01011$

$-0.00031$

0.00258

$-0.11051$

$-0.03823$

0.02780

0.01703

$-0.00063-0.01079$

$-0.00495$

0.01536

$-0.00096$

0.01386

$0.00037-0.00576$

$0.01741-0.00903-0$

$0.01122 \quad 0.00955$

0.00056

0.00053

$-0.07793$

0.03132

0.05347

$-0.04437$

$-0.00314-0.00293$

$-0.0350$

$-0.03518-0$
$0.00136-0$

$-0.04101-0.13453-0.04351$

0.01162

0.00815

$-0.0023$

$-0.00411-0.00796$

.01042

$-0.06007-0.02543$

0.00226

0.01009

$0.03274-0.00671$

$0.025260 .08692-0.00476$

$0.00985-0.00093$

$-0.01591-0.02510$

$\begin{array}{llll}0.02526 & 0.08692-0.00476\end{array}$

$0.17161 \quad 0.05757$

0.0060

$0.05670-0.06422$

$-0.03377$

$-0.06935$

.0031

0.03151
-0.02604

0.01977

0.00695

$-0.00397$

0.06332

0.09991

$0.00939-0.02604$

0.10831

0.05141

0.10563 


\begin{tabular}{|c|c|c|c|c|c|c|c|c|}
\hline H3 & $\mathrm{Y}$ & 0.04717 & 0.18577 & -0.03643 & 0.04513 & -0.06317 & 0.18096 & 0.26670 \\
\hline H3 & Z & -0.00088 & -0.00810 & 0.00819 & 0.00905 & -0.02790 & -0.05115 & \\
\hline H 4 & $\mathrm{X}$ & -0.01402 & -0.12706 & 0.04924 & 0.14272 & 0.03736 & -0.02193 & -0.04544 \\
\hline H 4 & $\mathrm{Y}$ & 0.01970 & 0.03878 & 0.01601 & 0.03616 & -0.09297 & -0.23102 & -0.37982 \\
\hline H4 & Z & -0.01145 & -0.03928 & 0.00547 & & & & \\
\hline C2 & $\mathrm{x}$ & 0.02149 & 0.09124 & 0.00707 & -0.04053 & 0.05400 & -0.03788 & -0.04719 \\
\hline C2 & $\mathrm{Y}$ & -0.01627 & -0.06013 & 0.02431 & -0.01113 & 0.12872 & 0.04112 & 0.08866 \\
\hline C2 & Z & -0.02088 & -0.10889 & 0.01337 & & -0.00444 & -0.04077 & \\
\hline H1 & $\mathrm{x}$ & 0.00560 & -0.07738 & -0.06537 & 0.19687 & -0.52421 & 0.10299 & 0.04872 \\
\hline H 1 & $\mathrm{Y}$ & -0.10552 & -0.57283 & 0.01526 & 0.13620 & -0.25635 & 0.06564 & 0.06130 \\
\hline H1 & Z & -0.05235 & -0.21365 & 0.08699 & -0.15990 & 0.40146 & -0.18579 & -0.19393 \\
\hline C3 & $\mathrm{x}$ & 0.00570 & 0.00366 & 0.00199 & -0.00405 & & -0.01361 & \\
\hline C3 & $\mathrm{Y}$ & 0.00756 & -0.01383 & -0.00435 & 0.01017 & -0.03654 & -0.01308 & -0.02844 \\
\hline C3 & Z & 0.00422 & 0.02877 & -0.00625 & 0.00484 & -0.01754 & 0.01069 & 0.01505 \\
\hline 04 & $\mathrm{x}$ & -0.00947 & -0.04061 & -0.0 & & -0.02376 & & \\
\hline 04 & $\mathrm{Y}$ & 0.0 & 0.02218 & 0.00258 & -0.00736 & & -0.00447 & -0.00397 \\
\hline 04 & Z & 0500 & 0.01660 & -0.00097 & -0.00359 & 0.00004 & -0.00097 & -0.00202 \\
\hline H8 & $\mathrm{x}$ & -0.0 & -0.1 & & -0 & & -0 . & -0 . \\
\hline H8 & $\mathrm{Y}$ & 0.0 & 0.0 & & & & & \\
\hline H8 & Z & -0.00141 & 0.0 & 0.0 & -0.00 & 0.01410 & -0.01963 & -0.0225 \\
\hline 05 & $\mathrm{x}$ & 558 & 0.0 & -0.0 & -0 . & -0.0 & & \\
\hline 05 & $\mathrm{Y}$ & -0 & -0.0 & & -0 & & & \\
\hline 05 & Z & 0.00784 & 0.03328 & -0.0 & 0.0 & -0.01066 & 432 & \\
\hline \multicolumn{2}{|c|}{ frequencies } & & & & & & & \\
\hline \multicolumn{2}{|c|}{ intensities } & 1.73 & 3.82 & & & 6.81 & 36 & 4.2 \\
\hline $\mathrm{Re} 1$ & $\mathrm{x}$ & 0.00011 & 0.0 & 25 & -0.0 & 0.000 & & -0.00 \\
\hline $\operatorname{Re} 1$ & $\mathrm{Y}$ & -0.00026 & -0.0 & & -0.0 & -0.00005 & -0 & 0 . \\
\hline e1 & Z & 0.00068 & 0.00024 & -0.0 & -0.0 & 0.00112 & 0013 & 0.00011 \\
\hline $\mathrm{N}$ & $\mathrm{x}$ & -0. & 0.0 & -0 & -0 . & -0.0 & & -0 . \\
\hline & $\mathrm{Y}$ & -0. & 0.0 & & & -0.00008 & -0 & \\
\hline N1 & Z & -0.0 & -0.03709 & -0.00370 & 119 & 0.02635 & -0 & -0.01058 \\
\hline C4 & $\mathrm{x}$ & -0. & -0.01903 & -0.0 & & 0.0 & & -0. \\
\hline C4 & $\mathrm{Y}$ & -0. & -0. & -0 . & -0 & 0 . & & -0 . \\
\hline C4 & Z & 297 & 3154 & 0197 & & -0.02415 & -0.00091 & 0. \\
\hline C7 & $\mathrm{x}$ & 0. & -0.0 & -0.0 & & & -0 & -0 . \\
\hline C & $\mathrm{Y}$ & & -0 & & -0 & -0 & & -0 . \\
\hline C7 & Z & 575 & -0.0 & -0.0 & -0.0 & -0.02969 & 0054 & 0 \\
\hline & $\mathrm{x}$ & -0. & -0.0 & -0. & & -0.0 & -0 . & \\
\hline & $\mathrm{Y}$ & -0 & & & & & -0 & -0 . \\
\hline & Z & -0.00566 & -0.02004 & 0031 & -0.0 & 0.044 & -0.00047 & 0. \\
\hline & $\mathrm{x}$ & & 0.57968 & & -0.0 & -0.19 & & \\
\hline & $\mathrm{Y}$ & & -0 & -0 . & & & -0 & -0 \\
\hline 18 & Z & & 0.2 & 938 & -0 . & -0.06 & & \\
\hline H21 & $\mathrm{x}$ & -0.0 & -0.40121 & -0.0 & & 0.0 & -0 & 0102 \\
\hline & $\mathrm{Y}$ & & & & & & & \\
\hline $\mathrm{H}$ & Z & & & & -0 . & -0. & 503 & -0 . \\
\hline H23 & $\mathrm{x}$ & -0.1 & -0.03224 & -0.0 & & -0.06 & -0 & -0.0 \\
\hline & $\mathrm{Y}$ & & & & & & -0 . & \\
\hline & Z & -0. & -0.20293 & -0. & -0 . & -0. & & -0 . \\
\hline H26 & $\mathrm{X}$ & & 0.16145 & 111 & -0.0 & 0.64396 & -0. & -0.04 \\
\hline & $\mathrm{Y}$ & & -0.0 & -0. & & -0 & & \\
\hline & Z & & & & -0 . & & -0 & -0 . \\
\hline 01 & $\mathrm{x}$ & & -0.00077 & 0.0 & & & -0 & \\
\hline 01 & $\mathrm{Y}$ & 0 & & -0.0 & & & & \\
\hline & Z & & & -0.0 & & -0. & & \\
\hline O2 & $\mathrm{x}$ & -0.0 & 0.0 & & & & -0 & -0. \\
\hline 02 & $\mathrm{Y}$ & 0.00189 & 0.00 & -0.00 & -0.00 & 0.00 & 23 & -0.0 \\
\hline $\mathrm{O} 2$ & Z & -0.00761 & -0.00642 & -0.00026 & & -0.00719 & -0.00081 & \\
\hline 03 & $\mathrm{x}$ & & -0.00372 & 0.00032 & -0.00790 & & -0.00471 & -0.0075 \\
\hline 03 & $\mathrm{Y}$ & 0.01856 & -0.00177 & -0.00375 & 0.0 & -0.00052 & 0.00422 & -0.0339 \\
\hline
\end{tabular}




\begin{tabular}{|c|c|c|c|c|c|c|c|c|}
\hline 03 & z & -0.00100 & -0.00296 & 0.01489 & -0.04143 & -0.00092 & 0.00080 & -0.00056 \\
\hline $\mathrm{C} 1$ & $\mathrm{x}$ & -0.03772 & 0.00568 & -0.00825 & 0.00346 & 0.00079 & 0.02132 & -0.09812 \\
\hline $\mathrm{C} 1$ & $\mathrm{Y}$ & -0.00058 & 0.00097 & -0.01557 & 0.01231 & 0.00171 & 0.01945 & 0.08463 \\
\hline $\mathrm{C} 1$ & Z & 0.00110 & 0.00872 & -0.04563 & 0.04048 & 0.00162 & 0.03954 & 0.02047 \\
\hline H3 & $\mathrm{x}$ & -0.02126 & 0.03579 & -0.11810 & 0.63768 & 0.00056 & -0.18106 & 0.49580 \\
\hline H3 & $\mathrm{Y}$ & -0.00550 & 0.01442 & -0.08528 & -0.15110 & 0.00566 & 0.22136 & -0.21652 \\
\hline H3 & Z & 0.00076 & -0.00744 & 0.01997 & -0.09511 & 0.00042 & 0.01927 & -0.04288 \\
\hline H4 & $\mathrm{x}$ & -0.03042 & -0.02595 & 0.14847 & -0.61424 & -0.00783 & 0.05596 & 0.57870 \\
\hline H4 & Y & 0.01106 & -0.01017 & 0.02302 & 0.13342 & 0.00404 & -0.04853 & -0.26194 \\
\hline H4 & Z & 0.01044 & -0.00767 & 0.02484 & -0.00072 & & -0.01237 & -0.10787 \\
\hline $\mathrm{C} 2$ & $\mathrm{x}$ & 0.00418 & -0.00338 & 0.01398 & 0.01664 & -0.00168 & 0.03446 & \\
\hline C2 & $\mathrm{Y}$ & -0.01185 & -0.00216 & 0.04294 & -0.03059 & -0.00539 & & \\
\hline C2 & Z & -0.00142 & -0.00211 & 0.02415 & 0.02507 & -0.00086 & -0.08313 & -0.01306 \\
\hline $\mathrm{H} 1$ & $\mathrm{x}$ & 0.01340 & 0.00214 & -0.01870 & -0.00445 & 0.00406 & -0.15524 & -0.00482 \\
\hline $\mathrm{H} 1$ & $\mathrm{Y}$ & -0.0 & 960 & -0.2 & & 0.02266 & & \\
\hline $\mathrm{H} 1$ & Z & -0.0 & 0.0 & -0.12 & & 0.00941 & & \\
\hline C3 & $\mathrm{x}$ & 0.00111 & 0.01381 & -0.07011 & -0.01591 & 0.00288 & 0.04512 & \\
\hline C3 & $\mathrm{Y}$ & -0.0 & -0.0 & & 0.01424 & & & \\
\hline C3 & Z & 102 & -0.0 & & & -0 & -0 & \\
\hline 04 & $\mathrm{x}$ & -0.0 & -0.01679 & 0.1 & 0.03245 & -0.00706 & 0.00237 & -0 \\
\hline 04 & $\mathrm{Y}$ & -0. & & -0.0 & -0.01384 & & & \\
\hline 04 & $z$ & & -0 & & & -0 & & \\
\hline H8 & $\mathrm{x}$ & 192 & 023 & -0.4 & -0.14694 & 0 & -0.43178 & -0 . \\
\hline H8 & Y & -0.0 & -0.0 & 0.2 & 0.0 & -0 & & \\
\hline H8 & $\mathrm{z}$ & & & -0 . & -0 . & & & \\
\hline 05 & $\mathrm{x}$ & & 0. & -0.0 & -0.01967 & 0327 & -0.02914 & -0 \\
\hline 05 & $\mathrm{Y}$ & -0. & & & 0.00609 & -0.0 & & \\
\hline 05 & Z & & & -0 & & & & \\
\hline & & & & & & & \\
\hline \multicolumn{2}{|c|}{$\begin{array}{l}\text { frequencies } \\
\text { intensities }\end{array}$} & & & & & & & \\
\hline $\operatorname{Re} 1$ & $\mathrm{x}$ & 0.00 & -0. & -0. & 0006 & -0.00030 & -0.00019 & \\
\hline Re1 & $\mathrm{Y}$ & & & & & & & \\
\hline Re1 & Z & -0. & -0 & -0 . & -0 & & 44 & \\
\hline N1 & $\mathrm{x}$ & 0.05992 & 597 & 0.0 & 0.00056 & -0.10005 & 2221 & -0 \\
\hline N1 & $\mathrm{Y}$ & -0.02404 & -0.0 & -0.0 & -0.00013 & & & \\
\hline N1 & z & & & & & & & -0 \\
\hline C4 & $\mathrm{x}$ & & 0. & & -0.00019 & 0.12731 & -0.16506 & 819 \\
\hline C4 & $\mathrm{Y}$ & & 0. & & -0.00006 & -0.02733 & 0.04005 & -0 \\
\hline C4 & Z & -0 . & -0 . & -0 & -0 & -0 & & \\
\hline C7 & $\mathrm{x}$ & & -0 & -0 . & $-0 . c$ & & 0.08593 & -0 \\
\hline C7 & Y & -0.0 & 0. & & 0.0 & -0.02921 & -0.02735 & \\
\hline C7 & Z & & & & -0 . & & & \\
\hline N2 & $\mathrm{x}$ & -0 & & & & -0 & -0 . & \\
\hline N2 & $\mathrm{Y}$ & 0.01567 & -0.00674 & -0.0 & 0.0 & 0.02476 & 0.01996 & -0 . \\
\hline N2 & Z & & & & & & & \\
\hline H18 & $\mathrm{x}$ & -0 & -0 & -0 . & -0 & -0 & & \\
\hline H18 & $\mathrm{Y}$ & 970 & 0.0 & & -0.00126 & 0 . & 0 & -0 \\
\hline & Z & & -0. & -0 . & & & & \\
\hline H21 & $\mathrm{x}$ & -0 & -0 & -0 . & -0 . & -0 . & & \\
\hline H21 & $\mathrm{Y}$ & & 0.0 & & & -0 & 093 & -0 . \\
\hline H 21 & Z & & & & & & -0 . & \\
\hline H23 & $\mathrm{x}$ & & -0.0 & -0.1 & $-0 . c$ & & & \\
\hline H23 & Y & -0.0 & & & & -0 & -0.00264 & -0 \\
\hline & Z & & -0.1 & -0.4 & -0.0 & -0 & -0.1 & \\
\hline H26 & $\mathrm{x}$ & & -0.0 & -0.3 & -0.0 & -0 & & \\
\hline H26 & $\mathrm{Y}$ & -0.0 & & & & & -0.00167 & -0 . \\
\hline & Z & & -0.03 & -0.1 & $-0.00 c$ & -0.09 & -0.05 & \\
\hline 01 & $\mathrm{x}$ & & -0.00112 & -0.00033 & & & -0.00077 & \\
\hline 01 & Y & & & -0.00052 & & -0.00184 & & -0.0001 \\
\hline 01 & Z & -0.00081 & 0.00022 & -0.0 & -0.00066 & -0 . & -0.00112 & 0 \\
\hline
\end{tabular}




\begin{tabular}{|c|c|c|c|c|c|c|c|c|}
\hline 02 & $\mathrm{x}$ & 0.00068 & 0.00034 & -0.00174 & 0.00030 & 0.00131 & 0.00154 & -0.00003 \\
\hline $\mathrm{O} 2$ & $\mathrm{Y}$ & -0.00118 & 0.00024 & -0.00017 & -0.00002 & -0.00086 & -0.00013 & \\
\hline 02 & Z & 0.00286 & 0.00010 & 0.00220 & 0.00144 & 0.00173 & -0.00145 & \\
\hline 03 & $\mathrm{x}$ & -0.00155 & 0.00304 & -0.00070 & -0.00839 & 0.00064 & -0.00024 & 0.00047 \\
\hline 03 & Y & & -0.00101 & 0.00166 & -0.00900 & 0.00077 & & \\
\hline 03 & Z & -0.00080 & 0.00068 & -0.00034 & -0.00173 & 0.00024 & -0.00075 & 0.0016 \\
\hline $\mathrm{C} 1$ & $\mathrm{x}$ & -0.01983 & -0.00990 & 0.00169 & -0.03429 & -0.00064 & -0.00021 & -0.00165 \\
\hline $\mathrm{C} 1$ & $\mathrm{Y}$ & 0.01733 & -0.00641 & 0.00329 & -0.07025 & -0.00067 & 0.00007 & 0.00447 \\
\hline $\mathrm{C} 1$ & Z & 0.00358 & -0.01519 & 0.00508 & -0.00728 & -0.00057 & -0.00034 & -0.0017 \\
\hline H3 & $\mathrm{x}$ & 0.10477 & 0.06451 & -0.01771 & 0.26476 & -0.00302 & 0.00075 & 0.0027 \\
\hline H3 & $\mathrm{Y}$ & -0.04911 & -0.08220 & 0.01901 & 0.51847 & 0.00147 & -0.00183 & -0.0304 \\
\hline H3 & Z & -0.00825 & -0.00513 & 0.00414 & -0.36026 & -0.00113 & 0.00026 & \\
\hline H4 & $\mathrm{x}$ & 0.11121 & -0.01103 & 0.00286 & 0.26278 & & & \\
\hline H 4 & Y & -0.04951 & 0.02691 & -0.01224 & 0.41212 & 0.00061 & -0.00076 & -0.0129 \\
\hline H4 & Z & -0.02116 & & -0.00772 & 0.45427 & & -0.00111 & -0.0 \\
\hline C2 & $\mathrm{x}$ & 0.00404 & -0.03315 & 0.01124 & -0.00109 & & & \\
\hline $\mathrm{C} 2$ & $\mathrm{Y}$ & -0.00455 & 0.04313 & -0.01432 & -0.00055 & -0.00043 & -0.00045 & -0.00 \\
\hline C2 & Z & -0.0 & 737 & -0.0 & & -0 & & \\
\hline $\mathrm{H} 1$ & $\mathrm{x}$ & 0.0 & 0.12701 & -0.0 & 0.00146 & -0.00082 & -0.00186 & -0. \\
\hline H1 & $\mathrm{Y}$ & 0.00651 & -0.16500 & 0.05294 & -0.01499 & 0.00189 & & \\
\hline H1 & Z & 0.0 & -0.2 & 616 & -0 & 0. & & \\
\hline C3 & $x$ & -0.0 & & -0.0 & -0 & -0. & -0 . & -0 . \\
\hline C3 & $\mathrm{Y}$ & 0.0 & -0.07111 & 0.02404 & 0.00079 & 0.00249 & 0.00465 & \\
\hline C3 & Z & -0.0 & -0.1 & 899 & -0.0 & -0. & -0. & -0 . \\
\hline 04 & & -0.0 & -0.0 & & & & & \\
\hline 04 & $\mathrm{Y}$ & 0.0 & 0.02373 & -0.00742 & -0.00045 & -0. & -0 . & -0.0 \\
\hline 04 & Z & 0.0 & 586 & -0.0 & -0.0 & -0 & & \\
\hline H8 & & -0.0 & -0.3 & & 34 & -0 . & -0 . & -0 . \\
\hline $\mathrm{H} 8$ & $\mathrm{Y}$ & 0.0 & 0.15129 & -0.04940 & -0.00182 & 048 & & \\
\hline H8 & $\mathrm{z}$ & 0.0 & -0.02 & & -0.00026 & -0 . & -0 . & -0 . \\
\hline 05 & & 0.0 & -0.0 & & & & & \\
\hline 05 & $\mathrm{Y}$ & -0.00 & 0.00669 & -0.00391 & -0.00025 & -0.0 & -0.0 & -0.03126 \\
\hline 05 & z & 0.00164 & 0.02193 & -0.00423 & 0.00561 & 0290 & & \\
\hline \multicolumn{2}{|c|}{ frequencies } & & & & & & & \\
\hline \multicolumn{2}{|c|}{ intensities } & & & & & & & \\
\hline $\operatorname{Re} 1$ & $\mathrm{x}$ & -0.0 & -0.00 & -0.00 & 0.0 & -0.0 & & -0 \\
\hline $\operatorname{Re} 1$ & Y & -0.0 & -0.00002 & 0.00001 & -0.00001 & 0000 & -0. & -0.00 \\
\hline $\operatorname{Re} 1$ & Z & 0.0 & -0.00001 & -0.00002 & -0.00001 & -0.0 & & \\
\hline N1 & $\mathrm{x}$ & 0.0 & & -0 & & -0 . & -0 & -0 . \\
\hline N1 & $\mathrm{Y}$ & 0.0 & -0.0 & 0001 & -0.0 & 13 & & \\
\hline N1 & Z & 0.0 & 0.00003 & -0.00003 & 0.00156 & -0.00094 & & $0.0020^{\circ}$ \\
\hline 4 & $\mathrm{x}$ & & -0.0 & & -0 & & & \\
\hline C4 & $\mathrm{Y}$ & -0.0 & 03 & -0.00045 & 255 & -0.01639 & -0 & \\
\hline C4 & Z & 0.0 & -0.00012 & 0.00159 & -0.03893 & 5445 & 0.0 & 0.00030 \\
\hline & $\mathrm{x}$ & & -0.0 & & & & & \\
\hline C7 & $\mathrm{Y}$ & -0.0 & & -0 & -0 & -0 & -0 . & -0 . \\
\hline C7 & Z & -0.0 & & -0.00023 & -0.00778 & -0.0 & -0.0 & 026 \\
\hline & $\mathrm{x}$ & & & -0.0 & -0.0 & -0.0 & -0 . & \\
\hline & $\mathrm{Y}$ & -0.0 & & & & & & \\
\hline N2 & $\mathrm{z}$ & -0.0 & & 0.000 & -0.00056 & -0.00166 & .75 & -0.0687 \\
\hline & $\mathrm{x}$ & -0.0 & & & & -0 & & \\
\hline & $\mathrm{Y}$ & & & & & -0 & & \\
\hline H18 & Z & 0.0 & -0.00 & & -0.01468 & 472 & $-0 . \varepsilon-2$. & -0.0 \\
\hline & $\mathrm{x}$ & -0.0 & 0.0 & -0.0 & 29 & -0.4 & -0.0 & \\
\hline H21 & $\mathrm{Y}$ & & -0.0 & & -0.13676 & & & \\
\hline H21 & Z & -0.0 & & -0.016 & 0.45521 & -0.6 & -0 & 0 \\
\hline II & $\mathrm{x}$ & -0.0 & 87 & -0.0 & -0.73432 & -0.5 & & -0.0 \\
\hline & $\mathrm{Y}$ & & & & & & & \\
\hline H23 & Z & & -0. & & & & -0.0 & 0.0052 \\
\hline H 26 & $\mathrm{X}$ & 0.00016 & -0.00010 & 0.00039 & 0.00759 & 0.00419 & 0.00968 & -0.31 \\
\hline
\end{tabular}




\begin{tabular}{|c|c|c|c|c|c|c|c|c|}
\hline H26 & Y & 0.00020 & -0.00004 & -0.00004 & -0.00006 & -0.00068 & 0.00017 & 0.00109 \\
\hline H26 & Z & 0.00045 & -0.00008 & -0.00164 & -0.01987 & -0.01140 & -0.02810 & 0.90512 \\
\hline 01 & $\mathrm{x}$ & 0.00006 & 0.00002 & 0.00001 & -0.00005 & 0.00000 & 0.00001 & 0.00000 \\
\hline 01 & Y & -0.00001 & 0.00005 & 0.00008 & 0.00016 & 0.00004 & 0.00007 & 0.00005 \\
\hline 01 & Z & 0.00000 & -0.00003 & 0.00015 & 0.00010 & 0.00004 & -0.00001 & 0.00001 \\
\hline $\mathrm{O} 2$ & $\mathrm{x}$ & 0.00005 & -0.00001 & 0.00010 & -0.00003 & 0.00003 & -0.00004 & 0.00010 \\
\hline 02 & Y & 0.00008 & -0.00005 & -0.00009 & -0.00005 & 0.00005 & 0.00001 & 0.00005 \\
\hline 02 & Z & 0.00000 & 0.00006 & -0.00001 & 0.00014 & -0.00008 & -0.00004 & -0.00010 \\
\hline 03 & $\mathrm{x}$ & 0.00216 & -0.00016 & 0.00038 & 0.00004 & 0.00008 & 0.00000 & -0.00003 \\
\hline 03 & Y & 0.00068 & -0.00006 & -0.00033 & 0.00001 & 0.00015 & -0.00008 & -0.00008 \\
\hline 03 & Z & -0.00023 & 0.00048 & -0.00008 & 0.00001 & 0.00004 & -0.00003 & 0.00001 \\
\hline $\mathrm{C} 1$ & $x$ & 0.02492 & -0.00438 & -0.00022 & -0.00010 & -0.00027 & 0.00015 & 0.00004 \\
\hline $\mathrm{C} 1$ & Y & 0.05826 & 0.01671 & -0.00066 & 0.00000 & -0.00031 & 0.00003 & 0.00001 \\
\hline $\mathrm{C} 1$ & $\mathrm{Z}$ & 0.01223 & -0.09041 & -0.00837 & -0.00052 & -0.00037 & 0.00009 & -0.00004 \\
\hline H3 & $\mathrm{X}$ & -0.21000 & 0.18085 & 0.01793 & 0.00138 & 0.00181 & -0.00060 & 0.00006 \\
\hline $\mathrm{H} 3$ & $\mathrm{Y}$ & -0.27454 & 0.25116 & 0.02895 & 0.00186 & 0.00233 & 0.00016 & 0.00010 \\
\hline H3 & Z & -0.63338 & 0.54331 & 0.05898 & 0.00425 & 0.00507 & -0.00068 & 0.00068 \\
\hline H4 & $\mathrm{x}$ & -0.11462 & -0.12432 & -0.00985 & -0.00052 & 0.00018 & 0.00021 & -0.00022 \\
\hline $\mathrm{H} 4$ & Y & -0.40184 & -0.44713 & -0.03996 & -0.00185 & 0.00075 & -0.00073 & 0.00031 \\
\hline $\mathrm{H} 4$ & Z & 0.49709 & 0.52543 & 0.04323 & 0.00214 & -0.00069 & -0.00050 & 0.00006 \\
\hline $\mathrm{C} 2$ & $\mathrm{x}$ & -0.00072 & 0.00546 & -0.05512 & -0.00023 & 0.00138 & 0.00011 & -0.00012 \\
\hline $\mathrm{C} 2$ & Y & -0.00053 & -0.00253 & 0.02983 & 0.00010 & -0.00075 & 0.00007 & 0.00006 \\
\hline $\mathrm{C} 2$ & Z & -0.00077 & 0.00515 & -0.05255 & -0.00025 & 0.00124 & 0.00011 & -0.00005 \\
\hline $\mathrm{H} 1$ & $x$ & 0.00933 & -0.06102 & 0.65268 & 0.00270 & -0.01623 & -0.00034 & 0.00084 \\
\hline $\mathrm{H} 1$ & Y & -0.00163 & 0.03064 & -0.33775 & -0.00131 & 0.00829 & -0.00029 & -0.00067 \\
\hline $\mathrm{H} 1$ & Z & 0.00765 & -0.05855 & 0.60235 & 0.00271 & -0.01466 & 0.00054 & 0.00106 \\
\hline $\mathrm{C} 3$ & $x$ & -0.00010 & -0.00053 & -0.00156 & -0.00006 & 0.00010 & -0.00041 & 0.00010 \\
\hline C3 & Y & -0.00017 & 0.00047 & 0.00063 & 0.00005 & -0.00004 & 0.00022 & -0.00005 \\
\hline $\mathrm{C} 3$ & Z & -0.00076 & -0.00067 & 0.00133 & -0.00009 & 0.00002 & -0.00092 & -0.00002 \\
\hline 04 & $\mathrm{x}$ & -0.00003 & 0.00001 & 0.00019 & -0.00002 & -0.00003 & 0.00019 & -0.00007 \\
\hline O4 & Y & -0.00001 & -0.00002 & -0.00005 & -0.00001 & 0.00003 & -0.00008 & 0.00002 \\
\hline 04 & Z & 0.00018 & 0.00013 & -0.00011 & -0.00003 & 0.00002 & 0.00011 & 0.00005 \\
\hline $\mathrm{H} 8$ & $\mathrm{x}$ & -0.00045 & -0.00073 & 0.00187 & -0.00007 & 0.00006 & -0.00067 & 0.00012 \\
\hline $\mathrm{H} 8$ & Y & 0.00019 & 0.00016 & -0.00065 & 0.00006 & -0.00004 & 0.00054 & -0.00027 \\
\hline H8 & Z & -0.00048 & -0.00002 & 0.00062 & 0.00029 & -0.00001 & 0.00031 & -0.00056 \\
\hline 05 & $\mathrm{x}$ & 0.00011 & 0.00025 & 0.00033 & 0.00003 & -0.00002 & 0.00013 & -0.00008 \\
\hline 05 & Y & -0.00013 & -0.00029 & -0.00013 & -0.00003 & 0.00001 & -0.00004 & 0.00001 \\
\hline 05 & Z & 0.00018 & 0.00026 & 0.00042 & 0.00004 & -0.00008 & 0.00083 & -0.00002 \\
\hline \multicolumn{2}{|c|}{ Erequencies } & 3741.35 & & & & & & \\
\hline atens & ities & 135.48 & & & & & & \\
\hline Re1 & $\mathrm{X}$ & 0.00000 & & & & & & \\
\hline Re1 & Y & 0.00000 & & & & & & \\
\hline Re1 & Z & 0.00000 & & & & & & \\
\hline N1 & $\mathrm{X}$ & 0.00004 & & & & & & \\
\hline N1 & Y & -0.00001 & & & & & & \\
\hline N1 & Z & 0.00007 & & & & & & \\
\hline $\mathrm{C} 4$ & $\mathrm{X}$ & -0.00003 & & & & & & \\
\hline $\mathrm{C} 4$ & Y & 0.00000 & & & & & & \\
\hline $\mathrm{C} 4$ & Z & -0.00003 & & & & & & \\
\hline C7 & $\mathrm{X}$ & 0.00003 & & & & & & \\
\hline C7 & $\mathrm{Y}$ & 0.00000 & & & & & & \\
\hline C7 & Z & 0.00005 & & & & & & \\
\hline $\mathrm{N} 2$ & $\mathrm{X}$ & 0.00000 & & & & & & \\
\hline N2 & Y & -0.00004 & & & & & & \\
\hline $\mathrm{N} 2$ & Z & 0.00004 & & & & & & \\
\hline H18 & $\mathrm{X}$ & -0.00070 & & & & & & \\
\hline H1 8 & Y & 0.00011 & & & & & & \\
\hline H18 & Z & -0.00084 & & & & & & \\
\hline H21 & $\mathrm{X}$ & 0.00019 & & & & & & \\
\hline H21 & $\mathrm{Y}$ & -0.00006 & & & & & & \\
\hline
\end{tabular}




\begin{tabular}{|c|c|c|}
\hline H 21 & Z & 0.00008 \\
\hline H23 & $\mathrm{x}$ & -0.00016 \\
\hline H23 & $\mathrm{Y}$ & 0.00013 \\
\hline H23 & Z & 0.00009 \\
\hline H26 & $\mathrm{x}$ & 0.00011 \\
\hline H26 & $\mathrm{Y}$ & 0.00013 \\
\hline H 26 & Z & -0.00066 \\
\hline 01 & $\mathrm{x}$ & -0.00003 \\
\hline 01 & $\mathrm{Y}$ & 0.00004 \\
\hline 01 & Z & -0.00001 \\
\hline 02 & $\mathrm{X}$ & 0.00002 \\
\hline 02 & $\mathrm{Y}$ & -0.00001 \\
\hline $\mathrm{O} 2$ & Z & -0.00006 \\
\hline 03 & $\mathrm{x}$ & -0.00004 \\
\hline 03 & $\mathrm{Y}$ & 0.00001 \\
\hline 03 & Z & -0.00001 \\
\hline $\mathrm{C} 1$ & $\mathrm{x}$ & -0.00006 \\
\hline $\mathrm{C} 1$ & $\mathrm{Y}$ & -0.00003 \\
\hline $\mathrm{C} 1$ & Z & 0.00000 \\
\hline H3 & $\mathrm{x}$ & 0.00019 \\
\hline H3 & $\mathrm{Y}$ & 0.00007 \\
\hline H3 & Z & 0.00035 \\
\hline H 4 & $\mathrm{x}$ & 0.00001 \\
\hline H 4 & Y & 0.00033 \\
\hline H 4 & Z & -0.00012 \\
\hline $\mathrm{C} 2$ & $\mathrm{x}$ & 0.00009 \\
\hline C2 & $\mathrm{Y}$ & -0.00009 \\
\hline C2 & Z & -0.00063 \\
\hline H 1 & $\mathrm{x}$ & 0.00077 \\
\hline $\mathrm{H} 1$ & $Y$ & -0.00016 \\
\hline H1 & Z & -0.00098 \\
\hline C3 & $\mathrm{x}$ & -0.00092 \\
\hline C3 & $\mathrm{Y}$ & 0.00063 \\
\hline C3 & Z & 0.00210 \\
\hline 04 & $\mathrm{x}$ & -0.00968 \\
\hline 04 & $\mathrm{Y}$ & 0.00978 \\
\hline 04 & Z & 0.05807 \\
\hline H8 & $\mathrm{x}$ & 0.14944 \\
\hline H8 & $\mathrm{Y}$ & -0.15666 \\
\hline H8 & Z & -0.94274 \\
\hline 05 & $\mathrm{x}$ & 0.00087 \\
\hline 05 & $\mathrm{Y}$ & -0.00033 \\
\hline 05 & Z & 0.00030 \\
\hline
\end{tabular}

Thermochemical Properties:

pressure: $\quad 1.0000 \mathrm{~atm}$

rotational symmetry number: 1

zero point energy: $\quad 93.620 \mathrm{kcal} / \mathrm{mol}$

$\begin{array}{rrrrrr}\text { temp }(\mathrm{K}) & \mathrm{Cp}(\mathrm{cal} / \mathrm{mol} \mathrm{K}) & \mathrm{S}(\mathrm{cal} / \mathrm{mol} \mathrm{K}) & \mathrm{H} & (\mathrm{kcal} / \mathrm{mol}) & \mathrm{G}(\mathrm{kcal} / \mathrm{mol}) \\ 0.00 & 7.9489 & 29.7093 & 0.0000 & 0.0000 \\ 298.15 & 50.4962 & 116.4984 & 8.9898 & -25.7441\end{array}$

end of program freq

start of program ch

Moments from quantum mechanical wavefunction:

Dipole Moments (Debye)
$\mathrm{X}=$
$-6.4813$
$\mathrm{Y}=$
$2.8751 \quad Z=$
-2.8377 Tot $=$
7.6372 
Final energy ........... -759.85584009889

Job WF26685 completed on CHENB071 at Sat Jan 24 02:32:48 2004

Reason for exit: Successful completion

Titan Program CPU Time : 010:25:33.4

Titan Program Wall Time: 019:14:15.0

TITAN Properties Program: (PC/x86)

Release 6.0 .6

Reason for exit: Successful completion

Properties Program CPU Time : 000:00:02.0

Properties Program Wall Time: 000:00:11.5 


\section{Scheme 5}

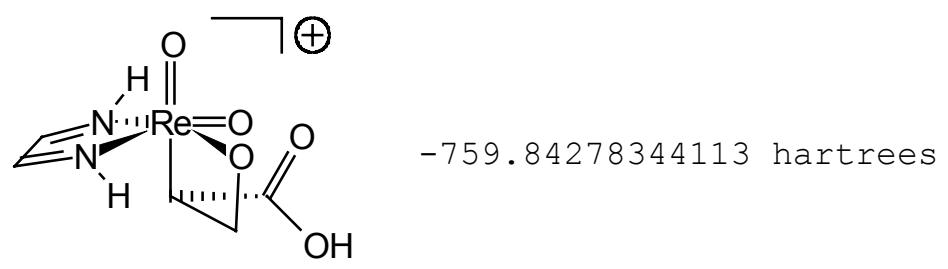

This higher energy structure is not shown explicitly in scheme 5.

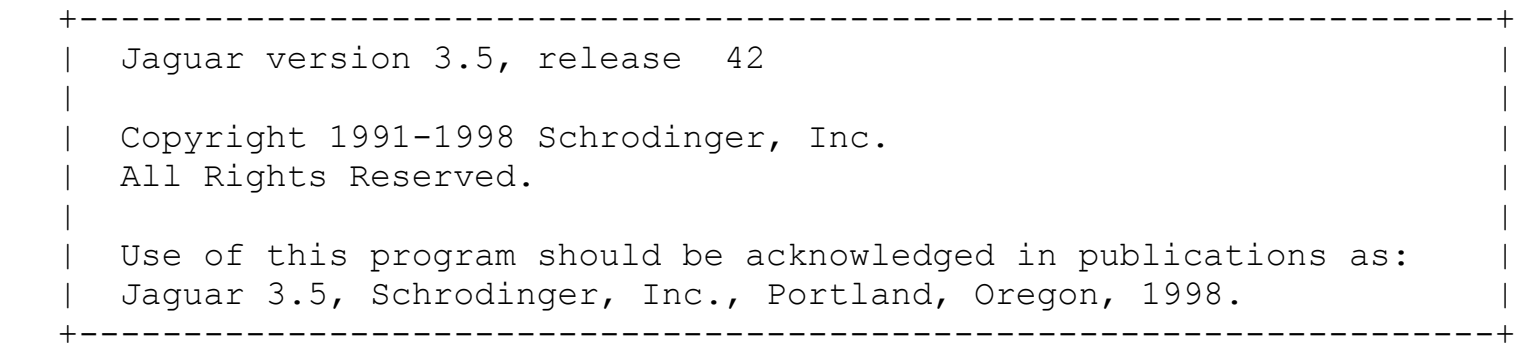

Job WF23865 started on CHENB071 at Fri Jan 23 09:51:24 2004

Jaguar version 3.5 , release 42

Job name:

Task:

Stoichiometry:

Molecular weight:

Molecular charge:

Spin multiplicity:

Point Group:

SCF method:

Basis set:

Basis functions:

Total electrons:

Alpha electrons:

Beta electrons:

Total orbitals:

Core orbitals:

Open-shell orbitals:

Occupied orbitals:

Shells:
WF23865

Geometry optimization

ReC $5 \mathrm{~N} 2 \mathrm{H} 8 \mathrm{O} 5$

$363.00 \mathrm{amu}$

1

1

C1

B3LYP

LACVP**

230

106

53

53

245

53

0

53

1

Non-default options chosen:

SCF calculation type: DFT

$\mathrm{DFT}=$ Becke_3 Parameter/HF+Slater+Becke88+VWN+LYP (B3LYP)

Geometry $\bar{w} \bar{l} \bar{l}$ be optimized in redundant internal coordinates

Numerical 2nd derivatives will be computed

Vibrational frequencies and related properties will be computed

Molecular symmetry not used

Initial Hessian: from previous calculation 


\begin{tabular}{|c|c|c|c|c|c|c|c|}
\hline Iter & Energy & & Gmax & & Grms & Dmax & Drms \\
\hline 1 & -759.818247 & * & 0.05567 & . & 0.01505 & 0.10826 & 0.03589 \\
\hline 2 & -759.837292 & & 0.01541 & . & 0.00533 & 0.11158 & 0.03335 \\
\hline 3 & -759.840645 & & 0.00721 & 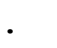 & 0.00235 & 0.13532 & 0.03585 \\
\hline 4 & -759.841680 & . & 0.00856 & . & 0.00230 & 0.18256 & 95 \\
\hline 5 & -759.841390 & . & 0.01579 & • & 0.00411 & 0.08189 & 0.02348 \\
\hline 6 & -759.841698 & & 0.01617 & . & 0.00353 & 0.13654 & 3587 \\
\hline$\star \star r$ & estarting opt & & ation fr & om & step & $6 \star \star$ & \\
\hline 6 & -759.839292 & 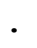 & 0.01617 & & 0.00353 & . 0.05417 & 0.01536 \\
\hline 8 & -759.842529 & & 0.00352 & . & 0.00124 &.$\quad 0.0$ & 48 \\
\hline$* \quad r$ & estarting opt & & ation fr & om & step & $8 \star \star$ & \\
\hline 8 & -759.842475 & . & 0.00352 & . & 0.00124 & .0 .03 & 0.0 \\
\hline 10 & -759.8 & & 0.00245 & $\cdot$ & 0.00 & 0.01 & 0 . \\
\hline 11 & -759.842732 & $\star$ & 0.00241 & . & 0.00077 & 0.02245 & 0.0 \\
\hline 12 & -759.842755 & * & 0.00225 & 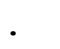 & 0.00059 & 0.04628 & 971 \\
\hline 13 & -759.842 & * & 0.00205 & • & & 0.01997 & 406 \\
\hline 14 & -759.842773 & $\star$ & 0.00161 & . & 0.00037 & 0.00465 & 0.00122 \\
\hline 15 & -759.842772 & \# & 0.00110 & • & 0.00028 & 0.00294 & 103 \\
\hline 16 & -759.8 & * & 0.00081 & . & & 0.00424 & 0.00137 \\
\hline 17 & -759.842780 & \# & 0.00030 & * & 0.00013 & 0.00293 & 0.00064 \\
\hline
\end{tabular}

energy change:

gradient maximum:

gradient rms:

step size: 0.00533

displacement maximum:

displacement rms:

predicted energy change:

step: $5.3321 \mathrm{E}-03$

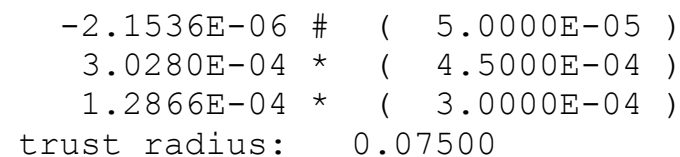

$2.9323 \mathrm{E}-03 \cdot(1.8000 \mathrm{E}-03)$

$6.3730 \mathrm{E}-04 \star(1.2000 \mathrm{E}-03)$

$-1.2617 \mathrm{E}-06$ geom step:

5.3321E-03 full

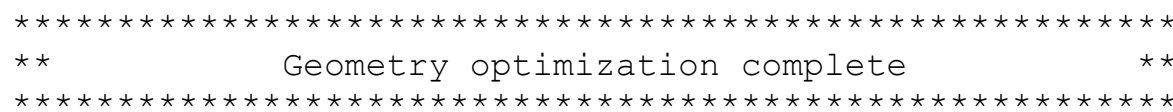

center of mass moved by:
$x$ :
$0.0000 \mathrm{E}+00$
$\mathrm{y}:$
$0.0000 \mathrm{E}+00$
$\mathrm{z}:$
$0.0000 \mathrm{E}+00$

final geometry:

$\begin{array}{lr}\text { atom } & \text { X } \\ \text { Re1 } & -0.0893423103 \\ \text { N1 } & -0.3067377087 \\ \text { H9 } & -1.2087983193 \\ \text { N2 } & 2.0230879442 \\ \text { H10 } & 2.9113256601 \\ \text { C1 } & 0.6700949427 \\ \text { H11 } & 0.5286957187 \\ \text { C2 } & 2.0023599985 \\ \text { H8 } & 2.8698500792 \\ \text { O1 } & 0.5710502722 \\ \text { C3 } & 0.5577090119 \\ \text { H7 } & 1.5324841729 \\ \text { H12 } & -0.2339867836 \\ \text { C4 } & 0.2645949435 \\ \text { H2 } & 1.1093583415 \\ \text { O2 } & 0.4609693391 \\ \text { O3 } & -1.7779474897 \\ \text { C5 } & -0.9835584586 \\ \text { O4 } & -1.3521721925 \\ \text { O5 } & -1.6564096835 \\ \text { H4 } & -2.4569987199\end{array}$

angstroms
y
-0.4015645930
0.1265814500
0.5088632060
-0.2811593370
-0.5382658308
0.1697151174
0.4888467610
-0.1583902133
-0.2546949023
0.1644966617
1.6384402075
1.9874380520
1.9963395044
1.8419864209
2.1788942327
-2.0325902715
-0.3858527707
2.5489563716
2.5903645817
3.1185150066
3.5333401680

$\mathrm{z}$

1. 4447006537

$-0.6228242389$

$-0.9206045566$

0.3629642982

0.7962922267

$-1.4532653476$

$-2.4838074857$

$-0.9076702352$

$-1.5586289892$

3.0949200095

3.0287882986

3.3770486028

3.6903668265

1.5492094521

0.9520802685

1. 3886878445

1. 5956078209

1.1071037138

$-0.0555445733$

2.1144187936

1. 7461422066 
calculating forces at perturbed geometry calculating forces at perturbed geometry calculating forces at perturbed geometry calculating forces at perturbed geometry calculating forces at perturbed geometry calculating forces at perturbed geometry calculating forces at perturbed geometry calculating forces at perturbed geometry calculating forces at perturbed geometry calculating forces at perturbed geometry calculating forces at perturbed geometry calculating forces at perturbed geometry calculating forces at perturbed geometry calculating forces at perturbed geometry calculating forces at perturbed geometry calculating forces at perturbed geometry calculating forces at perturbed geometry calculating forces at perturbed geometry calculating forces at perturbed geometry calculating forces at perturbed geometry calculating forces at perturbed geometry calculating forces at perturbed geometry calculating forces at perturbed geometry calculating forces at perturbed geometry calculating forces at perturbed geometry calculating forces at perturbed geometry calculating forces at perturbed geometry calculating forces at perturbed geometry calculating forces at perturbed geometry calculating forces at perturbed geometry calculating forces at perturbed geometry calculating forces at perturbed geometry calculating forces at perturbed geometry calculating forces at perturbed geometry calculating forces at perturbed geometry calculating forces at perturbed geometry calculating forces at perturbed geometry calculating forces at perturbed geometry calculating forces at perturbed geometry calculating forces at perturbed geometry calculating forces at perturbed geometry calculating forces at perturbed geometry calculating forces at perturbed geometry calculating forces at perturbed geometry calculating forces at perturbed geometry calculating forces at perturbed geometry calculating forces at perturbed geometry calculating forces at perturbed geometry calculating forces at perturbed geometry calculating forces at perturbed geometry calculating forces at perturbed geometry calculating forces at perturbed geometry calculating forces at perturbed geometry calculating forces at perturbed geometry calculating forces at perturbed geometry calculating forces at perturbed geometry calculating forces at perturbed geometry calculating forces at perturbed geometry calculating forces at perturbed geometry
1 of $126 \ldots$

2 of $126 \ldots$

3 of $126 \ldots$

4 of $126 \ldots$

5 of $126 \ldots$

6 of $126 \ldots$

7 of $126 \ldots$

8 of $126 \ldots$

9 of $126 \ldots$

10 of $126 \ldots$

11 of $126 \ldots$

12 of $126 \ldots$

13 of $126 \ldots$

14 of $126 \ldots$

15 of $126 \ldots$

16 of $126 \ldots$

17 of $126 \ldots$

18 of $126 \ldots$

19 of $126 \ldots$

20 of $126 \ldots$

21 of $126 \ldots$

22 of $126 \ldots$

23 of $126 \ldots$

24 of $126 \ldots$

25 of $126 \ldots$

26 of $126 \ldots$

27 of $126 \ldots$

28 of $126 \ldots$

29 of $126 \ldots$

30 of $126 \ldots$

31 of $126 \ldots$

32 of $126 \ldots$

33 of $126 \ldots$

34 of $126 \ldots$

35 of $126 \ldots$

36 of $126 \ldots$

37 of $126 \ldots$

38 of $126 \ldots$

39 of $126 \ldots$

40 of $126 \ldots$

41 of $126 \ldots$

42 of $126 \ldots$

43 of $126 \ldots$

44 of $126 \ldots$

45 of $126 \ldots$

46 of $126 \ldots$

47 of $126 \ldots$

48 of $126 \ldots$

49 of $126 \ldots$

50 of $126 \ldots$

51 of $126 \ldots$

52 of $126 \ldots$

53 of $126 \ldots$

54 of $126 \ldots$

55 of $126 \ldots$

56 of $126 \ldots$

57 of $126 \ldots$

58 of $126 \ldots$

59 of $126 \ldots$ 
calculating forces at perturbed geometry calculating forces at perturbed geometry calculating forces at perturbed geometry calculating forces at perturbed geometry calculating forces at perturbed geometry calculating forces at perturbed geometry calculating forces at perturbed geometry calculating forces at perturbed geometry calculating forces at perturbed geometry calculating forces at perturbed geometry calculating forces at perturbed geometry calculating forces at perturbed geometry calculating forces at perturbed geometry calculating forces at perturbed geometry calculating forces at perturbed geometry calculating forces at perturbed geometry calculating forces at perturbed geometry calculating forces at perturbed geometry calculating forces at perturbed geometry calculating forces at perturbed geometry calculating forces at perturbed geometry calculating forces at perturbed geometry calculating forces at perturbed geometry calculating forces at perturbed geometry calculating forces at perturbed geometry calculating forces at perturbed geometry calculating forces at perturbed geometry calculating forces at perturbed geometry calculating forces at perturbed geometry calculating forces at perturbed geometry calculating forces at perturbed geometry calculating forces at perturbed geometry calculating forces at perturbed geometry calculating forces at perturbed geometry calculating forces at perturbed geometry calculating forces at perturbed geometry calculating forces at perturbed geometry calculating forces at perturbed geometry calculating forces at perturbed geometry calculating forces at perturbed geometry calculating forces at perturbed geometry calculating forces at perturbed geometry 101 of $126 \ldots$ calculating forces at perturbed geometry 102 of $126 \ldots$ calculating forces at perturbed geometry 103 of $126 \ldots$ calculating forces at perturbed geometry 104 of $126 \ldots$ calculating forces at perturbed geometry 105 of $126 \ldots$ calculating forces at perturbed geometry 106 of $126 \ldots$ calculating forces at perturbed geometry 107 of $126 \ldots$ calculating forces at perturbed geometry 108 of $126 \ldots$ calculating forces at perturbed geometry 109 of $126 \ldots$ calculating forces at perturbed geometry 110 of $126 \ldots$ calculating forces at perturbed geometry 111 of $126 \ldots$ calculating forces at perturbed geometry 112 of $126 \ldots$ calculating forces at perturbed geometry 113 of $126 \ldots$ calculating forces at perturbed geometry 114 of $126 \ldots$ calculating forces at perturbed geometry 115 of $126 \ldots$ calculating forces at perturbed geometry 116 of $126 \ldots$ calculating forces at perturbed geometry 117 of $126 \ldots$ calculating forces at perturbed geometry 118 of $126 \ldots$ calculating forces at perturbed geometry 119 of $126 \ldots$ calculating forces at perturbed geometry 120 of $126 \ldots$ 
calculating forces at perturbed geometry 121 of $126 \ldots$ calculating forces at perturbed geometry 122 of $126 \ldots$ calculating forces at perturbed geometry 123 of $126 \ldots$ calculating forces at perturbed geometry 124 of $126 \ldots$ calculating forces at perturbed geometry 125 of $126 \ldots$ calculating forces at perturbed geometry 126 of $126 \ldots$

numerical derivatives complete;

calculation at normal geometry follows...

start of program freq

harmonic frequencies in $\mathrm{cm}^{\wedge}-1$, IR intensities in $\mathrm{km} / \mathrm{mol}$, and normal modes:

\begin{tabular}{|c|c|c|c|c|c|c|c|c|}
\hline Erequ & cies & 67.60 & 69.78 & 102.51 & 122.44 & 134.13 & 155.21 & 178.3 \\
\hline nten & ties & 5.39 & 5.39 & 4.23 & 4.21 & 2.03 & 6.53 & \\
\hline Re1 & $\mathrm{X}$ & -0.00101 & -0.00625 & 0.00192 & 0.01256 & 0.01304 & -0.00234 & 0.0174 \\
\hline $\operatorname{Re} 1$ & $\mathrm{Y}$ & 0.01206 & 0.00904 & -0.00434 & 0.01912 & -0.01512 & 0.01242 & \\
\hline Re1 & Z & 0.00206 & -0.00998 & -0.01027 & -0.00446 & -0.00083 & 0.00363 & \\
\hline N1 & $\mathrm{X}$ & 0.00076 & 0.04981 & -0.00081 & 0.01024 & 0.00942 & -0.01228 & -0.03 \\
\hline N1 & Y & -0.00179 & 0.01089 & 0.04353 & 0.00957 & 0.08130 & 0.03021 & -0.0 \\
\hline $\mathrm{N} 1$ & Z & -0.00381 & -0.00747 & -0.00221 & -0.01329 & 0.02969 & -0.00116 & -0.0 \\
\hline H9 & $\mathrm{X}$ & 0.00199 & 0.06739 & 0.00710 & 0.02582 & 0.03470 & -0.01557 & -0.0 \\
\hline $\mathrm{H} 9$ & Y & -0.00810 & 0.05065 & 0.08239 & 0.05855 & 0.17868 & 0.037 & -0 . \\
\hline $\mathrm{H} 9$ & Z & -0.01360 & -0.01451 & 0.02062 & 0.00019 & 0.07016 & 0.01 & -0 . \\
\hline N2 & $\mathrm{X}$ & -0.00626 & 0.02252 & -0.01934 & 0.01575 & -0.02338 & -0.01066 & -0 . \\
\hline N2 & Y & -0.02365 & -0.03204 & -0.05012 & -0.05247 & -0.08597 & -0.00640 & \\
\hline N2 & Z & -0.00001 & 0.04216 & -0.03230 & -0.04200 & 0.00145 & -0.03213 & \\
\hline $\mathrm{H} 10$ & $\mathrm{X}$ & -0.00909 & 0.00813 & -0.03122 & 0.01204 & -0.04260 & -0.00794 & -0 . \\
\hline $\mathrm{H} 10$ & Y & -0.02831 & -0.04735 & -0.09808 & -0.08342 & -0.16135 & -0.02747 & \\
\hline $\mathrm{H} 10$ & Z & 0.00314 & 0.062 & -0.03660 & -0.05269 & -0.00416 & -0.05 & \\
\hline $\mathrm{C} 1$ & $\mathrm{X}$ & 0.00078 & 0.06869 & -0.00933 & -0.00485 & 0.01039 & -0.02033 & -0 . \\
\hline $\mathrm{C} 1$ & Y & -0.02104 & -0.01967 & 0.03453 & -0.07472 & 0.10280 & 0.07121 & -0 . \\
\hline $\mathrm{C} 1$ & Z & -0.00472 & 0.01179 & -0.01316 & -0.033 & & -0.00 & -0 . \\
\hline $\mathrm{H} 11$ & $\mathrm{X}$ & 0.00433 & 0.09699 & -0.01010 & -0.00586 & 0.02340 & -0.03136 & -0 . \\
\hline $\mathrm{H} 1$ & Y & -0.03187 & -0.01976 & 0.06212 & -0.10414 & 0.19716 & 0.10 & -0 . \\
\hline $\mathrm{H} 1$ & Z & -0.00847 & 0.00 & -0.00436 & -0.04277 & & 0 & -0 \\
\hline $\mathrm{C} 2$ & $\mathrm{X}$ & -0.00303 & 0.05029 & -0.01808 & -0.00980 & -0.01014 & -0.01643 & -0 . \\
\hline $\mathrm{C} 2$ & Y & -0.02985 & -0.04683 & -0.02415 & -0.12790 & -0.00459 & 0.05876 & 0 \\
\hline $\mathrm{C} 2$ & Z & -0.00 & 0.04 & -0.030 & -0.04827 & & -0 & \\
\hline H8 & $\mathrm{X}$ & -0 & 0.06075 & -0.02368 & -0.03265 & -0.01040 & -0.01824 & \\
\hline $\mathrm{H} 8$ & Y & -0.04040 & -0.07272 & -0.04503 & -0.21756 & -0.00897 & 0.09204 & \\
\hline H8 & Z & 0 & 0.057 & -0.03388 & -0.06562 & 0.00 & -0.03 & \\
\hline O1 & $\mathrm{X}$ & 0 . & 0.00969 & -0.07377 & -0.01028 & 0 . & -0.02 & \\
\hline 01 & Y & 0.01015 & 0.01728 & 0.03145 & -0.02606 & 0.00645 & -0.02072 & -0 . \\
\hline 01 & Z & -0.00414 & -0.01810 & 0.01874 & 0.01859 & -0.02 & 0.03 & -0 \\
\hline C3 & $\mathrm{X}$ & 0 & 0.07198 & 0.01260 & -0.08894 & -0.04388 & 0.02 & -0 \\
\hline C3 & Y & 0 & 0.01598 & 0.01699 & -0.02276 & 0.00698 & -0.021 & -0 . \\
\hline C3 & Z & -0.0 & -0.04340 & -0.00058 & 0.07482 & -0.01109 & 0.03 & \\
\hline H7 & $\mathrm{X}$ & 0.06670 & 0.10730 & 0.03610 & -0.12973 & -0.07994 & 0.026 & -0 . \\
\hline H7 & Y & -0.01324 & -0.02224 & -0.02786 & 0.01939 & 0.07752 & -0.05264 & \\
\hline $\mathrm{H} 7$ & Z & -0 & -0.104 & -0.01940 & 0.14593 & 0 & 0.04 & \\
\hline $\mathrm{H} 12$ & $\mathrm{X}$ & 0.07286 & 0.12569 & 0.04278 & -0.14935 & -0.08895 & 0.02 & -0. \\
\hline $\mathrm{H} 12$ & Y & 0 & 0.06299 & 0.08150 & -0.09383 & -0.06522 & 0.00 & -0 . \\
\hline $\mathrm{H} 12$ & Z & -0.0 & -0.00473 & 0.00094 & 0.04117 & -0.02528 & 0.01 & -0 . \\
\hline $\mathrm{C} 4$ & $\mathrm{X}$ & 0.02758 & 0.00457 & 0.00933 & -0.02187 & -0.03384 & 0.05299 & \\
\hline $\mathrm{C} 4$ & $Y$ & 0.0 & 0.00690 & 0.00520 & 0.01112 & & -0.02 & \\
\hline C4 & $\mathrm{Z}$ & -0.01354 & -0.03054 & -0.00114 & 0.06418 & -0.01343 & 0.02507 & 0. \\
\hline $\mathrm{H} 2$ & $\mathrm{X}$ & 0.01227 & -0.03285 & 0.00242 & 0.00110 & -0.02558 & 0.05897 & 0 . \\
\hline $\mathrm{H} 2$ & $\mathrm{Y}$ & 0.0 & 0.02295 & & 0.04113 & & -0.01359 & 0. \\
\hline $\mathrm{H} 2$ & Z & -0 . & -0 & -0.01193 & 0.11174 & -0.00012 & 0.03816 & 0 \\
\hline
\end{tabular}




\begin{tabular}{|c|c|c|c|c|c|c|c|c|}
\hline 02 & $\mathrm{X}$ & -0.03358 & -0.03779 & 0.06501 & 0.00563 & -0.05615 & -0.02180 & -0.0536 \\
\hline $\mathrm{O} 2$ & $\mathrm{Y}$ & 00114 & -0.00202 & 0.01189 & 0.01631 & -0.04036 & 0.00658 & \\
\hline $\mathrm{O} 2$ & Z & 0.00563 & -0.00007 & 0.17027 & -0.02074 & 0.03221 & 0.02279 & 0.07978 \\
\hline 03 & $\mathrm{x}$ & -0.00115 & -0.00660 & -0.00420 & 0.01294 & 0.01778 & -0.00414 & 0.01270 \\
\hline 03 & $\mathrm{Y}$ & 0.04140 & 0.03429 & -0.04894 & 0.03161 & 0.06648 & 0.04347 & \\
\hline 03 & Z & 0.00057 & -0.01805 & -0.06964 & -0.01020 & 0.04270 & -0.01335 & -0.0534 \\
\hline C5 & $\mathrm{x}$ & 0.00752 & -0.03916 & 0.00477 & -0.02055 & -0.03295 & 0.03668 & -0.00392 \\
\hline C5 & $\mathrm{Y}$ & -0.02155 & -0.02840 & 0.01060 & -0.00498 & 0.01253 & -0.07713 & \\
\hline C5 & Z & -0.00058 & 0.03091 & 0.01747 & 0.03271 & -0.02432 & -0.01125 & \\
\hline 04 & $\mathrm{x}$ & 0.06238 & -0.12248 & -0.00570 & -0.00915 & -0.02907 & 0.02382 & -0.01057 \\
\hline 04 & $\mathrm{Y}$ & 0.06277 & -0.07371 & 0.03232 & -0.03257 & 0.01867 & -0.16557 & \\
\hline 04 & Z & -0.01442 & 0.05531 & 0.02133 & 0.02736 & -0.02764 & -0.01276 & \\
\hline 05 & $\mathrm{x}$ & -0.08868 & 0.02315 & 0.01223 & -0.03914 & -0.04132 & 0.02068 & 0619 \\
\hline 05 & $\mathrm{Y}$ & -0.17816 & -0.00504 & -0.00654 & & 0.01511 & & \\
\hline 05 & Z & 0.02521 & 0.05853 & 3254 & 0.01011 & -0.03325 & -0.04897 & \\
\hline H4 & $\mathrm{x}$ & -0.10064 & -0.01350 & 0.01080 & -0.03809 & -0.03773 & -0.00025 & \\
\hline $\mathrm{H} 4$ & $\mathrm{Y}$ & -0.20075 & -0.03677 & & -0.0 & & & \\
\hline H4 & Z & 0.02677 & 0.10263 & & & & -0.0 & \\
\hline & & 207.90 & 235.22 & & & & 339.24 & \\
\hline & & 8.48 & & & & & & \\
\hline Re1 & $\mathrm{x}$ & -0.02351 & 0.00453 & 0.00115 & -0.00070 & 0.00983 & -0.00 & 0.00 \\
\hline $\operatorname{Re} 1$ & $\mathrm{Y}$ & 0.00521 & 0.00774 & 0.00862 & -0.01818 & 0.00150 & 0200 & 529 \\
\hline $\operatorname{Re} 1$ & Z & -0.01655 & -0.0 & -0.0 & -0. & -0 & -0 . & \\
\hline N1 & $\mathrm{x}$ & 0.02263 & -0.04200 & -0.01370 & & -0.00886 & 0360 & \\
\hline N1 & $\mathrm{Y}$ & -0.01530 & 0.06188 & 0.00260 & & -0.04412 & -0 & \\
\hline N1 & Z & -0.0 & 211 & -0.0 & & -0. & & -0 . \\
\hline H9 & $\mathrm{x}$ & 0.02752 & -0.02173 & -0.0 & & -0.0 & -0 . & \\
\hline H9 & $\mathrm{Y}$ & -0.02877 & 0.11452 & & & -0.0 & -0 & \\
\hline H9 & Z & -0.0 & 670 & -0 . & & -0 & & -0 \\
\hline N2 & $\mathrm{x}$ & 0.03423 & -0.10585 & -0.0 & & -0.00945 & 0.04408 & -0 . \\
\hline N2 & $\mathrm{Y}$ & 0.01664 & 0.01699 & -0.0 & & -0.05939 & -0.01025 & \\
\hline & Z & -0.0 & 769 & & & 524 & -0 . & -0 \\
\hline $\mathrm{H} 10$ & $\mathrm{x}$ & 0.03516 & -0.14226 & -0.03963 & 0. & -0.00966 & 5863 & -0 . \\
\hline $\mathrm{H} 10$ & $\mathrm{Y}$ & 0.04003 & -0.01631 & -0.03373 & 973 & -0.06246 & 0.03330 & 061 \\
\hline $\mathrm{H} 10$ & Z & 646 & & & -0 . & & -0 & \\
\hline $\mathrm{C} 1$ & $\mathrm{x}$ & 3912 & -0.02696 & & -0 . & & -0 & \\
\hline $\mathrm{C} 1$ & $\mathrm{Y}$ & -0.01268 & -0.01112 & 1501 & -0.0 & 0.00133 & -0.01977 & -0.03060 \\
\hline $\mathrm{C} 1$ & Z & -0.01353 & & & & & & -0 . \\
\hline 11 & $\mathrm{x}$ & & & & -0 . & & -0 & \\
\hline H11 & $\mathrm{Y}$ & -0.02947 & -0.02603 & 0.0 & -0.13 & 0.00158 & -0.04035 & -0.09422 \\
\hline H11 & Z & -0.01976 & & & & & & -0 . \\
\hline C2 & $\mathrm{X}$ & & -0 & -0 . & -0 . & & & \\
\hline $\mathrm{C} 2$ & $\mathrm{Y}$ & 0.01699 & -0.06100 & -0.0 & -0.02 & 0.02854 & 0.01562 & -0.01124 \\
\hline $\mathrm{C} 2$ & Z & & & & -0 & & -0 . & -0 . \\
\hline H8 & $\mathrm{x}$ & & -0.0 & -0 . & -0 . & & -0 . & \\
\hline H8 & $\mathrm{Y}$ & 0.03735 & -0.14407 & -0.02273 & -0.0 & 0.09289 & 0.06912 & -0.02200 \\
\hline H8 & Z & -0.00302 & & 0.0 & -0 & 2315 & -0.0 & 1818 \\
\hline 01 & $\mathrm{x}$ & & & -0 . & -0 . & -0 . & & \\
\hline 01 & $\mathrm{Y}$ & -0.02810 & -0.0 & 0.0 & & 587 & 0.05240 & -0. \\
\hline 01 & Z & -0.04441 & -0.01555 & 0.00045 & -0.0 & 0.01280 & -0.04804 & 0.02245 \\
\hline & $\mathrm{x}$ & & & -0. & -0 & & -0 & \\
\hline C3 & $\mathrm{Y}$ & -0. & -0.0 & 0.0 & & & 940 & -0.0 \\
\hline C3 & Z & 0.02445 & 0.01187 & -0.01790 & 0.00386 & 0.02016 & 0.02440 & -0.01131 \\
\hline & $\mathrm{x}$ & -0.03 & 0.04378 & -0.1 & -0. & & -0 & \\
\hline & $\mathrm{Y}$ & & -0.01295 & & -0.00223 & -0.0 & & -0 \\
\hline H7 & Z & 0.05327 & -0.00470 & 0.10884 & 0.02737 & 0.02726 & 0.13046 & -0.02775 \\
\hline & $\mathrm{x}$ & -0.04131 & 0.05203 & -0.23619 & -0. & & -0 & \\
\hline & $\mathrm{Y}$ & -0.11529 & -0.01920 & -0.04557 & & & -0.05531 & \\
\hline $\mathrm{H} 1$ & Z & 0.02259 & 0.03397 & -0.13402 & -0.01711 & 0.02048 & -0.03483 & -0.00099 \\
\hline C4 & $\mathrm{X}$ & -0.00656 & 0.00927 & 0.03005 & 0.02352 & 0.02954 & 0.04153 & -0.00748 \\
\hline
\end{tabular}




C4
C4
H2
H2
H2
O2
O2
O2
O2
O3
O3
O3
C5
C5
C5
C5
O4
O4
O4
O4
O5
O5
O5
O5
H4
H4
H4

$-0.01378$

0.02704

$-0.00023$

$-0.01918$

0.03304

0.04879

0.02789

0.10665

$-0.01194$

$-0.00068$

0.11584

0.00733

$-0.00625$

0.01480

0.03049

$-0.01485$

0.00677

$-0.00791$

$-0.00124$

0.00076

$-0.00041$

0.00098

$-0.01317$

frequencies

intensities

Re1 $X$

Re1 Y

Re1 Z

N1 X

N1 Y

N1 Z

H9 X

H9 Y

H9 Z

N2 X

N2 Y

N2 Z

$\mathrm{H} 10 \mathrm{X}$

$\mathrm{H} 10 \mathrm{Y}$

H10 Z

C1 X

C1 Y

C1 Z

H11 X

H11 Y

H11 Z

C2

$\mathrm{C} 2$

C2

H 8

H 8

H8

01

01

01

C3

C3

C3

H7

$\mathrm{H} 7$

382.72

1.60

$-0.00968$

$-0.02299$

0.01229

0.03266

0.04553

0.05930

$-0.08391$

$-0.07417$

0.03769

$-0.01789$

$-0.10801$

0.01949

0.03975

0.04142

0.01894

$-0.08074$

0.07706

0.04304

$-0.07927$

0.00730

$0.00819-0.03509$

$0.02047-0.05615$

$0.00335 \quad 0.07243$

$0.00567-0.04181$

0.01074

0.04941

0.01839

0.00060

0.02310

0.01484

$-0.01943-0.01372$

0.00215

0.00421

.06714

0.00340

0.01081

$-0.01413$

0.05101

0.03982

$-0.00736-0.02919$

$0.00542-0.04473$

$0.03118-0.04576$

$-0.03728$

$-0.01404$

0.11876

$-0$

$-0.08476-0$

0.04121

$0.08140-0.0051$

$-0.06855$

0.14391

0.02366

$-0.00020$

0.05958

0.02439

0.02775

0.02651

0.01660

0.07855

0.00282

0.02997

0.00022

$-0.01580$

$-0.06848$

$0.02008-0.00912$

0.00418

0.07659

0.04354

0.01674

0.00110

$-0.00205$

0.00113

$-0.00943$

0.00024

0.01036

$-0.01365$

$-0.00086$

$-0.00912$

0.02473

$-0.06465$

0.13206

0.01195

0.02216

0.02497

0.02003

$-0.01890$

0.02140

$-0.02645$

0.01659

0.00610

$.00510-0.03984$

$0.00603-0.01805$

$-0.01805$

$0.06068-0.01996$

$0.01582-0.01407$

$0.05895-0.00887$

$0.05552-0.00164$

$0.03809-0.00530$

$-0.08018-0.11359$

$-0.01694-0.03228$

$\begin{array}{rr}0.01257 & 0.04108\end{array}$

$\begin{array}{lll}-0.01390 & 0.01118\end{array}$

$-0.10036-0.08694$

$\begin{array}{lll}-0.08660 & 0.02386\end{array}$

$0.03437-0.00816$

$0.01546-0.00854$

$0.00980-0.01892$

$0.01478-0.01990$

$\begin{array}{rr}-0.03208 & 0.01610\end{array}$

$0.01475-0.01574$

$\begin{array}{rrr}0.01174 & 0.00832\end{array}$

$-0.01338-0.00369$

$0.01455-0.01332$

$-0.023330 .01580$

$\begin{array}{ll}-0.07305 & 0.01712\end{array}$

$0.02429-0.00651$

443.88
1.22

480.27

539.37

16.78

5.20

0.00399

$0.00337-0.00155$

0.00061

$0.00408 \quad 0.00470$

$-0.00258$

$-0.00618$

$-0.00581$

$-0.03812$

$-0.00470$

0.12080

$0.02732-0.09942$

$-0.08658-0.03873$

$-0.00480$

0.01447

0.00131

0.01801

0.00277

0.04296

0.11390

0.01724

0.03781

0.02708

$-0.00667$

$-0.07506$

0.11644

0.10766

$0.00742-0.05225$

$0.00003 \quad 0.18705$

$0.00837-0.12191$

$0.00104 \quad 0.03698$

$0.00285-0.04770$

$0.01266-0.18575$

552.39
3.21

609.88

650.12

$0.00176-0.00199 \quad 0.00035$

$\begin{array}{lll}0.00498 & 0.00022 & 0.00134\end{array}$

$0.00648-0.00643-0.00603$

$\begin{array}{lll}0.03432 & 0.00514 & 0.00079\end{array}$

$-0.00419-0.01628-0.00591$

$\begin{array}{lll}0.03818 & 0.00569 & 0.00320\end{array}$

$\begin{array}{llll}0.03636 & 0.00634 & 0.00738\end{array}$

$0.02208-0.00104 \quad 0.02080$

$\begin{array}{lll}0.06748 & 0.01648 & 0.01531\end{array}$

$-0.03221-0.01174-0.00316$

$\begin{array}{lll}0.00022 & 0.01064 & 0.00151\end{array}$

$-0.02021$

0.00635

0.00298

0.05922

$-0.01054$

$-0.04303$

$-0.00324-0.00174$

$0.00639-0.00527$

$0.10100-0.00238$

0.03071

0.00692

0.01483

$.01983-0.00604$

0.00252

$0.01170-0.00869$

0.00925

$0.02908 \quad 0.00202$

0.00557

0.00137

0.01748

0.01007

0.06903

$-0.00374$

$-0.11122$

$-0.03542$

$0.35972-0.01880$

$0.09991-0.00334$

0.09241

0.05266

0.00163

0.01048

0.02197

$-0.02604-0.00239$

0.02197

0.00431

0.00687

$0.00141-0.01012$
$0.00343-0.05766$

$-0.01131$

0.06639

0.02261

0.02901

0.01046

$\begin{array}{rr}0.00195 & -0.06407 \\ -0.00269 & 0.01258\end{array}$

$\begin{array}{ll}0.00040 & -0.02700 \\ 0.00292 & -0.05293\end{array}$

$0.00292-0.05293$
$0.00872-0.02767$

$0.08429-0.00886$

0.06694

$0.00872-0.02767$
$0.00800-0.04551$

$-0.00849-0.00609$

0.00610

$-0.00708-0.04360$

0.05451

$0.02801-0.13583$ 


\begin{tabular}{|c|c|c|c|c|c|c|c|c|}
\hline H7 & z & 0.06963 & 0.00001 & -0.11270 & -0.01851 & -0.00132 & 0.11364 & -0.05671 \\
\hline H12 & $\mathrm{x}$ & -0.05001 & -0.00958 & -0.00710 & 0.03896 & -0.14825 & -0.07254 & 0.02058 \\
\hline H12 & $\mathrm{Y}$ & 0.05931 & -0.01365 & 0.07614 & -0.00105 & -0.05720 & 0.04663 & 0.04830 \\
\hline $\mathrm{H} 12$ & Z & -0.02067 & -0.00904 & -0.01735 & 0.06208 & -0.25365 & -0.08950 & 0.02308 \\
\hline C4 & $\mathrm{x}$ & 0.03134 & 0.00278 & -0.10370 & -0.02475 & 0.04552 & 0.07670 & -0.05289 \\
\hline C4 & $\mathrm{Y}$ & 0.05850 & 0.00997 & -0.11559 & 0.00096 & -0.00080 & -0.10059 & \\
\hline C4 & Z & 0.01768 & -0.00563 & -0.00281 & 0.02251 & -0.10227 & -0.00716 & \\
\hline H2 & $\mathrm{x}$ & 0.03247 & 0.00467 & -0.16027 & -0.02250 & 0.01723 & 0.07238 & -0.0155 \\
\hline H2 & $\mathrm{Y}$ & 0.02875 & 0.01448 & -0.09855 & 0.00493 & 0.02442 & -0.12288 & -0.24333 \\
\hline H2 & Z & 0.00424 & 0.00075 & -0.07141 & 0.02742 & -0.12629 & -0.02580 & \\
\hline $\mathrm{O} 2$ & $\mathrm{x}$ & 0.04528 & -0.04629 & -0.02943 & -0.00682 & 0.00945 & -0.00639 & -0.008 \\
\hline $\mathrm{O} 2$ & $\mathrm{Y}$ & -0.00575 & -0.01666 & -0.01512 & -0.00316 & 0.01338 & -0.00402 & \\
\hline 02 & Z & -0.03939 & 0.03189 & -0.00129 & -0.00207 & 0.00562 & -0.00447 & \\
\hline 03 & $\mathrm{x}$ & -0.01066 & 0.00725 & 0.00598 & -0.00361 & -0.00181 & 0.00068 & \\
\hline 03 & Y & 0.03581 & -0.00360 & -0.0 & -0.00531 & -0.00294 & -0.00115 & \\
\hline 03 & Z & 0.00145 & -0.00172 & -0.00116 & 0.00919 & 0.00234 & -0.00020 & -0 . \\
\hline C5 & $\mathrm{x}$ & 0.02814 & 0.00761 & -0.04585 & -0.01841 & 0.02469 & 0.05647 & \\
\hline C5 & $\mathrm{Y}$ & 800 & 723 & -0 & -0 & 2736 & -0 & \\
\hline C5 & $\mathrm{z}$ & & 0.0 & & -0. & & & \\
\hline 04 & $\mathrm{X}$ & 0.03289 & 0.01159 & 0608 & -0.00403 & 0.05418 & -0.06705 & 0271 \\
\hline 04 & $\mathrm{Y}$ & -0.03653 & -0.0 & -0.0 & 0.0 & -0.03689 & & \\
\hline 04 & z & & 0.0 & & -0 & & & \\
\hline 05 & $\mathrm{x}$ & -0.00613 & -0.0 & 985 & 0.02282 & -0.05880 & -0.04612 & 2000 \\
\hline 05 & $\mathrm{Y}$ & 0.00317 & 0.0 & 506 & -0.01415 & 0.04434 & -0.0 & \\
\hline 05 & Z & & & & & & -0 . & \\
\hline H 4 & $\mathrm{x}$ & -0.03819 & -0.01395 & 0.1 & & -0.09429 & 0.14737 & -0 . \\
\hline H4 & $\mathrm{Y}$ & -0.06627 & -0.01963 & & & -0.09742 & 0.11604 & -0 . \\
\hline H4 & Z & 0.0 & 0.0 & & & & -0.3 & \\
\hline \multicolumn{2}{|c|}{ Erequencies } & & & & & & & \\
\hline 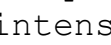 & ities & & & & & & & \\
\hline $\mathrm{Re} 1$ & $\mathrm{x}$ & 0.00 & -0.00518 & 0210 & 0.00 & 0009 & -0.00074 & 0 . \\
\hline $\operatorname{Re} 1$ & $\mathrm{Y}$ & 0.00 & -0.00521 & & & -0 & -0 . & \\
\hline $\operatorname{Re} 1$ & Z & 69 & -0 & 65 & 0. & -0 & -0 & \\
\hline N1 & $\mathrm{x}$ & -0.03646 & -0.00739 & 0.00472 & -0.07898 & -0.06974 & 0.04475 & 0. \\
\hline N1 & $\mathrm{Y}$ & -0.05171 & 0.00007 & -0.00423 & & & 0.01683 & -0 . \\
\hline N1 & z & -0.02677 & -0.0 & -0 . & 0. & -0 & 275 & \\
\hline H9 & $\mathrm{x}$ & 0.16191 & -0.01154 & 0.01725 & -0.13268 & 0.03322 & -0.06407 & 0 . \\
\hline H9 & $\mathrm{Y}$ & 0.59042 & -0.02169 & & -0.2 & & -0.29170 & \\
\hline & Z & & -0 & & -0 . & -0 & -0 . & \\
\hline N2 & $\mathrm{x}$ & -0.01632 & 0. & -0.0 & -0 . & -0.01736 & 0.00522 & -0 . \\
\hline N2 & $\mathrm{Y}$ & -0.03403 & 0.00014 & -0.00105 & -0.0 & 0.03410 & -0.03139 & -0.00061 \\
\hline & Z & -0.0 & -0.0 & & -0 . & & & \\
\hline 10 & $\mathrm{x}$ & & 0 & 020 & -0. & -0.17938 & 471 & -0 . \\
\hline H1O & $\mathrm{Y}$ & 0.35639 & 0.02474 & -0.01269 & 0.33195 & -0.36852 & 0.34472 & 0.00147 \\
\hline & Z & & & & & & & \\
\hline $\mathrm{C} 1$ & $\mathrm{x}$ & -0.0 & & & & & -0 . & -0 \\
\hline $\mathrm{C} 1$ & $\mathrm{Y}$ & -0.06210 & 0.0 & -0.00757 & 0.0 & -0.04259 & 0.04096 & -0.00430 \\
\hline & Z & & & & & & -0 & \\
\hline & $\mathrm{x}$ & & & -0 . & & -0.0 & & -0 . \\
\hline H11 & $\mathrm{Y}$ & 0.35404 & 0.0 & 0.00629 & -0.03020 & -0.10989 & 4915 & \\
\hline H11 & z & & & -0.0 & & & -0 & \\
\hline C2 & $\mathrm{x}$ & & & -0.0 & & & & \\
\hline C2 & $\mathrm{Y}$ & -0.02273 & -0.00751 & 0.00796 & -0. & 65 & -0.05873 & 0. \\
\hline C2 & $\mathrm{z}$ & -0.00781 & -0.00260 & 0.00414 & -0.0 & -0.02 & & \\
\hline & $\mathrm{x}$ & & & & & & -0 & \\
\hline H8 & $\mathrm{Y}$ & & 0.0 & & & -0.1 & 0.1 & \\
\hline & Z & 4325 & -0.00791 & 0.0 & -0. & -0.0 & 0.03083 & \\
\hline 01 & $\mathrm{x}$ & & & -0.01464 & -0.01063 & & 0.01438 & \\
\hline 01 & $\mathrm{Y}$ & & -0.03892 & & & -0.03577 & -0.04617 & -0.139 \\
\hline $\mathrm{O} 1$ & Z & 0.00467 & 0.19312 & -0.03770 & -0.0 & 0.01865 & 0.01506 & -0 . \\
\hline
\end{tabular}




\begin{tabular}{|c|c|c|c|c|c|c|c|c|}
\hline C3 & $\mathrm{x}$ & -0.00187 & 0.00649 & -0.00617 & 0.00979 & -0.04823 & -0.06029 & 0.03045 \\
\hline C3 & $\mathrm{Y}$ & -0.00137 & 0.03966 & -0.00593 & -0.01023 & 0.03524 & 0.05107 & 0.18171 \\
\hline C3 & Z & .00362 & -0.02063 & 0.00946 & 0.00876 & -0.04686 & -0.05365 & \\
\hline H7 & $\mathrm{x}$ & 0.00738 & -0.03876 & 0.02230 & -0.00168 & 0.02919 & 0.04264 & 0.00907 \\
\hline H7 & $\mathrm{Y}$ & -0.00321 & 0.13635 & -0.02083 & -0.01680 & 0.00312 & 0.00165 & 0.06006 \\
\hline H7 & Z & -0.01949 & 0.00656 & -0.05236 & 0.05111 & -0.24272 & -0.30552 & 0.19370 \\
\hline H12 & $\mathrm{x}$ & 0.01612 & -0.05605 & 0.04018 & -0.01055 & 0.08135 & 0.10796 & -0.07922 \\
\hline $\mathrm{H} 12$ & $\mathrm{Y}$ & 0.00481 & 0.07283 & -0.00559 & -0.01073 & 0.04522 & 0.06630 & 0.04498 \\
\hline $\mathrm{H} 12$ & Z & 0.02168 & -0.11637 & 0.06325 & -0.01427 & 0.09817 & 0.13371 & -0.04013 \\
\hline $\mathrm{C} 4$ & $\mathrm{x}$ & -0.00495 & 0.02781 & -0.05605 & 0.00976 & -0.05321 & -0.06408 & \\
\hline C4 & $\mathrm{Y}$ & 0.00668 & 0.03166 & -0.05431 & -0.02089 & 0.05408 & 0.05017 & 0.01267 \\
\hline C4 & Z & 0.00262 & -0.02878 & 0.00910 & -0.00453 & 0.02319 & & \\
\hline H2 & $\mathrm{X}$ & -0.01168 & 0.02244 & 0.05278 & 0.01145 & -0.02932 & -0.02588 & -0.04263 \\
\hline H2 & $\mathrm{Y}$ & 0.04544 & 0.02321 & -0.37805 & -0.03443 & 0.08997 & 0.09885 & -0.299 \\
\hline H2 & $\mathrm{Z}$ & 1376 & -0.0 & -0.0 & -0.01059 & & & \\
\hline $\mathrm{O} 2$ & $\mathrm{x}$ & 0.00653 & 0058 & -0.00172 & 0.00037 & -0.00543 & 0210 & \\
\hline $\mathrm{O} 2$ & $\mathrm{Y}$ & 0.01461 & 0.00835 & -0.01503 & -0.00763 & 0.00231 & 0.02107 & -0.01439 \\
\hline $\mathrm{O} 2$ & Z & -0.0 & -0.0 & & -0.0 & 463 & & \\
\hline 03 & $\mathrm{x}$ & 044 & 0.0 & 0. & 0.0 & 708 & -0 . & \\
\hline 03 & $\mathrm{Y}$ & -0.00167 & 0.00152 & 0.00168 & 0.00198 & 0.00147 & 0.00103 & -0.00029 \\
\hline 03 & Z & -0.0 & 0111 & -0.0 & -0.00368 & -0.0 & 260 & \\
\hline C5 & $\mathrm{x}$ & -0.0 & -0.0 & 0.1 & -0.0 & & & \\
\hline C5 & $\mathrm{Y}$ & -0.00949 & 0.06064 & 0.1 & 0.01188 & -0.00416 & 0100 & -0 \\
\hline C5 & Z & 0.00735 & -0.01910 & -0.0 & -0.0 & 2070 & 825 & -0 \\
\hline 04 & $\mathrm{x}$ & & & -0 & -0 . & -0 . & -0 . & \\
\hline 04 & $\mathrm{Y}$ & 0.00538 & -0.03147 & -0.0 & -0.00835 & 0338 & 0154 & \\
\hline 04 & $\mathrm{z}$ & -0.00266 & -0.03238 & 0.00 & -0.00818 & 4048 & 5333 & \\
\hline 05 & $\mathrm{x}$ & & -0 . & -0 . & -0 & & & \\
\hline 05 & $\mathrm{Y}$ & -0.0 & -0.0 & -0.0 & 645 & 2748 & -0 . & 0 \\
\hline 05 & Z & -0.0 & & & & -0 . & -0 . & \\
\hline H4 & $\mathrm{x}$ & & & 0 . & -0 & & & \\
\hline H 4 & $\mathrm{Y}$ & & 0.17109 & 0.2 & 1490 & -0.04041 & -0.05961 & \\
\hline H 4 & Z & 0.00578 & 0.11364 & -0.0 & 0.02615 & -0.12622 & -0.16373 & \\
\hline & & & & & & & & \\
\hline & & & & & & & & \\
\hline 21 & $\mathrm{X}$ & -0.00313 & 893 & 0.0 & -0.0 & -0.0 & 031 & \\
\hline Re1 & $\mathrm{Y}$ & 0.00414 & -0.01695 & -0.00678 & -0.00017 & -0.00136 & 0210 & -0.0001 \\
\hline $\operatorname{Re} 1$ & Z & -0.00056 & -0.00086 & -0.00014 & & & -0 . & -0.00015 \\
\hline & $\mathrm{x}$ & -0 & -0. & & & -0 . & -0 & \\
\hline N1 & $\mathrm{Y}$ & -0.0 & 145 & -0.00 & 05461 & -0.02333 & -0.00768 & -0 \\
\hline N1 & $\mathrm{z}$ & -0.00278 & 0.00495 & -0.01406 & 0.01598 & -0.00120 & -0.00308 & -0.00157 \\
\hline & $\mathrm{x}$ & 476 & -0. & & & & & \\
\hline H9 & $\mathrm{Y}$ & -0.0 & -0.03686 & 0 . & $-0.2 \xi$ & 2083 & 326 & \\
\hline H9 & Z & 0.00034 & 0.07343 & -0.19171 & -0.13495 & 0.05292 & 0.00696 & -0.00321 \\
\hline & $\mathrm{x}$ & & & -0.0 & & & & \\
\hline $\mathrm{N} 2$ & Y & -0. & -0.0 & & & -0 & & \\
\hline N2 & z & 0.00173 & 0.01152 & -0.03082 & 0.00236 & 0.00458 & -0.00076 & -0.01010 \\
\hline & $\mathrm{x}$ & & -0.04507 & & & & & \\
\hline & $\mathrm{Y}$ & & -0. & -0 & -0 . & 266 & -0 & \\
\hline H1O & z & 704 & 0.10410 & -0.25395 & -0.03552 & 0.01706 & -0.00207 & 4159 \\
\hline C1 & $\mathrm{x}$ & -0.0 & -0.04080 & & -0.02 & -0.0 & & \\
\hline $\mathrm{C} 1$ & $\mathrm{Y}$ & & & -0.0 & -0.1 & & & -0 \\
\hline $\mathrm{C} 1$ & Z & -0.0 & -0.03964 & 0.1 & -0.02420 & 0.0 & 519 & \\
\hline H11 & $\mathrm{x}$ & -0.0 & -0.11772 & & & -0.0 & & \\
\hline H11 & $\mathrm{Y}$ & -0.0 & & -0.1 & & & -0.0 & \\
\hline H11 & Z & -0.0 & -0.02815 & & & -0.0 & -0 & \\
\hline & $\mathrm{x}$ & 516 & 0.05446 & -0.12 & 0.0 & 0.00 & -0.0 & \\
\hline $\mathrm{C} 2$ & $\mathrm{Y}$ & -0.00729 & -0.01302 & & & -0.01160 & -0.00865 & \\
\hline $\mathrm{C} 2$ & Z & -0.00112 & & -0.01371 & & & -0.00470 & 0.0141 \\
\hline H8 & $\mathrm{X}$ & 0.01556 & 0.09775 & -0.22563 & -0.00104 & 0.01719 & 0.00391 & -0.1376 \\
\hline
\end{tabular}




\begin{tabular}{|c|c|c|c|c|c|c|c|c|}
\hline $\mathrm{H} 8$ & $\mathrm{Y}$ & 0.02253 & -0.03900 & 0.08170 & -0.01350 & -0.00820 & 0.05318 & -0.66502 \\
\hline H8 & Z & 0.00813 & 0.06181 & -0.13935 & 0.00286 & 0.01698 & -0.00197 & -0.06893 \\
\hline 01 & $\mathrm{X}$ & -0.00387 & -0.00510 & -0.00189 & 0.00630 & 0.00930 & 0.01624 & 0.00273 \\
\hline 01 & $\mathrm{Y}$ & 0.03719 & 0.01040 & 0.00274 & 0.00480 & 0.00111 & 0.01267 & 0.00047 \\
\hline 01 & Z & 0.03641 & -0.00414 & -0.00119 & -0.00153 & -0.00956 & 0.00214 & 0.00116 \\
\hline C3 & $\mathrm{x}$ & 0.03362 & 0.01031 & 0.00628 & -0.02510 & -0.04441 & -0.06892 & -0.01029 \\
\hline C3 & $\mathrm{Y}$ & -0.08678 & -0.00976 & -0.00242 & -0.00688 & -0.00312 & -0.01796 & -0.00254 \\
\hline C3 & Z & -0.02588 & 0.00607 & 0.00159 & -0.00902 & -0.00756 & -0.03688 & 0.00920 \\
\hline H7 & $\mathrm{x}$ & -0.06631 & -0.00767 & -0.00542 & 0.01283 & 0.02586 & 0.03035 & 0.00722 \\
\hline H7 & $\mathrm{Y}$ & 0.02435 & -0.01192 & 0.00107 & -0.03115 & -0.05872 & -0.07401 & -0.02361 \\
\hline H7 & Z & 0.15074 & 0.06343 & 0.03222 & -0.09503 & -0.15457 & -0.26779 & -0.01756 \\
\hline $\mathrm{H} 12$ & $\mathrm{x}$ & -0.09216 & -0.01613 & -0.01455 & & 0.06682 & 0.09292 & 0.02102 \\
\hline $\mathrm{H} 12$ & $\mathrm{Y}$ & -0.12780 & -0.02458 & -0.00926 & 0.04170 & 0.08787 & 0.09516 & 0.01015 \\
\hline $\mathrm{H} 12$ & Z & -0.15127 & -0.01672 & -0.01937 & 0.03525 & 0.07454 & 0.09308 & 0.04050 \\
\hline C4 & $\mathrm{x}$ & -0.06651 & -0.0 & -0.0 & & 0.05194 & & \\
\hline C4 & $\mathrm{Y}$ & 0.13174 & 1039 & 0.0 & 0.00909 & 0.00810 & 3559 & \\
\hline C4 & Z & 0.03436 & 0.00661 & 0.00644 & 0.01340 & 0.01218 & 0.04923 & -0.01305 \\
\hline H2 & $\mathrm{x}$ & -0.15 & -0.0 & -0 & 0.0 & & & \\
\hline H2 & $\mathrm{Y}$ & -0.42444 & -0.1 & -0. & -0.1 & -0 & -0 & -0 . \\
\hline H2 & Z & -0.37694 & -0.09612 & -0.05653 & 0.02603 & 0.04942 & 0.05258 & -0.03482 \\
\hline 02 & $\mathrm{X}$ & 312 & -0.0 & -0. & -0.0 & -0.00368 & 317 & 0008 \\
\hline $\mathrm{O} 2$ & $\mathrm{Y}$ & -0.0 & 0.2 & & & & -0 . & \\
\hline $\mathrm{O} 2$ & Z & -0.00233 & 415 & 185 & 0 . & 0.00061 & 0.00030 & 0009 \\
\hline 03 & $\mathrm{x}$ & 0.02957 & -0.0 & 0.0 & 0. & 8697 & -0 . & -0 . \\
\hline 03 & $\mathrm{Y}$ & -0 & 0 & & & $-0 . c$ & -0 & \\
\hline 03 & $\mathrm{z}$ & -0.0 & 226 & -0.0 & -0.00604 & -0.01739 & 0.01069 & 0066 \\
\hline C5 & $\mathrm{x}$ & -0.00505 & 0.0 & & -0.00400 & -0.00619 & -0.01169 & 0059 \\
\hline C5 & $\mathrm{Y}$ & -0 & -0 & -0 . & -0 . & -0 & -0 & -0 \\
\hline C5 & Z & 225 & 0. & & -0 . & -0 & -0 . & 0075 \\
\hline 04 & $\mathrm{x}$ & & -0.0 & -0 . & -0 . & -0 & -0 . & 0121 \\
\hline 04 & $\mathrm{Y}$ & & & & & & & \\
\hline 04 & Z & 292 & -0.0 & 0. & -0.00 & -0.02107 & -0.02612 & -0.00011 \\
\hline 05 & $\mathrm{x}$ & 643 & 0.0 & & -0. & -0 & -0 . & -0 . \\
\hline 05 & $\mathrm{Y}$ & -0.0 & 0. & -0 . & & & & \\
\hline 05 & Z & -0.03538 & 0.0 & -0.0 & 0.0 & 0.03020 & 4056 & 0.00390 \\
\hline H4 & $\mathrm{x}$ & 0.05147 & 0.0 & 424 & -0.0 & -0.01396 & -0 & 0.00414 \\
\hline H 4 & $\mathrm{Y}$ & -0.0 & -0 & -0 . & & & 0 & -0 \\
\hline H4 & Z & -0.07112 & -0.00833 & -0.00699 & -0.00979 & 0.00436 & -0.00094 & -0.015 \\
\hline \multicolumn{2}{|c|}{ Erequencies } & & & & & & & \\
\hline & & & & & & & & 03 \\
\hline Re1 & $\mathrm{x}$ & 0.00043 & -0.00025 & -0.0 & -0.0 & -0.00127 & -0.00060 & -0.00096 \\
\hline & $\mathrm{Y}$ & & & -0 . & -0 & & & \\
\hline 21 & z & -0.0 & 0 . & & & -0.00013 & -0 & -0 . \\
\hline N1 & $\mathrm{x}$ & 0.00030 & -0.0 & 0.02809 & -0.00195 & -0.03958 & -0.00042 & 0.00229 \\
\hline & $\mathrm{Y}$ & & & -0 & & & -0 . & \\
\hline & $\mathrm{z}$ & & -0 & -0 . & -0 . & & & \\
\hline H9 & $\mathrm{x}$ & -0.0 & 0.0 & -0. & -0.01007 & -0.28438 & -0.00410 & 0.00340 \\
\hline & $\mathrm{Y}$ & & & -0 . & -0 . & -0.1 & & \\
\hline & Z & -0 . & -0 & & & & & \\
\hline N2 & $\mathrm{x}$ & & -0.00253 & 0. & -0.00141 & -0.00978 & -0.00060 & -0.00016 \\
\hline & $\mathrm{Y}$ & & -0.0 & -0 & & & -0 & \\
\hline & Z & & -0.0 & & -0.0 & -0.0 & & \\
\hline & $\mathrm{x}$ & -0.0 & & -0.2 & & & & -0 . \\
\hline & $\mathrm{Y}$ & -0.0 & 0.0 & -0. & & -0.0 & & -0. \\
\hline & Z & & -0.0 & & -0.0 & -0.08564 & & \\
\hline $\mathrm{C} 1$ & $\mathrm{x}$ & & 0.0 & -0.0 & & 0.00872 & -0 & 0179 \\
\hline & $\mathrm{Y}$ & & 0.0 & -0. & -0.0 & 0.00010 & 005 & -0.00042 \\
\hline & Z & & 0.00090 & -0.01708 & -0.00054 & -0.02242 & -0.00058 & \\
\hline - & $\mathrm{x}$ & & -0.00956 & & & & 0.02388 & 0.00497 \\
\hline H11 & Y & -0.00715 & -0.00232 & -0 & -0 . & 05848 & -0.00563 & 0.00054 \\
\hline
\end{tabular}




\begin{tabular}{|c|c|c|c|c|c|c|c|c|}
\hline H11 & z & -0.00075 & 0.00151 & -0.04091 & -0.00283 & -0.13831 & -0.00623 & 0.00055 \\
\hline C2 & $\mathrm{x}$ & -0.00302 & 0.00099 & -0.02945 & 0.00122 & 0.01826 & 0.00207 & -0.00148 \\
\hline $\mathrm{C} 2$ & $\mathrm{Y}$ & -0.00979 & -0.00017 & 0.00955 & 0.00008 & 0.00327 & 0.00016 & 0.00003 \\
\hline $\mathrm{C} 2$ & Z & -0.00185 & 0.00063 & -0.01045 & -0.00020 & 0.00059 & -0.00069 & 0.00207 \\
\hline H8 & $\mathrm{x}$ & 0.00757 & 0.01644 & -0.35857 & 0.00528 & 0.07927 & -0.00142 & 0.00315 \\
\hline H8 & $\mathrm{Y}$ & 0.06532 & -0.00275 & 0.10779 & -0.00392 & -0.03337 & 0.00170 & -0.00014 \\
\hline H8 & Z & 0.00072 & 0.02188 & -0.47370 & 0.00574 & 0.08288 & -0.00651 & 0.0089 \\
\hline 01 & $\mathrm{x}$ & 0.02038 & -0.03307 & -0.00358 & -0.02944 & 0.00045 & 0.00827 & 0.0128 \\
\hline 01 & $\mathrm{Y}$ & 0.00328 & -0.00269 & 0.00023 & 0.00067 & -0.00180 & 0.00872 & \\
\hline 01 & Z & 0.01124 & 0.00831 & -0.00088 & 0.00525 & -0.00115 & 0.01723 & \\
\hline C3 & $\mathrm{x}$ & -0.06149 & 0.05290 & 0.00216 & 0.01486 & -0.00203 & 0.03478 & -0.03710 \\
\hline C3 & $\mathrm{Y}$ & -0.00511 & 0.00607 & -0.00053 & 0.00989 & -0.00070 & 0.03127 & \\
\hline C3 & $\mathrm{z}$ & 0.13039 & -0.00952 & -0.00220 & 0.00279 & 0.00040 & -0.02820 & -0 \\
\hline H7 & $\mathrm{x}$ & 0.06671 & -0.05589 & -0.00392 & -0.04571 & 0.00034 & 0.01579 & 0.03243 \\
\hline H7 & $\mathrm{Y}$ & -0.18697 & 0.38627 & 0.02423 & 0.38576 & 0.00207 & -0.28415 & \\
\hline H7 & $\mathrm{z}$ & -0.04134 & -0.07108 & -0.0 & -0.18775 & -0.01032 & & \\
\hline H12 & $\mathrm{x}$ & 0.14153 & -0.01737 & 0.00278 & 0.04922 & -0.00232 & 0.02210 & \\
\hline $\mathrm{H} 12$ & $\mathrm{Y}$ & .03672 & -0.45033 & -0.02655 & -0.47763 & 0.01495 & -0.06612 & -0 \\
\hline $\mathrm{H} 12$ & Z & & & & & -0.0 & & \\
\hline C4 & $\mathrm{X}$ & -0.00383 & -0.02635 & 0.0 & & 0.00306 & -0.07592 & \\
\hline C4 & $\mathrm{Y}$ & 0.03460 & 0.01221 & -0.00025 & 082 & -0.00134 & 0.02482 & -0 . \\
\hline C4 & Z & & -0.0 & & -0 & & -0 . & \\
\hline H2 & $\mathrm{x}$ & -0.0 & 0. & 0 . & & -0.0 & 2427 & -0 \\
\hline H2 & $\mathrm{Y}$ & -0.02615 & 0.0 & & -0. & & -0 & \\
\hline $\mathrm{H} 2$ & Z & -0 & 0.0 & & & -0 . & & -0 \\
\hline $\mathrm{O} 2$ & $\mathrm{x}$ & 0.00151 & 0.0 & -0. & -0 . & & & \\
\hline $\mathrm{O} 2$ & $\mathrm{Y}$ & -0.00653 & -0.0 & 0.0 & & -0 . & -0 . & \\
\hline $\mathrm{O} 2$ & Z & & -0. & -0 . & & -0 & & -0 \\
\hline 03 & $\mathrm{x}$ & -0.01023 & -0.00015 & 0.0 & & 0.006 & 0323 & \\
\hline 03 & Y & -0.00009 & 0.00058 & -0.0 & -0. & -0.00085 & 021 & \\
\hline 03 & Z & & 0. & & -0 & & -0 & -0 \\
\hline C5 & $\mathrm{x}$ & 853 & 0.0 & 0.0 & -0.05479 & 0.00192 & -0.06343 & \\
\hline C5 & $\mathrm{Y}$ & -0.00955 & -0.03782 & -0.0 & & -0.00191 & 0.00368 & \\
\hline C5 & Z & & -0 & -0 & & & & \\
\hline 04 & $\mathrm{x}$ & $\$ 31$ & -0.0 & -0 & & -0 & 844 & -0 \\
\hline 04 & $\mathrm{Y}$ & -0.00681 & -0.00058 & 0.0 & & 0.00114 & 0296 & 186 \\
\hline 04 & Z & & -0 & -0 . & & & & \\
\hline 05 & $\mathrm{x}$ & 207 & -0.0 & -0 & & -0 . & 44 & -0 \\
\hline 05 & $\mathrm{Y}$ & 0.00600 & 0.02760 & 0.0 & -0.0 & 0082 & -0.01061 & 259 \\
\hline 05 & Z & & & & & & & \\
\hline H4 & $\mathrm{x}$ & & & & -0 & & & -0 \\
\hline H4 & $\mathrm{Y}$ & -0.04802 & -0.16040 & -0.0 & & $-0 . c$ & -0.05059 & 638 \\
\hline H4 & z & -0.14227 & -0.46492 & -0.01864 & & -0.00604 & -0.14819 & \\
\hline \multirow{2}{*}{\multicolumn{2}{|c|}{$\begin{array}{l}\text { Erequencies } \\
\text { intensities }\end{array}$}} & & & & & & & \\
\hline & & & & & & & & \\
\hline $\operatorname{Re}$ & $\mathrm{x}$ & -0.00 & -0.0 & & & -0.0 & -0.0 & \\
\hline $\mathrm{R}$ & $\mathrm{Y}$ & -0.00 & 0. & -0 & & & -0. & \\
\hline e1 & Z & & -0 & -0 & & & -0 & \\
\hline & $\mathrm{x}$ & & & -0 & & -0 & & \\
\hline N1 & $\mathrm{Y}$ & 581 & -0 & -0 & -0 . & -0 & & \\
\hline & Z & & 0. & & & & -0. & \\
\hline $\mathrm{H}$ & $\mathrm{x}$ & & & & & -0.0 & & \\
\hline H9 & $\mathrm{Y}$ & & -0 & & & & -0 & \\
\hline & Z & -0.33474 & -0.0 & -0 . & -0 . & -0.0 & & -0.04006 \\
\hline & $\mathrm{X}$ & & & -0 . & & & & \\
\hline & $\mathrm{Y}$ & -0.0 & -0.0 & -0 & & -0.01 & -0 & \\
\hline N2 & Z & -0.07002 & -0.00143 & 0.05682 & 0.00100 & 0.10154 & 0.12055 & -0.0021 \\
\hline & $\mathrm{x}$ & -0.15063 & -0.00292 & & & & & \\
\hline & $\mathrm{Y}$ & -0.00 & & 0.8 & -0 & -0.00 & -0.01775 & \\
\hline 10 & Z & 0.34181 & 0.00861 & -0.19279 & -0.01 & 0.09159 & 0.03612 & -0.0 \\
\hline
\end{tabular}




\begin{tabular}{|c|c|c|c|c|c|c|c|c|}
\hline $\mathrm{C} 1$ & $\mathrm{x}$ & -0.03354 & -0.00115 & -0.07238 & -0.00290 & 0.15164 & -0.10821 & 0.00648 \\
\hline $\mathrm{C} 1$ & $\mathrm{Y}$ & 0.01255 & 0.00031 & 0.03238 & 0.00091 & -0.00697 & -0.00198 & 0.00027 \\
\hline $\mathrm{C} 1$ & Z & -0.01164 & -0.00061 & -0.06791 & -0.00228 & -0.07740 & 0.08861 & -0.00682 \\
\hline H11 & $\mathrm{x}$ & 0.28234 & 0.01374 & 0.40609 & 0.01461 & -0.12157 & 0.15046 & 0.00036 \\
\hline H11 & Y & -0.03523 & -0.00102 & -0.02521 & 0.00090 & 0.04493 & -0.04999 & 0.00410 \\
\hline H11 & Z & -0.06967 & -0.00343 & -0.16960 & -0.00529 & -0.03457 & 0.05556 & -0.00565 \\
\hline C2 & $\mathrm{x}$ & -0.08533 & -0.00164 & 0.07953 & 0.00353 & -0.04624 & -0.00848 & 0.00028 \\
\hline $\mathrm{C} 2$ & $\mathrm{Y}$ & 0.01831 & 0.00056 & -0.01560 & -0.00117 & 0.02082 & 0.02098 & -0.00104 \\
\hline $\mathrm{C} 2$ & Z & -0.00636 & -0.00074 & -0.00749 & 0.00164 & -0.13556 & -0.16596 & \\
\hline H8 & $\mathrm{x}$ & 0.27200 & 0.00457 & -0.10412 & -0.00640 & 0.08280 & 0.17973 & \\
\hline H8 & $\mathrm{Y}$ & -0.10520 & 0.00004 & 0.04813 & 0.00421 & -0.02201 & -0.04676 & 0.00354 \\
\hline H8 & Z & & 0.00819 & -0.29051 & -0.01286 & 0.02943 & & -0 \\
\hline 01 & $\mathrm{x}$ & -0.0 & 0.0 & 0.00038 & -0.00173 & 0.00005 & & \\
\hline 01 & $\mathrm{Y}$ & 058 & 0.0 & 0.00174 & -0.01479 & -0.00150 & 085 & -0 . \\
\hline 01 & Z & -0.0 & 0.0 & & -0.00662 & -0.00068 & & \\
\hline C3 & $\mathrm{x}$ & 0.0 & 0.0 & 0.0 & -0.01568 & -0.00076 & & -0 . \\
\hline C3 & $\mathrm{Y}$ & -0.0 & 0.0 & 0.0 & -0.03920 & 0.00190 & 0029 & \\
\hline C3 & Z & 0 & 0. & 0. & -0.0 & -0 . & & \\
\hline H7 & & -0 . & & & -0.32181 & -0.00223 & -0 . & \\
\hline H7 & Y & 139 & -0.08793 & -0.00881 & 0.33640 & -0.00556 & 0319 & -0 . \\
\hline H7 & Z & -0.0 & 0. & -0 & 0.4 & & & -0 \\
\hline $\mathrm{H} 12$ & & & -0 & -0 . & & & & -0 \\
\hline H12 & Y & 0. & -0. & -0.0 & 0.33820 & -0. & 177 & \\
\hline $\mathrm{H} 12$ & Z & 0. & -0. & -0.0 & 0.3 & & & -0 \\
\hline C4 & $\mathrm{X}$ & & -0 & & -0 & -0 & & -0 \\
\hline C4 & Y & -0.0 & 0.0 & -0.00 & -0.00079 & -0.00377 & 0021 & \\
\hline C4 & z & -0.0 & -0.0 & & 0.00436 & 0.00078 & -0 . & -0 \\
\hline $\mathrm{H} 2$ & $\mathrm{x}$ & & & -0 . & -0 & -0 . & -0 & \\
\hline H2 & $\mathrm{Y}$ & 0. & 0. & & 0.0 & 0290 & & -0 . \\
\hline $\mathrm{H} 2$ & Z & & & -0 . & -0 & 125 & -0 & \\
\hline 02 & $\mathrm{x}$ & -0 & & & & & & \\
\hline $\mathrm{O} 2$ & $\mathrm{Y}$ & 0. & -0.0 & 0.0 & -0.00086 & 0183 & 0105 & -0 . \\
\hline $\mathrm{O} 2$ & z & -0. & -0. & -0 & -0.00014 & -0 & -0 & -0 . \\
\hline 03 & $\mathrm{x}$ & & -0 . & -0 . & -0 & & & -0 \\
\hline 03 & $\mathrm{Y}$ & 0.0 & 0. & 0.0 & 0.00034 & 0051 & -0 & \\
\hline 03 & Z & -0.0 & & -0.0 & & -0 & & -0 \\
\hline C5 & $\mathrm{x}$ & -0 & & -0 . & 0 & -0 & & \\
\hline C5 & $\mathrm{Y}$ & 0.0 & -0.1 & 0.00268 & -0.01052 & 0.00090 & -0.00048 & -0.01 \\
\hline C5 & Z & -0.0 & -0.0 & -0.0 & -0.00426 & -0.00505 & 0 & \\
\hline 04 & $\mathrm{x}$ & & -0 . & & -0 & & -0 & -0 \\
\hline 04 & Y & -0 & & -0 & 0.0 & -0 & 096 & \\
\hline 04 & Z & 0.0 & 0.0 & & -0.00201 & 0.00293 & -0.00586 & -0 \\
\hline 05 & $\mathrm{x}$ & & -0 . & & -0 . & -0 & & \\
\hline 05 & $\mathrm{Y}$ & -0. & & -0. & 0.0 & 028 & -0 . & -0 \\
\hline 05 & Z & 0.0 & 0.0 & 0. & 0.00145 & 103 & -0 & -0. \\
\hline H4 & $\mathrm{x}$ & & -0 & & $-0 . c$ & & & -0 \\
\hline H4 & $\mathrm{Y}$ & -0 & & -0 . & & -0 . & & \\
\hline H4 & Z & -0.01183 & 0.40729 & -0.00605 & 0.04257 & -0.00356 & 0.00559 & 0.1208 \\
\hline \multicolumn{2}{|c|}{ requencies } & & & & & & & \\
\hline & ities & & & & & & & \\
\hline Re1 & $\mathrm{x}$ & & -0.00 & & & -0.0 & & \\
\hline Re1 & $\mathrm{Y}$ & -0.0 & & -0. & & & -0 & \\
\hline Re1 & Z & -0.0 & -0.0 & -0.00 & -0.00001 & 005 & -0 & \\
\hline N1 & $\mathrm{x}$ & -0.0 & & & & -0.00173 & -0.06444 & \\
\hline $\mathrm{N1}$ & $\mathrm{Y}$ & & & & -0.00035 & -0.00090 & & \\
\hline N1 & Z & -0.00 & & -0.0 & & 0.00329 & -0.01 & \\
\hline 115 & $\mathrm{x}$ & -0.0 & -0.0 & & -0.002 & -0.01541 & 0.85093 & -0 . \\
\hline $\mathrm{H} 9$ & $\mathrm{Y}$ & -0.00003 & & & & & -0.33938 & \\
\hline H9 & Z & & & & -0.00212 & -0.00699 & 0.28434 & \\
\hline N2 & $\mathrm{x}$ & -0.00016 & -0.0 & 013 & 0.00202 & 0.00022 & -0.00181 & -0 \\
\hline
\end{tabular}




\begin{tabular}{|c|c|c|c|c|c|c|c|c|}
\hline I2 & 1 & 0.00004 & -0.00007 & -0.00019 & -0.00025 & -0.00011 & 0.00064 & 0.0175 \\
\hline 2 & 2 & -0.00011 & -0.00019 & -0.00051 & -0.00358 & 0.00149 & -0.00122 & \\
\hline 10 & $x$ & 0.00178 & 0.00100 & 0.00205 & -0.02166 & 0.00184 & 0.02941 & 0.83064 \\
\hline 10 & Y & 0.00000 & -0.00075 & -0.00078 & 0.00731 & 0.00025 & -0.00931 & -0.23923 \\
\hline 10 & Z & 0.00090 & 0.00032 & 0.00177 & -0.01061 & 0.00056 & 0.01399 & \\
\hline 1 & $x$ & 0.00010 & 0.00009 & -0.00001 & 0.00030 & -0.01001 & 0.00254 & 0.000 \\
\hline & & -0.00009 & -0.00019 & -0.00010 & 0.00484 & 0.02401 & 0.00090 & -0.000 \\
\hline 1 & 2 & 0.00032 & 0.00061 & 0.00051 & -0.01721 & -0.07958 & -0.00406 & \\
\hline 11 & $x$ & -0.00058 & -0.00099 & -0.00099 & 0.02216 & 0.12692 & 0.00345 & -0.0000 \\
\hline 11 & & 0.00095 & 0.00163 & 0.00109 & -0.05929 & -0.27230 & -0.00694 & -0.00146 \\
\hline 11 & z & -0.00346 & -0.00562 & -0.00372 & 0.19452 & 0.87873 & 0.01379 & \\
\hline C2 & $\mathrm{x}$ & -0.00039 & -0.00057 & -0.00050 & -0.06576 & 0.01641 & 0.00053 & -0.00264 \\
\hline C2 & Y & 0.00005 & 0.00002 & -0.00002 & 0.00697 & -0.00219 & 0.00013 & 0.00002 \\
\hline C2 & Z & 0.00033 & 0.00049 & 0.00064 & 5152 & -0.00973 & 0.00067 & \\
\hline H8 & $x$ & 0.00420 & 0.00606 & 0.00518 & 0.74433 & -0.16572 & 0.00325 & \\
\hline H8 & Y & -0.00044 & -0.00068 & -0.00054 & -0.08339 & 0.01925 & -0.00081 & -0.00222 \\
\hline H8 & $z$ & -0.00306 & -0.00436 & -0.00373 & -0 & 0.11578 & -0.00153 & -0.00902 \\
\hline 01 & $x$ & 0.0 & 0.00051 & -0.00029 & -0 & & & \\
\hline 01 & $\mathrm{Y}$ & 0.00171 & 0.00022 & -0.00014 & 0003 & -0.00003 & 0.00010 & -0.00007 \\
\hline 01 & Z & -0.00 & 0.00000 & 0027 & 0.0 & 0. & 012 & -0 \\
\hline C3 & & 0.0 & -0.07634 & 0.04785 & & -0 & -0.00007 & -0.00008 \\
\hline C3 & $\mathrm{Y}$ & 0.0 & 0.00325 & -0.00142 & -0.00021 & 033 & -0.00008 & -0.00001 \\
\hline C3 & $\mathrm{Z}$ & 0.05 & 0.01942 & -0.00954 & -0.0 & 0.0 & 0005 & -0 . \\
\hline H7 & & -0.6 & 0.4 & -0.2 & 0 & -0 . & & \\
\hline H7 & Y & -0.23086 & 0.15638 & -0.09977 & 0064 & -0 & 00034 & 00035 \\
\hline H7 & z & -0.22524 & 0.15916 & -0.10452 & 0. & -0.0 & 0020 & 0072 \\
\hline $\mathrm{H} 12$ & & 0.4 & 0.4 & -0.2 & -0. & 0 & & -0 \\
\hline $\mathrm{H} 12$ & Y & -0.1 & -0.20026 & 0.12050 & 0157 & -0.00200 & -0.00017 & 0.00037 \\
\hline H12 & Z & -0.36 & -0.37895 & 0.23129 & 0. & -0.00329 & 0.00007 & 0091 \\
\hline C4 & & -0.0 & 0.03367 & 531 & -0.0 & 0 & -0.0 & -0 . \\
\hline C4 & Y & -0.00057 & 0.01216 & 0.01968 & -0.00019 & 0.00 & 0.00017 & -0.00001 \\
\hline C4 & Z & -0.00032 & -0.02369 & -0.03834 & 0.0 & -0.0 & -0.00017 & \\
\hline H2 & $x$ & 0.0 & -0.39639 & -0.63327 & 0.0 & -0 . & -0 & \\
\hline $\mathrm{H} 2$ & $\mathrm{Y}$ & 0.00523 & -0.14730 & -0.24006 & 0.0 & -0.00 & -0.00068 & 0.00127 \\
\hline $\mathrm{H} 2$ & Z & -0.0 & 0.27576 & 4760 & -0. & & -0.0 & -0 \\
\hline $\mathrm{O} 2$ & $\mathrm{x}$ & -0.0 & 0.00005 & 0 & 0.0 & 0.0 & & -0 \\
\hline 02 & $\mathrm{Y}$ & 0.00 & -0.00010 & 0.00006 & -0.0 & -0.00 & -0.00001 & 0004 \\
\hline $\mathrm{O} 2$ & Z & 0.000 & 0.00003 & 0.00000 & 0. & -0 & 0007 & -0.00001 \\
\hline 03 & $\mathrm{x}$ & -0.0 & -0.00008 & 0.0 & 0 & -0 & -0 & \\
\hline 03 & $\mathrm{Y}$ & 0.0 & -0.00001 & -0.00003 & -0.0 & 0.0 & 0.00003 & -0.0 \\
\hline 03 & Z & 0.00007 & 0.00007 & 0.00000 & 0.00002 & -0.00004 & 0.00003 & -0.00001 \\
\hline C5 & $\mathrm{x}$ & -0.00055 & -0.00021 & -0.00003 & 0.0 & & & \\
\hline C5 & $\mathrm{Y}$ & & 0.00 & 0083 & -0.0 & -0 & -0.00036 & \\
\hline C5 & Z & 0.00031 & 0.00006 & -0.00133 & -0.00009 & -0.00011 & 0.00117 & 0.00008 \\
\hline 04 & $\mathrm{x}$ & & 0.00004 & -0.00007 & 0.00000 & & -0.00067 & \\
\hline 04 & Y & -0.00 & -0.00013 & -0.00011 & 0.0 & -0 & 0.00021 & -0.00006 \\
\hline 04 & Z & -0.00005 & 0.00004 & 0.00027 & 0.00002 & 0.00007 & -0.00072 & -0.00007 \\
\hline 05 & $\mathrm{x}$ & & -0.00017 & -0.00008 & 0.00000 & -0.00002 & -0.00006 & \\
\hline 05 & $\mathrm{Y}$ & & & & -0.00001 & & 0.00000 & \\
\hline 05 & Z & -0.00024 & -0.00011 & 0.00036 & -0.00001 & 0.00006 & -0.00023 & -0.00013 \\
\hline H4 & $\mathrm{X}$ & -0.00089 & -0.00073 & 0.00011 & -0.00025 & & -0.00043 & \\
\hline H4 & $\mathrm{Y}$ & & 0.00030 & & 0.00018 & & 0.00082 & -0.001 \\
\hline H4 & Z & -0.00152 & -0.00152 & 0.00043 & 0.00006 & -0.00025 & 0.00061 & 0.00172 \\
\hline
\end{tabular}

$\begin{array}{crr}\text { frequencies } & 3742.21 \\ \text { intensities } & 122.04 \\ \text { Re1 X } & 0.00000 \\ \text { Re1 Y } & 0.00000 \\ \text { Re1 } & \text { Z } & 0.00000 \\ \text { N1 } & X & -0.00002 \\ \text { N1 } & \text { Y } & 0.00001\end{array}$




$\begin{array}{llr}\text { N1 } & \text { Z } & -0.00001 \\ \text { H9 } & \text { X } & 0.00055 \\ \text { H9 } & \text { Y } & 0.00067 \\ \text { H9 } & \text { Z } & 0.00088 \\ \text { N2 } & \text { X } & 0.00025 \\ \text { N2 } & \text { Y } & -0.00009 \\ \text { N2 } & \text { Z } & 0.00012 \\ \text { H10 } & \text { X } & -0.00356 \\ \text { H10 } & \text { Y } & 0.00110 \\ \text { H10 } & \text { Z } & -0.00181 \\ \text { C1 } & \text { X } & 0.00002 \\ \text { C1 } & \text { Y } & -0.00001 \\ \text { C1 } & \text { Z } & 0.00000 \\ \text { H11 } & \text { X } & 0.00000 \\ \text { H11 } & \text { Y } & -0.00006 \\ \text { H11 } & \text { Z } & 0.00009 \\ \text { C2 } & \text { X } & -0.00005 \\ \text { C2 } & \text { Y } & 0.00003 \\ \text { C2 } & \text { Z } & 0.00000 \\ \text { H8 } & \text { X } & 0.00009 \\ \text { H8 } & \text { Y } & -0.00011 \\ \text { H8 } & \text { Z } & -0.00007 \\ \text { O1 } & \text { X } & 0.00005 \\ \text { O1 } & \text { Y } & -0.00002 \\ \text { O1 } & \text { Z } & 0.00001 \\ \text { C3 } & \text { X } & 0.00001 \\ \text { C3 } & \text { Y } & -0.00004 \\ \text { C3 } & \text { Z } & 0.00013 \\ \text { H7 } & \text { X } & -0.00026 \\ \text { H7 } & \text { Y } & -0.00030 \\ \text { H7 } & \text { Z } & -0.00030 \\ \text { H12 } & \text { X } & 0.00111 \\ \text { H12 } & \text { Y } & -0.00061 \\ \text { H12 } & \text { Z } & -0.00098 \\ \text { C4 } & \text { X } & 0.00061 \\ \text { C4 } & \text { Y } & -0.00041 \\ \text { C4 } & \text { Z } & 0.00016 \\ \text { H2 } & \text { X } & -0.00007 \\ \text { H2 } & \text { Y } & 0.00082 \\ \text { H2 } & \text { Z } & 0.00046 \\ \text { O2 } & \text { X } & 0.00001 \\ \text { O2 } & \text { Y } & -0.00001 \\ \text { O2 } & \text { Z } & -0.00001 \\ \text { O3 } & \text { X } & -0.00004 \\ \text { O3 } & \text { Y } & -0.00001 \\ \text { O3 } & \text { Z } & 0.00000 \\ \text { C5 } & \text { X } & -0.00209 \\ \text { C5 } & \text { Y } & 0.00118 \\ \text { C5 } & \text { Z } & -0.00002 \\ \text { O4 } & \text { X } & 0.00007 \\ \text { O4 } & \text { Y } & -0.00023 \\ \text { O4 } & \text { Z } & -0.00093 \\ \text { O5 } & \text { X } & -0.04956 \\ \text { O5 } & \text { Y } & 0.02606 \\ \text { O5 } & \text { Z } & -0.02092 \\ \text { H4 } & \text { X } & 0.80125 \\ \text { H4 } & \text { Y } & -0.41863 \\ \text { H4 } & \text { Z } & 0.34376 \\ & & \end{array}$

Thermochemical Properties: 


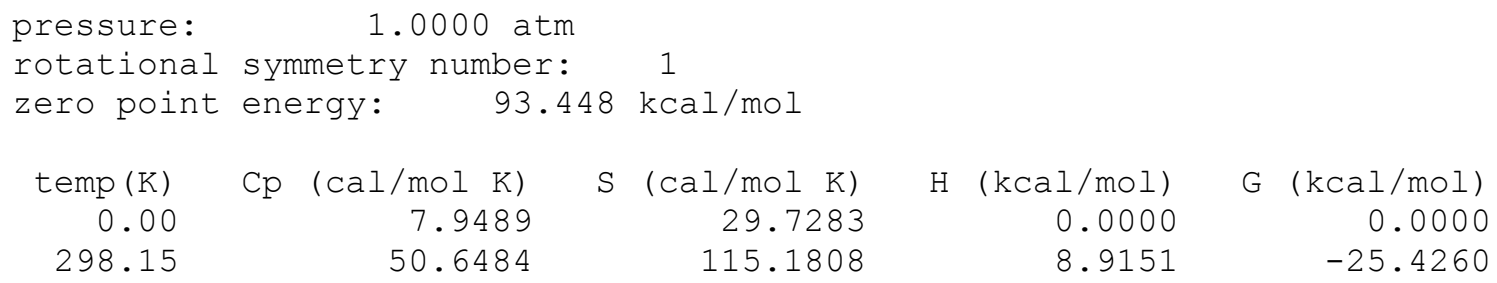

end of program freq

start of program ch

Moments from quantum mechanical wavefunction:

Dipole Moments (Debye)
$\mathrm{X}=$
5.3510
$\mathrm{Y}=$
3.3588
$\mathrm{Z}=$
-6.2809 Tot $=$
8.9087

Final energy .......... $\quad-759.84278344113$

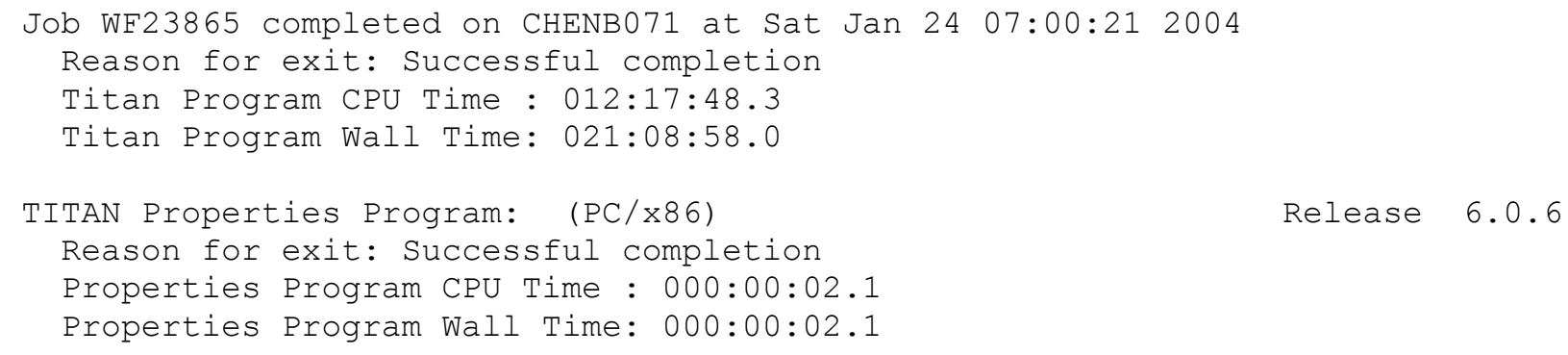




\section{Scheme 5}

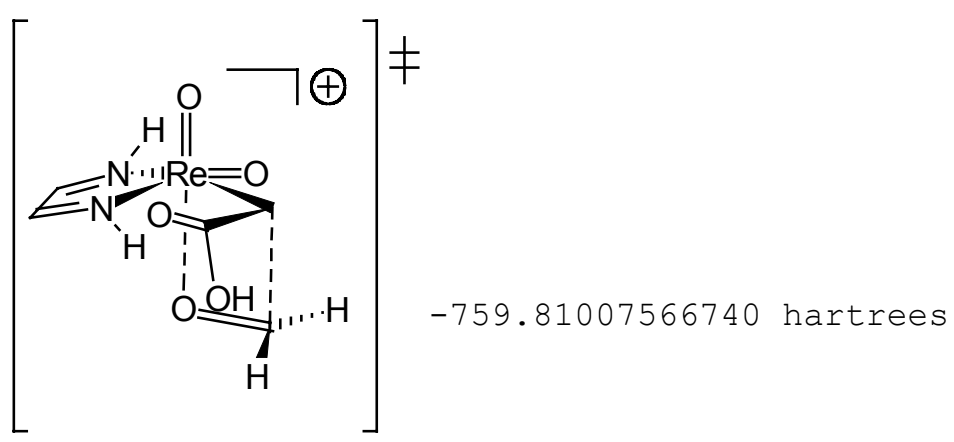

Jaguar version 3.5 , release 42
Copyright $1991-1998$ Schrodinger, Inc.
Al Rights Reserved.
| Use of this program should be acknowledged in publications as: |
Jaguar 3.5, Schrodinger, Inc., Portland, Oregon, 1998.

Job WF24992 started on CHENB071 at Mon Mar 10 17:32:23 2003

Jaguar version 3.5 , release 42

Job name:

Task:

Stoichiometry:

Molecular weight:

Molecular charge:

Spin multiplicity:

Point Group:

SCF method:

Basis set:

Basis functions:

Total electrons:

Alpha electrons:

Beta electrons:

Total orbitals:

Core orbitals:

Open-shell orbitals:

Occupied orbitals:

Shells:
WF24992

Transition-state search

$\mathrm{ReC} 5 \mathrm{~N} 2 \mathrm{H} 8 \mathrm{O} 5$

$363.00 \mathrm{amu}$

1

C1

B3LYP

LACVP**

230

106

53

53

245

53

0

53

1

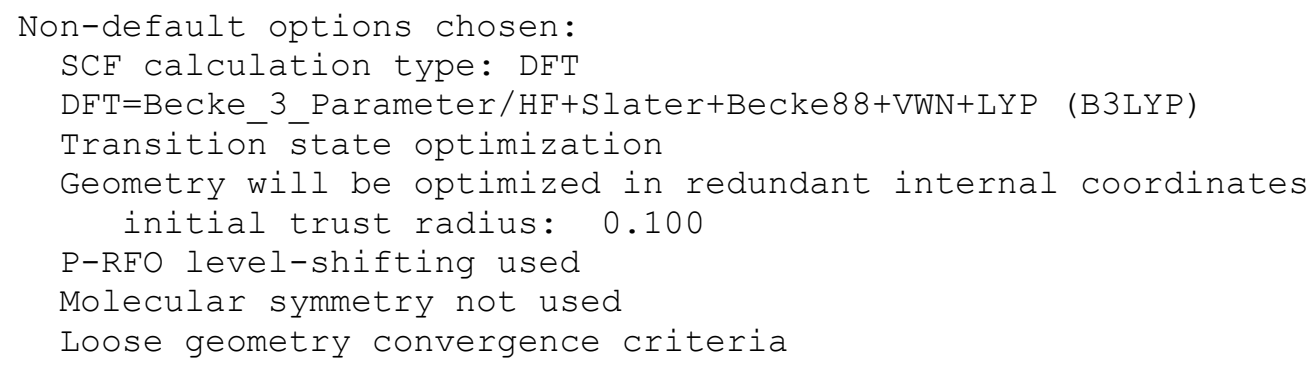


Initial Hessian: from previous calculation

Powell Hessian-updating used

\begin{tabular}{|c|c|c|c|c|c|c|c|}
\hline Iter & Energy & & Gmax & Grms & & Dmax & Drms \\
\hline 1 & -759.809588 & ‡ & 0.00433 & 0.00094 & * & 0.03750 & 0.01204 \\
\hline 2 & -759.809854 & - & 0.00441 & 0.00089 & * & 0.04636 & 0.01214 \\
\hline 3 & -759.810051 & * & 0.00404 & 0.00086 & $\star$ & 0.04283 & 0.01204 \\
\hline 4 & -759.810049 & \# & 0.00207 & 0.00066 & * & 0.05592 & 0.01204 \\
\hline 5 & -759.810071 & * & 0.00275 & 0.00079 & * & 0.05146 & 0.01210 \\
\hline 6 & -759.809985 & * & 0.00540 & 0.00116 & * & 0.04091 & 0.01206 \\
\hline 7 & -759.809912 & 夫 & 0.00581 & 0.00121 & 夫 & 0.04189 & 0.01206 \\
\hline 8 & -759.809970 & * & 0.00580 & 0.00105 & * & 0.03959 & 0.01209 \\
\hline 9 & -759.809705 & - & 0.00480 & 0.00118 & * & 085 & 0.01205 \\
\hline 10 & -759.809995 & . & 0.00153 & 0.00037 & 夫 & 0.05579 & 0.01204 \\
\hline 11 & -759.810078 & * & 0.00095 & 0.00033 & * & 0.06165 & 0.01204 \\
\hline 12 & -759.810183 & * & 0.00157 & 0.00051 & * & 5770 & 0.01204 \\
\hline 13 & -759.809980 & * & 0.00286 & 0.00088 & 夫 & 0.05898 & 0.01205 \\
\hline 14 & -759.810036 & * & 0.00270 & 0.00079 & $\star$ & 0.06433 & 0.01209 \\
\hline 15 & -759.810109 & * & 0.00071 & 0.00018 & $\#$ & 0.04315 & 0.00808 \\
\hline 16 & -759.810093 & * & 0.00071 & 0.00016 & $\#$ & 0.04433 & 0.01213 \\
\hline 17 & -759.810041 & 夫 & 0.00191 & 0.00052 & * & 0.02815 & 0.00600 \\
\hline 18 & -759.810104 & 夫 & 0.00100 & 0.00022 & \# & 0.04603 & 0.01204 \\
\hline 19 & -759.809980 & * & 0.00359 & 0.00092 & 夫 & 0.05823 & 0.01204 \\
\hline 20 & -759.810003 & * & 0.00328 & 0.00075 & * & 0.06623 & 0.01208 \\
\hline 21 & -759.810025 & * & 0.00264 & 0.00064 & * & 0.05484 & 0.01207 \\
\hline 22 & -759.810076 & * & 0.00044 & 0.00011 & $\#$ & 0.07045 & 0.01204 \\
\hline
\end{tabular}

energy change:

gradient maximum:

gradient rms:

step size: 0.10001

displacement maximum:

displacement rms:

predicted energy change:

step: $1.0001 \mathrm{E}-01$

$$
\begin{array}{r}
-5.1098 \mathrm{E}-05 \text { * } \\
4.3583 \mathrm{E}-04 \# \quad(2.5000 \mathrm{E}-04) \\
1.0942 \mathrm{E}-04 \# \quad(2.2500 \mathrm{E}-03) \\
1.5000 \mathrm{E}-03)
\end{array}
$$

trust radius: 0.10000

$$
\begin{gathered}
7.0445 \mathrm{E}-02 \cdot(9.0000 \mathrm{E}-03) \\
1.2040 \mathrm{E}-02 . \quad(6.0000 \mathrm{E}-03) \\
-5.8276 \mathrm{E}-05 \quad \text { geom step: }
\end{gathered}
$$

1.0001E-01 full

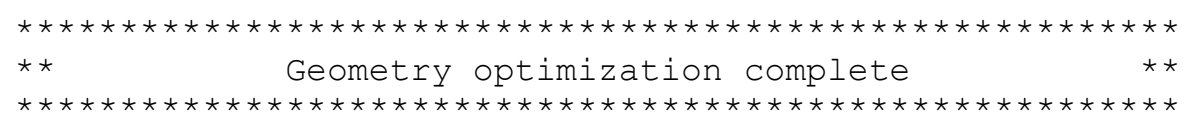

center of mass moved by:
$\mathrm{x}$ :
$0.0000 \mathrm{E}+00$
y:
$0.0000 E+00$
z :
$0.0000 \mathrm{E}+00$

final geometry:

$\begin{array}{lc}\text { atom } & \mathrm{x} \\ \text { Re1 } & 0.0918863110 \\ \mathrm{~N} 1 & -0.9467771563 \\ \mathrm{C} 4 & -2.2064742769 \\ \text { C7 } & -2.9494770423 \\ \mathrm{~N} 2 & -2.2610950132 \\ \mathrm{H} 18 & -0.3757521529 \\ \mathrm{H} 21 & -2.7445261259 \\ \mathrm{H} 23 & -4.0281593764 \\ \text { H26 } & -2.7656926411 \\ \text { O1 } & -0.3610335242 \\ \text { O2 } & 0.5881812895 \\ \text { O3 } & -0.2095321772\end{array}$

angstroms y

$-0.2350884085$

0.4829859554

0.6610992004

0.4391298905

0.0613930292

0.5926853340

0.9527794049

0.5955178439

$-0.0938727304$

$-1.7877403948$

$-0.3676400871$

1. 8790075415
1.2682134151

$-0.6563085104$

$-0.6928399328$

0.5807128325

1. 5838617716

$-1.5062484092$

$-1.5949786997$

0.6148392730

2.4578682900

0.7276686455

2.8833450965

1.5467234546 


$\begin{array}{llrr}\text { C1 } & 0.9351043458 & 2.1821099646 & 1.0382104361 \\ \text { H3 } & 1.7617339499 & 2.4521444094 & 1.7052479371 \\ \text { H4 } & 0.9950227731 & 2.5114040203 & -0.0053405692 \\ \text { C2 } & 1.8464800633 & 0.1806770910 & 0.5082042786 \\ \text { H1 } & 2.6844409182 & 0.2592975153 & 1.1975786250 \\ \text { C3 } & 2.2376336676 & 0.1424975165 & -0.9238307702 \\ \text { O4 } & 3.5292432142 & -0.1648945537 & -1.0496360745 \\ \text { H8 } & 3.7473458405 & -0.2111967662 & -1.9984261816 \\ \text { O5 } & 1.4990015178 & 0.3701469147 & -1.8714184387\end{array}$

Final energy ........... $\quad-759.81007566740$

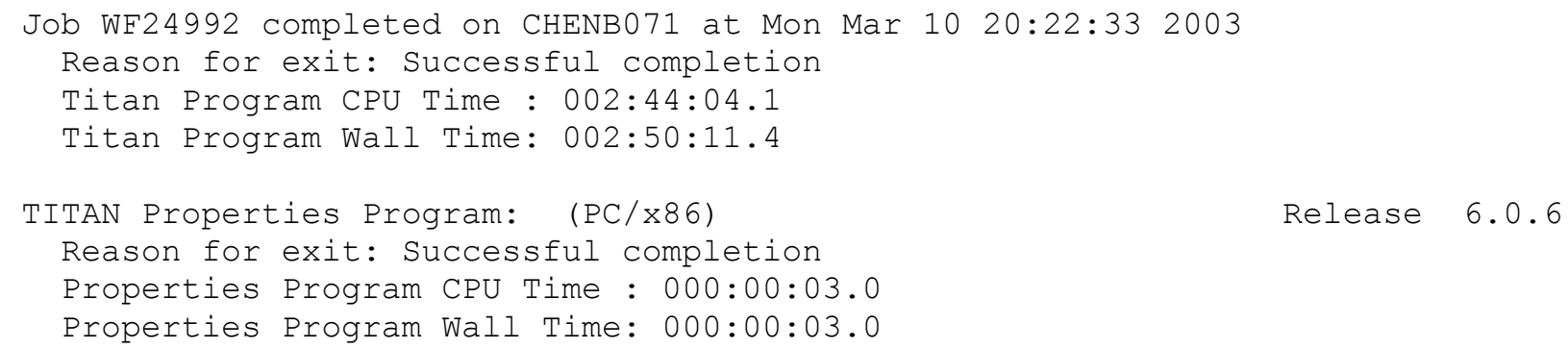

Job WF19608 started on CHENB071 at Thu Jan 22 08:24:31 2004 Jaguar version 3.5, release 42

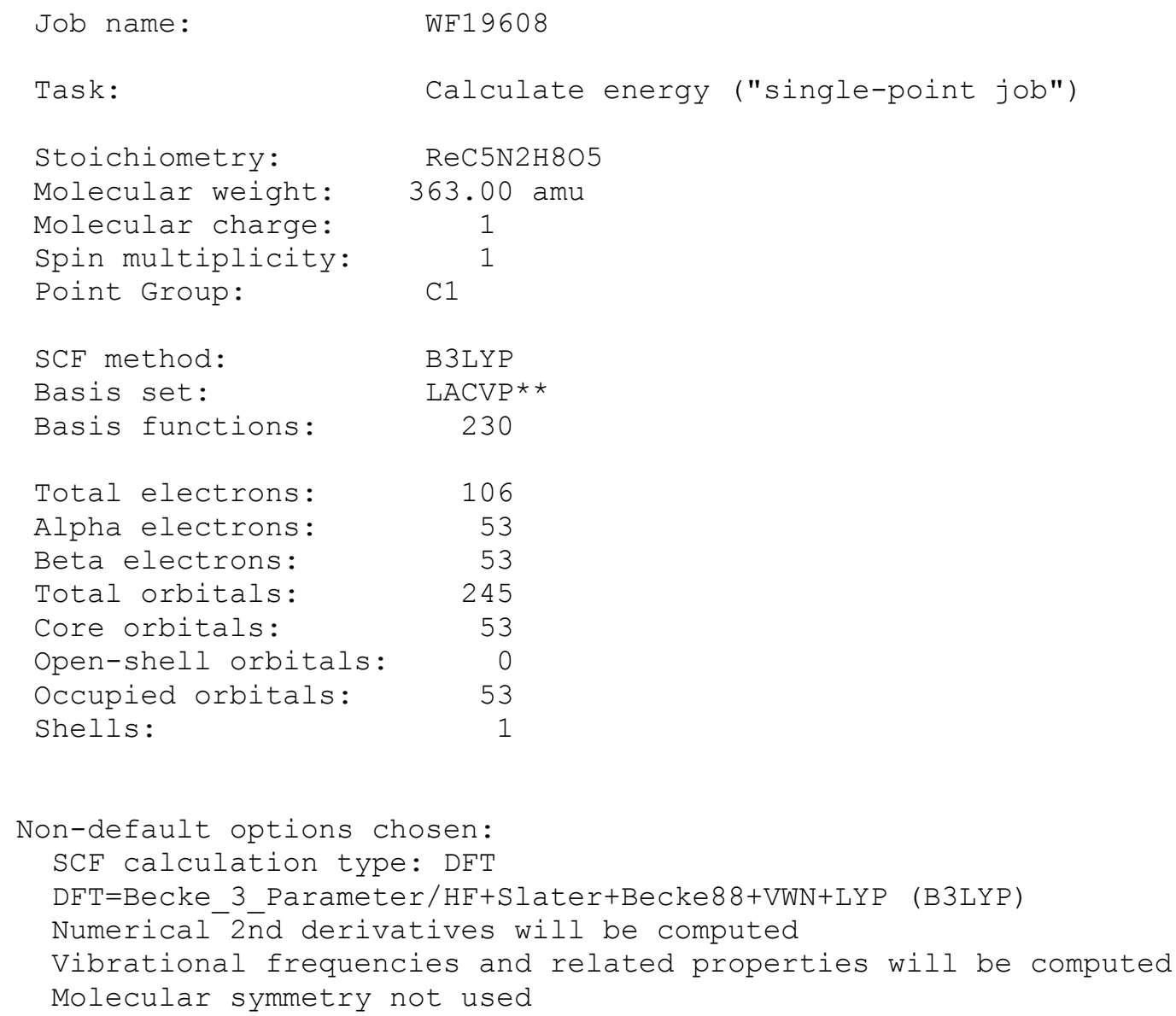

calculating forces at perturbed geometry 1 of $126 \ldots$ 
calculating forces at perturbed geometry calculating forces at perturbed geometry calculating forces at perturbed geometry calculating forces at perturbed geometry calculating forces at perturbed geometry calculating forces at perturbed geometry calculating forces at perturbed geometry calculating forces at perturbed geometry calculating forces at perturbed geometry calculating forces at perturbed geometry calculating forces at perturbed geometry calculating forces at perturbed geometry calculating forces at perturbed geometry calculating forces at perturbed geometry calculating forces at perturbed geometry calculating forces at perturbed geometry calculating forces at perturbed geometry calculating forces at perturbed geometry calculating forces at perturbed geometry calculating forces at perturbed geometry calculating forces at perturbed geometry calculating forces at perturbed geometry calculating forces at perturbed geometry calculating forces at perturbed geometry calculating forces at perturbed geometry calculating forces at perturbed geometry calculating forces at perturbed geometry calculating forces at perturbed geometry calculating forces at perturbed geometry calculating forces at perturbed geometry calculating forces at perturbed geometry calculating forces at perturbed geometry calculating forces at perturbed geometry calculating forces at perturbed geometry calculating forces at perturbed geometry calculating forces at perturbed geometry calculating forces at perturbed geometry calculating forces at perturbed geometry calculating forces at perturbed geometry calculating forces at perturbed geometry calculating forces at perturbed geometry calculating forces at perturbed geometry calculating forces at perturbed geometry calculating forces at perturbed geometry calculating forces at perturbed geometry calculating forces at perturbed geometry calculating forces at perturbed geometry calculating forces at perturbed geometry calculating forces at perturbed geometry calculating forces at perturbed geometry calculating forces at perturbed geometry calculating forces at perturbed geometry calculating forces at perturbed geometry calculating forces at perturbed geometry calculating forces at perturbed geometry calculating forces at perturbed geometry calculating forces at perturbed geometry calculating forces at perturbed geometry calculating forces at perturbed geometry calculating forces at perturbed geometry calculating forces at perturbed geometry
2 of $126 \ldots$

3 of $126 \ldots$

4 of $126 \ldots$

5 of $126 \ldots$

6 of $126 \ldots$

7 of $126 \ldots$

8 of $126 \ldots$

9 of $126 \ldots$ 10 of $126 \ldots$

11 of $126 \ldots$ 12 of $126 \ldots$ 13 of $126 \ldots$ 14 of $126 \ldots$ 15 of $126 \ldots$ 16 of $126 \ldots$ 17 of $126 \ldots$ 18 of $126 \ldots$ 19 of $126 \ldots$ 20 of $126 \ldots$ 21 of $126 \ldots$ 22 of $126 \ldots$ 23 of $126 \ldots$ 24 of $126 \ldots$ 25 of $126 \ldots$ 26 of $126 \ldots$ 27 of $126 \ldots$ 28 of $126 \ldots$ 29 of $126 \ldots$ 30 of $126 \ldots$ 31 of $126 \ldots$ 32 of $126 \ldots$ 33 of $126 \ldots$ 34 of $126 \ldots$ 35 of $126 \ldots$ 36 of $126 \ldots$ 37 of $126 \ldots$ 38 of $126 \ldots$ 39 of $126 \ldots$ 40 of $126 \ldots$ 41 of $126 \ldots$ 42 of $126 \ldots$ 43 of $126 \ldots$ 44 of $126 \ldots$ 45 of $126 \ldots$ 46 of $126 \ldots$ 47 of $126 \ldots$ 48 of $126 \ldots$ 49 of $126 \ldots$ 50 of $126 \ldots$ 51 of $126 \ldots$ 52 of $126 \ldots$ 53 of $126 \ldots$ 54 of $126 \ldots$ 55 of $126 \ldots$ 56 of $126 \ldots$ 57 of $126 \ldots$ 58 of $126 \ldots$ 59 of $126 \ldots$ 60 of $126 \ldots$ 61 of $126 \ldots$ 62 of $126 \ldots$ 
calculating forces at perturbed geometry calculating forces at perturbed geometry calculating forces at perturbed geometry calculating forces at perturbed geometry calculating forces at perturbed geometry calculating forces at perturbed geometry calculating forces at perturbed geometry calculating forces at perturbed geometry calculating forces at perturbed geometry calculating forces at perturbed geometry calculating forces at perturbed geometry calculating forces at perturbed geometry calculating forces at perturbed geometry calculating forces at perturbed geometry calculating forces at perturbed geometry calculating forces at perturbed geometry calculating forces at perturbed geometry calculating forces at perturbed geometry calculating forces at perturbed geometry calculating forces at perturbed geometry calculating forces at perturbed geometry calculating forces at perturbed geometry calculating forces at perturbed geometry calculating forces at perturbed geometry calculating forces at perturbed geometry calculating forces at perturbed geometry calculating forces at perturbed geometry calculating forces at perturbed geometry calculating forces at perturbed geometry calculating forces at perturbed geometry calculating forces at perturbed geometry calculating forces at perturbed geometry calculating forces at perturbed geometry calculating forces at perturbed geometry calculating forces at perturbed geometry calculating forces at perturbed geometry calculating forces at perturbed geometry calculating forces at perturbed geometry calculating forces at perturbed geometry 101 of $126 \ldots$ calculating forces at perturbed geometry 102 of $126 \ldots$ calculating forces at perturbed geometry 103 of $126 \ldots$ calculating forces at perturbed geometry 104 of $126 \ldots$ calculating forces at perturbed geometry 105 of $126 \ldots$ calculating forces at perturbed geometry 106 of $126 \ldots$ calculating forces at perturbed geometry 107 of $126 \ldots$ calculating forces at perturbed geometry 108 of $126 \ldots$ calculating forces at perturbed geometry 109 of $126 \ldots$ calculating forces at perturbed geometry 110 of $126 \ldots$ calculating forces at perturbed geometry 111 of $126 \ldots$ calculating forces at perturbed geometry 112 of $126 \ldots$ calculating forces at perturbed geometry 113 of $126 \ldots$ calculating forces at perturbed geometry 114 of $126 \ldots$ calculating forces at perturbed geometry 115 of $126 \ldots$ calculating forces at perturbed geometry 116 of $126 \ldots$ calculating forces at perturbed geometry 117 of $126 \ldots$ calculating forces at perturbed geometry 118 of $126 \ldots$ calculating forces at perturbed geometry 119 of $126 \ldots$ calculating forces at perturbed geometry 120 of $126 \ldots$ calculating forces at perturbed geometry 121 of $126 \ldots$ calculating forces at perturbed geometry 122 of $126 \ldots$ calculating forces at perturbed geometry 123 of $126 \ldots$ 
calculating forces at perturbed geometry 124 of $126 \ldots$ calculating forces at perturbed geometry 125 of $126 \ldots$ calculating forces at perturbed geometry 126 of $126 \ldots$

numerical derivatives complete;

calculation at normal geometry follows...

start of program freq

harmonic frequencies in $\mathrm{cm}^{\wedge}-1$, IR intensities in $\mathrm{km} / \mathrm{mol}$, and normal modes:

\begin{tabular}{|c|c|c|c|c|c|c|c|c|}
\hline frequ & cies & -160.88 & 71.61 & 80.33 & 116.17 & 125.21 & 156.51 & 172.5 \\
\hline inten & ties & 35.52 & 2.07 & 1.76 & 0.69 & 3.75 & 1.52 & \\
\hline $\operatorname{Re} 1$ & $\mathrm{X}$ & 0.00806 & -0.00624 & -0.00588 & -0.00565 & 0.00530 & 0.00068 & 0.0025 \\
\hline Re1 & Y & -0.01116 & -0.00801 & -0.01107 & -0.01829 & -0.00921 & 0.00518 & 0.002 \\
\hline $\operatorname{Re} 1$ & Z & -0.00891 & 0.00207 & 0.02008 & -0.00983 & 0.00534 & 0.00688 & -0 . \\
\hline N1 & $\mathrm{X}$ & 0.00901 & 0.00531 & 0.06093 & -0.02141 & 0.00522 & -0.02462 & -0 . \\
\hline $\mathrm{N} 1$ & $Y$ & 0.02335 & -0.01463 & -0.02290 & -0.05222 & 0.00483 & -0.02237 & -0 . \\
\hline N1 & Z & 0.02796 & -0.00472 & -0.01717 & 0.00403 & -0.00876 & -0.017 & -0 . \\
\hline $\mathrm{C} 4$ & $\mathrm{X}$ & 0.00763 & 0.01013 & 0.06686 & -0.01483 & 0.01604 & -0.03649 & -0 . \\
\hline $\mathrm{C} 4$ & Y & 0.01251 & 0.01717 & 0.00603 & 0.00701 & 0.08459 & -0.10029 & -0 . \\
\hline C4 & Z & 0.01479 & -0.00644 & -0.05747 & 0.03930 & 0.014 & -0.026 & -0 \\
\hline C7 & $\mathrm{X}$ & -0.00863 & 0.00990 & 0.02908 & 0.00862 & 0.02 & -0.021 & -0 \\
\hline C7 & Y & 0.01158 & 0.05708 & 0.02687 & 0.05237 & 0.09 & -0.020 & \\
\hline C7 & Z & 0.00 & 0.000 & -0.07 & 0.06147 & $0.01 \varepsilon$ & -0.0 & \\
\hline N2 & $\mathrm{X}$ & -0.01959 & 0.003 & 0.000 & 0.019 & 0.00 & -0 & \\
\hline $\mathrm{N} 2$ & Y & 0.00656 & 0.044 & $0.02 \varepsilon$ & 0.03709 & -0.001 & 0.07 & \\
\hline N2 & Z & 0.012 & -0.000 & -0.05 & 0.047 & $-0.00^{\circ}$ & 0.02 & \\
\hline H18 & $\mathrm{X}$ & 0.01 & 0.01 & 0.09 & -0.034 & -0.00 & -0.0 & -0 . \\
\hline $\mathrm{H} 18$ & Y & 0.00720 & -0.05748 & -0.026 & -0.05232 & -0.018 & -0.05 & -0 . \\
\hline H18 & Z & 0.031 & -0.008 & 0.003 & -0.004 & -0.01 & -0.03 & -0 \\
\hline H21 & $\mathrm{X}$ & 0.01 & 0.014 & 0.10 & -0.02446 & 0 & -0.0 & -0 \\
\hline $\mathrm{H} 21$ & Y & -0.000 & 0.01126 & 0.016 & 0.03218 & 0.12 & -0.20 & -0 . \\
\hline H21 & Z & 0.00 & -0.011 & -0.07 & 0.053 & 0. & -0.0 & -0 \\
\hline H23 & $\mathrm{X}$ & -0.009 & 0.015 & 0.0 & 0.015 & 0 . & -0.0 & \\
\hline $\mathrm{H} 23$ & Y & 0.01524 & 0. & 0 & 0.09585 & 0.17 & -0.03 & \\
\hline H23 & Z & -0.01 & 0.0 & -0.1 & 0 . & 0 . & -0 & \\
\hline H26 & $\mathrm{X}$ & -0.030 & 0.00568 & -0.02 & 0.03935 & 0 . & -0.0 & \\
\hline H26 & Y & 0.0 & 0.070 & 0 & 0.06808 & 0.001 & 0.1 & \\
\hline H26 & Z & 0 & 0.00 & -0.0 & 0.06 & -0.00 & 0 & \\
\hline 01 & $\mathrm{X}$ & 0.009 & -0.02771 & -0.02449 & -0.01276 & 0.04 & -0.07 & \\
\hline 01 & Y & -0 & -0.00618 & -0.02939 & -0.03646 & -0.011 & 0.044 & -0 . \\
\hline $\mathrm{O} 1$ & Z & -0.0 & 0 . & 0 & 0.047 & -0.01 & -0.0 & \\
\hline 02 & $\mathrm{X}$ & -0.01293 & 0 & 0.06666 & -0.04301 & 0.02 & 0.0 & \\
\hline 02 & Y & 0.05017 & -0.01069 & 0.029 & 0.05911 & -0.026 & -0.07 & \\
\hline 02 & Z & 0.003 & -0 & 0 & 0.0 & -0 . & -0.0 & -0 \\
\hline 03 & $X$ & -0.03474 & 02183 & -0.01207 & 0.00580 & -0.05 & 0.02 & -0 . \\
\hline 03 & Y & 0.08011 & -0.00726 & -0.001 & 0.02153 & -0.03265 & -0.00 & -0 \\
\hline 03 & Z & 0.0 & 0 & 0.0 & -0.08440 & 0.0 & 0.0 & -0 \\
\hline $\mathrm{C} 1$ & $\mathrm{X}$ & -0.09542 & 0.02457 & -0.01816 & 0.01496 & -0.118 & -0.03882 & -0 \\
\hline $\mathrm{C} 1$ & Y & 0.15642 & -0.01707 & 0.009 & 0.00108 & 0.001 & 0.020 & -0 \\
\hline $\mathrm{C} 1$ & $\mathrm{Z}$ & 0.06519 & -0.00023 & 0.022 & -0.05964 & -0.11 & -0.05 & \\
\hline H3 & $\mathrm{X}$ & -0.05218 & 0.02857 & -0.01118 & 0.00455 & -0.07877 & 0.02193 & -0.08 \\
\hline $\mathrm{H} 3$ & Y & 0.08838 & -0.02600 & -0.0096 & 0.00895 & 0.11621 & 0.073 & -0.0 \\
\hline H3 & Z & 0.04410 & -0.00189 & 0.0212 & -0.04847 & -0.20965 & -0.15307 & \\
\hline $\mathrm{H} 4$ & $\mathrm{X}$ & -0.05962 & 0.02019 & -0.01793 & 0.05201 & -0.24515 & -0.14409 & 0.08 \\
\hline $\mathrm{H} 4$ & Y & 0.06376 & -0.00408 & 0.01350 & -0.04731 & -0.05515 & -0.05507 & \\
\hline $\mathrm{H} 4$ & Z & 0.03509 & 0.00367 & 0.02331 & -0.07326 & -0.13765 & -0.08610 & \\
\hline $\mathrm{C} 2$ & $\mathrm{x}$ & 0.00586 & -0.00491 & -0.02908 & 0.01060 & -0.01350 & 0.00073 & -0.00 \\
\hline $\mathrm{C} 2$ & Y & -0.11761 & -0.01430 & -0.02197 & -0.05052 & 0.06466 & -0.04808 & 0.0 \\
\hline $\mathrm{C} 2$ & Z & -0.00894 & -0.01024 & -0.04524 & 0.01131 & -0.00122 & -0.01520 & -0.0 \\
\hline
\end{tabular}




$\begin{array}{ll}\text { H1 } & \text { X1 } \\ \text { H1 } & \\ \text { C3 } & X \\ \text { C3 } & Y \\ \text { C3 } & \\ \text { O4 } & \text { X } \\ \text { O4 } & \text { Y } \\ \text { O4 } & \\ \text { H8 } & \text { X } \\ \text { H8 } & \\ \text { H8 } & \\ \text { O5 } & \text { X } \\ \text { O5 } & Y \\ \text { O5 } & \end{array}$

\begin{tabular}{|c|c|c|}
\hline-0.00408 & -0.00513 & -0.01782 \\
\hline 0.00456 & -0.02161 & -0.04304 \\
\hline-0.00884 & -0.00953 & -0.05628 \\
\hline 0.00843 & 0.00132 & -0.02702 \\
\hline-0.05568 & -0.00135 & 0.02471 \\
\hline-0.00445 & -0.00833 & -0.04716 \\
\hline 0.01883 & 0.04570 & -0.03252 \\
\hline-0.00487 & 0.17470 & 0.00570 \\
\hline-0.01759 & 0.00215 & -0.05103 \\
\hline 0.02113 & 0.05145 & -0.02650 \\
\hline 0.03561 & 0.17625 & 0.04277 \\
\hline-0.01903 & 0.00362 & -0.05161 \\
\hline 0.00223 & -0.02393 & -0.00695 \\
\hline-0.03227 & -0.12905 & 0.08223 \\
\hline 0.00342 & -0.01978 & -0.05078 \\
\hline
\end{tabular}

$-0.00510-0$

$-0.02210-0.00954-0.00390$

$-0.04222$

0.10688

$-0.03784$

0.03110

0.03004

$0.00400-0.00282-0.00372$

0.03159

$0.00112 \quad 0.03065$

0.00525

0.02908

0.00789

$-0.00268$

0.01123

0.01788

$0.00365-0.00843$

0.00082

$\begin{array}{llll}0.03145 & -0.00318 & 0.03583 & 0.00022\end{array}$

$\begin{array}{lllll}0.03156 & -0.02058 & 0.00267 & -0.03299\end{array}$

$\begin{array}{llll}0.00975 & 0.03422 & 0.01263 & 0.03833\end{array}$

$\begin{array}{llll}0.04245 & 0.00942 & 0.06147 & 0.01765\end{array}$

$\begin{array}{lllll}0.09727 & -0.06248 & 0.04042 & -0.07939\end{array}$

0.009110 .03904

$0.01667 \quad 0.04476$

$\begin{array}{llll}0.04200 & 0.01408 & 0.05187 & 0.02254\end{array}$

$\begin{array}{lllll}0.11186 & -0.02019 & 0.04217 & -0.01265\end{array}$

$0.02915-0.01237-0.01252-0.01581$

\begin{tabular}{|c|c|c|c|c|c|c|c|c|}
\hline freque & cies & 180.86 & 210.09 & 239.60 & 258.13 & 267.60 & 279.82 & 296.91 \\
\hline intens & ties & 5.51 & 12.19 & 13.74 & 6.93 & 4.84 & 14.81 & 3.56 \\
\hline $\operatorname{Re} 1$ & $\mathrm{X}$ & -0.01290 & -0.02123 & 0.00754 & -0.01191 & 0.00385 & 0.00383 & -0.02170 \\
\hline Re1 & $\mathrm{Y}$ & 0.00401 & 0.00158 & 0.00919 & -0.00319 & 0.01094 & -0.00910 & 0.01729 \\
\hline Re1 & Z & -0.00506 & -0.00175 & 0.02150 & -0.00188 & -0.02256 & -0.00419 & 0.00164 \\
\hline N1 & $\mathrm{X}$ & -0.01276 & 0.06829 & 0.00399 & 0.01213 & 0.05099 & 0.07826 & 0.02906 \\
\hline N1 & $\mathrm{Y}$ & 0.05497 & -0.00748 & 0.01112 & 0.02521 & -0.00154 & 0.00542 & -0.08147 \\
\hline N1 & Z & 0.01179 & 0.00475 & -0.02765 & -0.03131 & 0.02791 & 0.10672 & 0.03371 \\
\hline C4 & $\mathrm{X}$ & -0.01528 & 0.06370 & 0.00629 & 0.01023 & 0.04996 & 0.07632 & 0.03907 \\
\hline C4 & Y & 0.05788 & -0.04613 & 0.02844 & 0.00035 & -0.01405 & -0.03038 & -0.01986 \\
\hline C4 & Z & 0.04334 & 0.02253 & 0.00337 & -0.01884 & 0.02225 & 0.04447 & 0.0 \\
\hline C7 & $\mathrm{X}$ & -0.01377 & 0.10263 & 0.02368 & 0.05066 & 0.03216 & -0.02201 & 0.0 \\
\hline C7 & Y & -0.05524 & -0.02532 & -0.00380 & -0.01920 & -0.01978 & -0.01504 & 0.04 \\
\hline $\mathrm{C} 7$ & Z & 0.02726 & 0.05107 & 0.00786 & -0.00071 & 0.01129 & -0.00961 & 0.01 \\
\hline N2 & $\mathrm{X}$ & -0.00593 & 0.12870 & 0.02350 & 0.08327 & 0.02189 & -0.09843 & -0.04530 \\
\hline N2 & Y & -0.06970 & -0.00590 & -0.01866 & 0.00315 & 0.00635 & 0.04342 & -0.07 \\
\hline N2 & Z & 0.01632 & 0.04027 & 0.00291 & -0.01308 & 0.02581 & 0.06503 & 0.0 \\
\hline $\mathrm{H} 18$ & $\mathrm{X}$ & -0.03439 & 0.07491 & -0.01742 & 0.02060 & 0.06691 & 0.11513 & 0.04721 \\
\hline H1 8 & Y & 0.11052 & -0.02723 & 0.02188 & 0.03459 & -0.00319 & 0.01130 & -0.14 \\
\hline H18 & Z & 0.00548 & 0.00162 & -0.03761 & -0.02300 & 0.03639 & 0.12 & 0.0 \\
\hline H21 & $\mathrm{X}$ & -0.02703 & 0.03256 & -0.01362 & -0.01495 & 0.06009 & 0.13559 & 0.06403 \\
\hline H21 & Y & 0.12449 & -0.08701 & 0.05503 & -0.00273 & -0.01821 & -0.04210 & -0.03 \\
\hline H21 & Z & 0.07171 & 0.02837 & 0.02328 & -0.00502 & 0.01522 & 0.006 & 0.0 \\
\hline H23 & $\mathrm{X}$ & -0.02412 & 0.10490 & 0.02127 & 0.04855 & 0.02862 & -0.02752 & 0.02412 \\
\hline $\mathrm{H} 23$ & Y & -0.12464 & -0.02479 & -0.02039 & -0.04500 & -0.04037 & -0.02830 & 0.1 \\
\hline H23 & Z & 0.01993 & 0.08714 & 0.01460 & 0.03504 & -0.01365 & -0.11529 & 0.0 \\
\hline H26 & $\mathrm{X}$ & -0.01113 & 0.16670 & 0.01802 & 0.10607 & 0.01784 & -0.17088 & -0.06700 \\
\hline H26 & Y & -0.16285 & 0.01374 & -0.04730 & -0.00104 & -0.00070 & 0.07354 & -0.05 \\
\hline H26 & Z & -0.00296 & 0.06528 & -0.00538 & -0.00095 & 0.02197 & 0.02888 & -0.00160 \\
\hline 01 & $\mathrm{X}$ & -0.05365 & 0.00614 & -0.04867 & -0.01969 & -0.09012 & -0.04045 & 0.08412 \\
\hline O1 & $\mathrm{Y}$ & -0.00746 & 0.00270 & 0.05293 & 0.00728 & 0.00504 & 0.02357 & 0.00 \\
\hline 01 & $\mathrm{Z}$ & 0.05728 & -0.03617 & -0.05660 & -0.03086 & 0.06700 & -0.05625 & -0.07026 \\
\hline $\mathrm{O} 2$ & $\mathrm{X}$ & 0.10185 & -0.05564 & -0.02783 & 0.13220 & -0.03746 & 0.03774 & 0.05068 \\
\hline $\mathrm{O} 2$ & Y & 0.03526 & -0.01524 & -0.04320 & -0.00655 & 0.04461 & 0.00245 & 0.02 \\
\hline $\mathrm{O} 2$ & Z & -0.03799 & 0.00924 & 0.02955 & -0.04658 & -0.00648 & -0.01016 & -0.01522 \\
\hline 03 & X & -0.00746 & 0.00561 & -0.10211 & -0.01349 & -0.03860 & -0.02073 & 0.01023 \\
\hline 03 & $Y$ & -0.00386 & 0.02899 & -0.03664 & 0.02782 & -0.08056 & 0.01130 & 0.00475 \\
\hline O3 & Z & 0.00002 & 0.01629 & -0.11169 & -0.05017 & 0.07267 & -0.07357 & 0.04053 \\
\hline $\mathrm{C} 1$ & $\mathrm{X}$ & -0.00069 & 0.00070 & -0.04247 & -0.00635 & -0.05984 & 0.00753 & 0.01252 \\
\hline $\mathrm{C} 1$ & Y & -0.01623 & 0.03033 & -0.03593 & 0.02815 & -0.06339 & 0.00046 & -0.06288 \\
\hline $\mathrm{C} 1$ & Z & 0.00149 & 0.00984 & 0.01504 & -0.01945 & 0.00766 & -0.02046 & -0.00784 \\
\hline H3 & $\mathrm{X}$ & 0.00247 & 0.01155 & -0.10659 & -0.02490 & -0.04952 & -0.02197 & 0.04240 \\
\hline H3 & Y & -0.03781 & 0.01077 & -0.07767 & 0.04985 & -0.01021 & -0.00911 & -0.07884 \\
\hline H3 & Z & 0.00553 & 0.00470 & 0.10952 & -0.00458 & -0.02973 & 0.02125 & -0.04027 \\
\hline H4 & $\mathrm{X}$ & 0.00198 & -0.00605 & 0.06547 & 0.02970 & -0.13163 & 0.05685 & -0.01189 \\
\hline
\end{tabular}




H4
H4
C2
C2
C2
H1
H1
H1
C3
C3
C3
O4
O4
O4
H8
H8
H8
O5
O5
O5

$Y$
$Z$
$X$
$Y$
$Z$
$X$
$Y$
$Z$
$X$
$Y$
$Z$
$X$
$Y$
$Z$
$X$
$Y$
$Z$
$X$
$Y$
$Z$

\begin{abstract}
0.00243
0.00771

0.05407

$-0.02054$

$-0.01471$

$-0.03979$

$-0.03225$

$-0.03019$

$-0.02472-0$.

$0.03854-0$.

$-0.01126$

$-0.02347$

$0.05213-0$.

$-0.00548-0.00835$

0.05634

0.11269

0.02875

$-0.01129$

0.02115

0.07083

$-0.03817$

0.08379

0.03806

$-0.01457$

0.02665

$-0.05735-0.06667$
\end{abstract}

frequencies

intensities

Re1 $x$

Re1 Y

Re1 Z

N1 X

N1 Y

N1 Z

C $4 \quad X$

C4 Y

C 4 Z

C7 $\mathrm{X}$

C7 Y

C7 Z

N2 X

N2 Y

N2 Z

H18 X

H18 Y

H18 Z

H21 X

H21 Y

H21 Z

H23

H23

H23

H2 6

H26 Y

H26 Z

O1 X

O1 Y

O1 Z

O2 X

$\mathrm{O} 2 \mathrm{Y}$

$02 \mathrm{Z}$

$03 \mathrm{X}$

$03 \mathrm{Y}$

$03 \mathrm{Z}$

C1 $\mathrm{X}$

C1 Y
328.32

3. 41

354.32

13.36

$\begin{array}{lll}-0.00433 & 0.01662\end{array}$

$-0.00839-0.00778$

0.00467

0.00368

$-0.00399$

0.12512

$-0.01505$

$0.01308-0.03881$

$-0.02592-0.00865$

$\begin{array}{ll}-0.02869 & 0.00799\end{array}$

$-0.02012-0.00940$

$-0.00915$

$-0.02702$

0.01092

$-0.01129$

0.00316

0.02601

0.00699

$0.09455-0.05593$

$0.01080-0.02385$

$-0.00986-0.03594$

$0.15386-0.07320$

$0.01409-0.05467$

$-0.04164-0.02195$

$\begin{array}{lll}-0.09830 & 0.00723\end{array}$

$-0.03364-0.00229$

$-0.01437$

0.01684

$-0.06469$

$-0.00268$

0.04829

0.06002

0.02405

$0.00626-0$

$0.16565-0.07793$

$0.08561-0.08918$

$-0.01850$

0.05413

$-0.04819-0.08695$

0.00709

0.09905

$$
-0.00476
$$$$
0.12828-0
$$

0.00994

0.02333

$-0.03311$

0.02446

0.01666

$-0.01734-0$
$0.03051-0$

0.00957

$-0.09692$

$-0.02364$
0.02232
$-0.01431-0.02604$

$\begin{array}{rr}0.02925 & -0.03448 \\ 0.01998 & 0.01544\end{array}$

$-0.07146-0.02838$

$-0.01678$

0.05748

0.00424

$-0.03355$

0.06890

$-0.02016$

$-0.02393$

$-0.00550$

0.07077

$0.02754-0.02521$

0.00851

0.00556

$-0.05746$

0.01609

0.01180

$-0.06321$

0.01468

0.00729

0.05136

$-0.06351$

0.01758

0.00279

$-0.06217$

$-0.00264$

0.10272

384.85

19.30

0.01302

0.01790

$-0.01202$

0.01473

$-0.02354$

0.00282

0.02069

0.01764

0.01458

0.00189

$-0.01876$

$-0.00262$

404.07

2.72

0.00642

0.00082

$-0.00292$

0.01660

$0.11910-0.04513$

$0.02504 \quad 0.15798$

$-0.01400$

$-0.03481$

0.00751

0.00430

$-0.02335$

0.01276

$-0.04673$

0.089310 .00895

$0.02465-0.02484$

0.08573

0.01781

0.02341

$-0.01946$

$-0.02665$

$-0.11355$

$\begin{array}{lll}0.00897 & -0.00617 & 0.02831\end{array}$

0.03014

$-0.06686$

0.20082

$0.00119-0.00129$

$0.03224-0.04881$

0.08178

$0.06416-0.35278$

0.02091

0.02310

$-0.08393$

$-0.05814$

$0.05354-0.04635$

$0.35469-0.02022$

$0.09174 \quad 0.07542$

$\begin{array}{ll}0.00241 & 0.19911\end{array}$

$0.03820-0.01224$

$0.01091-0.05489$

0.00044

0.04578

$-0.02650$

$-0.02459$

0.00510

$0.01554-0.00577$

$0.04830-0.00792$

0.04654

0.00260

$-0.02516-0.01068$

0.00107

$-0.00299$

$-0.03693$

0.06045

$\begin{array}{rrr}0.00032 & -0.02112 & 0.00620 \\ -0.01926 & 0.00164 & -0.00793 \\ -0.04387 & -0.01542 & -0.04568\end{array}$

0.02112

$\begin{array}{rrr}-0.00032 & -0.02112 & 0.00620 \\ -0.01926 & 0.00164 & -0.00793 \\ -0.04387 & -0.01542 & -0.04568\end{array}$

$\begin{array}{rrr}-0.00032 & -0.02112 & 0.00620 \\ -0.01926 & 0.00164 & -0.00793 \\ -0.04387 & -0.01542 & -0.04568\end{array}$

$\begin{array}{rrr}-0.00032 & -0.02112 & 0.00620 \\ -0.01926 & 0.00164 & -0.00793 \\ -0.04387 & -0.01542 & -0.04568\end{array}$

$-0.04387-0.01542$

$0.02856-0.08988$

$-0.01022-0.01706$

$\begin{array}{ll}-0.01618 & 0.01446\end{array}$

$0.05536-0.07095$

$0.00046 \quad 0.00092$

$\begin{array}{rrr}-0.01344 & 0.00938\end{array}$

$0.05962-0.08162$

$-0.005440 .00743$

$\begin{array}{ll}-0.01671 & 0.02503\end{array}$

$0.01995-0.02826$

$0.00266-0.00141$

$\begin{array}{ll}-0.02153 & 0.02807\end{array}$

$\begin{array}{ll}0.00264 & 0.00170\end{array}$

$0.03055-0.04489$

$\begin{array}{ll}-0.00194 & 0.00446\end{array}$

$\begin{array}{ll}-0.03162 & 0.05763\end{array}$

$0.03660-0.05333$

$\begin{array}{ll}-0.00843 & 0.01347\end{array}$

$\begin{array}{ll}-0.00305 & 0.01303\end{array}$

$-0.01066 \quad 0.01576$

$499.36 \quad 625.32$

$73.39 \quad 54.03$

$-0.00649-0.00261$

0.007360 .00041

0.000630 .00056

$\begin{array}{cc}-0.00846 & 0.00002\end{array}$

$0.00895 \quad 0.00241$

0.075130 .00488

$-0.00945-0.00225$

$-0.01140-0.00013$

$0.00248 \quad 0.00539$

$-0.01580-0.00869$

$\begin{array}{lll}0.01798 & 0.00147\end{array}$

$\begin{array}{rr}-0.00339 & 0.00200\end{array}$

$0.02487-0.00236$

$\begin{array}{ll}-0.00415 & 0.00097\end{array}$

$-0.03961-0.00311$

$0.02837-0.01176$

$-0.01091-0.01100$

$0.09373-0.00651$

$0.03107-0.00003$

$-0.04439-0.01260$

$\begin{array}{ll}-0.03132 & 0.00021\end{array}$

$-0.01067-0.01006$

$0.04008-0.00664$ 


\begin{tabular}{|c|c|c|c|c|c|c|c|c|}
\hline $\mathrm{C} 1$ & Z & -0.00597 & 0.00276 & -0.00795 & -0.00887 & -0.00144 & 0.00295 & 0.00678 \\
\hline H3 & $\mathrm{X}$ & -0.00238 & 0.02925 & 0.00310 & 0.00290 & 0.00798 & -0.06295 & -0.04396 \\
\hline H3 & $\mathrm{Y}$ & -0.11032 & -0.02621 & -0.08450 & -0.04528 & -0.08548 & 0.22234 & 0.16197 \\
\hline 13 & Z & -0.01768 & -0.02015 & -0.02030 & 0.00118 & -0.00658 & 0.04076 & -0.00151 \\
\hline 4 & $\mathrm{x}$ & -0.02095 & -0.01237 & -0.01213 & 0.02355 & 0.01185 & -0.02633 & \\
\hline 4 & $\mathrm{Y}$ & -0.09486 & -0.03875 & -0.06987 & -0.01585 & -0.04963 & 0.13040 & -0.1 \\
\hline & Z & -0.00533 & -0.00364 & -0.01515 & -0.00780 & -0.00181 & 0.00995 & -0 \\
\hline 2 & $\mathrm{X}$ & -0.00758 & -0.02429 & -0.03256 & -0.00247 & -0.00092 & -0.00419 & 0 \\
\hline 2 & $\mathrm{Y}$ & -0.03270 & -0.01768 & -0.12284 & -0.01206 & 0.00864 & -0.03105 & -0 \\
\hline 2 & Z & -0.00767 & -0.00376 & -0.00985 & -0.00259 & -0.01500 & -0.01161 & \\
\hline 1 & $\mathrm{X}$ & -0.00633 & -0.00668 & 0.00223 & 0.00439 & -0.00621 & -0.00 & \\
\hline 1 & $\mathrm{Y}$ & -0 & 0.025 & -0.17 & -0 . & -0.00 & -0 . & -0 \\
\hline 1 & Z & -0 & -0.03090 & -0.04794 & -0.008 & -0.00631 & -0.00799 & \\
\hline $\mathrm{C} 3$ & $\mathrm{X}$ & -0 & -0.05153 & -0.077 & -0.01 & 0.01194 & -0.00 & -0 \\
\hline C3 & $\mathrm{Y}$ & -0 & 0 . & -0 . & -0 & 0.0 & -0 . & \\
\hline C3 & Z & -0.01102 & -0.00174 & -0.00039 & -0 & -0.00682 & -0.00 & 0 \\
\hline O4 & $\mathrm{x}$ & 0.00461 & -0.04631 & -0 & -0.00942 & 0.01153 & -0.00219 & -0 \\
\hline 04 & $\mathrm{Y}$ & & & & & -0.005 & -0.0 & \\
\hline 04 & Z & 0 . & 0.0728 & 0.108 & 0.019 & 0.00790 & 0.016 & -0.0 \\
\hline H8 & $\mathrm{X}$ & 0 . & 0 . & 0 . & & 0 & 0 . & -0 \\
\hline H8 & $\mathrm{Y}$ & & 0.00 & & 0.00 & -0.035 & 0.06 & -0 \\
\hline H8 & Z & 0.00502 & 0.090 & 0.136 & 0.025 & 0.01217 & 0.021 & -0.0 \\
\hline 05 & $\mathrm{X}$ & & -0 & -0.036 & -0.00 & -0.00046 & -0.0 & \\
\hline & $\mathrm{Y}$ & 0.0 & 0.0 & 0 & 0 & -0.001 & 0.0 & -0 \\
\hline & Z & -0.012 & -0.01 & -0.01 & -0.0 & -0.001 & -0.0 & -0 \\
\hline
\end{tabular}

\begin{tabular}{|c|c|c|c|c|c|c|c|c|}
\hline & 638.60 & 704.47 & 722.81 & 729.33 & 757.55 & 803.59 & 830.45 \\
\hline \multicolumn{2}{|c|}{ intensities } & 58.14 & 37.60 & 122.81 & 1.67 & 19.02 & 30.39 & 100.47 \\
\hline $\operatorname{Re} 1$ & $\mathrm{x}$ & 0.00145 & -0.00575 & -0.00183 & -0.00666 & 0.00601 & 0.00106 & \\
\hline $\operatorname{Re} 1$ & $\mathrm{Y}$ & -0.00025 & -0.00159 & 0.00055 & -0.00220 & -0.00005 & 0.00070 & 0.00381 \\
\hline $\operatorname{Re} 1$ & Z & -0.00307 & 0.00177 & 0.00066 & 0.00302 & 0.00021 & & \\
\hline N1 & $\mathrm{x}$ & & & -0.00737 & & & & \\
\hline N1 & $\mathrm{Y}$ & & & -0.02110 & & 0.00063 & -0.00497 & \\
\hline N1 & z & 921 & -0.00315 & -0.000 & 0.00052 & -0.00627 & 0.07102 & -0.00688 \\
\hline C4 & $\mathrm{x}$ & -0.0 & & & -0.00 & & & \\
\hline C4 & $\mathrm{Y}$ & -0. & -0.0 & -0.0 & & & & \\
\hline C4 & Z & & 0.0 & -0.0 & 0.00212 & -0.001 & -0.13 & \\
\hline C7 & $\mathrm{x}$ & -0.0 & & -0 & & -0.0 & & \\
\hline C7 & $\mathrm{Y}$ & & -0.0 & -0 & & -0.00 & -0 & -0 . \\
\hline C7 & Z & & -0.0 & -0 & & 0.00 & -0 & \\
\hline N2 & $\mathrm{x}$ & 0. & -0. & -0 . & 0.0 & -0.0 & -0 . & \\
\hline $\mathrm{N} 2$ & Y & & -0.0 & -0.0 & 60 & -0.0 & -0. & -0 . \\
\hline & $\mathrm{z}$ & 2 & & -0. & & & & \\
\hline & $\mathrm{x}$ & -0 . & -0 & & -0 . & 0 . & & -0 \\
\hline 18 & $\mathrm{Y}$ & -0. & & & -0 . & & -0. & \\
\hline 18 & $\mathrm{z}$ & -0. & -0. & & -0 . & 0.0 & 0 & -0.0 \\
\hline & $\mathrm{x}$ & & & & -0 . & -0. & -0 & \\
\hline 21 & $\mathrm{Y}$ & -0 . & & & -0 . & & & \\
\hline H21 & Z & & & & -0.0 & & -0.14 & \\
\hline & $\mathrm{x}$ & -0.0 & & & -0.0 & & & \\
\hline 23 & $\mathrm{Y}$ & -0 & & & -0 . & & -0 . & \\
\hline H23 & Z & & & 0.0 & -0.03 & 0.8 & -0.0 & \\
\hline H26 & $\mathrm{x}$ & & & & -0.02 & & -0.2 & \\
\hline H26 & $\mathrm{Y}$ & -0.0 & & & -0 . & & & \\
\hline H 26 & Z & -0.0 & 0.0 & 0.1 & -0.05 & 0.0 & -0.0 & \\
\hline 01 & $\mathrm{x}$ & -0.0 & & -0.0 & & & -0 & \\
\hline & $\mathrm{Y}$ & & -0.0 & & & & & \\
\hline $\mathrm{O} 1$ & Z & & -0.0 & & -0.00 & -0.0 & 0.0 & \\
\hline 2 & $\mathrm{x}$ & 0.0 & -0.0 & & & -0.00 & -0.00 & .0 \\
\hline & Y & & & & & & & \\
\hline 02 & Z & -0.00647 & -0.00322 & 0.00050 & -0.00615 & 0.00583 & -0.00515 & 0.0104 \\
\hline
\end{tabular}




\begin{tabular}{|c|c|c|c|c|c|c|c|c|}
\hline 03 & $\mathrm{x}$ & -0.00549 & -0.00911 & 0.00138 & 0.00138 & -0.00649 & 0.00226 & 0.02279 \\
\hline 03 & $\mathrm{Y}$ & 0.00811 & 0.01458 & 0.00043 & -0.00101 & 0.00118 & -0.00208 & -0.01354 \\
\hline 03 & Z & .00206 & 0.00434 & 0.00531 & -0.00094 & 0.00012 & -0.00065 & -0.00954 \\
\hline C1 & $\mathrm{x}$ & 0.00362 & 0.01666 & -0.00453 & -0.01587 & -0.00300 & -0.00181 & -0.00477 \\
\hline C1 & Y & -0.00779 & -0.00722 & 0.01074 & 0.01575 & 0.00729 & -0.00551 & -0.01321 \\
\hline $\mathrm{C} 1$ & Z & 0.00727 & 0.03411 & -0.01603 & -0.03392 & -0.00340 & 0.00348 & 0.01172 \\
\hline H3 & $\mathrm{x}$ & -0.03891 & -0.13473 & 0.04698 & 0.11241 & 0.00266 & -0.00461 & 0 \\
\hline H3 & Y & 0.12391 & 0.44854 & -0.13929 & -0.36466 & 0.02352 & 0.01040 & -0.03006 \\
\hline H3 & Z & 0.00835 & 0.04337 & -0.02344 & -0.04649 & -0.01702 & 0.00126 & \\
\hline H4 & $\mathrm{x}$ & 0.02852 & 0.09903 & -0.05217 & -0.11025 & -0.02266 & & \\
\hline H4 & $\mathrm{Y}$ & -0.13199 & -0.44507 & 0.18980 & 0.41028 & 0.00866 & -0.04129 & -0.12481 \\
\hline H4 & Z & -0.02939 & -0.09345 & 0.03607 & 0.08065 & -0.00432 & & \\
\hline C2 & $\mathrm{x}$ & 0.01949 & 0.09287 & 0.05137 & 0.13467 & -0.08853 & -0.00200 & \\
\hline C2 & $\mathrm{Y}$ & 0.01600 & 0.01713 & -0.00983 & -0.02088 & -0.01009 & -0.01431 & -0 . \\
\hline C2 & Z & 234 & & & 0.04213 & -0.04607 & & \\
\hline $\mathrm{H} 1$ & $\mathrm{x}$ & 5499 & 0.1 & 0.08527 & & -0.16480 & -0 & -0 \\
\hline H1 & $\mathrm{Y}$ & 0.01432 & -0.02834 & -0.00032 & -0.01810 & -0.60217 & 0.08616 & \\
\hline $\mathrm{H} 1$ & Z & 992 & -0. & -0.0 & -0.03477 & 1408 & -0 . & -0 \\
\hline C3 & $\mathrm{x}$ & -0.0 & & -0.0 & & & & \\
\hline C3 & Y & -0.02661 & 779 & 0.00730 & 0.08763 & 0.14928 & 1922 & \\
\hline C3 & Z & 063 & -0.0 & -0.0 & -0.0 & 945 & & \\
\hline 04 & $\mathrm{x}$ & -0.0 & & -0. & -0.0 & -0 . & & \\
\hline 04 & Y & -0. & -0.0 & -0. & -0.03641 & -0.04930 & 0330 & -0 \\
\hline 04 & Z & -0.0 & 0.0 & 0.0 & 2187 & -0.01126 & & \\
\hline H8 & $\mathrm{x}$ & -0 . & & & & -0 & & \\
\hline H8 & $\mathrm{Y}$ & 473 & 0.2 & 0.0 & 0.2 & 0.22505 & & \\
\hline H8 & $\mathrm{z}$ & -0.0 & 0.0 & 0.0 & 0.04865 & -0.02925 & -0 & -0 \\
\hline 05 & $\mathrm{x}$ & & -0 & -0 & -0 & -0 & & \\
\hline 05 & $\mathrm{Y}$ & -0.0 & -0. & -0.0 & -0.01666 & -0.05395 & -0 . & -0.03576 \\
\hline 05 & Z & -0.06164 & -0.0 & -0.0 & -0.03412 & 0.02262 & -0 . & \\
\hline \multicolumn{2}{|c|}{ frequencies } & & & & & & & \\
\hline \multicolumn{2}{|c|}{ intensities } & & & & & & & \\
\hline $\operatorname{Re} 1$ & $\mathrm{x}$ & 0.00 & 0.00 & -0.0 & -0 . & & & \\
\hline Re1 & $\mathrm{Y}$ & 039 & 0.0 & -0.0 & -0.01675 & -0.00659 & -0 & \\
\hline $\operatorname{Re} 1$ & Z & 025 & 0.0 & & -0.01227 & 0.01543 & -0 . & \\
\hline N1 & $\mathrm{x}$ & 73 & -0 & -0 . & -0 & & & \\
\hline N1 & Y & 136 & -0.0 & 0.0 & -0.00111 & -0.00518 & 023 & -0.06 \\
\hline N1 & Z & 640 & 0.0 & -0.0 & 0.00163 & 0680 & 240 & -0 \\
\hline C4 & $\mathrm{x}$ & & -0 . & -0 . & -0 & & & \\
\hline C4 & Y & & -0. & -0 . & & 383 & -0 & \\
\hline C4 & Z & 0.02897 & & & 0.01662 & $-0 . c$ & -0 . & \\
\hline C7 & $\mathrm{x}$ & 99 & & & & & & \\
\hline C7 & $\mathrm{Y}$ & -0 & & & -0.0 & -0 . & & \\
\hline C7 & Z & -0.0 & -0.09852 & -0.0 & -0.00728 & 0769 & 0.03073 & 303 \\
\hline N2 & $\mathrm{x}$ & & & -0 & & & & \\
\hline N2 & $\mathrm{Y}$ & -0 . & & & & -0 & & \\
\hline N2 & Z & -0.0 & -0.0 & -0.0 & -0.00666 & -0.00014 & -0.01738 & -0.00187 \\
\hline H18 & $\mathrm{x}$ & & -0.3 & & & -0 & & \\
\hline & $\mathrm{Y}$ & -0 & & & -0 & & & \\
\hline H18 & Z & -0.1 & -0.1 & -0.0 & 0.0 & -0.00888 & -0 & \\
\hline H21 & $\mathrm{x}$ & -0. & -0. & -0. & -0.0 & & & \\
\hline H21 & $\mathrm{Y}$ & -0.3 & -0. & -0. & -0.0 & -0 & & \\
\hline H21 & Z & -0. & & & & -0 & & -0 \\
\hline H23 & $\mathrm{x}$ & & & & & $-0 . c$ & -0.1 & \\
\hline H23 & $\mathrm{Y}$ & & & & -0.01150 & -0.00399 & & \\
\hline H23 & Z & & -0.25772 & -0.0 & -0.01910 & 0.03810 & -0.17637 & -0 \\
\hline & $\mathrm{x}$ & & -0.2 & -0 & -0 & & & \\
\hline H26 & $\mathrm{Y}$ & & & & & & & \\
\hline H26 & Z & & -0.16055 & -0.00887 & -0.01602 & & & \\
\hline 01 & $\mathrm{x}$ & -0.00312 & -0.00 & 0.0 & 0.06140 & 0.02113 & 0.00027 & -0 \\
\hline
\end{tabular}




\begin{tabular}{|c|c|c|c|c|c|c|c|c|}
\hline 01 & $\mathrm{Y}$ & -0.01093 & -0.01266 & 0.01438 & 0.20382 & 0.07241 & 0.00189 & -0.01036 \\
\hline 01 & Z & 0.00035 & -0.00365 & 0.00211 & 0.07202 & 0.02239 & 0.00105 & -0.00559 \\
\hline 02 & $\mathrm{x}$ & -0.00084 & -0.00453 & -0.01891 & 0.02251 & -0.06260 & 0.00007 & 0.00058 \\
\hline 02 & $\mathrm{Y}$ & -0.00016 & 0.00218 & 0.00395 & -0.00454 & 0.01883 & -0.00009 & 0.00089 \\
\hline $\mathrm{O} 2$ & Z & -0.00199 & -0.01517 & -0.03972 & 0.06923 & -0.21266 & -0.00025 & 0.00263 \\
\hline 03 & $\mathrm{x}$ & 0.00095 & 0.00051 & -0.00378 & 0.00105 & 0.00202 & -0.00102 & -0.01414 \\
\hline 03 & $\mathrm{Y}$ & -0.00162 & -0.00037 & 0.00198 & 0.00010 & -0.00450 & 0.00064 & 0.01262 \\
\hline 03 & Z & -0.00377 & 0.00171 & 0.00135 & 0.00030 & -0.00119 & 0.00044 & 0.00867 \\
\hline $\mathrm{C} 1$ & $\mathrm{x}$ & 0.00173 & 0.00171 & 0.00150 & -0.00187 & 0.00008 & 0.00186 & 0.02726 \\
\hline $\mathrm{C} 1$ & $\mathrm{Y}$ & 0.00264 & 0.00310 & 0.00249 & -0.00465 & -0.00338 & -0.00169 & -0.03385 \\
\hline $\mathrm{C} 1$ & Z & 0.00061 & -0.00176 & 0.00486 & 0.00057 & 0.00013 & -0.00048 & -0.01446 \\
\hline H3 & $\mathrm{x}$ & 0.00312 & 0.00142 & -0.00788 & -0.00719 & -0.00500 & & \\
\hline H3 & $\mathrm{Y}$ & -0.01643 & -0.00630 & 0.02748 & 0.01766 & 0.01455 & & \\
\hline H3 & Z & 0.00662 & 0.00127 & 0.00595 & 0.00006 & 0.00040 & 0.00035 & \\
\hline H4 & $\mathrm{x}$ & 0.00008 & -0.0 & 0.00761 & -0.00742 & -0.00157 & -0 & \\
\hline H4 & Y & -0.00325 & 0.0 & -0.06047 & 0.01827 & 0.02758 & & \\
\hline H4 & Z & -0.00078 & 0.00365 & -0.01244 & 0.00612 & 0.00924 & 0343 & \\
\hline C2 & $\mathrm{x}$ & 179 & -0.0 & 0.0 & 674 & -0 & & \\
\hline $\mathrm{C} 2$ & Y & 0.0 & -0.0 & & -0.0 & -0 & -0 & -0 \\
\hline C2 & Z & -0.00027 & 0.02264 & -0.17571 & 0.00156 & 0.02984 & 0057 & -0.00599 \\
\hline $\mathrm{H} 1$ & $\mathrm{x}$ & 0. & -0. & 0.2 & -0.00134 & -0 & & -0 \\
\hline $\mathrm{H} 1$ & & -0. & -0. & -0. & -0.0 & & & \\
\hline $\mathrm{H} 1$ & Z & -0.0 & 0.0 & -0.4 & 0.01193 & 6482 & -0 & \\
\hline C3 & $\mathrm{x}$ & -0.0 & -0.0 & 0.0 & -0.00129 & -0 & & \\
\hline C3 & Y & -0 & & & & & & \\
\hline C3 & Z & -0.0 & -0.0 & 0.0 & 0175 & -0.00631 & -0 & -0 \\
\hline 04 & $\mathrm{x}$ & 0.0 & & -0.0 & & 0.01719 & & -0 \\
\hline 04 & Y & -0 & -0 . & & -0 & -0 & & \\
\hline 04 & $\mathrm{Z}$ & 0 & -0 & 0. & & -0 & & -0 \\
\hline H8 & $\mathrm{x}$ & & & $-0.2+x+2$ & & & & \\
\hline H8 & $\mathrm{Y}$ & 0 . & & & -0 & -0 . & & -0 \\
\hline H8 & Z & 0. & -0 & -0.0 & 256 & -0 & -0 & \\
\hline 05 & $\mathrm{x}$ & -0. & -0 & & -0 & -0 . & & \\
\hline 05 & $\mathrm{Y}$ & & -0 . & -0. & & 260 & & -0 \\
\hline 05 & Z & 0.00168 & -0.00441 & 0.06160 & & -0.01118 & & \\
\hline \multicolumn{2}{|c|}{ Erequencies } & & & & & & & \\
\hline \multicolumn{2}{|c|}{ intensities } & & & & & 4.81 & & \\
\hline $\operatorname{Re} 1$ & $\mathrm{x}$ & -0.00093 & -0 & -0.0 & -0.00 & 0.00007 & -0 & -0.0 \\
\hline $\mathrm{Re}$ & $\mathrm{Y}$ & & -0 . & -0 . & & & & \\
\hline $\operatorname{Re} 1$ & z & 26 & & -0.0 & -0.00030 & 0009 & 117 & \\
\hline N1 & $\mathrm{x}$ & -0.0 & -0.0 & 0.0 & 0.02599 & 0114 & 505 & -0.01265 \\
\hline N1 & $\mathrm{Y}$ & -0. & & -0 . & & -0 & & \\
\hline N1 & z & -0 & -0 & 0 & -0.02235 & -0 & -0 & -0 \\
\hline C4 & $\mathrm{x}$ & 641 & -0.0 & 0. & -0.00500 & -0.00096 & -0 & -0.00306 \\
\hline C4 & $\mathrm{Y}$ & & -0 & & -0 & & & \\
\hline C4 & $\mathrm{z}$ & & & -0 . & & & & \\
\hline C7 & $\mathrm{x}$ & 0. & -0.0 & 0.0 & -0.02381 & -0.00175 & -0.00280 & -0.00435 \\
\hline C7 & $\mathrm{Y}$ & & & -0 & -0.00363 & & & \\
\hline C7 & $\mathrm{Z}$ & & -0 . & & & & & \\
\hline N2 & $\mathrm{x}$ & 0. & -0.0 & & 3624 & 0.0 & 124 & \\
\hline N2 & $\mathrm{Y}$ & -0. & & -0. & & -0 & & \\
\hline N2 & Z & -0. & -0.0 & & -0.0 & -0 & -0 & \\
\hline $\mathrm{H} 18$ & $\mathrm{x}$ & & & -0.574 & & 0 & & \\
\hline H18 & $\mathrm{Y}$ & & -0. & & -0.016 & -0 & -0 & \\
\hline H18 & z & & & -0.2 & & & & \\
\hline H21 & $\mathrm{x}$ & -0.00460 & -0.2 & & -0.01180 & -0.0 & -0 & \\
\hline & $\mathrm{Y}$ & 56 & -0.0 & 0.0 & -0.01 & -0.00024 & -0.0 & \\
\hline H21 & z & -0.06427 & & -0.24378 & & & & \\
\hline H23 & $\mathrm{x}$ & -0.00756 & -0.00223 & & -0.02151 & -0.00203 & & -0.00348 \\
\hline H23 & Y & -0.05296 & 0.01354 & -0.0 & -0.13744 & -0.00709 & -0.02546 & 0.0 \\
\hline
\end{tabular}




\begin{tabular}{|c|c|c|c|c|c|c|c|c|}
\hline $\mathrm{H} 23$ & z & -0.00600 & -0.08118 & 0.13681 & 0.53587 & 0.01815 & 0.09451 & -0.08373 \\
\hline H26 & & 0.00326 & 0.04854 & -0.09103 & -0.61173 & -0.02256 & -0.09438 & -0.02865 \\
\hline H26 & Y & 0.07080 & 0.00249 & 0.01763 & 0.19996 & 0.00999 & 0.03101 & 0.01327 \\
\hline H26 & Z & 0.01281 & 0.01528 & -0.03241 & -0.37090 & -0.01314 & -0.05435 & -0.01557 \\
\hline 01 & $\mathrm{x}$ & 0.00015 & 0.00106 & 0.00265 & -0.00075 & -0.00007 & -0.00112 & -0.00110 \\
\hline 01 & $\mathrm{Y}$ & 0.00348 & 0.00298 & 0.00423 & -0.00304 & -0.00016 & -0.00490 & -0.00260 \\
\hline 01 & Z & 0.00259 & & 0.00203 & -0.00069 & -0.00013 & -0.00161 & -0.00060 \\
\hline $\mathrm{O} 2$ & $\mathrm{x}$ & -0.00052 & -0.00449 & 0.00115 & -0.00004 & -0.00014 & -0.00148 & 0.00002 \\
\hline $\mathrm{O} 2$ & Y & -0.00221 & -0.00043 & 0.00004 & -0.00016 & 0.00017 & -0.00065 & -0.00276 \\
\hline 02 & Z & -0.00190 & -0.01445 & 0.00776 & 0.00148 & & & -0.00101 \\
\hline 03 & $\mathrm{x}$ & 0.02906 & 0.00736 & 0.00027 & 0.00011 & 0.02756 & -0.00085 & 0.13782 \\
\hline 03 & $\mathrm{Y}$ & -0.02782 & -0.0 & -0.00338 & 0.00116 & -0.00095 & & \\
\hline 03 & Z & -0.01673 & -0.00313 & 0.00303 & -0.00138 & 0.05671 & -0.00334 & -0.06335 \\
\hline $\mathrm{C} 1$ & $\mathrm{x}$ & -0.06164 & -0.01654 & -0.00323 & 0.00051 & -0.05067 & 0.00216 & -0.12918 \\
\hline $\mathrm{C} 1$ & Y & 7304 & 0.0 & 0.01402 & -0.00192 & 0.00525 & -0. & \\
\hline $\mathrm{C} 1$ & Z & 3058 & 0.0 & -0.00075 & 0.00345 & -0.10616 & 0936 & \\
\hline H3 & $\mathrm{x}$ & 0.21204 & 0.04096 & 0.01531 & 0.00813 & -0.44716 & 0.01725 & -0.26816 \\
\hline H3 & $\mathrm{Y}$ & -0.5 & -0.1 & -0.0 & 1395 & -0.1 & 2337 & \\
\hline H3 & Z & -0.0 & -0.0 & 0.0 & -0.0 & & -0 & \\
\hline H4 & $\mathrm{x}$ & 0.14753 & 0.05589 & 0.03842 & -0.02357 & 0.59298 & -0.04139 & -0.35332 \\
\hline H4 & $\mathrm{Y}$ & -0.4 & -0.1 & -0.0 & 0406 & 3073 & -0 & -0 \\
\hline H4 & Z & -0.1 & -0. & -0. & & -0 . & & \\
\hline C2 & $\mathrm{x}$ & -0. & 0.0 & 0.0 & -0.01545 & 0486 & & \\
\hline $\mathrm{C} 2$ & Y & 799 & 0.0 & 0. & -0.00041 & 096 & & \\
\hline $\mathrm{C} 2$ & Z & 48 & -0 & -0 & & -0 & & \\
\hline $\mathrm{H} 1$ & $\mathrm{x}$ & 042 & -0.2 & -0.14 & 0.06222 & -0.00485 & -0 & \\
\hline $\mathrm{H} 1$ & Y & -0.0 & -0.0 & -0.02 & -0.01018 & -0.02535 & & -0 \\
\hline $\mathrm{H} 1$ & $\mathrm{Z}$ & -0 & & & -0 & & & \\
\hline C3 & $\mathrm{x}$ & -0. & 0. & & & -0 & -0 & -0 \\
\hline C3 & $\mathrm{Y}$ & 0. & -0 & -0 & & & & -0 \\
\hline C3 & $\mathrm{z}$ & 10 & -0 . & -0 . & & & & \\
\hline 04 & $\mathrm{x}$ & 560 & -0. & -0.0 & -0.00764 & 0711 & & \\
\hline 04 & $\mathrm{Y}$ & -0.0 & & & & -0 & -0 & -0 \\
\hline 04 & $\mathrm{z}$ & & -0 . & -0 . & -0 & & & -0 \\
\hline H8 & $\mathrm{x}$ & -0.06440 & 0.3 & 0.1 & 0.08476 & -0.04161 & -0 & 5924 \\
\hline H8 & $\mathrm{Y}$ & 0.02212 & -0.0 & -0.0 & -0.02052 & & & -0 . \\
\hline H8 & Z & -0 & & & & -0 & -0 & \\
\hline 05 & $\mathrm{x}$ & -0.0 & & & & -0.0 & -0 & \\
\hline 05 & $\mathrm{Y}$ & 132 & -0.0 & -0. & -0.0 & & & \\
\hline 05 & z & -0.01592 & 0 & & & -0 & -0 & -0 \\
\hline \multicolumn{2}{|c|}{ frequencies } & & & & & & & \\
\hline \multicolumn{2}{|c|}{ intensities } & & & & & & & \\
\hline & $\mathrm{X}$ & & & & & 13 & -0.0 & \\
\hline $\operatorname{Re} 1$ & $\mathrm{Y}$ & -0.0 & 0.0 & 0. & -0.00095 & 0001 & -0.00006 & -0.0 \\
\hline $\operatorname{Re} 1$ & Z & & & & -0. & -0 . & & \\
\hline N1 & $\mathrm{x}$ & -0 . & -0 & -0 . & & & & -0 \\
\hline N1 & $\mathrm{Y}$ & 0.0 & 0. & 0. & -0.00026 & -0.01480 & -0.01825 & 374 \\
\hline N1 & z & -0.0 & -0 & -0 . & & & & \\
\hline C4 & $\mathrm{x}$ & -0 . & -0 . & -0 . & -0 . & -0 & -0 & \\
\hline C4 & $\mathrm{Y}$ & -0.0 & -0.0 & -0.0 & & 1079 & 2222 & -0 . \\
\hline C4 & Z & & & & & & & \\
\hline C7 & $\mathrm{x}$ & -0. & & & & -0 & & \\
\hline C7 & $\mathrm{Y}$ & -0. & & & & & -0 & 721 \\
\hline C7 & Z & & -0. & -0.0 & & -0.1 & & -0 \\
\hline N2 & $\mathrm{x}$ & & -0. & -0.0 & -0.00071 & & -0 . & \\
\hline N2 & $\mathrm{Y}$ & -0.0 & & & & -0.0 & 2916 & -0 . \\
\hline $1 \sqrt{2}$ & $\mathrm{z}$ & & -0. & -0 & & 0 & -0 & \\
\hline H18 & $\mathrm{x}$ & & & & & & & \\
\hline H18 & Y & -0.06166 & -0.00278 & -0.04880 & & & & -0.0227 \\
\hline H1 8 & Z & 0.12132 & 0.00336 & 0.09 & 0.00938 & -0.04984 & -0.05432 & 0.0 \\
\hline
\end{tabular}




\begin{tabular}{|c|c|c|c|c|c|c|c|c|}
\hline H21 & X & 0.52707 & & 0.40674 & 0.00427 & 0.02390 & 0.07647 & 0.00302 \\
\hline H21 & $\mathrm{Y}$ & -0.00934 & -0.00208 & -0.01740 & -0.00318 & 0.01531 & 0.03012 & -0.00431 \\
\hline H21 & Z & 0.25065 & -0.02559 & -0.14514 & -0.00528 & -0.07500 & -0.16166 & \\
\hline 23 & $\mathrm{X}$ & -0.03886 & 0.01197 & 0.09630 & 0.00009 & -0.06817 & 0.09091 & -0.01195 \\
\hline H23 & $\mathrm{Y}$ & 0.12982 & -0.02078 & -0.14075 & -0.00323 & -0.01272 & 0.02207 & -0.00606 \\
\hline H23 & Z & -0.46579 & & 0.46089 & & & & \\
\hline 26 & $\mathrm{X}$ & -0.20669 & 0.02408 & 0.27432 & -0.00132 & 0.01884 & 0.02620 & 0.00004 \\
\hline H26 & $\mathrm{Y}$ & 0.06307 & -0.00840 & -0.08495 & -0.00320 & -0.03088 & 0.01017 & -0.00300 \\
\hline H26 & Z & -0.11550 & 0.01665 & 0.15072 & -0.00131 & 0.09638 & -0.05057 & \\
\hline $\mathrm{O} 1$ & $\mathrm{X}$ & -0.00028 & -0.00123 & 0.00013 & 0.00106 & & 0.00061 & \\
\hline $\mathrm{O} 1$ & $\mathrm{Y}$ & -0.00069 & -0.00368 & -0.00048 & 0.00186 & 0.00121 & 0.00089 & 0.00195 \\
\hline $\mathrm{O} 1$ & z & 0.00020 & -0.00153 & -0.00021 & 0.00040 & 0.00036 & -0.00041 & 0.00127 \\
\hline $\mathrm{O} 2$ & $\mathrm{x}$ & -0.00103 & -0.00116 & 0.00011 & -0.00092 & -0.00077 & & \\
\hline $\mathrm{O} 2$ & $\mathrm{Y}$ & -0.00 & -0.00018 & -0.00006 & 0.00124 & -0.00021 & -0.00025 & \\
\hline $\mathrm{O} 2$ & Z & -0.00 & 0.00230 & -0.00258 & -0.00101 & -0.00105 & -0.00172 & 0.00036 \\
\hline 03 & $\mathrm{x}$ & -0.0 & -0.0 & & -0.0 & & & \\
\hline 03 & $\mathrm{Y}$ & -0.0 & 0229 & -0.00014 & -0.00260 & -0.0 & & -0.0 \\
\hline 03 & z & & 0778 & -0.00022 & & -0.0 & -0.0 & -0.0 \\
\hline C1 & $\mathrm{x}$ & & & -0 . & & & -0 . & \\
\hline C1 & $\mathrm{Y}$ & 0.01221 & 0.00099 & & & -0. & & \\
\hline $\mathrm{C} 1$ & z & -0.01008 & -0.00562 & 0.00026 & -0.04824 & & & -0. \\
\hline H3 & $\mathrm{X}$ & & 0 . & & -0 . & & & \\
\hline H3 & $\mathrm{Y}$ & & 2569 & -0.0 & -0.2 & & & -0 \\
\hline H3 & Z & -0.04260 & -0.02399 & -0.00283 & & -0.0 & -0.00463 & \\
\hline H4 & $\mathrm{x}$ & & & & -0 . & -0 & & -0 \\
\hline H4 & $\mathrm{Y}$ & & 1091 & -0. & -0.2 & -0 . & & -0 . \\
\hline H4 & z & -0.01001 & -0.00064 & -0.0 & -0.15264 & & 087 & -0 . \\
\hline C2 & $\mathrm{x}$ & -0 . & & -0 . & -0 & -0 & & \\
\hline C2 & $\mathrm{Y}$ & -0.0 & -0.00448 & -0.00119 & -0 . & & -0 & -0 . \\
\hline $\mathrm{C} 2$ & z & 0073 & -0.07106 & 0.01333 & 0. & & 0013 & 1148 \\
\hline $\mathrm{H} 1$ & $\mathrm{x}$ & -0 . & -0 & & -0 . & & -0 & \\
\hline $\mathrm{H} 1$ & & & & & & -0 & & -0 \\
\hline H1 & z & -0 . & 799 & -0 & & -0 . & 00 & 7180 \\
\hline C3 & $\mathrm{x}$ & & -0 & & & & -0 & -0 \\
\hline C3 & $\mathrm{Y}$ & -0 . & & -0 . & -0 . & -0 . & & \\
\hline C3 & Z & & & -0 & & & -0.02218 & -0 \\
\hline 04 & $\mathrm{x}$ & & & -0 & & -0 . & & \\
\hline 04 & $\mathrm{Y}$ & -0 & -0 & & -0 & & -0 . & -0 \\
\hline 04 & Z & & -0.0 & 78 & -0 . & & & 993 \\
\hline H8 & $\mathrm{x}$ & & & -0 & & & & -0 . \\
\hline H8 & $\mathrm{Y}$ & & -0. & & -0 . & -0 & & \\
\hline H8 & z & -0.0 & & -0.0 & & & -0 & -0 \\
\hline 05 & $\mathrm{x}$ & & & -0 & -0 . & -0 . & & \\
\hline 0 & $\mathrm{Y}$ & & -0. & & & & -0 . & -0 . \\
\hline 05 & z & -0.00608 & -0.01617 & -0.00446 & -0.0 & -0 . & 0.01 & 0.1 \\
\hline \multicolumn{2}{|c|}{ fequencies } & & & & & & & \\
\hline & & & & & & & & \\
\hline Re1 & $\mathrm{X}$ & 0.00003 & 0.00000 & -0.00001 & & -0 & 0010 & -0.00001 \\
\hline & $\mathrm{Y}$ & & -0.0 & & -0 . & & & \\
\hline & Z & -0 & & & & & & \\
\hline N1 & $\mathrm{X}$ & 002 & -0.00019 & 0.00145 & 0312 & -0.0 & -0.04259 & 0.00043 \\
\hline N1 & $\mathrm{Y}$ & & -0.00004 & -0.0 & -0 . & -0 . & -0 . & \\
\hline & Z & & & & & & & -0 . \\
\hline C4 & $\mathrm{x}$ & & & -0.02673 & -0.03422 & & & 0037 \\
\hline C4 & $\mathrm{Y}$ & & -0.000 & 0.01336 & & -0.0 & -0.00109 & 0.00012 \\
\hline C4 & Z & & & -0.0 & & & & \\
\hline C7 & $\mathrm{x}$ & -0.0 & -0.00065 & & -0.0 & & & -0.00569 \\
\hline C7 & $\mathrm{Y}$ & 008 & 0.00013 & -0.01048 & 0.0 & $-0.0 c$ & 0.00002 & 0.00166 \\
\hline C7 & Z & & & & & & -0.00003 & \\
\hline N2 & $\mathrm{x}$ & -0.00018 & 0.00004 & -0.00295 & 0.00199 & -0.00017 & -0.00037 & -0.03052 \\
\hline
\end{tabular}




\begin{tabular}{|c|c|c|c|c|c|c|c|c|}
\hline N2 & $\mathrm{Y}$ & 0.00002 & 0.00000 & 0.00067 & -0.00071 & 0.00016 & 0.00002 & -0.01212 \\
\hline & $z$ & 0.00032 & -0.00017 & -0.00084 & 0.00131 & -0.00047 & -0.00014 & \\
\hline H18 & $\mathrm{x}$ & 0.00114 & 0.00206 & 0.00963 & 0.00944 & 0.00058 & 0.49448 & -0.00317 \\
\hline 18 & $\mathrm{Y}$ & -0.00030 & 0.00081 & 0.00188 & 0.00228 & 0.00028 & 0.11385 & -0.00063 \\
\hline 18 & & & -0.00218 & & -0.01441 & & & \\
\hline 21 & $x$ & -0.00062 & -0.00351 & 0.27606 & 0.36954 & -0.01763 & -0.00743 & -0 \\
\hline 21 & $\mathrm{Y}$ & -0.00020 & 0.00212 & -0.15034 & -0.20550 & 0.00985 & 0.00451 & 0.00045 \\
\hline 21 & Z & -0.00105 & -0.00591 & 0.46446 & 0.64012 & -0.03075 & -0.01718 & -0.00147 \\
\hline 23 & & -0.00061 & 0.00883 & -0.75955 & 0.55041 & -0.07250 & 0.00548 & \\
\hline 23 & Y & -0.00043 & -0.00123 & 0.10905 & -0.07749 & 0.01018 & -0.00004 & -0.00152 \\
\hline 23 & z & -0.00001 & -0.00022 & 0.02794 & -0.02719 & 0.00346 & -0.00320 & -0.003 \\
\hline 26 & & 0.00059 & -0.00031 & 0.01015 & -0.00611 & -0.00054 & 0.00292 & \\
\hline 26 & Y & 0.00002 & -0.00008 & 0.00350 & -0.00242 & -0.00033 & 0.00147 & \\
\hline H26 & Z & -0.00172 & 0.00068 & -0.01877 & 0.01248 & 0.00158 & -0.00592 & -0.82 \\
\hline 01 & $\mathrm{x}$ & 0.00008 & 0.00000 & 0.00000 & -0.0 & -0.00016 & -0.00019 & \\
\hline 01 & $\mathrm{Y}$ & 0.00018 & -0.00002 & 0.00000 & 0.00005 & -0.00012 & -0.00018 & -0 \\
\hline $\mathrm{O} 1$ & Z & -0.00008 & -0.00005 & 0.00004 & -0.00008 & -0.00005 & -0.00024 & \\
\hline 02 & $x$ & 0.0 & 0.00 & 0.0 & & -0 & & \\
\hline & y & 0.00022 & 0.00006 & 0.0 & & -0 & -0.00002 & \\
\hline $\mathrm{O} 2$ & Z & 0.00013 & 0.00011 & 0004 & 0014 & 0 & -0.00017 & \\
\hline 03 & $\mathrm{X}$ & 0.00115 & 0.00038 & 0.00002 & -0.0 & 0.0 & -0.00038 & \\
\hline 03 & Y & 0.00110 & 0.00009 & 0.00000 & -0 & 0.0 & -0.00004 & \\
\hline 03 & Z & -0.00047 & 0.00073 & 0.00002 & 0008 & -0.0 & 0.00008 & \\
\hline $\mathrm{C} 1$ & $\mathrm{x}$ & 378 & -0.04100 & -0.00063 & 0.00028 & 0.00345 & 0.00029 & -0 \\
\hline $\mathrm{C} 1$ & Y & 0.03032 & 0.00241 & 0.00001 & 0.0 & 0.00054 & -0.00006 & -0 . \\
\hline C1 & Z & -0.02030 & -0.08824 & -0.00136 & 0047 & 0.00694 & 0.00014 & -0 . \\
\hline H3 & $\mathrm{X}$ & -0.5 & 0.50472 & 0.00765 & -0.00389 & -0.0 & -0.00122 & \\
\hline H3 & & -0.1 & 0.15828 & 0225 & -0.0 & -0.01410 & -0.00039 & \\
\hline H3 & Z & -0.42279 & 0.41267 & 0.00647 & -0.00339 & -0.03980 & -0.00072 & 0.00087 \\
\hline H4 & & -0.02729 & -0.02810 & -0.00041 & -0.0 & 0.0 & 0.00 & \\
\hline H4 & & -0.1 & -0.18859 & -0.00267 & 0.0 & 0.0 & 0.00029 & \\
\hline H 4 & Z & 0.67246 & 0.62159 & 0.00923 & -0.00194 & -0.03970 & -0.00296 & -0.00115 \\
\hline $\mathrm{C} 2$ & $\mathrm{x}$ & -0.00156 & 0.00503 & -0.00247 & & 0.06090 & -0.00039 & \\
\hline C2 & & -0 & 0.00058 & -0.0 & 0.0 & 0.0 & -0.00034 & \\
\hline $\mathrm{C} 2$ & Z & -0.00063 & 0.00461 & -0.00196 & 0.00393 & 0.05216 & 0.00061 & 0.00008 \\
\hline $\mathrm{H} 1$ & $\mathrm{x}$ & & -0.05830 & 0.02810 & -0.05571 & -0.72435 & 0.00184 & \\
\hline $\mathrm{H} 1$ & $\mathrm{Y}$ & & -0.00684 & 0.0 & -0 & -0 & .78 & \\
\hline $\mathrm{H} 1$ & Z & 0.00915 & -0.04954 & 0.02366 & -0.04680 & -0.60931 & 0.00352 & -0.00253 \\
\hline C3 & $\mathrm{X}$ & 0.00000 & -0.00010 & -0.00015 & 0.00009 & 0.00174 & -0.00127 & -0.00009 \\
\hline C3 & $\mathrm{Y}$ & & & & & -0. & & \\
\hline C3 & Z & 0.00006 & -0.00042 & -0.00010 & -0.00023 & -0.00130 & -0.00411 & 0.00002 \\
\hline 04 & $\mathrm{x}$ & -0.00005 & 0.00001 & 0.00002 & 0.00006 & -0.00005 & 0.00071 & \\
\hline 04 & $\mathrm{Y}$ & -0.0 & -0.00002 & 0.00000 & -0.0 & & -0.00021 & \\
\hline 04 & Z & 03 & 0.00004 & -0.00002 & -0.00009 & 0.00 & 0.00094 & -0.00018 \\
\hline H8 & $\mathrm{X}$ & -0.0 & -0.00014 & -0.00021 & -0.00051 & -0.00141 & -0.00167 & -0.00071 \\
\hline H8 & $\mathrm{Y}$ & -0.00008 & -0.00023 & -0.00004 & & & 0.00015 & \\
\hline H8 & Z & -0.00014 & -0.00056 & 0.00055 & 0.00088 & -0.00086 & -0.00526 & \\
\hline 05 & $\mathrm{x}$ & 0.00002 & 0.00014 & 0.00002 & -0.000 & -0.00 & 0.00085 & 0.00005 \\
\hline 05 & $\mathrm{Y}$ & & -0.00012 & -0.00002 & -0.00003 & 0.00016 & -0.00036 & \\
\hline 05 & Z & -0.00001 & 0.00011 & 0.00010 & 0.00010 & -0.00035 & 0.00270 & 0.0000 \\
\hline
\end{tabular}

$\begin{array}{ccr}\text { frequencies } & 3741.39 \\ \text { intensities } & 193.71 \\ \text { Re1 X } & -0.00001 \\ \text { Re1 } & \text { Y } & 0.00000 \\ \text { Re1 } & \text { Z } & 0.00001 \\ \text { N1 } & X & 0.00026 \\ \text { N1 } & Y & 0.00005 \\ \text { N1 } & \text { Z } & -0.00029 \\ \text { C4 } & \text { X } & -0.00011 \\ \text { C4 } & \text { Y } & 0.00003\end{array}$




\begin{tabular}{|c|c|c|}
\hline C4 & Z & -0.00010 \\
\hline C7 & $\mathrm{X}$ & 0.00001 \\
\hline C7 & $\mathrm{Y}$ & -0.00002 \\
\hline C7 & Z & 0.00004 \\
\hline N2 & $\mathrm{x}$ & -0.00005 \\
\hline N2 & $\mathrm{Y}$ & -0.00001 \\
\hline N2 & Z & 0.00016 \\
\hline H18 & $\mathrm{x}$ & -0.00376 \\
\hline H18 & $\mathrm{Y}$ & -0.00042 \\
\hline H18 & Z & 0.00347 \\
\hline H 21 & $\mathrm{x}$ & 0.00067 \\
\hline H 21 & $\mathrm{Y}$ & -0.00035 \\
\hline H 21 & Z & 0.00099 \\
\hline H23 & $\mathrm{x}$ & 0.00024 \\
\hline H23 & $\mathrm{Y}$ & -0.00002 \\
\hline H23 & Z & 0.00019 \\
\hline H26 & $\mathrm{x}$ & 0.00110 \\
\hline H 26 & $\mathrm{Y}$ & 0.00017 \\
\hline H26 & Z & -0.00217 \\
\hline $\mathrm{O} 1$ & $\mathrm{x}$ & 0.00003 \\
\hline 01 & $\mathrm{Y}$ & 0.00000 \\
\hline 01 & z & 0.00003 \\
\hline $\mathrm{O} 2$ & $\mathrm{x}$ & -0.00001 \\
\hline $\mathrm{O} 2$ & Y & 0.00001 \\
\hline 02 & Z & -0.00016 \\
\hline 03 & $\mathrm{x}$ & 0.00011 \\
\hline 03 & $\mathrm{Y}$ & 0.00000 \\
\hline 03 & Z & -0.00005 \\
\hline $\mathrm{C} 1$ & $\mathrm{x}$ & -0.00004 \\
\hline $\mathrm{C} 1$ & $\mathrm{Y}$ & 0.00005 \\
\hline $\mathrm{C} 1$ & Z & 0.00009 \\
\hline H3 & $\mathrm{x}$ & -0.00017 \\
\hline H3 & $\mathrm{Y}$ & -0.00022 \\
\hline H3 & Z & -0.00009 \\
\hline H4 & $\mathrm{x}$ & -0.00011 \\
\hline H4 & Y & 0.00033 \\
\hline H4 & Z & -0.00031 \\
\hline C2 & $\mathrm{x}$ & 0.00022 \\
\hline C2 & $\mathrm{Y}$ & 0.00005 \\
\hline C2 & Z & -0.00070 \\
\hline $\mathrm{H} 1$ & $\mathrm{x}$ & 0.00086 \\
\hline $\mathrm{H} 1$ & $\mathrm{Y}$ & -0.00088 \\
\hline H1 & Z & -0.00070 \\
\hline C3 & $\mathrm{x}$ & -0.00092 \\
\hline C3 & $\mathrm{Y}$ & 0.00016 \\
\hline C3 & Z & 0.00209 \\
\hline 04 & $\mathrm{x}$ & -0.01529 \\
\hline 04 & $\mathrm{Y}$ & 0.00338 \\
\hline 04 & Z & 0.05768 \\
\hline H8 & $\mathrm{x}$ & 0.23806 \\
\hline H8 & $\mathrm{Y}$ & -0.05130 \\
\hline H8 & Z & -0.93601 \\
\hline 05 & $\mathrm{x}$ & 0.00082 \\
\hline 05 & $\mathrm{Y}$ & -0.00025 \\
\hline 05 & z & 0.00036 \\
\hline
\end{tabular}

Thermochemical Properties:

pressure: $\quad 1.0000$ atm

rotational symmetry number: 1

vibrational frequencies below $10.0 \mathrm{~cm}-1$ excluded: 1 


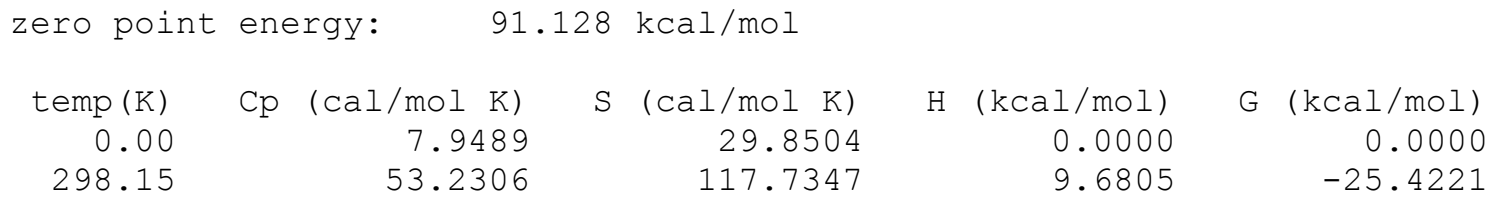

end of program freq

start of program ch

Moments from quantum mechanical wavefunction:

Dipole Moments (Debye)
$\mathrm{X}=$
$-4.0540 \quad \mathrm{Y}=$
4.4975
$\mathrm{Z}=$
-3.7173 Tot $=$
7.1050

Final energy ........... $\quad-759.81007687334$

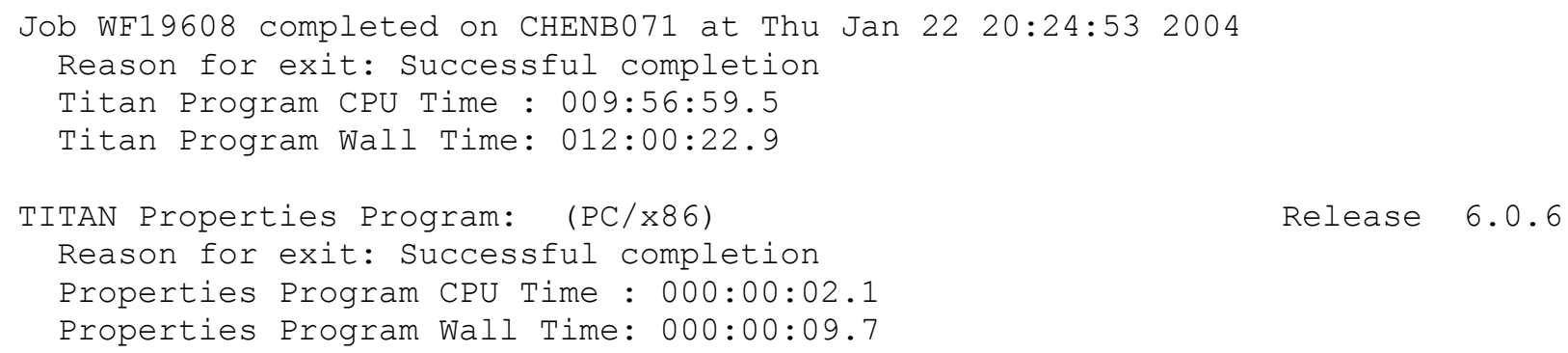




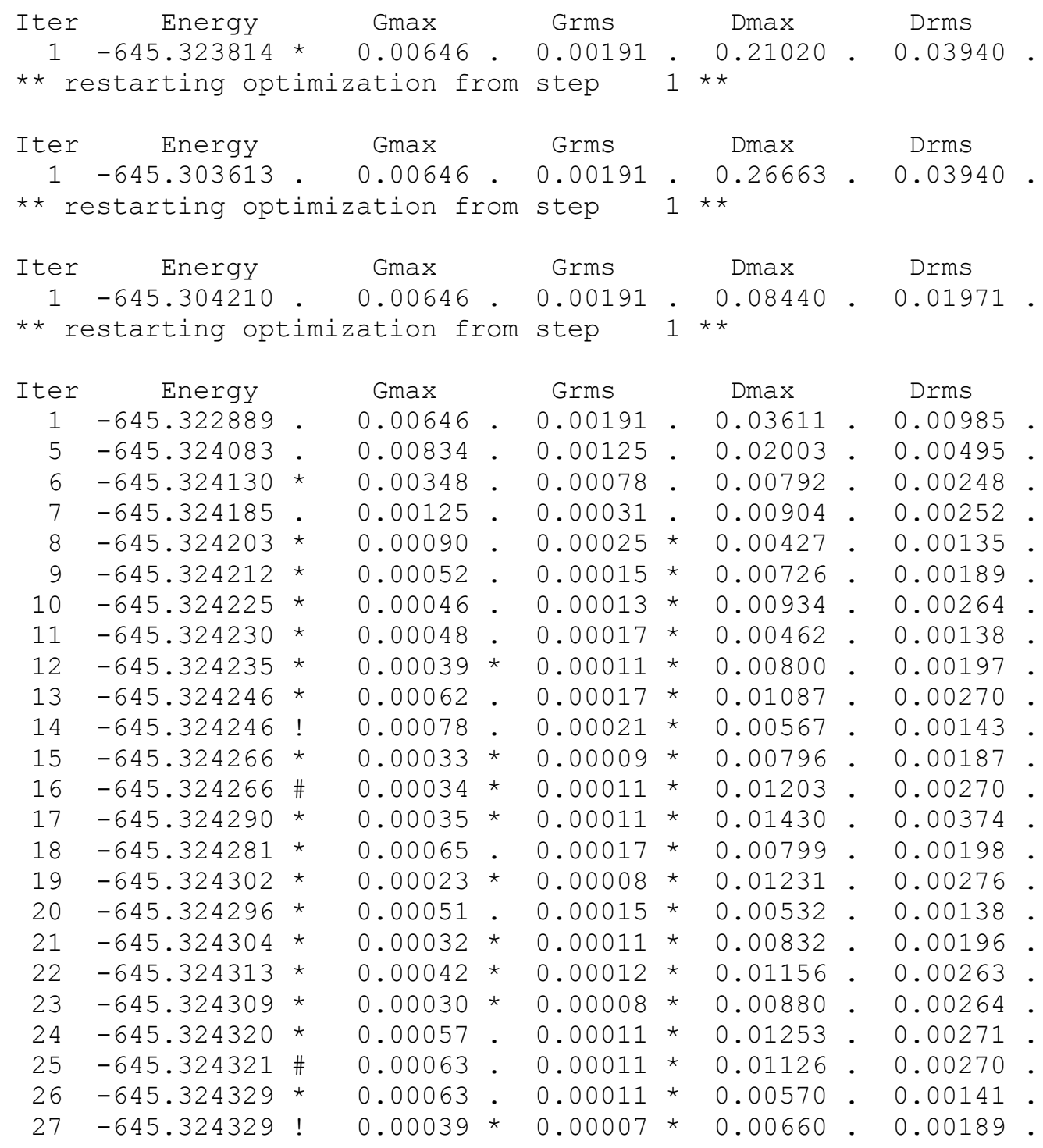

energy change:

gradient maximum:

gradient rms:

step size: 0.01438

displacement maximum:

displacement rms:

predicted energy change:

step: $1.4381 \mathrm{E}-02$

$$
\begin{aligned}
& 2.0693 \mathrm{E}-07 !(5.0000 \mathrm{E}-05) \\
& 3.8742 \mathrm{E}-04 *(4.5000 \mathrm{E}-04) \\
& 7.0200 \mathrm{E}-05 *(3.0000 \mathrm{E}-04)
\end{aligned}
$$

trust radius: 0.01414

$$
6.5994 \mathrm{E}-03 \cdot(1.8000 \mathrm{E}-03)
$$$$
1.8884 \mathrm{E}-03 .(1.2000 \mathrm{E}-03)
$$$$
-1.8617 \mathrm{E}-06 \text { geom step: }
$$

1.4381E-02 full

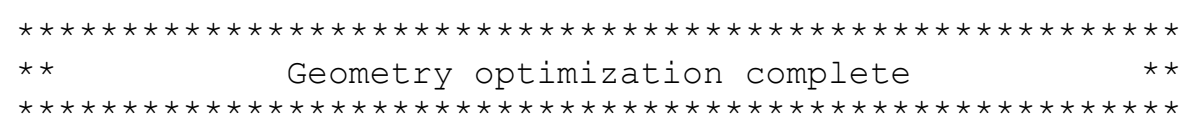

center of mass moved by:
$x$ :
$0.0000 \mathrm{E}+00$
$\mathrm{y}:$
$0.0000 \mathrm{E}+00$
z :
$0.0000 \mathrm{E}+00$

final geometry:

$\begin{array}{lc}\text { atom } & x \\ \text { C1 } & -0.7197507227 \\ \text { N2 } & -0.7107798222 \\ \text { C7 } & -0.7048067577 \\ \text { N1 } & -0.5977309833\end{array}$

angstroms

Y

0.7155374397

1. 0960729298

$-0.7502286406$

$-1.4048435870$
Z

$-1.4902285642$

$-0.2685261109$

$-1.6556003162$

$-0.5563553941$ 


$\begin{array}{lrrr}\text { Re1 } & -0.6749261021 & -0.3645847076 & 1.4180795612 \\ \text { O1 } & -0.9096780184 & -1.9396784032 & 2.0218084057 \\ \text { O2 } & -1.5266643185 & 0.8337535621 & 2.2751159459 \\ \text { C3 } & 1.1701537201 & 0.0184093993 & 1.7213648249 \\ \text { C10 } & 1.6802157803 & 1.4123864051 & 1.9452785812 \\ \text { O3 } & 1.1628532789 & 2.4009049678 & 1.4636931480 \\ \text { O4 } & 2.7631216832 & 1.4058154454 & 2.7218032806 \\ \text { H9 } & -0.7672373189 & -1.2246080654 & -2.6330375510 \\ \text { H17 } & -0.5974094926 & -2.4242891282 & -0.6041856700 \\ \text { H19 } & -0.6549977013 & 2.0930024737 & -0.0480874549 \\ \text { H22 } & -0.7173405124 & 1.3950126738 & -2.3399840647 \\ \text { H3 } & 3.0520125818 & 2.3258766332 & 2.8656577394 \\ \text { H4 } & 1.8841472298 & -0.7889106801 & 1.8933897909\end{array}$

Final energy ........... -645.32432866803

Job WF31 completed on CHENB071 at Wed Mar 12 22:18:58 2003

Reason for exit: Successful completion

Titan Program CPU Time : 002:17:53.3

Titan Program Wall Time: 004:43:40.2

TITAN Properties Program: (PC/x86)
Reason for exit: Successful completion
Properties Program CPU Time : 000:00:01.8
Properties Program Wall Time: 000:00:26.9

Release 6.0 .6

Job WF24510 started on CHENB091 at Mon Jan 12 09:06:57 2004

Jaguar version 3.5, release 42

Job name:

Task:

Stoichiometry: Molecular weight:

Molecular charge:

Spin multiplicity:

Point Group:

SCF method:

Basis set:

Basis functions:

Total electrons:

Alpha electrons:

Beta electrons:

Total orbitals:

Core orbitals:

Open-shell orbitals:

Occupied orbitals:

Shells:
WF2 4510

Calculate energy ("single-point job")

ReC 4N2H6O4

332.99 amu

1

1

C1

B3LYP

LACVP**

192

90

45

45

205

45

0

45

1

Non-default options chosen:

SCF calculation type: DFT

$\mathrm{DFT}=$ Becke_3_Parameter/HF+Slater+Becke88+VWN+LYP (B3LYP)

Numerical 2 nd derivatives will be computed

Vibrational frequencies and related properties will be computed

Molecular symmetry not used 
calculating forces at perturbed geometry calculating forces at perturbed geometry calculating forces at perturbed geometry calculating forces at perturbed geometry calculating forces at perturbed geometry calculating forces at perturbed geometry calculating forces at perturbed geometry calculating forces at perturbed geometry calculating forces at perturbed geometry calculating forces at perturbed geometry calculating forces at perturbed geometry calculating forces at perturbed geometry calculating forces at perturbed geometry calculating forces at perturbed geometry calculating forces at perturbed geometry calculating forces at perturbed geometry calculating forces at perturbed geometry calculating forces at perturbed geometry calculating forces at perturbed geometry calculating forces at perturbed geometry calculating forces at perturbed geometry calculating forces at perturbed geometry calculating forces at perturbed geometry calculating forces at perturbed geometry calculating forces at perturbed geometry calculating forces at perturbed geometry calculating forces at perturbed geometry calculating forces at perturbed geometry calculating forces at perturbed geometry calculating forces at perturbed geometry calculating forces at perturbed geometry calculating forces at perturbed geometry calculating forces at perturbed geometry calculating forces at perturbed geometry calculating forces at perturbed geometry calculating forces at perturbed geometry calculating forces at perturbed geometry calculating forces at perturbed geometry calculating forces at perturbed geometry calculating forces at perturbed geometry calculating forces at perturbed geometry calculating forces at perturbed geometry calculating forces at perturbed geometry calculating forces at perturbed geometry calculating forces at perturbed geometry calculating forces at perturbed geometry calculating forces at perturbed geometry calculating forces at perturbed geometry calculating forces at perturbed geometry calculating forces at perturbed geometry calculating forces at perturbed geometry calculating forces at perturbed geometry calculating forces at perturbed geometry calculating forces at perturbed geometry calculating forces at perturbed geometry calculating forces at perturbed geometry calculating forces at perturbed geometry calculating forces at perturbed geometry
1 of $102 \ldots$

2 of $102 \ldots$

3 of $102 \ldots$

4 of $102 \ldots$

5 of $102 \ldots$

6 of $102 \ldots$

7 of $102 \ldots$

8 of $102 \ldots$

9 of $102 \ldots$ 10 of $102 \ldots$ 11 of $102 \ldots$ 12 of $102 \ldots$ 13 of $102 \ldots$ 14 of $102 \ldots$ 15 of $102 \ldots$ 16 of $102 \ldots$ 17 of $102 \ldots$ 18 of $102 \ldots$ 19 of $102 \ldots$ 20 of $102 \ldots$ 21 of $102 \ldots$ 22 of $102 \ldots$ 23 of $102 \ldots$ 24 of $102 \ldots$ 25 of $102 \ldots$ 26 of $102 \ldots$ 27 of $102 \ldots$ 28 of $102 \ldots$ 29 of $102 \ldots$ 30 of $102 \ldots$ 31 of $102 \ldots$ 32 of $102 \ldots$ 33 of $102 \ldots$ 34 of $102 \ldots$ 35 of $102 \ldots$ 36 of $102 \ldots$ 37 of $102 \ldots$ 38 of $102 \ldots$ 39 of $102 \ldots$ 40 of $102 \ldots$ 41 of $102 \ldots$ 42 of $102 \ldots$ 43 of $102 \ldots$ 44 of $102 \ldots$ 45 of $102 \ldots$ 46 of $102 \ldots$ 47 of $102 \ldots$ 48 of $102 \ldots$ 49 of $102 \ldots$ 50 of $102 \ldots$ 51 of $102 \ldots$ 52 of $102 \ldots$ 53 of $102 \ldots$ 54 of $102 \ldots$ 55 of $102 \ldots$ 56 of $102 \ldots$ 57 of $102 \ldots$ 58 of $102 \ldots$ 
calculating forces at perturbed geometry calculating forces at perturbed geometry calculating forces at perturbed geometry calculating forces at perturbed geometry calculating forces at perturbed geometry calculating forces at perturbed geometry calculating forces at perturbed geometry calculating forces at perturbed geometry calculating forces at perturbed geometry calculating forces at perturbed geometry calculating forces at perturbed geometry calculating forces at perturbed geometry calculating forces at perturbed geometry calculating forces at perturbed geometry calculating forces at perturbed geometry calculating forces at perturbed geometry calculating forces at perturbed geometry calculating forces at perturbed geometry calculating forces at perturbed geometry calculating forces at perturbed geometry calculating forces at perturbed geometry calculating forces at perturbed geometry calculating forces at perturbed geometry calculating forces at perturbed geometry calculating forces at perturbed geometry calculating forces at perturbed geometry calculating forces at perturbed geometry calculating forces at perturbed geometry calculating forces at perturbed geometry calculating forces at perturbed geometry calculating forces at perturbed geometry calculating forces at perturbed geometry calculating forces at perturbed geometry calculating forces at perturbed geometry calculating forces at perturbed geometry calculating forces at perturbed geometry calculating forces at perturbed geometry calculating forces at perturbed geometry calculating forces at perturbed geometry calculating forces at perturbed geometry calculating forces at perturbed geometry calculating forces at perturbed geometry 100 of $102 \ldots$ calculating forces at perturbed geometry 101 of $102 \ldots$ calculating forces at perturbed geometry 102 of $102 \ldots$

numerical derivatives complete;

calculation at normal geometry follows...

start of program freq

harmonic frequencies in $\mathrm{cm}^{\wedge}-1$, IR intensities in $\mathrm{km} / \mathrm{mol}$, and normal modes:

\begin{tabular}{|c|c|c|c|c|c|c|c|c|}
\hline \multicolumn{2}{|c|}{ frequencies } & 52.37 & 85.16 & 100.37 & 147.65 & 171.44 & 205.26 & 230.2 \\
\hline into & ities & 4.67 & 4.89 & 2.60 & 0.95 & 1.72 & 1.30 & \\
\hline C1 & $\mathrm{X}$ & 0.03675 & 0.02756 & -0.11902 & -0.02271 & -0.08187 & -0.01117 & -0.02 \\
\hline $\mathrm{C} 1$ & $Y$ & -0.00442 & 0.04245 & -0.04290 & -0.00964 & 0.02894 & & -0.0 \\
\hline $\mathrm{C} 1$ & Z & -0.00034 & 0 . & -0.02032 & -0.00 & 0.01241 & -0 . & \\
\hline N2 & $\mathrm{X}$ & 0.000 & -0.043 & -0.12482 & -0.007 & 0.000 & -0 . & \\
\hline N2 & $Y$ & -0.00246 & 0.02202 & -0.02207 & 0.001 & 0.016 & & -0 \\
\hline N2 & Z & -0.00049 & 0.03472 & -0.02534 & -0.00953 & 0.01322 & -0.0 & \\
\hline C7 & $\mathrm{X}$ & 0.07271 & 0.11272 & 0.02918 & -0.02904 & -0.04653 & 0.02563 & -0 \\
\hline
\end{tabular}

59 of $102 \ldots$ 60 of $102 \ldots$ 61 of $102 \ldots$ 62 of $102 \ldots$ 63 of $102 \ldots$ 64 of $102 \ldots$ 65 of $102 \ldots$ 66 of $102 \ldots$ 67 of $102 \ldots$ 68 of $102 \ldots$ 69 of $102 \ldots$ 70 of $102 \ldots$ 71 of $102 \ldots$ 72 of $102 \ldots$ 73 of $102 \ldots$ 74 of $102 \ldots$ 75 of $102 \ldots$ 76 of $102 \ldots$ 77 of $102 \ldots$ 78 of $102 \ldots$ 79 of $102 \ldots$ 80 of $102 \ldots$ 81 of $102 \ldots$ 82 of $102 \ldots$ 83 of $102 \ldots$ 84 of $102 \ldots$ 85 of $102 \ldots$ 86 of $102 \ldots$ 87 of $102 \ldots$ 88 of $102 \ldots$ 89 of $102 \ldots$ 90 of $102 \ldots$ 91 of $102 \ldots$ 92 of $102 \ldots$ 93 of $102 \ldots$ 94 of $102 \ldots$ 95 of $102 \ldots$ 96 of $102 \ldots$ 97 of $102 \ldots$ 98 of $102 \ldots$ 99 of $102 \ldots$ 


C7
C7
N1
N1
N1
Re1
Re1
Re1
O1
O1
O1
O2
O2
O2
C3
C3
C3
C10
C10
C10
O3
O3
O3
O4
O4
O4
H9
H9
H9
H17
H17
H17
H19
H19
H19
H22
H22
H22
H3
H3
H3
H4
H4
H4

$\begin{array}{rrrr}-0.00444 & 0.04684 & -0.04343 & -0.01078 \\ 0.00136 & 0.00269 & -0.00419 & 0.00889 \\ 0.05144 & 0.08961 & 0.09371 & -0.00304 \\ -0.00174 & 0.03231 & -0.01696 & -0.00175\end{array}$

$\begin{array}{lllll}-0.00174 & 0.03231 & -0.01696 & -0.00175\end{array}$

$0.00510-0.00369$

$-0.01021-0.01670$

$0.00239-0.00513$

$0.00230 \quad 0.01240$

$-0.01793$

$0.00414-0.01657$

$0.00244-0.01221$

$-0.02528-0.02354$

$0.00036-0.02072$

$-0.011010 .02990$

$-0.01063-0.01263$

$-0.00430-0.00511$

$0.02101-0.01307-0$

$-0.00222$

$-0.00579-0$.

0.01681

$0.00485-0.06034$

$-0.07724 \quad 0.06222$

$0.00496-0.01742$

$0.10645-0.12841$

$0.10406-0.00770$

$-0.01694-0.00034$

$-0.14146-0.02746$

$0.11174 \quad 0.18742$

$-0.00586$

0.06103

$-0.00047-0.00896$

0.07341

0.14510

0.00426

$0.00349-0.00941$

$0.01579-0.00422$

$-0.00622-0.00362$

$\begin{array}{rr}-0.00128 & 0.08271\end{array}$

$0.01594-0.01070$

$-0.00659$

0.03123

0.01566

$0.01938-0.04396$

$0.01558-0.05179$

$0.01016-0.02646$

$-0.01712$

$-0.01619$

0.01344

$-0.02747$

0.02713

0.01745

$-0.01849$

0.04547

0.00003

$-0.05443$

0.04797

0.07422

0.06788

0.02505

0.04752

0.04244

0.02334

0.11626

0.07601

0.02190

0.05842

$-0.03682$

0.01303

$0.05948-0.02185$

$0.00069 \quad 0.01573$

$0.19234-0.00308$

$-0.00166$

0.03353

0.00501

$-0.03322$

$-0.02384$

$-0.01804$

0.01950

$-0.00219$

0.02179

$-0.09749$

$-0.23782$

$-0.00831$

$-0.00083$

0.02180

0.00541

0.00102

0.04048

$-0.00549$

0.04574

0.02916

$0.05768-0$

$-0.05380-0.02351$

$-0.00128$

0.03995

0.01345

0.11371

$-0.01782$

$-0.00153$

$-0.15667-0.06202$

$-0.01853-0.01702$

$-0.00838-0.01045$

0.03395

$-0.01825$

$-0.20885-0.03140$

$-0.05300-0.01936$

$-0.02840-0.01428$

$\begin{array}{rrr}0.00113 & 0.12678\end{array}$

$-0.05988-0.05806$

0.07915

0.01131

0.00050

$-0.02955$

$-0.03443$

$-0.04308$

0.05241

0.02697

0.02812

0.00454

$0.05007-0.00329$

$0.03336-0.00352 \quad 0.02703$

$\begin{array}{llll}0.02199 & 0.04328 & -0.01235\end{array}$

$-0.00641$

0.01443

0.02035

0.00309

$-0.00461$

$-0.00087$

$0.00188 \quad 0.00016$

$-0.06786$

0.00788

$-0.00387$

$-0.05647$

0.01081

$-0.09182$

0.00207

$-0.03505$

0.13263

0.00869

$-0.02273$

0.03072

$-0.00592$

$-0.06498$

$-0.04330$

0.03282

0.05833

$-0.00286$

$-0.08405$

0.03125

0.00542

0.06229

0.02243

$-0.01747$

$-0.01859$

0.01363

0.02760

$-0.16488$

0.03325

0.01550

0.03171

0.07467

$-0.10492$

$-0.02781$

$-0.03765$

0.00894

$187-0.01782$

$0.01345-0.15911$

$0.03471-0.01171$

$0.15151-0.03928$

$\begin{array}{lll}0.03262 & 0.05925\end{array}$

$0.06828 \quad 0.02618$

$-0.10044-0.00727$

$-0.00061-0.00063$

$\begin{array}{ll}0.00062 & 0.05942\end{array}$

$-0.05098-0.02779$

$\begin{array}{ll}-0.01513 & 0.01478\end{array}$

$0.00138 \quad 0.07249$

$-0.02184-0.00044$

$\begin{array}{ll}-0.01322 & 0.05477\end{array}$

0.020620 .10210

$0.01997 \quad 0.02061$

$\begin{array}{ll}-0.02905 & 0.01722\end{array}$

$-0.035850 .02663$

$-0.00032-0.00094$

$0.07087-0.00861$

$0.03707 \quad 0.00748$

$0.02060 \quad 0.00645$

$0.02322 \quad 0.04004$

$0.04445-0.01208$

$-0.00175-0.00424$

$\begin{array}{ll}-0.06189 & 0.00442\end{array}$

$0.04911-0.02510$

$\begin{array}{lll}0.00387 & 0.05101\end{array}$

$-0.03166-0.05938$

$\begin{array}{lll}0.03843 & 0.00607\end{array}$

$-0.014760 .04066$

$\begin{array}{ll}-0.03414 & 0.03952\end{array}$

$-0.04316$

0.01700

0.01772

$\begin{array}{ll}0.05533 & 0.01772 \\ 0.00441 & 0.03989\end{array}$

frequencies

intensities

$\mathrm{C} 1 \mathrm{X}$

260.25

3.95

296.13

3.12

$-0.01070$

0.00005

309.95

330.53

1.85

0.28

340.64

0.70

383.08

1.04

$0.01231-0.00013$

$-0.04888-0.01706$

0.00752

0.01231

0.00127

$0.00500-0.08011-0.00858-0.00445$

$0.04015-0.04703$

0.01429

$0.09665-0.01134-0.01082$

$-0.08230$

\section{$0.04223 \quad 0.10065$}

0.04322

0.00444

0.12061

0.00967

$-0.01852$

$-0.02782$

$-0.01082$

433.49

0.11

0.11945

0.00364

0.00111

$0.03533 \quad 0.10258$

$-0.02508$

0.00332

0.01160

$-0.10685-0.0074$

$-0.02098$

0.0277

$0.00316-0.00688-0.00326$

$-0.01593$

$-0.00971$

$-0.00756$

0.00316

0.05429

$-0.01564$

0.00415

$-0.00892$

$\begin{array}{llll}0.03770 & -0.05772 & 0.02799\end{array}$

0.00597

0.08060

0.0012

$0.10120-0.00930-0.01027$

$0.00427-0.02098$

$0.02098 \quad 0.00253$

$\begin{array}{llll}0.07951 & 0.09251 & -0.07017\end{array}$

0.01404

0.01057

.01190

$-0.01196-0.0076$

$0.00174-0.01194$

Re1 Y

\begin{abstract}
0.00145
\end{abstract}




\begin{tabular}{|c|c|c|c|c|c|c|c|c|}
\hline $\operatorname{Re} 1$ & z & 0.00201 & -0.03078 & 0.01021 & 0.00192 & 0.02433 & 0.00473 & -0.00021 \\
\hline 01 & $\mathrm{x}$ & -0.04078 & 0.07262 & 0.05838 & 0.00833 & 0.04531 & 0.02749 & -0.00960 \\
\hline 01 & $\mathrm{Y}$ & 0.00008 & -0.01403 & -0.01269 & -0.03544 & -0.06933 & -0.01722 & -0.00025 \\
\hline 01 & Z & -0.04266 & -0.02111 & 0.00370 & -0.04941 & -0.12743 & -0.00369 & 0.00340 \\
\hline 02 & $\mathrm{x}$ & -0.10494 & 0.06457 & 0.03454 & 0.04677 & 0.01230 & -0.00466 & 0.01138 \\
\hline $\mathrm{O} 2$ & $\mathrm{Y}$ & -0.03485 & 0.04596 & 0.05033 & 0.00252 & 0.10564 & 0.03340 & 0.00983 \\
\hline $\mathrm{O} 2$ & Z & -0.05158 & -0.00440 & -0.00702 & 0.03669 & -0.10881 & -0.03620 & -0.01361 \\
\hline C3 & $\mathrm{x}$ & 0.02933 & -0.01593 & -0.03108 & -0.01317 & -0.01104 & 0.06076 & -0.00768 \\
\hline C3 & $\mathrm{Y}$ & -0.01627 & -0.00295 & 0.00523 & -0.00037 & 0.00338 & & \\
\hline C3 & Z & -0.10944 & 0.04291 & 0.00690 & 0.02469 & 0.00596 & -0.10096 & \\
\hline C10 & $\mathrm{x}$ & -0.01104 & -0.00419 & -0.03036 & -0.00737 & -0.01611 & 0.09223 & -0.00672 \\
\hline C10 & $\mathrm{Y}$ & -0.02252 & -0.00325 & 0.00341 & & 0.00528 & -0.02796 & \\
\hline $\mathrm{C} 10$ & Z & -0.05059 & 0.01672 & -0.01507 & & -0.00791 & 0.01111 & \\
\hline 03 & $\mathrm{x}$ & -0.03684 & 0.00171 & -0.00989 & 0.00374 & -0.00703 & 0.01138 & -0.00269 \\
\hline 03 & $\mathrm{Y}$ & -0.00841 & -0.01088 & 0.01791 & 0.00239 & 0.01104 & -0.05802 & \\
\hline 03 & $\mathrm{z}$ & 0.00461 & -0.00628 & -0.0 & -0.0 & -0.00230 & & \\
\hline 04 & $\mathrm{x}$ & -0.04409 & 0.00801 & -0.03073 & & -0.01573 & 0.07189 & 0018 \\
\hline 04 & $\mathrm{Y}$ & -0.0 & 243 & -0.0 & 938 & -0.02740 & 0.11983 & \\
\hline 04 & Z & -0.0 & -0.0 & -0 & -0 & -0. & & \\
\hline H9 & $\mathrm{x}$ & -0.07231 & 0.0 & -0.1 & -0.0 & 0.07985 & -0.04303 & \\
\hline H9 & $\mathrm{Y}$ & -0.03577 & -0.0 & 0.0 & -0.10 & -0.00173 & & \\
\hline H9 & Z & & & -0 & & -0 & -0 & \\
\hline H17 & $\mathrm{x}$ & 0.0 & 0.0 & & & -0 . & & \\
\hline H17 & $\mathrm{Y}$ & 0.0 & -0.0 & -0 & & & & \\
\hline H17 & Z & & & -0 . & & & -0 & \\
\hline H19 & $\mathrm{x}$ & 0.07316 & 0. & & & -0 & 508 & \\
\hline H19 & $\mathrm{Y}$ & 0.00851 & -0. & -0 & & -0 & -0 . & \\
\hline H19 & Z & & & & -0 . & & & \\
\hline H22 & $\mathrm{x}$ & -0.03061 & -0.0 & -0 & -0 . & 68 & & \\
\hline H22 & Y & -0.02454 & -0.00723 & & -0 . & & & \\
\hline H22 & Z & & & & -0 & -0 & & \\
\hline H3 & $\mathrm{x}$ & -0.07 & 0.01924 & 0.0 & & 0.00796 & -0.10674 & \\
\hline H3 & $\mathrm{Y}$ & -0.05772 & 0.01496 & -0.0 & & -0.03796 & 0.17686 & -0.0 \\
\hline H3 & $\mathrm{Z}$ & & -0 & & -0 & & & \\
\hline H4 & $\mathrm{x}$ & 348 & -0. & -0. & -0 . & & 752 & -0 \\
\hline H4 & $\mathrm{Y}$ & -0.01159 & -0.0 & 0.0 & & 1077 & -0.0 & \\
\hline H4 & Z & -0.1 & 0. & -0 . & & -0. & -0 . & \\
\hline \multicolumn{2}{|c|}{ requencies } & & & & & & & \\
\hline & ities & & & & & & & .66 \\
\hline $\mathrm{C} 1$ & $\mathrm{x}$ & -0.00 & -0.0 & 27 & & & & \\
\hline $\mathrm{C} 1$ & $\mathrm{Y}$ & 0.00804 & 0.0 & 157 & -0.00249 & 0.00458 & 0.00876 & -0.10443 \\
\hline $\mathrm{C} 1$ & Z & & & & & -0 & -0 . & \\
\hline $\mathrm{N}$ & $\mathrm{x}$ & & & & & & & \\
\hline N2 & $\mathrm{Y}$ & 0.17 & 056 & 514 & 0.0 & -0.01173 & -0.01003 & 8287 \\
\hline & Z & -0.00 & & -0 & -0 & & -0 & \\
\hline & $\mathrm{x}$ & & & & -0 & & & \\
\hline C7 & $\mathrm{Y}$ & -0.02 & & & & & 41 & -0 . \\
\hline $\mathrm{C} 7$ & Z & & & & -0 & & & \\
\hline & $\mathrm{x}$ & & -0.0 & -0 & & & & \\
\hline N1 & $\mathrm{Y}$ & -0.1 & -0 & -0 . & -0 . & & & \\
\hline & Z & -0.0 & & -0 . & -0 . & -0.0 & & -0 . \\
\hline $\mathrm{Re}]$ & $\mathrm{x}$ & & & -0 & -0 & -0.0 & & \\
\hline $\operatorname{Re} 1$ & $\mathrm{Y}$ & -0.0 & & -0 & -0 & & -0 . & \\
\hline $\operatorname{Re1}$ & Z & & & -0 . & & -0.0 & & \\
\hline $\mathrm{O} 1$ & $\mathrm{X}$ & & -0 & -0 . & & & & \\
\hline 01 & $\mathrm{Y}$ & & -0 & -0 & & -0.0 & -0 . & -0 \\
\hline 01 & Z & -0.01310 & 0.01172 & -0.00227 & -0.00965 & 0.00343 & -0.00284 & 0.01130 \\
\hline & $\mathrm{x}$ & & & & -0.0 & -0.00090 & & \\
\hline $\mathrm{O} 2$ & $\mathrm{Y}$ & & -0.0 & & & & -0 & -0.0 \\
\hline 02 & Z & -0.00751 & -0.00947 & 0.00385 & 0.00363 & 0.00492 & 0.00439 & $-0.007 \varepsilon$ \\
\hline
\end{tabular}




C3
C3
C3
C10
C10
C10
O3
O3
O3
O4
O4
O4
H9
H9
H9
H17
H17
H17
H19
H19
H19
H22
H22
H22
H3
H3
H3
H4
H4
H4

$-0.00820-0.04207$ $\begin{array}{ll}-0.00932 & 0.08593\end{array}$

$-0.01096-0.04480$

$-0.00211-0.04459$

$\begin{array}{ll}-0.00924 & 0.02270\end{array}$

$\begin{array}{rr}-0.00197 & 0.12212\end{array}$

$0.00999-0.03764$

$0.00197-0.06172$

$0.00828-0.05558-0$

$-0.00426 \quad 0.02708$

$0.01174-0.02143$

$-0.00110$

0.03649

0.01154

0.09812

$-0.01012$

0.01384

0.00738

0.00082

$0.00904-0.01292$

$-0.15905-0.00453$

$-0.14252-0.00703$

$\begin{array}{rr}-0.01556 & 0.01255 \\ 0.19293 & -0.00138\end{array}$

$-0.10101$

$-0.02222$

$0.00689-0$.

-0.01807
0.00221

$-0.10127$

$0.00080-0$

$-0.01814$

$0.40826-0$

0.02208

$-0.07811$

$-0.03894-0.36974$

$-0.01586$

$-0.01730$

$0.02162-0$

$-0.01633$

0.06864

$-0.39214$

frequencies

intensities

$\mathrm{C} 1$

C1

C1

N2

N2

N2

C 7

C7

C 7

N1

N1

N1

Re1 X

Re1 Y

Re1 Z

O1 X

01 Y

01 Z

02

02

O2

C3

C3

C3 Z

C10 X

C10 Y

C10 Z

$03 \mathrm{x}$

861.54

873.91

0.00465

0.02255

$-0.00970-0.03735-0$

0.00782

0.03103

0.00354

0.00526

0.09933

$-0.00299-0.02505$

$-0.00182$

0.036

0.00374

0.02106

$-0.00053-0.00712$

$0.00017-0.00078$

$0.00238-0.00033$

$\begin{array}{ll}-0.00349 & 0.00041\end{array}$

$\begin{array}{ll}-0.00037 & 0.00091\end{array}$

$-0.01214-0.00333$

0.01754

0.00313

0.00328

0.00352

$-0.00843-0.00202$

$-0.00538-0.00589-0$

$-0.02086$

0.00991

$-0.04352$

$0.14813-0.01181$

0.00630

0.09832

$-0.02320$

$0.00695-0.15635-0.01136$

$\begin{array}{lllll}.02553 & -0.01013 & 0.00942 & -0.01909 & -0.00815\end{array}$

$\begin{array}{llllll}0.06701 & 0.08424 & -0.00601 & 0.08015 & -0.00170\end{array}$

$\begin{array}{llllll}0.06372 & 0.04347 & 0.00394 & -0.05630 & -0.00066\end{array}$

$\begin{array}{llllll}-0.01887 & -0.09838 & -0.00119 & -0.09676 & 0.00055\end{array}$

$-0.09538-0.06502$

$0.00516-0.00576-0.00057$

$-0.04140$

0.02520

0.0031

$4-0$

$0.04799-0.00002$

.06082

0.00795

$-0.00010$

$0.04799-0$

0.00002

481

00200

$0.02447 \quad 0.07988-0.00427$

1822

0.00186

$-0.00833-0$

0.07988

0.02503

0.00112

$0.01303 \quad 0.00441$

$-0.03331$

0.00949

$0.00079-0.00127$

0.03713

$-0.00824$

$-0.08136$

$-0.01328$

0.00054

$-0.44562$

$0.01218-0.12242$

$-0.01410-0.00189-0.0077$

$-0.04688$

0.13349

$\begin{array}{lll}-0.01637 & -0.00569 & 0.00782\end{array}$

0.00143

0.08707

$-0.02555-0.02655$

$-0.53986$

0.00712

0.20755

$0.01783 \quad 0.00893$

0.02156

$-0.01443$

$-0.17346$

$\begin{array}{llllll}0.01316 & -0.01032 & -0.03179 & 0.02550 & -0.19831\end{array}$

$-0.00870-0.00572$

0.02079

$0.01504-0.11155$

$0.00504-0.00164$

0.00536

$-0.00064$

0.09827

$-0.09247-0.10227$

0.00485

$-0.04890-0.00587$

$0.58378-0.34537$

0.00485

0.05763

0.00600

$-0.02412$

0.05868

0.00117

$-0.17080-0.01015$

0.07706

0.01208

0.03876

$-0.41549-0.02720$

0.05689

$0.32103-0.08428$

0.06789

0.01462

951.55

982.68

1018.21

1037.93

1061.11

3.86

102.45

68.54

17.80

$\begin{array}{lll}-0.01286 & -0.08378 & 0.00137\end{array}$

$-0.00805-0.02129-0.01471$

0.00181

0.01503

0.00429

$-0.00549$

0.11849

$0.14971-0.00261-0.01554-0.02317$

$-0.00016-0$

$-0.03204$

$-0.00470-0$.

0.00012

0.00210

0.00083

$-0.00401$

$-0.00683$

$0.00095-0.07345$

$0.01208-0.00146$

0.03144

0.01932

$-0.00441$

0.00292

0.00694

$-0.00159$

$-0.00391$

$-0.00178$

$-0.00570$

$-0.00449$

$0.01263-0.13682$

0.01203

$0.00123-0.01959$

$0.00368-0.06484$

0.01114

$-0.00073-0.00276$

$-0.00124$

0.01724

0.01008

0.00086

$-0.03573$

$-0.00459$

$0.00424 \quad 0.00525$

$-0.00187$

$0.01470-0.00038$

$0.00018-0.00841$

$-0.00670-0.00051$

$-0.00573$

$-0.00373$

0.00766

0.02330

$0.00610-0.00398$

$0.00056-0.00136$

0.00273

$0.00883 \quad 0.00045$

$0.00003-0.00373$

$-0.02013$

$0.00357-0.00064$

$0.01049-0.02251$

$-0.15006$

$-0.01198-0.00044$

$-0.00551$

0.01100

0.05507

$-0.02741$

$-0.00010$

$0.01600-0.00331$

0.08305

0.06438

0.00079

0.01489

0.00342

$-0.11982$

$-0.08625$

0.00017

$0.00514-0.08397$

0.08723

0.01158

0.01242

0.15082

0.01081

$-0.00671$

0.086490 .00498

0.04675

0.00484

0.01202

0.00167

$-0.07159-0.00694$ 


\begin{tabular}{|c|c|c|c|c|c|c|c|c|}
\hline 03 & $\mathrm{Y}$ & 0.02231 & 0.00026 & -0.06055 & -0.00309 & 0.00522 & -0.00894 & 0.00189 \\
\hline 03 & Z & 0.01505 & -0.00121 & 0.03636 & 0.00152 & -0.00255 & 0.00522 & 0.00002 \\
\hline 04 & $\mathrm{x}$ & -0.02276 & 0.00083 & -0.05453 & -0.00286 & -0.00272 & -0.00807 & -0.0006 \\
\hline 04 & $\mathrm{Y}$ & -0.00198 & -0.00140 & -0.01341 & -0.00162 & 0.00005 & -0.00028 & 0.00043 \\
\hline 04 & Z & 0.01198 & -0.00225 & -0.03047 & -0.00097 & -0.00295 & -0.00549 & -0.00059 \\
\hline H9 & $\mathrm{x}$ & 0.03314 & -0.19865 & 0.00862 & -0.02080 & 0.01156 & -0.00135 & \\
\hline H9 & $\mathrm{Y}$ & -0.00050 & -0.04711 & 0.03103 & -0.32278 & 0.04044 & 0.05127 & \\
\hline H9 & & -0.01135 & -0.01243 & -0.00489 & 0.01561 & -0.00207 & -0.01149 & -0.01356 \\
\hline H17 & & -0.02775 & -0.59675 & 0.00568 & 0.07611 & 0.01181 & -0.00597 & \\
\hline H17 & Y & 0.00355 & 0.01511 & 0.00230 & -0.05037 & -0.00405 & -0.00413 & \\
\hline H17 & Z & 0.00870 & 0.09721 & -0.02837 & 0.26671 & -0.00813 & -0.00942 & -0.02007 \\
\hline H19 & $\mathrm{x}$ & 7850 & & -0.00467 & 0.00302 & 0.00181 & & \\
\hline H19 & Y & 0.00743 & 0.01142 & 0.00636 & -0.02219 & -0.00552 & 0.00593 & -0.03129 \\
\hline H19 & z & -0.01209 & -0.00531 & -0.02716 & 0.28282 & -0.01187 & -0.01493 & \\
\hline H22 & $\mathrm{x}$ & 472 & & & -0.08082 & -0.02979 & & \\
\hline H22 & $\mathrm{Y}$ & -0. & -0.0 & -0.03047 & 0.31086 & -0.00003 & -0.04146 & -0 . \\
\hline H22 & Z & 0.00167 & 0.02452 & -0.00951 & 0.08382 & -0.00293 & -0.02191 & -0. \\
\hline H3 & $\mathrm{x}$ & 546 & 0.0 & -0.1 & -0.0 & 1278 & -0 . & \\
\hline H3 & $\mathrm{Y}$ & -0.0 & -0.0 & & & -0 & & -0 \\
\hline H3 & Z & 0.00587 & 0.0 & -0.14719 & -0.00715 & 0.01083 & -0.01561 & \\
\hline H4 & $\mathrm{x}$ & 676 & -0.0 & & 5902 & -0.04728 & 4923 & -0 \\
\hline H 4 & $\mathrm{Y}$ & -0.0 & & & & -0 . & & \\
\hline H4 & Z & -0.6 & 0. & 0.0 & -0.00070 & 0.07861 & 3721 & -0 . \\
\hline \multicolumn{2}{|c|}{ requencies } & & & & & & & \\
\hline \multicolumn{2}{|c|}{ intensities } & 9.44 & & & & & 1.78 & \\
\hline $\mathrm{C} 1$ & $\mathrm{x}$ & -0.02221 & -0.0 & -0.00 & -0.00096 & -0.00060 & 0.00185 & \\
\hline $\mathrm{C} 1$ & $\mathrm{Y}$ & -0.0 & & -0 & -0.0 & -0 & & -0 \\
\hline $\mathrm{C} 1$ & Z & 0 & -0 & -0 & 0 & 234 & -0 . & -0 \\
\hline N2 & $\mathrm{x}$ & -0 & & & & & & -0 \\
\hline N2 & Y & -0 & & & 0 . & -0 . & -0 & \\
\hline N2 & Z & -0.0 & 0. & -0.0 & 0.0 & -0.001 & 7328 & -0 . \\
\hline C7 & $\mathrm{x}$ & 0.1 & -0 & & & & & -0 \\
\hline C7 & Y & & -0 . & & -0 & -0 & & -0 \\
\hline C7 & Z & -0.0 & -0. & -0.0 & 0.0 & 0418 & 2074 & -0 \\
\hline N1 & $\mathrm{x}$ & -0.0 & -0. & & -0.0 & & & -0 \\
\hline N1 & $\mathrm{Y}$ & -0 & -0 . & & & & & \\
\hline N1 & Z & 0.0 & 0.0 & -0.01566 & -0.01128 & -0.00151 & -0 . & \\
\hline Re1 & $\mathrm{x}$ & -0.0 & -0.0 & -0.00032 & 0.00002 & 0224 & -0 . & \\
\hline Re1 & $\mathrm{Y}$ & & -0 . & & & & & \\
\hline Re1 & z & -0. & -0 . & & -0.0 & 061 & -0 & \\
\hline 01 & $\mathrm{x}$ & -0.0 & -0. & 0. & -0.00042 & 0199 & -0 & \\
\hline 01 & $\mathrm{Y}$ & & & & -0 . & & & \\
\hline 01 & z & -0 & -0 & -0. & & & 111 & -0 . \\
\hline $\mathrm{O} 2$ & $\mathrm{x}$ & 0.0 & -0.0 & 0.0 & 0.00049 & 298 & 081 & 0 \\
\hline 02 & $\mathrm{Y}$ & -0.0 & & & & & & \\
\hline 02 & Z & -0 . & & -0 & -0 & -0 & -0 & -0 \\
\hline C3 & $\mathrm{x}$ & -0.0 & 0. & -0. & -0.00074 & -0.11973 & -0 & -0 . \\
\hline C3 & Y & -0. & & & & & & \\
\hline C3 & Z & & & -0 . & -0 & -0 & -0 & -0 \\
\hline $\mathrm{C} 10$ & $\mathrm{x}$ & & & -0.0 & -0.00205 & 0.02482 & 0291 & 703 \\
\hline C10 & $\mathrm{Y}$ & -0.0 & & & & & & \\
\hline C10 & Z & -0.0 & & & & -0 & & \\
\hline 03 & $\mathrm{x}$ & -0.0 & & -0. & -0.0 & 2674 & -0 . & -0 \\
\hline 03 & $\mathrm{Y}$ & & -0.0 & & & -0.0 & -0 & \\
\hline 03 & Z & & & -0.0 & -0.0 & & & \\
\hline 04 & $\mathrm{x}$ & 0. & -0.1 & & & -0.03589 & -0 . & -0 \\
\hline & Y & 0. & & & -0.00 & 0.0 & -0.0 & \\
\hline 04 & Z & & -0.08663 & & & -0.02807 & -0.00113 & \\
\hline H9 & $\mathrm{x}$ & -0.67828 & -0.00165 & -0.01560 & & & & \\
\hline $\mathrm{H} 9$ & Y & 0.01207 & -0.01 & 0.30934 & -0.48696 & -0.00762 & -0.38632 & \\
\hline
\end{tabular}




\begin{tabular}{|c|c|c|c|c|c|c|c|c|}
\hline H9 & z & 0.04750 & 0.00026 & -0.16562 & 0.22982 & 0.00790 & 0.23974 & -0.00715 \\
\hline H17 & $\mathrm{x}$ & 0.51824 & 0.01122 & -0.00183 & -0.04047 & -0.01122 & 0.02209 & -0.00102 \\
\hline H17 & $\mathrm{Y}$ & 0.00364 & -0.00029 & -0.01566 & 0.07740 & 0.00270 & -0.03252 & 0.00117 \\
\hline H17 & Z & -0.03409 & -0.00169 & 0.37059 & -0.62024 & -0.01315 & 0.35112 & -0.01550 \\
\hline H19 & $\mathrm{x}$ & 0.09922 & -0.01698 & -0.09053 & -0.05335 & -0.00518 & 0.03357 & 0.00462 \\
\hline H19 & $\mathrm{Y}$ & -0.00760 & -0.00110 & -0.10635 & -0.03472 & 0.00057 & 0.05732 & -0.00378 \\
\hline H19 & Z & 0.00256 & 0.03332 & 0.61916 & 0.37466 & -0.01509 & -0.35820 & \\
\hline H22 & $\mathrm{x}$ & 0.05765 & 0.00924 & 0.04484 & 0.01538 & 0.00115 & -0.00957 & -0.00113 \\
\hline H22 & $\mathrm{Y}$ & -0.01850 & -0.01286 & -0.42431 & -0.24197 & 0.00969 & -0.35171 & 0.00753 \\
\hline H22 & Z & -0.00829 & -0.01221 & -0.35162 & -0.17504 & & -0.35098 & \\
\hline H3 & $\mathrm{x}$ & 0.00285 & 0.26794 & 0.00612 & -0.00536 & 0.43767 & -0.01006 & -0.38512 \\
\hline H3 & $\mathrm{Y}$ & -0.00097 & -0.15365 & -0.00075 & 0.00331 & -0.16490 & 0.00302 & \\
\hline H3 & Z & 0.00220 & 0.20677 & 0.00644 & -0.00328 & & -0.00956 & \\
\hline H4 & $\mathrm{x}$ & 0.00843 & -0.31903 & 0.01888 & 0.00709 & 0.33969 & 0.00259 & \\
\hline H 4 & $\mathrm{Y}$ & 0.00593 & -0.33486 & 0.01930 & & & 0.00200 & \\
\hline H4 & Z & -0.00942 & -0.06009 & 0.00395 & 0.00136 & 0.11514 & 0.00114 & \\
\hline \multicolumn{2}{|c|}{ Erequencies } & & & & & & 98.57 & \\
\hline & & & & & & & & \\
\hline $\mathrm{C} 1$ & $\mathrm{X}$ & -0.00097 & -0.00077 & 0.00264 & 0.00016 & 0.00000 & 0.00003 & 0.00004 \\
\hline $\mathrm{C} 1$ & $\mathrm{Y}$ & -0.09251 & -0.08298 & 0.05490 & 0.0 & 0.00063 & 3373 & \\
\hline & Z & & -0.1 & & & -0 . & -0 . & \\
\hline N2 & $\mathrm{x}$ & 0.00191 & 0.00043 & -0.00065 & -0.00180 & -0.00007 & -0.00011 & -0.00010 \\
\hline & $\mathrm{Y}$ & 0.0 & 219 & -0.03195 & -0.0 & -0.00015 & -0.00114 & \\
\hline & Z & -0.0 & & -0. & -0 . & -0 . & & \\
\hline C7 & $\mathrm{x}$ & 0.00217 & -0.01239 & -0.00980 & 0.0 & -0.00002 & 0.00362 & -0 \\
\hline C7 & $\mathrm{Y}$ & 0.08627 & 0.12127 & 0.07678 & -0.00 & -0.00025 & 2503 & \\
\hline C & Z & & -0 & -0 . & -0 . & -0 . & & \\
\hline N1 & $\mathrm{x}$ & -0 & 0. & 787 & -0 . & 02 & 0020 & \\
\hline & $\mathrm{Y}$ & -0 & -0. & -0. & -0 & & -0 . & \\
\hline & Z & -0 & & & & -0 & -0 & \\
\hline Re1 & $\mathrm{x}$ & -0.0 & 0.0 & -0.0 & -0.0 & -0.000 & -0 & \\
\hline Re1 & $\mathrm{Y}$ & -0.0 & & & & -0 . & -0 . & \\
\hline e1 & Z & & 0. & & & -0 . & -0 & \\
\hline 01 & $\mathrm{x}$ & 0.00031 & 0.00036 & -0.00025 & -0.00 & -0.00026 & -0.00003 & -0 \\
\hline 01 & $\mathrm{Y}$ & 0.00259 & 0.00121 & -0.00208 & -0.0 & -0.00046 & -0.00008 & -0 \\
\hline 01 & Z & & -0.0 & -0 . & & & & \\
\hline $\mathrm{O} 2$ & $\mathrm{x}$ & 0.00050 & 0.0 & 0.00057 & 47 & -0.00023 & 00007 & -0 \\
\hline 02 & $\mathrm{Y}$ & -0.00118 & -0.00079 & -0.00138 & -0 & 0.0 & -0 . & \\
\hline $\mathrm{O} 2$ & Z & & -0 & & -0 & & -0 & \\
\hline C3 & $\mathrm{x}$ & 0083 & -0. & -0. & & 80 & -0 & -0 \\
\hline C3 & $\mathrm{Y}$ & -0.00016 & -0.00202 & -0.00027 & -0.0 & -0.06064 & 0027 & 047 \\
\hline & Z & & -0. & & & & & \\
\hline 10 & $\mathrm{x}$ & & -0. & 402 & -0 . & 165 & -0 & -0 \\
\hline C10 & $\mathrm{Y}$ & -0.00225 & 0.00291 & -0.00662 & 0.1 & 0.00141 & 0.0 & -0 . \\
\hline C10 & Z & & -0.0 & & -0 . & & -0 . & \\
\hline 03 & $\mathrm{x}$ & -0. & & -0 . & & -0 & & \\
\hline 03 & $\mathrm{Y}$ & 0.00278 & -0.00326 & 0.00482 & -0.11801 & 0.00031 & -0.00004 & -0.00001 \\
\hline 03 & Z & & & -0.0 & & & & \\
\hline & $\mathrm{x}$ & -0 & & -0 . & & -0 & & \\
\hline 04 & $\mathrm{Y}$ & -0.00091 & 0.0 & 0035 & -0.01014 & -0.00011 & 0002 & -0.00004 \\
\hline 04 & Z & & & -0.0 & & -0.0 & & \\
\hline & $\mathrm{x}$ & & & -0.0 & & & -0 . & \\
\hline H9 & $\mathrm{Y}$ & -0.31636 & -0.1 & -0.1 & -0. & & -0 & \\
\hline & Z & & -0.0 & -0. & & & -0 & \\
\hline H17 & $\mathrm{x}$ & & & & & & & \\
\hline H17 & $\mathrm{Y}$ & -0.01836 & -0.08 & -0.0 & -0.0 & -0.0 & 008 & -0 \\
\hline & Z & & & -0.0 & -0. & -0.0 & 223 & -0 \\
\hline H19 & $\mathrm{x}$ & -0.02615 & & -0.00785 & & -0.00026 & 0.00146 & \\
\hline H19 & $\mathrm{Y}$ & -0.06067 & & & -0.01451 & & 0.01733 & \\
\hline 19 & Z & 0.29433 & 0.06787 & -0.00652 & 0.028 & 0.00030 & 0.00347 & 0.0 \\
\hline
\end{tabular}




\begin{tabular}{|c|c|c|c|c|c|c|c|c|}
\hline H22 & $\mathrm{X}$ & 0.01018 & 0.00292 & -0.00711 & -0.00014 & 0.00017 & -0.00094 & -0.00117 \\
\hline H22 & $\mathrm{Y}$ & 0.23959 & 0.09865 & -0.19091 & -0.00574 & -0.00572 & -0.40170 & -0.44396 \\
\hline $\mathrm{H} 22$ & Z & 0.31767 & 0.01432 & -0.01641 & 0.00299 & 0.00695 & 0.50113 & 0.54185 \\
\hline H3 & $\mathrm{X}$ & -0.00420 & 0.00153 & 0.00305 & -0.06889 & -0.00071 & -0.00001 & 0.00024 \\
\hline H3 & $Y$ & 0.00134 & 0.00078 & -0.00171 & 0.03519 & 0.00135 & -0.00029 & 0.00085 \\
\hline H3 & $\mathrm{Z}$ & -0.00350 & 0.00236 & 0.00419 & -0.06046 & -0.00017 & -0.00013 & -0.00005 \\
\hline $\mathrm{H} 4$ & $\mathrm{X}$ & 0.00258 & -0.00364 & 0.00293 & -0.04447 & -0.62325 & 0.00245 & 0.00589 \\
\hline H4 & Y & 0.00026 & 0.00313 & 0.00341 & -0.05738 & 0.70865 & -0.00302 & -0.00640 \\
\hline H 4 & $\mathrm{Z}$ & -0.00418 & 0.00871 & 0.00242 & -0.01363 & -0.15061 & 0.00051 & 0.00152 \\
\hline \multicolumn{2}{|c|}{ frequencies } & 3530.66 & 3550.77 & 3737.12 & & & & \\
\hline \multicolumn{2}{|c|}{ intensities } & 132.23 & 121.03 & 178.22 & & & & \\
\hline C1 & $\mathrm{X}$ & -0.00004 & 0.00010 & -0.00002 & & & & \\
\hline $\mathrm{C} 1$ & Y & 0.00000 & -0.00044 & 0.00002 & & & & \\
\hline $\mathrm{C} 1$ & $\mathrm{Z}$ & 0.00602 & -0.00040 & 0.00006 & & & & \\
\hline N2 & $\mathrm{X}$ & -0.00294 & 0.00003 & -0.00002 & & & & \\
\hline N2 & Y & -0.06898 & -0.00101 & -0.00008 & & & & \\
\hline $\mathrm{N} 2$ & $\mathrm{Z}$ & -0.01921 & -0.00006 & -0.00004 & & & & \\
\hline C7 & $\mathrm{X}$ & -0.00018 & 0.00056 & 0.00001 & & & & \\
\hline $\mathrm{C} 7$ & Y & 0.00050 & -0.00075 & -0.00004 & & & & \\
\hline C7 & $\mathrm{Z}$ & -0.00028 & 0.00581 & 0.00013 & & & & \\
\hline N1 & $\mathrm{X}$ & -0.00001 & -0.00063 & -0.00002 & & & & \\
\hline N1 & Y & -0.00026 & 0.07154 & 0.00030 & & & & \\
\hline $\mathrm{N} 1$ & $\mathrm{Z}$ & 0.00018 & -0.00089 & -0.00004 & & & & \\
\hline $\operatorname{Re1}$ & $\mathrm{X}$ & -0.00003 & -0.00002 & 0.00000 & & & & \\
\hline $\operatorname{Re1}$ & Y & 0.00000 & -0.00003 & 0.00000 & & & & \\
\hline $\operatorname{Re1}$ & Z & 0.00001 & 0.00000 & 0.00001 & & & & \\
\hline 01 & $\mathrm{X}$ & 0.00006 & 0.00005 & 0.00002 & & & & \\
\hline 01 & Y & 0.00010 & 0.00012 & 0.00006 & & & & \\
\hline 01 & Z & -0.00007 & 0.00004 & 0.00000 & & & & \\
\hline $\mathrm{O} 2$ & $\mathrm{X}$ & 0.00014 & 0.00006 & 0.00004 & & & & \\
\hline 02 & Y & -0.00005 & -0.00007 & -0.00005 & & & & \\
\hline $\mathrm{O} 2$ & Z & -0.00003 & -0.00008 & -0.00006 & & & & \\
\hline $\mathrm{C} 3$ & $\mathrm{X}$ & 0.00001 & 0.00003 & 0.00007 & & & & \\
\hline C3 & Y & 0.00006 & 0.00006 & 0.00061 & & & & \\
\hline $\mathrm{C} 3$ & $\mathrm{Z}$ & 0.00007 & 0.00005 & 0.00001 & & & & \\
\hline C10 & $\mathrm{X}$ & -0.00005 & 0.00010 & -0.00088 & & & & \\
\hline $\mathrm{C} 10$ & Y & 0.00084 & 0.00007 & -0.00194 & & & & \\
\hline $\mathrm{C} 10$ & $\mathrm{Z}$ & -0.00012 & 0.00002 & -0.00036 & & & & \\
\hline 03 & $\mathrm{X}$ & 0.00003 & -0.00003 & 0.00061 & & & & \\
\hline 03 & Y & -0.00068 & -0.00002 & -0.00042 & & & & \\
\hline 03 & $\mathrm{Z}$ & -0.00004 & -0.00005 & 0.00051 & & & & \\
\hline 04 & $\mathrm{X}$ & 0.00011 & 0.00003 & -0.01927 & & & & \\
\hline 04 & Y & -0.00011 & 0.00022 & -0.05567 & & & & \\
\hline 04 & Z & 0.00011 & -0.00001 & -0.01007 & & & & \\
\hline H9 & $\mathrm{X}$ & 0.00054 & -0.00112 & 0.00013 & & & & \\
\hline H9 & Y & 0.00290 & -0.00942 & -0.00042 & & & & \\
\hline H9 & Z & 0.00462 & -0.01528 & -0.00073 & & & & \\
\hline H17 & $\mathrm{X}$ & 0.00021 & 0.00232 & 0.00025 & & & & \\
\hline H17 & Y & 0.00962 & -0.95845 & -0.00369 & & & & \\
\hline H1 7 & Z & 0.00042 & -0.04008 & -0.00024 & & & & \\
\hline H19 & $\mathrm{X}$ & 0.04133 & 0.00073 & -0.00043 & & & & \\
\hline H19 & Y & 0.93664 & 0.00897 & 0.00124 & & & & \\
\hline $\mathrm{H} 19$ & Z & 0.20254 & 0.00158 & -0.00057 & & & & \\
\hline H22 & $\mathrm{X}$ & 0.00067 & 0.00009 & 0.00016 & & & & \\
\hline H22 & Y & 0.01156 & -0.00350 & 0.00028 & & & & \\
\hline $\mathrm{H} 22$ & Z & -0.01165 & 0.00398 & -0.00032 & & & & \\
\hline H3 & $\mathrm{X}$ & -0.00102 & -0.00100 & 0.30374 & & & & \\
\hline H3 & Y & -0.00087 & -0.00360 & 0.90500 & & & & \\
\hline H3 & Z & -0.00012 & -0.00070 & 0.15549 & & & & \\
\hline H4 & $\mathrm{X}$ & 0.00172 & 0.00043 & 0.00083 & & & & \\
\hline
\end{tabular}


$\begin{array}{rrrrr}\mathrm{H} 4 & \mathrm{Y} & -0.00198 & -0.00068 & -0.00006 \\ \mathrm{H} 4 & \mathrm{Z} & 0.00038 & -0.00025 & 0.00063\end{array}$

Thermochemical Properties:

pressure: $\quad 1.0000 \mathrm{~atm}$

rotational symmetry number: 1

zero point energy: $\quad 72.322 \mathrm{kcal} / \mathrm{mol}$

$\begin{array}{rrrrr}\text { temp }(\mathrm{K}) & \mathrm{Cp}(\mathrm{cal} / \mathrm{mol} \mathrm{K}) & \mathrm{S}(\mathrm{cal} / \mathrm{mol} \mathrm{K}) & \mathrm{H} \quad(\mathrm{kcal} / \mathrm{mol}) & \mathrm{G}(\mathrm{kcal} / \mathrm{mol}) \\ 0.00 & 7.9489 & 28.9823 & 0.0000 & 0.0000 \\ 298.15 & 42.9353 & 107.5539 & 7.7658 & -24.3014\end{array}$

end of program freq

start of program ch

Moments from quantum mechanical wavefunction:

Dipole Moments (Debye)
$\mathrm{X}=$
$2.6612 \quad \mathrm{Y}=$
0.3833
$\mathrm{Z}=$
-9.1020 Tot $=$
9.4908

Final energy ........... -645.32433089403

Job WF24510 completed on CHENB091 at Tue Jan 13 03:52:02 2004

Reason for exit: Successful completion

Titan Program CPU Time : 017:17:23.2

Titan Program Wall Time: 018:45:06.2

TITAN Properties Program: (PC/x86)

Release 6.0 .6

Reason for exit: Successful completion

Properties Program CPU Time : 000:00:03.6

Properties Program Wall Time: 000:00:04.1 


\section{Scheme 5}

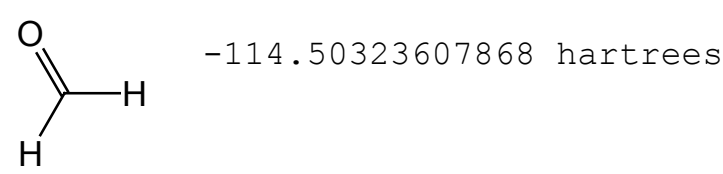

Jaguar version 3.5, release 42

Copyright 1991-1998 Schrodinger, Inc.

All Rights Reserved.

Use of this program should be acknowledged in publications as:

| Jaguar 3.5, Schrodinger, Inc., Portland, Oregon, 1998.

Job WF13309 started on CHENB071 at Wed Mar 12 10:21:21 2003

Jaguar version 3.5, release 42

Job name:

Task:

Stoichiometry:

Molecular weight:

Molecular charge:

Spin multiplicity:

Point Group:

SCF method:

Basis set:

Basis functions:

Total electrons:

Alpha electrons:

Beta electrons:

Total orbitals:

Core orbitals:

Open-shell orbitals:

Occupied orbitals:

Shells:
WF13309

Geometry optimization

$\mathrm{CH} 2 \mathrm{O}$

$30.01 \mathrm{amu}$

0

$\mathrm{c} 2 \mathrm{v}$

B3LYP

LACVP**

40

16

8

40

8

0

8

1

Non-default options chosen:

SCF calculation type: DFT

$\mathrm{DFT}=$ Becke_3_Parameter/HF+Slater+Becke88+VWN+LYP (B3LYP)

Geometry $\bar{w} \bar{l} \bar{l}$ be optimized in redundant internal coordinates

Initial Hessian: from previous calculation

Powell Hessian-updating used

\begin{tabular}{|c|c|c|c|c|c|c|}
\hline Iter & Energy & & Gmax & Grms & Dmax & Drms \\
\hline 1 & -114.501994 & * & 0.02527 & 0.00963 & 0.08380 & 0.03844 \\
\hline 2 & -114.503219 & . & 0.00347 & 0.00133 & 0.00455 & 0.00265 \\
\hline 3 & -114.503228 & $\star$ & 0.00074 & 0.00027 & 0.00076 & 0.00038 \\
\hline 4 & -114.503236 & $\star$ & 0.00004 & 0.00001 & 0.00002 & 0.00001 \\
\hline
\end{tabular}




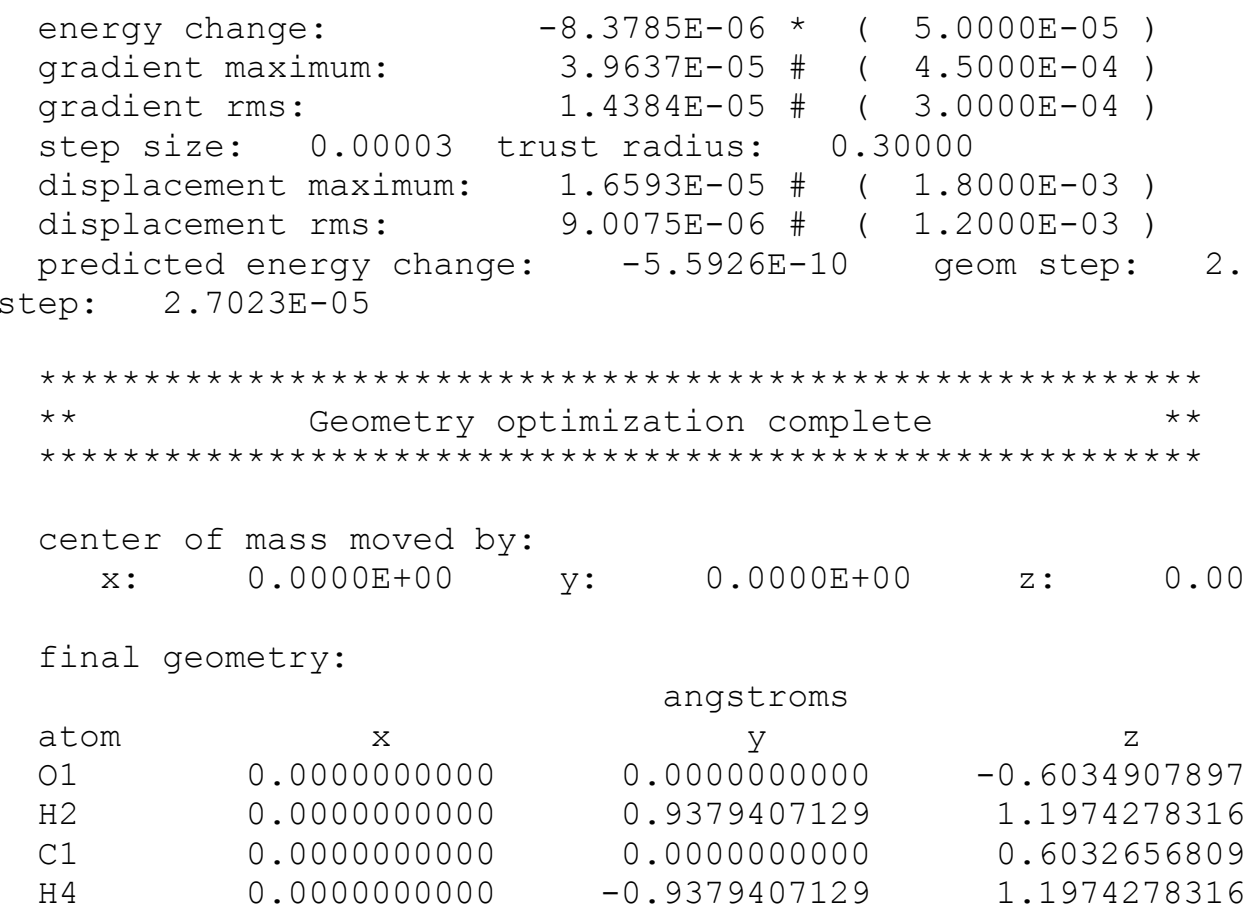

Final energy ............ -114.50323607868

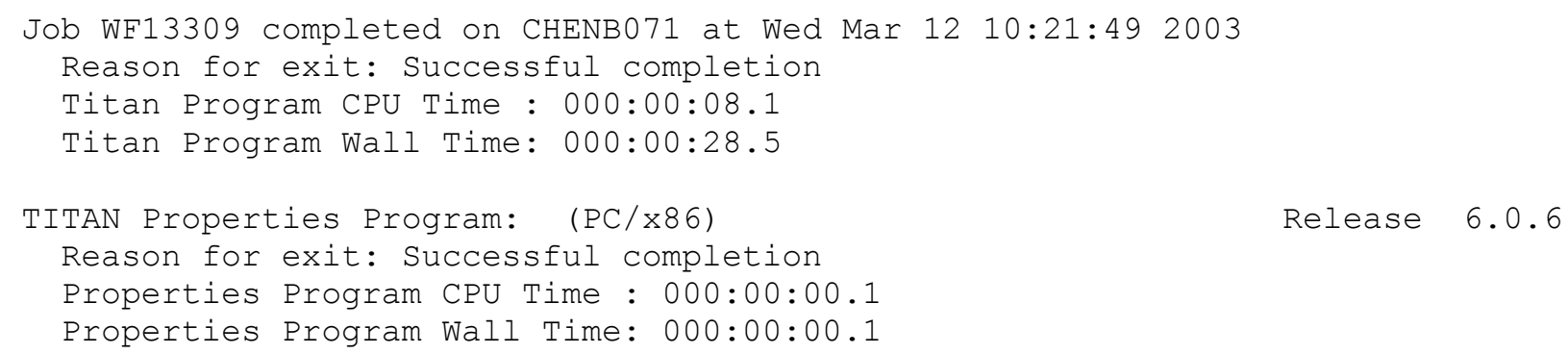




\section{Scheme 5}

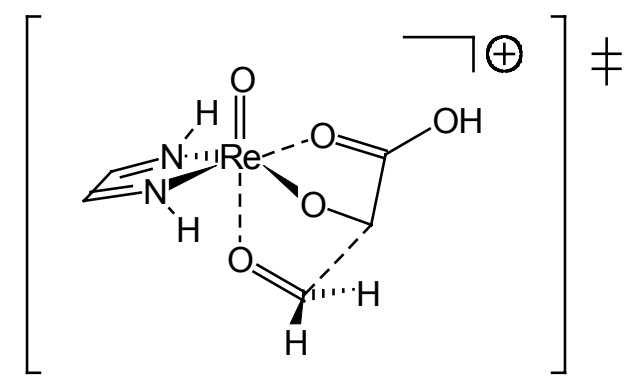

-759.84900112109 hartrees

Jaguar version 3.5 , release 42

| Copyright 1991-1998 Schrodinger, Inc.

I All Rights Reserved.

Use of this program should be acknowledged in publications as:

I Jaguar 3.5, Schrodinger, Inc., Portland, Oregon, 1998.

Job WF13610 started on CHENB071 at Tue Mar 11 17:39:26 2003

Jaguar version 3.5, release 42

$\begin{array}{lc}\text { Job name: } & \text { WF13610 } \\ \text { Task: } & \text { Transition-state search } \\ \text { Stoichiometry: } & \text { ReC5N2H805 } \\ \text { Molecular weight: } & 363.00 \text { amu } \\ \text { Molecular charge: } & 1 \\ \text { Spin multiplicity: } & 1 \\ \text { Point Group: } & \text { C1 } \\ & \\ \text { SCF method: } & \text { B3LYP } \\ \text { Basis set: } & \text { LACVP** } \\ \text { Basis functions: } & 230 \\ & \\ \text { Total electrons: } & 106 \\ \text { Alpha electrons: } & 53 \\ \text { Beta electrons: } & 53 \\ \text { Total orbitals: } & 245 \\ \text { Core orbitals: } & 53 \\ \text { Open-shell orbitals: } & 0 \\ \text { Occupied orbitals: } & 53 \\ \text { Shells: } & 1\end{array}$

Non-default options chosen:

SCF calculation type: DFT

DFT=Becke_3_Parameter/HF+Slater+Becke88+VWN+LYP (B3LYP)

Transition state optimization

Geometry will be optimized in redundant internal coordinates initial trust radius: 0.100

P-RFO level-shifting used

Molecular symmetry not used

Loose geometry convergence criteria

Initial Hessian: from previous calculation 
Powell Hessian-updating used

\begin{tabular}{|c|c|c|c|c|c|c|c|}
\hline [ter & Energy & & Gmax & Grms & & Dmax & Drms \\
\hline 1 & -759.833971 & * & 0.03051 & 0.01246 & & 0.02831 & 0.01189 \\
\hline 2 & -759.841464 & & 0.02329 & 0.00774 & & 0.03154 & 0.01189 \\
\hline 3 & -759.845691 & . & 0.00936 & 0.00413 & & 0.02727 & 0.01187 \\
\hline 4 & -759.847667 & . & 0.00899 & 0.00220 & & 0.06115 & 0.01190 \\
\hline 5 & -759.847999 & . & 0.01255 & 0.00192 & & 0.07709 & 0.01188 \\
\hline 6 & -759.848031 & * & 0.00791 & 0.00146 & A & 0.06852 & 0.01189 \\
\hline 7 & -759.848403 & . & 0.00686 & 0.00128 & & 0.04443 & 0.01187 \\
\hline 8 & -759.848666 & & 0.00599 & 0.00124 & & 0.03111 & 0.01193 \\
\hline 9 & -759.848726 & * & 0.00714 & 0.00173 & & 0.06528 & 0.01190 \\
\hline 10 & -759.848843 & $\star$ & 0.01281 & 0.00213 & & 0.06664 & 0.01188 \\
\hline 11 & -759.848894 & 夫 & 0.00445 & 0.00106 & . & 0.07673 & 0.01189 \\
\hline 12 & -759.848928 & 夫 & 0.00332 & 0.00065 & & 0.06433 & 0.01189 \\
\hline 13 & -759.848943 & * & 0.00277 & 0.00071 & 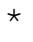 & 0.07251 & 0.01189 \\
\hline 14 & -759.849001 & $\star$ & 0.00118 & 0.00028 & $\#$ & 0.02940 & 0.00503 \\
\hline
\end{tabular}

energy change:

gradient maximum:

gradient rms:

step size: 0.04241

$-5.8543 \mathrm{E}-05 *(2.5000 \mathrm{E}-04)$

$1.1819 \mathrm{E}-03 *(2.2500 \mathrm{E}-03)$

2.8451E-04 \# (1.5000E-03)

trust radius: 0.10000

displacement maximum: 2.9401E-02 . (9.0000E-03)

displacement rms: $\quad 5.0326 \mathrm{E}-03 *(6.0000 \mathrm{E}-03)$

predicted energy change: $\quad-7.4061 \mathrm{E}-06$ geom step: $4.2405 \mathrm{E}-02$ full

step: $\quad 4.2405 \mathrm{E}-02$

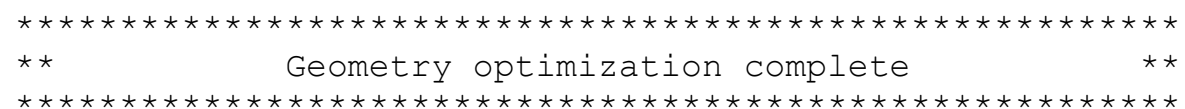

center of mass moved by:
$\mathrm{x}$ :
$0.0000 \mathrm{E}+00$
$\mathrm{y}:$
$0.0000 \mathrm{E}+00$
$\mathrm{z}:$
$0.0000 \mathrm{E}+00$

final geometry:

\begin{tabular}{lr} 
atom & \multicolumn{1}{c}{ x } \\
Re1 & 0.8855022123 \\
N2 & 1.5428388624 \\
C10 & 0.9219169886 \\
C1 & 0.1061081930 \\
N1 & 0.1307204958 \\
O1 & 2.1590050479 \\
O2 & -0.6721073398 \\
O3 & -1.0322999788 \\
C4 & -1.0496116281 \\
H19 & -1.8574216637 \\
C7 & -1.9150580011 \\
H21 & -1.9213854546 \\
C15 & -0.1949370800 \\
O6 & 0.7862117291 \\
O7 & -0.3236503067 \\
H4 & -2.8192449753 \\
H8 & 0.3316966181 \\
H12 & -0.3297784779 \\
H20 & -0.4143093494 \\
H22 & 1.0602978409 \\
H26 & 2.2261496721
\end{tabular}

angstroms
y
-0.8420355151
0.8841385489
1.9742668931
1.8677663057
0.7025975085
-1.5517122401
-1.9099676101
-0.0182799710
-2.7777593153
-3.4638018554
-0.8123950624
-1.1822259132
-2.8451861551
-1.9523502699
-3.7122835854
-0.9963538392
-3.5147035506
0.6018226581
2.7248458408
2.9211563046
0.9410888975

z

0.0449994572

1.0266997279

0.6798232934

$-0.5219949096$

$-1.1051487514$

$-0.7768608375$

$-0.4170417725$

1.3633185082

0.5624980652

0.3434775363

1.7564225670

2.7913804814

1.6466382976

1.7072966782

2. 6410367947

1. 1601520722

3. 3295746517

$-2.0092816602$

$-0.9415965429$

1.1951253734

1.7810765686 
Final energy ............ $\quad-759.84900112109$

Job WF13610 completed on CHENB071 at Tue Mar 11 19:49:59 2003

Reason for exit: Successful completion

Titan Program CPU Time : 002:06:29.6

Titan Program Wall Time: 002:10:33.2

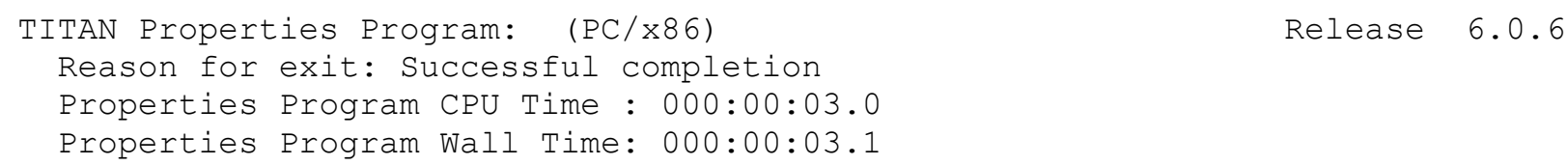

Job name:

Task:

Stoichiometry:

Molecular weight:

Molecular charge:

Spin multiplicity:

Point Group:

SCF method:

Basis set:

Basis functions:

Total electrons:

Alpha electrons:

Beta electrons:

Total orbitals:

Core orbitals:

Open-shell orbitals:

Occupied orbitals:

Shells:
WF23928

Calculate energy ("single-point job")

ReC5N2H8O
363.00 amu
1
1
C1
B3LYP
LACVP *
230
106
53
53
245
53
0
53
1

Non-default options chosen:

SCF calculation type: DFT

$\mathrm{DFT}=$ Becke 3 Parameter $/ \mathrm{HF}+\mathrm{Slater}+\mathrm{Becke} 88+\mathrm{VWN}+\mathrm{LYP}$ (B3LYP)

Numerical $2 \bar{n} d$ derivatives will be computed

Vibrational frequencies and related properties will be computed

Molecular symmetry not used

$\begin{array}{llll}\text { calculating forces at perturbed geometry } & 1 \text { of } 126 \ldots \\ \text { calculating forces at perturbed geometry } & 2 \text { of } 126 \ldots \\ \text { calculating forces at perturbed geometry } & 3 \text { of } 126 \ldots \\ \text { calculating forces at perturbed geometry } & 4 \text { of } 126 \ldots \\ \text { calculating forces at perturbed geometry } & 5 \text { of } 126 \ldots \\ \text { calculating forces at perturbed geometry } & 6 \text { of } 126 \ldots \\ \text { calculating forces at perturbed geometry } & 7 \text { of } 126 \ldots \\ \text { calculating forces at perturbed geometry } & 8 \text { of } 126 \ldots \\ \text { calculating forces at perturbed geometry } & 9 \text { of } 126 \ldots \\ \text { calculating forces at perturbed geometry } & 10 \text { of } 126 \ldots\end{array}$


calculating forces at perturbed geometry calculating forces at perturbed geometry calculating forces at perturbed geometry calculating forces at perturbed geometry calculating forces at perturbed geometry calculating forces at perturbed geometry calculating forces at perturbed geometry calculating forces at perturbed geometry calculating forces at perturbed geometry calculating forces at perturbed geometry calculating forces at perturbed geometry calculating forces at perturbed geometry calculating forces at perturbed geometry calculating forces at perturbed geometry calculating forces at perturbed geometry calculating forces at perturbed geometry calculating forces at perturbed geometry calculating forces at perturbed geometry calculating forces at perturbed geometry calculating forces at perturbed geometry calculating forces at perturbed geometry calculating forces at perturbed geometry calculating forces at perturbed geometry calculating forces at perturbed geometry calculating forces at perturbed geometry calculating forces at perturbed geometry calculating forces at perturbed geometry calculating forces at perturbed geometry calculating forces at perturbed geometry calculating forces at perturbed geometry calculating forces at perturbed geometry calculating forces at perturbed geometry calculating forces at perturbed geometry calculating forces at perturbed geometry calculating forces at perturbed geometry calculating forces at perturbed geometry calculating forces at perturbed geometry calculating forces at perturbed geometry calculating forces at perturbed geometry calculating forces at perturbed geometry calculating forces at perturbed geometry calculating forces at perturbed geometry calculating forces at perturbed geometry calculating forces at perturbed geometry calculating forces at perturbed geometry calculating forces at perturbed geometry calculating forces at perturbed geometry calculating forces at perturbed geometry calculating forces at perturbed geometry calculating forces at perturbed geometry calculating forces at perturbed geometry calculating forces at perturbed geometry calculating forces at perturbed geometry calculating forces at perturbed geometry calculating forces at perturbed geometry calculating forces at perturbed geometry calculating forces at perturbed geometry calculating forces at perturbed geometry calculating forces at perturbed geometry calculating forces at perturbed geometry calculating forces at perturbed geometry
11 of $126 \ldots$ 12 of $126 \ldots$ 13 of $126 \ldots$ 14 of $126 \ldots$ 15 of $126 \ldots$ 16 of $126 \ldots$ 17 of $126 \ldots$ 18 of $126 \ldots$ 19 of $126 \ldots$ 20 of $126 \ldots$ 21 of $126 \ldots$ 22 of $126 \ldots$ 23 of $126 \ldots$ 24 of $126 \ldots$ 25 of $126 \ldots$ 26 of $126 \ldots$ 27 of $126 \ldots$ 28 of $126 \ldots$ 29 of $126 \ldots$ 30 of $126 \ldots$ 31 of $126 \ldots$ 32 of $126 \ldots$ 33 of $126 \ldots$ 34 of $126 \ldots$ 35 of $126 \ldots$ 36 of $126 \ldots$ 37 of $126 \ldots$ 38 of $126 \ldots$ 39 of $126 \ldots$ 40 of $126 \ldots$ 41 of $126 \ldots$ 42 of $126 \ldots$ 43 of $126 \ldots$ 44 of $126 \ldots$ 45 of $126 \ldots$ 46 of $126 \ldots$ 47 of $126 \ldots$ 48 of $126 \ldots$ 49 of $126 \ldots$ 50 of $126 \ldots$ 51 of $126 \ldots$ 52 of $126 \ldots$ 53 of $126 \ldots$ 54 of $126 \ldots$ 55 of $126 \ldots$ 56 of $126 \ldots$ 57 of $126 \ldots$ 58 of $126 \ldots$ 59 of $126 \ldots$ 60 of $126 \ldots$ 61 of $126 \ldots$ 62 of $126 \ldots$ 63 of $126 \ldots$ 64 of $126 \ldots$ 65 of $126 \ldots$ 66 of $126 \ldots$ 67 of $126 \ldots$ 68 of $126 \ldots$ 69 of $126 \ldots$ 70 of $126 \ldots$ 71 of $126 \ldots$ 
calculating forces at perturbed geometry calculating forces at perturbed geometry calculating forces at perturbed geometry calculating forces at perturbed geometry calculating forces at perturbed geometry calculating forces at perturbed geometry calculating forces at perturbed geometry calculating forces at perturbed geometry calculating forces at perturbed geometry calculating forces at perturbed geometry calculating forces at perturbed geometry calculating forces at perturbed geometry calculating forces at perturbed geometry calculating forces at perturbed geometry calculating forces at perturbed geometry calculating forces at perturbed geometry calculating forces at perturbed geometry calculating forces at perturbed geometry calculating forces at perturbed geometry calculating forces at perturbed geometry calculating forces at perturbed geometry calculating forces at perturbed geometry calculating forces at perturbed geometry calculating forces at perturbed geometry calculating forces at perturbed geometry calculating forces at perturbed geometry calculating forces at perturbed geometry calculating forces at perturbed geometry calculating forces at perturbed geometry calculating forces at perturbed geometry 101 calculating forces at perturbed geometry 102 of $126 \ldots$ calculating forces at perturbed geometry 103 of $126 \ldots$ calculating forces at perturbed geometry 104 of $126 \ldots$ calculating forces at perturbed geometry 105 of $126 \ldots$ calculating forces at perturbed geometry 106 of $126 \ldots$ calculating forces at perturbed geometry 107 of $126 \ldots$ calculating forces at perturbed geometry 108 of $126 \ldots$ calculating forces at perturbed geometry 109 of $126 \ldots$ calculating forces at perturbed geometry 110 of $126 \ldots$ calculating forces at perturbed geometry 111 of $126 \ldots$ calculating forces at perturbed geometry 112 of $126 \ldots$ calculating forces at perturbed geometry 113 of $126 \ldots$ calculating forces at perturbed geometry 114 of $126 \ldots$ calculating forces at perturbed geometry 115 of $126 \ldots$ calculating forces at perturbed geometry 116 of $126 \ldots$ calculating forces at perturbed geometry 117 of $126 \ldots$ calculating forces at perturbed geometry 118 of $126 \ldots$ calculating forces at perturbed geometry 119 of $126 \ldots$ calculating forces at perturbed geometry 120 of $126 \ldots$ calculating forces at perturbed geometry 121 of $126 \ldots$ calculating forces at perturbed geometry 122 of $126 \ldots$ calculating forces at perturbed geometry 123 of $126 \ldots$ calculating forces at perturbed geometry 124 of $126 \ldots$ calculating forces at perturbed geometry 125 of $126 \ldots$ calculating forces at perturbed geometry 126 of $126 \ldots$

numerical derivatives complete;

calculation at normal geometry follows...

start of program freq

72 of $126 \ldots$

73 of $126 \ldots$ 75 of $126 \ldots$ 76 of $126 \ldots$ 77 of $126 \ldots$ 79 of $126 \ldots$ 80 of $126 \ldots$ 81 of $126 \ldots$ 83 of $126 \ldots$ 84 of $126 \ldots$ 86 of $126 \ldots$ 87 of $126 \ldots$ 89 of $126 \ldots$ 90 of $126 \ldots$ 91 of $126 \ldots$ 93 of $126 \ldots$ 94 of $126 \ldots$ 5 of $126 \ldots$ 97 of $126 \ldots$ 98 of $126 \ldots$ 99 of $126 \ldots$ 100 of $126 \ldots$

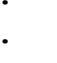
. 
harmonic frequencies in $\mathrm{cm}^{\wedge}-1$, IR intensities in $\mathrm{km} / \mathrm{mol}$, and normal modes:

\begin{tabular}{|c|c|c|c|c|c|c|c|c|}
\hline \multirow{2}{*}{\multicolumn{2}{|c|}{$\begin{array}{l}\text { frequencies } \\
\text { intensities }\end{array}$}} & -184.48 & 38.71 & 95.72 & 107.92 & 147.16 & 163.35 & 187.5 \\
\hline & & 32.75 & 2.78 & 6.67 & 7.39 & 2.04 & 6.03 & \\
\hline Re1 & $\mathrm{X}$ & 0.02156 & 0.00540 & 0.01387 & 0.01903 & -0.00431 & 0.00524 & -0.007 \\
\hline Re1 & $\mathrm{Y}$ & -0.01088 & -0.00394 & -0.00475 & -0.00096 & -0.00262 & 0.00124 & \\
\hline Re1 & $\mathrm{Z}$ & -0.01232 & -0.00984 & -0.01861 & 0.00418 & -0.00801 & 0.00512 & -0.006 \\
\hline N2 & $\mathrm{X}$ & -0.04500 & -0.00498 & -0.01013 & -0.03710 & -0.09218 & 0.03561 & \\
\hline N2 & Y & -0.02178 & -0.01700 & -0.03350 & 0.03412 & -0.01819 & -0.00762 & -0 \\
\hline $\mathrm{N} 2$ & $\mathrm{Z}$ & 0.02381 & 0.00650 & 0.02885 & -0.02180 & 0.07472 & -0.02489 & -0.0 \\
\hline C10 & $\mathrm{x}$ & -0.00805 & -0.02407 & -0.05436 & -0.08030 & -0.07406 & -0.03480 & -0.0 \\
\hline $\mathrm{C} 10$ & Y & -0.00185 & -0.01862 & -0.04063 & 0.00507 & -0.01750 & -0.02796 & -0 \\
\hline C10 & $\mathrm{Z}$ & -0.00254 & 0.03442 & 0.08599 & -0.03955 & 0.04389 & 0.02765 & \\
\hline $\mathrm{C} 1$ & $\mathrm{X}$ & -0.00858 & -0.02695 & -0.04913 & -0.08292 & 0.06752 & -0.06476 & -0 \\
\hline $\mathrm{C} 1$ & Y & -0.00255 & -0.00746 & -0.01471 & -0.04570 & 0.01056 & -0.035 & -0 \\
\hline C1 & $\mathrm{Z}$ & 0.01147 & 0.03666 & 0.07987 & -0.03248 & -0.05470 & 0.05 & \\
\hline N1 & $\mathrm{x}$ & -0.03531 & -0.01628 & -0.00670 & -0.02051 & 0.10681 & -0.00 & \\
\hline N1 & Y & -0.02191 & 0.00267 & 0.01293 & -0.04869 & 0.01462 & -0.01 & \\
\hline N1 & Z & 0.03369 & 0.01625 & 0.025 & -0.02273 & -0.059 & 0.0 & -0 \\
\hline 01 & $\mathrm{X}$ & 0.01630 & -0.00413 & 0.01245 & 0.03074 & -0.012 & -0.02 & -0 \\
\hline 01 & $Y$ & -0.00684 & -0.00244 & 0.00111 & 0.00853 & -0.01653 & -0.082 & -0 \\
\hline 01 & Z & -0.015 & -0.02539 & -0.025 & 0.01419 & -0.008 & 0.02 & \\
\hline 02 & $\mathrm{x}$ & -0.03549 & -0.00439 & 0.00706 & -0.01679 & -0.03173 & -0.01 & \\
\hline 02 & Y & 0.03737 & 0.00520 & -0.00908 & 0.05605 & 0.02914 & 0.02 & -0 \\
\hline 02 & $\mathrm{Z}$ & 0.01 & 0.01080 & -0.008 & 0.00 & 0.02 & 0 & -0 \\
\hline 03 & $\mathrm{x}$ & -0.09334 & 0.05683 & -0.02831 & 0.05819 & -0.003 & -0.01 & \\
\hline 03 & $Y$ & 0.05844 & -0.00986 & -0.00936 & 0.00617 & 0.014 & 0.04 & -0 \\
\hline 03 & Z & 0.06 & 0.10 & -0.0 & 0.06 & & -0 & \\
\hline $\mathrm{C} 4$ & $\mathrm{X}$ & 0.049 & -0.00957 & -0.01673 & -0.05524 & -0.022 & -0.03 & \\
\hline $\mathrm{C} 4$ & Y & -0.05322 & 0.02414 & 0.03443 & 0.06836 & 0.03584 & 0.05 & -0 \\
\hline $\mathrm{C} 4$ & Z & -0.050 & 0.03390 & 0.01 & -0.00 & 0.04 & 0 . & -0 \\
\hline H19 & $\mathrm{X}$ & 0.071 & -0.00124 & -0.02856 & -0.07813 & -0.024 & -0.03 & \\
\hline $\mathrm{H} 19$ & Y & -0.074 & 0.01259 & 0.041 & 0.09724 & 0.03569 & 0.06 & -0 . \\
\hline H19 & $\mathrm{Z}$ & -0.063 & 0.03 & 0.03 & -0.008 & & 0 & -0 \\
\hline C7 & $\mathrm{X}$ & -0.0 & -0.07385 & 0.039 & 0.04604 & 0.007 & -0.01 & \\
\hline C7 & $\mathrm{Y}$ & 0.084 & 0.01267 & -0.04604 & 0.0033 & 0.00282 & 0.06 & -0 \\
\hline $\mathrm{C} 7$ & Z & 0.0 & -0.13488 & 0.02 & 0.046 & 0.02 & -0 & \\
\hline $\mathrm{H} 21$ & $\mathrm{X}$ & -0 & -0.35938 & 0.150 & 0.04385 & 0.01 & 0 & \\
\hline $\mathrm{H} 21$ & Y & 0.054 & 0.02338 & -0.03825 & -0.02357 & 0.01 & 0.09 & -0 \\
\hline H21 & Z & 0 & -0.13337 & 0.03 & 0.03 & 0.02 & -0 & \\
\hline C15 & $\mathrm{X}$ & -0.000 & 0.01116 & -0.020 & -0.03239 & 0 . & 0.0 & \\
\hline C15 & $\mathrm{Y}$ & 0.009 & 0.01620 & 0.044 & 0.00112 & 0.00 & 0.01 & \\
\hline C15 & $\mathrm{Z}$ & -0.008 & 0 & 0.023 & -0.01915 & 0.01 & 0.0 & -0 \\
\hline 06 & $\mathrm{X}$ & -0.040 & 0.01530 & 0.006 & 0.00480 & 0.02 & 0 . & -0 \\
\hline 06 & $\mathrm{Y}$ & 0.059 & 0.00654 & 0.014 & -0.04814 & -0.003 & 0.0 & \\
\hline 06 & $\mathrm{Z}$ & 0 & -0.00 & -0.005 & -0.00971 & -0.00 & 0.0 & -0 \\
\hline 07 & $\mathrm{X}$ & 0.012 & 0 & -0.059 & -0.07186 & 0.056 & 0.08 & -0 \\
\hline 07 & $\mathrm{Y}$ & -0.004 & 0.03439 & 0.095 & -0.02117 & -0.01686 & -0.06 & \\
\hline 07 & $\mathrm{Z}$ & -0 & 0 . & 0 & -0.04556 & -0.0 & -0.0 & -0 \\
\hline $\mathrm{H} 4$ & $\mathrm{X}$ & -0.043 & 0.07 & -0.032 & 0.02767 & 0.00252 & -0.03 & \\
\hline H4 & Y & -0.009 & 0.04450 & -0.047 & 0.0572 & -0.00488 & 0.06 & -0 \\
\hline $\mathrm{H} 4$ & $\mathrm{Z}$ & 0.0 & -0.3 & 0.136 & 0.057 & 0.031 & -0.0 & \\
\hline H8 & $\mathrm{X}$ & -0.00513 & 0.01189 & -0.05225 & -0.05411 & 0.08368 & 0.07 & -0 \\
\hline $\mathrm{H} 8$ & $\mathrm{Y}$ & 0.011 & 0.03 & 0.097 & -0.08297 & -0.03368 & -0.06 & \\
\hline H8 & $\mathrm{Z}$ & -0.00439 & 0.02812 & 0.05460 & -0.04468 & -0.02368 & -0.04 & 0 . \\
\hline $\mathrm{H} 12$ & $\mathrm{X}$ & -0.05825 & -0.01455 & -0.00785 & -0.03081 & 0.21381 & -0.026 & 0.065 \\
\hline $\mathrm{H} 12$ & $Y$ & -0.03574 & 0.01311 & 0.03654 & -0.09050 & 0.03773 & -0.01 & \\
\hline $\mathrm{H} 12$ & Z & 0.04742 & 0.01441 & 0.02400 & -0.01296 & -0.11658 & 0.01833 & -0 . \\
\hline $\mathrm{H} 2 \mathrm{O}$ & $\mathrm{X}$ & 0.00359 & -0.03685 & -0.08079 & -0.13136 & 0.13459 & -0.12745 & -0.040 \\
\hline $\mathrm{H} 2 \mathrm{O}$ & $Y$ & -0.00466 & -0.00609 & -0.01473 & -0.07722 & 0.02537 & -0.05 & -0 \\
\hline $\mathrm{H} 2 \mathrm{O}$ & Z & -0.00621 & 0 & 0.11952 & -0.03645 & -0.10732 & 0.09544 & 0 \\
\hline
\end{tabular}




\begin{tabular}{|c|c|c|c|c|c|c|c|c|}
\hline 122 & $\mathrm{X}$ & 0.02711 & -0.03694 & -0.09339 & -0.11440 & -0.15007 & -0.06691 & -0.0806 \\
\hline 22 & $\mathrm{Y}$ & 00056 & -0.02845 & -0.06214 & 0.01927 & -0.03328 & -0.03612 & -0 \\
\hline H22 & Z & -0.01751 & 0.05541 & 0.13576 & -0.05696 & 0.09344 & 0.05083 & 0.06268 \\
\hline $\mathrm{H} 2$ & $\mathrm{x}$ & -0.05111 & -0.00674 & -0.01703 & -0.03381 & -0.18745 & 0.06524 & 0.02559 \\
\hline & $\mathrm{Y}$ & -0.03150 & -0.02597 & -0.05032 & 0.07560 & -0.03771 & & \\
\hline & Z & 0.02963 & 0.00860 & 0.03639 & -0.02807 & 0.16208 & -0.05289 & -0.02879 \\
\hline \multicolumn{2}{|c|}{ Erequencies } & 192.67 & 218.84 & 258.86 & 267.07 & 322.08 & 340.84 & 388. \\
\hline & itis & 26.49 & 2.60 & 10.69 & 8.44 & 8.33 & 29.03 & \\
\hline e1 & $\mathrm{x}$ & 0.01194 & -0.00967 & 0.00061 & -0.00671 & 0.01058 & 0.00556 & $0.007^{\circ}$ \\
\hline $\mathrm{R}$ & $\mathrm{Y}$ & 0.00535 & -0.00599 & -0.01354 & 0.00663 & 0.00339 & -0.01917 & 0.001 \\
\hline e1 & z & 0.00153 & -0.01644 & -0.00074 & 0.00649 & -0.01841 & 0.01321 & -0.00531 \\
\hline N2 & $\mathrm{x}$ & 0.00982 & -0.00006 & -0.08532 & 0.05099 & 0.02782 & 0.01048 & -0.00765 \\
\hline & $\mathrm{Y}$ & 0.00964 & -0.04460 & 0.0 & -0.05641 & 0.05212 & 0.05786 & \\
\hline & Z & -0.01361 & -0.01517 & 0.0 & -0.01273 & -0.03909 & -0.00249 & \\
\hline C10 & $\mathrm{x}$ & 0.04423 & 0.03939 & 0.00702 & -0.00213 & -0.02680 & -0.01220 & 0.00975 \\
\hline $\mathrm{C} 10$ & $\mathrm{Y}$ & 0.02279 & -0.01981 & 0.0 & -0.07811 & 0.03908 & 4166 & 1290 \\
\hline $\mathrm{C} 10$ & Z & -0.03933 & -0.00014 & -0.0 & 0.00425 & & & \\
\hline $\mathrm{C} 1$ & $\mathrm{x}$ & 0.02594 & 0.01770 & 0.01658 & 0.01041 & -0.00493 & -0.01916 & -0.01356 \\
\hline $\mathrm{C} 1$ & $\mathrm{Y}$ & 0.01142 & 0.01243 & 0.0 & -0.0 & 0.05017 & 0.01972 & -0.00243 \\
\hline $\mathrm{C} 1$ & Z & -0.02214 & 0.0 & -0.0 & -0. & & & \\
\hline & $\mathrm{x}$ & -0.00530 & -0.0 & -0.0 & 0.04536 & 0.02430 & 2326 & \\
\hline N1 & $\mathrm{Y}$ & -0.0 & 1625 & 0.0 & -0.06684 & 0.05717 & 2557 & 0324 \\
\hline $\mathrm{N}$ & Z & 234 & 0.0 & & -0 & -0 & & -0 \\
\hline 01 & $\mathrm{x}$ & 4024 & 0.1 & -0.0 & -0.0 & 0.00936 & & 2 \\
\hline 01 & $\mathrm{Y}$ & -0.06919 & 0.0 & -0.0 & 0.0 & -0.00727 & 1093 & -0 \\
\hline 01 & Z & -0.0 & 0.1 & -0.0 & -0.0 & -0.01399 & -0.0 & 0 \\
\hline $\mathrm{O} 2$ & $\mathrm{x}$ & -0.02305 & -0.0 & -0 & -0 & & 1331 & -0 \\
\hline $\mathrm{O} 2$ & $\mathrm{Y}$ & 0.05449 & 0.0 & -0. & & -0.07908 & -0 & \\
\hline $\mathrm{O} 2$ & Z & 221 & 0.0 & -0 . & -0 & 0 & -0 . & \\
\hline 03 & $\mathrm{x}$ & -0.09757 & -0.00198 & & 0.0 & -0.00603 & 0.00801 & \\
\hline 03 & $\mathrm{Y}$ & -0.05248 & -0.00322 & 0. & -0.01 & -0.01457 & -0.01519 & -0.13785 \\
\hline 03 & Z & & 0 & -0 . & & 0 & & -0 \\
\hline C4 & $\mathrm{x}$ & -0.03064 & -0.04317 & & 0. & -0.03750 & -0.08057 & \\
\hline C4 & $\mathrm{Y}$ & 0.07684 & 0.04351 & 0.0 & & -0.01189 & 0.01177 & -0.05084 \\
\hline C4 & Z & & 0.0 & -0 & -0 & & -0 . & -0 \\
\hline & $\mathrm{x}$ & 279 & -0.0 & & & -0.05945 & -0 . & \\
\hline H19 & $\mathrm{Y}$ & 0.06593 & 0.0 & -0.02705 & -0.02361 & 0.01570 & 0.05725 & -0.08094 \\
\hline & Z & & & & -0.0 & & -0 . & -0 \\
\hline C & $\mathrm{x}$ & -0 . & & & & -0.01554 & -0 & -0 \\
\hline C7 & $\mathrm{Y}$ & -0.15467 & -0.02890 & -0.01477 & -0.03639 & -0.00672 & -0.00368 & 0.01581 \\
\hline C7 & Z & & & -0.0 & & & & \\
\hline $\mathrm{H}$ & $\mathrm{x}$ & -0 . & & & & -0. & & -0 \\
\hline 21 & $\mathrm{Y}$ & -0.19266 & -0.03207 & 0. & -0.02552 & 0.00626 & -0.03337 & 0.10567 \\
\hline H21 & Z & & & -0 . & & & & \\
\hline & $\mathrm{x}$ & & -0 & & -0 & -0.0 & -0 . & -0 \\
\hline 15 & $\mathrm{Y}$ & & & & & -0.01786 & 508 & -0.01834 \\
\hline C15 & Z & & 776 & & & & -0 & \\
\hline & $\mathrm{x}$ & & -0.0 & -0 & -0 . & -0.07 & -0 . & \\
\hline 06 & $\mathrm{Y}$ & & 0. & & & 0.01825 & 2927 & \\
\hline 06 & Z & 0.01852 & -0.00163 & 0.0 & 0. & 0.04605 & -0.07216 & 0.01650 \\
\hline 07 & $\mathrm{x}$ & & & & & & & \\
\hline 07 & $\mathrm{Y}$ & & -0.0 & -0.0 & -0.0 & -0.10 & 597 & \\
\hline 07 & Z & -0.02400 & -0.01189 & -0.04027 & -0.04164 & 0.00417 & 0.01181 & 0.04210 \\
\hline & $\mathrm{x}$ & & & & & -0.02015 & -0 & \\
\hline & $\mathrm{Y}$ & & -0.01211 & -0.016 & -0.046 & & & \\
\hline H 4 & Z & -0.00001 & 0.01628 & -0.04282 & -0.00989 & 0.02799 & 0.03631 & 0.00543 \\
\hline & $\mathrm{x}$ & -0.00041 & 0.05419 & -0.06834 & -0.08749 & -0.14435 & & \\
\hline & $\mathrm{Y}$ & -0.01650 & -0.00191 & -0.00124 & & -0.06087 & 0.20938 & \\
\hline H8 & Z & -0.01255 & -0.02165 & 0.02447 & 0.03878 & 0.10419 & -0.07755 & -0.11820 \\
\hline $\mathrm{H} 12$ & $\mathrm{X}$ & -0.04222 & -0.06309 & -0.11737 & 0.08629 & 0.06674 & 0.03595 & 0.00853 \\
\hline
\end{tabular}




$\begin{array}{rcrrrrrrr}\text { H12 } & \text { Y } & -0.03065 & 0.04339 & -0.00225 & -0.06826 & 0.08037 & 0.01995 & -0.00003 \\ \text { H12 } & \text { Z } & 0.03344 & 0.01113 & 0.08114 & -0.05376 & -0.02988 & 0.00039 & 0.00169 \\ \text { H20 } & \text { X } & 0.02890 & 0.03924 & 0.07786 & -0.00368 & 0.00699 & -0.03478 & -0.04621 \\ \text { H20 } & \text { Y } & 0.00618 & 0.03397 & 0.06302 & -0.08287 & 0.05290 & 0.00803 & -0.01571 \\ \text { H20 } & \text { Z } & -0.03560 & 0.02661 & -0.06391 & 0.01050 & -0.00264 & -0.00468 & 0.02843 \\ \text { H22 } & \text { X } & 0.07857 & 0.07970 & 0.05916 & -0.04517 & -0.06746 & -0.02012 & 0.04875 \\ \text { H22 } & \text { Y } & 0.03033 & -0.03006 & 0.05597 & -0.08124 & 0.02311 & 0.05075 & 0.02577 \\ \text { H22 } & \text { Z } & -0.06284 & 0.00718 & -0.03528 & 0.02053 & 0.06628 & -0.00929 & -0.04370 \\ \text { H26 } & \text { X } & 0.01028 & 0.00456 & -0.13617 & 0.06410 & 0.02191 & 0.01890 & 0.02264 \\ \text { H26 } & \text { Y } & 0.00999 & -0.08497 & -0.00225 & -0.04839 & 0.04849 & 0.08860 & 0.01147 \\ \text { H26 } & \text { Z } & -0.01368 & -0.01601 & 0.09973 & -0.02532 & -0.03390 & -0.01198 & -0.01867\end{array}$

\begin{tabular}{|c|c|c|c|c|c|c|c|c|}
\hline freque & ncies & 409.10 & 418.49 & 426.95 & 446.52 & 478.02 & 536.18 & 549.17 \\
\hline intens & ities & 21.87 & 17.47 & 73.94 & 1.83 & 3.07 & 38.93 & 12.22 \\
\hline $\operatorname{Re} 1$ & $\mathrm{X}$ & -0.00735 & -0.00870 & -0.00334 & -0.01151 & 0.00706 & 0.00453 & 0.00648 \\
\hline $\operatorname{Re} 1$ & $\mathrm{Y}$ & -0.02302 & -0.02297 & -0.00254 & -0.00028 & 0.00262 & 0.00823 & -0.00083 \\
\hline Re1 & Z & 0.00282 & 0.00528 & -0.00066 & -0.01740 & 0.01061 & 0.00362 & 0.00556 \\
\hline $\mathrm{N} 2$ & $\mathrm{x}$ & 0.02194 & 0.02358 & 0.01846 & -0.00657 & -0.10233 & -0.04873 & 0.06613 \\
\hline N2 & $\mathrm{Y}$ & 0.04426 & 0.03584 & 0.00853 & 0.07631 & -0.09106 & -0.02480 & \\
\hline $\mathrm{N} 2$ & Z & -0.04892 & -0.03527 & 0.00112 & 0.10752 & -0.00239 & -0.09083 & 0.06443 \\
\hline $\mathrm{C} 10$ & $\mathrm{x}$ & -0.01720 & -0.02435 & -0.00484 & 0.06999 & 0.06048 & -0.03317 & 0.0108 \\
\hline C10 & $\mathrm{Y}$ & 0.03922 & 0.02359 & -0.00527 & 0.07195 & -0.01934 & 0.03110 & -0.0 \\
\hline $\mathrm{C} 10$ & Z & 0.00925 & 0.01620 & 0.01118 & -0.01284 & -0.06265 & 0.00034 & 0.0 \\
\hline $\mathrm{C} 1$ & $\mathrm{X}$ & -0.04887 & -0.00899 & 0.01316 & -0.02877 & -0.07973 & 0.00598 & 0.0 \\
\hline C1 & $\mathrm{Y}$ & 0.03236 & 0.03155 & -0.00367 & -0.07877 & 0.02062 & 0.01967 & -0.0 \\
\hline $\mathrm{C} 1$ & Z & 0.03274 & 0.00355 & -0.00788 & 0.06557 & 0.03062 & 0.02100 & -0.0 \\
\hline N1 & $\mathrm{X}$ & 0.06905 & 0.02251 & -0.01141 & 0.09358 & 0.03768 & 0.07137 & -0.04 \\
\hline N1 & $\mathrm{Y}$ & 0.06382 & 0.03887 & -0.00617 & -0.06348 & 0.07999 & -0.04301 & 0.0 \\
\hline N1 & Z & -0.02820 & -0.00740 & 0.00166 & 0.02455 & -0.08754 & 0.11617 & -0.0 \\
\hline 01 & $\mathrm{x}$ & 0.01563 & 0.01142 & -0.00374 & 0.00930 & -0.01612 & 0.00222 & -0.00080 \\
\hline 01 & $\mathrm{Y}$ & 0.02579 & 0.02525 & 0.00235 & -0.00138 & -0.00405 & 0.00432 & -0.00 \\
\hline 01 & $\mathrm{Z}$ & -0.00120 & -0.00480 & -0.00782 & 0.01724 & -0.01847 & 0.00419 & \\
\hline $\mathrm{O} 2$ & $\mathrm{X}$ & -0.04320 & 0.01592 & 0.05332 & 0.00622 & 0.02168 & -0.08251 & -0.06546 \\
\hline $\mathrm{O} 2$ & $\mathrm{Y}$ & 0.07551 & 0.01333 & -0.04445 & -0.02518 & -0.04066 & -0.07371 & -0.05 \\
\hline 02 & $\mathrm{Z}$ & 0.03469 & -0.03088 & -0.03698 & -0.01917 & -0.02085 & -0.02668 & 0.0 \\
\hline 03 & $\mathrm{X}$ & -0.02213 & 0.00953 & -0.00919 & -0.00250 & 0.00150 & -0.00369 & -0.01204 \\
\hline 03 & $\mathrm{Y}$ & 0.07932 & -0.08827 & 0.01420 & 0.00646 & -0.00455 & 0.00615 & 0 \\
\hline 03 & Z & 0.00 & -0.01185 & 0.02142 & 0.01229 & 0.00656 & -0.00504 & -0.0 \\
\hline $\mathrm{C} 4$ & $\mathrm{X}$ & 0.04966 & 0.03584 & 0.00195 & -0.00047 & -0.00727 & -0.02514 & -0.00674 \\
\hline $\mathrm{C} 4$ & $\mathrm{Y}$ & -0.03425 & 0.05315 & 0.02319 & 0.01378 & 0.01089 & -0.03234 & -0.05 \\
\hline $\mathrm{C} 4$ & Z & -0.01595 & 0.00734 & 0.00564 & 0.01794 & 0.00996 & 0.00543 & \\
\hline $\mathrm{H} 19$ & $\mathrm{X}$ & 0.10228 & 0.05783 & 0.02871 & 0.01235 & -0.01302 & -0.04305 & -0.05579 \\
\hline H19 & $\mathrm{Y}$ & -0.08356 & 0.01652 & -0.00652 & -0.00522 & 0.01078 & -0.05004 & -0.03 \\
\hline $\mathrm{H} 19$ & Z & -0.05337 & 0.04606 & 0.00242 & 0.02964 & 0.02972 & 0.11435 & 0.1 \\
\hline $\mathrm{C} 7$ & $\mathrm{X}$ & 0.06623 & -0.08019 & -0.01044 & -0.00526 & -0.00581 & 0.01097 & 0.01309 \\
\hline C7 & $\mathrm{Y}$ & -0.04732 & 0.04554 & 0.00364 & 0.00313 & 0.00509 & -0.00490 & -0.01 \\
\hline $\mathrm{C} 7$ & Z & -0.04910 & 0.05245 & 0.01064 & 0.00604 & 0.01680 & 0.00594 & 0.0 \\
\hline H21 & $\mathrm{X}$ & 0.07195 & -0.09567 & 0.00400 & -0.00383 & -0.04164 & -0.06632 & -0.14272 \\
\hline H21 & $\mathrm{Y}$ & -0.06999 & 0.08901 & -0.03123 & -0.00878 & 0.07163 & 0.13104 & 0.26 \\
\hline $\mathrm{H} 21$ & Z & -0.05848 & 0.06954 & -0.00255 & 0.00187 & 0.04102 & 0.05495 & 0.11 \\
\hline C15 & $\mathrm{X}$ & 0.01762 & 0.04892 & -0.00681 & 0.01235 & -0.01760 & -0.01250 & -0.02758 \\
\hline C15 & $\mathrm{Y}$ & -0.00804 & 0.06725 & 0.03630 & 0.01376 & 0.02375 & 0.04038 & 0.06 \\
\hline C15 & $\mathrm{Z}$ & 0.00106 & -0.00939 & 0.01167 & 0.01385 & 0.01406 & 0.00406 & 0.0 \\
\hline 06 & $\mathrm{X}$ & 0.00181 & 0.07929 & -0.00068 & 0.01133 & 0.00585 & 0.02972 & 0.03013 \\
\hline 06 & $\mathrm{Y}$ & 0.01867 & 0.03688 & 0.02379 & -0.00023 & 0.00669 & -0.00821 & 0.02649 \\
\hline 06 & Z & 0.02161 & 0.02901 & 0.02801 & 0.00783 & -0.00524 & -0.02438 & -0.07107 \\
\hline 07 & $\mathrm{X}$ & -0.02441 & -0.01931 & -0.03456 & 0.00086 & 0.00083 & -0.00416 & -0.01888 \\
\hline 07 & $\mathrm{Y}$ & 0.00232 & 0.02255 & 0.02547 & -0.00636 & -0.00757 & 0.01297 & 0.00504 \\
\hline 07 & $\mathrm{Z}$ & 0.00928 & -0.07015 & -0.00169 & -0.00686 & -0.01155 & -0.02881 & -0.05174 \\
\hline $\mathrm{H} 4$ & $\mathrm{X}$ & 0.12363 & -0.12222 & -0.02601 & -0.01666 & 0.01664 & 0.08885 & 0.17357 \\
\hline $\mathrm{H} 4$ & $Y$ & -0.16479 & 0.12804 & 0.04214 & 0.02771 & -0.03669 & -0.15118 & -0.31914 \\
\hline
\end{tabular}




\begin{tabular}{|c|c|c|c|c|c|c|c|c|}
\hline H4 & Z & -0.09746 & 0.08744 & 0.02132 & 0.01500 & -0.00336 & -0.06422 & -0.12566 \\
\hline H8 & $\mathrm{X}$ & 0.06056 & -0.15815 & 0.55777 & -0.03418 & 0.01747 & 0.02284 & -0.04133 \\
\hline H8 & Y & -0.10181 & 0.03463 & -0.55001 & -0.00495 & -0.04202 & -0.04593 & -0.05989 \\
\hline & Z & -0.04299 & 0.05640 & -0.40227 & 0.02642 & -0.01810 & -0.03868 & -0.01 \\
\hline 12 & $\mathrm{X}$ & 0.09255 & 0.06951 & -0.01140 & 0.02859 & -0.04620 & 0.20377 & \\
\hline 12 & Y & 0.09114 & 0.06319 & -0.00373 & -0.08429 & 0.10016 & -0.10084 & \\
\hline 2 & Z & -0.04319 & -0.03407 & 0.00089 & 0.05984 & -0.04714 & 0.05765 & -0.0 \\
\hline 20 & $\mathrm{X}$ & -0.10589 & 0.01469 & 0.03090 & -0.19940 & -0.21858 & & \\
\hline 20 & Y & 0.01291 & 0.03831 & 0.00337 & -0.16624 & 0.00837 & -0.01 & \\
\hline 20 & Z & 0.06278 & -0.01342 & -0.01607 & 0.10200 & 622 & -0.12 & \\
\hline 2 & $\mathrm{X}$ & & -0.0342 & -0.04790 & 0.13 & 0.26 & & \\
\hline 22 & Y & & 0.0175 & -0.00992 & & -0.00 & & -0 \\
\hline 22 & Z & 0 & 0.0310 & 0.03189 & -0.17 & -0.14 & & \\
\hline 26 & $\mathrm{X}$ & & 0.04 & -0.01358 & & -0.0 & & -0 \\
\hline 26 & Y & & & & & -0.12 & -0 & \\
\hline 26 & Z & -0.10823 & -0.0570 & 0.03015 & & -0.07 & & \\
\hline & & & & 674.92 & & 742.68 & & \\
\hline & & & & 4.70 & 125 & & & \\
\hline e1 & $\mathrm{X}$ & 0.00 & 0.00 & -0.00079 & & 0.0 & -0.0 & -0 \\
\hline$e 1$ & Y & & 0.002 & 0.00134 & & -0.00 & -0 . & \\
\hline e1 & Z & 0. & -0.001 & -0.00098 & -0.00 & 0.00600 & -0 . & -0 . \\
\hline 2 & $\mathrm{X}$ & & 0.03 & -0.00035 & & & & \\
\hline 2 & Y & & 0. & -0.00276 & & -0 . & -0 & -0 \\
\hline 2 & Z & 0. & 0. & -0.00446 & 0 . & 0 & -0 & \\
\hline 10 & $\mathrm{X}$ & -0 & -0.00 & -0.00020 & -0 . & -0 . & & \\
\hline 10 & Y & -0 & -0.0 & -0.00310 & -0 . & -0.00 & & -0 \\
\hline 10 & Z & -0 & 0 & 0.00234 & 0 & -0.01 & -0 & -0 \\
\hline & $\mathrm{X}$ & -0 & -0.000 & 0 & -0 . & -0 . & -0 & -0 \\
\hline $\mathrm{C} 1$ & Y & 0 & 0 & -0 & -0.01 & & & \\
\hline 1 & Z & -0 & -0.00 & 0463 & 0 & -0 & & \\
\hline 1 & $\mathrm{X}$ & & 0. & 0194 & -0 . & 0 & & -0 \\
\hline 1 & Y & 0.0 & 0 & -0 & & -0 . & & \\
\hline 1 & Z & -0 & -0.03 & -0 & -0 . & 0 & & \\
\hline 1 & $\mathrm{X}$ & -0 & -0.008 & 0 . & & -0 & & \\
\hline 01 & Y & 0.0 & 0 & -0 & -0 . & 0 & & -0 \\
\hline 01 & Z & -0 & 0.0 & 0 & & 0 & & \\
\hline 02 & $\mathrm{X}$ & -0.10307 & -0.09 & 0 . & -0 . & -0 . & -0 & \\
\hline $\mathrm{O} 2$ & Y & -0 & -0 & & -0 . & 0 & & \\
\hline 02 & Z & 0223 & -0.1 & & -0 . & -0.08567 & 0 & \\
\hline 03 & $\mathrm{X}$ & & -0.00 & & -0 . & 0 & -0 & -0 \\
\hline 03 & Y & 0.0 & & & & -0 . & -0 & \\
\hline 03 & Z & 0.01459 & 0.00714 & 0 . & & 0 & & -0 \\
\hline C4 & $\mathrm{X}$ & -0 & -0 & 0 & & & & -0 \\
\hline C4 & Y & -0.04577 & & -0 & -0 . & & -0 & \\
\hline $\mathrm{C} 4$ & Z & 0.03747 & -0.04713 & -0.02191 & -0 . & 0 . & -0 & \\
\hline H19 & $\mathrm{X}$ & 0.0 & 0.0 & 0.1 & -0 . & -0 & -0 & \\
\hline & Y & -0.13900 & -0. & -0.22541 & & & & -0 \\
\hline H19 & Z & 0.12660 & -0.01235 & -0.13160 & & 0 & & -0 \\
\hline C7 & $\mathrm{X}$ & -0.0 & -0.00 & -0 & 0 & -0.0 & -0 & \\
\hline $\mathrm{C} 7$ & Y & 0.00 & -0 & -0.0 & -0.00 & 0 & & -0 \\
\hline $\mathrm{C} 7$ & Z & -0.03435 & 0.01084 & -0.029 & -0.005 & -0.01083 & -0 & \\
\hline H21 & $\mathrm{X}$ & 0.17978 & -0.03461 & 0.1 & 0 & & & -0 \\
\hline $\mathrm{H} 21$ & Y & -0.35434 & 0 & -0.24 & $-0.042 \varepsilon$ & -0.1271 & -0.132 & \\
\hline H21 & Z & -0.16496 & 0.02930 & -0.11 & -0.01982 & -0.05826 & -0.064 & \\
\hline C15 & $\mathrm{X}$ & 0.08005 & -0.03225 & -0.12 & -0.014 & -0.00403 & -0.030 & \\
\hline C15 & Y & -0.03754 & 0.00247 & 0.13762 & 0.013 & 0.012 & 0.02247 & -0 . \\
\hline C15 & Z & -0.05603 & 0.03724 & 0.08587 & & 0.07160 & 0.00035 & -0 \\
\hline 06 & $\mathrm{X}$ & -0.00305 & & 0.04368 & & & & -0 \\
\hline 06 & Y & 0.03735 & -0.07735 & -0.05181 & -0.00948 & 0.06715 & -0.00793 & -0 . \\
\hline 06 & Z & 0.00865 & 0.1 & -0.02042 & 0.00899 & -0.12641 & 0.04927 & -0 \\
\hline
\end{tabular}




\begin{tabular}{|c|c|c|c|c|c|c|c|c|}
\hline 07 & $\mathrm{x}$ & -0.02532 & 0.03944 & 0.03145 & 0.00516 & -0.01788 & 0.00989 & -0.00047 \\
\hline 07 & $\mathrm{Y}$ & 0.03257 & -0.02176 & -0.00655 & -0.00579 & -0.08591 & 0.01090 & 0.00279 \\
\hline 07 & Z & -0.02955 & 0.05398 & -0.03908 & 0.00541 & 0.07692 & -0.01656 & -0.00132 \\
\hline H 4 & $\mathrm{x}$ & -0.18634 & 0.00698 & -0.12879 & -0.00673 & -0.06938 & -0.01371 & 0.00152 \\
\hline H 4 & $\mathrm{Y}$ & 0.35536 & -0.03140 & 0.23371 & 0.01374 & 0.12349 & 0.05173 & -0.00899 \\
\hline H4 & Z & 0.11597 & -0.00184 & 0.07840 & 0.00223 & 0.04217 & -0.01452 & 0.00381 \\
\hline H8 & $\mathrm{x}$ & -0.03467 & 0.04929 & -0.01626 & 0.01258 & -0.04673 & 0.04256 & -0.00382 \\
\hline H8 & $\mathrm{Y}$ & -0.02987 & 0.09619 & 0.02943 & -0.00611 & -0.18820 & 0.03436 & 0.00482 \\
\hline H8 & Z & -0.00416 & 0.01504 & -0.00425 & -0.00115 & 0.13357 & -0.05504 & \\
\hline H12 & $\mathrm{x}$ & -0.03405 & -0.08817 & -0.04071 & 0.38072 & -0.00110 & 0.06201 & \\
\hline $\mathrm{H} 12$ & $\mathrm{Y}$ & 0.02359 & 0.00978 & -0.01183 & 0.14830 & -0.03604 & 0.00920 & 0.03374 \\
\hline $\mathrm{H} 12$ & $\mathrm{z}$ & -0.01453 & 0.00986 & 0.02174 & -0.26769 & 0.02493 & -0.02290 & -0.20234 \\
\hline H2O & $\mathrm{x}$ & -0.03918 & -0.04933 & -0.01663 & 0.31651 & -0.02070 & -0.03678 & \\
\hline H2O & $\mathrm{Y}$ & 0.00876 & 0.00729 & -0.00730 & 0.11522 & -0.00123 & -0.00334 & 0.06931 \\
\hline H2O & Z & 0.04159 & 4698 & 0.00920 & -0.1 & -0.01193 & & \\
\hline H22 & $\mathrm{x}$ & -0.02479 & -0.0 & -0.0 & & -0.01903 & -0 . & \\
\hline H22 & $\mathrm{Y}$ & -0.00621 & -0.01554 & -0.00917 & 0.08546 & -0.01544 & -0.01497 & -0.07023 \\
\hline H22 & Z & 0.00232 & 483 & 783 & -0.2 & & & \\
\hline H26 & $\mathrm{x}$ & -0.01192 & -0.0 & -0 & & -0 . & -0 & \\
\hline H 26 & $\mathrm{Y}$ & 0.01823 & 0.02467 & -0.01315 & 0.15482 & 0.01480 & -0.02530 & -0.00931 \\
\hline H 26 & Z & 0.03676 & 0.06976 & 0.01112 & -0.28867 & & & \\
\hline & & & & & & 1026.22 & & \\
\hline & iti & .63 & .86 & & & .59 & .78 & \\
\hline$e 1$ & $\mathrm{X}$ & -0.00323 & -0.00 & & -0.0 & -0.0 & -0 & -0 . \\
\hline e1 & $\mathrm{Y}$ & -0.00033 & -0.00074 & -0.00055 & 0 & -0.00004 & 0835 & 0285 \\
\hline e1 & z & -0.00463 & -0.00013 & 0.00115 & & -0.00008 & 0958 & \\
\hline & $\mathrm{x}$ & & -0 & -0 . & & -0 & & \\
\hline N2 & $\mathrm{Y}$ & -0.02804 & -0.0 & -0. & -0. & -0.01207 & 0414 & \\
\hline & Z & 0.08 & 0.0 & & & 0.03136 & -0 & -0 \\
\hline 10 & $\mathrm{x}$ & -0.0 & 0. & & & & -0 & -0 \\
\hline C10 & $\mathrm{Y}$ & -0.10438 & 0.03835 & & & 0.03111 & -0 & -0 . \\
\hline $\mathrm{C} 10$ & Z & -0.05712 & -0.01406 & -0 & & -0.0 & -0 . & \\
\hline C1 & $\mathrm{x}$ & -0.0 & 0. & & -0 . & -0 & & -0 . \\
\hline $\mathrm{C} 1$ & $\mathrm{Y}$ & 0.09636 & 0.04082 & 0.0 & 0.03 & -0.02938 & -0.00540 & 0034 \\
\hline $\mathrm{C} 1$ & Z & -0.07893 & -0.05965 & & -0.0 & & & \\
\hline N1 & $\mathrm{x}$ & 343 & -0. & -0 . & -0 . & & & \\
\hline N1 & $\mathrm{Y}$ & 0.04062 & -0.02181 & -0.0 & -0.0 & 0.01246 & 0490 & -0 . \\
\hline N1 & Z & 0.07047 & 0.02008 & & -0.0 & -0.01575 & -0 & -0 . \\
\hline & $\mathrm{x}$ & & & & & & & \\
\hline 01 & $\mathrm{Y}$ & 57 & -0.0 & -0.0 & -0.0 & -0.0 & -0.09725 & -0 . \\
\hline 01 & Z & 649 & -0.0 & -0 . & -0.0 & -0.00159 & -0.11299 & -0 \\
\hline & $\mathrm{X}$ & & & -0 . & -0 & & & \\
\hline $\mathrm{O} 2$ & $\mathrm{Y}$ & -0.0 & 0 . & -0 . & & 099 & -0 . & \\
\hline $\mathrm{O} 2$ & Z & 0.01005 & -0.00329 & -0.00487 & -0.00416 & -0.00147 & -0.00095 & -0.01539 \\
\hline & $\mathrm{x}$ & & -0.0 & & & & -0 & \\
\hline & Y & & -0 & & & -0 . & & -0 \\
\hline 03 & Z & 0.00112 & 0.00022 & -0.0 & 0. & -0.00012 & 0.00315 & -0.00865 \\
\hline & $\mathrm{x}$ & & -0.0 & & & & & \\
\hline & $\mathrm{Y}$ & -0.0 & -0 & & -0 & -0 . & -0 . & \\
\hline C4 & Z & 00517 & -0.0 & & & 02 & -0 & 149 \\
\hline & $\mathrm{x}$ & -0.0 & -0.0 & & & -0 & -0 & \\
\hline & $\mathrm{Y}$ & & -0.0 & & -0.0 & -0 & & -0 . \\
\hline & Z & 0.0 & -0.0 & & & & -0. & \\
\hline & $\mathrm{x}$ & 94 & & -0. & -0.0 & -0.0 & 423 & -0 . \\
\hline & $\mathrm{Y}$ & -0.00 & & -0. & & & & \\
\hline C7 & Z & & & -0 & -0 & & -0 . & \\
\hline & $\mathrm{x}$ & -0.00 & 0.0 & -0 & -0.0 & 93 & -0 & \\
\hline H21 & $\mathrm{Y}$ & & -0.02378 & & & -0.00729 & 0.12776 & \\
\hline$\angle \perp$ & Z & & -0.01013 & & & -0.00283 & & -0.18361 \\
\hline 15 & $\mathrm{X}$ & 0.00453 & -0.01199 & 0.02739 & 0.00107 & 0.00051 & 0.00927 & -0 . \\
\hline
\end{tabular}




\begin{tabular}{|c|c|c|c|c|c|c|c|c|}
\hline C15 & $\mathrm{Y}$ & 0.00147 & -0.00465 & 0.01484 & 0.00079 & 0.00077 & 0.00075 & -0.00012 \\
\hline C15 & Z & -0.00022 & -0.00957 & 0.02933 & -0.00043 & 0.00071 & 0.00606 & -0.00971 \\
\hline 06 & $\mathrm{x}$ & -0.00679 & 0.03984 & -0.12193 & -0.01508 & 0.00092 & 0.00322 & -0.00141 \\
\hline 06 & $\mathrm{Y}$ & -0.00797 & 0.03031 & -0.09686 & -0.01177 & 0.00090 & 0.00010 & -0.00809 \\
\hline 06 & Z & 0.01049 & 0.01075 & -0.02211 & -0.00003 & 0.00062 & 0.00537 & 0.00248 \\
\hline 07 & $\mathrm{x}$ & 0.00182 & -0.00401 & 0.01179 & 0.00028 & 0.00033 & 0.00270 & -0.00432 \\
\hline 07 & $\mathrm{Y}$ & 0.00905 & -0.01571 & 0.04976 & 0.00606 & -0.00009 & 0.00175 & -0.00394 \\
\hline 07 & Z & -0.00934 & 0.01601 & -0.05205 & -0.00807 & 0.00038 & 0.00286 & -0.00434 \\
\hline H4 & $\mathrm{x}$ & 0.00868 & 0.01744 & & -0.01366 & 0.00128 & & \\
\hline H4 & $\mathrm{Y}$ & -0.01425 & -0.02808 & 0.04288 & 0.02054 & 0.00124 & & -0.45955 \\
\hline H4 & Z & -0.00672 & -0.01478 & 0.02603 & 0.01184 & -0.00227 & 0.05482 & -0.21622 \\
\hline H8 & $\mathrm{x}$ & 0.00210 & & -0.00687 & 0.00269 & -0.00145 & & \\
\hline H8 & $\mathrm{Y}$ & 0.00710 & 0.00086 & 0.00905 & 0.01724 & -0.00413 & -0. & \\
\hline H8 & Z & -0.00916 & 0.00289 & -0.02579 & -0.01474 & 0.00319 & 0.03021 & -0.05758 \\
\hline $\mathrm{H} 12$ & $\mathrm{x}$ & -0.17108 & 0.32688 & 0.10941 & -0.00383 & -0.18019 & -0.01637 & \\
\hline H12 & & -0.23291 & -0.0 & & -0.25224 & -0.02230 & & \\
\hline $\mathrm{H} 12$ & Z & 0.22224 & -0.17124 & -0.06785 & -0.01088 & 0.09608 & 0656 & \\
\hline $\mathrm{H} 2 \mathrm{O}$ & $\mathrm{x}$ & -0.14299 & -0.44192 & -0.1 & -0.07181 & 0.40627 & 1247 & \\
\hline H2O & & & -0.1 & -0 & -0. & & & \\
\hline H2O & Z & -0.0 & 0.1 & & -0.35425 & -0.26574 & & \\
\hline H22 & $\mathrm{x}$ & 0.0 & -0.2 & -0.1 & 0.28087 & -0.45654 & -0 . & \\
\hline H22 & $\mathrm{Y}$ & -0. & -0 & -0 . & -0 & -0 & & \\
\hline H22 & Z & -0.1 & 0 & & & 2972 & & \\
\hline H26 & $\mathrm{x}$ & 0.2 & & & & & & \\
\hline H26 & $\mathrm{Y}$ & & -0 & & -0 & & & \\
\hline H26 & Z & -0.09246 & -0.2 & -0.0 & 0.03248 & -0.1 & & \\
\hline \multicolumn{2}{|c|}{ frequencies } & & & & & & & \\
\hline \multicolumn{2}{|c|}{ intensities } & & & & & & & \\
\hline $\operatorname{Re} 1$ & $\mathrm{X}$ & -0.00074 & & & & & & -0 \\
\hline $\operatorname{Re} 1$ & $\mathrm{Y}$ & -0 & & & & & & \\
\hline $\operatorname{Re} 1$ & Z & 0.0 & -0.0 & 0.00 & 0.000 & 0.00034 & -0.00038 & -0.00019 \\
\hline N2 & $\mathrm{x}$ & 0.0 & -0.00913 & -0.01 & 0.02 & 0.02436 & 0.00217 & \\
\hline N2 & $\mathrm{Y}$ & -0 & & -0 & -0.02 & -0 & & \\
\hline N2 & Z & & -0 & -0 . & & .794 & & \\
\hline C10 & $\mathrm{x}$ & -0.0 & 0.0 & & -0.01571 & -0.01418 & -0.00286 & -0.02995 \\
\hline & $\mathrm{Y}$ & & -0 & -0 & & & & \\
\hline C10 & Z & & -0 . & -0 . & -0.0 & -0 & & -0 \\
\hline $\mathrm{C} 1$ & $\mathrm{x}$ & -0 & & & -0.00021 & 0022 & -0 . & -0.03 \\
\hline $\mathrm{C} 1$ & $\mathrm{Y}$ & & -0 . & -0 . & -0 . & -0 . & & \\
\hline $\mathrm{C} 1$ & $\mathrm{z}$ & -0 . & -0 . & -0 . & -0. & -0 . & & -0 \\
\hline N1 & $\mathrm{x}$ & 57 & -0 & & & 441 & & 069 \\
\hline N1 & $\mathrm{Y}$ & -0 . & & & & & & \\
\hline N1 & z & -0 & & & & & & \\
\hline 01 & $\mathrm{x}$ & & & & -0.00132 & -0.00043 & -0 & -0 . \\
\hline 01 & $\mathrm{Y}$ & & -0 . & -0. & & -0.0 & & \\
\hline 01 & z & & -0 . & -0 . & & -0 & & \\
\hline 02 & $\mathrm{x}$ & -0.0 & & -0. & -0.0 & 0 & & \\
\hline 02 & $\mathrm{Y}$ & -0.1 & & -0 & -0. & & -0 . & \\
\hline 02 & Z & & -0 & & & -0 & & \\
\hline 03 & $\mathrm{x}$ & & -0 & & & -0 . & & \\
\hline 03 & $\mathrm{Y}$ & & & & -0.0 & & & -0 \\
\hline 03 & z & -0.0 & & -0. & & -0 & & \\
\hline C4 & $\mathrm{x}$ & & 0.0 & & & -0 . & -0 & \\
\hline C4 & $\mathrm{Y}$ & 3956 & -0. & & & -0 . & & \\
\hline $\mathrm{C} 4$ & $\mathrm{Z}$ & -0.0 & & & & & & \\
\hline & $\mathrm{x}$ & & & -0 . & -0.0 & & & \\
\hline H19 & $\mathrm{Y}$ & 0.15480 & -0.16003 & & 0.01311 & -0.02481 & -0.32253 & -0.00524 \\
\hline H19 & z & -0.28774 & & & -0.00 & & & \\
\hline C7 & $\mathrm{x}$ & -0.0 & & -0 . & -0.0 & & -0 . & \\
\hline C7 & Y & -0.00187 & -0.01988 & 0.00219 & 0.00903 & -0.00966 & -0.00379 & 0.00103 \\
\hline
\end{tabular}




\begin{tabular}{|c|c|c|c|c|c|c|c|c|}
\hline C7 & z & 0.00444 & -0.01179 & 0.00359 & -0.07231 & 0.08025 & 0.00037 & -0.00171 \\
\hline H21 & $\mathrm{x}$ & -0.01822 & -0.04444 & 0.00133 & 0.33653 & -0.37274 & 0.00529 & 0.00582 \\
\hline H21 & Y & -0.05002 & 0.10551 & -0.01905 & 0.22219 & -0.24760 & 0.00549 & 0.01049 \\
\hline H21 & Z & -0.01496 & 0.03461 & -0.00454 & 0.00047 & -0.00122 & 0.00199 & 0.00226 \\
\hline C15 & $\mathrm{x}$ & -0.02081 & -0.06831 & 0.00283 & -0.00183 & -0.00009 & 0.00999 & -0.01482 \\
\hline C15 & Y & -0.01625 & -0.03238 & 0.00182 & -0.00298 & 0.00093 & 0.05313 & -0.01643 \\
\hline C15 & Z & -0.01743 & -0.03798 & 0.00077 & 0.00140 & -0.00161 & -0.07178 & 0.00308 \\
\hline 06 & $\mathrm{x}$ & 0.01990 & 0.03445 & -0.00302 & 0.00017 & -0.00328 & -0.00702 & 0.00371 \\
\hline 06 & $\mathrm{Y}$ & 0.02070 & 0.03489 & -0.00392 & -0.00004 & -0.00285 & -0.00981 & 0.00290 \\
\hline 06 & Z & -0.00819 & 0.00282 & 0.00198 & 0.00049 & 0.00085 & 0.00426 & \\
\hline 07 & $\mathrm{x}$ & -0.01635 & -0.04538 & 0.00328 & 0.00096 & 0.00221 & 0.01157 & 0.00346 \\
\hline 07 & $\mathrm{Y}$ & -0.03265 & -0.05 & 0.00370 & 0.00223 & 0.00258 & -0.03041 & \\
\hline 07 & $\mathrm{Z}$ & 0.02118 & 0.00294 & -0.00060 & -0.00148 & -0.00025 & 0.05660 & \\
\hline H4 & $\mathrm{x}$ & 0.00931 & -0.05596 & 0.00794 & -0.20107 & 0.22300 & -0.00930 & 0.00422 \\
\hline H4 & $\mathrm{Y}$ & 976 & & -0.0 & -0.26168 & & & \\
\hline H4 & Z & -0.02231 & 0. & -0.00837 & 0.24630 & -0.27301 & 0.00794 & -0 . \\
\hline H8 & $\mathrm{x}$ & 0.10143 & 0.21503 & -0.01577 & -0.00179 & -0.01137 & -0.04459 & -0.01161 \\
\hline $\mathrm{H} 8$ & Y & 897 & 0.5 & -0.0 & -0.00924 & -0.02286 & -0.14849 & \\
\hline H8 & Z & -0.1 & -0.3 & 0.0 & 0.00367 & 1844 & & \\
\hline $\mathrm{H} 12$ & $\mathrm{x}$ & -0.01143 & -0.00770 & -0.09074 & -0.05666 & -0.04762 & -0.00463 & -0.1 \\
\hline $\mathrm{H} 12$ & Y & 0. & & & & 7997 & & \\
\hline $\mathrm{H} 12$ & $\mathrm{z}$ & & & & & & & \\
\hline H2O & $\mathrm{x}$ & -0. & 0. & -0.1 & -0.11499 & -0.10471 & -0 & \\
\hline H2O & Y & 005 & -0 & -0.3 & -0.19696 & -0.1 & & \\
\hline H2O & z & -0 & -0 & -0 . & -0.2 & -0 . & & \\
\hline $\mathrm{H} 22$ & $\mathrm{X}$ & -0.0 & 0.0 & 0.2 & -0.22306 & -0.20300 & 1007 & \\
\hline H22 & Y & 0.0 & -0.0 & -0.2 & & 0.16044 & -0 . & -0 \\
\hline H22 & $\mathrm{z}$ & 0. & -0 . & & -0 & -0 . & & \\
\hline $\mathrm{H} 26$ & $\mathrm{X}$ & 0. & -0 . & -0. & & 0. & & \\
\hline H26 & $\mathrm{Y}$ & -0 & & & -0 . & -0 . & & \\
\hline H 26 & z & -0.0 & -0 . & 0 . & & & & \\
\hline \multicolumn{2}{|c|}{ requencies } & & & & & & & \\
\hline \multicolumn{2}{|c|}{ ntensities } & & & & & & & \\
\hline $\operatorname{Re} 1$ & $\mathrm{x}$ & -0.00007 & -0.0 & -0.00 & -0.00067 & -0.00017 & -0.00037 & -0 . \\
\hline $\operatorname{Re} 1$ & $\mathrm{Y}$ & 0.00116 & & -0.0 & -0.00135 & & & \\
\hline Re1 & z & -0. & 0. & & & -0 . & & \\
\hline N2 & $\mathrm{x}$ & -0.0 & 0.0 & 0.0 & 0.04648 & 0.01498 & 4950 & -0.0 \\
\hline N2 & $\mathrm{Y}$ & 0.0 & -0.1 & 0.0 & -0.02602 & -0.0 & & \\
\hline N2 & Z & -0 . & & & & & & -0 \\
\hline C10 & $\mathrm{x}$ & 774 & -0 . & -0.0 & -0.09269 & -0.02803 & -0.07746 & \\
\hline C10 & Y & -0. & & -0.0 & 5906 & 0.0 & 2994 & -0 \\
\hline C10 & Z & & & -0 . & -0 . & -0 & & \\
\hline $\mathrm{C} 1$ & $\mathrm{x}$ & & -0 & & & 751 & -0 & -0 \\
\hline $\mathrm{C} 1$ & $\mathrm{Y}$ & -0.0 & & 0. & 0. & 839 & -0 & -0 \\
\hline $\mathrm{C} 1$ & Z & -0. & -0 & & & & & \\
\hline N1 & $\mathrm{x}$ & -0 . & & -0 . & -0 & -0 & -0 & \\
\hline N1 & $\mathrm{Y}$ & 0 & -0. & 0.0 & -0.03935 & -0.00738 & 908 & 367 \\
\hline N1 & Z & & -0 & -0. & -0 & -0 & & \\
\hline 01 & $\mathrm{x}$ & & & & & & & \\
\hline 01 & Y & -0.0 & & -0.00 & 26 & -0.0 & 027 & -0 \\
\hline 01 & Z & & -0 & -0.0 & -0 & & & \\
\hline 02 & $\mathrm{x}$ & -0. & & & & -0 & & \\
\hline 02 & $\mathrm{Y}$ & -0.0 & & -0.0 & & -0.0 & -0. & -0 . \\
\hline 02 & Z & & -0.0 & & -0.0 & -0.0 & & \\
\hline 03 & $\mathrm{x}$ & -0.0 & -0.0 & & & -0.0 & & \\
\hline 03 & Y & -0. & -0. & -0.0 & & -0 & 619 & -0 \\
\hline 03 & Z & & & & -0.0 & 0.0 & -0 & \\
\hline C4 & $\mathrm{x}$ & & -0.00564 & -0.00724 & & & & \\
\hline C4 & Y & & & & & -0.01095 & & \\
\hline C4 & Z & 0.00582 & 0.00140 & 81 & -0.01687 & 0.09244 & -0.00608 & -0 \\
\hline
\end{tabular}




\begin{tabular}{|c|c|c|c|c|c|c|c|c|}
\hline H19 & $\mathrm{x}$ & 0.09525 & 0.00356 & -0.00261 & -0.00531 & 0.04467 & -0.00473 & -0.01759 \\
\hline H19 & $\mathrm{Y}$ & -0.05929 & -0.01294 & 0.01755 & -0.04314 & 0.10464 & -0.00467 & -0.05466 \\
\hline H19 & Z & .15988 & 0.01036 & -0.04966 & 0.05919 & -0.12987 & 0.00600 & 0.05587 \\
\hline C7 & $\mathrm{x}$ & 0.02631 & 0.02982 & 0.07162 & -0.00466 & 0.05680 & -0.01644 & 0.13031 \\
\hline C7 & Y & 0.01261 & 0.01623 & 0.04478 & -0.00549 & 0.03250 & -0.01108 & 0.09897 \\
\hline C7 & Z & -0.01384 & -0.01506 & -0.03519 & 0.00280 & -0.02868 & 0.00856 & -0.05970 \\
\hline H21 & $\mathrm{x}$ & 0.06483 & & 0.40094 & -0.12534 & -0.25685 & 0.05930 & -0.33844 \\
\hline H 21 & $\mathrm{Y}$ & 0.06270 & 0.08746 & 0.26576 & -0.06727 & -0.06650 & 0.01331 & -0.09518 \\
\hline H 21 & Z & 0.00422 & 0.01086 & 0.03656 & -0.01380 & -0.05532 & 0.01391 & -0.11457 \\
\hline C15 & $\mathrm{x}$ & -0.14936 & -0.00278 & & -0.00136 & -0.06104 & & \\
\hline C15 & $\mathrm{Y}$ & -0.14879 & -0.00778 & 0.04677 & -0.04141 & 0.06984 & -0.00302 & -0.01974 \\
\hline C15 & Z & 0428 & 0.01212 & -0.02150 & 0.05624 & -0.17237 & & \\
\hline 06 & $\mathrm{x}$ & 5018 & 0.00293 & -0.01167 & 0.00861 & -0.00042 & -0.00040 & \\
\hline 06 & $\mathrm{Y}$ & 0.03770 & 0.00160 & -0.01477 & 0.01193 & -0.01384 & 0.00049 & \\
\hline 06 & Z & & -0.0 & -0.0 & -0.00920 & 0.02024 & 0060 & \\
\hline 07 & $\mathrm{X}$ & & -0.0 & -0.00 & -0.00291 & 0.01265 & -0.001 & -0 \\
\hline 07 & $\mathrm{Y}$ & 5498 & 0.0 & -0.01602 & 0.01178 & -0.02214 & 0.00101 & \\
\hline 07 & Z & -0.0 & -0.0 & & -0.01937 & 4736 & -0 . & \\
\hline H4 & $\mathrm{x}$ & & & & -0.0 & -0 . & & \\
\hline H4 & $\mathrm{Y}$ & 033 & 0.0 & 0.3 & -0.07556 & -0.09608 & 0.02656 & -0.14873 \\
\hline H4 & Z & -0. & -0.0 & -0 & 9790 & 3119 & -0 . & \\
\hline H8 & $\mathrm{x}$ & -0. & & & & -0 & & \\
\hline H8 & Y & -0 & 0.0 & 0.0 & 0.04783 & -0 & 1131 & \\
\hline H8 & Z & 0. & -0.0 & -0.0 & -0.04522 & 3377 & -0. & -0 \\
\hline $\mathrm{H} 12$ & & & -0. & & -0.0 & -0 . & & \\
\hline $\mathrm{H} 12$ & Y & -0.0 & 0.3 & -0.12 & -0.15209 & -0.01895 & -0.03042 & \\
\hline $\mathrm{H} 12$ & Z & 0700 & -0.0 & -0.00 & -0.05018 & -0.00375 & & \\
\hline H2O & $\mathrm{x}$ & & & -0 & -0 . & & & \\
\hline $\mathrm{H} 20$ & Y & -0 . & & -0 . & -0 . & -0 . & 86 & \\
\hline $\mathrm{H} 2 \mathrm{O}$ & Z & -0 & & -0 & -0 . & -0 & & \\
\hline H22 & $\mathrm{x}$ & -0. & -0 & & & & & -0 \\
\hline $\mathrm{H} 22$ & Y & 0. & 0 & -0.0 & -0.12322 & -0.01201 & -0 . & \\
\hline H22 & Z & -0. & -0. & & & & & \\
\hline H26 & $\mathrm{x}$ & -0. & -0 . & & & & & -0 \\
\hline H26 & $\mathrm{Y}$ & 0.03502 & 0.29383 & -0.12706 & -0.15537 & -0.01024 & 4062 & 0 \\
\hline H26 & z & -0.00323 & 0.06516 & 0.00095 & 0.05278 & 0.02277 & 0.05480 & -0 . \\
\hline \multicolumn{2}{|c|}{ requencies } & & & & & & & \\
\hline \multicolumn{2}{|c|}{ ntensities } & & & & 8.24 & 5.94 & & \\
\hline Re1 & X & -0.0 & & -0.0 & 0. & -0 & -0 & \\
\hline Re1 & Y & 0 & & 0 & 0.00006 & -0 & & \\
\hline Re1 & Z & 0.0 & 0.0 & -0.0 & -0.00001 & -0.00001 & -0.00002 & -0 . \\
\hline N2 & $\mathrm{x}$ & & & -0 . & -0 & & & \\
\hline N2 & $\mathrm{Y}$ & & & -0 . & 0.0 & 08 & & -0 \\
\hline N2 & z & -0.0 & 0.0 & -0.0 & -0.00013 & 0.00010 & 4860 & -0.01927 \\
\hline $\mathrm{C} 10$ & $\mathrm{x}$ & & & & -0.0 & -0 & -0 & \\
\hline C10 & Y & -0 . & -0 . & & -0 . & -0 & & \\
\hline $\mathrm{C} 10$ & $\mathrm{z}$ & & & & -0.03194 & -0 & 0033 & -0 . \\
\hline C1 & $\mathrm{x}$ & & & & & & & \\
\hline $\mathrm{C} 1$ & $\mathrm{Y}$ & -0 . & & -0 & -0.04393 & -0 & -0 . & -0 \\
\hline $\mathrm{C} 1$ & Z & -0. & & & 0.02368 & 0. & -0.00089 & \\
\hline N1 & $\mathrm{x}$ & & & -0.0 & -0.0 & -0 & & \\
\hline N1 & $\mathrm{Y}$ & & -0. & & & & & \\
\hline N1 & Z & & -0. & -0.0 & & -0.00022 & 0.02292 & \\
\hline 01 & $\mathrm{x}$ & & & -0. & -0.0 & 0.0 & 0.00020 & -0 \\
\hline 01 & $\mathrm{Y}$ & -0.0 & -0.0 & & -0.0 & & & \\
\hline 01 & Z & -0.0 & -0. & -0 . & & 0. & -0.00003 & \\
\hline 02 & $\mathrm{x}$ & -0.0 & 0.0 & -0 & -0.00 & -0.00004 & 0.00 & \\
\hline $\mathrm{O} 2$ & Y & -0.00029 & & -0.00006 & -0.00004 & & -0.00003 & \\
\hline $\mathrm{O} 2$ & Z & & -0.00037 & & & -0.00027 & & \\
\hline $\mathrm{O} 3$ & $\mathrm{x}$ & 0.00116 & -0.00036 & -0.0 & 0.00000 & -0.00014 & -0.00001 & 0.0 \\
\hline
\end{tabular}




\begin{tabular}{|c|c|c|c|c|c|c|c|c|}
\hline O3 & $\mathrm{Y}$ & 0.00059 & 0.00011 & -0.00002 & 0.00007 & -0.00020 & 0.00004 & -0.00003 \\
\hline 03 & Z & -0.00052 & -0.00067 & -0.00002 & 0.00002 & 0.00003 & -0.00002 & -0.00002 \\
\hline C4 & $\mathrm{x}$ & -0.00093 & -0.00092 & -0.00032 & -0.00162 & 0.06471 & 0.00008 & 0.00015 \\
\hline C4 & $\mathrm{Y}$ & -0.00021 & -0.00050 & -0.00022 & -0.00118 & 0.05102 & 0.00005 & 0.00031 \\
\hline C4 & Z & -0.00035 & -0.00001 & -0.00021 & -0.00077 & 0.02076 & 0.00019 & -0.00055 \\
\hline H19 & $\mathrm{x}$ & 0.00587 & 0.00558 & 0.00319 & 0.01752 & -0.71379 & 0.00078 & -0.00282 \\
\hline H19 & $\mathrm{Y}$ & 0.00360 & 0.00322 & 0.00280 & 0.01470 & -0.59679 & 0.00037 & -0.00211 \\
\hline H19 & Z & 0.00066 & 0.00070 & 0.00093 & 0.00487 & -0.19881 & -0.00014 & -0.00080 \\
\hline C7 & $\mathrm{x}$ & 0.04453 & 0.04743 & 0.00000 & 0.00017 & 0.00064 & -0.00007 & -0.00010 \\
\hline C7 & $\mathrm{Y}$ & 0.02786 & -0.00912 & 0.00010 & 0.00016 & 0.00036 & -0.00012 & 0.00020 \\
\hline C7 & Z & -0.02356 & 0.08408 & -0.00011 & -0.00009 & 0.00042 & -0.00006 & 0.00010 \\
\hline H21 & $\mathrm{x}$ & 0.01569 & -0.00368 & 0.00022 & 0.00014 & 0.00001 & 0.00002 & -0.00007 \\
\hline H21 & $\mathrm{Y}$ & -0.23334 & 0.22027 & -0.00075 & -0.00149 & -0.00071 & 0.00009 & 0.00013 \\
\hline H21 & Z & 0.66256 & -0.61168 & 0.00145 & 0.00333 & 0.00043 & 0.00007 & -0.00038 \\
\hline C15 & $\mathrm{x}$ & 0.00049 & 0.00033 & 0.00012 & 0.00031 & -0.00283 & -0.00031 & 0.00046 \\
\hline C15 & $\mathrm{Y}$ & 0.00033 & -0.00012 & 0.00003 & 0.00003 & 0.00064 & -0.00007 & -0.00017 \\
\hline C15 & Z & 0.00028 & 0.00052 & 0.00010 & 0.00033 & -0.00391 & -0.00023 & 0.00060 \\
\hline 06 & $\mathrm{x}$ & -0.00019 & -0.00030 & -0.00001 & -0.00007 & -0.00085 & 0.00013 & -0.00015 \\
\hline 06 & $\mathrm{Y}$ & -0.00042 & -0.00018 & 0.00000 & -0.00004 & -0.00074 & 0.00010 & -0.00006 \\
\hline 06 & Z & 0.00000 & -0.00009 & 0.00005 & 0.00002 & -0.00009 & -0.00001 & -0.00004 \\
\hline 07 & $\mathrm{x}$ & -0.00001 & 0.00002 & 0.00000 & 0.00009 & 0.00046 & -0.00015 & 0.00043 \\
\hline 07 & $\mathrm{Y}$ & -0.00001 & 0.00018 & -0.00001 & 0.00007 & 0.00020 & -0.00008 & 0.00027 \\
\hline 07 & Z & 0.00014 & -0.00034 & -0.00001 & 0.00005 & 0.00037 & -0.00010 & 0.00029 \\
\hline H4 & $\mathrm{x}$ & -0.54702 & -0.55294 & -0.00005 & -0.00279 & -0.00847 & 0.00059 & 0.00025 \\
\hline H4 & $\mathrm{Y}$ & -0.11025 & -0.11427 & 0.00003 & -0.00082 & -0.00103 & 0.00003 & -0.00023 \\
\hline H4 & Z & -0.38413 & -0.37245 & -0.00002 & -0.00206 & -0.00527 & 0.00044 & -0.00020 \\
\hline H8 & $\mathrm{x}$ & -0.00062 & -0.00073 & -0.00020 & -0.00182 & -0.00239 & 0.00273 & -0.00692 \\
\hline H8 & $\mathrm{Y}$ & -0.00023 & 0.00071 & 0.00000 & -0.00032 & -0.00249 & 0.00100 & -0.00197 \\
\hline H8 & Z & -0.00125 & -0.00020 & -0.00028 & -0.00214 & -0.00106 & 0.00284 & -0.00722 \\
\hline H12 & $\mathrm{x}$ & -0.00050 & 0.00011 & 0.00740 & 0.00476 & 0.00144 & -0.15830 & -0.40112 \\
\hline H12 & $\mathrm{Y}$ & -0.00002 & 0.00038 & -0.00051 & -0.00056 & -0.00005 & -0.03652 & -0.09253 \\
\hline $\mathrm{H} 12$ & Z & -0.00021 & -0.00028 & 0.01108 & 0.00837 & 0.00297 & -0.31104 & -0.79308 \\
\hline H2O & $\mathrm{x}$ & 0.00217 & -0.00035 & -0.34154 & -0.30506 & -0.00916 & -0.00448 & -0.00597 \\
\hline H2O & $\mathrm{Y}$ & -0.00351 & 0.00071 & 0.55978 & 0.49325 & 0.01462 & 0.00580 & 0.00678 \\
\hline H2O & z & 0.00189 & -0.00032 & -0.27492 & -0.24999 & -0.00747 & -0.00302 & -0.00383 \\
\hline H22 & $\mathrm{x}$ & -0.00069 & -0.00015 & -0.08050 & 0.09568 & 0.00189 & -0.00159 & -0.00015 \\
\hline H22 & $\mathrm{Y}$ & -0.00273 & -0.00084 & -0.55105 & 0.61422 & 0.01254 & -0.01095 & 0.00207 \\
\hline H22 & Z & -0.00140 & -0.00047 & -0.30108 & 0.34221 & 0.00714 & -0.00784 & 0.00199 \\
\hline H26 & $\mathrm{x}$ & -0.00055 & -0.00052 & 0.00859 & -0.00695 & -0.00119 & -0.59944 & 0.23606 \\
\hline H26 & $\mathrm{Y}$ & -0.00010 & -0.00016 & 0.00253 & -0.00267 & -0.00014 & -0.04398 & 0.01700 \\
\hline H26 & Z & -0.00010 & -0.00035 & 0.01114 & -0.00889 & -0.00163 & -0.66138 & 0.25870 \\
\hline \multicolumn{2}{|c|}{ frequencies } & 3780.50 & & & & & & \\
\hline ntens & ities & 205.06 & & & & & & \\
\hline $\operatorname{Re} 1$ & $\mathrm{x}$ & -0.00001 & & & & & & \\
\hline Re1 & $\mathrm{Y}$ & 0.00000 & & & & & & \\
\hline $\operatorname{Re} 1$ & Z & 0.00001 & & & & & & \\
\hline N2 & $\mathrm{x}$ & -0.00038 & & & & & & \\
\hline N2 & $\mathrm{Y}$ & 0.00013 & & & & & & \\
\hline N2 & Z & -0.00049 & & & & & & \\
\hline $\mathrm{C} 10$ & $\mathrm{x}$ & 0.00016 & & & & & & \\
\hline $\mathrm{C} 10$ & $\mathrm{Y}$ & -0.00029 & & & & & & \\
\hline $\mathrm{C} 10$ & Z & 0.00012 & & & & & & \\
\hline C1 & $\mathrm{x}$ & 0.00001 & & & & & & \\
\hline $\mathrm{C} 1$ & $\mathrm{Y}$ & -0.00036 & & & & & & \\
\hline $\mathrm{C} 1$ & Z & -0.00020 & & & & & & \\
\hline N1 & $\mathrm{x}$ & 0.00026 & & & & & & \\
\hline N1 & $\mathrm{Y}$ & 0.00031 & & & & & & \\
\hline N1 & Z & 0.00051 & & & & & & \\
\hline 01 & $\mathrm{x}$ & -0.00003 & & & & & & \\
\hline 01 & $\mathrm{Y}$ & 0.00003 & & & & & & \\
\hline
\end{tabular}




\begin{tabular}{|c|c|c|}
\hline O1 & Z & 0.00002 \\
\hline 02 & $\mathrm{x}$ & 0.00000 \\
\hline $\mathrm{O} 2$ & $\mathrm{Y}$ & 0.00001 \\
\hline $\mathrm{O} 2$ & Z & 0.00007 \\
\hline 03 & $\mathrm{x}$ & -0.00011 \\
\hline 03 & $\mathrm{Y}$ & -0.00022 \\
\hline 03 & Z & 0.00009 \\
\hline C4 & $\mathrm{x}$ & 0.00104 \\
\hline C4 & Y & -0.00044 \\
\hline C4 & Z & 0.00104 \\
\hline H19 & $\mathrm{x}$ & -0.00210 \\
\hline H19 & $\mathrm{Y}$ & -0.00131 \\
\hline H19 & Z & -0.00014 \\
\hline C7 & $\mathrm{x}$ & 0.00026 \\
\hline C7 & $\mathrm{Y}$ & 0.00030 \\
\hline C7 & Z & 0.00005 \\
\hline H 21 & $\mathrm{x}$ & -0.00013 \\
\hline H 21 & $\mathrm{Y}$ & 0.00013 \\
\hline H 21 & Z & 0.00089 \\
\hline C15 & $\mathrm{X}$ & -0.00193 \\
\hline C15 & $\mathrm{Y}$ & -0.00050 \\
\hline C15 & z & -0.00189 \\
\hline 06 & $\mathrm{x}$ & 0.00003 \\
\hline 06 & Y & -0.00013 \\
\hline 06 & Z & 0.00023 \\
\hline 07 & $\mathrm{X}$ & -0.03983 \\
\hline 07 & Y & -0.01024 \\
\hline 07 & Z & -0.04378 \\
\hline H 4 & $\mathrm{x}$ & -0.00049 \\
\hline H 4 & $\mathrm{Y}$ & -0.00093 \\
\hline H4 & Z & -0.00082 \\
\hline H8 & $\mathrm{x}$ & 0.64481 \\
\hline H8 & $\mathrm{Y}$ & 0.17670 \\
\hline H8 & Z & 0.69835 \\
\hline $\mathrm{H} 12$ & $\mathrm{x}$ & -0.00329 \\
\hline $\mathrm{H} 12$ & $\mathrm{Y}$ & -0.00070 \\
\hline $\mathrm{H} 12$ & Z & -0.00710 \\
\hline $\mathrm{H} 2 \mathrm{O}$ & $\mathrm{x}$ & -0.00124 \\
\hline H2O & $\mathrm{Y}$ & 0.00158 \\
\hline H 20 & Z & -0.00089 \\
\hline H 22 & $\mathrm{x}$ & 0.00019 \\
\hline H22 & $\mathrm{Y}$ & 0.00163 \\
\hline H22 & Z & 0.00115 \\
\hline H 26 & $\mathrm{x}$ & 0.00494 \\
\hline H 26 & $\mathrm{Y}$ & 0.00021 \\
\hline H26 & Z & 0.00534 \\
\hline
\end{tabular}

Thermochemical Properties:

pressure: $1.0000 \mathrm{~atm}$

rotational symmetry number: 1

vibrational frequencies below $10.0 \mathrm{~cm}-1$ excluded: 1 zero point energy: $\quad 91.617 \mathrm{kcal} / \mathrm{mol}$

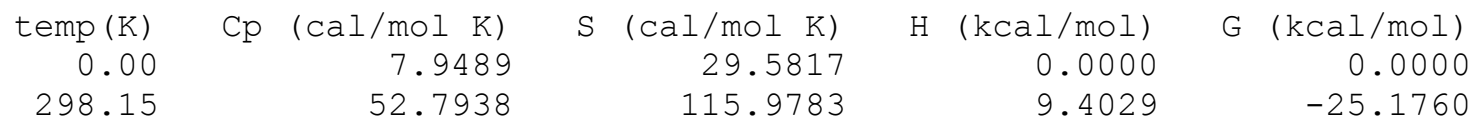

end of program freq 
start of program ch

Moments from quantum mechanical wavefunction:

Dipole Moments (Debye)

$\mathrm{X}=-1.6275 \quad \mathrm{Y}=$

$6.8417 \quad \mathrm{Z}=$

1.9407 Tot $=$

7.2955

Final energy .......... -759.84900275441

Job WF23928 completed on CHENB071 at Fri Jan 23 06:08:26 2004 Reason for exit: Successful completion

Titan Program CPU Time : 010:40:51.7

Titan Program Wall Time: 013:00:09.7

TITAN Properties Program: (PC/x86)

Release 6.0 .6

Reason for exit: Successful completion

Properties Program CPU Time : 000:00:02.0

Properties Program Wall Time: 000:00:02.4 


\section{Scheme 5}

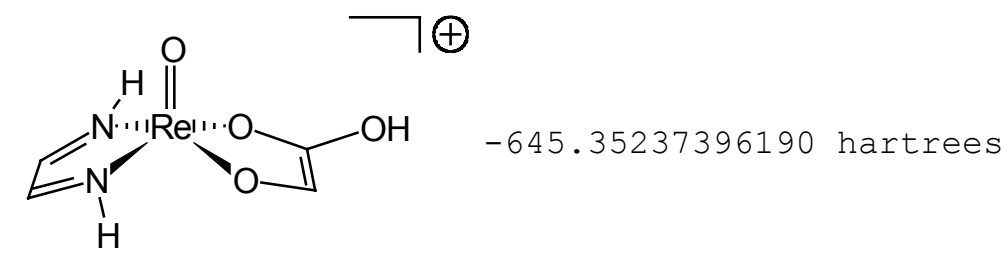

Jaguar version 3.5, release 42

Copyright 1991-1998 Schrodinger, Inc.

All Rights Reserved.

Use of this program should be acknowledged in publications as:

| Jaguar 3.5, Schrodinger, Inc., Portland, Oregon, 1998.

Job WF32256 started on CHENB071 at Wed Mar 12 11:58:03 2003

Jaguar version 3.5, release 42

Job name:

Task:

Stoichiometry:

Molecular weight:

Molecular charge:

Spin multiplicity:

Point Group:

SCF method:

Basis set:

Basis functions:

Total electrons:

Alpha electrons:

Beta electrons:

Total orbitals:

Core orbitals:

Open-shell orbitals:

Occupied orbitals:

Shells:
WF32256

Geometry optimization

ReC $4 \mathrm{~N} 2 \mathrm{H} 6 \mathrm{O} 4$

$332.99 \mathrm{amu}$

1

C1

B3LYP

LACVP**

192

90

45

45

205

45

45

Non-default options chosen:

SCF calculation type: DFT

$\mathrm{DFT}=$ Becke_3_Parameter/HF+Slater+Becke88+VWN+LYP (B3LYP)

Geometry $\bar{w} \bar{l} l$ be optimized in redundant internal coordinates

Molecular symmetry not used

Initial Hessian: from previous calculation

Powell Hessian-updating used

$\begin{array}{cccccc}\text { Iter } & \text { Energy } & \text { Gmax } & \text { Grms } & \text { Dmax } & \text { Drms } \\ 1 & -645.331206 & 0.05093 & 0.01457 & 0.22721 & 0.03906 .\end{array}$




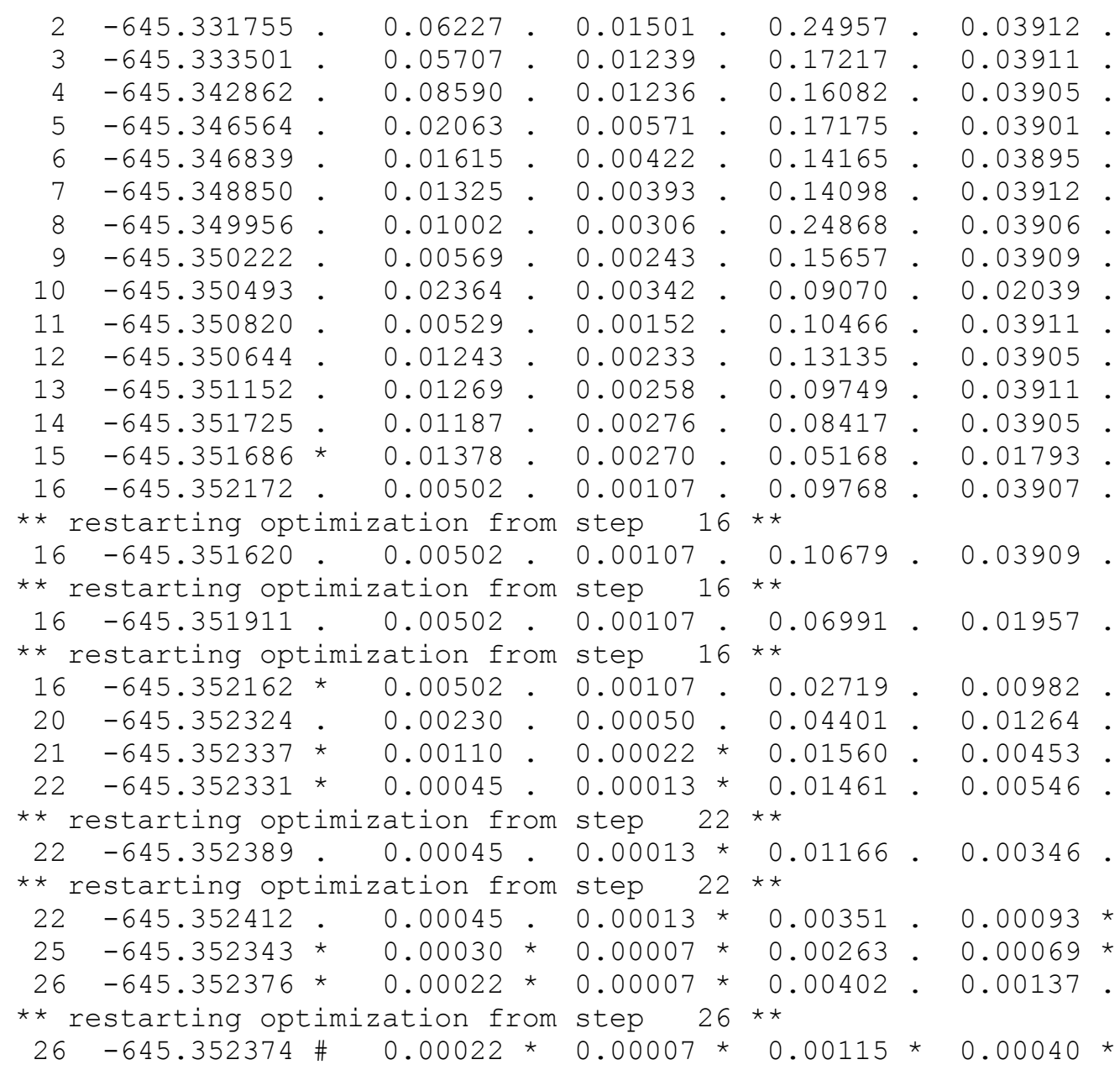

energy change:

gradient maximum:

gradient rms:

step size:

0.00309

displacement maximum:

displacement rms:

predicted energy change:

step: $\quad 3.0927 \mathrm{E}-03$

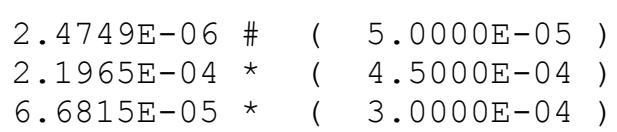

trust radius:
$1.1535 \mathrm{E}-03$ * $(1.8000 \mathrm{E}-03)$

$4.0263 \mathrm{E}-04 \star(1.2000 \mathrm{E}-03)$

3.0927E-03 full

center of mass moved by:
$\mathrm{x}$ :
$-2.2204 \mathrm{E}-16$
Y :
$2.2204 E-16$
$\mathrm{z}:$
$0.0000 \mathrm{E}+00$

final geometry:

$\begin{array}{lr}\text { atom } & x \\ \text { Re1 } & 0.8785834384 \\ \text { N1 } & 0.9678178597 \\ \text { C4 } & -0.2186354016 \\ \text { C7 } & -1.3162285596 \\ \text { N2 } & -0.9805313806 \\ \text { O2 } & 0.6888829657 \\ \text { O3 } & 2.5976459776 \\ \text { C1 } & 2.8016592981 \\ \text { C10 } & 1.7015562069\end{array}$

angstroms

$$
\text { Y }
$$

$-0.9364741099$

$-0.0359162585$

0.2125152183

0.0748829308

$-0.2474274607$

$-0.2257076121$

0.0880723329

0.5789365968

0.4024744395

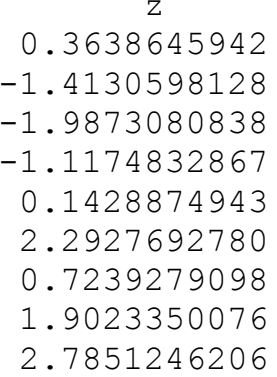




$\begin{array}{lrrr}\text { O4 } & 1.7427329014 & 0.7960477159 & 4.0365945080 \\ \text { H2 } & 3.7706346010 & 0.9922235738 & 2.1651507132 \\ \text { O5 } & 0.8575080421 & -2.5878436395 & 0.0312588740 \\ \text { H1 } & -1.7080813067 & -0.2571003423 & 0.8537940403 \\ \text { H14 } & -2.3467067002 & 0.2476143888 & -1.4082612199 \\ \text { H15 } & -0.2993159562 & 0.5333523196 & -3.0201626527 \\ \text { H19 } & 1.8201246543 & 0.1749481998 & -1.9276179885 \\ \text { H20 } & 0.9419102038 & 0.5170111405 & 4.5145647400\end{array}$

Final energy ........... -645.35237396190

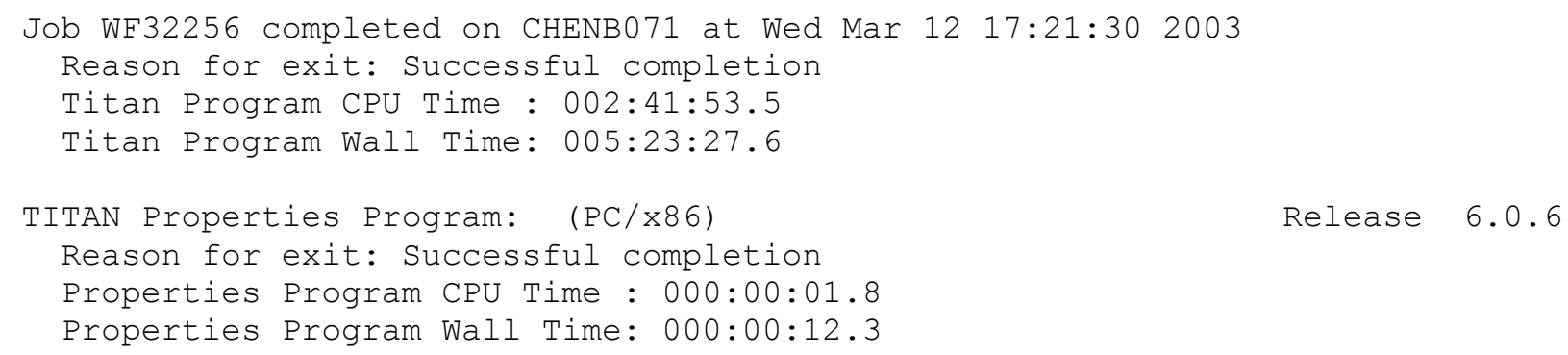

Release 6.0 .6

Job WF15803 started on CHENB071 at Wed Jan 21 18:08:53 2004 Jaguar version 3.5, release 42

Job name:

Task:

Stoichiometry:

Molecular weight:

Molecular charge:

Spin multiplicity:

Point Group:

SCF method:

Basis set:

Basis functions:

Total electrons:

Alpha electrons:

Beta electrons:

Total orbitals:

Core orbitals:

Open-shell orbitals:

Occupied orbitals:

Shells:
WF15803

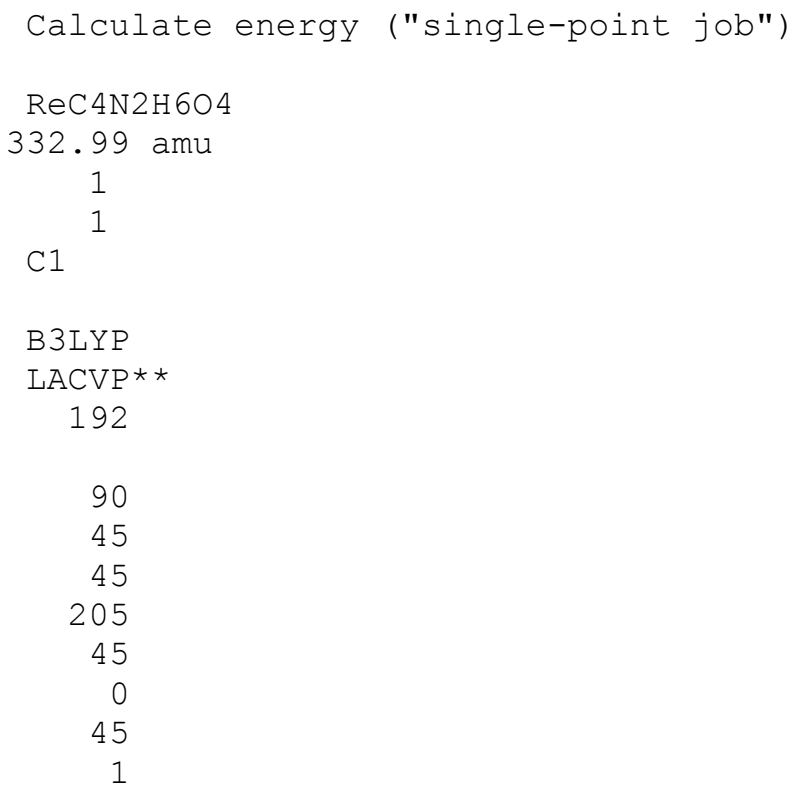

\footnotetext{
Non-default options chosen: SCF calculation type: DFT $\mathrm{DFT}=$ Becke_3_Parameter/HF+Slater+Becke88+VWN+LYP (B3LYP) Numerical $2 \bar{n} d$ derivatives will be computed Vibrational frequencies and related properties will be computed Molecular symmetry not used
} 
calculating forces at perturbed geometry calculating forces at perturbed geometry calculating forces at perturbed geometry calculating forces at perturbed geometry calculating forces at perturbed geometry calculating forces at perturbed geometry calculating forces at perturbed geometry calculating forces at perturbed geometry calculating forces at perturbed geometry calculating forces at perturbed geometry calculating forces at perturbed geometry calculating forces at perturbed geometry calculating forces at perturbed geometry calculating forces at perturbed geometry calculating forces at perturbed geometry calculating forces at perturbed geometry calculating forces at perturbed geometry calculating forces at perturbed geometry calculating forces at perturbed geometry calculating forces at perturbed geometry calculating forces at perturbed geometry calculating forces at perturbed geometry calculating forces at perturbed geometry calculating forces at perturbed geometry calculating forces at perturbed geometry calculating forces at perturbed geometry calculating forces at perturbed geometry calculating forces at perturbed geometry calculating forces at perturbed geometry calculating forces at perturbed geometry calculating forces at perturbed geometry calculating forces at perturbed geometry calculating forces at perturbed geometry calculating forces at perturbed geometry calculating forces at perturbed geometry calculating forces at perturbed geometry calculating forces at perturbed geometry calculating forces at perturbed geometry calculating forces at perturbed geometry calculating forces at perturbed geometry calculating forces at perturbed geometry calculating forces at perturbed geometry calculating forces at perturbed geometry calculating forces at perturbed geometry calculating forces at perturbed geometry calculating forces at perturbed geometry calculating forces at perturbed geometry calculating forces at perturbed geometry calculating forces at perturbed geometry calculating forces at perturbed geometry calculating forces at perturbed geometry calculating forces at perturbed geometry calculating forces at perturbed geometry calculating forces at perturbed geometry calculating forces at perturbed geometry calculating forces at perturbed geometry calculating forces at perturbed geometry calculating forces at perturbed geometry calculating forces at perturbed geometry calculating forces at perturbed geometry calculating forces at perturbed geometry
3 of $102 \ldots$

4 of $102 \ldots$

5 of $102 \ldots$

6 of $102 \ldots$

7 of $102 \ldots$

8 of $102 \ldots$

9 of $102 \ldots$

10 of $102 \ldots$

11 of $102 \ldots$

12 of $102 \ldots$

13 of $102 \ldots$

14 of $102 \ldots$

15 of $102 \ldots$

16 of $102 \ldots$

17 of $102 \ldots$

18 of $102 \ldots$

19 of $102 \ldots$

20 of $102 \ldots$

21 of $102 \ldots$

22 of $102 \ldots$

23 of $102 \ldots$

24 of $102 \ldots$

25 of $102 \ldots$

26 of $102 \ldots$

27 of $102 \ldots$

28 of $102 \ldots$

29 of $102 \ldots$

30 of $102 \ldots$

31 of $102 \ldots$

32 of $102 \ldots$

33 of $102 \ldots$

34 of $102 \ldots$

35 of $102 \ldots$

36 of $102 \ldots$

37 of $102 \ldots$

38 of $102 \ldots$

39 of $102 \ldots$

40 of $102 \ldots$

41 of $102 \ldots$

42 of $102 \ldots$

43 of $102 \ldots$

44 of $102 \ldots$

45 of $102 \ldots$

46 of $102 \ldots$

47 of $102 \ldots$

48 of $102 \ldots$

49 of $102 \ldots$

50 of $102 \ldots$

51 of $102 \ldots$

52 of $102 \ldots$

53 of $102 \ldots$

54 of $102 \ldots$

55 of $102 \ldots$

56 of $102 \ldots$

57 of $102 \ldots$

58 of $102 \ldots$

59 of $102 \ldots$

60 of $102 \ldots$

61 of $102 \ldots$

62 of $102 \ldots$

63 of $102 \ldots$ 
calculating forces at perturbed geometry calculating forces at perturbed geometry calculating forces at perturbed geometry calculating forces at perturbed geometry calculating forces at perturbed geometry calculating forces at perturbed geometry calculating forces at perturbed geometry calculating forces at perturbed geometry calculating forces at perturbed geometry calculating forces at perturbed geometry calculating forces at perturbed geometry calculating forces at perturbed geometry calculating forces at perturbed geometry calculating forces at perturbed geometry calculating forces at perturbed geometry calculating forces at perturbed geometry calculating forces at perturbed geometry calculating forces at perturbed geometry calculating forces at perturbed geometry calculating forces at perturbed geometry calculating forces at perturbed geometry calculating forces at perturbed geometry calculating forces at perturbed geometry calculating forces at perturbed geometry calculating forces at perturbed geometry calculating forces at perturbed geometry calculating forces at perturbed geometry calculating forces at perturbed geometry calculating forces at perturbed geometry calculating forces at perturbed geometry calculating forces at perturbed geometry calculating forces at perturbed geometry calculating forces at perturbed geometry calculating forces at perturbed geometry calculating forces at perturbed geometry calculating forces at perturbed geometry calculating forces at perturbed geometry calculating forces at perturbed geometry 101 of $102 \ldots$ calculating forces at perturbed geometry 101 of $102 \ldots$ calculating forces at perturbed geometry 102 of $102 \ldots$
64 of $102 \ldots$ 65 of $102 \ldots$ 66 of $102 \ldots$ 67 of $102 \ldots$ 68 of $102 \ldots$ 69 of $102 \ldots$ 70 of $102 \ldots$ 71 of $102 \ldots$ 72 of $102 \ldots$ 73 of $102 \ldots$ 74 of $102 \ldots$ 75 of $102 \ldots$ 76 of $102 \ldots$ 77 of $102 \ldots$ 78 of $102 \ldots$ 79 of $102 \ldots$ 80 of $102 \ldots$ 81 of $102 \ldots$ 82 of $102 \ldots$ 83 of $102 \ldots$ 84 of $102 \ldots$ 85 of $102 \ldots$ 86 of $102 \ldots$ 87 of $102 \ldots$ 88 of $102 \ldots$ 89 of $102 \ldots$ 90 of $102 \ldots$ 91 of $102 \ldots$ 92 of $102 \ldots$ 93 of $102 \ldots$ 94 of $102 \ldots$ 95 of $102 \ldots$ 96 of $102 \ldots$ 97 of $102 \ldots$ 98 of $102 \ldots$ 99 of $102 \ldots$ 102 of $102 .$.

numerical derivatives complete;

calculation at normal geometry follows...

start of program freq

harmonic frequencies in $\mathrm{cm}^{\wedge}-1$, IR intensities in $\mathrm{km} / \mathrm{mol}$, and normal modes:

$\begin{array}{rrrrrrrrr}\text { frequencies } & 73.09 & 94.05 & 136.49 & 157.87 & 218.17 & 228.43 & 271.59 \\ \text { intensities } & 6.80 & 3.39 & 3.14 & 5.32 & 0.06 & 0.86 & 13.99 \\ \text { Re1 } & \mathrm{X} & -0.00631 & -0.00350 & 0.01701 & -0.00821 & -0.01185 & -0.00737 & 0.00392 \\ \text { Re1 } & Y & 0.02059 & 0.02162 & 0.01141 & 0.00214 & -0.00286 & 0.01317 & -0.00826 \\ \text { Re1 } & \mathrm{Z} & 0.00334 & -0.00039 & -0.01110 & 0.00694 & 0.00504 & 0.00001 & -0.01644 \\ \text { N1 } & \mathrm{X} & 0.00664 & -0.01788 & -0.02903 & 0.04097 & -0.00724 & -0.00015 & -0.03935 \\ \text { N1 } & \mathrm{Y} & -0.05888 & 0.03456 & -0.06098 & 0.02789 & -0.06855 & 0.02556 & -0.03447 \\ \text { N1 } & \mathrm{Z} & -0.04252 & 0.01437 & -0.05373 & 0.04105 & -0.02634 & -0.00247 & -0.04479 \\ \text { C4 } & \mathrm{X} & 0.01076 & -0.02712 & -0.06143 & 0.04619 & 0.00053 & 0.00438 & -0.03509 \\ \text { C4 } & \mathrm{Y} & -0.09072 & -0.05065 & -0.08856 & -0.04095 & -0.03531 & 0.02116 & 0.05028 \\ \text { C4 } & \mathrm{Z} & -0.06018 & -0.00982 & -0.00513 & -0.00558 & -0.02247 & -0.01536 & -0.02576 \\ \text { C7 } & \mathrm{X} & 0.00544 & -0.03256 & -0.01749 & 0.02861 & 0.00554 & -0.00327 & -0.02707 \\ \text { C7 } & \mathrm{Y} & -0.04888 & -0.10187 & -0.00544 & -0.07193 & 0.05353 & -0.00142 & 0.02754 \\ \text { C7 } & \text { Z } & -0.05771 & -0.02589 & 0.06132 & -0.03815 & -0.00223 & -0.02953 & -0.03010\end{array}$




\begin{tabular}{|c|c|c|c|c|c|c|c|c|}
\hline N2 & $\mathrm{x}$ & -0.00177 & -0.03568 & 0.04677 & 0.00040 & 0.00433 & -0.01359 & -0.01216 \\
\hline N2 & $\mathrm{Y}$ & 0.02051 & -0.05963 & 0.07197 & -0.01417 & 0.06292 & 0.00984 & -0.05985 \\
\hline N2 & Z & -0.03809 & -0.01377 & 0.06560 & -0.00947 & 0.00218 & -0.01964 & -0.05383 \\
\hline $\mathrm{O} 2$ & $\mathrm{x}$ & 0.01147 & -0.01410 & 0.00648 & 0.05094 & -0.01950 & 0.05036 & 0.01144 \\
\hline $\mathrm{O} 2$ & $\mathrm{Y}$ & -0.01354 & 0.06138 & -0.05345 & -0.07180 & 0.00410 & -0.15441 & 0.01305 \\
\hline 02 & Z & 0.01466 & -0.01986 & 0.00734 & 0.05261 & 0.04984 & 0.04179 & 0.07645 \\
\hline 03 & $\mathrm{x}$ & -0.02848 & 0.05235 & 0.01990 & -0.00833 & -0.03455 & -0.00712 & 0.00275 \\
\hline 03 & $\mathrm{Y}$ & 0.05995 & -0.09497 & -0.02074 & -0.00154 & -0.02213 & -0.02783 & 0.06380 \\
\hline 03 & z & -0.01376 & 0.03778 & 0.03381 & 0.00811 & 0.00965 & -0.02211 & \\
\hline $\mathrm{C} 1$ & $\mathrm{x}$ & -0.00476 & 0.05865 & -0.00412 & -0.01019 & -0.01644 & 0.00834 & \\
\hline $\mathrm{C} 1$ & $\mathrm{Y}$ & 0.01906 & -0.10431 & -0.01922 & 0.05063 & -0.01630 & -0.05262 & -0.02518 \\
\hline $\mathrm{C} 1$ & $\mathrm{z}$ & 0.00070 & 0.04149 & 0.04040 & -0.01490 & 0.00415 & -0.00949 & \\
\hline C10 & $\mathrm{x}$ & 0.02276 & 0.01845 & -0.02194 & 0.02027 & -0.00564 & 0.02253 & \\
\hline C10 & $\mathrm{Y}$ & -0.03691 & -0.00750 & -0.01643 & 0.00800 & 0.00022 & -0.06513 & -0.00358 \\
\hline C10 & Z & 0.02237 & 755 & 0.01826 & & 0.02187 & & \\
\hline 04 & $\mathrm{x}$ & 0.06152 & 0740 & -0.07839 & 0.03636 & & -0 & \\
\hline 04 & $\mathrm{Y}$ & -0.11994 & -0.00202 & 0.02276 & 0.04925 & 0.03193 & 0.07255 & \\
\hline 04 & Z & 710 & 633 & 762 & & 131 & -0 . & \\
\hline H2 & $\mathrm{x}$ & -0.00317 & & -0.0 & -0.0 & -0 & & \\
\hline H2 & $\mathrm{Y}$ & 0.02095 & -0.18524 & -0.02933 & 0.10341 & -0.02445 & -0.05652 & -0.07327 \\
\hline H2 & Z & -0.00889 & 704 & 211 & -0.0 & -0.01422 & -0 & \\
\hline 05 & $\mathrm{x}$ & -0.0 & & -0.0 & -0 & & & \\
\hline 05 & $\mathrm{Y}$ & 0.00838 & 2759 & 0.01531 & 0. & 0.01841 & 0366 & 1310 \\
\hline 05 & Z & 0.06744 & -0.0 & -0.02434 & -0. & -0.10806 & & \\
\hline $\mathrm{H} 1$ & $\mathrm{x}$ & -0.0 & -0 & & -0 . & & & \\
\hline $\mathrm{H} 1$ & $\mathrm{Y}$ & 0.0 & -0.0 & 0.1 & -0.0 & 0. & -0 . & -0 . \\
\hline H1 & z & -0.04 & -0.02296 & & -0.0 & & -0 . & \\
\hline H14 & $\mathrm{x}$ & & -0 . & -0 . & & & & \\
\hline H14 & $\mathrm{Y}$ & -0 & -0 & -0.0 & -0 . & & -0 . & \\
\hline $\mathrm{H} 14$ & z & -0 & -0 & & -0 . & & -0 . & \\
\hline $\mathrm{H}$ & $\mathrm{x}$ & & -0 . & -0 & & & & \\
\hline H15 & $\mathrm{Y}$ & -0.1 & -0 & -0.1 & -0.0 & -0.063 & 2733 & \\
\hline H15 & Z & -0.0 & -0 & -0 & -0. & -0 & -0 . & \\
\hline H19 & $\mathrm{x}$ & & -0 & -0 . & & -0 & & \\
\hline H19 & $\mathrm{Y}$ & -0.08118 & 265 & -0.0 & & -0.1 & 5165 & -0.0 \\
\hline H19 & Z & -0.04882 & 0.0 & -0.1 & & -0.03663 & & -0.0 \\
\hline $\mathrm{H} 2 \mathrm{C}$ & $\mathrm{x}$ & & -0 & -0 . & & & -0 . & \\
\hline H2O & $\mathrm{Y}$ & -0.1 & 0.0 & -0. & & & & \\
\hline H2O & Z & 0.05319 & -0.01943 & -0.01003 & & 0.04979 & -0.02383 & 0.10186 \\
\hline \multicolumn{2}{|c|}{ Erequencies } & & & & & & & \\
\hline \multicolumn{2}{|c|}{ intensities } & 19.65 & .13 & .63 & & & & .55 \\
\hline & $\mathrm{x}$ & 0.0 & & -0.01 & & & & \\
\hline 1 & $\mathrm{Y}$ & .00608 & -0.0 & -0.0 & -0.0 & 89 & & \\
\hline Re1 & Z & 0.02750 & 0.00085 & -0.00904 & 0.02255 & -0.00557 & -0.00684 & -0.00264 \\
\hline & $\mathrm{x}$ & & & -0.0 & -0 . & & & \\
\hline & $\mathrm{Y}$ & -0 . & & & & -0.0 & & -0 \\
\hline N1 & Z & -0.04147 & 1577 & 0.03728 & -0.02138 & -0.05600 & 0.07155 & 00032 \\
\hline C4 & $\mathrm{x}$ & & & -0.0 & -0.05 & & & \\
\hline & $\mathrm{Y}$ & & -0 & -0 . & & & -0 . & \\
\hline C4 & Z & -0.01486 & 212 & -0.01650 & -0.07477 & 0.01676 & -0.00 & 461 \\
\hline C7 & $\mathrm{x}$ & -0.0 & & -0.0 & -0.0 & & -0.0 & \\
\hline & $\mathrm{Y}$ & & -0.0 & -0 & -0. & -0.0 & & \\
\hline C7 & Z & -0.0 & 0.0 & -0.02 & -0.0 & & & \\
\hline & $\mathrm{x}$ & -0.0 & & 0.0 & -0.0 & -0.0 & -0.0 & \\
\hline & $\mathrm{Y}$ & & & & & -0.00219 & -0.0 & \\
\hline N2 & Z & -0.04464 & & & -0.067 & & -0. & \\
\hline & $\mathrm{x}$ & 0.04944 & -0.0 & 0.1 & -0 & & & \\
\hline & $\mathrm{Y}$ & -0.01319 & & 0.02807 & & & & \\
\hline $\mathrm{O} 2$ & Z & -0.04452 & -0.01074 & -0.02630 & -0.00507 & & -0.00602 & \\
\hline 03 & $\mathrm{X}$ & 0.02140 & -0.09081 & 0.03412 & -0.8 & -0.14090 & -0.07362 & -0 . \\
\hline
\end{tabular}




\begin{tabular}{|c|c|c|c|c|c|c|c|c|}
\hline 03 & $\mathrm{Y}$ & 0.04199 & 0.12385 & 0.05235 & -0.02034 & -0.08117 & -0.02714 & -0.02991 \\
\hline & Z & -0.03726 & -0.06352 & 0.03384 & -0.01299 & -0.00295 & -0.00857 & -0.01209 \\
\hline $\mathrm{C} 1$ & $\mathrm{X}$ & 0.05733 & 0.03048 & 0.08309 & -0.02330 & -0.00627 & -0.01907 & -0.01844 \\
\hline $\mathrm{C} 1$ & $\mathrm{Y}$ & -0.01508 & -0.11828 & 0.03791 & -0.01519 & -0.00746 & -0.01773 & 0.03055 \\
\hline $\mathrm{C} 1$ & Z & -0.01129 & 0.01687 & 0.02776 & -0.00956 & -0.03188 & -0.01046 & -0.03101 \\
\hline C10 & $\mathrm{x}$ & 0.04975 & 0.01933 & 0.07199 & -0.00444 & 0.04639 & 0.01372 & 0.02346 \\
\hline C10 & $\mathrm{Y}$ & -0.02044 & -0.06543 & 0.03224 & -0.00376 & 0.06006 & 0.00824 & -0.02595 \\
\hline $\mathrm{C} 10$ & Z & -0.02361 & 0.00787 & 0.03115 & -0.00632 & 0.03266 & 0.01903 & 0.02102 \\
\hline 04 & $\mathrm{x}$ & -0.08104 & -0.00826 & -0.06466 & 0.00705 & -0.04268 & -0.00690 & 0.00845 \\
\hline 04 & $\mathrm{Y}$ & -0.01951 & 0.02944 & -0.00098 & -0.00133 & 0.00599 & 0.01390 & -0.05121 \\
\hline 04 & Z & -0.02432 & -0.02299 & 0.04871 & -0.00738 & 0.06480 & 0.02700 & 0.03249 \\
\hline $\mathrm{H} 2$ & $\mathrm{x}$ & 0.06519 & 0.08214 & 9688 & -0.01514 & 0.06386 & & \\
\hline H2 & $\mathrm{Y}$ & -0.03402 & -0.25255 & 0.02570 & -0.02048 & -0.09051 & -0.03781 & 0.16133 \\
\hline H2 & Z & -0.01191 & 0.03486 & 0.00353 & -0.03795 & -0.17401 & -0.07785 & -0.10690 \\
\hline 05 & $\mathrm{x}$ & -0.01416 & -0.0 & & & -0.00286 & & \\
\hline 05 & $\mathrm{Y}$ & 0.02774 & -0.0 & -0.0 & -0.00416 & -0.00185 & -0.00237 & \\
\hline 05 & Z & -0.07234 & -0.02057 & 0.00469 & -0.02026 & 0.00891 & 0.01199 & 0576 \\
\hline $\mathrm{H} 1$ & $\mathrm{x}$ & -0.0 & 0. & & & -0.0 & 5893 & \\
\hline $\mathrm{H} 1$ & $\mathrm{Y}$ & -0.1 & & & & & & \\
\hline $\mathrm{H} 1$ & Z & -0.07417 & 3243 & 084 & -0.07193 & 0.00298 & -0.00910 & -0.00692 \\
\hline $\mathrm{H} 14$ & $\mathrm{X}$ & -0.0 & 624 & -0. & & & & \\
\hline H14 & $\mathrm{Y}$ & & -0.0 & -0 & & & & \\
\hline H14 & Z & -0.00739 & 389 & -0 & -0.0 & -0.01692 & 1188 & -0 \\
\hline H15 & $\mathrm{x}$ & 1447 & 0.0 & & -0.0 & -0.01671 & 2609 & \\
\hline H15 & $\mathrm{Y}$ & & -0. & -0 . & & & & \\
\hline H15 & Z & 418 & 0.0 & -0.0 & -0.0 & 0.05537 & -0.06346 & \\
\hline H19 & $\mathrm{x}$ & -0.0 & 0.0 & -0.0 & -0.0 & & 0.00155 & \\
\hline $\mathrm{H}$ & $\mathrm{Y}$ & -0 & & & & & & \\
\hline H19 & Z & -0 & 0.0 & & -0 . & -0. & & \\
\hline H2O & $\mathrm{x}$ & -0 & -0. & -0 & & & & \\
\hline H2O & $\mathrm{Y}$ & -0.0 & & -0 . & & -0 & -0 . & \\
\hline $\mathrm{H} 20$ & z & & -0.04353 & & & & & \\
\hline \multicolumn{2}{|c|}{ Erequencies } & & & & & & & \\
\hline \multicolumn{2}{|c|}{ intensities } & 1.70 & & & & & & 9.44 \\
\hline $\operatorname{Re} 1$ & $\mathrm{x}$ & 0.01392 & -0.0 & -0.0 & 0.0 & 0.00027 & -0.00230 & -0.00208 \\
\hline e. & $\mathrm{Y}$ & 37 & -0. & & -0 . & 00349 & & \\
\hline $\operatorname{Re} 1$ & Z & -0.01166 & 0.0 & -0.00181 & 0.00219 & -0.00337 & 0.00253 & 0.00188 \\
\hline & $\mathrm{x}$ & -0.01094 & 0.0 & -0.0 & & -0.03360 & -0.0 & \\
\hline & $\mathrm{Y}$ & -0 & 0. & -0 . & & & & \\
\hline & Z & 0.09269 & -0.0 & 0.0 & -0.05 & 0.06616 & 5667 & -0.02261 \\
\hline C4 & $\mathrm{x}$ & 0.01880 & 0.00981 & -0.01644 & 0.00959 & -0.01112 & -0.00073 & -0.01081 \\
\hline & $\mathrm{Y}$ & & & -0 . & & & & \\
\hline C4 & z & & 0 . & -0. & -0.0 & 44 & 902 & -0 \\
\hline C7 & $\mathrm{x}$ & -0.06711 & 0.0 & -0.00353 & 0.00724 & 0.01051 & 0.00500 & 5609 \\
\hline & $\mathrm{Y}$ & & & & & & & \\
\hline & $\mathrm{z}$ & -0 . & & -0 . & & -0.0 & -0 . & \\
\hline N2 & $\mathrm{x}$ & -0.11700 & -0.03994 & 0.08816 & -0.06 & 0.07183 & 0.05993 & 1879 \\
\hline & $\mathrm{Y}$ & & & -0 . & & & & \\
\hline & Z & & & -0 & & -0.01 & -0 & \\
\hline 02 & $\mathrm{x}$ & -0.00610 & 0.0 & & 0.0 & -0.01068 & 0.04650 & 240 \\
\hline 02 & $\mathrm{Y}$ & -0.0 & -0.0 & -0. & -0. & & -0 & \\
\hline 02 & Z & -0.0 & -0.1 & & & & -0 & -0 . \\
\hline 03 & $\mathrm{x}$ & & -0.06759 & -0.0 & 0. & -0.062 & 0 & 187 \\
\hline & $\mathrm{Y}$ & & & & 0.0 & -0.00 & $-0 . c$ & \\
\hline & Z & & & & & & & \\
\hline $\mathrm{C} 1$ & $\mathrm{x}$ & -0.00343 & -0.0 & & & & -0. & -0 \\
\hline & $\mathrm{Y}$ & & 0.0 & -0 & -0.0 & 89 & -0.0 & -0 . \\
\hline & z & & & & & & -0.02340 & \\
\hline $\mathrm{C} 10$ & $\mathrm{x}$ & -0.01283 & -0.01065 & -0.04904 & -0.06072 & & -0.05373 & -0.00464 \\
\hline $\mathrm{C} 10$ & $\mathrm{Y}$ & -0.00417 & -0.00574 & 0.10915 & 0.14 & -0.03792 & 0.02611 & -0 \\
\hline
\end{tabular}




\begin{tabular}{|c|c|c|c|c|c|c|c|c|}
\hline $\mathrm{C} 10$ & z & -0.00227 & -0.02951 & -0.05181 & -0.05251 & -0.02683 & 0.04073 & 0.01100 \\
\hline 04 & $\mathrm{x}$ & 0.00397 & 0.06456 & 0.01538 & 0.00786 & -0.01156 & 0.01480 & 0.00174 \\
\hline 04 & $\mathrm{Y}$ & 0.00077 & 0.00290 & -0.04352 & -0.04122 & -0.01763 & 0.02659 & 0.00685 \\
\hline 04 & Z & -0.00615 & -0.04574 & -0.02232 & 0.00115 & -0.07882 & 0.08963 & 0.01753 \\
\hline H2 & $\mathrm{x}$ & -0.00706 & -0.00135 & 0.11917 & 0.10501 & 0.03489 & -0.06841 & -0.01308 \\
\hline H2 & $\mathrm{Y}$ & -0.00192 & -0.01654 & -0.30177 & -0.29205 & 0.08016 & -0.07409 & 0.00105 \\
\hline H2 & Z & 0.02818 & 0.10123 & 0.09182 & 0.11703 & -0.06197 & 0.00404 & -0.00235 \\
\hline 05 & $\mathrm{x}$ & -0.00629 & 0.00405 & -0.00026 & -0.00049 & -0.00205 & 0.00361 & -0.00066 \\
\hline 05 & $\mathrm{Y}$ & -0.00125 & -0.00325 & -0.00173 & 0.00046 & -0.00532 & 0.00036 & -0.00027 \\
\hline 05 & Z & 0.00390 & -0.00050 & -0.00009 & -0.00095 & 0.00185 & -0.00515 & \\
\hline $\mathrm{H} 1$ & $\mathrm{x}$ & -0.18624 & -0.06307 & 0.17303 & -0.16372 & -0.00951 & 0.03331 & -0.15686 \\
\hline $\mathrm{H} 1$ & $\mathrm{Y}$ & -0.07314 & -0.00467 & 0.15504 & -0.21870 & & -0.19502 & \\
\hline $\mathrm{H} 1$ & Z & -0.06261 & -0.00454 & 0.04572 & -0.06752 & -0.10498 & -0.04820 & \\
\hline H14 & $\mathrm{x}$ & -0.04771 & 0.01006 & -0.01217 & 0.01034 & -0.02882 & -0.02337 & \\
\hline H14 & $\mathrm{Y}$ & -0.11438 & -0.0 & 0.1 & -0.11393 & & & \\
\hline H14 & Z & -0.16427 & -0.0 & 0. & -0.07942 & 0.00843 & & \\
\hline H15 & $\mathrm{x}$ & 0.12525 & -0.04385 & 0.08409 & -0.06842 & 0.03581 & 0.04671 & -0.02707 \\
\hline H15 & $\mathrm{Y}$ & 432 & 0. & & -0.1 & -0.1 & -0 . & \\
\hline H15 & z & & & & -0.0 & -0.0 & -0 . & -0 \\
\hline H19 & $\mathrm{x}$ & 0.00947 & 898 & -0.01673 & 0.01309 & -0.02658 & -0.02119 & 1578 \\
\hline H19 & $\mathrm{Y}$ & 0.0 & 0.0 & 0.1 & -0. & -0.37259 & -0 & \\
\hline H19 & Z & & -0. & & & & -0 . & \\
\hline H2O & $\mathrm{X}$ & 0.02343 & 927 & -0.0 & -0.0 & 0.00095 & 4461 & 202 \\
\hline H2O & $\mathrm{Y}$ & -0.01264 & 0.2 & 0.1 & & -0.09043 & & \\
\hline $\mathrm{H} 20$ & Z & & & -0 . & & & & \\
\hline & & & & & & & \\
\hline \multicolumn{2}{|c|}{$\begin{array}{l}\text { Erequencles } \\
\text { intensities }\end{array}$} & & & & & & & \\
\hline $\operatorname{Re} 1$ & $\mathrm{x}$ & -0.00014 & -0.00087 & -0.00460 & -0.00026 & 0. & -0 & \\
\hline Re1 & $\mathrm{Y}$ & 0.00077 & 0.0 & -0.0 & 0.0 & & -0 & -0 \\
\hline Re1 & Z & -0.00066 & -0.0 & 0 & 0. & & -0 & \\
\hline N1 & $\mathrm{x}$ & -0.00335 & -0.01128 & 0.1 & 0.0 & -0.00453 & -0 . & 4602 \\
\hline N1 & $\mathrm{Y}$ & 0.00001 & 0.04772 & & -0.0 & & -0 & -0 \\
\hline & Z & 0.0 & 0.0 & -0 . & -0 . & & & \\
\hline C4 & $\mathrm{x}$ & -0.00058 & 0.00238 & -0.0 & -0.00002 & -0.00058 & -0.00186 & -0 \\
\hline C4 & $\mathrm{Y}$ & -0.00886 & -0.07523 & -0.0 & & & & -0 . \\
\hline C4 & Z & -0.0 & -0.0 & & 0.0 & -0.0 & -0 . & \\
\hline C7 & $\mathrm{x}$ & 0.00504 & 0.01733 & -0.12946 & -0.00355 & 0.00459 & -0.00032 & 3168 \\
\hline $\mathrm{C} 7$ & $\mathrm{Y}$ & -0.01037 & -0.08436 & & -0.10821 & -0.00119 & 021 & \\
\hline & Z & -0.0 & -0 & -0 . & -0 . & -0 & -0 & -0 \\
\hline & $\mathrm{x}$ & & 0 & & & -0.0 & 585 & \\
\hline N2 & $\mathrm{Y}$ & 0.00369 & 0.0 & 0.0 & 0.0 & 0.00092 & 024 & 480 \\
\hline & Z & & & -0 . & & & & \\
\hline $\mathrm{O} 2$ & $\mathrm{x}$ & 02 & 0 . & -0.0 & -0.0 & -0.10502 & -0.0 & \\
\hline $\mathrm{O} 2$ & $\mathrm{Y}$ & -0.01597 & -0.00117 & -0.00428 & 0.0 & -0.06394 & -0.00372 & 1066 \\
\hline 02 & Z & & -0.0 & -0 & & & & \\
\hline & $\mathrm{x}$ & -0.0 & & & -0 . & -0 . & -0 & \\
\hline 03 & $\mathrm{Y}$ & 0.02137 & -0.00623 & -0.00117 & -0.00034 & -0.00490 & -0.00088 & 0335 \\
\hline 03 & Z & & & -0 & & & & \\
\hline & $\mathrm{X}$ & & -0. & & & & & \\
\hline $\mathrm{C} 1$ & $\mathrm{Y}$ & -0.14069 & 0.01651 & -0.00279 & -0.00 & 0.05720 & 565 & -0 \\
\hline & Z & & -0.0 & -0.0 & -0.0 & & & \\
\hline $\mathrm{C} 10$ & $\mathrm{x}$ & -0.0 & & & -0 & & & \\
\hline $\mathrm{C} 10$ & $\mathrm{Y}$ & & -0.0 & & -0. & -0.00 & 182 & -0 \\
\hline & Z & -0.0 & & & -0.0 & -0.00 & -0 & \\
\hline & $\mathrm{x}$ & & & & -0.0 & & & \\
\hline 04 & $\mathrm{Y}$ & -0.0 & & & -0 & & & \\
\hline & Z & 59 & 0.0 & & -0. & & & -0 . \\
\hline & $\mathrm{x}$ & -0.21894 & & & & & & \\
\hline H2 & $\mathrm{Y}$ & & -0.07589 & & & & -0.00350 & \\
\hline $\mathrm{H} 2$ & Z & -0.21641 & 0.03018 & -0.02 & -0.0 & -0.28755 & -0.00615 & -0 . \\
\hline
\end{tabular}




\begin{tabular}{|c|c|c|c|c|c|c|c|c|}
\hline 05 & $\mathrm{x}$ & 0.00019 & 0.00161 & 0.00433 & 0.00074 & -0.00099 & 0.00162 & -0.00004 \\
\hline 05 & $\mathrm{Y}$ & 0.00127 & -0.00011 & 0.00314 & -0.00054 & -0.01124 & 0.23416 & 0.00798 \\
\hline 05 & $\mathrm{Z}$ & 0.00153 & 0.00268 & -0.00301 & -0.00077 & -0.00195 & 0.04577 & 0.00136 \\
\hline $\mathrm{H} 1$ & $\mathrm{x}$ & -0.00965 & -0.10307 & 0.15338 & -0.05068 & -0.00242 & 0.01571 & -0.12641 \\
\hline $\mathrm{H} 1$ & & -0.01415 & -0.22872 & -0.25473 & -0.13618 & 0.00674 & -0.01263 & 0.10906 \\
\hline $\mathrm{H} 1$ & Z & -0.00617 & -0.09590 & -0.00786 & -0.05683 & 0.00578 & 0.01023 & -0.19198 \\
\hline H14 & $\mathrm{x}$ & 0.01148 & 0.07727 & -0.13727 & 0.05521 & 0.00632 & -0.00341 & \\
\hline $\mathrm{H} 14$ & $\mathrm{Y}$ & 0.06052 & 0.51792 & 0.05426 & 0.53182 & 0.00174 & -0.00372 & 0.03605 \\
\hline $\mathrm{H} 14$ & z & 0.01901 & 0.14671 & 0.01848 & 0.15616 & -0.00390 & 0.00668 & -0.33075 \\
\hline H15 & $\mathrm{x}$ & 0.00523 & 0.04246 & -0.03144 & & 0.00624 & & \\
\hline H15 & $\mathrm{Y}$ & 0.07331 & 0.50785 & 0.01153 & -0.53041 & 0.00354 & -0.00486 & -0.02921 \\
\hline H15 & $\mathrm{z}$ & 0.01740 & 5716 & 0.15297 & -0.16857 & -0.00303 & -0.00674 & \\
\hline H19 & $\mathrm{X}$ & -0.00073 & -0.02711 & -0.00414 & 0.01860 & -0.00417 & 0.00556 & \\
\hline H19 & $\mathrm{Y}$ & -0.00153 & -0.27125 & 0.19440 & 0.13453 & -0.02175 & -0.01335 & \\
\hline H19 & Z & 0584 & -0.1 & -0.1 & 0.07845 & -0.00349 & & \\
\hline H2O & $\mathrm{x}$ & 574 & 0.0 & -0.0 & 0.00039 & 0.05624 & 0478 & \\
\hline H2O & $\mathrm{Y}$ & -0.02777 & -0.00013 & 0.00214 & -0.00113 & 0.10599 & -0.00939 & \\
\hline $\mathrm{H} 20$ & $\mathrm{z}$ & -0.01372 & 0.0 & & & & -0 & \\
\hline \multicolumn{2}{|c|}{ frequencies } & 1133.07 & & & & & 1383.54 & \\
\hline \multicolumn{2}{|c|}{ intensities } & 111 & & & & & 7.51 & \\
\hline $\operatorname{Re} 1$ & $\mathrm{x}$ & -0.0 & 0.0 & -0.0 & -0 . & -0 . & 0.00011 & -0 \\
\hline Re1 & $\mathrm{Y}$ & 0072 & 0.0 & -0.00 & -0.00006 & -0.00054 & 0.00002 & -0 \\
\hline Re1 & Z & -0.00182 & -0.0 & -0.00152 & -0.00108 & -0.00086 & -0.00026 & -0 \\
\hline N1 & $\mathrm{x}$ & -0.0 & & -0.0 & -0.03393 & -0 & -0 . & \\
\hline N1 & $\mathrm{Y}$ & 0071 & -0.0 & 0.00312 & 0.01035 & 0.01709 & 0.03053 & -0 \\
\hline N1 & Z & -0.0 & 0.0 & -0.01618 & -0.01480 & -0.03774 & -0.07252 & \\
\hline C4 & $\mathrm{x}$ & & -0 . & & & & 2883 & \\
\hline C4 & $\mathrm{Y}$ & -0 & & -0 & -0.0 & 1198 & -0 & \\
\hline C4 & Z & & -0 & & & & & -0 \\
\hline C7 & $\mathrm{x}$ & & & & -0 & & -0 & \\
\hline C7 & $\mathrm{Y}$ & -0 & -0 & -0. & -0.00724 & 2054 & 0395 & \\
\hline C7 & Z & & & & & & & -0 \\
\hline N2 & $\mathrm{x}$ & & -0 . & -0 . & -0 & -0 & & \\
\hline N2 & $\mathrm{Y}$ & 919 & & & 0867 & 0.02385 & 2468 & -0 \\
\hline N2 & Z & -0.0 & -0.0 & -0.0 & -0.0 & -0.07509 & & \\
\hline $\mathrm{O} 2$ & $\mathrm{x}$ & -0 . & -0 . & & -0 & -0 & -0 & -0 \\
\hline 02 & Y & -0.0 & -0.0 & & & -0.0 & 0133 & -0 \\
\hline 02 & Z & -0.0 & & & & & 0004 & -0 \\
\hline 03 & $\mathrm{x}$ & & -0 & & -0 & & & \\
\hline 03 & Y & -0 . & -0 . & -0 & -0 & 842 & -0 & \\
\hline 03 & Z & -0 & -0. & -0. & -0.08785 & -0. & 0971 & -0 \\
\hline $\mathrm{C} 1$ & $\mathrm{x}$ & -0 & & & & & & \\
\hline $\mathrm{C} 1$ & $\mathrm{Y}$ & & & & & & & \\
\hline $\mathrm{C} 1$ & z & 808 & 0.0 & 0 . & 0 . & 7679 & 2313 & 011 \\
\hline & $\mathrm{x}$ & & -0 & -0 . & & & & \\
\hline & $\mathrm{Y}$ & & -0 & & & & -0 & \\
\hline C10 & Z & -0 & -0 & & -0.02526 & 0 & 0513 & 371 \\
\hline 04 & $\mathrm{x}$ & & -0 . & & & & & \\
\hline 04 & Y & & -0 . & -0 . & -0 . & -0 . & 049 & \\
\hline 04 & Z & & -0. & -0.0 & -0.00 & -0.03972 & -0.00354 & -0 \\
\hline $\mathrm{H} 2$ & $\mathrm{x}$ & -0. & & & -0.16 & & & \\
\hline H2 & $\mathrm{Y}$ & & & & & -0 & -0 & \\
\hline H2 & Z & & & & & -0 & -0 & -0 \\
\hline 05 & $\mathrm{x}$ & & -0 & & -0.0 & -0.0 & -0 & \\
\hline 05 & $\mathrm{Y}$ & & & & -0.0 & & & \\
\hline 05 & Z & -0. & & & & & 0163 & -0 \\
\hline $\mathrm{H} 1$ & $\mathrm{x}$ & -0. & -0.2 & -0.2 & 0.1 & & -0.32733 & \\
\hline $\mathrm{H} 1$ & $\mathrm{Y}$ & & & & -0.08128 & -0.10295 & 0.16141 & \\
\hline H1 & Z & -0.09878 & -0.30676 & -0.25540 & & & -0.36154 & \\
\hline H14 & $\mathrm{X}$ & 0.03367 & -0.12374 & -0.0 & 0.04938 & 0.04668 & 0.04014 & , \\
\hline
\end{tabular}




\begin{tabular}{|c|c|c|c|c|c|c|c|c|}
\hline $\mathrm{H} 14$ & $\mathrm{Y}$ & -0.04107 & -0.14698 & -0.10971 & 0.02470 & -0.00479 & 0.07585 & 0.01692 \\
\hline H14 & $\mathrm{Z}$ & 0.04108 & 0.47895 & 0.38823 & -0.12980 & -0.02220 & -0.30262 & -0.07189 \\
\hline H15 & $\mathrm{x}$ & 0.05503 & -0.42733 & 0.49398 & -0.10713 & -0.04663 & 0.29289 & -0.15770 \\
\hline H15 & $\mathrm{Y}$ & -0.04634 & 0.05287 & -0.06950 & -0.01334 & -0.01743 & -0.03356 & 0.01919 \\
\hline H15 & Z & 0.03030 & 0.02188 & -0.00893 & 0.01809 & 0.02922 & 0.01912 & -0.03520 \\
\hline H19 & $\mathrm{x}$ & -0.10229 & 0.22884 & -0.25785 & 0.08657 & 0.09296 & 0.28854 & -0.14568 \\
\hline H19 & $\mathrm{Y}$ & 0.02891 & -0.13894 & 0.17730 & -0.08492 & -0.07983 & -0.17090 & \\
\hline H19 & $\mathrm{z}$ & -0.07247 & 0.23590 & -0.29790 & 0.15055 & 0.19501 & 0.37062 & -0.22819 \\
\hline H2O & $\mathrm{x}$ & -0.20356 & 0.00630 & 0.09515 & 0.11080 & 0.04470 & -0.00855 & \\
\hline H2O & $\mathrm{Y}$ & -0.25417 & 0.00731 & 0.12033 & 0.15537 & 0.04374 & & \\
\hline $\mathrm{H} 20$ & z & -0.50104 & 0.01545 & 0.23387 & 0.30300 & 0.06648 & -0.02995 & -0.06080 \\
\hline \multicolumn{2}{|c|}{ frequencies } & 1486.44 & 1507.97 & 1547.56 & & 3234.43 & & \\
\hline \multicolumn{2}{|c|}{ intensities } & 191.14 & 5.99 & 371.13 & 577.21 & 7.99 & & \\
\hline $\operatorname{Re} 1$ & $\mathrm{x}$ & 0.00047 & -0.00084 & -0.00070 & -0.00010 & -0.00003 & -0.00007 & -0. \\
\hline $\operatorname{Re} 1$ & $\mathrm{Y}$ & -0.0 & -0.00007 & 0.00048 & 0.00015 & -0.00003 & -0 . & \\
\hline $\operatorname{Re} 1$ & Z & 0.00146 & 0.00069 & -0.00044 & 0.00031 & -0.00004 & 0.00000 & -0.00004 \\
\hline N1 & $\mathrm{x}$ & -0.0 & 7881 & -0.025 & 0.01504 & 0017 & & \\
\hline N1 & $\mathrm{Y}$ & 0.0 & -0.0 & -0.00944 & -0.00055 & & -0 . & \\
\hline N1 & Z & -0.03526 & 0.03764 & 0.02482 & 0.00048 & -0.00049 & 0.00103 & -0.00150 \\
\hline C4 & $\mathrm{x}$ & -0.0 & -0.1 & 0.1 & -0.04953 & 0.00326 & -0 . & \\
\hline C4 & $\mathrm{Y}$ & -0. & 0.0 & & & -0 . & & \\
\hline C4 & Z & 893 & -0.05693 & -0.06 & 0.01480 & 0.03304 & -0.04419 & \\
\hline C7 & $\mathrm{x}$ & 181 & 0.02657 & -0.0 & 0.02723 & -0.03932 & & \\
\hline C7 & $\mathrm{Y}$ & & -0.0 & -0 & & & -0 & \\
\hline C7 & Z & -0.0 & 0.13863 & 0 & -0.03994 & -0.01166 & 1218 & \\
\hline N2 & $\mathrm{x}$ & -0.0 & -0.01968 & 0. & -0.00500 & 0.00093 & -0. & -0 \\
\hline N2 & Y & & & -0 . & -0 . & -0 & & \\
\hline N2 & Z & -0 & -0.0 & -0.0 & 15 & 24 & 003 & -0 \\
\hline 02 & $\mathrm{x}$ & & 0.0 & & & & & \\
\hline 02 & $\mathrm{Y}$ & & 0. & & & & & \\
\hline $\mathrm{O} 2$ & Z & 50 & -0.0 & 0 . & 06 & -0 & -0 & \\
\hline 03 & $\mathrm{x}$ & -0 & -0.0 & & & & & \\
\hline 03 & Y & -0 . & -0 & & -0 . & & -0 & \\
\hline 03 & Z & -0.04876 & -0.0 & 0.0 & -0.01011 & -0.00035 & -0 & \\
\hline $\mathrm{C} 1$ & $\mathrm{x}$ & & 0.0 & & -0.04048 & & & \\
\hline $\mathrm{C} 1$ & Y & & 0. & & & & & \\
\hline $\mathrm{C} 1$ & Z & 66 & 0. & -0 & & & 135 & \\
\hline C10 & $\mathrm{x}$ & -0. & -0.0 & -0.0 & 0.0 & -0.0 & & -0 \\
\hline & $\mathrm{Y}$ & -0 . & -0. & -0 . & -0 . & -0 & -0 & \\
\hline C10 & Z & & & -0 . & -0.2 & & & \\
\hline 04 & $\mathrm{x}$ & 0.0 & 0.0 & & -0.01038 & 046 & & \\
\hline 04 & $\mathrm{Y}$ & & & & & & & \\
\hline 04 & z & -0 & -0 & & & -0. & -0 . & -0 \\
\hline H2 & $\mathrm{x}$ & 3964 & 0.0 & 0. & 0. & -0.64683 & -0 . & -0 . \\
\hline H2 & $\mathrm{Y}$ & & & & & & & \\
\hline H2 & Z & & & -0 . & -0 . & -0 . & -0 & -0 \\
\hline 05 & $\mathrm{x}$ & -0 & 0.0 & & 01 & -0.0 & 002 & \\
\hline 05 & Y & & & & & & & \\
\hline 05 & Z & & -0 & & -0 . & & & \\
\hline $\mathrm{H} 1$ & $\mathrm{x}$ & 229 & 0.0 & $-0.0 s$ & 2692 & -0.00695 & 705 & \\
\hline $\mathrm{H} 1$ & $\mathrm{Y}$ & -0.0 & -0.0 & & -0.0 & & & \\
\hline H1 & Z & & & -0.1 & & & & \\
\hline H14 & $\mathrm{x}$ & -0.0 & & & -0.0 & 0.4 & -0.4 & -0 \\
\hline & $\mathrm{Y}$ & -0.0 & 0.0 & & -0.01 & -0.075 & & \\
\hline H1 4 & Z & & -0.29523 & & & & -0.1 & \\
\hline H15 & $\mathrm{x}$ & & 0.33100 & -0.2 & & -0.0 & 3837 & -0 \\
\hline & $\mathrm{Y}$ & -0.0 & 0.0 & 0.0 & -0.00 & 51 & -0.1 & \\
\hline H15 & Z & & -0.10150 & -0.04218 & & -0.38152 & 0.50572 & \\
\hline H19 & $\mathrm{x}$ & & -0.00801 & -0.13775 & & -0.00391 & & \\
\hline H19 & Y & -0.03663 & 0.04054 & 0.0 & -0.01380 & -0.00153 & 0.00282 & -0 \\
\hline
\end{tabular}




\begin{tabular}{|c|c|c|c|c|}
\hline H19 & Z & 0.10362 & -0.10289 & -0.13000 \\
\hline $\mathrm{H} 20$ & $\mathrm{X}$ & -0.10357 & -0.00904 & -0.03179 \\
\hline H2O & Y & -0.15129 & -0.01342 & -0.03948 \\
\hline $\mathrm{H} 2 \mathrm{O}$ & Z & -0.30539 & -0.02918 & -0.06255 \\
\hline \multicolumn{2}{|c|}{ frequencies } & 3568.00 & 3571.79 & 3754.29 \\
\hline \multicolumn{2}{|c|}{ intensities } & 121.70 & 54.14 & 307.10 \\
\hline Re1 & $\mathrm{X}$ & 0.00000 & 0.00003 & 0.00001 \\
\hline $\operatorname{Re} 1$ & Y & -0.00001 & 0.00001 & -0.00001 \\
\hline Re1 & Z & -0.00004 & 0.00001 & 0.00002 \\
\hline N1 & $\mathrm{x}$ & -0.06022 & 0.00842 & -0.00017 \\
\hline $\mathrm{N} 1$ & Y & -0.01344 & 0.00196 & 0.00015 \\
\hline N1 & Z & 0.03351 & -0.00519 & -0.00019 \\
\hline $\mathrm{C} 4$ & $\mathrm{X}$ & 0.00167 & 0.00101 & 0.00058 \\
\hline $\mathrm{C} 4$ & Y & -0.00091 & 0.00025 & -0.00008 \\
\hline $\mathrm{C} 4$ & Z & 0.00293 & -0.00105 & -0.00014 \\
\hline $\mathrm{C} 7$ & $\mathrm{X}$ & 0.00066 & -0.00280 & -0.00026 \\
\hline $\mathrm{C} 7$ & Y & 0.00013 & 0.00052 & -0.00012 \\
\hline $\mathrm{C} 7$ & Z & -0.00130 & -0.00183 & 0.00050 \\
\hline N2 & $\mathrm{X}$ & -0.00647 & -0.04844 & 0.00008 \\
\hline N2 & Y & -0.00025 & -0.00129 & 0.00008 \\
\hline N2 & Z & 0.00731 & 0.05080 & -0.00056 \\
\hline $\mathrm{O} 2$ & $\mathrm{X}$ & 0.00007 & -0.00029 & 0.00003 \\
\hline 02 & Y & -0.00008 & -0.00002 & 0.00009 \\
\hline $\mathrm{O} 2$ & Z & -0.00004 & -0.00009 & 0.00026 \\
\hline 03 & $\mathrm{X}$ & 0.00015 & -0.00001 & -0.00011 \\
\hline 03 & Y & 0.00004 & 0.00020 & -0.00011 \\
\hline 03 & Z & 0.00037 & -0.00010 & -0.00004 \\
\hline $\mathrm{C} 1$ & $\mathrm{X}$ & 0.00009 & -0.00014 & -0.00110 \\
\hline $\mathrm{C} 1$ & Y & -0.00010 & -0.00005 & -0.00003 \\
\hline $\mathrm{C} 1$ & Z & -0.00037 & 0.00030 & 0.00107 \\
\hline $\mathrm{C} 10$ & $\mathrm{X}$ & -0.00027 & 0.00046 & 0.00222 \\
\hline $\mathrm{C} 10$ & Y & 0.00001 & 0.00006 & 0.00084 \\
\hline $\mathrm{C} 10$ & Z & 0.00022 & -0.00032 & -0.00097 \\
\hline O4 & $\mathrm{X}$ & 0.00001 & 0.00030 & 0.04867 \\
\hline 04 & Y & -0.00001 & 0.00014 & 0.01605 \\
\hline O4 & Z & -0.00016 & -0.00001 & -0.03147 \\
\hline $\mathrm{H} 2$ & $\mathrm{X}$ & 0.00110 & -0.00052 & 0.00141 \\
\hline $\mathrm{H} 2$ & Y & 0.00038 & -0.00050 & 0.00085 \\
\hline $\mathrm{H} 2$ & Z & 0.00006 & 0.00018 & 0.00033 \\
\hline 05 & $\mathrm{X}$ & 0.00002 & -0.00003 & -0.00001 \\
\hline 05 & Y & 0.00028 & -0.00041 & 0.00018 \\
\hline 05 & Z & 0.00013 & -0.00008 & -0.00005 \\
\hline $\mathrm{H} 1$ & $\mathrm{X}$ & 0.09552 & 0.67488 & -0.00356 \\
\hline $\mathrm{H} 1$ & Y & 0.00185 & 0.01130 & 0.00017 \\
\hline $\mathrm{H} 1$ & Z & -0.09382 & -0.66974 & 0.00403 \\
\hline $\mathrm{H} 14$ & $X$ & 0.00147 & 0.01195 & 0.00078 \\
\hline H14 & Y & -0.00039 & -0.00108 & 0.00010 \\
\hline $\mathrm{H} 14$ & Z & 0.00077 & 0.00296 & 0.00032 \\
\hline H15 & $\mathrm{X}$ & -0.00063 & 0.00024 & 0.00004 \\
\hline H15 & Y & 0.00366 & 0.00058 & -0.00038 \\
\hline H15 & Z & -0.01373 & 0.00113 & 0.00110 \\
\hline H19 & $\mathrm{X}$ & 0.80017 & -0.11280 & -0.00032 \\
\hline H19 & Y & 0.19313 & -0.02735 & -0.00013 \\
\hline H19 & Z & -0.47596 & 0.06611 & -0.00044 \\
\hline H2O & $\mathrm{X}$ & -0.00056 & -0.00451 & -0.78780 \\
\hline $\mathrm{H} 2 \mathrm{O}$ & Y & -0.00006 & -0.00143 & -0.26581 \\
\hline $\mathrm{H} 2 \mathrm{O}$ & Z & 0.00063 & 0.00264 & 0.4933 \\
\hline
\end{tabular}

$\begin{array}{llll}0.01172 & 0.00399 & -0.00679 & 0.00774\end{array}$

$0.04884-0.00110-0.00088-0.00024$

$\begin{array}{llll}0.13355 & -0.00095 & -0.00083 & 0.00084\end{array}$ 


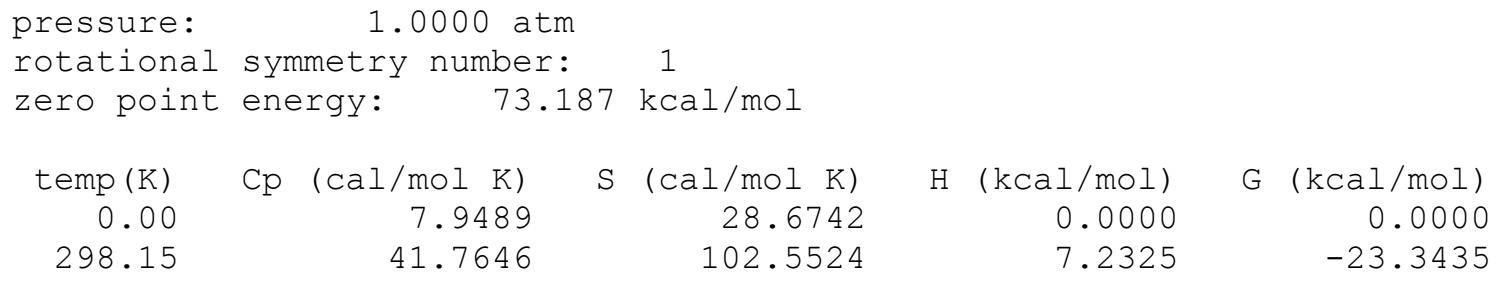

end of program freq

start of program ch

Moments from quantum mechanical wavefunction:

Dipole Moments (Debye)
$\mathrm{X}=$
$-2.0627$
$\mathrm{Y}=$
5.0841
$\mathrm{Z}=$
0.5804 Tot $=$
5.5172

Final energy ........... -645.35238780547

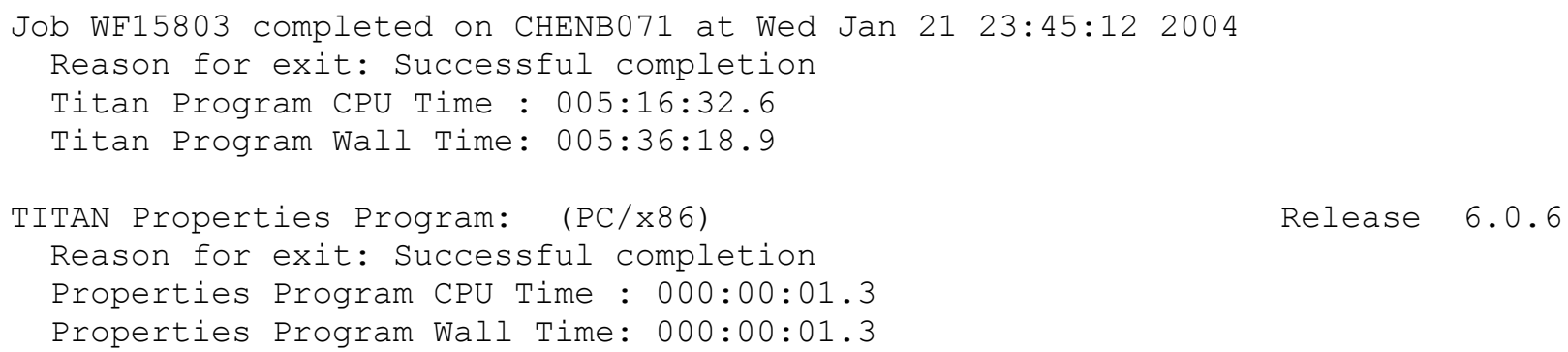




\author{
Spartan '02 \\ build 116 (May 14 2002)
}

Wavefunction Developers:

B.J. Deppmeier, A.J. Driessen, T.S. Hehre, W.J. Hehre,

J.A. Johnson, P.E. Klunzinger, J.M. Leonard, I.N. Pham

W.J. Pietro, Jianguo Yu

Q-Chem Developers:

J. Kong, C.A. White, A.I. Krylov, C.D. Sherrill,

R.D. Adamson, T.R. Furlani, M.S. Lee, A.M. Lee,

S.R. Gwaltney, T.R. Adams, C. Ochsenfeld, A.T.B. Gilbert,

G.S. Kedziora, V.A. Rassolov, D. R. Maurice, N. Nair,

Y. Shao, N.A. Besley, P.E. Maslen, J.P. Dombroski,

H. Dachsel, W.M. Zhang, P.P. Korambath, J. Baker,

E.F. C. Byrd, T. Van Voorhis, M. Oumi, S. Hirata,

C.P. Hsu, N. Ishikawa, J. Florian, A. Warshel,

B.G. Johnson, P.M.W. Gill, M. Head-Gordon, J.A. Pople

$\begin{array}{lll}\text { Wavefunction Inc. } & \text { Sales: } & \text { sales@wavefun.com } \\ \text { Irvine CA } & \text { Support: } & \text { support@wavefun.com } \\ & \text { Web: } & \text { www.wavefun.com }\end{array}$

Copyright () $1995-2002$

Spartan '02 Mechanics Program: (PC/x86)

Release 116

Reason for exit: Successful completion

Mechanics CPU Time : 000:00:00.2

Mechanics Wall Time: 000:00:00.5

Spartan '02 Quantum Mechanics Program: (PC/x86) Release 116

Job type: Geometry optimization.

Method: RB3LYP

Basis set: LACVP**

Number of shells: 112

Number of basis functions: 339

SCF model:

A restricted hybrid HF-DFT SCF calculation will be performed using Pulay DIIS extrapolation

Optimization:

$\begin{array}{rc}\text { Step } & \text { Energy } \\ 1 & -878.6756006 \\ 2 & -878.6737382 \\ 3 & -878.6730976 \\ 4 & -878.6868975 \\ 5 & -878.6867401 \\ 6 & -878.6903969 \\ 7 & -878.6910311 \\ 8 & -878.6917098 \\ 9 & -878.6924761 \\ 10 & -878.6928279 \\ 11 & -878.6934197 \\ 12 & -878.6938138\end{array}$

$\begin{array}{rr}\text { Max Grad. } & \text { Max Dist. } \\ 0.031130 & 0.148997 \\ 0.031199 & 0.124695 \\ 0.032532 & 0.120532 \\ 0.011282 & 0.178914 \\ 0.011538 & 0.164122 \\ 0.005454 & 0.190284 \\ 0.002511 & 0.073613 \\ 0.003394 & 0.139180 \\ 0.002393 & 0.143421 \\ 0.001850 & 0.146256 \\ 0.001497 & 0.149802 \\ 0.001215 & 0.158891\end{array}$




$\begin{array}{llll}13 & -878.6943419 & 0.001233 & 0.163421 \\ 14 & -878.6944558 & 0.000939 & 0.066420 \\ 15 & -878.6944519 & 0.000453 & 0.017664 \\ 16 & -878.6945171 & 0.000322 & 0.025807 \\ 17 & -878.6945190 & 0.000389 & 0.022841 \\ 18 & -878.6944744 & 0.000412 & 0.008346 \\ 19 & -878.6944700 & 0.000155 & 0.001411 \\ 20 & -878.6946553 & 0.000064 & 0.000833\end{array}$

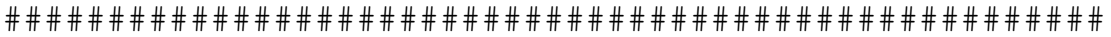 \\ \# Entering optman.exe on Thu May 15 07:39:33 2003 \#

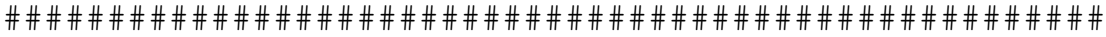

\author{
Cartesian Hessian Update \\ Hessian Updated using BFGS Update
}

** GEOMETRY OPTIMIZATION IN DELOCALIZED INTERNAL COORDiNATES ** Searching for a Minimum

Optimization Cycle: 20

\begin{tabular}{|c|c|c|c|c|}
\hline & & Coor & nates & roms ) \\
\hline & $\mathrm{OM}$ & X & Y & Z \\
\hline 1 & $\mathrm{Re}$ & 1.261780 & -0.065892 & -0.830529 \\
\hline 2 & $\mathrm{~N}$ & -0.223687 & 1.324962 & -0.271093 \\
\hline 3 & $\mathrm{C}$ & -2.302936 & 3.070405 & 0.224663 \\
\hline 4 & C & 0.012195 & 2.650267 & -0.217754 \\
\hline 5 & $\mathrm{C}$ & -1.476038 & 0.828933 & -0.052780 \\
\hline 6 & $\mathrm{C}$ & -2.537178 & 1.693565 & 0.197104 \\
\hline 7 & C & -1.014216 & 3.558278 & 0.018231 \\
\hline 8 & $\mathrm{H}$ & 1.046793 & 2.944247 & -0.355463 \\
\hline 9 & $\mathrm{H}$ & -3.535076 & 1.309529 & 0.367716 \\
\hline 10 & $\mathrm{H}$ & -0.798889 & 4.619970 & 0.044074 \\
\hline 11 & $\mathrm{H}$ & -3.124977 & 3.752837 & 0.414049 \\
\hline 12 & $\mathrm{C}$ & -1.576415 & -0.647649 & -0.083977 \\
\hline 13 & $\mathrm{C}$ & -1.489286 & -3.411785 & -0.128192 \\
\hline 14 & $\mathrm{C}$ & -2.747017 & -1.368783 & 0.133100 \\
\hline 15 & $\mathrm{~N}$ & -0.402562 & -1.296370 & -0.324391 \\
\hline 16 & $\mathrm{C}$ & -0.348312 & -2.640589 & -0.324984 \\
\hline 17 & $\mathrm{C}$ & -2.701722 & -2.764382 & 0.100339 \\
\hline 18 & $\mathrm{H}$ & -3.683676 & -0.860563 & 0.325771 \\
\hline 19 & $\mathrm{H}$ & 0.637160 & -3.067466 & -0.472221 \\
\hline 20 & $\mathrm{H}$ & -3.608900 & -3.337304 & 0.262114 \\
\hline 21 & $\mathrm{H}$ & -1.418913 & -4.492989 & -0.147729 \\
\hline 22 & 0 & 1.400028 & -0.071134 & -2.506468 \\
\hline 23 & 0 & 2.306987 & -1.375522 & 0.086576 \\
\hline 24 & 0 & 2.543003 & 1.113781 & -0.029438 \\
\hline 25 & $\mathrm{C}$ & 3.527287 & -0.929699 & 0.725314 \\
\hline 26 & $\mathrm{H}$ & 3.363822 & -0.968929 & 1.808530 \\
\hline 27 & $\mathrm{C}$ & 3.816880 & 0.487336 & 0.246264 \\
\hline 28 & $\mathrm{H}$ & 4.409997 & 0.490257 & -0.675649 \\
\hline 29 & $\mathrm{H}$ & 4.327369 & -1.629326 & 0.465068 \\
\hline 30 & $\mathrm{H}$ & 4.336497 & 1.084013 & 1.001754 \\
\hline
\end{tabular}

Energy is $\quad-878.694655276$

Hessian Updated using BFGS Update 
internal optimization with constraints (0)

84 Hessian modes will be used to form the next step Hessian Eigenvalues:

$\begin{array}{llllll}0.002417 & 0.003333 & 0.009889 & 0.011621 & 0.015630 & 0.021585 \\ 0.022090 & 0.024192 & 0.024616 & 0.024896 & 0.026263 & 0.027119 \\ 0.027376 & 0.028842 & 0.028914 & 0.029291 & 0.029648 & 0.032978 \\ 0.033574 & 0.036176 & 0.037424 & 0.038640 & 0.041659 & 0.043850 \\ 0.046552 & 0.048522 & 0.053269 & 0.058298 & 0.075780 & 0.087985 \\ 0.094644 & 0.102043 & 0.110440 & 0.125574 & 0.136490 & 0.137120 \\ 0.137499 & 0.138022 & 0.140226 & 0.142414 & 0.144426 & 0.149444 \\ 0.153455 & 0.155297 & 0.157554 & 0.161347 & 0.180460 & 0.195370 \\ 0.210290 & 0.213282 & 0.218447 & 0.223221 & 0.229024 & 0.230494 \\ 0.253432 & 0.266774 & 0.269422 & 0.270756 & 0.288366 & 0.294285 \\ 0.296029 & 0.300359 & 0.302327 & 0.303260 & 0.305332 & 0.310290 \\ 0.312800 & 0.313277 & 0.314349 & 0.318368 & 0.319619 & 0.322800 \\ 0.326532 & 0.332053 & 0.348231 & 0.348376 & 0.361368 & 0.368110 \\ 0.370788 & 0.375041 & 0.390715 & 0.422149 & 0.465676 & 0.799696\end{array}$

Minimum Search - Taking Simple RFO Step

Searching for Lamda that Minimizes Along All modes

Value Taken Lamda $=-0.00000011$

Step Taken. Stepsize is 0.002551

$\begin{array}{lrrr} & \text { Maximum } & \text { Tolerance } & \text { Cnvgd? } \\ \text { Gradient } & 0.000064 & 0.000300 & \text { YES } \\ \text { Displacement } & 0.000833 & 0.001200 & \text { YES } \\ \text { Energy change } & -0.000185 & 0.000001 & \text { NO }\end{array}$

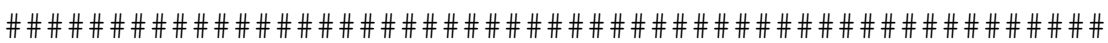

\# Entering anlman.exe on Thu May 15 07:39:34 2003 \#

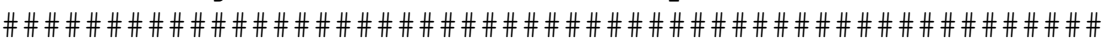

Analysis of SCF Wavefunction

Cartesian Multipole Moments

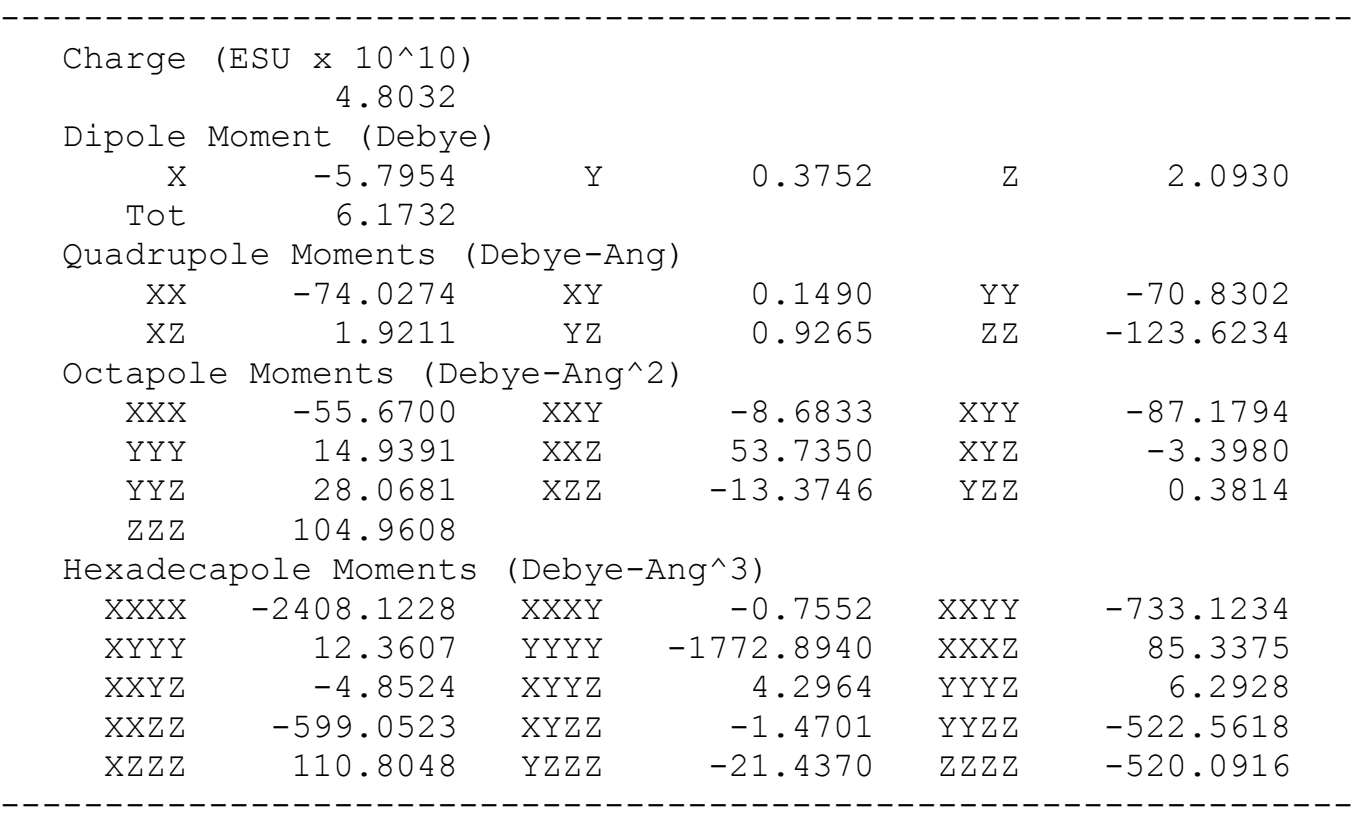


Reason for exit: Sucessful completion

Quantum Mechanics Program CPU Time : 012:05:26.5

Quantum Mechanics Program Wall Time: 023:38:59.6

Spartan '02 Properties Program: (PC/x86)

Release 116

Reason for exit: Successful completion

Properties Program CPU Time : 000:00:08.7

Properties Program Wall Time: 000:01:45.0

Spartan '02 Quantum Mechanics Program: (PC/x86)

Release 116

Job type: Frequency calculation.

Method: RB3LYP

Basis set: LACVP**

Number of shells: 112

Number of basis functions: 339

SCF model:

A restricted hybrid HF-DFT SCF calculation will be performed using Pulay DIIS extrapolation

$\begin{array}{llll}\text { SCF total energy: } & -878.6944617 & \text { hartrees } \\ \text { SCF total energy: } & -878.6944590 \text { hartrees } \\ \text { SCF total energy: } & -878.6944528 \text { hartrees } \\ \text { SCF total energy: } & -878.6945120 \text { hartrees } \\ \text { SCF total energy: } & -878.6945321 \text { hartrees } \\ \text { SCF total energy: } & -878.6944636 \text { hartrees } \\ \text { SCF total energy: } & -878.6944570 \text { hartrees } \\ \text { SCF total energy: } & -878.6944844 \text { hartrees } \\ \text { SCF total energy: } & -878.6944788 \text { hartrees } \\ \text { SCF total energy: } & -878.6944836 \text { hartrees } \\ \text { SCF total energy: } & -878.6944749 \text { hartrees } \\ \text { SCF total energy: } & -878.6944769 \text { hartrees } \\ \text { SCF total energy: } & -878.6944814 \text { hartrees } \\ \text { SCF total energy: } & -878.6944724 \text { hartrees } \\ \text { SCF total energy: } & -878.6944752 \text { hartrees } \\ \text { SCF total energy: } & -878.6944810 \text { hartrees } \\ \text { SCF total energy: } & -878.6944783 \text { hartrees } \\ \text { SCF total energy: } & -878.6944747 \text { hartrees } \\ \text { SCF total energy: } & -878.6944822 \text { hartrees } \\ \text { SCF total energy: } & -878.6944731 \text { hartrees } \\ \text { SCF total energy: } & -878.6944796 \text { hartrees } \\ \text { SCF total energy: } & -878.6944732 \text { hartrees } \\ \text { SCF total energy: } & -878.6944742 \text { hartrees } \\ \text { SCF total energy: } & -878.6944761 \text { hartrees } \\ \text { SCF total energy: } & -878.6944776 \text { hartrees } \\ \text { SCF total energy: } & -878.6944804 \text { hartrees } \\ \text { SCF total energy: } & -878.6944742 \text { hartrees } \\ \text { SCF total energy: } & -878.6944751 \text { hartrees } \\ \text { SCF total energy: } & -878.6944820 \text { hartrees } \\ \text { SCF total energy: } & -878.6944755 \text { hartrees } \\ \text { SCF total energy: } & -878.6944789 \text { hartrees } \\ \text { SCF total energy: } & -878.6944793 \text { hartrees } \\ \text { SCF total energy: } & -878.6944746 \text { hartrees } \\ \text { SCF total energy: } & -878.6944788 \text { hartrees } \\ \text { SCF total energy: } & -878.6944778 \text { hartrees } \\ \text { SCF total energy: } & -878.6944762 \text { hartrees } \\ \text { SCF total energy: } & -878.6944748 \text { hartrees } \\ \text { SCF total energy: } & -878.6944792 \text { hartrees } \\ & -8749 & \end{array}$


-878.6944723 hartrees -878.6944755 hartrees -878.6944853 hartrees -878.6944790 hartrees -878.6944802 hartrees -878.6944785 hartrees -878.6944800 hartrees -878.6944782 hartrees -878.6944774 hartrees -878.6944804 hartrees -878.6944751 hartrees -878.6944783 hartrees -878.6944777 hartrees -878.6944776 hartrees -878.6944851 hartrees -878.6944781 hartrees -878.6944797 hartrees -878.6944818 hartrees -878.6944780 hartrees -878.6944781 hartrees -878.6944797 hartrees -878.6944768 hartrees -878.6944778 hartrees -878.6944730 hartrees -878.6944833 hartrees -878.6944787 hartrees -878.6944788 hartrees -878.6944826 hartrees -878.6944805 hartrees -878.6944808 hartrees -878.6944706 hartrees -878.6944794 hartrees -878.6944746 hartrees -878.6944821 hartrees -878.6944790 hartrees -878.6944804 hartrees -878.6944723 hartrees -878.6944818 hartrees -878.6944727 hartrees -878.6944802 hartrees -878.6944786 hartrees -878.6944809 hartrees -878.6944764 hartrees -878.6944788 hartrees -878.6944733 hartrees -878.6944818 hartrees -878.6944789 hartrees -878.6944540 hartrees -878.6944638 hartrees -878.6944767 hartrees -878.6944651 hartrees -878.6944555 hartrees -878.6944616 hartrees -878.6944761 hartrees -878.6944791 hartrees -878.6944835 hartrees -878.6944779 hartrees -878.6944784 hartrees -878.6944773 hartrees -878.6944788 hartrees -878.6944763 hartrees 
-878.6944773 hartrees -878.6944728 hartrees -878.6944809 hartrees -878.6944767 hartrees -878.6944747 hartrees -878.6944793 hartrees -878.6944750 hartrees -878.6944737 hartrees -878.6944822 hartrees -878.6944777 hartrees -878.6944800 hartrees -878.6944753 hartrees -878.6944783 hartrees -878.6944802 hartrees -878.6944764 hartrees -878.6944735 hartrees -878.6944764 hartrees -878.6944833 hartrees -878.6944700 hartrees -878.6944831 hartrees -878.6944755 hartrees -878.6944786 hartrees -878.6944810 hartrees -878.6944805 hartrees -878.6944771 hartrees -878.6944793 hartrees -878.6944779 hartrees -878.6944806 hartrees -878.6944836 hartrees -878.6944902 hartrees -878.6944779 hartrees -878.6944739 hartrees -878.6944737 hartrees -878.6944778 hartrees -878.6944795 hartrees -878.6944898 hartrees -878.6944746 hartrees -878.6945294 hartrees -878.6944819 hartrees -878.6944763 hartrees -878.6945200 hartrees -878.6945322 hartrees -878.6944811 hartrees -878.6944752 hartrees -878.6944783 hartrees -878.6944825 hartrees -878.6944802 hartrees -878.6944810 hartrees -878.6944765 hartrees -878.6944738 hartrees -878.6944800 hartrees -878.6944711 hartrees -878.6944809 hartrees -878.6944777 hartrees -878.6944816 hartrees -878.6944852 hartrees -878.6944773 hartrees -878.6944787 hartrees -878.6944763 hartrees -878.6944782 hartrees -878.6944824 hartrees 


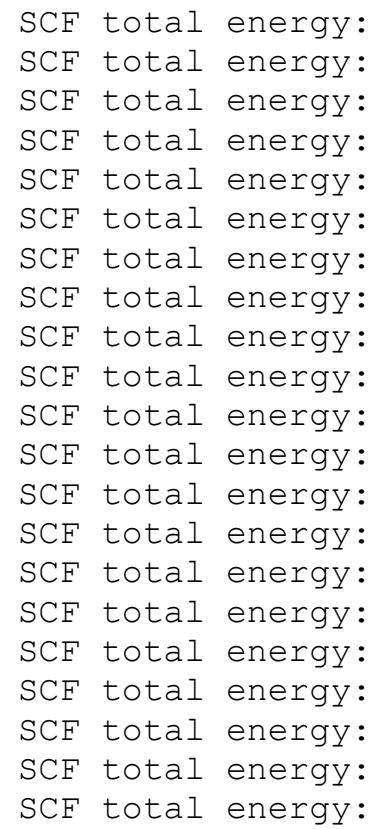

\begin{abstract}
-878.6944803 hartrees -878.6944807 hartrees -878.6944756 hartrees -878.6944869 hartrees -878.6944770 hartrees -878.6944788 hartrees -878.6944815 hartrees -878.6944688 hartrees -878.6944846 hartrees -878.6944744 hartrees -878.6944749 hartrees -878.6944787 hartrees -878.6944757 hartrees -878.6944871 hartrees -878.6944721 hartrees -878.6944795 hartrees -878.6944789 hartrees -878.6944833 hartrees -878.6944773 hartrees -878.6944811 hartrees -878.6944761 hartrees
\end{abstract}

Reason for exit: Sucessful completion

Quantum Mechanics Program CPU Time : 079:57:04.6

Quantum Mechanics Program Wall Time: 081:16:33.5

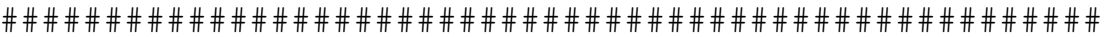 \# Entering vibman.exe on Wed Jan 14 04:35:26 2004 \#

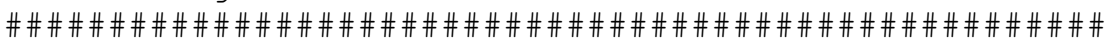

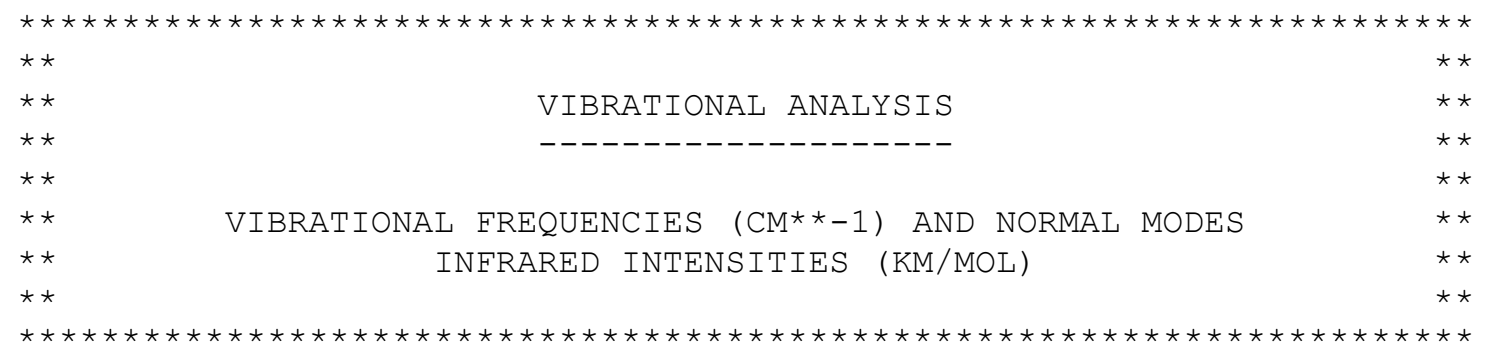

\begin{tabular}{|c|c|c|c|c|c|c|c|c|c|}
\hline \multicolumn{2}{|c|}{ Frequency: } & \multicolumn{2}{|l|}{50.63} & \multicolumn{3}{|c|}{56.99} & \multicolumn{3}{|c|}{85.67} \\
\hline IR AC & tive: & YES & & & YES & & & YES & \\
\hline IR Ir & tens: & 0.593 & & & 0.302 & & & 5.217 & \\
\hline Ramar & Active: & YES & & & YES & & & YES & \\
\hline & X & $\mathrm{Y}$ & Z & $\mathrm{X}$ & $\mathrm{Y}$ & Z & $\mathrm{X}$ & $\mathrm{Y}$ & Z \\
\hline $\mathrm{Re}$ & 0.003 & -0.018 & -0.026 & 0.005 & 0.015 & -0.027 & 0.000 & 0.002 & -0.054 \\
\hline $\mathrm{N}$ & 0.001 & -0.006 & -0.074 & -0.012 & 0.005 & -0.044 & 0.006 & -0.006 & -0.020 \\
\hline C & 0.066 & -0.002 & 0.190 & -0.016 & -0.005 & -0.019 & 0.030 & -0.008 & 0.080 \\
\hline $\mathrm{C}$ & 0.007 & -0.005 & -0.096 & -0.035 & 0.011 & -0.128 & 0.043 & -0.017 & 0.152 \\
\hline C & 0.023 & -0.003 & 0.053 & 0.009 & -0.003 & 0.047 & -0.014 & -0.001 & -0.11 \\
\hline $\mathrm{C}$ & 0.060 & 0.000 & 0.200 & 0.011 & -0.010 & 0.075 & -0.010 & 0.000 & -0.10 \\
\hline $\mathrm{C}$ & 0.038 & -0.004 & 0.028 & -0.041 & 0.006 & -0.130 & 0.061 & -0.018 & 0.23 \\
\hline $\mathrm{H}$ & -0.008 & -0.006 & -0.197 & -0.048 & 0.025 & -0.184 & 0.058 & -0.030 & 0.22 \\
\hline $\mathrm{H}$ & 0.083 & 0.001 & 0.329 & 0.029 & -0.019 & 0.154 & -0.032 & 0.006 & -0.20 \\
\hline $\mathrm{H}$ & 0.043 & -0.005 & 0.010 & -0.061 & 0.011 & -0.207 & 0.096 & -0.027 & 0.39 \\
\hline $\mathrm{H}$ & 0.094 & -0.001 & 0.309 & -0.017 & -0.010 & -0.007 & 0.039 & -0.007 & 0.11 \\
\hline C & 0.006 & 0.000 & 0.022 & 0.024 & -0.003 & 0.071 & -0.013 & -0.001 & -0.11 \\
\hline $\mathrm{C}$ & -0.039 & 0.000 & -0.109 & 0.024 & -0.001 & -0.014 & 0.060 & 0.000 & 0.23 \\
\hline C & 0.001 & 0.007 & 0.024 & 0.061 & -0.011 & 0.227 & -0.003 & -0.001 & -0.0 \\
\hline & -0.010 & -0.006 & -0.035 & -0.005 & 0.007 & -0.090 & 0.001 & -0.001 & -0.0 \\
\hline
\end{tabular}




$\begin{array}{lrrr}\mathrm{C} & -0.032 & -0.008 & -0.097 \\ \mathrm{C} & -0.022 & 0.007 & -0.044 \\ \mathrm{H} & 0.013 & 0.012 & 0.067 \\ \mathrm{H} & -0.044 & -0.022 & -0.137 \\ \mathrm{H} & -0.027 & 0.013 & -0.048 \\ \mathrm{H} & -0.057 & -0.001 & -0.164 \\ \mathrm{O} & 0.075 & -0.157 & -0.019 \\ \mathrm{O} & -0.043 & 0.085 & 0.169 \\ \mathrm{O} & -0.027 & 0.069 & -0.101 \\ \mathrm{C} & -0.089 & 0.165 & 0.203 \\ \mathrm{H} & -0.182 & 0.322 & 0.195 \\ \mathrm{C} & -0.044 & 0.092 & 0.024 \\ \mathrm{H} & -0.008 & -0.045 & 0.046 \\ \mathrm{H} & -0.072 & 0.122 & 0.369 \\ \mathrm{H} & -0.062 & 0.183 & -0.038\end{array}$

\begin{tabular}{lrrr} 
Frequency: & \multicolumn{3}{c}{97.57} \\
IR Active: & \multicolumn{3}{c}{0.116} \\
IR Intens : & \multicolumn{3}{c}{ YES } \\
Raman Active: & \multicolumn{3}{c}{ Z } \\
& X & Y r \\
Re & -0.002 & -0.010 & -0.007 \\
N & -0.031 & 0.027 & -0.117 \\
C & 0.013 & 0.012 & 0.143 \\
C & -0.045 & 0.029 & -0.163 \\
C & -0.005 & 0.022 & 0.001 \\
C & 0.024 & 0.011 & 0.160 \\
C & -0.025 & 0.022 & -0.050 \\
H & -0.066 & 0.044 & -0.268 \\
H & 0.056 & 0.000 & 0.314 \\
H & -0.033 & 0.024 & -0.086 \\
H & 0.038 & 0.004 & 0.276 \\
C & -0.002 & 0.022 & -0.014 \\
C & 0.039 & 0.019 & 0.080 \\
C & -0.025 & 0.013 & -0.157 \\
N & 0.027 & 0.026 & 0.108 \\
C & 0.052 & 0.027 & 0.177 \\
C & -0.003 & 0.012 & -0.119 \\
H & -0.061 & 0.003 & -0.307 \\
H & 0.080 & 0.046 & 0.299 \\
H & -0.023 & 0.006 & -0.236 \\
H & 0.056 & 0.020 & 0.137 \\
O & 0.027 & -0.004 & -0.003 \\
O & 0.039 & -0.038 & -0.082 \\
O & -0.064 & -0.020 & 0.094 \\
C & 0.039 & -0.056 & -0.076 \\
H & 0.089 & -0.215 & -0.074 \\
C & -0.041 & 0.026 & 0.105 \\
H & 0.012 & 0.184 & 0.139 \\
H & 0.065 & 0.023 & -0.203 \\
H & -0.131 & -0.022 & 0.207 \\
& &
\end{tabular}

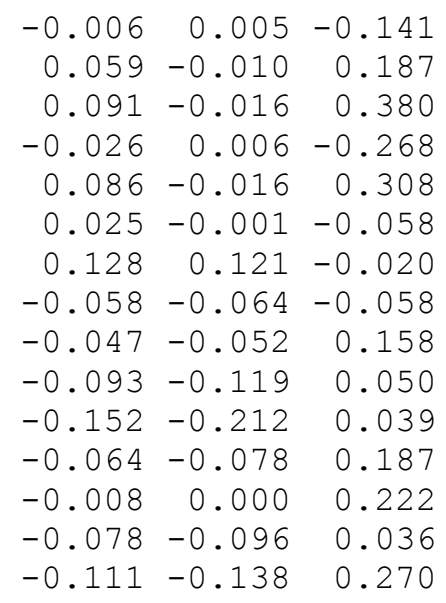

128.64
YES
0.320
YES

$0.003-0.024-0.003$

$\begin{array}{llll}0.010 & -0.025 & 0.008\end{array}$

$\begin{array}{llll}0.042 & 0.012 & -0.024\end{array}$

$\begin{array}{lll}0.045 & -0.032 & 0.042\end{array}$

$0.002-0.004-0.018$

$\begin{array}{llll}0.009 & 0.017 & -0.053\end{array}$

$0.063-0.013 \quad 0.039$

$\begin{array}{rrrr}0.057 & -0.058 & 0.074\end{array}$

$\begin{array}{rrrr}-0.008 & 0.040 & -0.097\end{array}$

$\begin{array}{llll}0.089 & -0.019 & 0.074\end{array}$

$0.050 \quad 0.028-0.046$

$-0.011-0.003-0.003$

$-0.095-0.001-0.020$

$\begin{array}{lll}-0.019 & 0.031 & 0.058\end{array}$

$-0.027-0.029-0.038$

$-0.078-0.033-0.058$

$\begin{array}{lll}-0.063 & 0.030 & 0.056\end{array}$

$\begin{array}{lll}0.009 & 0.062 & 0.110\end{array}$

$-0.101-0.072-0.104$

$\begin{array}{lll}-0.069 & 0.056 & 0.109\end{array}$

$-0.127-0.003-0.037$

$\begin{array}{llll}0.024 & -0.029 & 0.003\end{array}$

$\begin{array}{lll}0.095 & 0.039-0.028\end{array}$

$\begin{array}{rrr}-0.115 & 0.055 & 0.057\end{array}$

$0.126 \quad 0.079-0.112$

$0.268-0.147-0.100$

$\begin{array}{lll}-0.061 & 0.208 & 0.117\end{array}$

$\begin{array}{lll}0.043 & 0.451 & 0.183\end{array}$

$0.1690 .216-0.340$

$\begin{array}{llll}-0.243 & 0.180 & 0.269\end{array}$

$\begin{array}{rrr}0.037 & 0.003 & 0.126 \\ 0.036 & -0.002 & 0.122 \\ -0.017 & -0.001 & -0.128 \\ 0.050 & 0.013 & 0.182 \\ 0.049 & -0.004 & 0.184 \\ 0.092 & 0.001 & 0.391 \\ 0.044 & 0.018 & -0.051 \\ -0.045 & 0.000 & 0.002 \\ -0.037 & -0.005 & 0.023 \\ -0.111 & 0.007 & 0.124 \\ -0.228 & 0.034 & 0.109 \\ -0.060 & -0.006 & 0.123 \\ 0.008 & -0.027 & 0.166 \\ -0.083 & 0.001 & 0.227 \\ -0.111 & 0.003 & 0.152\end{array}$

$\begin{array}{ccc} & 144.86 \\ & \text { YES } \\ & 0.548 \\ & \text { YES } & \\ \mathrm{X} & \mathrm{Y} & \mathrm{Z}\end{array}$

$\begin{array}{llll}0.033 & 0.005 & 0.020\end{array}$

$\begin{array}{lll}-0.072 & 0.032 & -0.119\end{array}$

$\begin{array}{llll}-0.116 & -0.045 & 0.130\end{array}$

$\begin{array}{llll}-0.126 & 0.041 & -0.043\end{array}$

$\begin{array}{llll}-0.061 & 0.004 & -0.109\end{array}$

$-0.077-0.045-0.002$

$\begin{array}{llll}-0.137 & -0.004 & 0.099\end{array}$

$\begin{array}{llll}-0.146 & 0.097 & -0.070\end{array}$

$\begin{array}{lll}-0.055 & -0.090 & 0.013\end{array}$

$\begin{array}{lll}-0.156 & -0.002 & 0.191\end{array}$

$\begin{array}{lll}-0.117 & -0.081 & 0.253\end{array}$

$-0.057 \quad 0.013-0.111$

$\begin{array}{lll}-0.089 & 0.024 & 0.087\end{array}$

$\begin{array}{lll}-0.057 & 0.043-0.012\end{array}$

$\begin{array}{lll}-0.065 & 0.002-0.119\end{array}$

$-0.095-0.001-0.050$

$\begin{array}{llll}-0.073 & 0.044 & 0.115\end{array}$

$\begin{array}{llll}-0.039 & 0.068 & 0.002\end{array}$

$-0.115-0.036-0.078$

$\begin{array}{lll}-0.063 & 0.065 & 0.232\end{array}$

$\begin{array}{lll}-0.091 & 0.023 & 0.175\end{array}$

$0.278-0.058 \quad 0.041$

$\begin{array}{rrrr}-0.030 & 0.004 & 0.071\end{array}$

$0.051-0.002-0.004$

$0.091-0.046-0.122$

$0.276-0.060-0.098$

$0.066-0.048-0.156$

$-0.054-0.067-0.232$

$0.016-0.075-0.274$

$0.182-0.065-0.225$

$\begin{array}{rrr} & 185.98 \\ & \text { YES } \\ & 3.060 \\ \text { Y } & \text { YES } \\ -0.050 & 0.004 & 0.022 \\ 0.002 & -0.059 & -0.023 \\ 0.175 & 0.063 & -0.009 \\ 0.121 & -0.082 & -0.045\end{array}$

$$
\begin{array}{r}
192.17 \\
\text { YES } \\
1.010 \\
\text { YES }
\end{array}
$$$$
\begin{array}{lll}
X & Y
\end{array}
$$$$
0.014-0.007-0.055
$$$$
\begin{array}{llll}
-0.004 & -0.027 & 0.204
\end{array}
$$$$
\begin{array}{llll}
-0.094 & 0.007 & -0.133
\end{array}
$$$$
\begin{array}{lll}
-0.021 & -0.023 & 0.191
\end{array}
$$ 


$\begin{array}{rrr}0.012 & -0.064 & 0.025 \\ 0.029 & -0.058 & 0.068 \\ 0.014 & -0.065 & -0.085 \\ 0.009 & -0.078 & -0.074 \\ 0.036 & -0.047 & 0.124 \\ 0.008 & -0.063 & -0.158 \\ 0.038 & -0.054 & 0.024 \\ -0.019 & -0.054 & -0.021 \\ -0.173 & -0.036 & 0.103 \\ -0.076 & 0.021 & -0.055 \\ -0.033 & -0.094 & -0.009 \\ -0.126 & -0.102 & 0.060 \\ -0.160 & 0.021 & 0.015 \\ -0.052 & 0.088 & -0.113 \\ -0.156 & -0.178 & 0.069 \\ -0.195 & 0.073 & 0.006 \\ -0.215 & -0.040 & 0.176 \\ -0.011 & 0.296 & -0.005 \\ 0.109 & -0.004 & -0.095 \\ 0.004 & 0.017 & 0.016 \\ -0.026 & 0.106 & 0.102 \\ -0.262 & 0.302 & 0.077 \\ 0.022 & 0.018 & -0.068 \\ -0.081 & -0.121 & -0.134 \\ 0.042 & 0.076 & 0.394 \\ 0.126 & 0.081 & -0.193\end{array}$

$\begin{array}{rrr}-0.017 & -0.013 & -0.023 \\ 0.059 & 0.072 & 0.002 \\ 0.195 & -0.008 & -0.048 \\ 0.149 & -0.177 & -0.039 \\ 0.030 & 0.155 & 0.034 \\ 0.261 & -0.021 & -0.060 \\ 0.227 & 0.119 & 0.015 \\ -0.030 & -0.030 & -0.045 \\ 0.109 & -0.052 & 0.021 \\ 0.006 & -0.087 & -0.045 \\ -0.013 & -0.008 & -0.046 \\ 0.064 & -0.002 & -0.017 \\ 0.084 & -0.092 & -0.002 \\ -0.021 & -0.130 & -0.057 \\ 0.090 & 0.054 & -0.003 \\ 0.112 & -0.128 & 0.021 \\ 0.159 & -0.049 & 0.066 \\ 0.196 & 0.111 & 0.046 \\ -0.074 & 0.010 & 0.073 \\ -0.111 & 0.028 & 0.101 \\ -0.004 & 0.039 & -0.083 \\ 0.126 & 0.101 & -0.064 \\ -0.066 & 0.011 & -0.188 \\ -0.294 & -0.045 & -0.333 \\ -0.033 & 0.028 & -0.145 \\ 0.142 & 0.014 & -0.337\end{array}$

\begin{tabular}{rrr}
\multicolumn{3}{c}{258.00} \\
Y YES \\
X & YES \\
Y & $Z$ \\
-0.010 & -0.007 & -0.012 \\
0.119 & 0.080 & -0.039 \\
-0.037 & -0.116 & -0.008 \\
0.015 & 0.104 & -0.001 \\
0.174 & -0.010 & -0.031 \\
0.090 & -0.123 & -0.053 \\
-0.069 & -0.001 & 0.045 \\
-0.016 & 0.197 & -0.016 \\
0.123 & -0.215 & -0.093 \\
-0.166 & 0.017 & 0.095 \\
-0.102 & -0.194 & -0.013 \\
0.186 & 0.003 & 0.013 \\
-0.068 & 0.018 & -0.016 \\
0.124 & 0.122 & 0.014 \\
0.129 & -0.081 & 0.030 \\
0.023 & -0.094 & 0.045 \\
-0.017 & 0.129 & -0.036 \\
0.172 & 0.204 & 0.017 \\
-0.011 & -0.179 & 0.061 \\
-0.079 & 0.212 & -0.087 \\
-0.182 & 0.010 & -0.048 \\
-0.078 & 0.049 & -0.026 \\
-0.116 & 0.025 & 0.166 \\
-0.127 & -0.007 & 0.175 \\
-0.013 & -0.003 & -0.033 \\
0.167 & 0.008 & -0.009 \\
-0.083 & -0.009 & -0.097 \\
-0.288 & -0.030 & -0.227 \\
-0.060 & -0.009 & -0.164 \\
0 & & \\
0.010 &
\end{tabular}

$\begin{array}{rrr}-0.018 & 0.001 & 0.190 \\ -0.058 & 0.002 & 0.024 \\ -0.063 & -0.013 & 0.002 \\ -0.011 & -0.016 & 0.272 \\ -0.066 & -0.005 & -0.035 \\ -0.071 & -0.011 & -0.067 \\ -0.131 & 0.020 & -0.339 \\ -0.011 & 0.018 & 0.207 \\ -0.051 & 0.037 & -0.051 \\ -0.039 & 0.019 & 0.066 \\ 0.006 & 0.043 & 0.223 \\ -0.007 & 0.041 & 0.175 \\ -0.074 & 0.022 & -0.134 \\ -0.043 & 0.020 & 0.045 \\ 0.007 & 0.040 & 0.260 \\ -0.108 & 0.013 & -0.334 \\ -0.064 & 0.037 & -0.165 \\ 0.393 & -0.043 & -0.025 \\ -0.031 & -0.022 & -0.036 \\ -0.069 & 0.022 & 0.017 \\ -0.052 & 0.024 & -0.017 \\ -0.090 & 0.064 & -0.021 \\ -0.059 & 0.003 & -0.059 \\ -0.127 & -0.030 & -0.102 \\ -0.035 & 0.026 & 0.031 \\ 0.002 & 0.012 & -0.109\end{array}$

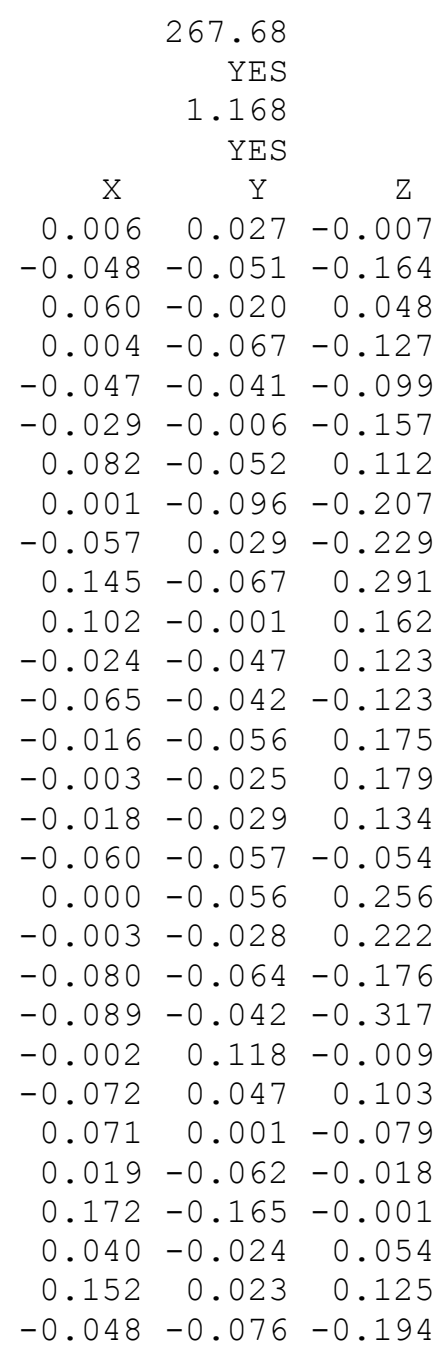




\begin{tabular}{lrrr} 
Frequency: & \multicolumn{3}{c}{ 295.03 } \\
IR Active: & \multicolumn{3}{c}{ YES } \\
IR Intens: & \multicolumn{3}{c}{ Y I S } \\
Raman Active: & \multicolumn{3}{c}{ Y } \\
R & Z \\
Re & 0.011 & -0.114 & -0.019 \\
N & -0.036 & 0.045 & 0.051 \\
C & -0.112 & 0.100 & 0.010 \\
C & -0.081 & 0.058 & 0.046 \\
C & -0.059 & 0.095 & 0.042 \\
C & -0.077 & 0.094 & 0.047 \\
C & -0.102 & 0.070 & 0.003 \\
H & -0.090 & 0.089 & 0.044 \\
H & -0.066 & 0.066 & 0.052 \\
H & -0.085 & 0.068 & -0.037 \\
H & -0.117 & 0.104 & -0.023 \\
C & -0.044 & 0.098 & -0.006 \\
C & 0.120 & 0.079 & -0.008 \\
C & 0.001 & 0.035 & -0.018 \\
N & -0.030 & 0.114 & -0.030 \\
C & 0.061 & 0.132 & -0.029 \\
C & 0.098 & 0.036 & -0.013 \\
H & -0.031 & -0.029 & -0.010 \\
H & 0.091 & 0.200 & -0.012 \\
H & 0.132 & -0.012 & 0.002 \\
H & 0.169 & 0.083 & 0.015 \\
O & -0.027 & 0.489 & -0.035 \\
O & -0.041 & 0.028 & 0.290 \\
O & 0.012 & -0.062 & -0.154 \\
C & 0.112 & 0.043 & 0.030 \\
H & 0.354 & -0.019 & 0.059 \\
C & 0.021 & 0.078 & 0.017 \\
H & 0.151 & 0.135 & 0.098 \\
H & 0.053 & 0.056 & -0.182 \\
H & -0.147 & 0.148 & 0.079
\end{tabular}

\begin{tabular}{rrr}
\multicolumn{3}{c}{321.00} \\
\multicolumn{3}{c}{ Y YES } \\
Y YES \\
0.066 & 0.011 & -0.045 \\
-0.089 & -0.066 & 0.018 \\
-0.016 & 0.078 & -0.004 \\
-0.041 & -0.084 & 0.020 \\
-0.130 & 0.003 & 0.033 \\
-0.084 & 0.080 & 0.041 \\
0.015 & -0.012 & -0.013 \\
-0.024 & -0.128 & 0.050 \\
-0.101 & 0.131 & 0.073 \\
0.098 & -0.027 & -0.037 \\
0.024 & 0.131 & -0.022 \\
-0.130 & 0.002 & 0.026 \\
0.002 & 0.006 & -0.008 \\
-0.100 & -0.074 & 0.022 \\
-0.078 & 0.067 & 0.016 \\
-0.041 & 0.077 & 0.004 \\
-0.035 & -0.078 & 0.008 \\
-0.121 & -0.114 & 0.033 \\
-0.026 & 0.111 & 0.013 \\
-0.001 & -0.131 & 0.008 \\
0.080 & 0.012 & -0.024 \\
-0.234 & -0.099 & -0.070 \\
0.006 & 0.075 & 0.161 \\
-0.079 & -0.065 & 0.360 \\
0.094 & 0.006 & 0.046 \\
0.196 & 0.069 & 0.061 \\
0.022 & -0.046 & -0.076 \\
-0.331 & -0.113 & -0.298 \\
0.038 & -0.036 & -0.013 \\
0.326 & -0.022 & -0.310
\end{tabular}

Frequency:

IR Active:

IR Intens:

Raman Active:
423.44

YES

5.531

YES

$$
\begin{array}{r}
429.57 \\
\text { YES } \\
0.368 \\
\text { YES }
\end{array}
$$

Z

$$
0.077-0.008
$$$$
0.036-0.026
$$$$
0.021
$$$$
0.182
$$

$0.020 \quad 0.016$

0.154

$-0.035-0.009-0.120$

$\begin{array}{llll}-0.010 & 0.003 & 0.036\end{array}$

$\begin{array}{llll}-0.043 & 0.027 & -0.154\end{array}$

$-0.011-0.001-0.033$

$\begin{array}{lll}-0.073 & 0.023-0.300\end{array}$

$\begin{array}{llll}-0.088 & 0.034 & -0.376\end{array}$

$0.001-0.002-0.090$

$\begin{array}{llll}0.065 & 0.016 & 0.347\end{array}$

$\begin{array}{llll}-0.026 & 0.005 & 0.021\end{array}$

$\begin{array}{llll}0.009 & 0.006 & 0.007\end{array}$

$-0.035-0.021-0.057$

$\begin{array}{llll}-0.006 & 0.026 & 0.051\end{array}$

$\begin{array}{llll}-0.019 & 0.030 & -0.047\end{array}$

$\begin{array}{rrr}0.003 & -0.024 & 0.043\end{array}$

$-0.053-0.033-0.106$

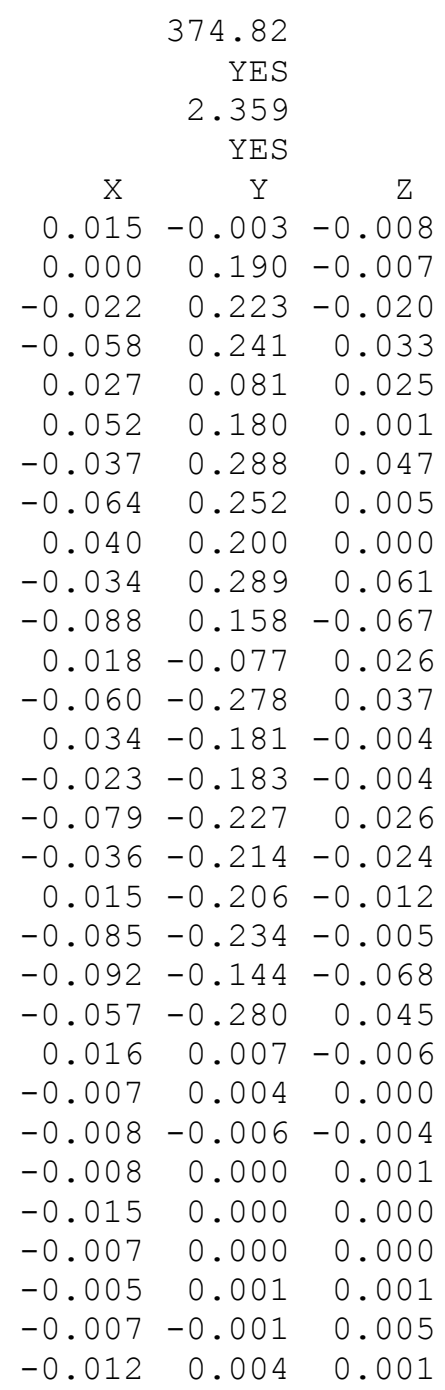

\begin{tabular}{rrr}
\multicolumn{3}{c}{433.68} \\
Y & YES \\
X & YES \\
0.027 & -0.002 & 0.014 \\
-0.029 & 0.004 & -0.122 \\
-0.013 & 0.005 & -0.069 \\
0.028 & -0.015 & 0.165 \\
-0.033 & 0.004 & -0.141 \\
0.031 & -0.004 & 0.164 \\
-0.011 & 0.002 & -0.065 \\
0.079 & -0.041 & 0.450 \\
0.082 & -0.010 & 0.422 \\
-0.014 & 0.003 & -0.100 \\
-0.023 & 0.009 & -0.126 \\
-0.034 & 0.002 & -0.138 \\
-0.014 & 0.001 & -0.080 \\
0.025 & -0.003 & 0.142 \\
-0.025 & 0.002 & -0.089 \\
0.025 & 0.007 & 0.149 \\
-0.008 & -0.003 & -0.038 \\
0.068 & -0.008 & 0.356
\end{tabular} 


$\begin{array}{rrrr}\mathrm{H} & -0.033 & 0.028 & -0.115 \\ \mathrm{H} & 0.025 & -0.040 & 0.104 \\ \mathrm{H} & 0.041 & 0.008 & 0.014 \\ \mathrm{O} & -0.095 & 0.007 & 0.013 \\ \mathrm{O} & -0.186 & -0.146 & -0.043 \\ \mathrm{O} & -0.130 & 0.180 & -0.104 \\ \mathrm{C} & -0.226 & -0.019 & -0.127 \\ \mathrm{H} & -0.173 & -0.056 & -0.121 \\ \mathrm{C} & -0.234 & 0.049 & -0.071 \\ \mathrm{H} & -0.197 & 0.075 & -0.047 \\ \mathrm{H} & -0.155 & 0.078 & -0.163 \\ \mathrm{H} & -0.189 & -0.074 & -0.002\end{array}$

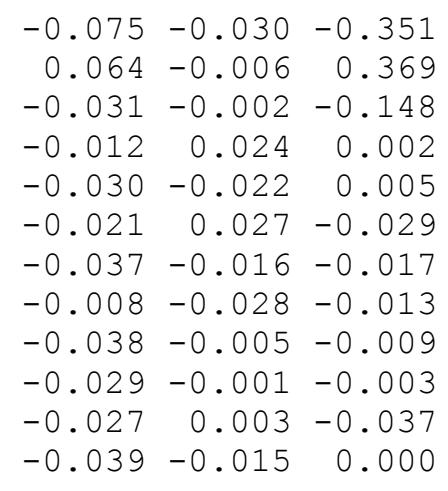

Frequency:
IR Active:
IR Intens:

Raman Active:

$$
\begin{array}{r}
457.78 \\
\text { YES } \\
0.061
\end{array}
$$

YES

$$
\begin{array}{r}
489.75 \\
\text { YES } \\
2.324 \\
\text { YES }
\end{array}
$$

Z

$\begin{array}{rrr}0.015 & -0.001 & 0.012\end{array}$

$-0.039-0.011-0.151$

$\begin{array}{llll}-0.030 & 0.001 & -0.163\end{array}$

$-0.012-0.024-0.070$

$\begin{array}{llll}0.019 & -0.009 & 0.153\end{array}$

$\begin{array}{lll}-0.002-0.003 & 0.043\end{array}$

$\begin{array}{rlll}0.043-0.033 & 0.179\end{array}$

$-0.032-0.020-0.187$

$\begin{array}{llll}-0.004 & 0.000 & 0.039\end{array}$

$\begin{array}{llll}0.089 & -0.044 & 0.356\end{array}$

$\begin{array}{llll}-0.073 & 0.024 & -0.426\end{array}$

$\begin{array}{llll}0.024 & 0.004 & 0.154\end{array}$

$\begin{array}{llll}0.046 & 0.024 & 0.183\end{array}$

$\begin{array}{llll}0.003 & 0.006 & 0.049\end{array}$

$\begin{array}{lll}-0.034 & 0.015-0.157\end{array}$

$\begin{array}{llll}-0.006 & 0.023-0.063\end{array}$

$\begin{array}{rrr}-0.030 & 0.010 & -0.172\end{array}$

$\begin{array}{llll}0.003 & 0.004 & 0.052\end{array}$

$\begin{array}{lll}-0.026 & 0.023-0.179\end{array}$

$\begin{array}{llll}-0.077 & 0.000 & -0.443\end{array}$

$\begin{array}{lll}0.088 & 0.025 & 0.365\end{array}$

$\begin{array}{lll}-0.034 & 0.002 & 0.013\end{array}$

$-0.027-0.033-0.015$

$\begin{array}{llll}-0.018 & 0.039 & -0.024\end{array}$

$-0.041-0.004-0.026$

$-0.032-0.009-0.025$

$\begin{array}{llll}-0.041 & 0.010-0.016\end{array}$

$-0.0350 .013-0.013$

$\begin{array}{lll}-0.023 & 0.017-0.028\end{array}$

$-0.025-0.018-0.003$

\begin{tabular}{lrrr} 
Frequency: & \multicolumn{3}{c}{590.52} \\
IR Active: & \multicolumn{3}{c}{ YES } \\
IR Intens: & \multicolumn{3}{c}{ I.368 } \\
Raman Active: & YES \\
& X & Y & Z \\
Re & -0.001 & -0.036 & 0.002 \\
N & 0.010 & -0.002 & -0.012 \\
C & -0.014 & 0.003 & -0.007 \\
C & 0.003 & -0.003 & -0.013 \\
C & 0.005 & 0.015 & -0.001 \\
C & -0.002 & 0.003 & 0.008 \\
C & -0.005 & -0.012 & 0.011
\end{tabular}

$\begin{array}{rrrr}0.078 & 0.029 & 0.421\end{array}$

$-0.010-0.006-0.058$

$\begin{array}{llll}-0.021 & 0.000 & -0.136\end{array}$

$\begin{array}{lll}-0.048 & 0.007 & 0.013\end{array}$

$-0.058-0.059-0.021$

$\begin{array}{llll}-0.038 & 0.071 & -0.049\end{array}$

$-0.078-0.009-0.046$

$-0.057-0.021-0.044$

$\begin{array}{llll}-0.079 & 0.017 & -0.027\end{array}$

$\begin{array}{lll}-0.063 & 0.025-0.017\end{array}$

$-0.048 \quad 0.030-0.058$

$\begin{array}{lll}-0.061 & -0.031 & 0.000\end{array}$

$\begin{array}{ccc}-0.001 & -0.002 & 0.000\end{array}$

$\begin{array}{lll}0.192 & 0.089-0.037\end{array}$

$0.019-0.142-0.029$

$0.0840 .142-0.014$

$\begin{array}{lll}0.192 & 0.003-0.020\end{array}$

$0.176-0.134-0.029$

$\begin{array}{rrr}-0.033 & 0.013 & 0.027\end{array}$

$\begin{array}{llll}0.047 & 0.250 & -0.053\end{array}$

$0.231-0.279-0.065$

$\begin{array}{rrrr}-0.190 & 0.043 & 0.056\end{array}$

$-0.072-0.246-0.064$

$\begin{array}{llll}-0.191 & 0.025 & 0.024\end{array}$

$0.037 \quad 0.010-0.020$

$\begin{array}{llll}-0.190 & -0.110 & 0.031\end{array}$

$\begin{array}{llll}-0.185 & 0.108 & 0.029\end{array}$

$\begin{array}{lll}-0.065 & 0.149 & 0.013\end{array}$

$-0.034-0.1360 .019$

$\begin{array}{lll}-0.261 & -0.247 & 0.059\end{array}$

$\begin{array}{llll}-0.013 & 0.259 & 0.059\end{array}$

$\begin{array}{lll}0.041 & -0.249 & 0.040\end{array}$

$0.1940 .023-0.039$

$\begin{array}{lll}0.002 & 0.013 & 0.002\end{array}$

$0.018-0.020-0.004$

$\begin{array}{lll}-0.015 & -0.022 & 0.012\end{array}$

$\begin{array}{lll}0.014 & 0.022 & 0.003\end{array}$

$\begin{array}{lll}-0.004 & 0.047 & 0.001\end{array}$

\begin{tabular}{|c|c|c|}
\hline & 559.02 & \\
\hline & YES & \\
\hline & 0.001 & \\
\hline & YES & \\
\hline X & $\mathrm{Y}$ & Z \\
\hline 0.000 & 0.002 & 0.000 \\
\hline 0.003 & -0.012 & 0.054 \\
\hline-0.021 & 0.015 & -0.094 \\
\hline-0.032 & -0.004 & -0.130 \\
\hline 0.026 & -0.009 & 0.167 \\
\hline 0.005 & 0.008 & 0.058 \\
\hline 0.030 & -0.009 & 0.147 \\
\hline-0.089 & 0.022 & -0.447 \\
\hline-0.039 & 0.023 & -0.146 \\
\hline 0.047 & -0.012 & 0.149 \\
\hline-0.077 & 0.033 & -0.394 \\
\hline-0.025 & -0.003 & -0.165 \\
\hline-0.030 & -0.003 & -0.146 \\
\hline-0.004 & 0.010 & -0.059 \\
\hline-0.005 & -0.011 & -0.053 \\
\hline 0.030 & -0.011 & 0.123 \\
\hline 0.023 & 0.011 & 0.097 \\
\hline 0.041 & 0.018 & 0.136 \\
\hline 0.091 & 0.008 & 0.446 \\
\hline 0.078 & 0.016 & 0.393 \\
\hline-0.048 & -0.004 & -0.147 \\
\hline 0.000 & -0.004 & 0.001 \\
\hline 0.004 & 0.001 & 0.000 \\
\hline-0.003 & 0.003 & -0.003 \\
\hline 0.004 & -0.007 & 0.005 \\
\hline 0.012 & -0.015 & 0.006 \\
\hline-0.007 & -0.005 & 0.001 \\
\hline-0.007 & -0.014 & 0.001 \\
\hline 0.006 & -0.001 & -0.006 \\
\hline-0.005 & 0.000 & -0.005 \\
\hline & 670.84 & \\
\hline & YES & \\
\hline & 1.950 & \\
\hline & YES & \\
\hline $\mathrm{X}$ & Y & Z \\
\hline-0.001 & 0.004 & -0.001 \\
\hline 0.161 & -0.110 & -0.045 \\
\hline-0.180 & 0.127 & 0.045 \\
\hline-0.003 & -0.121 & 0.002 \\
\hline 0.100 & 0.117 & -0.025 \\
\hline 0.058 & 0.110 & -0.009 \\
\hline-0.080 & -0.176 & 0.002 \\
\hline
\end{tabular}

$-0.003 \quad 0.020-0.005$

$-0.0120 .036-0.011$

$\begin{array}{lll}0.032 & 0.032 & 0.033\end{array}$

$\begin{array}{lll}-0.013 & 0.034 & -0.010\end{array}$

\begin{tabular}{rrr}
\multicolumn{4}{c}{659.08} \\
\multicolumn{4}{c}{0.168} \\
YES \\
YES \\
0.001 & 0.000 & 0.001 \\
-0.042 & -0.103 & 0.001 \\
0.043 & 0.107 & -0.002 \\
-0.235 & -0.043 & 0.047 \\
0.049 & -0.214 & -0.023 \\
0.270 & 0.033 & -0.055 \\
-0.044 & 0.224 & 0.016
\end{tabular}

$$
\begin{array}{r}
559.02 \\
\text { YES } \\
0.001 \\
\text { YES }
\end{array}
$$




$\mathrm{H}$
$\mathrm{H}$
$\mathrm{H}$
$\mathrm{H}$
$\mathrm{C}$
$\mathrm{C}$
$\mathrm{C}$
$\mathrm{N}$
$\mathrm{C}$
$\mathrm{C}$
$\mathrm{H}$
$\mathrm{H}$
$\mathrm{H}$
$\mathrm{H}$
$\mathrm{O}$
$\mathrm{O}$
$\mathrm{O}$
$\mathrm{C}$
$\mathrm{H}$
$\mathrm{C}$
$\mathrm{H}$
$\mathrm{H}$
$\mathrm{H}$

$\begin{array}{rrr}0.003 & 0.002 & -0.011 \\ 0.005 & -0.013 & 0.016 \\ 0.000 & -0.013 & 0.028 \\ -0.012 & 0.009 & -0.016 \\ -0.005 & 0.016 & 0.001 \\ 0.004 & -0.010 & -0.011 \\ 0.002 & 0.002 & -0.007 \\ -0.010 & 0.001 & 0.012 \\ -0.004 & 0.000 & 0.013 \\ 0.013 & 0.000 & 0.008 \\ -0.007 & -0.012 & -0.015 \\ -0.004 & 0.004 & 0.008 \\ 0.012 & 0.004 & 0.015 \\ 0.001 & -0.010 & -0.032 \\ -0.002 & 0.011 & -0.001 \\ -0.190 & 0.130 & -0.096 \\ 0.175 & 0.066 & 0.175 \\ -0.163 & 0.134 & -0.161 \\ -0.343 & 0.335 & -0.184 \\ 0.220 & 0.111 & 0.001 \\ 0.321 & 0.317 & 0.061 \\ -0.326 & -0.129 & 0.062 \\ 0.206 & -0.170 & 0.251\end{array}$

Freque
IR A
IR Int
Raman
Re
N
C
C
C
C
C
H
H
H
H
C
C
C
N
C
C
H
H
H
H
O
O
O
C
H
C
H
H
H

$\begin{array}{rrr}-0.230 & -0.077 & 0.045 \\ 0.240 & 0.122 & -0.039 \\ 0.133 & 0.192 & -0.011 \\ -0.147 & -0.134 & 0.034 \\ -0.075 & -0.206 & 0.019 \\ 0.073 & 0.215 & -0.012 \\ -0.266 & 0.068 & 0.056 \\ 0.023 & -0.110 & -0.002 \\ 0.231 & -0.072 & -0.049 \\ -0.024 & 0.117 & 0.004 \\ -0.230 & 0.149 & 0.036 \\ 0.224 & -0.100 & -0.052 \\ 0.135 & -0.142 & -0.043 \\ -0.116 & 0.202 & 0.012 \\ 0.001 & -0.001 & 0.003 \\ -0.012 & 0.007 & -0.005 \\ 0.002 & -0.016 & 0.004 \\ -0.009 & 0.009 & -0.011 \\ -0.022 & 0.033 & -0.012 \\ 0.010 & 0.007 & -0.003 \\ 0.027 & 0.034 & 0.007 \\ -0.021 & -0.013 & 0.010 \\ -0.014 & -0.002 & 0.021\end{array}$

\begin{tabular}{rrr}
\multicolumn{3}{c}{686.02} \\
YES \\
Y & YES \\
0.068 & $\mathrm{Y}$ \\
0.006 & -0.020 & -0.006 \\
-0.138 & 0.164 & 0.036 \\
0.159 & -0.188 & -0.036 \\
0.122 & 0.170 & -0.013 \\
-0.108 & -0.009 & 0.036 \\
-0.182 & -0.128 & 0.024 \\
0.090 & 0.086 & -0.019 \\
0.177 & 0.000 & -0.019 \\
-0.254 & 0.057 & 0.038 \\
-0.215 & 0.145 & 0.040 \\
0.206 & -0.134 & -0.035 \\
-0.074 & -0.025 & 0.025 \\
0.058 & -0.001 & -0.014 \\
-0.147 & 0.089 & 0.024 \\
-0.082 & -0.098 & 0.013 \\
0.099 & -0.107 & -0.021 \\
0.087 & 0.112 & -0.013 \\
-0.189 & 0.008 & 0.030 \\
0.139 & -0.027 & -0.012 \\
0.146 & 0.020 & -0.020 \\
-0.141 & -0.017 & 0.027 \\
-0.005 & 0.014 & -0.001 \\
0.028 & 0.092 & -0.031 \\
0.035 & 0.185 & 0.020 \\
0.005 & -0.057 & 0.043 \\
0.063 & -0.287 & 0.045 \\
-0.022 & -0.049 & 0.039 \\
-0.155 & -0.281 & -0.044 \\
0.002 & 0.004 & -0.143 \\
0.218 & -0.063 & -0.122 \\
& &
\end{tabular}

$\begin{array}{rrr}-0.059 & 0.067 & 0.025 \\ 0.147 & -0.118 & -0.026 \\ 0.108 & -0.213 & -0.039 \\ -0.141 & 0.177 & 0.047 \\ -0.109 & 0.127 & 0.040 \\ 0.081 & -0.196 & -0.013 \\ -0.085 & 0.153 & 0.016 \\ -0.212 & -0.129 & 0.047 \\ 0.019 & -0.161 & -0.012 \\ 0.235 & 0.149 & -0.045 \\ -0.220 & -0.098 & 0.018 \\ 0.117 & 0.061 & -0.013 \\ 0.215 & 0.183 & -0.048 \\ -0.183 & -0.219 & 0.044 \\ 0.001 & -0.003 & -0.001 \\ 0.029 & 0.074 & -0.028 \\ 0.007 & 0.097 & 0.003 \\ 0.008 & -0.044 & 0.033 \\ 0.058 & -0.197 & 0.037 \\ -0.029 & -0.040 & 0.024 \\ -0.113 & -0.189 & -0.028 \\ 0.007 & 0.002 & -0.099 \\ 0.103 & -0.019 & -0.089\end{array}$

\begin{tabular}{rrr}
\multicolumn{4}{c}{691.69} \\
YES \\
X 37.024 \\
YES & Y & $Z$ \\
0.007 & -0.026 & 0.015 \\
0.018 & -0.034 & -0.003 \\
-0.014 & 0.033 & 0.005 \\
-0.042 & -0.027 & 0.011 \\
0.033 & -0.015 & -0.013 \\
0.071 & 0.017 & -0.008 \\
-0.018 & 0.017 & 0.006 \\
-0.054 & -0.004 & -0.011 \\
0.078 & -0.009 & -0.029 \\
0.033 & 0.007 & -0.031 \\
-0.054 & -0.012 & -0.012 \\
0.038 & -0.007 & -0.023 \\
-0.026 & 0.050 & 0.013 \\
0.064 & -0.062 & -0.005 \\
0.061 & 0.066 & -0.010 \\
-0.045 & 0.091 & 0.005 \\
-0.073 & -0.071 & 0.009 \\
0.102 & 0.008 & -0.001 \\
-0.081 & 0.017 & -0.014 \\
-0.086 & -0.056 & -0.001 \\
0.112 & 0.062 & -0.023 \\
0.008 & 0.018 & -0.003 \\
0.026 & 0.392 & -0.132 \\
-0.043 & 0.047 & -0.042 \\
-0.049 & -0.066 & 0.036 \\
0.015 & -0.475 & 0.034 \\
-0.103 & -0.118 & 0.043 \\
-0.258 & -0.451 & -0.053 \\
-0.152 & -0.063 & -0.299 \\
0.101 & 0.001 & -0.210
\end{tabular}




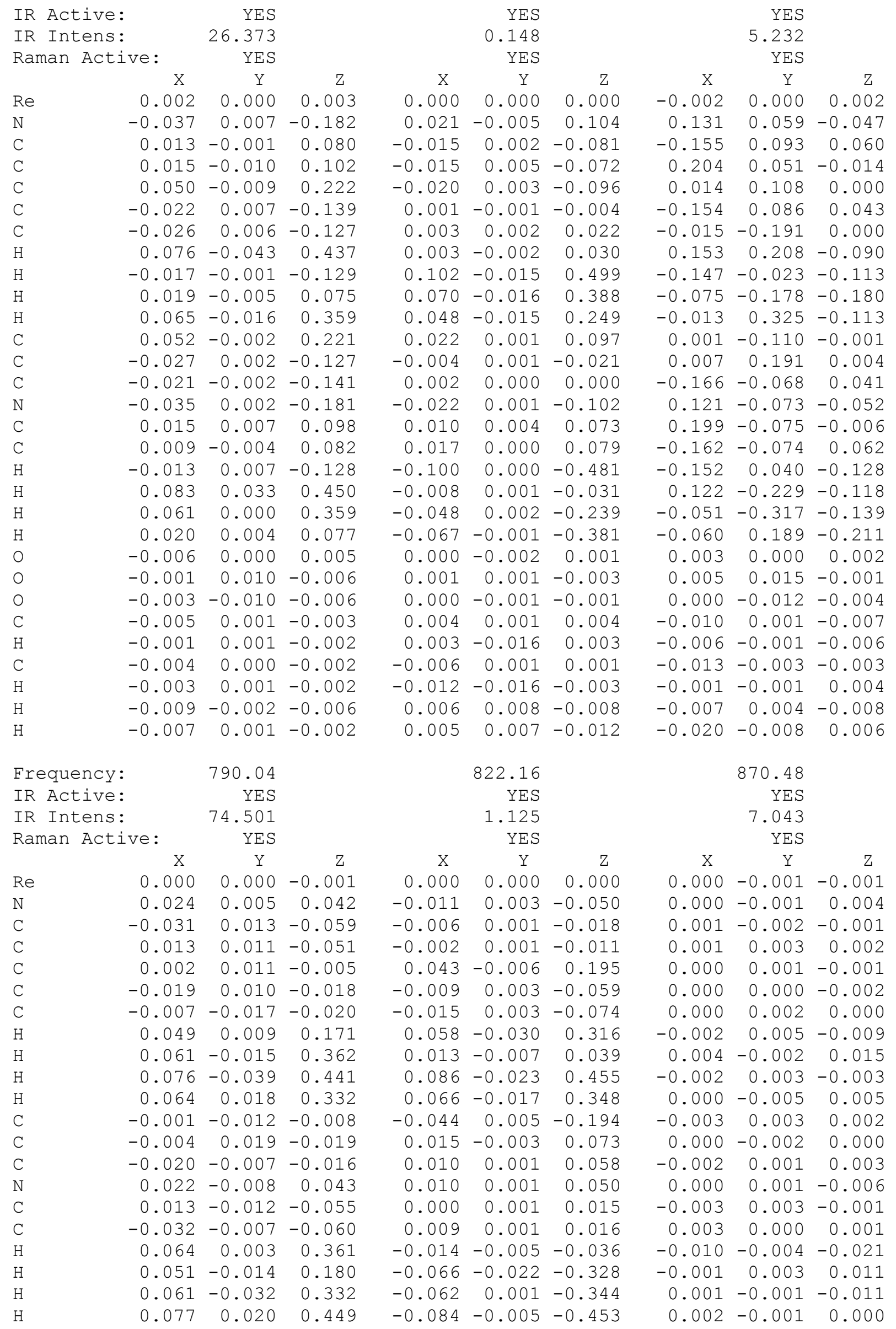




$\mathrm{O}$
$\mathrm{O}$
$\mathrm{O}$
$\mathrm{C}$
$\mathrm{H}$
$\mathrm{C}$
$\mathrm{H}$
$\mathrm{H}$
$\mathrm{H}$

$\begin{array}{rrr}0.001 & 0.000 & 0.001 \\ 0.000 & 0.007 & -0.001 \\ -0.002 & -0.004 & -0.001 \\ -0.001 & 0.000 & 0.000 \\ -0.003 & 0.000 & 0.000 \\ -0.001 & -0.003 & 0.000 \\ 0.002 & -0.001 & 0.002 \\ -0.003 & -0.002 & 0.000 \\ -0.007 & 0.000 & 0.002\end{array}$

$\begin{array}{rrr}0.000 & 0.001 & 0.000 \\ 0.001 & -0.001 & 0.002 \\ -0.001 & 0.000 & -0.001 \\ -0.001 & -0.001 & -0.001 \\ -0.001 & 0.001 & -0.001 \\ 0.001 & -0.001 & 0.000 \\ 0.000 & 0.003 & 0.000 \\ 0.001 & 0.000 & 0.002 \\ 0.000 & 0.000 & 0.000\end{array}$

$\begin{array}{rrr}0.000 & 0.004 & 0.006 \\ 0.095 & 0.068 & 0.064 \\ -0.078 & 0.082 & -0.006 \\ 0.006 & -0.094 & -0.073 \\ -0.158 & 0.412 & -0.071 \\ 0.003 & -0.111 & -0.054 \\ 0.172 & 0.362 & 0.063 \\ -0.013 & -0.306 & 0.402 \\ -0.247 & -0.381 & 0.336\end{array}$

Frequency:

IR Active:

IR Intens:

Raman Active:

$\begin{array}{lrrr} & X & Y & Z \\ \text { Re } & 0.000 & 0.000 & 0.000 \\ \text { N } & -0.003 & 0.001 & -0.022 \\ \text { C } & 0.002 & 0.000 & 0.000 \\ \text { C } & 0.009 & -0.004 & 0.054 \\ \text { C } & 0.012 & -0.004 & 0.064 \\ \text { C } & -0.023 & 0.005 & -0.107 \\ \text { C } & 0.008 & -0.004 & 0.041 \\ \text { H } & -0.057 & 0.034 & -0.297 \\ \text { H } & 0.103 & -0.010 & 0.528 \\ \text { H } & -0.065 & 0.014 & -0.347 \\ \text { H } & 0.021 & 0.000 & 0.078 \\ \text { C } & -0.011 & -0.001 & -0.063 \\ \text { C } & -0.009 & 0.000 & -0.033 \\ \text { C } & 0.023 & -0.001 & 0.100 \\ \text { N } & 0.004 & 0.000 & 0.022 \\ \text { C } & -0.007 & -0.002 & -0.056 \\ \text { C } & -0.004 & 0.000 & 0.003 \\ \text { H } & -0.099 & 0.005 & -0.491 \\ \text { H } & 0.062 & 0.026 & 0.297 \\ \text { H } & -0.025 & 0.005 & -0.091 \\ \text { H } & 0.050 & 0.001 & 0.300 \\ \text { O } & 0.000 & 0.000 & -0.002 \\ \text { O } & -0.004 & -0.001 & -0.002 \\ \text { O } & -0.001 & 0.000 & 0.000 \\ \text { C } & 0.001 & 0.000 & 0.003 \\ \text { H } & 0.002 & -0.008 & 0.002 \\ \text { C } & 0.005 & 0.004 & 0.001 \\ \text { H } & 0.001 & -0.003 & -0.002 \\ \text { H } & -0.005 & -0.003 & -0.006 \\ \text { H } & 0.005 & 0.013 & -0.007 \\ & & & \end{array}$

\begin{tabular}{rrr}
\multicolumn{3}{c}{919.30} \\
YES & 1.291 \\
X & YES & $Z$ \\
0.000 & 0.000 & 0.000 \\
-0.001 & 0.000 & -0.010 \\
0.002 & -0.001 & -0.011 \\
0.006 & -0.004 & 0.048 \\
0.001 & -0.001 & 0.013 \\
-0.016 & 0.003 & -0.082 \\
0.008 & -0.003 & 0.044 \\
-0.056 & 0.031 & -0.283 \\
0.098 & -0.011 & 0.495 \\
-0.061 & 0.015 & -0.324 \\
0.022 & -0.004 & 0.089 \\
0.000 & 0.001 & 0.016 \\
0.010 & 0.001 & 0.042 \\
-0.019 & 0.002 & -0.086 \\
-0.002 & 0.000 & -0.012 \\
0.006 & 0.003 & 0.057 \\
0.003 & 0.001 & -0.015 \\
0.105 & -0.010 & 0.525 \\
-0.069 & -0.028 & -0.323 \\
0.031 & -0.002 & 0.113 \\
-0.056 & 0.000 & -0.321 \\
0.000 & 0.000 & -0.002 \\
0.007 & -0.001 & 0.004 \\
0.009 & -0.001 & 0.002 \\
-0.007 & 0.007 & -0.006 \\
0.005 & 0.005 & -0.004 \\
-0.010 & -0.005 & 0.000 \\
-0.002 & -0.006 & 0.005 \\
0.008 & 0.024 & -0.008 \\
-0.002 & -0.022 & 0.009
\end{tabular}

\begin{tabular}{rrr}
\multicolumn{3}{c}{1007.67} \\
\multicolumn{3}{c}{ YES } \\
2.620 \\
YES \\
Y & \multicolumn{1}{c}{$\mathrm{Y}$} & $\mathrm{Z}$ \\
0.000 & 0.001 & 0.000 \\
-0.008 & 0.003 & 0.021 \\
-0.005 & 0.003 & 0.067 \\
-0.006 & 0.008 & -0.140 \\
0.006 & -0.003 & 0.015 \\
0.000 & 0.001 & -0.056 \\
0.010 & 0.002 & 0.070 \\
0.171 & -0.121 & 0.748 \\
0.074 & -0.038 & 0.251 \\
-0.080 & 0.026 & -0.320
\end{tabular}

921.92

YES

126.225

YES

\begin{tabular}{rrr}
$X$ & \multicolumn{1}{c}{$Y$} & $Z$ \\
0.004 & -0.002 & 0.005 \\
-0.005 & 0.006 & 0.000 \\
-0.003 & 0.003 & 0.000 \\
0.006 & -0.001 & 0.002 \\
0.007 & 0.005 & -0.002 \\
0.005 & 0.005 & -0.008 \\
0.001 & -0.012 & 0.005 \\
0.003 & -0.004 & -0.016 \\
0.020 & -0.008 & 0.043 \\
-0.011 & -0.010 & -0.032 \\
0.004 & 0.009 & 0.007 \\
0.007 & -0.005 & 0.004 \\
0.003 & 0.015 & 0.008 \\
0.004 & -0.005 & -0.017 \\
-0.007 & -0.007 & -0.001 \\
0.005 & 0.004 & 0.006 \\
-0.006 & -0.004 & 0.001 \\
0.030 & 0.005 & 0.082 \\
-0.001 & 0.007 & -0.039 \\
0.001 & -0.012 & 0.010 \\
-0.003 & 0.015 & -0.062 \\
-0.002 & 0.000 & -0.017 \\
-0.151 & -0.006 & -0.092 \\
-0.195 & 0.064 & -0.043 \\
0.159 & -0.169 & 0.133 \\
-0.091 & -0.083 & 0.095 \\
0.232 & 0.113 & -0.008 \\
0.050 & 0.162 & -0.111 \\
-0.169 & -0.586 & 0.250 \\
0.053 & 0.478 & -0.191
\end{tabular}

1010.93

YES

6.303

YES

$\begin{array}{rrr}X & Y & Z \\ 0.000 & 0.004 & 0.004 \\ -0.003 & 0.003 & -0.002 \\ -0.003 & 0.001 & -0.039 \\ 0.010 & -0.002 & 0.068 \\ -0.003 & -0.001 & -0.010 \\ 0.010 & 0.003 & 0.029 \\ -0.007 & -0.006 & -0.031 \\ -0.071 & 0.037 & -0.380 \\ -0.027 & 0.018 & -0.137 \\ 0.018 & -0.015 & 0.146\end{array}$




$\mathrm{H}$
$\mathrm{C}$
$\mathrm{C}$
$\mathrm{C}$
$\mathrm{N}$
$\mathrm{C}$
$\mathrm{C}$
$\mathrm{H}$
$\mathrm{H}$
$\mathrm{H}$
$\mathrm{H}$
$\mathrm{O}$
$\mathrm{O}$
$\mathrm{O}$
$\mathrm{C}$
$\mathrm{H}$
$\mathrm{C}$
$\mathrm{H}$
$\mathrm{H}$
$\mathrm{H}$

$\begin{array}{rrr}0.011 & -0.001 & 0.043 \\ 0.008 & 0.000 & 0.018 \\ 0.011 & -0.006 & 0.084 \\ 0.002 & 0.001 & -0.059 \\ -0.011 & -0.003 & 0.020 \\ -0.003 & -0.004 & -0.143 \\ -0.012 & -0.005 & 0.059 \\ 0.091 & 0.037 & 0.262 \\ 0.192 & 0.112 & 0.739 \\ -0.104 & 0.019 & -0.319 \\ -0.099 & -0.011 & -0.398 \\ -0.001 & 0.000 & 0.012 \\ -0.010 & 0.005 & -0.006 \\ 0.015 & -0.001 & 0.005 \\ 0.016 & -0.002 & 0.006 \\ -0.005 & 0.013 & 0.003 \\ -0.020 & 0.003 & -0.008 \\ 0.003 & 0.018 & 0.005 \\ 0.015 & -0.005 & 0.018 \\ -0.032 & -0.004 & 0.007\end{array}$

\begin{tabular}{|c|c|c|c|}
\hline Frequ & & 1029.89 & \\
\hline IR Ac & & YES & \\
\hline IR In & & 114.016 & \\
\hline Raman & ve: & YES & \\
\hline & $\mathrm{X}$ & $\mathrm{Y}$ & $\mathrm{Z}$ \\
\hline $\mathrm{Re}$ & -0.003 & 0.001 & -0.012 \\
\hline $\mathrm{N}$ & 0.041 & -0.028 & -0.006 \\
\hline $\mathrm{C}$ & -0.010 & 0.007 & 0.008 \\
\hline $\mathrm{C}$ & 0.004 & 0.002 & -0.004 \\
\hline C & -0.006 & -0.015 & 0.000 \\
\hline C & -0.041 & -0.022 & 0.003 \\
\hline C & 0.008 & 0.047 & -0.001 \\
\hline $\mathrm{H}$ & 0.008 & 0.003 & 0.010 \\
\hline $\mathrm{H}$ & -0.040 & -0.021 & 0.033 \\
\hline $\mathrm{H}$ & 0.049 & 0.045 & 0.000 \\
\hline $\mathrm{H}$ & -0.031 & -0.002 & -0.031 \\
\hline C & -0.003 & -0.005 & 0.000 \\
\hline C & -0.003 & -0.012 & -0.008 \\
\hline $\mathrm{C}$ & -0.015 & 0.005 & -0.004 \\
\hline N & 0.020 & 0.009 & -0.003 \\
\hline C & -0.018 & 0.008 & 0.003 \\
\hline $\mathrm{C}$ & 0.019 & 0.009 & 0.011 \\
\hline $\mathrm{H}$ & -0.018 & -0.019 & 0.045 \\
\hline $\mathrm{H}$ & -0.028 & -0.014 & 0.002 \\
\hline $\mathrm{H}$ & 0.001 & 0.009 & -0.083 \\
\hline $\mathrm{H}$ & 0.011 & -0.011 & 0.047 \\
\hline O & -0.004 & 0.000 & 0.115 \\
\hline 0 & 0.172 & 0.023 & 0.081 \\
\hline O & 0.099 & -0.050 & 0.031 \\
\hline C & -0.228 & -0.253 & -0.006 \\
\hline $\mathrm{H}$ & -0.154 & -0.311 & 0.025 \\
\hline C & -0.031 & 0.264 & -0.085 \\
\hline $\mathrm{H}$ & -0.069 & 0.217 & -0.127 \\
\hline $\mathrm{H}$ & -0.309 & -0.375 & 0.009 \\
\hline $\mathrm{H}$ & -0.044 & 0.477 & -0.237 \\
\hline
\end{tabular}

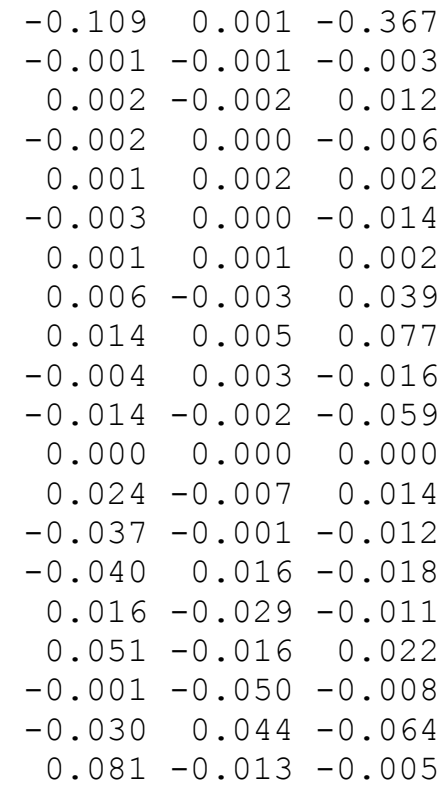

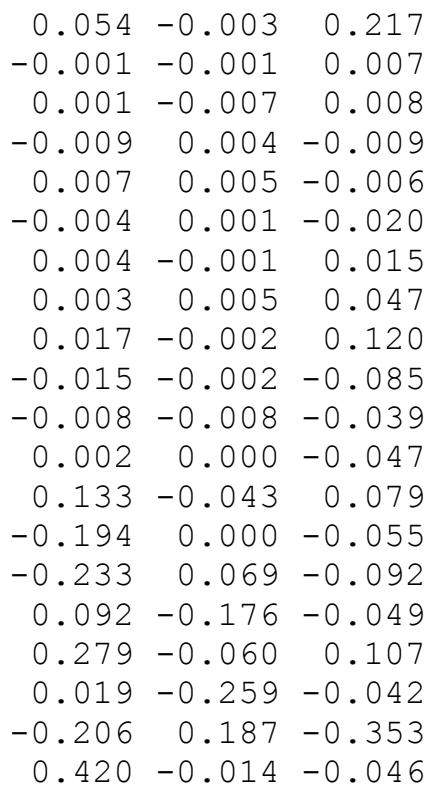

$\begin{array}{lrrr}\text { Frequency: } & 1041.24 & 1046.43 & 1057.50 \\ \text { IR Active: } & \text { YES } & \text { YES } & \text { YES } \\ \text { IR Intens: } & 21.740 & 101.763 & 12.850 \\ \text { Raman Active: } & \text { YES } & \text { YES } & \text { YES }\end{array}$




\begin{tabular}{|c|c|c|c|c|c|c|c|c|c|}
\hline & X & $\mathrm{Y}$ & Z & X & $Y$ & Z & X & $\mathrm{Y}$ & Z \\
\hline $\mathrm{Re}$ & 0.000 & 0.004 & 0.001 & 0.010 & -0.001 & -0.063 & -0.002 & -0.001 & -0.015 \\
\hline $\mathrm{N}$ & 0.201 & -0.151 & -0.057 & -0.154 & 0.122 & 0.044 & 0.219 & -0.156 & -0.058 \\
\hline C & -0.124 & 0.112 & 0.023 & -0.036 & 0.001 & -0.003 & -0.059 & 0.042 & 0.008 \\
\hline C & 0.134 & 0.039 & -0.019 & -0.002 & -0.003 & 0.017 & 0.030 & -0.016 & -0.001 \\
\hline C & -0.032 & -0.154 & 0.001 & 0.037 & 0.040 & -0.008 & -0.011 & -0.065 & 0.000 \\
\hline $\mathrm{C}$ & -0.204 & -0.108 & 0.040 & 0.112 & 0.019 & -0.018 & -0.214 & -0.120 & 0.040 \\
\hline C & 0.043 & 0.239 & 0.003 & 0.010 & -0.121 & -0.013 & 0.049 & 0.248 & 0.004 \\
\hline $\mathrm{H}$ & 0.161 & -0.007 & -0.044 & -0.005 & -0.071 & -0.119 & 0.036 & -0.023 & -0.031 \\
\hline $\mathrm{H}$ & -0.212 & -0.154 & 0.021 & 0.176 & -0.145 & -0.070 & -0.226 & -0.135 & 0.037 \\
\hline $\mathrm{H}$ & 0.097 & 0.252 & -0.003 & 0.227 & -0.156 & -0.064 & 0.208 & 0.243 & -0.043 \\
\hline $\mathrm{H}$ & -0.148 & 0.102 & 0.065 & -0.034 & -0.022 & 0.069 & -0.109 & -0.006 & 0.048 \\
\hline $\mathrm{C}$ & 0.013 & -0.156 & -0.004 & 0.022 & -0.002 & -0.008 & 0.000 & -0.013 & 0.002 \\
\hline C & -0.016 & 0.267 & 0.006 & -0.006 & 0.130 & -0.014 & 0.012 & -0.267 & 0.010 \\
\hline C & 0.213 & -0.140 & -0.044 & 0.107 & -0.069 & -0.018 & -0.242 & 0.136 & 0.053 \\
\hline $\mathrm{N}$ & -0.237 & -0.140 & 0.057 & -0.133 & -0.075 & 0.033 & 0.296 & 0.164 & -0.064 \\
\hline C & -0.127 & 0.052 & 0.021 & 0.019 & -0.014 & 0.015 & -0.066 & 0.044 & 0.002 \\
\hline C & 0.135 & 0.097 & -0.027 & -0.008 & 0.002 & 0.003 & 0.034 & 0.013 & -0.015 \\
\hline $\mathrm{H}$ & 0.213 & -0.185 & -0.057 & 0.119 & -0.053 & -0.042 & -0.299 & 0.061 & 0.051 \\
\hline $\mathrm{H}$ & -0.159 & 0.012 & 0.036 & 0.008 & 0.020 & -0.134 & -0.089 & -0.045 & 0.090 \\
\hline $\mathrm{H}$ & 0.169 & 0.085 & -0.018 & 0.003 & -0.008 & -0.003 & 0.026 & 0.024 & 0.033 \\
\hline $\mathrm{H}$ & -0.075 & 0.287 & -0.007 & -0.028 & 0.136 & 0.068 & 0.095 & -0.276 & -0.075 \\
\hline 0 & 0.001 & -0.001 & -0.013 & -0.061 & 0.011 & 0.725 & -0.020 & 0.003 & 0.223 \\
\hline O & -0.010 & -0.004 & -0.010 & -0.046 & 0.009 & -0.011 & -0.071 & 0.005 & -0.036 \\
\hline O & -0.019 & 0.008 & -0.001 & -0.062 & -0.010 & -0.029 & -0.058 & 0.004 & -0.022 \\
\hline C & 0.014 & 0.031 & -0.001 & 0.034 & 0.078 & -0.015 & 0.071 & 0.072 & 0.009 \\
\hline $\mathrm{H}$ & 0.045 & 0.040 & 0.002 & 0.041 & 0.061 & -0.019 & 0.054 & 0.077 & 0.000 \\
\hline C & 0.013 & -0.034 & 0.011 & 0.066 & -0.089 & 0.073 & 0.038 & -0.079 & 0.038 \\
\hline $\mathrm{H}$ & 0.015 & -0.025 & 0.015 & -0.105 & -0.037 & -0.021 & -0.003 & -0.060 & 0.021 \\
\hline $\mathrm{H}$ & 0.027 & 0.056 & -0.023 & 0.067 & 0.125 & -0.028 & 0.058 & 0.071 & -0.012 \\
\hline $\mathrm{H}$ & 0.004 & -0.056 & 0.033 & 0.172 & -0.094 & -0.003 & 0.043 & -0.081 & 0.032 \\
\hline Frequency: & & 1061.43 & & & .088 .53 & & & 1099.06 & \\
\hline IR Active: & & YES & & & YES & & & YES & \\
\hline IR Intens: & & 3.587 & & & 6.591 & & & 6.687 & \\
\hline Raman Activ & ve: & YES & & & YES & & & YES & \\
\hline & $\mathrm{X}$ & $Y$ & Z & $\mathrm{X}$ & $Y$ & Z & X & Y & Z \\
\hline $\mathrm{Re}$ & 0.000 & -0.002 & 0.002 & -0.002 & 0.000 & -0.002 & 0.000 & 0.000 & -0.002 \\
\hline $\mathrm{N}$ & -0.188 & 0.115 & 0.043 & 0.075 & 0.010 & -0.013 & 0.064 & -0.013 & -0.015 \\
\hline C & -0.172 & 0.111 & 0.045 & 0.129 & -0.041 & -0.027 & 0.046 & 0.001 & -0.007 \\
\hline C & 0.241 & 0.066 & -0.017 & -0.128 & 0.015 & 0.010 & 0.006 & 0.057 & -0.006 \\
\hline $\mathrm{C}$ & -0.036 & -0.184 & -0.005 & 0.002 & 0.022 & 0.002 & -0.033 & -0.107 & 0.004 \\
\hline $\mathrm{C}$ & 0.165 & 0.041 & -0.032 & -0.028 & 0.048 & 0.008 & -0.003 & 0.062 & 0.003 \\
\hline $\mathrm{C}$ & -0.022 & -0.110 & -0.022 & -0.036 & -0.084 & 0.012 & -0.058 & -0.071 & 0.012 \\
\hline $\mathrm{H}$ & 0.297 & -0.166 & -0.225 & -0.212 & 0.330 & 0.158 & -0.048 & 0.283 & 0.082 \\
\hline $\mathrm{H}$ & 0.203 & -0.049 & -0.037 & -0.125 & 0.299 & 0.029 & -0.173 & 0.505 & 0.052 \\
\hline $\mathrm{H}$ & -0.114 & -0.093 & 0.118 & -0.095 & -0.084 & -0.028 & -0.271 & -0.036 & 0.033 \\
\hline $\mathrm{H}$ & -0.246 & 0.042 & 0.019 & 0.300 & 0.157 & -0.050 & 0.137 & 0.110 & -0.027 \\
\hline C & 0.014 & -0.196 & 0.002 & -0.003 & -0.013 & 0.003 & 0.020 & -0.109 & -0.006 \\
\hline C & 0.005 & -0.033 & 0.022 & -0.027 & 0.093 & 0.017 & 0.046 & -0.072 & -0.014 \\
\hline C & -0.093 & 0.014 & 0.019 & -0.035 & -0.049 & 0.006 & 0.010 & 0.059 & 0.000 \\
\hline $\mathrm{N}$ & 0.118 & 0.050 & -0.022 & 0.075 & -0.016 & -0.014 & -0.060 & -0.007 & 0.014 \\
\hline C & -0.228 & 0.081 & 0.014 & -0.127 & -0.006 & 0.004 & -0.004 & 0.057 & 0.010 \\
\hline C & 0.191 & 0.097 & -0.044 & 0.134 & 0.026 & -0.026 & -0.040 & 0.008 & 0.006 \\
\hline $\mathrm{H}$ & -0.132 & -0.066 & 0.021 & -0.168 & -0.310 & 0.035 & 0.217 & 0.461 & -0.050 \\
\hline $\mathrm{H}$ & -0.303 & -0.133 & 0.240 & -0.246 & -0.328 & 0.183 & 0.068 & 0.267 & -0.089 \\
\hline $\mathrm{H}$ & 0.269 & 0.009 & -0.012 & 0.285 & -0.196 & -0.053 & -0.114 & 0.121 & 0.029 \\
\hline $\mathrm{H}$ & 0.048 & -0.021 & -0.118 & -0.104 & 0.100 & -0.035 & 0.262 & -0.062 & -0.028 \\
\hline O & 0.002 & -0.001 & -0.031 & 0.000 & 0.000 & 0.021 & -0.002 & 0.001 & 0.029 \\
\hline O & -0.002 & -0.001 & -0.002 & -0.004 & 0.000 & -0.003 & 0.001 & 0.002 & -0.001 \\
\hline O & 0.018 & -0.002 & 0.006 & -0.003 & 0.001 & -0.001 & -0.003 & 0.001 & 0.000 \\
\hline
\end{tabular}


$\mathrm{C}$
$\mathrm{H}$
$\mathrm{C}$
$\mathrm{H}$
$\mathrm{H}$
$\mathrm{H}$

Fregu
IR
IR
Raman
Re
N
C
C
C
C
C
H
H
H
H
C
C
C
N
C
C
H
H
H
H
O
O
O
C
H
C
H
H
H

$$
\begin{array}{rrr}
0.005 & -0.011 & 0.005 \\
-0.008 & -0.001 & 0.004 \\
-0.019 & 0.012 & -0.009 \\
-0.002 & 0.016 & 0.000 \\
0.004 & -0.015 & 0.017 \\
-0.025 & 0.010 & -0.002
\end{array}
$$

Frequency:

1134.80

YES

9.928

YES

$\mathrm{X}$

0.000

$Y$

$-0.027$

0.000

$-0.013$

0.069

0.034

$-0.082-0.008$

$0.064 \quad 0.018-0.012$

$\begin{array}{lll}-0.053 & -0.071 & 0.007\end{array}$

$0.065-0.017-0.012$

$\begin{array}{rrr}-0.105 & 0.055 & 0.046\end{array}$

$0.023-0.276-0.007$

$0.528-0.106-0.116$

$\begin{array}{llll}0.108 & 0.195 & -0.014\end{array}$

$0.062-0.029-0.013$

$0.070 \quad 0.009-0.012$

$\begin{array}{rrr}-0.044 & 0.077 & 0.009\end{array}$

$\begin{array}{lll}-0.036 & -0.066 & 0.011\end{array}$

$\begin{array}{lll}-0.083 & 0.017 & 0.010\end{array}$

$\begin{array}{lll}-0.019 & -0.031 & 0.003\end{array}$

$\begin{array}{llll}0.054 & 0.276 & -0.011\end{array}$

$\begin{array}{lll}-0.113 & -0.046 & 0.053\end{array}$

$0.076-0.191-0.014$

$\begin{array}{llll}0.566 & 0.049 & -0.119\end{array}$

$\begin{array}{llll}0.004 & 0.000 & -0.028\end{array}$

$\begin{array}{lll}0.003 & -0.001 & 0.008\end{array}$

$0.006-0.001-0.003$

$0.004-0.007-0.018$

$\begin{array}{lll}-0.055 & 0.017-0.026\end{array}$

$\begin{array}{rrr}-0.005 & 0.008 & 0.016\end{array}$

$-0.047-0.020-0.010$

$\begin{array}{lll}0.034 & 0.001 & 0.047\end{array}$

$0.0490 .005-0.019$

\begin{abstract}
$0.004 \quad 0.004$
$0.007 \quad 0.002$

$0.004-0.005$

$0.005-0.008$

0.002

0.003

0.002

0.001
\end{abstract}

1136.95

YES

0.831

YES

$x$
0.000

$\mathrm{Y}$
0.001

$0.008-0.002$

$0.002 \quad 0.003$

0.004

0.000

$-0.007-0.010$

0.001

$-0.005$

0.001

$-0.004$

$-0.028$

0.005

$-0.008$

$-0.011$

0.007

0.004

0.013

0.003

$-0.010-0$

0.001

0.000

$0.010-0.006$

$0.014-0.001$

$\begin{array}{ll}0.004 & 0.008\end{array}$

$0.008-0.001$

$\begin{array}{rr}-0.004 & 0.001\end{array}$$$
-0.019
$$

$-0.104$

$-0.016$

0.014

$0.060-0$

$-0.421$

$-0.035$

$-0.446-0.174$

$0.225-0.060$

0.409

0.093

1205.92

YES

5.845

YES

$\mathrm{x}$

0.000

$Y$

0.010

0.000

0.010

$-0.020-0.037$

0.002$$
\text { ( }
$$

$-0.011$

0.000

0.000

0.034

0.024

$0.038-0.012-0.007$

$\begin{array}{lll}-0.036 & 0.143 & 0.030\end{array}$

$\begin{array}{llll}-0.102 & 0.266 & 0.030\end{array}$

$0.441-0.087-0.096$

$\begin{array}{lll}-0.283 & -0.348 & 0.043\end{array}$

$-0.001-0.006 \quad 0.000$

$-0.037-0.006$

0.006

$\begin{array}{rrr}-0.031 & 0.138 & 0.026\end{array}$

$\begin{array}{rrr}-0.098 & 0.288 & 0.026\end{array}$

$0.341-0.073-0.075$

$\begin{array}{lll}-0.289 & -0.380 & 0.044\end{array}$

$\begin{array}{rrr}-0.011 & 0.000 & 0.002\end{array}$

0.027

$0.009-0.005$ $\begin{array}{rrr}-0.001 & 0.002 & -0.002 \\ 0.000 & 0.003 & -0.002 \\ 0.004 & -0.002 & 0.004 \\ -0.007 & -0.001 & -0.002 \\ -0.002 & 0.001 & -0.001 \\ 0.013 & -0.003 & -0.002\end{array}$

1154.38

YES

0.564

YES

$\begin{array}{ccc}X & Y & Z \\ 0.000 & 0.002 & -0.001\end{array}$

$-0.007-0.073-0.004$

$-0.008-0.063-0.001$

$0.057-0.030-0.007$

$\begin{array}{rrr}-0.010 & 0.069 & 0.004\end{array}$

$\begin{array}{llll}0.021 & 0.067 & -0.001\end{array}$

$\begin{array}{lll}-0.029 & 0.052 & 0.006\end{array}$

$0.119-0.252-0.076$

$\begin{array}{lll}-0.034 & 0.226 & 0.014\end{array}$

$\begin{array}{llll}-0.386 & 0.124 & 0.092\end{array}$

$\begin{array}{lll}-0.226 & -0.345 & 0.033\end{array}$

$\begin{array}{llll}0.016 & 0.069 & -0.003\end{array}$

$\begin{array}{llll}0.035 & 0.047 & -0.004\end{array}$

$\begin{array}{lll}-0.014 & 0.066 & 0.003\end{array}$

$\begin{array}{rrr}0.001 & -0.070 & 0.002\end{array}$

$\begin{array}{lll}-0.057 & -0.024 & 0.004\end{array}$

$\begin{array}{lll}-0.001 & -0.062 & 0.000\end{array}$

$\begin{array}{llll}0.053 & 0.209 & -0.011\end{array}$

$\begin{array}{lll}-0.144 & -0.245 & 0.082\end{array}$

$0.171-0.345-0.036$

$\begin{array}{rrrr}0.403 & 0.080 & -0.091\end{array}$

$\begin{array}{lll}-0.001 & -0.001 & 0.013\end{array}$

$\begin{array}{rrr}-0.004 & -0.001 & 0.004\end{array}$

$0.002-0.004-0.004$

$0.006-0.003-0.012$

$\begin{array}{llll}-0.026 & 0.024 & -0.016\end{array}$

$\begin{array}{lll}-0.003 & 0.002 & 0.014\end{array}$

$-0.037-0.013-0.007$

$\begin{array}{lll}0.023 & 0.000 & 0.031\end{array}$

$0.027 \quad 0.013-0.017$

1233.69

YES

4. 981

YES

$\begin{array}{ll}X & Y\end{array}$

Z

$\begin{array}{llll}0.000 & 0.000 & 0.000\end{array}$

$0.0010 .001-0.001$

$\begin{array}{lll}0.000 & 0.001 & 0.000\end{array}$

$\begin{array}{llll}0.001 & -0.001 & 0.000\end{array}$

$-0.002-0.002 \quad 0.000$

$\begin{array}{llll}0.000 & -0.001 & 0.000\end{array}$

$\begin{array}{rrr}-0.001 & 0.000 & 0.000\end{array}$

$0.001-0.002-0.001$

$\begin{array}{llll}-0.001 & 0.003 & 0.000\end{array}$

$\begin{array}{llll}-0.004 & 0.001 & 0.000\end{array}$

$\begin{array}{lll}0.001 & 0.002 & 0.000\end{array}$

$\begin{array}{lll}-0.002 & 0.002 & 0.000\end{array}$

$\begin{array}{lll}-0.001 & 0.000 & 0.000\end{array}$ 


$\mathrm{C}$
$\mathrm{N}$
$\mathrm{C}$
$\mathrm{C}$
$\mathrm{H}$
$\mathrm{H}$
$\mathrm{H}$
$\mathrm{H}$
$\mathrm{O}$
$\mathrm{O}$
$\mathrm{O}$
$\mathrm{C}$
$\mathrm{H}$
$\mathrm{C}$
$\mathrm{H}$
$\mathrm{H}$
$\mathrm{H}$

$\begin{array}{rrr}0.008 & 0.017 & -0.001 \\ -0.004 & 0.012 & 0.000 \\ 0.004 & 0.021 & 0.002 \\ 0.029 & -0.036 & -0.006 \\ 0.124 & 0.243 & -0.028 \\ 0.047 & 0.133 & -0.030 \\ 0.235 & -0.364 & -0.044 \\ -0.420 & -0.035 & 0.086 \\ 0.001 & 0.000 & -0.009 \\ 0.000 & 0.000 & 0.000 \\ 0.000 & 0.001 & 0.000 \\ 0.000 & 0.000 & 0.000 \\ -0.003 & -0.002 & -0.001 \\ 0.000 & 0.000 & 0.000 \\ 0.004 & -0.004 & 0.002 \\ -0.001 & 0.000 & -0.001 \\ -0.001 & 0.001 & 0.000\end{array}$

Frequency:

IR Active:

IR Intens:

Raman Active:

1253.70

YES

13.309

YES

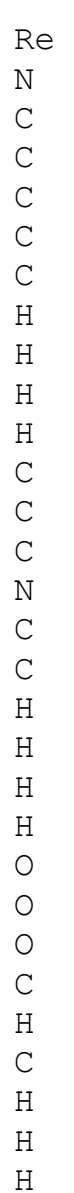

$$
\begin{array}{rrr}
X & \multicolumn{1}{c}{Y} & Z \\
0.000 & 0.000 & -0.001 \\
-0.004 & -0.003 & 0.000 \\
0.000 & 0.001 & 0.000 \\
0.001 & 0.005 & 0.000 \\
-0.003 & -0.001 & 0.001 \\
0.002 & -0.001 & -0.001 \\
0.001 & -0.001 & 0.000 \\
-0.003 & 0.025 & 0.008 \\
0.011 & -0.022 & -0.003 \\
0.003 & -0.001 & -0.003 \\
0.006 & 0.009 & -0.001 \\
-0.004 & 0.003 & 0.001 \\
-0.001 & 0.000 & 0.000 \\
0.002 & 0.002 & 0.000 \\
-0.001 & -0.001 & -0.001 \\
0.002 & 0.000 & 0.000 \\
0.000 & -0.002 & 0.000 \\
0.006 & 0.010 & -0.002 \\
-0.002 & -0.010 & 0.000 \\
0.000 & -0.002 & -0.002 \\
0.004 & 0.001 & -0.002 \\
-0.002 & 0.000 & 0.004 \\
0.019 & -0.020 & 0.005 \\
0.013 & 0.028 & 0.028 \\
0.016 & 0.040 & -0.066 \\
0.118 & 0.308 & -0.046 \\
-0.023 & -0.052 & 0.042 \\
0.170 & -0.420 & 0.160 \\
-0.316 & -0.333 & -0.061 \\
-0.367 & 0.506 & -0.192
\end{array}
$$

$$
\begin{array}{rrr}
-0.005 & -0.037 & 0.000 \\
0.009 & -0.008 & -0.002 \\
0.002 & -0.017 & -0.002 \\
-0.017 & 0.043 & 0.004 \\
-0.135 & -0.292 & 0.030 \\
-0.045 & -0.139 & 0.028 \\
-0.260 & 0.435 & 0.050 \\
0.356 & 0.034 & -0.073 \\
0.001 & 0.000 & -0.006 \\
-0.001 & 0.000 & -0.001 \\
-0.001 & 0.000 & 0.000 \\
0.001 & 0.001 & -0.001 \\
0.001 & 0.002 & -0.001 \\
0.000 & 0.000 & 0.001 \\
0.004 & -0.007 & 0.002 \\
-0.002 & -0.003 & 0.001 \\
-0.002 & 0.005 & -0.002
\end{array}
$$

\begin{tabular}{rrr}
\multicolumn{3}{c}{1303.32} \\
YES \\
X & \multicolumn{1}{r}{ YES } \\
Y & $Z$ \\
0.000 & 0.000 & 0.000 \\
-0.023 & 0.006 & 0.007 \\
-0.020 & -0.054 & 0.002 \\
-0.052 & -0.026 & 0.010 \\
0.160 & 0.085 & -0.030 \\
-0.063 & 0.063 & 0.015 \\
0.024 & 0.010 & -0.005 \\
0.076 & -0.522 & -0.067 \\
-0.137 & 0.250 & 0.038 \\
-0.153 & 0.042 & 0.034 \\
-0.001 & -0.031 & 0.001 \\
0.144 & -0.101 & -0.031 \\
0.011 & -0.010 & -0.004 \\
-0.067 & -0.045 & 0.013 \\
-0.013 & -0.026 & 0.005 \\
-0.050 & 0.046 & 0.013 \\
-0.011 & 0.050 & 0.002 \\
-0.189 & -0.278 & 0.041 \\
0.156 & 0.586 & -0.078 \\
0.003 & 0.029 & 0.003 \\
-0.134 & -0.020 & 0.030 \\
0.001 & 0.000 & -0.005 \\
0.004 & -0.003 & 0.003 \\
0.004 & 0.004 & 0.003 \\
-0.001 & 0.002 & -0.005 \\
-0.003 & 0.015 & -0.006 \\
-0.002 & -0.003 & 0.001 \\
0.004 & -0.020 & 0.006 \\
-0.013 & -0.010 & -0.006 \\
-0.026 & 0.027 & -0.008 \\
& &
\end{tabular}

$$
\begin{array}{rrr}
0.001 & 0.001 & 0.000 \\
0.000 & -0.001 & -0.001 \\
0.001 & 0.002 & 0.000 \\
0.000 & 0.000 & -0.001 \\
-0.001 & -0.003 & -0.002 \\
0.001 & 0.003 & -0.002 \\
-0.001 & 0.002 & 0.005 \\
0.000 & 0.000 & -0.001 \\
-0.001 & 0.000 & 0.007 \\
-0.016 & 0.017 & 0.029 \\
-0.019 & 0.014 & 0.030 \\
-0.001 & -0.026 & -0.036 \\
-0.418 & -0.330 & -0.110 \\
0.037 & -0.015 & -0.057 \\
0.358 & -0.252 & 0.135 \\
0.423 & 0.378 & 0.158 \\
-0.279 & 0.239 & -0.050
\end{array}
$$

1312.65

YES

16.583

YES

$\begin{array}{ccc}\mathrm{X} & \text { YES } & \mathrm{Z} \\ 0.000 & -0.001 & 0.000\end{array}$

$0.101 \quad 0.176-0.010$

$0.043 \quad 0.065-0.006$

$0.000-0.164-0.006$

$\begin{array}{rrr}-0.049 & 0.013 & 0.012\end{array}$

$0.015-0.102-0.006$

$\begin{array}{llll}-0.107 & 0.018 & 0.019\end{array}$

$0.101-0.520-0.073$

$\begin{array}{lll}-0.163 & 0.354 & 0.044\end{array}$

$0.085-0.021-0.010$

$\begin{array}{lll}-0.062 & -0.066 & 0.010\end{array}$

$\begin{array}{llll}0.074 & -0.020 & -0.017\end{array}$

$\begin{array}{llll}0.113 & 0.002 & -0.019\end{array}$

$\begin{array}{lll}-0.039 & -0.110 & 0.007\end{array}$

$\begin{array}{lll}-0.084 & 0.181 & 0.014\end{array}$

$\begin{array}{lll}-0.027 & -0.152 & 0.003\end{array}$

$\begin{array}{llll}-0.036 & 0.079 & 0.008\end{array}$

$\begin{array}{llll}0.185 & 0.316 & -0.036\end{array}$

$\begin{array}{llll}-0.130 & -0.396 & 0.056\end{array}$

$0.050-0.058-0.011$

$\begin{array}{llll}-0.131 & -0.019 & 0.017\end{array}$

$0.000 \quad 0.002-0.003$

$\begin{array}{lll}0.004 & 0.001 & 0.000\end{array}$

$0.000 \quad 0.006 \quad 0.002$

$\begin{array}{lll}0.000 & 0.002 & 0.001\end{array}$

$0.005-0.002 \quad 0.002$

$-0.001-0.008-0.002$

$\begin{array}{llll}-0.003 & 0.008 & -0.003\end{array}$

$-0.017-0.016-0.004$

$\begin{array}{lll}-0.041 & 0.035 & -0.010\end{array}$

1359.28

1351.79

YES

33.940

YES

$\begin{array}{ll}X & Y\end{array}$

$\begin{array}{lll}0.000 & 0.000 & 0.000\end{array}$

$\begin{array}{lll}-0.032 & -0.018 & 0.007\end{array}$
YES

12.262

YES

$\begin{array}{ll}X & Y\end{array}$

$\begin{array}{lll}0.000 & 0.000 & 0.000\end{array}$ $\begin{array}{lll}-0.114 & -0.165 & 0.013\end{array}$ 
Frequency:

IR Active:

IR Intens:

Raman Active

$\begin{array}{rrr}-0.018 & 0.067 & 0.006 \\ 0.025 & -0.041 & -0.006 \\ -0.007 & -0.165 & -0.004 \\ 0.019 & -0.095 & -0.007 \\ -0.067 & -0.003 & 0.012 \\ 0.091 & -0.255 & -0.043 \\ -0.219 & 0.507 & 0.057 \\ 0.118 & -0.038 & -0.020 \\ 0.083 & 0.204 & -0.007 \\ 0.013 & 0.166 & -0.001 \\ -0.064 & 0.011 & 0.011 \\ 0.027 & 0.089 & -0.005 \\ 0.020 & -0.092 & -0.002 \\ 0.029 & 0.034 & -0.005 \\ -0.025 & -0.063 & 0.005 \\ -0.273 & -0.479 & 0.055 \\ 0.112 & 0.226 & -0.040 \\ 0.059 & -0.210 & -0.012 \\ 0.125 & 0.025 & -0.019 \\ 0.001 & 0.000 & 0.001 \\ 0.000 & 0.000 & -0.001 \\ 0.000 & 0.000 & -0.001 \\ 0.002 & 0.002 & 0.000 \\ 0.007 & 0.010 & 0.001 \\ -0.002 & 0.001 & 0.001 \\ 0.002 & -0.012 & 0.004 \\ -0.016 & -0.016 & -0.006 \\ 0.003 & -0.006 & 0.003\end{array}$

1364.82

YES

3.764

YES

$\begin{array}{ll}X & Y\end{array}$

$0.000-0.002$

$0.001-0.001$

$0.006-0.005-0.001$

$0.003-0.001-0.001$

$\begin{array}{rrrr}-0.015 & -0.001 & 0.003\end{array}$

$\begin{array}{rrrr}0.003 & 0.000 & -0.001\end{array}$

$\begin{array}{lll}-0.002 & -0.001 & 0.000\end{array}$

$\begin{array}{rrr}-0.010 & 0.050 & 0.006\end{array}$

$0.015-0.030-0.004$

$0.000-0.002 \quad 0.000$

$\begin{array}{llll}-0.014 & -0.021 & 0.002\end{array}$

$\begin{array}{lll}0.015 & 0.002 & -0.003\end{array}$

$\begin{array}{rrr}0.001 & -0.001 & 0.000\end{array}$

$\begin{array}{lll}-0.002 & 0.002 & 0.000\end{array}$

$\begin{array}{llll}-0.001 & 0.000 & -0.001\end{array}$

$\begin{array}{lll}-0.002 & -0.003 & 0.001\end{array}$

$\begin{array}{rrrr}-0.007 & 0.004 & 0.002\end{array}$

$\begin{array}{lll}-0.023 & -0.038 & 0.005\end{array}$

$0.016 \quad 0.043-0.007$

$0.014-0.031-0.005$

$0.008-0.001-0.001$

$0.0010 .000-0.008$

$\begin{array}{llll}-0.019 & 0.017-0.020\end{array}$

$\begin{array}{lll}0.031 & 0.017 & 0.002\end{array}$

$\begin{array}{lll}-0.066 & -0.058 & 0.004\end{array}$

$\begin{array}{llll}0.284 & 0.295 & 0.062\end{array}$

$0.047-0.088$

$$
\begin{array}{rrr}
-0.073 & -0.052 & 0.012 \\
-0.036 & 0.068 & 0.012 \\
0.161 & -0.033 & -0.035 \\
-0.029 & 0.006 & 0.006 \\
0.032 & 0.000 & -0.006 \\
0.096 & -0.428 & -0.061 \\
-0.161 & 0.342 & 0.048 \\
0.082 & -0.008 & -0.016 \\
0.196 & 0.294 & -0.026 \\
-0.123 & 0.012 & 0.027 \\
-0.011 & 0.001 & 0.003 \\
0.024 & -0.003 & -0.005 \\
0.004 & 0.021 & -0.003 \\
0.045 & 0.028 & -0.011 \\
0.044 & -0.049 & -0.009 \\
0.160 & 0.254 & -0.034 \\
-0.156 & -0.486 & 0.067 \\
-0.130 & 0.233 & 0.024 \\
-0.095 & -0.005 & 0.016 \\
0.000 & 0.000 & -0.002 \\
-0.004 & 0.002 & -0.003 \\
0.005 & 0.002 & 0.000 \\
-0.007 & -0.007 & 0.001 \\
0.036 & 0.034 & 0.009 \\
0.005 & -0.013 & 0.005 \\
-0.040 & 0.065 & -0.023 \\
0.038 & 0.044 & 0.003 \\
-0.037 & 0.058 & -0.025
\end{array}
$$

1389.95

YES

6.414

YES

$\begin{array}{cc}X & Y \\ 0.001 & 0.000\end{array}$

$-0.001 \quad 0.001$

$0.000-0.001$

$\begin{array}{rr}-0.001 & 0.001\end{array}$

$\begin{array}{rr}0.001 & 0.001\end{array}$

$0.001-0.001$

$\begin{array}{rr}-0.001 & 0.000\end{array}$

$0.003-0.011$

$\begin{array}{rr}-0.001 & 0.005\end{array}$

$0.005-0.001$

$\begin{array}{lll}0.004 & 0.004 & 0.000\end{array}$

$0.003 \quad 0.001-0.001$

$\begin{array}{rrrr}0.003 & 0.000 & -0.001\end{array}$

$\begin{array}{lll}-0.002 & -0.003 & 0.000\end{array}$

$\begin{array}{rrr}-0.001 & 0.003 & 0.001\end{array}$

$\begin{array}{llll}0.000 & -0.001 & 0.000\end{array}$

$\begin{array}{cccc}-0.002 & 0.001 & 0.001\end{array}$

$\begin{array}{rrr}0.002 & 0.005 & 0.000\end{array}$

$-0.002-0.006-0.001$

$\begin{array}{llll}-0.002 & 0.002 & -0.003\end{array}$

$\begin{array}{lll}-0.009 & -0.001 & 0.003\end{array}$

$\begin{array}{lll}0.001 & 0.000 & 0.005\end{array}$

$\begin{array}{llll}-0.020 & 0.021 & -0.028\end{array}$

$\begin{array}{llll}-0.026 & -0.013 & 0.003\end{array}$

$-0.039-0.107-0.002$

$\begin{array}{lll}0.462 & 0.499 & 0.091\end{array}$

$\begin{array}{rrrr}-0.010 & 0.094 & -0.008\end{array}$ $\begin{array}{llll}-0.080 & -0.018 & 0.015\end{array}$

$\begin{array}{lll}0.032 & 0.147 & 0.002\end{array}$

$0.093-0.141-0.024$

$\begin{array}{rrrr}-0.003 & 0.020 & 0.001\end{array}$

$0.077-0.007-0.013$

$\begin{array}{lll}-0.053 & 0.460 & 0.047\end{array}$

$\begin{array}{llll}-0.070 & 0.191 & 0.020\end{array}$

$\begin{array}{lll}-0.037 & 0.020 & 0.001\end{array}$

$\begin{array}{llll}0.133 & 0.263-0.016\end{array}$

$0.187 \quad 0.133-0.038$

$0.094-0.003-0.016$

$\begin{array}{lll}-0.022 & -0.027 & 0.004\end{array}$

$\begin{array}{llll}-0.106 & 0.180 & 0.019\end{array}$

$\begin{array}{llll}-0.010 & -0.178 & 0.004\end{array}$

$\begin{array}{llll}-0.109 & 0.059 & 0.022\end{array}$

$\begin{array}{lll}-0.175 & -0.317 & 0.039\end{array}$

$\begin{array}{lll}-0.026 & -0.212 & 0.009\end{array}$

$0.179-0.419-0.038$

$-0.008-0.017-0.006$

$\begin{array}{lll}-0.002 & 0.000 & 0.002\end{array}$

$\begin{array}{llll}0.001 & 0.000 & 0.002\end{array}$

$-0.003-0.002 \quad 0.000$

$\begin{array}{rrr}0.004 & 0.004 & 0.000\end{array}$

$-0.031-0.036-0.006$

$\begin{array}{rrrr}-0.001 & 0.004 & -0.004\end{array}$

$\begin{array}{rrr}0.017 & -0.009 & 0.007\end{array}$

$\begin{array}{lll}-0.011 & -0.014 & 0.002\end{array}$

$0.016-0.022 \quad 0.006$

1467.69

YES

3.008

YES

$\begin{array}{ccc}X & Y & Z \\ 0.000 & 0.000 & 0.000\end{array}$

$0.029-0.049-0.009$

$-0.016-0.101-0.001$

$\begin{array}{lll}0.012 & 0.096 & 0.004\end{array}$

$\begin{array}{rrr}-0.068 & 0.042 & 0.015\end{array}$

$0.050 \quad 0.035-0.009$

$\begin{array}{rrr}-0.091 & 0.019 & 0.018\end{array}$

$0.144-0.343-0.058$

$0.078-0.007-0.015$

$0.453-0.083-0.091$

$\begin{array}{llll}0.256 & 0.227 & -0.041\end{array}$

$\begin{array}{lll}-0.068 & -0.028 & 0.013\end{array}$

$\begin{array}{lll}-0.082 & -0.004 & 0.015\end{array}$

$\begin{array}{llll}0.039 & -0.044 & -0.008\end{array}$

$0.037 \quad 0.039-0.008$

$\begin{array}{lll}-0.005 & -0.097 & 0.004\end{array}$

$\begin{array}{lll}-0.002 & 0.096 & 0.001\end{array}$

$0.0820 .019-0.016$

$0.1750 .329-0.056$

$0.207-0.222-0.041$

$0.4250 .035-0.080$

$0.000 \quad 0.000-0.001$

$\begin{array}{rrr}-0.001 & 0.001-0.001\end{array}$

$-0.001-0.001-0.001$

$\begin{array}{rrr}0.000 & 0.000 & -0.001\end{array}$

$-0.002-0.005-0.001$

$\begin{array}{lll}0.001 & 0.001 & 0.000\end{array}$ 


$\begin{array}{rrrr}\mathrm{H} & -0.277 & 0.454 & -0.158 \\ \mathrm{H} & 0.298 & 0.361 & 0.006 \\ \mathrm{H} & -0.231 & 0.425 & -0.187\end{array}$

\begin{tabular}{|c|c|c|c|}
\hline Freq & & 1491.04 & \\
\hline IR A & & YES & \\
\hline IR II & & 51.907 & \\
\hline Ramal & ve: & YES & \\
\hline & X & $Y$ & Z \\
\hline $\mathrm{Re}$ & 0.000 & 0.001 & 0.000 \\
\hline $\mathrm{N}$ & -0.023 & -0.078 & -0.001 \\
\hline C & -0.009 & -0.074 & -0.001 \\
\hline C & 0.077 & 0.033 & -0.012 \\
\hline C & -0.084 & 0.066 & 0.020 \\
\hline $\mathrm{C}$ & 0.075 & -0.026 & -0.016 \\
\hline $\mathrm{C}$ & -0.136 & 0.055 & 0.029 \\
\hline $\mathrm{H}$ & 0.089 & 0.054 & -0.016 \\
\hline $\mathrm{H}$ & 0.037 & 0.117 & -0.004 \\
\hline $\mathrm{H}$ & 0.455 & -0.048 & -0.088 \\
\hline $\mathrm{H}$ & 0.277 & 0.276 & -0.044 \\
\hline C & 0.097 & 0.058 & -0.020 \\
\hline C & 0.150 & 0.041 & -0.029 \\
\hline C & -0.082 & -0.013 & 0.017 \\
\hline N & 0.012 & -0.086 & 0.000 \\
\hline $\mathrm{C}$ & -0.073 & 0.052 & 0.012 \\
\hline C & 0.002 & -0.085 & -0.001 \\
\hline $\mathrm{H}$ & -0.029 & 0.122 & 0.008 \\
\hline $\mathrm{H}$ & -0.099 & 0.035 & 0.023 \\
\hline $\mathrm{H}$ & -0.269 & 0.333 & 0.055 \\
\hline $\mathrm{H}$ & -0.501 & -0.001 & 0.094 \\
\hline 0 & 0.000 & -0.001 & -0.002 \\
\hline 0 & 0.000 & -0.002 & 0.001 \\
\hline 0 & 0.001 & -0.002 & -0.001 \\
\hline $\mathrm{C}$ & 0.001 & 0.002 & -0.001 \\
\hline $\mathrm{H}$ & -0.010 & -0.009 & -0.003 \\
\hline $\mathrm{C}$ & 0.000 & -0.001 & 0.001 \\
\hline $\mathrm{H}$ & -0.008 & 0.006 & -0.004 \\
\hline $\mathrm{H}$ & -0.002 & -0.002 & 0.001 \\
\hline $\mathrm{H}$ & 0.000 & -0.001 & 0.002 \\
\hline
\end{tabular}

$\begin{array}{rrr}0.278 & -0.457 & 0.164 \\ 0.268 & 0.212 & 0.072 \\ 0.189 & -0.168 & 0.075\end{array}$

0.164

0.072

1505.46

YES

68.956

YES

$\begin{array}{rrr}X & Y & Z \\ 0.000 & 0.000 & 0.000 \\ -0.074 & -0.008 & 0.015 \\ -0.045 & 0.034 & 0.010 \\ 0.043 & -0.096 & -0.014 \\ 0.078 & 0.057 & -0.014 \\ 0.026 & -0.119 & -0.010 \\ 0.037 & 0.041 & -0.005 \\ -0.116 & 0.475 & 0.060 \\ -0.153 & 0.346 & 0.044 \\ -0.168 & 0.091 & 0.036 \\ 0.015 & 0.131 & 0.001 \\ -0.074 & 0.070 & 0.016 \\ -0.029 & 0.048 & 0.005 \\ -0.041 & -0.115 & 0.008 \\ 0.075 & -0.018 & -0.015 \\ -0.055 & -0.091 & 0.013 \\ 0.051 & 0.024 & -0.010 \\ 0.196 & 0.339 & -0.038 \\ 0.165 & 0.452 & -0.055 \\ -0.010 & 0.146 & 0.004 \\ 0.167 & 0.075 & -0.032 \\ 0.000 & -0.001 & -0.001 \\ 0.000 & 0.000 & 0.001 \\ -0.001 & -0.001 & -0.001 \\ 0.001 & -0.005 & 0.004 \\ -0.039 & 0.044 & -0.002 \\ -0.004 & -0.004 & 0.002 \\ 0.037 & 0.037 & 0.025 \\ -0.001 & 0.019 & -0.057 \\ 0.040 & 0.013 & -0.039\end{array}$

1535.41

YES

26.991

0.949

IR Active:

IR Intens:

Raman Active:

YES

x. $x$

0.000

$0.000 \quad 0.000$

$0.000-0.001$

$0.001-0.001$

$0.000 \quad 0.003$

$\begin{array}{ll}0.001 & 0.000\end{array}$

$-0.002$

0.001

$0.002-0.003$

$\begin{array}{ll}-0.001 & 0.004\end{array}$

0.005

$0.000-0.001$

0.004

0.004

$0.001-0.004$

$-0.002-0.001$

$0.001 \quad 0.002$

$-0.001$

0.000

0.002

0.002
O. $\begin{gathered}\text { Z } \\ 0.000\end{gathered}$

0.000

0.000

0.000

0.000

0.000

0.000

0.000

0.000

-0.001
-0.001

0.000

0.000

0.000

0.000

0.000

\section{YES}

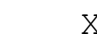

\subsection{0}

0.000

0.074

0.032

0.021

$-0.093$

0.11

$-0.040-0.178$

$-0.0490 .100$

0.084

$0.050-0.43$

$0.120-0.364-0.035$

$\begin{array}{lll}-0.131 & -0.058 & 0.021\end{array}$

$\begin{array}{lll}-0.151 & -0.199 & 0.023\end{array}$

$\begin{array}{lll}-0.019 & 0.179 & 0.005\end{array}$

$0.0920 .071-0.018$

$\begin{array}{lll}-0.060 & -0.090 & 0.011\end{array}$

$0.069-0.039-0.012$

$\begin{array}{lll}-0.102 & -0.101 & 0.021\end{array}$
0.019 $\begin{array}{rrr}-0.008 & -0.006 & -0.006 \\ 0.000 & -0.001 & 0.003 \\ -0.007 & -0.002 & 0.007\end{array}$

1511.53

YES

2.468

YES

$\begin{array}{rrr}\text { X } & Y & Z \\ 0.000 & 0.000 & 0.000 \\ -0.004 & 0.000 & 0.001 \\ -0.002 & 0.003 & 0.001 \\ 0.002 & -0.005 & -0.001 \\ 0.005 & 0.002 & -0.001 \\ 0.001 & -0.007 & 0.000 \\ 0.004 & 0.001 & -0.001 \\ -0.008 & 0.029 & 0.004 \\ -0.009 & 0.018 & 0.002 \\ -0.014 & 0.005 & 0.003 \\ -0.002 & 0.004 & 0.001 \\ -0.004 & 0.006 & 0.001 \\ -0.001 & 0.003 & 0.000 \\ -0.003 & -0.007 & 0.001 \\ 0.005 & -0.002 & -0.001 \\ -0.004 & -0.005 & 0.001 \\ 0.003 & 0.001 & -0.001 \\ 0.011 & 0.021 & -0.002 \\ 0.011 & 0.030 & -0.003 \\ -0.002 & 0.010 & -0.001 \\ 0.007 & 0.005 & -0.001 \\ 0.000 & 0.000 & 0.000 \\ -0.010 & 0.000 & -0.006 \\ 0.010 & 0.000 & 0.002 \\ -0.028 & 0.043 & -0.042 \\ 0.433 & -0.352 & 0.029 \\ 0.039 & 0.025 & -0.006 \\ -0.304 & -0.235 & -0.205 \\ 0.051 & -0.115 & 0.543 \\ -0.311 & -0.047 & 0.277\end{array}$

1615.38

YES

5.140

YES

$\begin{array}{ccc}X & Y\end{array}$

$\begin{array}{rrr}0.000 & 0.000 & 0.000\end{array}$

$\begin{array}{lll}-0.086 & -0.138 & 0.009\end{array}$

$0.2320 .231-0.036$

$\begin{array}{lll}0.047 & 0.151 & 0.000\end{array}$

$0.1190 .043-0.023$

$\begin{array}{lll}-0.086 & -0.107 & 0.013\end{array}$

$\begin{array}{llll}-0.208 & -0.090 & 0.036\end{array}$

$0.156-0.196-0.051$

$\begin{array}{llll}-0.106 & -0.094 & 0.020\end{array}$

$0.192-0.189-0.044$

$-0.266-0.413 \quad 0.036$

$0.074-0.038-0.016$

$\begin{array}{lll}-0.149 & 0.080 & 0.029\end{array}$

$\begin{array}{lll}-0.055 & 0.076 & 0.012\end{array}$

$\begin{array}{rrr}-0.048 & 0.108 & 0.008\end{array}$

$0.023-0.112-0.002$ 


$\begin{array}{lrrr}\mathrm{C} & -0.001 & 0.001 & 0.000 \\ \mathrm{H} & -0.004 & -0.008 & 0.001 \\ \mathrm{H} & -0.001 & -0.004 & 0.001 \\ \mathrm{H} & 0.003 & -0.005 & -0.001 \\ \mathrm{H} & 0.002 & -0.001 & 0.000 \\ \mathrm{O} & 0.000 & 0.000 & -0.002 \\ \mathrm{O} & -0.004 & 0.001 & -0.005 \\ \mathrm{O} & -0.009 & 0.000 & 0.000 \\ \mathrm{C} & -0.023 & 0.037 & -0.033 \\ \mathrm{H} & 0.324 & -0.272 & 0.020 \\ \mathrm{C} & -0.056 & -0.040 & 0.009 \\ \mathrm{H} & 0.393 & 0.309 & 0.271 \\ \mathrm{H} & 0.014 & -0.104 & 0.415 \\ \mathrm{H} & 0.382 & 0.093 & -0.388\end{array}$

\begin{tabular}{lrrr} 
Frequency: & \multicolumn{3}{c}{1622.90} \\
IR Active: & \multicolumn{3}{c}{ YES } \\
IR Intens: & \multicolumn{3}{c}{ Y 10} \\
Raman Active: & \multicolumn{3}{c}{ Y } \\
& X & Y \\
Re & 0.000 & 0.001 & 0.000 \\
N & -0.074 & -0.100 & 0.009 \\
C & 0.158 & 0.185 & -0.024 \\
C & 0.025 & 0.120 & 0.003 \\
C & 0.111 & 0.044 & -0.022 \\
C & -0.052 & -0.116 & 0.006 \\
C & -0.124 & -0.075 & 0.021 \\
H & 0.100 & -0.126 & -0.034 \\
H & -0.118 & 0.019 & 0.025 \\
H & 0.084 & -0.132 & -0.021 \\
H & -0.212 & -0.288 & 0.031 \\
C & -0.137 & 0.074 & 0.030 \\
C & 0.168 & -0.122 & -0.033 \\
C & 0.058 & -0.157 & -0.013 \\
N & 0.084 & -0.154 & -0.015 \\
C & -0.018 & 0.171 & 0.000 \\
C & -0.193 & 0.277 & 0.040 \\
H & 0.148 & -0.018 & -0.033 \\
H & -0.163 & -0.167 & 0.049 \\
H & 0.246 & -0.438 & -0.050 \\
H & -0.153 & -0.171 & 0.028 \\
O & 0.000 & -0.001 & 0.000 \\
O & -0.001 & -0.003 & 0.001 \\
O & 0.001 & -0.003 & -0.001 \\
C & 0.002 & 0.001 & 0.000 \\
H & -0.005 & -0.004 & -0.001 \\
C & -0.001 & 0.000 & 0.001 \\
H & -0.002 & 0.004 & 0.000 \\
H & -0.001 & -0.001 & 0.001 \\
H & 0.003 & -0.001 & -0.001
\end{tabular}

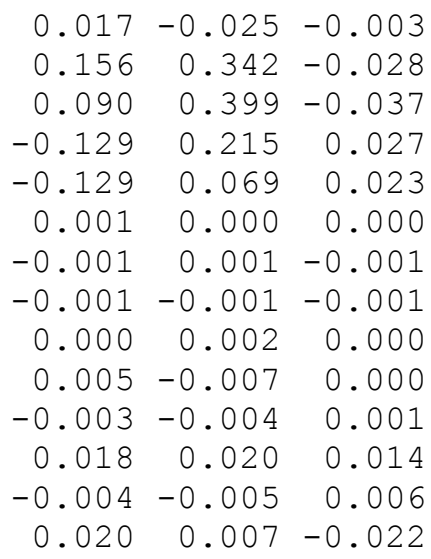

\begin{tabular}{rrr}
\multicolumn{3}{c}{1646.83} \\
Y & YES \\
X & YES \\
-0.001 & 0.000 & 0.000 \\
-0.041 & 0.054 & 0.011 \\
0.063 & -0.089 & -0.016 \\
0.102 & -0.143 & -0.027 \\
0.110 & -0.163 & -0.029 \\
-0.107 & 0.208 & 0.030 \\
-0.163 & 0.083 & 0.035 \\
0.004 & 0.265 & 0.018 \\
0.112 & -0.382 & -0.034 \\
0.260 & 0.013 & -0.047 \\
0.122 & -0.047 & -0.025 \\
0.134 & 0.150 & -0.026 \\
-0.177 & -0.064 & 0.034 \\
-0.133 & -0.196 & 0.026 \\
-0.050 & -0.048 & 0.011 \\
0.119 & 0.130 & -0.024 \\
0.077 & 0.080 & -0.015 \\
0.153 & 0.371 & -0.028 \\
-0.022 & -0.258 & 0.014 \\
0.126 & 0.039 & -0.023 \\
0.264 & -0.039 & -0.048 \\
0.001 & 0.000 & -0.003 \\
0.001 & -0.001 & 0.000 \\
0.001 & 0.000 & 0.000 \\
0.000 & 0.000 & 0.000 \\
0.000 & 0.000 & 0.000 \\
0.000 & 0.000 & 0.000 \\
0.000 & 0.000 & 0.000 \\
0.000 & 0.000 & 0.000 \\
0.000 & 0.000 & 0.000 \\
& & \\
0.01 &
\end{tabular}

$\begin{array}{rrr}0.150 & -0.181 & -0.031 \\ -0.055 & 0.096 & 0.013 \\ 0.132 & 0.133 & -0.037 \\ -0.154 & 0.322 & 0.032 \\ 0.165 & 0.120 & -0.030 \\ -0.001 & 0.000 & 0.000 \\ 0.000 & 0.001 & 0.001 \\ 0.000 & -0.001 & 0.000 \\ 0.000 & -0.001 & -0.001 \\ -0.004 & -0.002 & -0.001 \\ -0.001 & 0.001 & -0.001 \\ 0.004 & 0.001 & 0.002 \\ 0.001 & 0.000 & 0.001 \\ 0.003 & -0.001 & -0.002\end{array}$

1659.00 YES 23.593 YES

$\begin{array}{rrr}X & Y & Z \\ 0.000 & 0.000 & 0.000 \\ 0.059 & -0.073 & -0.016 \\ -0.082 & 0.074 & 0.020 \\ -0.115 & 0.178 & 0.031 \\ -0.207 & 0.118 & 0.048 \\ 0.139 & -0.195 & -0.036 \\ 0.184 & -0.091 & -0.040 \\ 0.008 & -0.317 & -0.024 \\ -0.038 & 0.307 & 0.017 \\ -0.251 & -0.020 & 0.045 \\ -0.093 & 0.093 & 0.021 \\ 0.220 & 0.088 & -0.046 \\ -0.194 & -0.067 & 0.037 \\ -0.159 & -0.173 & 0.032 \\ -0.068 & -0.063 & 0.014 \\ 0.133 & 0.161 & -0.027 \\ 0.093 & 0.060 & -0.018 \\ 0.068 & 0.295 & -0.011 \\ -0.039 & -0.301 & 0.020 \\ 0.099 & 0.086 & -0.018 \\ 0.248 & -0.044 & -0.044 \\ 0.000 & 0.000 & -0.001 \\ 0.000 & 0.000 & 0.000 \\ 0.000 & 0.000 & 0.000 \\ 0.001 & 0.001 & 0.000 \\ -0.007 & -0.004 & -0.002 \\ 0.000 & 0.000 & 0.000 \\ -0.001 & 0.003 & 0.000 \\ -0.001 & -0.002 & -0.001 \\ 0.003 & -0.002 & 0.000\end{array}$

3105.88 YES 10.914 YES

$\begin{array}{ccc}X & Y & Z \\ 0.000 & 0.000 & 0.000 \\ 0.000 & 0.000 & 0.000 \\ 0.000 & 0.000 & 0.000 \\ 0.000 & 0.000 & 0.000 \\ 0.000 & 0.000 & 0.000\end{array}$




$\begin{array}{lrrr}\text { C } & 0.000 & 0.000 & 0.000 \\ \mathrm{C} & 0.000 & 0.000 & 0.000 \\ \mathrm{H} & -0.001 & 0.000 & 0.000 \\ \mathrm{H} & 0.000 & 0.000 & 0.000 \\ \mathrm{H} & 0.000 & 0.000 & 0.000 \\ \mathrm{H} & -0.001 & 0.001 & 0.000 \\ \mathrm{C} & 0.000 & 0.000 & 0.000 \\ \mathrm{C} & 0.000 & 0.000 & 0.000 \\ \mathrm{C} & 0.000 & 0.000 & 0.000 \\ \mathrm{~N} & 0.000 & 0.000 & 0.000 \\ \mathrm{C} & 0.000 & 0.000 & 0.000 \\ \mathrm{C} & 0.000 & 0.000 & 0.000 \\ \mathrm{H} & -0.001 & 0.001 & 0.000 \\ \mathrm{H} & 0.000 & 0.000 & 0.000 \\ \mathrm{H} & -0.002 & -0.001 & 0.000 \\ \mathrm{H} & 0.000 & 0.000 & 0.000 \\ \mathrm{O} & 0.000 & 0.000 & 0.000 \\ \mathrm{O} & 0.001 & 0.000 & 0.000 \\ \mathrm{O} & 0.002 & -0.001 & 0.001 \\ \mathrm{C} & -0.002 & -0.005 & 0.023 \\ \mathrm{H} & 0.042 & 0.006 & -0.286 \\ \mathrm{H} & 0.051 & 0.022 & -0.038 \\ \mathrm{C} & -0.439 & 0.006 & 0.733 \\ \mathrm{H} & -0.034 & 0.034 & 0.017 \\ \mathrm{H} & -0.187 & -0.233 & -0.298 \\ \mathrm{H} & & \end{array}$

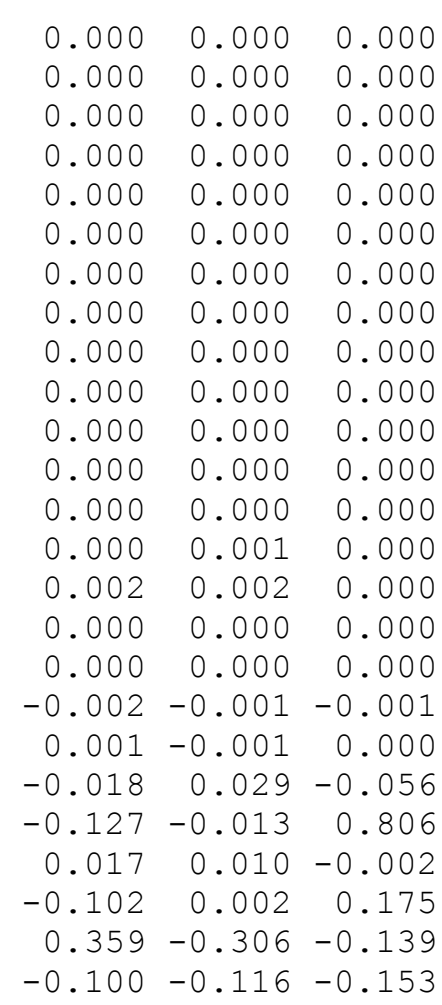

Frequency:
IR Active:

IR Intens:

Raman Active:

\subsection{5}

YES

22.321

YES

$$
0.000 \quad 0.000
$$

$0.000 \quad 0.000$

$0.000 \quad 0.000$

$0.000 \quad 0.000$

$0.000 \quad 0.000$

$0.000 \quad 0.000$

$0.000 \quad 0.000$

0.0010 .000

$0.000 \quad 0.000$

$0.000 \quad 0.000$

$0.001-0.001$

$0.000 \quad 0.000$

$0.000 \quad 0.000$

$0.000 \quad 0.000$

$0.000 \quad 0.000$

$0.000 \quad 0.000$

$0.000 \quad 0.000$

$0.000 \quad 0.000$

$-0.001$

0.000

0.002

0.000

0.001

0.000

0.001

0.000

0.000

0.000

$-0.001$

0.000

$0.037-0.028$

$-0.054-0.017$

$-0.007-0.030$

$-0.212-0.008$

$-0.389$

0.295

0.343

0.357

Z

0.000

0.000

0.000

0.000

0.000

0.000

0.000

0.000

0.000

0.000

0.000

0.000

0.000

0.000

0.000

0.000

0.000

0.000

0.000

0.000

0.000

0.000

0.000

0.000

$-0.043$

0.377

$-0.065$

0.340

0.132

0.429

\author{
3219.44 \\ YES \\ 0.773 \\ YES
}

$x$
0.000
0.000

$x$

$Y$

0.000

$-0.060$

0.007

0.000

0.

0.000

0.000

0.000

0.0490 .014

$0.003-0.001$

$-0.001$

0.023

$0.000 \quad 0.000$

$0.006-0.004$

$-0.002-0.023-0.001$

$\begin{array}{lll}-0.081 & -0.025 & 0.012\end{array}$

$\begin{array}{lll}-0.259 & -0.101 & 0.049\end{array}$

$\begin{array}{lll}0.049 & 0.271 & 0.004\end{array}$

$0.682-0.557-0.159$

$\begin{array}{lll}0.000 & 0.000 & 0.000\end{array}$

$\begin{array}{llll}0.000 & -0.003 & 0.000\end{array}$

$\begin{array}{lll}-0.009 & 0.004 & 0.002\end{array}$

$\begin{array}{rrr}0.000 & 0.000 & 0.000\end{array}$

$\begin{array}{rrr}-0.001 & 0.001 & 0.000\end{array}$

$\begin{array}{llll}0.008 & 0.005 & -0.002\end{array}$

$0.102-0.054-0.022$

$0.012-0.005-0.002$

$\begin{array}{lll}-0.096 & -0.062 & 0.019\end{array}$

$-0.003$

0.038

0.000

0.000

0.000

0.000

0.000

0.000

0.000

0.000

0.000

0.000

0.000

0.000

0.000

0.000

$0.000-0.001$

$\begin{array}{llll}0.000 & 0.000 & 0.000\end{array}$

0.000

0.000

0.000

$\begin{array}{llll}0.000 & 0.000 & 0.000\end{array}$

$-0.001-0.001-0.001$

$\begin{array}{rrr}0.000 & 0.000 & 0.000 \\ 0.000 & 0.000 & 0.000 \\ 0.000 & 0.000 & 0.000 \\ 0.000 & 0.000 & 0.000 \\ 0.000 & 0.000 & 0.000 \\ 0.000 & 0.000 & 0.000 \\ 0.000 & 0.000 & 0.000 \\ 0.000 & 0.000 & 0.000 \\ 0.000 & 0.000 & 0.000 \\ 0.000 & 0.000 & 0.000 \\ 0.000 & 0.000 & 0.000 \\ 0.000 & 0.000 & 0.000 \\ 0.000 & 0.000 & 0.000 \\ 0.001 & 0.000 & 0.000 \\ 0.000 & 0.000 & 0.000 \\ 0.000 & 0.000 & 0.000 \\ 0.000 & 0.000 & 0.000 \\ 0.000 & 0.000 & -0.001 \\ 0.000 & 0.000 & 0.000 \\ -0.045 & 0.037 & 0.039 \\ 0.040 & 0.013 & -0.293 \\ -0.014 & -0.030 & -0.051 \\ -0.122 & -0.005 & 0.192 \\ 0.498 & -0.440 & -0.171 \\ 0.288 & 0.349 & 0.420\end{array}$

\begin{tabular}{rrr}
\multicolumn{4}{c}{3223.16} \\
\multicolumn{3}{c}{ YES } \\
X & YES \\
\hline & $Y$ & $Z$ \\
0.000 & 0.000 & 0.000 \\
0.000 & 0.000 & 0.000 \\
-0.011 & 0.009 & 0.003 \\
0.002 & 0.001 & 0.000 \\
0.000 & 0.000 & 0.000 \\
-0.005 & -0.002 & 0.001 \\
-0.001 & -0.006 & 0.000 \\
-0.027 & -0.008 & 0.004 \\
0.051 & 0.021 & -0.010 \\
0.012 & 0.066 & 0.001 \\
0.124 & -0.102 & -0.029 \\
-0.001 & 0.000 & 0.000 \\
0.000 & 0.029 & 0.000 \\
0.026 & -0.011 & -0.005 \\
0.000 & 0.000 & 0.000 \\
0.010 & -0.005 & -0.002 \\
-0.061 & -0.040 & 0.012 \\
-0.287 & 0.152 & 0.061 \\
-0.118 & 0.051 & 0.019 \\
0.700 & 0.453 & -0.139 \\
0.023 & -0.341 & -0.003 \\
0.000 & 0.000 & 0.000 \\
0.000 & 0.000 & 0.000 \\
0.000 & 0.000 & 0.000 \\
0.000 & 0.000 & 0.000 \\
0.000 & 0.000 & -0.003 \\
0.000 & 0.000 & 0.000 \\
0.000 & 0.000 & 0.000 \\
0.000 & 0.000 & 0.000 \\
0.000 & -0.001 & -0.001
\end{tabular}




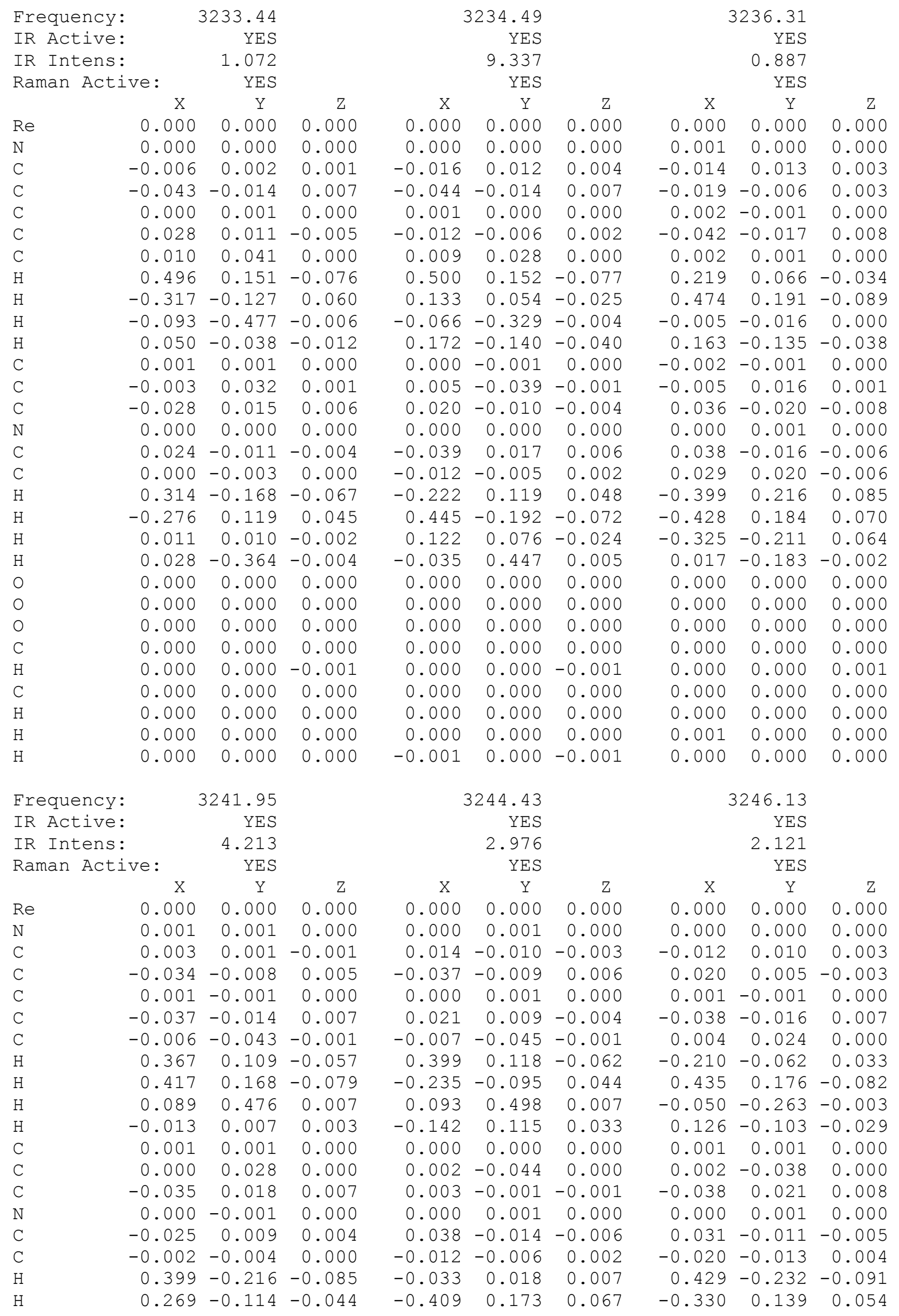




\begin{tabular}{lrrrrrrrrr}
$\mathrm{H}$ & 0.037 & 0.027 & -0.007 & 0.116 & 0.073 & -0.023 & 0.211 & 0.136 & -0.042 \\
$\mathrm{H}$ & 0.021 & -0.310 & -0.002 & -0.034 & 0.484 & 0.004 & -0.030 & 0.417 & 0.004 \\
$\mathrm{O}$ & 0.000 & 0.000 & -0.001 & 0.000 & 0.000 & -0.001 & 0.000 & 0.000 & 0.000 \\
$\mathrm{O}$ & 0.000 & 0.000 & 0.000 & 0.000 & 0.000 & 0.000 & 0.000 & 0.000 & 0.000 \\
$\mathrm{O}$ & 0.000 & 0.000 & 0.000 & 0.000 & 0.000 & 0.000 & 0.000 & 0.000 & 0.000 \\
$\mathrm{C}$ & 0.000 & 0.000 & 0.000 & 0.000 & 0.000 & 0.000 & 0.000 & 0.000 & 0.000 \\
$\mathrm{H}$ & 0.000 & 0.000 & 0.000 & 0.000 & 0.000 & -0.001 & 0.000 & 0.000 & -0.002 \\
$\mathrm{C}$ & 0.000 & 0.000 & 0.000 & 0.000 & 0.000 & 0.000 & 0.000 & 0.000 & 0.000 \\
$\mathrm{H}$ & -0.001 & 0.000 & 0.001 & 0.000 & 0.000 & 0.000 & 0.000 & 0.000 & 0.000 \\
$\mathrm{H}$ & 0.000 & 0.000 & 0.000 & 0.001 & 0.000 & 0.000 & 0.000 & 0.000 & 0.000 \\
$\mathrm{H}$ & 0.000 & 0.000 & 0.000 & 0.000 & 0.000 & -0.001 & 0.000 & -0.001 & -0.001 \\
\multicolumn{2}{l}{ STANDARD THERMODYNAMIC QUANTITIES AT } & $298.18 \mathrm{~K}$ & AND & 1.00 & ATM &
\end{tabular}

This Molecule has 0 Imaginary Frequencies

Zero point vibrational energy: $\quad 144.640 \mathrm{kcal} / \mathrm{mol}$

\begin{tabular}{|c|c|c|c|c|c|c|}
\hline Atom & 1 & Element & $\mathrm{Re}$ & Has & Mass & 186.95600 \\
\hline Atom & 2 & Element & $\mathrm{N}$ & Has & Mass & 14.00307 \\
\hline Atom & 3 & Element & $\mathrm{C}$ & Has & Mass & 12.00000 \\
\hline Atom & 4 & Element & $\mathrm{C}$ & Has & Mass & 12.00000 \\
\hline Atom & 5 & Element & $\mathrm{C}$ & Has & Mass & 12.00000 \\
\hline Atom & 6 & Element & $\mathrm{C}$ & Has & Mass & 12.00000 \\
\hline Atom & 7 & Element & $\mathrm{C}$ & Has & Mass & 12.00000 \\
\hline Atom & 8 & Element & $\mathrm{H}$ & Has & Mass & 1.00783 \\
\hline Atom & 9 & Element & $\mathrm{H}$ & Has & Mass & 1.00783 \\
\hline Atom & 10 & Element & $\mathrm{H}$ & Has & Mass & 1.00783 \\
\hline Atom & 11 & Element & $\mathrm{H}$ & Has & Mass & 1.00783 \\
\hline Atom & 12 & Element & $\mathrm{C}$ & Has & Mass & 12.00000 \\
\hline Atom & 13 & Element & $\mathrm{C}$ & Has & Mass & 12.00000 \\
\hline Atom & 14 & Element & $\mathrm{C}$ & Has & Mass & 12.0000 \\
\hline Atom & 15 & Element & $\mathrm{N}$ & Has & Mass & 14.00307 \\
\hline Atom & 16 & Element & $\mathrm{C}$ & Has & Mass & 12.00000 \\
\hline Atom & 17 & Element & $\mathrm{C}$ & Has & Mass & 12.00000 \\
\hline Atom & 18 & Element & $\mathrm{H}$ & Has & Mass & 1.00783 \\
\hline Atom & 19 & Element & $\mathrm{H}$ & Has & Mass & 1.00783 \\
\hline Atom & 20 & Element & $\mathrm{H}$ & Has & Mass & $1.00^{\circ}$ \\
\hline Atom & 21 & Element & $\mathrm{H}$ & Has & Mass & 1.00783 \\
\hline Atom & 22 & Element & 0 & Has & Mass & 15.99491 \\
\hline Atom & 23 & Element & 0 & Has & Mass & 15.99491 \\
\hline Atom & 24 & Element & 0 & Has & Mass & 15.99491 \\
\hline Atom & 25 & Element & $\mathrm{C}$ & Has & Mass & 12.0000 \\
\hline Atom & 26 & Element & $\mathrm{H}$ & Has & Mass & 1.00783 \\
\hline Atom & 27 & Element & $\mathrm{C}$ & Has & Mass & 12.00000 \\
\hline Atom & 28 & Element & $\mathrm{H}$ & Has & Mass & 1.00783 \\
\hline Atom & 29 & Element & $\mathrm{H}$ & Has & Mass & 1.0078 \\
\hline Atom & 30 & Element & $\mathrm{H}$ & Has & Mass & 1.00783 \\
\hline Molec & ar & Mass: & 419 & & 830 & \\
\hline
\end{tabular}

Principal axes and moments of inertia in atomic units:

Principal axes and moments or inertia in atomic units:

Eigenvalues -- $\quad 3893.979575092 .983137910 .99094$

$\begin{array}{lrrr}X & 0.99331 & 0.01538 & 0.11447 \\ Y & -0.01436 & 0.99985 & -0.00974 \\ Z & -0.11461 & 0.00803 & 0.99338\end{array}$

Rotational Symmetry Number is 1

The Molecule is an Asymmetric Top

Translational Enthalpy: $\quad 0.889 \mathrm{kcal} / \mathrm{mol}$

Rotational Enthalpy: $\quad 0.889 \mathrm{kcal} / \mathrm{mol}$

Vibrational Enthalpy: $\quad 152.423 \mathrm{kcal} / \mathrm{mol}$

gas constant (RT): $\quad 0.593 \mathrm{kcal} / \mathrm{mol}$

Translational Entropy: $\quad 43.989 \mathrm{cal} / \mathrm{mol} . \mathrm{K}$ 
Rotational Entropy:

Vibrational Entropy:

Total Enthalpy:

Total Entropy:
$33.417 \mathrm{cal} / \mathrm{mol} \cdot \mathrm{K}$

$48.086 \mathrm{cal} / \mathrm{mol} . \mathrm{K}$

$154.794 \mathrm{kcal} / \mathrm{mol}$

$125.492 \mathrm{cal} / \mathrm{mol} . \mathrm{K}$

Spartan '02 Properties Program: (PC/x86)

Release

116

Reason for exit: Successful completion

Properties Program CPU Time : 000:00:09.1

Properties Program Wall Time: 000:00:09.2 


\author{
Spartan ' 02 \\ build 116 (May 14 2002)
}

Wavefunction Developers:

B.J. Deppmeier, A.J. Driessen, T.S. Hehre, W.J. Hehre,

J.A. Johnson, P.E. Klunzinger, J.M. Leonard, I.N. Pham

W.J. Pietro, Jianguo Yu

Q-Chem Developers:

J. Kong, C.A. White, A.I. Krylov, C.D. Sherrill,

R.D. Adamson, T.R. Furlani, M.S. Lee, A.M. Lee,

S.R. Gwaltney, T.R. Adams, C. Ochsenfeld, A.T.B. Gilbert,

G.S. Kedziora, V.A. Rassolov, D. R. Maurice, N. Nair,

Y. Shao, N.A. Besley, P.E. Maslen, J.P. Dombroski,

H. Dachsel, W.M. Zhang, P.P. Korambath, J. Baker,

E.F. C. Byrd, T. Van Voorhis, M. Oumi, S. Hirata,

C.P. Hsu, N. Ishikawa, J. Florian, A. Warshel,

B.G. Johnson, P.M.W. Gill, M. Head-Gordon, J.A. Pople

$\begin{array}{lll}\text { Wavefunction Inc. } & \text { Sales: } & \text { sales@wavefun.com } \\ \text { Irvine CA } & \text { Support: } & \text { support@wavefun.com } \\ & \text { Web: } & \text { www.wavefun.com }\end{array}$

Copyright (c) $1995-2002$

Spartan '02 Quantum Mechanics Program: (PC/x86)

Release 116

Job type: Geometry optimization.

Method: RB3LYP

Basis set: LACVP**

Number of shells: 112

Number of basis functions: 339

SCF model:

A restricted hybrid HF-DFT SCF calculation will be performed using Pulay DIIS extrapolation

Optimization:

Step

$$
\begin{aligned}
& \text { Energy } \\
& -878.6203645 \\
& -878.6344844 \\
& -878.6410618 \\
& -878.6437298 \\
& -878.6448111 \\
& -878.6454403 \\
& -878.6458503 \\
& -878.6460362 \\
& -878.6464426 \\
& -878.6467817 \\
& -878.6468753 \\
& -878.6467855 \\
& -878.6467611 \\
& -878.6470538 \\
& -878.6467850 \\
& -878.6469375 \\
& -878.6470855 \\
& -878.6468109
\end{aligned}
$$

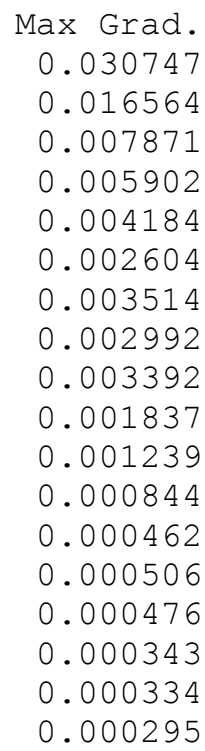

Max Dist. 0.099575

0.091459

0.152127

0.164490

0.160902

0.135923

0.105017

0.102135

0.109475

0.075043

0.049974

0.023770

0.014851

0.009291

0.016054

0.007994

0.009415

0.004842 


$\begin{array}{llll}19 & -878.6468092 & 0.000298 & 0.003428 \\ 20 & -878.6468137 & 0.000255 & 0.002602 \\ 21 & -878.6468056 & 0.000160 & 0.003680 \\ 22 & -878.6468090 & 0.000127 & 0.002096 \\ 23 & -878.6469751 & 0.000076 & 0.001565 \\ 24 & -878.6468443 & 0.000044 & 0.001336 \\ 25 & -878.6468337 & 0.000030 & 0.001453 \\ 26 & -878.6469285 & 0.000018 & 0.000740\end{array}$

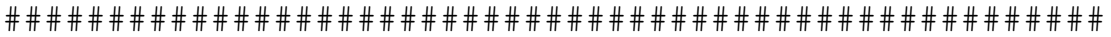 \\ \# Entering optman.exe on Fri May 16 10:07:29 2003 \#

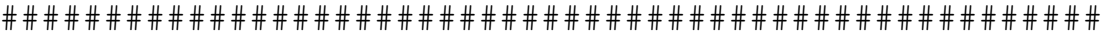

\author{
Cartesian Hessian Update \\ Hessian Updated using BFGS Update
}

** GEOMETRY OPTIMIZATION IN DELOCALIZED INTERNAL COORDiNATES ** Searching for a Minimum

Optimization Cycle: 26

\begin{tabular}{|c|c|c|c|c|}
\hline \multirow{2}{*}{\multicolumn{2}{|c|}{ ATOM }} & \multicolumn{3}{|c|}{ Coordinates (Angstroms) } \\
\hline & & $\mathrm{X}$ & Y & Z \\
\hline 1 & $\mathrm{Re}$ & 1.640337 & 0.139507 & -0.907432 \\
\hline 2 & $\mathrm{~N}$ & -0.232542 & 1.383776 & -0.472366 \\
\hline 3 & $\mathrm{C}$ & -1.355405 & 0.758293 & -0.050200 \\
\hline 4 & $\mathrm{C}$ & -1.289003 & -0.715401 & -0.044198 \\
\hline 5 & $\mathrm{~N}$ & -0.141874 & -1.235033 & -0.531339 \\
\hline 6 & O & 2.195174 & 1.617690 & -1.551107 \\
\hline 7 & O & 2.028978 & -1.076936 & -2.044111 \\
\hline 8 & 0 & 1.555408 & 0.082969 & 1.045326 \\
\hline 9 & $\mathrm{C}$ & 2.925584 & -0.023198 & 1.452742 \\
\hline 10 & $\mathrm{H}$ & 3.054716 & -0.847348 & 2.162127 \\
\hline 11 & $\mathrm{H}$ & 3.243939 & 0.911354 & 1.926771 \\
\hline 12 & $\mathrm{C}$ & 3.549221 & -0.261862 & 0.085592 \\
\hline 13 & $\mathrm{H}$ & 3.809320 & -1.295858 & -0.134830 \\
\hline 14 & $\mathrm{H}$ & 4.311939 & 0.429526 & -0.268196 \\
\hline 15 & $\mathrm{C}$ & 0.007266 & -2.566484 & -0.637021 \\
\hline 16 & $\mathrm{H}$ & 0.939062 & -2.911466 & -1.068773 \\
\hline 17 & $\mathrm{C}$ & -0.985227 & -3.449733 & -0.226943 \\
\hline 18 & $\mathrm{H}$ & -0.827327 & -4.517445 & -0.324575 \\
\hline 19 & C & -2.165424 & -2.929960 & 0.299622 \\
\hline 20 & $\mathrm{H}$ & -2.959188 & -3.589242 & 0.634700 \\
\hline 21 & $\mathrm{C}$ & -2.323187 & -1.548449 & 0.383915 \\
\hline 22 & $\mathrm{H}$ & -3.239705 & -1.130694 & 0.780427 \\
\hline 23 & $\mathrm{C}$ & -2.493202 & 1.487597 & 0.293725 \\
\hline 24 & $\mathrm{H}$ & -3.393902 & 0.982206 & 0.618064 \\
\hline 25 & $\mathrm{C}$ & -0.206459 & 2.723682 & -0.571423 \\
\hline 26 & $\mathrm{H}$ & 0.719617 & 3.155581 & -0.931387 \\
\hline 27 & $\mathrm{C}$ & -1.307168 & 3.507450 & -0.241976 \\
\hline 28 & $\mathrm{H}$ & -1.246346 & 4.584978 & -0.340131 \\
\hline 29 & C & -2.467520 & 2.877846 & 0.200810 \\
\hline 30 & $\mathrm{H}$ & -3.347080 & 3.456656 & 0.462186 \\
\hline
\end{tabular}

Energy is $\quad-878.646928520$

Hessian Updated using BFGS Update 
internal optimization with constraints (0)

84 Hessian modes will be used to form the next step Hessian Eigenvalues:

$\begin{array}{llllll}0.001131 & 0.002475 & 0.003687 & 0.004716 & 0.011580 & 0.012206 \\ 0.016547 & 0.018201 & 0.018437 & 0.021141 & 0.023194 & 0.024806 \\ 0.026460 & 0.027349 & 0.028151 & 0.028210 & 0.029519 & 0.029990 \\ 0.031200 & 0.032549 & 0.033441 & 0.034643 & 0.037420 & 0.037966 \\ 0.040917 & 0.042410 & 0.045420 & 0.050130 & 0.063078 & 0.067210 \\ 0.087346 & 0.093938 & 0.105276 & 0.109447 & 0.122958 & 0.129073 \\ 0.130539 & 0.132549 & 0.134395 & 0.135329 & 0.136323 & 0.142254 \\ 0.145683 & 0.150326 & 0.165177 & 0.172272 & 0.178044 & 0.188531 \\ 0.217204 & 0.219226 & 0.233455 & 0.235334 & 0.256576 & 0.267925 \\ 0.274643 & 0.295392 & 0.300951 & 0.304435 & 0.311111 & 0.324294 \\ 0.330319 & 0.332049 & 0.337944 & 0.343921 & 0.353332 & 0.359180 \\ 0.362453 & 0.374438 & 0.391208 & 0.405088 & 0.417075 & 0.419737 \\ 0.435915 & 0.448839 & 0.454015 & 0.498094 & 0.510124 & 0.571786 \\ 0.578311 & 0.611200 & 0.638824 & 0.700073 & 0.761422 & 0.792788\end{array}$

Minimum Search - Taking Simple RFO Step

Searching for Lamda that Minimizes Along All modes Value Taken Lamda $=-0.00000002$

Step Taken. Stepsize is 0.001167

$\begin{array}{lrrr} & \text { Maximum } & \text { Tolerance } & \text { Cnvgd? } \\ \text { Gradient } & 0.000018 & 0.000300 & \text { YES } \\ \text { Displacement } & 0.000740 & 0.001200 & \text { YES } \\ \text { Energy change } & -0.000095 & 0.000001 & \text { NO }\end{array}$

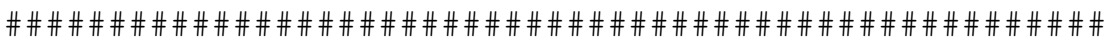

\# Entering anlman.exe on Fri May 16 10:07:29 2003 \#

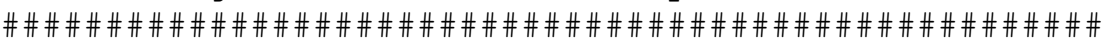

Analysis of SCF Wavefunction

Cartesian Multipole Moments

\begin{tabular}{|c|c|c|c|c|c|}
\hline \multicolumn{6}{|c|}{$\begin{array}{r}\text { Charge (ESU x 10^10) } \\
4.8032\end{array}$} \\
\hline \multicolumn{6}{|c|}{ Dipole Moment (Debye) } \\
\hline $\mathrm{X}$ & -3.6048 & $\mathrm{Y}$ & -0.4313 & Z & 1.6264 \\
\hline Tot & 3.9781 & & & & \\
\hline \multicolumn{6}{|c|}{ Quadrupole Moments (Debye-Ang) } \\
\hline XX & -80.3543 & $X Y$ & -3.4278 & YY & -73.8819 \\
\hline XZ & 2.4172 & $\mathrm{YZ}$ & -0.9315 & $\mathrm{ZZ}$ & -121.8540 \\
\hline \multicolumn{6}{|c|}{ Octapole Moments (Debye-Ang^2) } \\
\hline XXX & -85.3704 & $\mathrm{XXY}$ & -5.3104 & $X Y Y$ & -87.9267 \\
\hline YYY & -18.3452 & $\mathrm{XXZ}$ & 72.1846 & $\mathrm{XYZ}$ & 2.4492 \\
\hline YYZ & 29.9429 & $\mathrm{XZZ}$ & -28.4881 & YZZ & -5.5325 \\
\hline ZZZ & 111.9794 & & & & \\
\hline \multicolumn{6}{|c|}{ Hexadecapole Moments (Debye-Ang^3) } \\
\hline XXXX & -2273.7070 & XXXY & -2.7440 & XXYY & -706.5213 \\
\hline XYYY & -5.7212 & YYYY & -1795.9093 & XXXZ & 152.1404 \\
\hline XXYZ & 6.2597 & XYYZ & 34.7387 & YYYZ & 15.6806 \\
\hline XXZZ & -565.6149 & XYZZ & 14.6720 & YYZZ & -544.6431 \\
\hline XZZZ & 194.7294 & YZZZ & 14.3955 & ZZZZ & -661.3821 \\
\hline
\end{tabular}

Total job wall time: $5.4 \mathrm{e}+007 \mathrm{~s}$ 
Reason for exit: Sucessful completion

Quantum Mechanics Program CPU Time : 015:00:01.0

Quantum Mechanics Program Wall Time: 015:02:39.7

Spartan '02 Properties Program: (PC/x86)

Release

116

Reason for exit: Successful completion

Properties Program CPU Time : 000:00:08.9

Properties Program Wall Time: 000:00:08.9

Release 116

Job type: Frequency calculation.

Method: RB3LYP

Basis set: LACVP**

Number of shells: 112

Number of basis functions: 339

SCF model:

A restricted hybrid HF-DFT SCF calculation will be performed using Pulay DIIS extrapolation

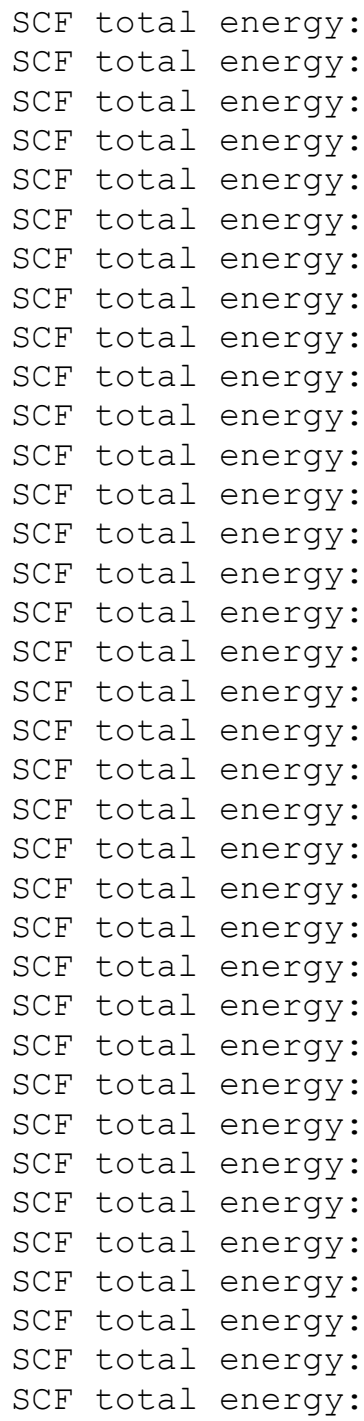

$$
\begin{aligned}
& -878.6468492 \text { hartrees } \\
& -878.6469666 \text { hartrees } \\
& -878.6468339 \text { hartrees } \\
& -878.6468470 \text { hartrees } \\
& -878.6469490 \text { hartrees } \\
& -878.6469903 \text { hartrees } \\
& -878.6468181 \text { hartrees } \\
& -878.6468666 \text { hartrees } \\
& -878.6468709 \text { hartrees } \\
& -878.6468491 \text { hartrees } \\
& -878.6468709 \text { hartrees } \\
& -878.6468569 \text { hartrees } \\
& -878.6468559 \text { hartrees } \\
& -878.6468549 \text { hartrees } \\
& -878.6468537 \text { hartrees } \\
& -878.6468582 \text { hartrees } \\
& -878.6468563 \text { hartrees } \\
& -878.6468547 \text { hartrees } \\
& -878.6468569 \text { hartrees } \\
& -878.6468589 \text { hartrees } \\
& -878.6468593 \text { hartrees } \\
& -878.6468513 \text { hartrees } \\
& -878.6468574 \text { hartrees } \\
& -878.6468549 \text { hartrees } \\
& -878.6468575 \text { hartrees } \\
& -878.6468536 \text { hartrees } \\
& -878.6468560 \text { hartrees } \\
& -878.6468600 \text { hartrees } \\
& -878.6468619 \text { hartrees } \\
& -878.6468667 \text { hartrees } \\
& -878.6468593 \text { hartrees } \\
& -878.6468591 \text { hartrees } \\
& -878.6470024 \text { hartrees } \\
& -878.6468480 \text { hartrees } \\
& -878.6468591 \text { hartrees } \\
& -878.6468548 \text { hartrees }
\end{aligned}
$$


-878.6468501 hartrees -878.6468613 hartrees -878.6468457 hartrees -878.6469880 hartrees -878.6468586 hartrees -878.6468606 hartrees -878.6468529 hartrees -878.6468151 hartrees -878.6468192 hartrees -878.6468141 hartrees -878.6468104 hartrees -878.6468076 hartrees -878.6468525 hartrees -878.6468590 hartrees -878.6468542 hartrees -878.6468563 hartrees -878.6468553 hartrees -878.6468584 hartrees -878.6468516 hartrees -878.6468642 hartrees -878.6468607 hartrees -878.6468639 hartrees -878.6468530 hartrees -878.6468568 hartrees -878.6468609 hartrees -878.6468564 hartrees -878.6468579 hartrees -878.6468537 hartrees -878.6468619 hartrees -878.6468542 hartrees -878.6468601 hartrees -878.6468702 hartrees -878.6468735 hartrees -878.6468501 hartrees -878.6468591 hartrees -878.6468566 hartrees -878.6468660 hartrees -878.6468519 hartrees -878.6468558 hartrees -878.6468560 hartrees -878.6468514 hartrees -878.6468626 hartrees -878.6468541 hartrees -878.6468563 hartrees -878.6468636 hartrees -878.6468545 hartrees -878.6468621 hartrees -878.6468594 hartrees -878.6468524 hartrees -878.6468566 hartrees -878.6468617 hartrees -878.6468523 hartrees -878.6468521 hartrees -878.6468620 hartrees -878.6468529 hartrees -878.6468605 hartrees -878.6468526 hartrees -878.6468612 hartrees -878.6468576 hartrees -878.6468584 hartrees -878.6468545 hartrees 
-878.6468613 hartrees -878.6468479 hartrees -878.6468528 hartrees -878.6468611 hartrees -878.6468604 hartrees -878.6468594 hartrees -878.6468598 hartrees -878.6468560 hartrees -878.6468634 hartrees -878.6468510 hartrees -878.6468599 hartrees -878.6468564 hartrees -878.6468550 hartrees -878.6468545 hartrees -878.6468582 hartrees -878.6468549 hartrees -878.6468614 hartrees -878.6468584 hartrees -878.6468576 hartrees -878.6468591 hartrees -878.6468602 hartrees -878.6468477 hartrees -878.6468608 hartrees -878.6468584 hartrees -878.6468548 hartrees -878.6468569 hartrees -878.6468551 hartrees -878.6468575 hartrees -878.6468621 hartrees -878.6468612 hartrees -878.6468561 hartrees -878.6468582 hartrees -878.6468546 hartrees -878.6468588 hartrees -878.6468571 hartrees -878.6468581 hartrees -878.6468530 hartrees -878.6468537 hartrees -878.6468576 hartrees -878.6468496 hartrees -878.6468611 hartrees -878.6468599 hartrees -878.6468553 hartrees -878.6468565 hartrees -878.6468636 hartrees -878.6468549 hartrees -878.6468584 hartrees -878.6468590 hartrees -878.6468602 hartrees -878.6468558 hartrees -878.6468600 hartrees -878.6468495 hartrees -878.6468591 hartrees -878.6468574 hartrees -878.6468625 hartrees -878.6468509 hartrees -878.6468558 hartrees -878.6468628 hartrees -878.6468526 hartrees -878.6468547 hartrees -878.6468517 hartrees 


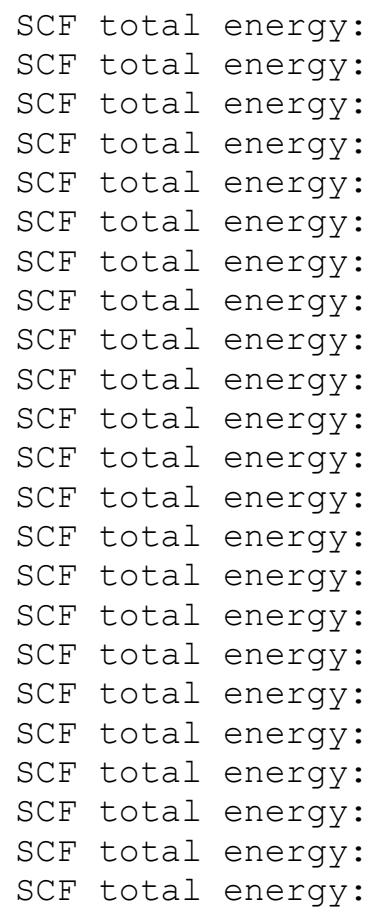

-878.6468525 hartrees

-878.6468575 hartrees

-878.6468537 hartrees

-878.6468536 hartrees

-878.6468568 hartrees

-878.6468514 hartrees

-878.6468626 hartrees

-878.6468527 hartrees

-878.6468601 hartrees

-878.6468507 hartrees

-878.6468605 hartrees

-878.6468534 hartrees

-878.6468511 hartrees

-878.6468540 hartrees

-878.6468593 hartrees

-878.6468552 hartrees

-878.6468562 hartrees

-878.6468519 hartrees

-878.6468594 hartrees

-878.6468553 hartrees

-878.6468571 hartrees

-878.6468614 hartrees

-878.6468582 hartrees

Reason for exit: Sucessful completion

Quantum Mechanics Program CPU Time : 085:25:52.2

Quantum Mechanics Program Wall Time: 086:36:14.5

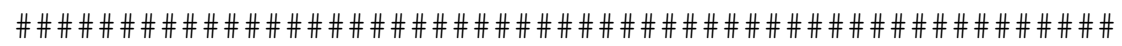

\# Entering vibman.exe on Sat Jan 17 22:58:25 2004 \#

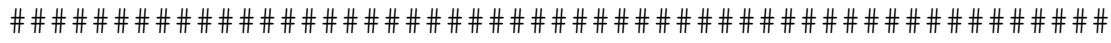

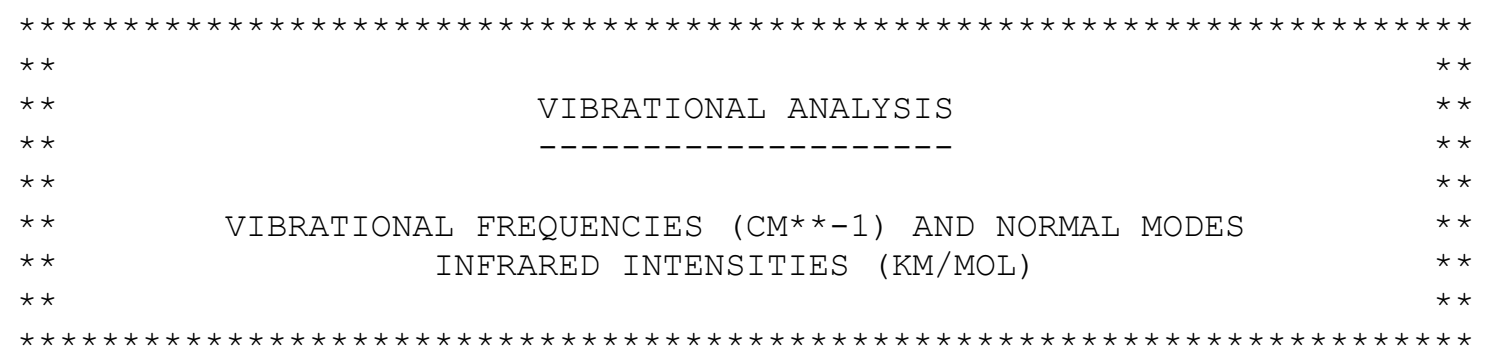

Frequency:

IR Active:

IR Intens:

Raman Active:
46.22

YES

1.776

YES

$$
X \quad Y
$$

$\mathrm{Re}$

$\mathrm{N}$

C

C

$\mathrm{N}$

O

0

O

C

$\mathrm{H}$

$\mathrm{H}$

C

$\mathrm{H}$

$\mathrm{H}$

$$
-0.014-0.015
$$

$-0.011-0.011$

$-0.026 \quad 0.012$

$-0.008-0.003$

$-0.003-0.018$

$-0.005-0.066-0.132$

$\begin{array}{lll}-0.151 & -0.028 & 0.128\end{array}$

$0.075-0.018-0.010$

$\begin{array}{lll}0.203 & 0.142 & 0.053\end{array}$

$\begin{array}{llll}0.178 & 0.239 & 0.112\end{array}$

$\begin{array}{llll}0.357 & 0.114 & 0.005\end{array}$

$\begin{array}{llll}0.138 & 0.194 & 0.094\end{array}$

$\begin{array}{lll}0.056 & 0.279 & 0.157\end{array}$

0.233

0.209

0.089

$-0.010$

0.041

0.002

58.22

YES

0.050

YES

$\begin{array}{rrr}X & Y & Z \\ 0.003 & 0.009 & -0.006 \\ 0.004 & 0.007 & -0.044 \\ -0.022 & 0.026 & 0.049 \\ -0.029 & 0.032 & 0.066 \\ 0.025 & -0.002 & -0.097 \\ -0.019 & 0.043 & 0.059 \\ -0.018 & 0.058 & -0.015 \\ 0.021 & -0.145 & -0.016 \\ 0.017 & -0.198 & 0.063 \\ 0.006 & -0.290 & -0.024 \\ 0.010 & -0.264 & 0.190 \\ 0.035 & -0.023 & 0.083 \\ -0.003 & 0.053 & 0.005 \\ 0.099 & 0.024 & 0.196\end{array}$

$\mathrm{x}$

$-0.022$

$$
0
$$


$\mathrm{C}$
$\mathrm{H}$
$\mathrm{C}$
$\mathrm{H}$
$\mathrm{C}$
$\mathrm{H}$
$\mathrm{C}$
$\mathrm{H}$
$\mathrm{C}$
$\mathrm{H}$
$\mathrm{C}$
$\mathrm{H}$
$\mathrm{C}$
$\mathrm{H}$
$\mathrm{C}$
$\mathrm{H}$

Freque
IR A
IR I
Raman
Re
N
C
C
N
O
O
O
C
H
H
C
H
H
C
H
C
H
C
H
C
H
C
H
C
H
C
H
C
H

$$
\begin{array}{rrr}
0.026 & -0.040 & -0.080 \\
0.030 & -0.051 & -0.097 \\
0.048 & -0.045 & -0.111 \\
0.071 & -0.061 & -0.157 \\
0.041 & -0.028 & -0.083 \\
0.058 & -0.032 & -0.107 \\
0.012 & -0.006 & -0.025 \\
0.009 & 0.005 & -0.012 \\
-0.051 & 0.047 & 0.157 \\
-0.063 & 0.069 & 0.243 \\
-0.017 & -0.007 & -0.050 \\
-0.003 & -0.030 & -0.131 \\
-0.040 & 0.024 & 0.053 \\
-0.043 & 0.026 & 0.049 \\
-0.060 & 0.054 & 0.168 \\
-0.080 & 0.082 & 0.262
\end{array}
$$

$$
\begin{array}{rrr}
0.023 & -0.014 & 0.005 \\
0.021 & -0.023 & 0.003 \\
0.023 & -0.005 & 0.037 \\
0.022 & -0.007 & 0.057 \\
0.022 & 0.007 & 0.048 \\
0.019 & 0.016 & 0.080 \\
0.022 & 0.009 & 0.022 \\
0.019 & 0.022 & 0.040 \\
0.042 & -0.017 & -0.119 \\
0.067 & -0.038 & -0.220 \\
-0.018 & 0.031 & 0.107 \\
-0.037 & 0.047 & 0.180 \\
-0.002 & 0.020 & 0.036 \\
-0.014 & 0.031 & 0.069 \\
0.033 & -0.009 & -0.092 \\
0.050 & -0.023 & -0.171
\end{array}
$$

\begin{tabular}{|c|c|c|c|c|c|c|c|c|}
\hline Frequency: & 162.66 & & & 166.04 & & & 203.04 & \\
\hline IR Active: & YES & & & YES & & & YES & \\
\hline IR Intens: & 2.619 & & & 0.855 & & & 0.250 & \\
\hline Raman Active: & YES & & & YES & & & YES & \\
\hline 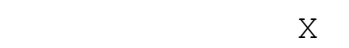 & $Y$ & Z & $\mathrm{X}$ & $Y$ & Z & $\mathrm{X}$ & Y & Z \\
\hline 0.084 & -0.032 & 0.022 & 0.020 & 0.087 & -0.019 & 0.024 & -0.014 & 0.053 \\
\hline-0.166 & 0.021 & 0.054 & -0.118 & -0.084 & -0.039 & 0.019 & -0.082 & -0.178 \\
\hline-0.118 & 0.037 & 0.052 & -0.100 & -0.084 & -0.039 & 0.058 & -0.065 & -0.171 \\
\hline
\end{tabular}

$\begin{array}{ccc} & 119.16 & \\ & \text { YES } & \\ & 0.759 & \\ & \text { YES } & \\ \mathrm{X} & \mathrm{Y} & \mathrm{Z}\end{array}$

$\begin{array}{llll}0.000 & -0.001 & 0.003\end{array}$

$\begin{array}{rrrr}-0.021 & 0.019 & 0.105\end{array}$

$\begin{array}{llll}-0.009 & -0.003 & 0.017\end{array}$

$\begin{array}{llll}-0.024 & 0.011 & 0.056\end{array}$

$0.006-0.003-0.036$

$-0.002-0.066-0.147$

$\begin{array}{llll}-0.077 & -0.049 & 0.095\end{array}$

$\begin{array}{lll}0.134 & 0.243 & 0.020\end{array}$

$\begin{array}{llll}-0.016 & 0.011 & 0.010\end{array}$

$0.065-0.129-0.038$

$\begin{array}{llll}-0.201 & 0.046 & 0.066\end{array}$

$\begin{array}{lll}-0.031 & -0.060 & 0.003\end{array}$

$\begin{array}{lll}-0.014 & -0.083 & 0.009\end{array}$

$-0.057-0.072-0.022$

$0.053-0.034-0.171$

$0.094-0.051-0.277$

$0.048-0.032-0.161$

$0.090-0.057-0.285$

$\begin{array}{lll}-0.027 & 0.012 & 0.050\end{array}$

$\begin{array}{llll}-0.053 & 0.026 & 0.116\end{array}$

$\begin{array}{llll}-0.060 & 0.034 & 0.156\end{array}$

$\begin{array}{llll}-0.110 & 0.068 & 0.306\end{array}$

$0.027-0.052-0.144$

$0.049-0.089-0.292$

$\begin{array}{lll}-0.030 & 0.031 & 0.176\end{array}$

$\begin{array}{lll}-0.046 & 0.062 & 0.283\end{array}$

$\begin{array}{lll}-0.009 & -0.002 & 0.080\end{array}$

$\begin{array}{rrrr}-0.015 & 0.007 & 0.135\end{array}$

$0.031-0.056-0.129$

$0.061-0.095-0.267$

$\begin{array}{llll}-0.008 & 0.021 & 0.005 \\ -0.005 & 0.021 & -0.007\end{array}$ 


$\begin{array}{lrrr}\mathrm{C} & -0.094 & 0.001 & 0.065 \\ \mathrm{~N} & -0.085 & 0.012 & 0.064 \\ \mathrm{O} & 0.058 & -0.037 & -0.027 \\ \mathrm{O} & 0.164 & -0.064 & -0.038 \\ \mathrm{O} & 0.027 & -0.046 & 0.051 \\ \mathrm{C} & 0.042 & -0.004 & 0.004 \\ \mathrm{H} & -0.003 & 0.026 & -0.008 \\ \mathrm{H} & 0.053 & -0.009 & 0.008 \\ \mathrm{C} & 0.090 & 0.014 & -0.015 \\ \mathrm{H} & 0.073 & 0.042 & -0.036 \\ \mathrm{H} & 0.130 & 0.019 & -0.020 \\ \mathrm{C} & -0.108 & 0.048 & -0.002 \\ \mathrm{H} & -0.093 & 0.096 & 0.001 \\ \mathrm{C} & -0.124 & 0.023 & -0.087 \\ \mathrm{H} & -0.123 & 0.030 & -0.147 \\ \mathrm{C} & -0.128 & -0.009 & -0.096 \\ \mathrm{H} & -0.135 & -0.033 & -0.169 \\ \mathrm{C} & -0.115 & -0.022 & -0.001 \\ \mathrm{H} & -0.122 & -0.058 & -0.002 \\ \mathrm{C} & -0.093 & 0.127 & -0.037 \\ \mathrm{H} & -0.018 & 0.155 & -0.044 \\ \mathrm{C} & -0.242 & 0.073 & -0.015 \\ \mathrm{H} & -0.318 & 0.034 & -0.006 \\ \mathrm{C} & -0.227 & 0.147 & -0.120 \\ \mathrm{H} & -0.270 & 0.170 & -0.187 \\ \mathrm{C} & -0.154 & 0.172 & -0.142 \\ \mathrm{H} & -0.129 & 0.220 & -0.233\end{array}$

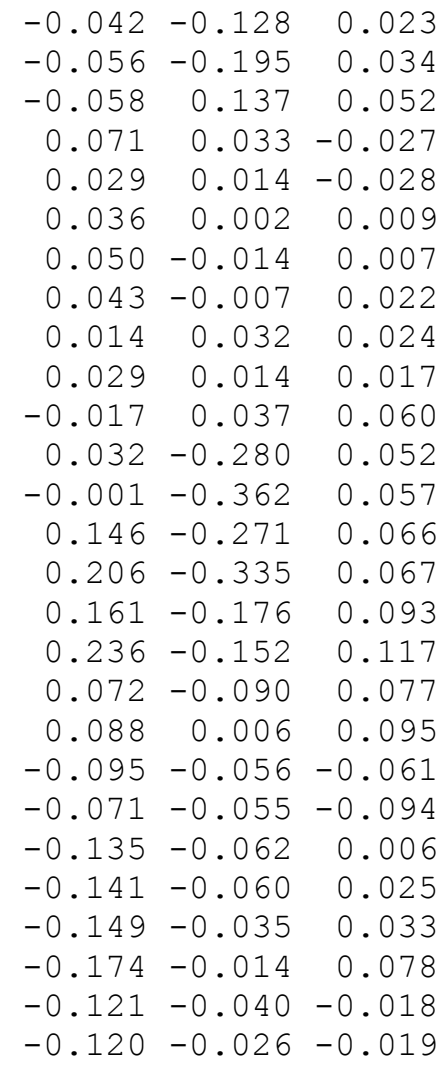

\begin{tabular}{rrr}
\multicolumn{3}{c}{252.31} \\
YES \\
X & 0.528 \\
YES \\
-0.038 & 0.009 & 0.025 \\
0.062 & -0.020 & -0.190 \\
0.039 & -0.018 & -0.146 \\
-0.013 & 0.027 & 0.053 \\
-0.025 & 0.041 & 0.115 \\
-0.083 & -0.021 & -0.082 \\
0.320 & -0.219 & -0.159 \\
0.024 & -0.006 & 0.026 \\
0.030 & -0.003 & 0.052 \\
0.013 & -0.034 & 0.012 \\
0.035 & -0.036 & 0.109 \\
0.057 & 0.087 & 0.046 \\
-0.039 & 0.205 & 0.039 \\
0.159 & 0.122 & 0.100 \\
-0.012 & 0.046 & 0.030 \\
-0.017 & 0.068 & 0.063 \\
0.027 & 0.007 & -0.143 \\
0.073 & -0.017 & -0.302 \\
-0.018 & 0.021 & -0.025 \\
-0.019 & 0.009 & -0.064 \\
-0.053 & 0.040 & 0.126 \\
-0.085 & 0.044 & 0.210 \\
0.039 & -0.051 & -0.163 \\
0.032 & -0.079 & -0.244 \\
0.070 & -0.020 & -0.119 \\
0.105 & -0.027 & -0.194 \\
0.013 & 0.014 & 0.152 \\
-0.012 & 0.049 & 0.352
\end{tabular}

$\begin{array}{rrr}0.058 & -0.072 & -0.154 \\ 0.060 & -0.053 & -0.160 \\ 0.025 & -0.011 & 0.066 \\ -0.044 & 0.051 & 0.074 \\ -0.054 & 0.142 & 0.037 \\ -0.147 & 0.093 & -0.076 \\ -0.177 & 0.057 & -0.133 \\ -0.229 & 0.102 & -0.041 \\ -0.049 & 0.065 & -0.129 \\ -0.073 & 0.111 & -0.167 \\ 0.030 & 0.066 & -0.168 \\ 0.042 & -0.038 & -0.156 \\ 0.074 & -0.034 & -0.224 \\ -0.037 & -0.006 & 0.008 \\ -0.069 & 0.018 & 0.066 \\ -0.083 & 0.003 & 0.129 \\ -0.163 & 0.041 & 0.310 \\ -0.011 & -0.047 & -0.018 \\ -0.028 & -0.054 & 0.023 \\ 0.039 & 0.024 & -0.059 \\ 0.082 & 0.047 & -0.039 \\ -0.038 & -0.040 & -0.155 \\ -0.065 & -0.079 & -0.219 \\ -0.081 & 0.067 & 0.025 \\ -0.137 & 0.114 & 0.113 \\ -0.052 & 0.108 & 0.108 \\ -0.087 & 0.197 & 0.285\end{array}$

\begin{tabular}{rrr}
\multicolumn{4}{c}{264.26} \\
\multicolumn{3}{c}{2.855} \\
$X$ & YES \\
-0.048 & 0.022 & 0.000 \\
0.001 & -0.085 & 0.140 \\
0.087 & -0.071 & 0.113 \\
0.124 & -0.124 & -0.037 \\
0.151 & -0.051 & -0.087 \\
-0.034 & -0.012 & -0.054 \\
0.170 & -0.065 & -0.146 \\
0.069 & 0.078 & 0.003 \\
0.049 & 0.010 & 0.055 \\
0.069 & -0.051 & 0.021 \\
0.019 & -0.010 & 0.112 \\
0.034 & 0.070 & 0.065 \\
-0.034 & 0.151 & 0.072 \\
0.098 & 0.095 & 0.105 \\
0.092 & 0.016 & -0.099 \\
0.148 & 0.066 & -0.188 \\
-0.084 & 0.033 & 0.069 \\
-0.184 & 0.123 & 0.166 \\
-0.106 & -0.068 & 0.054 \\
-0.218 & -0.067 & 0.141 \\
0.032 & -0.174 & -0.089 \\
0.035 & -0.268 & -0.146 \\
0.102 & 0.047 & 0.136 \\
0.159 & 0.091 & 0.212 \\
-0.068 & -0.050 & 0.085 \\
-0.143 & -0.069 & 0.140 \\
-0.020 & 0.020 & -0.112 \\
-0.064 & 0.036 & -0.266 \\
& &
\end{tabular}




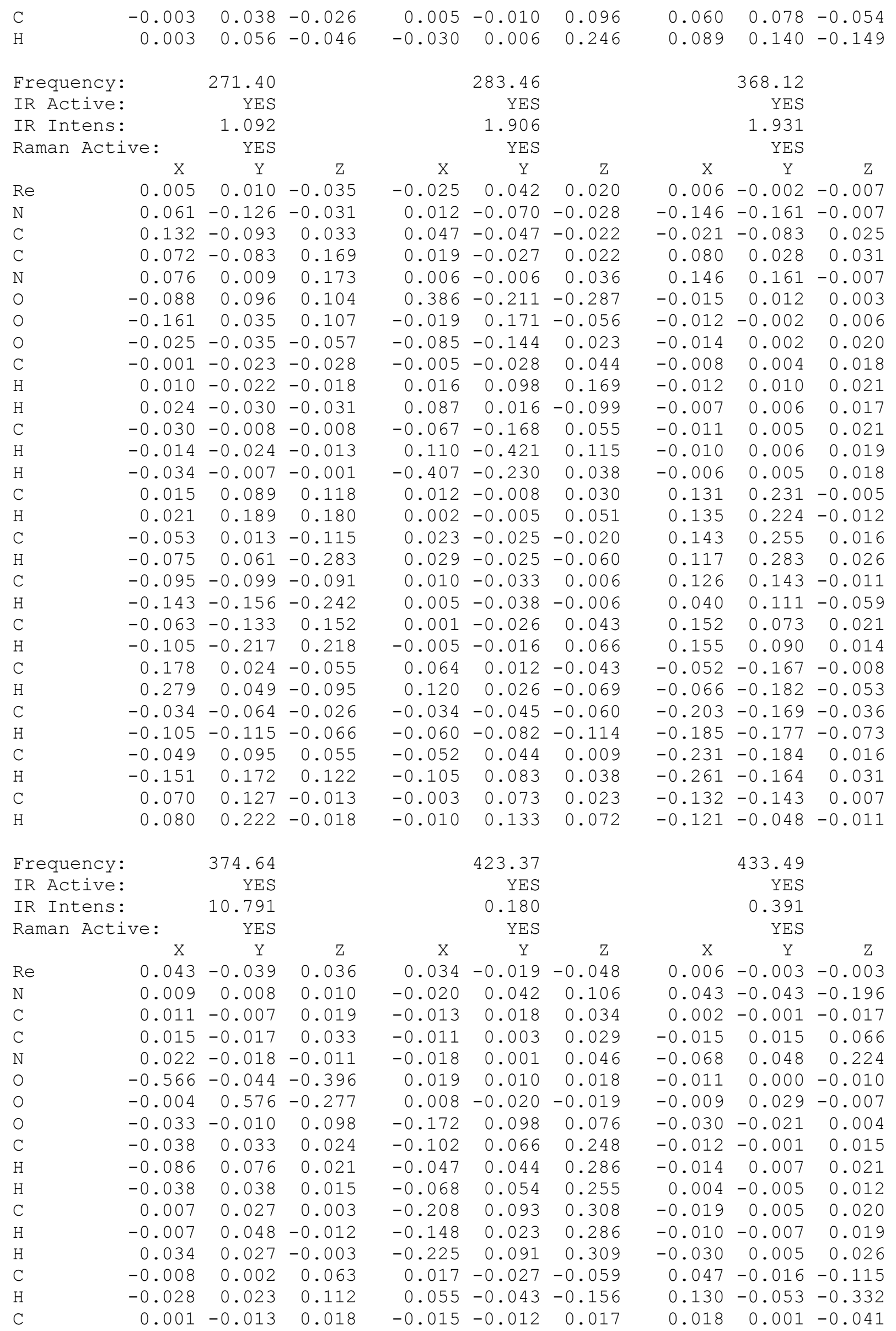


$\mathrm{H}$
$\mathrm{C}$
$\mathrm{H}$
$\mathrm{C}$
$\mathrm{H}$
$\mathrm{C}$
$\mathrm{H}$
$\mathrm{C}$
$\mathrm{H}$
$\mathrm{C}$
$\mathrm{H}$
$\mathrm{C}$
$\mathrm{H}$

Frequency:

IR Active:

IR Intens:

Raman Active

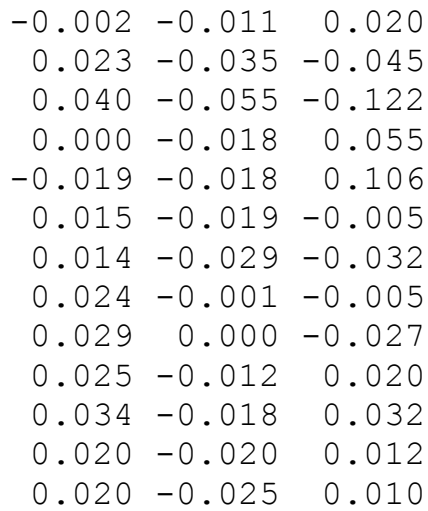

$0.020-0.025$

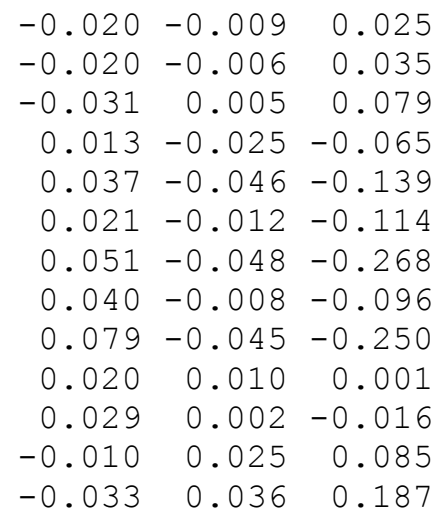

$\begin{array}{lll}-0.033 & 0.036 & 0.187\end{array}$

$\begin{array}{rrr}0.051 & -0.018 & -0.140 \\ -0.049 & 0.038 & 0.147 \\ -0.103 & 0.070 & 0.287 \\ 0.054 & -0.033 & -0.148 \\ 0.149 & -0.108 & -0.439 \\ -0.027 & 0.022 & 0.107 \\ -0.077 & 0.072 & 0.331 \\ -0.013 & 0.020 & 0.070 \\ -0.041 & 0.055 & 0.203 \\ -0.014 & 0.016 & 0.072 \\ -0.036 & 0.042 & 0.189 \\ 0.029 & -0.037 & -0.140 \\ 0.062 & -0.075 & -0.288\end{array}$

0.486

YES

7. 355

YES

$$
\mathrm{X}
$$

Z

$0.012-0.005-0.010$

$0.025-0.043-0.112$

$0.045-0.043-0.145$

$0.033-0.023-0.147$

$0.011-0.007-0.075$

$0.013-0.004-0.002$

$0.019-0.019-0.001$

$\begin{array}{lll}-0.064 & 0.031 & 0.024\end{array}$

$\begin{array}{llll}-0.043 & 0.028 & 0.079\end{array}$

$\begin{array}{lll}-0.022 & 0.024 & 0.097\end{array}$

$\begin{array}{llll}-0.027 & 0.024 & 0.078\end{array}$

$\begin{array}{llll}-0.082 & 0.037 & 0.100\end{array}$

$\begin{array}{llll}-0.069 & 0.020 & 0.098\end{array}$

$\begin{array}{llll}-0.090 & 0.038 & 0.105\end{array}$

$\begin{array}{llll}-0.054 & 0.029 & 0.128\end{array}$

$\begin{array}{rrr}-0.149 & 0.068 & 0.374\end{array}$

$0.036-0.016-0.089$

$0.062-0.036-0.141$

$0.0150 .009-0.019$

$0.0170 .013-0.005$

$\begin{array}{lll}-0.042 & 0.045 & 0.118\end{array}$

$\begin{array}{llll}-0.104 & 0.103 & 0.311\end{array}$

$\begin{array}{llll}-0.020 & 0.030 & 0.161\end{array}$

$\begin{array}{llll}-0.070 & 0.088 & 0.413\end{array}$

$\begin{array}{lll}-0.044 & 0.025 & 0.157\end{array}$

$\begin{array}{rrrr}-0.111 & 0.094 & 0.441\end{array}$

$0.007-0.022-0.079$

$0.007-0.026-0.122$

$0.015-0.010-0.052$

$0.023-0.004-0.082$

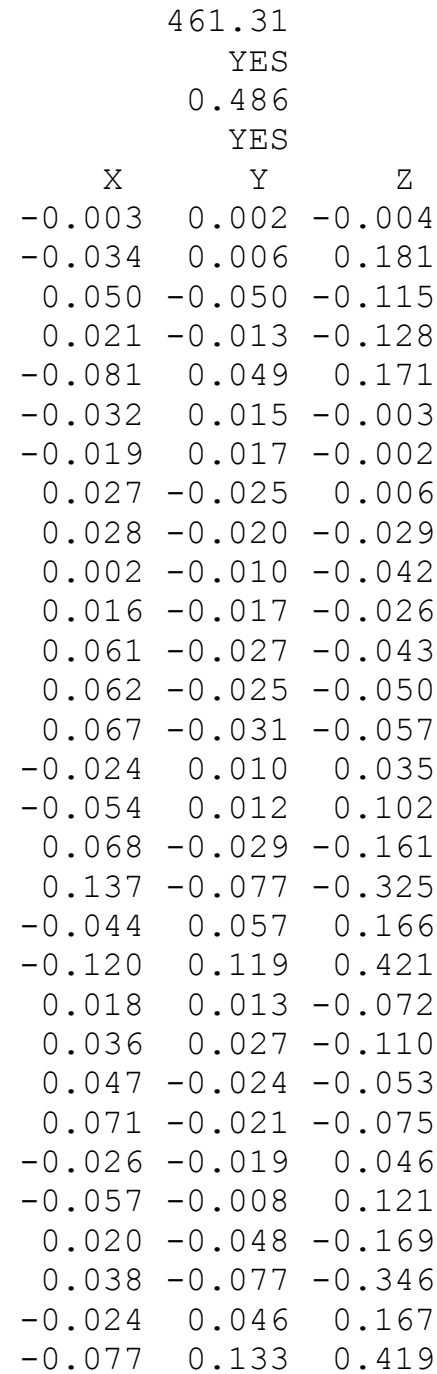

$\mathrm{Y}$

$\mathrm{X}$

$\begin{array}{lll}-0.011 & 0.009 & -0.036\end{array}$

$-0.004-0.010-0.031$

$0.006-0.002-0.006$

$\begin{array}{lll}-0.002 & -0.001 & 0.003\end{array}$

$0.004 \quad 0.007-0.023$

$\begin{array}{lll}-0.004 & 0.028 & 0.017\end{array}$
557.61

YES

0.031

YES

$\begin{array}{rrr} & 557.61 \\ & \text { YES } \\ \text { X } 0.031 & \\ \text { YES } & \\ 0.000 & 0.000 & -0.001 \\ -0.011 & 0.015 & 0.042 \\ -0.037 & 0.040 & 0.161 \\ 0.053 & -0.033 & -0.158 \\ 0.017 & -0.006 & -0.039 \\ -0.001 & 0.002 & 0.001\end{array}$

487.87

YES

0.440

\begin{tabular}{rrr}
\multicolumn{3}{c}{487.87} \\
Y & 0.440 \\
YES & Y & Z \\
-0.007 & -0.004 & -0.001 \\
-0.081 & 0.191 & -0.053 \\
-0.110 & 0.124 & -0.111 \\
0.164 & -0.110 & 0.030 \\
0.193 & -0.041 & 0.086 \\
0.053 & -0.021 & 0.016 \\
-0.027 & 0.036 & -0.013 \\
0.008 & 0.006 & 0.006 \\
0.001 & 0.000 & -0.003 \\
-0.003 & -0.001 & -0.008 \\
-0.007 & 0.002 & 0.000 \\
0.010 & -0.006 & -0.007 \\
0.006 & -0.002 & -0.005 \\
0.013 & -0.008 & -0.015 \\
0.127 & 0.076 & 0.058 \\
0.153 & 0.189 & 0.089 \\
-0.011 & 0.034 & -0.053 \\
-0.092 & 0.132 & -0.138 \\
-0.075 & -0.105 & 0.025 \\
-0.219 & -0.135 & 0.036 \\
0.061 & -0.200 & 0.067 \\
0.009 & -0.350 & 0.116 \\
-0.203 & 0.034 & -0.060 \\
-0.360 & -0.025 & -0.051 \\
0.041 & 0.149 & 0.004 \\
0.128 & 0.224 & 0.079 \\
0.055 & -0.031 & -0.028 \\
0.176 & -0.111 & -0.010 \\
-0.100 & -0.076 & 0.030 \\
-0.134 & -0.193 & 0.123 \\
& &
\end{tabular}

647.21

YES

9.181

YES

$0.001 \quad 0.002 \quad 0.000$

$-0.256-0.017-0.055$

$\begin{array}{llll}-0.057 & 0.110 & -0.028\end{array}$

$\begin{array}{lll}0.108 & -0.033 & 0.033\end{array}$

$0.016-0.229 \quad 0.057$

$0.009-0.001 \quad 0.001$ 


$\begin{array}{lrrr}\mathrm{O} & -0.034 & -0.027 & 0.012 \\ \mathrm{O} & -0.041 & -0.013 & 0.360 \\ \mathrm{C} & -0.121 & 0.071 & 0.155 \\ \mathrm{H} & -0.368 & 0.143 & 0.007 \\ \mathrm{H} & -0.255 & 0.103 & 0.187 \\ \mathrm{C} & 0.336 & -0.132 & -0.050 \\ \mathrm{H} & 0.406 & -0.205 & -0.102 \\ \mathrm{H} & 0.281 & -0.209 & -0.258 \\ \mathrm{C} & 0.011 & -0.008 & -0.007 \\ \mathrm{H} & 0.008 & -0.024 & -0.008 \\ \mathrm{C} & -0.003 & -0.007 & 0.017 \\ \mathrm{H} & -0.019 & 0.007 & 0.030 \\ \mathrm{C} & 0.003 & -0.023 & -0.012 \\ \mathrm{H} & 0.014 & -0.027 & -0.030 \\ \mathrm{C} & -0.014 & -0.007 & 0.007 \\ \mathrm{H} & -0.015 & 0.002 & 0.015 \\ \mathrm{C} & 0.011 & 0.014 & 0.009 \\ \mathrm{H} & 0.001 & 0.018 & 0.037 \\ \mathrm{C} & 0.009 & -0.012 & -0.003 \\ \mathrm{H} & 0.018 & -0.002 & 0.013 \\ \mathrm{C} & 0.009 & 0.001 & 0.014 \\ \mathrm{H} & -0.012 & 0.018 & 0.034 \\ \mathrm{C} & 0.028 & 0.003 & -0.011 \\ \mathrm{H} & 0.030 & 0.000 & -0.018 \\ & & \end{array}$

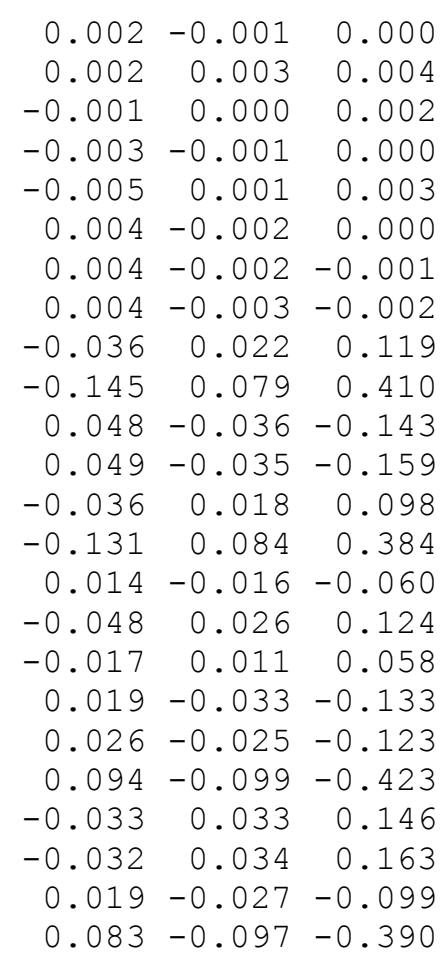

Frequency

IR Active:

IR Intens:

Raman Active:
663.44

YES

2.325

YES

$\mathrm{X}$

$-0.002-0.001$

$0.072-0.102$

$-0.169-0.185$

$-0.140-0.209$

$\begin{array}{lll}-0.104 & 0.005 & -0.042\end{array}$

$\begin{array}{lll}-0.010 & -0.006 & 0.001\end{array}$

$-0.004-0.008-0.003$

$\begin{array}{lll}-0.002 & 0.001 & 0.000\end{array}$

$\begin{array}{lll}-0.002 & 0.001 & 0.003\end{array}$

$\begin{array}{lll}-0.003 & 0.002 & 0.003\end{array}$

$\begin{array}{rrr}-0.001 & 0.001 & 0.003 \\ 0.005 & -0.003 & 0.000\end{array}$

$\begin{array}{lll}0.005 & -0.003 & 0.000\end{array}$

$\begin{array}{rrrr}-0.001 & 0.003 & 0.001\end{array}$

$0.010-0.003-0.004$

$\begin{array}{llll}-0.176 & 0.128 & -0.081\end{array}$

$-0.220 \quad 0.032-0.058$

$\begin{array}{llll}0.153 & 0.233 & -0.007\end{array}$

$\begin{array}{lll}0.223 & 0.156 & 0.040\end{array}$

$\begin{array}{lll}0.112 & -0.006 & 0.045\end{array}$

$-0.193-0.094-0.023$

$\begin{array}{llll}0.183 & -0.143 & 0.088\end{array}$

$\begin{array}{lll}0.232 & 0.027 & 0.074\end{array}$

$-0.167 \quad 0.116-0.060$

$\begin{array}{llll}0.031 & 0.202 & -0.059\end{array}$

$\begin{array}{llll}0.156 & -0.115 & 0.061\end{array}$

$\begin{array}{llll}0.032 & -0.187 & 0.042\end{array}$

$0.197 \quad 0.205-0.002$

$\begin{array}{llll}0.164 & 0.228 & -0.029\end{array}$

$\begin{array}{llll}-0.081 & 0.105 & -0.042\end{array}$

$\begin{array}{lll}-0.117 & -0.194 & 0.002\end{array}$
667.52

YES

8.332

YES

$\mathrm{X}$

$-0.005$

$Y$

0.201

$0.003-0.002$

0.032

0.047

$0.090-0.043$

$0.100-0.049$

0.024

0.031

$0.023-0.236 \quad 0.062$

$\begin{array}{llll}-0.003 & 0.000 & 0.003\end{array}$

$\begin{array}{llll}-0.002 & 0.006 & 0.002\end{array}$

$\begin{array}{lll}-0.037 & 0.023-0.038\end{array}$

$\begin{array}{lll}-0.015 & 0.014 & 0.041\end{array}$

$-0.037-0.034-0.017$

$\begin{array}{lll}-0.010 & -0.025 & 0.110\end{array}$

$\begin{array}{llll}0.071 & -0.010 & 0.003\end{array}$

$\begin{array}{lll}0.145 & -0.113 & 0.024\end{array}$

$-0.063-0.045-0.030$

$-0.186-0.053-0.055$

$\begin{array}{lll}-0.117 & 0.127-0.087\end{array}$

$\begin{array}{llll}-0.121 & 0.011 & -0.041\end{array}$

$\begin{array}{lll}0.075 & -0.212 & 0.076\end{array}$

$\begin{array}{lll}-0.009 & 0.276 & -0.069\end{array}$

$\begin{array}{llll}-0.078 & 0.257 & -0.091\end{array}$

$\begin{array}{llll}0.216 & 0.025 & 0.069\end{array}$

$\begin{array}{llll}0.153 & -0.170 & 0.107\end{array}$

$0.040-0.222 \quad 0.065$

$\begin{array}{llll}0.171 & -0.166 & 0.069\end{array}$

$\begin{array}{llll}-0.012 & 0.202-0.054\end{array}$

$\begin{array}{llll}-0.140 & 0.129-0.085\end{array}$

$-0.070 \quad 0.066-0.027$

$\begin{array}{llll}0.173 & -0.094 & 0.053\end{array}$

$-0.238-0.049-0.043$

$\begin{array}{lll}-0.221 & 0.072-0.081\end{array}$

$\begin{array}{rrr}-0.002 & 0.009 & 0.000 \\ 0.007 & -0.001 & 0.007 \\ 0.003 & -0.001 & -0.007 \\ 0.005 & 0.004 & -0.001 \\ -0.002 & 0.004 & -0.015 \\ -0.009 & 0.003 & -0.002 \\ -0.016 & 0.011 & -0.001 \\ -0.004 & 0.006 & 0.006 \\ -0.175 & -0.047 & -0.049 \\ -0.110 & 0.131 & -0.075 \\ -0.126 & 0.005 & -0.045 \\ 0.050 & -0.195 & 0.062 \\ -0.023 & 0.265 & -0.068 \\ -0.076 & 0.252 & -0.080 \\ 0.194 & 0.039 & 0.058 \\ 0.136 & -0.148 & 0.096 \\ 0.007 & 0.226 & -0.049 \\ -0.190 & 0.136 & -0.077 \\ -0.017 & -0.206 & 0.041 \\ 0.173 & -0.105 & 0.062 \\ 0.033 & -0.135 & 0.044 \\ -0.225 & 0.033 & -0.050 \\ 0.301 & 0.017 & 0.060 \\ 0.297 & -0.046 & 0.066\end{array}$

706.68

YES

37.518

YES

$\begin{array}{ccc}X & Y & Z \\ -0.001 & 0.006 & -0.011 \\ 0.022 & 0.003 & 0.001 \\ 0.011 & -0.006 & 0.010 \\ 0.006 & -0.006 & 0.010 \\ 0.001 & -0.020 & 0.002\end{array}$

$0.014-0.004-0.003$

$\begin{array}{lll}-0.002 & -0.001 & 0.008\end{array}$

$\begin{array}{lll}0.121 & -0.080 & 0.202\end{array}$

$0.001-0.022-0.082$

$\begin{array}{lll}0.062 & 0.193 & 0.146\end{array}$

$\begin{array}{llll}-0.043 & 0.164 & -0.394\end{array}$

$-0.186-0.018-0.004$

$\begin{array}{llll}-0.508 & 0.437 & -0.112\end{array}$

$\begin{array}{rrr}0.395 & 0.116 & 0.088\end{array}$

$-0.020-0.006-0.004$

$\begin{array}{rrr}-0.020 & 0.007 & 0.003\end{array}$

$-0.008-0.002-0.006$

$\begin{array}{rrr}0.009 & -0.022 & 0.008\end{array}$

$\begin{array}{llll}-0.001 & 0.020 & -0.002\end{array}$

$\begin{array}{rrr}-0.013 & 0.019 & 0.002\end{array}$

$\begin{array}{llll}0.023 & -0.004 & 0.003\end{array}$

$\begin{array}{lll}0.018 & -0.021 & 0.005\end{array}$

$0.012-0.027 \quad 0.005$

$\begin{array}{llll}0.029 & -0.020 & 0.007\end{array}$

$0.0010 .025-0.005$

$\begin{array}{lll}-0.009 & 0.022 & 0.001\end{array}$

$-0.0040 .008-0.006$

$\begin{array}{rrr}0.023-0.009 & 0.008\end{array}$

$-0.023-0.004-0.002$

$\begin{array}{lll}-0.023 & 0.016 \quad 0.002\end{array}$ 


\begin{tabular}{|c|c|c|c|c|c|c|c|c|}
\hline Frequency: & \multicolumn{2}{|l|}{742.21} & \multicolumn{3}{|c|}{760.14} & \multicolumn{3}{|c|}{768.96} \\
\hline IR Active: & YES & & & YES & & & YES & \\
\hline IR Intens: & 19.743 & & & 0.056 & & & 14.420 & \\
\hline Raman Active: & YES & & & YES & & & YES & \\
\hline $\mathrm{X}$ & $\mathrm{Y}$ & Z & $\mathrm{X}$ & Y & Z & $X$ & $\mathrm{Y}$ & Z \\
\hline 0.000 & 0.000 & 0.001 & 0.000 & 0.000 & 0.000 & 0.002 & 0.003 & 0.005 \\
\hline 0.047 & -0.052 & -0.176 & -0.023 & 0.025 & 0.099 & -0.003 & -0.002 & -0.004 \\
\hline-0.054 & 0.040 & 0.219 & 0.022 & -0.018 & -0.092 & -0.002 & 0.002 & 0.001 \\
\hline-0.062 & 0.057 & 0.221 & -0.029 & 0.016 & 0.085 & -0.001 & 0.002 & 0.002 \\
\hline 0.068 & -0.041 & -0.179 & 0.029 & -0.019 & -0.097 & 0.001 & 0.001 & -0.002 \\
\hline 0.001 & -0.004 & 0.000 & 0.000 & -0.001 & 0.000 & 0.004 & 0.003 & -0.010 \\
\hline 0.006 & -0.001 & 0.003 & 0.000 & -0.002 & 0.000 & -0.006 & 0.003 & -0.005 \\
\hline-0.003 & 0.003 & -0.003 & 0.001 & 0.004 & -0.001 & -0.089 & 0.049 & -0.108 \\
\hline-0.012 & 0.010 & 0.008 & 0.001 & 0.000 & 0.000 & -0.017 & -0.001 & 0.074 \\
\hline-0.020 & -0.006 & -0.012 & 0.003 & 0.001 & 0.003 & 0.176 & 0.093 & 0.314 \\
\hline-0.011 & -0.004 & 0.032 & 0.001 & 0.002 & -0.003 & 0.029 & 0.129 & -0.190 \\
\hline 0.006 & 0.006 & 0.000 & 0.000 & -0.001 & 0.000 & 0.063 & -0.157 & 0.013 \\
\hline 0.025 & -0.025 & 0.017 & 0.000 & 0.001 & -0.004 & -0.310 & 0.360 & -0.077 \\
\hline-0.043 & -0.005 & -0.006 & 0.006 & 0.000 & -0.002 & 0.718 & -0.055 & -0.023 \\
\hline-0.028 & 0.012 & 0.109 & -0.031 & 0.015 & 0.068 & 0.002 & 0.000 & 0.001 \\
\hline-0.138 & 0.076 & 0.408 & 0.013 & -0.014 & -0.054 & 0.001 & 0.006 & 0.007 \\
\hline 0.030 & -0.045 & -0.128 & 0.009 & -0.003 & -0.018 & 0.001 & -0.002 & -0.001 \\
\hline-0.019 & -0.018 & 0.030 & 0.140 & -0.088 & -0.373 & -0.003 & 0.001 & 0.004 \\
\hline-0.037 & 0.040 & 0.081 & -0.024 & 0.016 & 0.076 & 0.000 & -0.002 & 0.001 \\
\hline-0.093 & 0.100 & 0.316 & 0.081 & -0.059 & -0.243 & 0.003 & 0.000 & 0.006 \\
\hline 0.042 & -0.015 & -0.141 & 0.002 & -0.002 & 0.007 & -0.002 & 0.001 & -0.003 \\
\hline 0.046 & -0.037 & -0.165 & 0.156 & -0.117 & -0.459 & -0.002 & 0.003 & -0.001 \\
\hline 0.022 & -0.032 & -0.141 & 0.004 & 0.000 & -0.001 & -0.002 & 0.003 & -0.002 \\
\hline 0.038 & -0.026 & -0.142 & -0.103 & 0.103 & 0.471 & -0.004 & 0.002 & -0.005 \\
\hline-0.016 & 0.014 & 0.107 & 0.013 & -0.014 & -0.070 & -0.002 & -0.004 & 0.003 \\
\hline-0.095 & 0.088 & 0.421 & -0.005 & 0.012 & 0.027 & -0.001 & -0.004 & 0.004 \\
\hline 0.037 & -0.019 & -0.129 & -0.009 & 0.001 & 0.022 & -0.001 & -0.002 & -0.001 \\
\hline-0.003 & 0.024 & 0.044 & -0.086 & 0.085 & 0.363 & -0.006 & 0.000 & -0.003 \\
\hline-0.031 & 0.025 & 0.082 & 0.020 & -0.020 & -0.075 & 0.002 & 0.001 & 0.001 \\
\hline-0.089 & 0.071 & 0.338 & -0.047 & 0.059 & 0.229 & 0.001 & -0.001 & 0.002 \\
\hline Frequency: & 786.06 & & & 789.23 & & & 821.26 & \\
\hline IR Active: & YES & & & YES & & & YES & \\
\hline IR Intens: & 8.252 & & & 77.686 & & & 1.234 & \\
\hline Raman Active: & YES & & & YES & & & YES & \\
\hline $\mathrm{X}$ & $\mathrm{Y}$ & Z & $\mathrm{X}$ & $\mathrm{Y}$ & Z & X & $\mathrm{Y}$ & Z \\
\hline-0.001 & 0.001 & -0.002 & 0.000 & 0.000 & -0.001 & 0.000 & 0.000 & 0.000 \\
\hline 0.042 & -0.128 & 0.040 & -0.004 & -0.004 & 0.048 & 0.012 & -0.010 & -0.053 \\
\hline-0.059 & -0.088 & -0.020 & -0.006 & -0.010 & -0.003 & -0.043 & 0.043 & 0.194 \\
\hline 0.081 & 0.070 & -0.018 & 0.007 & 0.008 & 0.002 & 0.058 & -0.042 & -0.189 \\
\hline 0.132 & -0.023 & 0.042 & 0.000 & 0.005 & 0.043 & -0.018 & 0.011 & 0.050 \\
\hline-0.008 & -0.006 & 0.001 & -0.001 & 0.000 & 0.000 & 0.000 & -0.001 & 0.000 \\
\hline 0.003 & 0.008 & 0.005 & 0.000 & 0.002 & 0.001 & -0.003 & -0.002 & -0.002 \\
\hline 0.006 & -0.004 & 0.010 & 0.000 & 0.000 & -0.001 & -0.001 & -0.002 & -0.001 \\
\hline-0.003 & 0.001 & 0.000 & 0.004 & -0.003 & -0.002 & 0.000 & 0.000 & 0.001 \\
\hline-0.005 & 0.008 & 0.004 & 0.004 & -0.001 & -0.001 & 0.000 & 0.000 & 0.000 \\
\hline-0.005 & 0.007 & -0.010 & 0.003 & -0.002 & -0.003 & 0.000 & 0.000 & 0.001 \\
\hline-0.004 & 0.000 & -0.001 & -0.001 & 0.000 & 0.000 & 0.000 & 0.000 & 0.000 \\
\hline-0.008 & 0.007 & -0.007 & 0.001 & -0.001 & -0.003 & -0.002 & 0.001 & 0.001 \\
\hline 0.005 & 0.004 & 0.006 & 0.001 & 0.001 & 0.001 & 0.003 & 0.000 & -0.002 \\
\hline 0.188 & -0.059 & 0.096 & 0.037 & -0.015 & -0.038 & -0.010 & 0.003 & 0.008 \\
\hline 0.291 & 0.052 & -0.044 & -0.041 & 0.042 & 0.185 & 0.108 & -0.058 & -0.308 \\
\hline-0.123 & -0.135 & 0.010 & -0.005 & -0.018 & -0.022 & -0.022 & 0.021 & 0.072 \\
\hline-0.069 & -0.155 & -0.236 & -0.161 & 0.083 & 0.407 & 0.154 & -0.091 & -0.419 \\
\hline-0.072 & 0.159 & -0.036 & 0.012 & 0.004 & -0.062 & 0.000 & -0.002 & 0.014 \\
\hline 0.262 & 0.199 & -0.164 & -0.088 & 0.097 & 0.312 & 0.110 & -0.078 & -0.315 \\
\hline
\end{tabular}




$\mathrm{C}$
$\mathrm{H}$
$\mathrm{C}$
$\mathrm{H}$
$\mathrm{C}$
$\mathrm{H}$
$\mathrm{C}$
$\mathrm{H}$
$\mathrm{C}$
$\mathrm{H}$

$$
\begin{array}{rrr}
-0.083 & 0.163 & -0.039 \\
-0.062 & 0.018 & -0.199 \\
-0.175 & 0.060 & -0.035 \\
-0.052 & 0.083 & -0.148 \\
0.091 & -0.184 & 0.073 \\
-0.014 & -0.282 & -0.030 \\
0.115 & 0.138 & 0.010 \\
0.117 & 0.121 & -0.172 \\
-0.168 & 0.045 & -0.032 \\
-0.178 & -0.270 & -0.123
\end{array}
$$

\begin{tabular}{lrrr} 
Frequency: & \multicolumn{3}{c}{908.54} \\
IR Active: & YES \\
IR Intens: & 0.165 \\
Raman Active: & YES \\
& X & Y & Z \\
Re & 0.000 & 0.000 & 0.000 \\
N & -0.005 & 0.005 & 0.019 \\
C & 0.014 & -0.014 & -0.059 \\
C & -0.021 & 0.015 & 0.060 \\
N & 0.009 & -0.003 & -0.021 \\
O & -0.001 & -0.002 & 0.001 \\
O & -0.003 & -0.003 & -0.003 \\
O & 0.000 & 0.000 & 0.000 \\
C & 0.000 & 0.000 & 0.000 \\
H & 0.001 & 0.002 & 0.003 \\
H & 0.000 & 0.002 & -0.004 \\
C & 0.000 & 0.001 & 0.000 \\
H & 0.004 & -0.001 & -0.007 \\
H & 0.001 & 0.001 & 0.003 \\
C & -0.022 & 0.012 & 0.059 \\
H & 0.124 & -0.057 & -0.326 \\
C & -0.017 & 0.007 & 0.039 \\
H & 0.113 & -0.076 & -0.328 \\
C & 0.003 & -0.003 & -0.004 \\
H & -0.030 & 0.023 & 0.105 \\
C & 0.036 & -0.025 & -0.106 \\
H & -0.173 & 0.134 & 0.529 \\
C & -0.019 & 0.020 & 0.092 \\
H & 0.097 & -0.096 & -0.433 \\
C & 0.010 & -0.012 & -0.046 \\
H & -0.054 & 0.062 & 0.249 \\
C & 0.007 & -0.008 & -0.033 \\
H & -0.065 & 0.071 & 0.281 \\
C & 0.001 & 0.001 & 0.000 \\
H & 0.016 & -0.012 & -0.068
\end{tabular}

$$
\begin{array}{rrr}
0.000 & 0.011 & -0.027 \\
-0.117 & 0.081 & 0.315 \\
-0.012 & 0.000 & -0.027 \\
-0.090 & 0.088 & 0.353 \\
0.020 & -0.032 & -0.041 \\
-0.043 & 0.015 & 0.179 \\
0.017 & 0.010 & -0.021 \\
-0.090 & 0.124 & 0.432 \\
-0.004 & -0.009 & -0.067 \\
-0.095 & 0.055 & 0.332
\end{array}
$$

\begin{tabular}{rrr}
\multicolumn{4}{c}{912.84} \\
YES \\
Y & .238 \\
YES \\
Y & $Z$ \\
-0.001 & -0.001 & 0.000 \\
0.001 & 0.000 & -0.002 \\
-0.001 & 0.000 & 0.001 \\
0.000 & 0.000 & -0.001 \\
0.000 & 0.000 & -0.001 \\
-0.006 & -0.008 & 0.008 \\
-0.005 & -0.005 & -0.006 \\
-0.001 & 0.029 & -0.001 \\
-0.042 & -0.057 & -0.021 \\
0.093 & 0.176 & 0.293 \\
0.005 & 0.131 & -0.398 \\
0.052 & 0.022 & 0.018 \\
0.272 & -0.083 & -0.470 \\
-0.188 & 0.159 & 0.570 \\
0.000 & 0.000 & 0.002 \\
0.002 & -0.003 & -0.005 \\
0.000 & 0.001 & 0.000 \\
0.001 & 0.001 & -0.005 \\
0.000 & 0.000 & 0.000 \\
-0.002 & 0.000 & 0.002 \\
0.000 & 0.000 & -0.001 \\
-0.003 & 0.001 & 0.007 \\
0.001 & 0.000 & -0.003 \\
-0.005 & 0.003 & 0.015 \\
-0.001 & 0.001 & 0.002 \\
0.003 & 0.000 & -0.008 \\
-0.001 & 0.000 & 0.001 \\
0.002 & -0.003 & -0.011 \\
0.001 & 0.000 & 0.000 \\
0.000 & 0.001 & 0.003
\end{tabular}

999.79

YES

3.926

YES

\begin{tabular}{crr}
$X$ & \multicolumn{1}{c}{$Y$} & $Z$ \\
0.003 & 0.005 & -0.001 \\
0.001 & 0.001 & 0.000 \\
0.000 & -0.003 & -0.007 \\
-0.008 & 0.001 & 0.020 \\
-0.008 & 0.004 & 0.014 \\
-0.016 & -0.050 & 0.021 \\
-0.012 & -0.009 & -0.013 \\
-0.004 & 0.000 & 0.001 \\
0.005 & -0.001 & 0.000
\end{tabular}

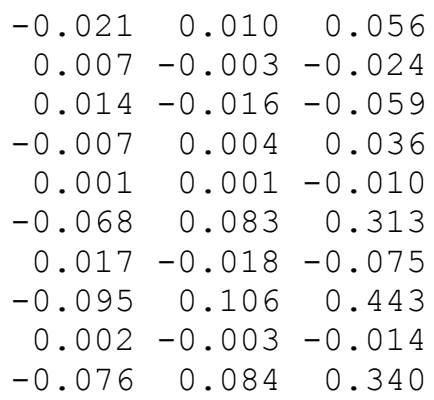




$\begin{array}{lrrr}\mathrm{H} & -0.058 & 0.036 & -0.103 \\ \mathrm{H} & -0.033 & 0.005 & -0.127 \\ \mathrm{C} & 0.048 & -0.027 & 0.053 \\ \mathrm{H} & -0.030 & 0.024 & 0.181 \\ \mathrm{H} & 0.011 & 0.017 & 0.225 \\ \mathrm{C} & 0.012 & -0.019 & -0.063 \\ \mathrm{H} & -0.155 & -0.029 & 0.310 \\ \mathrm{C} & -0.007 & 0.030 & 0.045 \\ \mathrm{H} & 0.084 & -0.021 & -0.240 \\ \mathrm{C} & -0.008 & -0.004 & 0.024 \\ \mathrm{H} & 0.009 & -0.053 & -0.149 \\ \mathrm{C} & 0.010 & -0.018 & -0.029 \\ \mathrm{H} & -0.053 & 0.015 & 0.154 \\ \mathrm{C} & -0.015 & 0.006 & -0.002 \\ \mathrm{H} & -0.034 & -0.006 & -0.016 \\ \mathrm{C} & -0.010 & -0.007 & 0.013 \\ \mathrm{H} & -0.081 & -0.069 & -0.060 \\ \mathrm{C} & 0.013 & 0.002 & -0.007 \\ \mathrm{H} & 0.010 & 0.010 & 0.041 \\ \mathrm{C} & -0.002 & -0.003 & -0.003 \\ \mathrm{H} & -0.014 & -0.033 & 0.033\end{array}$

\begin{tabular}{lrrr} 
Frequency: & \multicolumn{3}{c}{1007.33} \\
IR Active: & \multicolumn{3}{c}{ YES } \\
IR Intens: & \multicolumn{3}{c}{ Y2.836 } \\
Raman Active: & YES \\
& X & Y & Z \\
Re & -0.001 & -0.014 & 0.012 \\
N & -0.007 & 0.002 & 0.006 \\
C & 0.002 & 0.000 & 0.007 \\
C & 0.011 & 0.003 & 0.004 \\
N & -0.006 & 0.018 & -0.002 \\
O & 0.060 & 0.204 & -0.084 \\
O & -0.065 & -0.040 & -0.073 \\
O & 0.070 & -0.035 & -0.017 \\
C & -0.019 & -0.003 & 0.210 \\
H & 0.204 & -0.016 & 0.392 \\
H & 0.149 & -0.005 & 0.104 \\
C & -0.094 & 0.065 & -0.140 \\
H & 0.119 & -0.086 & -0.511 \\
H & -0.056 & -0.003 & -0.395 \\
C & 0.005 & -0.008 & -0.022 \\
H & -0.051 & 0.015 & 0.124 \\
C & -0.015 & -0.009 & 0.014 \\
H & 0.016 & -0.031 & -0.069 \\
C & -0.004 & 0.003 & 0.006 \\
H & 0.012 & -0.009 & -0.041 \\
C & 0.018 & -0.008 & -0.002 \\
H & -0.005 & -0.011 & 0.056 \\
C & 0.004 & -0.009 & -0.021 \\
H & -0.023 & 0.020 & 0.108 \\
C & 0.011 & -0.016 & -0.051 \\
H & -0.094 & 0.053 & 0.284 \\
C & 0.003 & 0.013 & 0.027 \\
H & 0.042 & -0.026 & -0.121 \\
C & -0.009 & 0.006 & 0.024 \\
H & 0.027 & -0.048 & -0.143 \\
& & &
\end{tabular}

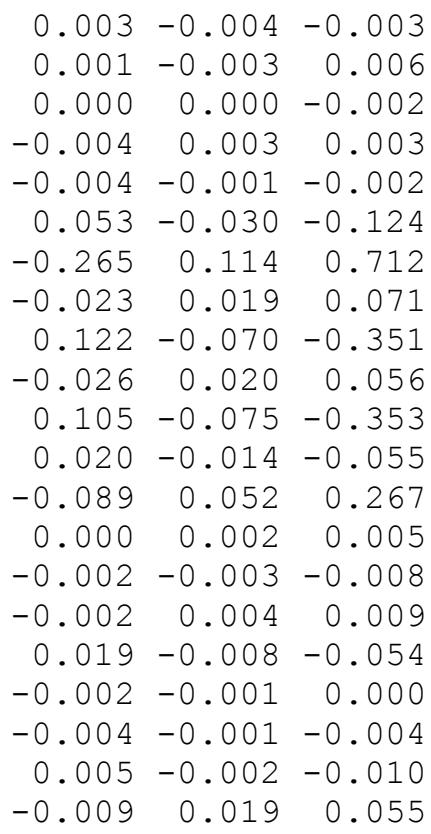

$\begin{array}{llll}-0.009 & 0.019 & 0.055\end{array}$

1013.58
YES
55.161
YES

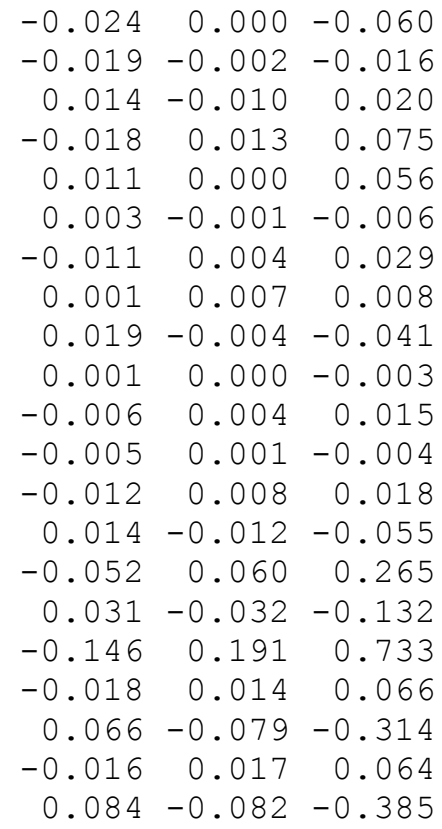
Frequency:
1033.16
YES
1034.22
$\mathrm{YES}$
.872
IR Intens:
26.016 


\begin{tabular}{|c|c|c|c|}
\hline Raman & Active: & YES & \\
\hline & $X$ & Y & Z \\
\hline $\mathrm{Re}$ & 0.000 & 0.000 & 0.000 \\
\hline $\mathrm{N}$ & 0.278 & -0.028 & 0.064 \\
\hline $\mathrm{C}$ & 0.015 & 0.041 & 0.002 \\
\hline C & 0.023 & 0.022 & -0.006 \\
\hline $\mathrm{N}$ & -0.031 & 0.138 & -0.030 \\
\hline O & -0.006 & 0.004 & -0.003 \\
\hline 0 & 0.002 & -0.003 & 0.002 \\
\hline 0 & 0.044 & -0.032 & -0.027 \\
\hline $\mathrm{C}$ & -0.065 & 0.042 & 0.001 \\
\hline $\mathrm{H}$ & -0.076 & 0.029 & -0.024 \\
\hline $\mathrm{H}$ & -0.062 & 0.038 & -0.004 \\
\hline C & 0.011 & -0.008 & 0.025 \\
\hline $\mathrm{H}$ & -0.012 & 0.015 & 0.052 \\
\hline $\mathrm{H}$ & 0.003 & -0.001 & 0.054 \\
\hline $\mathrm{C}$ & -0.036 & 0.002 & 0.001 \\
\hline $\mathrm{H}$ & -0.001 & -0.011 & -0.101 \\
\hline C & -0.084 & -0.104 & -0.051 \\
\hline $\mathrm{H}$ & -0.180 & -0.055 & 0.193 \\
\hline C & -0.011 & -0.023 & 0.060 \\
\hline $\mathrm{H}$ & 0.100 & -0.108 & -0.272 \\
\hline C & 0.132 & -0.039 & 0.031 \\
\hline $\mathrm{H}$ & 0.099 & -0.010 & 0.161 \\
\hline C & -0.110 & 0.228 & -0.049 \\
\hline $\mathrm{H}$ & -0.151 & 0.172 & -0.231 \\
\hline $\mathrm{C}$ & -0.002 & -0.029 & -0.022 \\
\hline $\mathrm{H}$ & -0.081 & -0.008 & 0.168 \\
\hline C & -0.163 & -0.184 & 0.070 \\
\hline $\mathrm{H}$ & -0.107 & -0.276 & -0.289 \\
\hline C & -0.001 & -0.020 & -0.070 \\
\hline $\mathrm{H}$ & -0.108 & 0.085 & 0.367 \\
\hline
\end{tabular}

\begin{tabular}{rrr}
\multicolumn{1}{c}{ YES } & \multicolumn{1}{c}{ Y } & \multicolumn{1}{c}{$Z$} \\
0.000 & 0.000 & 0.000 \\
-0.022 & 0.002 & -0.013 \\
-0.003 & -0.001 & 0.008 \\
-0.002 & 0.000 & 0.005 \\
-0.002 & 0.009 & -0.010 \\
0.000 & -0.003 & 0.001 \\
0.001 & 0.001 & 0.001 \\
-0.010 & 0.007 & 0.006 \\
0.014 & -0.009 & -0.001 \\
0.015 & -0.006 & 0.003 \\
0.011 & -0.008 & 0.000 \\
-0.002 & 0.001 & -0.004 \\
0.003 & -0.003 & -0.010 \\
-0.001 & 0.000 & -0.009 \\
0.009 & -0.005 & -0.014 \\
-0.041 & 0.011 & 0.112 \\
-0.024 & 0.006 & 0.053 \\
0.098 & -0.071 & -0.296 \\
0.025 & -0.014 & -0.078 \\
-0.154 & 0.104 & 0.436 \\
-0.003 & 0.006 & 0.040 \\
0.079 & -0.061 & -0.211 \\
0.000 & -0.009 & 0.049 \\
0.077 & -0.069 & -0.240 \\
0.007 & -0.006 & -0.024 \\
-0.026 & 0.047 & 0.167 \\
-0.004 & 0.032 & 0.070 \\
0.098 & -0.077 & -0.381 \\
0.020 & -0.021 & -0.094 \\
-0.117 & 0.131 & 0.529
\end{tabular}

1060.28 YES

0.055

YES
Raman Active: $\quad$ YES

\begin{tabular}{lrrr} 
Frequency: & \multicolumn{3}{c}{1051.82} \\
IR Active: & \multicolumn{3}{c}{ YES } \\
IR Intens: & \multicolumn{3}{c}{ I060 } \\
Raman Active: & YES \\
& X & Y & Z \\
Re & 0.001 & -0.001 & 0.008 \\
N & 0.214 & -0.037 & 0.039 \\
C & 0.005 & 0.007 & 0.002 \\
C & -0.004 & -0.007 & 0.009 \\
N & 0.076 & -0.253 & 0.058 \\
O & 0.025 & 0.097 & -0.042 \\
O & -0.082 & -0.049 & -0.085 \\
O & -0.235 & 0.146 & 0.167 \\
C & 0.309 & -0.190 & -0.095 \\
H & 0.260 & -0.133 & -0.065 \\
H & 0.200 & -0.161 & -0.032 \\
C & -0.037 & 0.018 & -0.046 \\
H & 0.037 & -0.061 & -0.099 \\
H & 0.041 & 0.006 & -0.125 \\
C & -0.024 & 0.004 & -0.011 \\
H & -0.016 & 0.052 & 0.017 \\
C & 0.155 & 0.162 & 0.027 \\
H & 0.204 & 0.137 & -0.013 \\
C & 0.007 & -0.010 & -0.007 \\
H & -0.015 & 0.015 & 0.057 \\
C & -0.218 & 0.059 & -0.083 \\
H & -0.200 & 0.126 & -0.124 \\
C & -0.078 & 0.170 & -0.050 \\
& & &
\end{tabular}

\begin{tabular}{rrr}
\multicolumn{1}{c}{ YES } & \multicolumn{1}{c}{ Y } & \multicolumn{1}{c}{$Z$} \\
0.000 & 0.002 & -0.001 \\
-0.090 & 0.010 & -0.035 \\
0.006 & -0.008 & 0.005 \\
0.024 & 0.008 & -0.001 \\
-0.067 & 0.279 & -0.083 \\
-0.006 & -0.019 & 0.007 \\
0.000 & -0.009 & 0.000 \\
-0.224 & 0.138 & 0.138 \\
0.307 & -0.190 & -0.057 \\
0.297 & -0.139 & 0.003 \\
0.222 & -0.162 & -0.007 \\
-0.044 & 0.024 & -0.073 \\
0.056 & -0.070 & -0.177 \\
0.027 & 0.003 & -0.183 \\
-0.002 & -0.011 & 0.016 \\
0.015 & -0.073 & -0.083 \\
-0.165 & -0.190 & -0.044 \\
-0.221 & -0.179 & 0.116 \\
-0.013 & 0.015 & 0.026 \\
0.063 & -0.035 & -0.160 \\
0.236 & -0.069 & 0.083 \\
0.199 & -0.143 & 0.173 \\
0.034 & -0.062 & 0.014 \\
0.097 & -0.020 & 0.073 \\
0.010 & -0.012 & 0.016 \\
0.064 & -0.007 & -0.046 \\
0.051 & 0.048 & -0.014 \\
0.055 & 0.057 & 0.068 \\
-0.031 & 0.006 & 0.007 \\
-0.011 & -0.024 & -0.084 \\
& &
\end{tabular}

\subsection{5} YES

2. 111 YES

\begin{tabular}{rrr} 
X & \multicolumn{1}{c}{$Y$} & $Z$ \\
0.000 & 0.001 & -0.001 \\
0.031 & -0.055 & 0.020 \\
-0.007 & -0.011 & 0.000 \\
0.019 & 0.016 & 0.003 \\
0.054 & -0.021 & 0.023 \\
-0.002 & -0.007 & 0.004 \\
0.000 & 0.000 & 0.002 \\
-0.003 & 0.001 & 0.003 \\
0.004 & -0.002 & 0.001 \\
0.004 & -0.001 & 0.003 \\
0.004 & -0.002 & 0.001 \\
0.001 & 0.000 & -0.005 \\
-0.007 & 0.006 & 0.005 \\
-0.011 & 0.002 & 0.007 \\
-0.079 & 0.081 & -0.053 \\
-0.001 & 0.382 & -0.031 \\
-0.083 & -0.049 & -0.015 \\
-0.134 & -0.008 & -0.060 \\
0.074 & -0.102 & 0.050 \\
0.345 & -0.030 & 0.126 \\
0.012 & 0.051 & -0.007 \\
0.084 & 0.276 & -0.049 \\
-0.053 & -0.029 & -0.005
\end{tabular}




$\begin{array}{rrrr}\mathrm{H} & -0.152 & 0.131 & -0.110 \\ \mathrm{C} & -0.010 & 0.029 & -0.005 \\ \mathrm{H} & -0.060 & -0.007 & -0.023 \\ \mathrm{C} & -0.107 & -0.139 & 0.013 \\ \mathrm{H} & -0.087 & -0.167 & -0.003 \\ \mathrm{C} & 0.016 & -0.008 & -0.004 \\ \mathrm{H} & -0.004 & -0.003 & 0.052\end{array}$

\begin{tabular}{lrrr} 
Frequency: & \multicolumn{3}{c}{1104.06} \\
IR Active: & \multicolumn{3}{c}{4.746} \\
IR Intens: \\
Raman Active: \\
Y Y S \\
Re & -0.001 & -0.002 & 0.000 \\
N & -0.043 & 0.019 & -0.013 \\
C & -0.051 & -0.087 & 0.009 \\
C & -0.073 & -0.065 & -0.009 \\
N & 0.031 & -0.047 & 0.019 \\
O & 0.005 & 0.010 & -0.004 \\
O & 0.003 & 0.005 & 0.004 \\
O & 0.002 & 0.001 & -0.001 \\
C & -0.002 & -0.001 & 0.002 \\
H & -0.002 & 0.005 & 0.006 \\
H & 0.008 & -0.003 & -0.002 \\
C & 0.001 & 0.001 & -0.002 \\
H & -0.001 & 0.001 & 0.003 \\
H & -0.005 & 0.000 & -0.002 \\
C & 0.039 & 0.045 & 0.000 \\
H & 0.106 & 0.259 & 0.009 \\
C & -0.085 & -0.022 & -0.023 \\
H & -0.245 & 0.153 & -0.131 \\
C & 0.034 & -0.033 & 0.021 \\
H & 0.184 & 0.006 & 0.061 \\
C & 0.034 & 0.057 & -0.001 \\
H & 0.166 & 0.508 & -0.064 \\
C & 0.043 & 0.046 & -0.002 \\
H & 0.446 & 0.232 & 0.057 \\
C & 0.027 & 0.053 & -0.005 \\
H & 0.198 & 0.144 & -0.002 \\
C & -0.006 & -0.083 & 0.018 \\
H & 0.191 & -0.218 & 0.100 \\
C & -0.027 & 0.023 & -0.013 \\
H & -0.016 & 0.142 & -0.037 \\
& & &
\end{tabular}

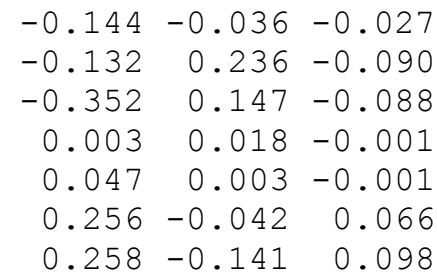

1139.96
YES
10.202
$\mathrm{YES}$

$0.000 \quad 0.000-0.001$

$-0.057-0.035-0.004$

$0.036-0.052 \quad 0.018$

$\begin{array}{lll}0.055 & -0.025 & 0.024\end{array}$

$0.0260 .065-0.003$

$\begin{array}{llll}-0.002 & -0.008 & 0.004\end{array}$

$\begin{array}{lll}0.005 & 0.002 & 0.006\end{array}$

$0.005-0.003-0.004$

$\begin{array}{llll}-0.006 & 0.003 & 0.010\end{array}$

$\begin{array}{rrr}-0.002 & 0.005 & 0.016\end{array}$

$\begin{array}{llll}0.012 & -0.004 & 0.013\end{array}$

$0.006-0.004-0.018$

$\begin{array}{llll}-0.040 & 0.030 & 0.045\end{array}$

$\begin{array}{lll}-0.048 & 0.012 & 0.054\end{array}$

$\begin{array}{lll}-0.068 & 0.038 & -0.034\end{array}$

$\begin{array}{llll}-0.055 & 0.104 & -0.032\end{array}$

$\begin{array}{llll}0.041 & -0.046 & 0.025\end{array}$

$\begin{array}{llll}0.360 & -0.411 & 0.218\end{array}$

$0.007 \quad 0.035-0.007$

$\begin{array}{rrrr}0.175 & 0.091 & 0.038\end{array}$

$-0.084-0.028-0.023$

$\begin{array}{lll}-0.148 & -0.216 & 0.015\end{array}$

$\begin{array}{llll}0.012 & 0.090 & -0.017\end{array}$

$\begin{array}{lll}0.193 & 0.175 & 0.001\end{array}$

$\begin{array}{llll}-0.051 & 0.062 & -0.027\end{array}$

$\begin{array}{llll}-0.115 & 0.036 & -0.031\end{array}$

$\begin{array}{lll}0.052 & -0.035 & 0.021\end{array}$

$\begin{array}{lll}0.459 & -0.301 & 0.173\end{array}$

$-0.034-0.013-0.006$

$\begin{array}{lll}-0.065 & -0.196 & 0.037\end{array}$

\begin{tabular}{rrr}
\multicolumn{3}{c}{1177.21} \\
\multicolumn{3}{c}{1.481} \\
YES \\
YES \\
0.001 & 0.001 & 0.000 \\
0.001 & -0.002 & 0.000 \\
0.001 & 0.002 & 0.000 \\
0.001 & 0.002 & 0.000 \\
-0.002 & 0.000 & 0.000 \\
-0.002 & -0.005 & 0.001 \\
-0.003 & -0.002 & -0.002 \\
0.024 & 0.047 & -0.002 \\
-0.079 & -0.133 & 0.002 \\
-0.301 & 0.391 & 0.263 \\
0.481 & -0.182 & -0.291 \\
0.033 & 0.053 & -0.005
\end{tabular}

$\begin{array}{rrr}-0.325 & -0.152 & -0.038 \\ -0.102 & 0.067 & -0.042 \\ -0.415 & -0.087 & -0.054 \\ 0.039 & 0.104 & -0.015 \\ -0.032 & 0.167 & -0.053 \\ 0.121 & -0.064 & 0.043 \\ 0.092 & -0.368 & 0.107\end{array}$

1146.47

YES

20.063

YES

$\begin{array}{lll}X & Y\end{array}$

$\begin{array}{rrr}-0.002 & 0.001 & 0.001\end{array}$

$-0.005-0.004-0.003$

$0.003-0.002 \quad 0.001$

$\begin{array}{lll}0.004 & -0.002 & 0.002\end{array}$

$\begin{array}{llll}0.004 & 0.003 & 0.000\end{array}$

$\begin{array}{llll}-0.002 & -0.008 & 0.001\end{array}$

$\begin{array}{lll}0.009 & 0.003 & 0.003\end{array}$

$0.012-0.004-0.018$

$-0.016-0.016-0.071$

$-0.0440 .025-0.066$

$\begin{array}{llll}-0.095 & 0.050-0.153\end{array}$

$\begin{array}{rrrr}-0.039 & 0.046 & 0.178\end{array}$

$0.390-0.279-0.399$

$0.415-0.132-0.574$

$-0.007 \quad 0.005-0.005$

$\begin{array}{lll}-0.006 & 0.020 & 0.003\end{array}$

$\begin{array}{llll}0.002 & -0.003 & 0.002\end{array}$

$\begin{array}{llll}0.031 & -0.036 & 0.017\end{array}$

$\begin{array}{lll}0.002 & 0.001 & 0.001\end{array}$

$\begin{array}{llll}0.021 & 0.007 & 0.004\end{array}$

$-0.008-0.002-0.002$

$\begin{array}{lll}-0.011 & -0.012 & 0.000\end{array}$

$\begin{array}{llll}0.002 & 0.007 & -0.001\end{array}$

$\begin{array}{llll}0.019 & 0.014 & 0.000\end{array}$

$\begin{array}{lll}-0.006 & 0.005 & -0.002\end{array}$

$-0.018-0.003-0.006$

$\begin{array}{lll}0.005 & -0.001 & 0.002\end{array}$

$0.043-0.026 \quad 0.015$

$-0.003-0.002 \quad 0.000$

$-0.006-0.022 \quad 0.004$

\begin{tabular}{rrr}
\multicolumn{4}{c}{1195.30} \\
\multicolumn{3}{c}{6.878} \\
YES & YES \\
0.000 & 0.001 & 0.000 \\
-0.005 & -0.016 & 0.003 \\
0.011 & 0.005 & 0.001 \\
0.003 & 0.013 & -0.001 \\
-0.014 & -0.007 & -0.003 \\
-0.001 & -0.003 & 0.001 \\
-0.001 & -0.001 & -0.002 \\
0.000 & 0.001 & 0.000 \\
-0.002 & -0.003 & 0.000 \\
-0.004 & 0.006 & 0.006 \\
0.007 & -0.002 & -0.007 \\
0.001 & 0.001 & 0.000
\end{tabular}




$\begin{array}{lrrr}\text { H } & 0.002 & -0.002 & 0.006 \\ \mathrm{H} & -0.004 & -0.002 & -0.010 \\ \mathrm{C} & -0.025 & 0.056 & -0.025 \\ \mathrm{H} & 0.042 & 0.279 & -0.020 \\ \mathrm{C} & -0.010 & -0.049 & 0.008 \\ \mathrm{H} & 0.197 & -0.290 & 0.131 \\ \mathrm{C} & 0.047 & 0.044 & 0.006 \\ \mathrm{H} & 0.417 & 0.160 & 0.105 \\ \mathrm{C} & -0.063 & -0.035 & -0.012 \\ \mathrm{H} & -0.109 & -0.174 & 0.001 \\ \mathrm{C} & -0.028 & -0.068 & 0.010 \\ \mathrm{H} & -0.163 & -0.138 & -0.008 \\ \mathrm{C} & 0.059 & -0.018 & 0.019 \\ \mathrm{H} & 0.265 & 0.088 & 0.030 \\ \mathrm{C} & -0.046 & -0.017 & -0.007 \\ \mathrm{H} & -0.318 & 0.157 & -0.106 \\ \mathrm{C} & 0.038 & 0.053 & -0.003 \\ \mathrm{H} & 0.094 & 0.442 & -0.088\end{array}$

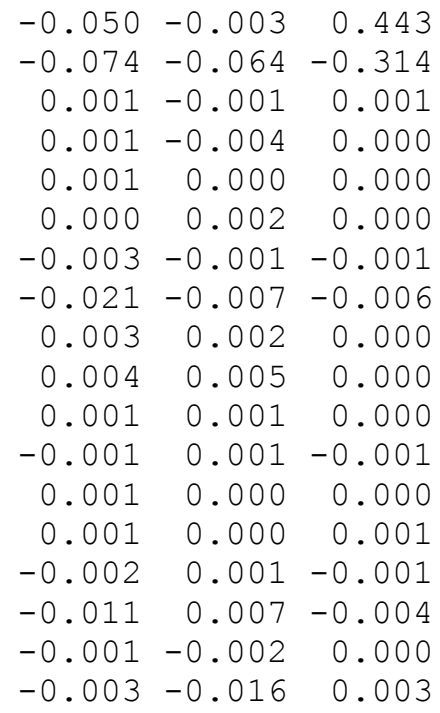

1218.08
YES
2.882
YES
$\mathrm{X} \quad \mathrm{Y}$

$\begin{array}{lll}0.000 & 0.000 & 0.000\end{array}$

$\begin{array}{lll}0.000 & 0.001 & 0.001\end{array}$

$\begin{array}{llll}0.000 & -0.001 & 0.000\end{array}$

$0.000 \quad 0.000 \quad 0.000$

$0.000 \quad 0.000 \quad 0.000$

$0.000 \quad 0.000 \quad 0.000$

$\begin{array}{lll}-0.001 & 0.000 & 0.000\end{array}$

$\begin{array}{llll}-0.011 & -0.021 & 0.006\end{array}$

$\begin{array}{llll}-0.029 & -0.038 & -0.007\end{array}$

$\begin{array}{llll}0.500 & -0.266 & 0.247\end{array}$

$\begin{array}{lll}-0.478 & 0.275 & -0.285\end{array}$

$\begin{array}{llll}0.047 & 0.069 & -0.009\end{array}$

$\begin{array}{lll}-0.002 & -0.009 & 0.394\end{array}$

$-0.118-0.028-0.228$

$0.000-0.001 \quad 0.000$

$0.000-0.002-0.001$

$\begin{array}{llll}0.000 & 0.000 & 0.000\end{array}$

$\begin{array}{llll}0.000 & 0.000 & 0.000\end{array}$

$\begin{array}{lll}0.000 & 0.000 & 0.000\end{array}$

$\begin{array}{llll}-0.003 & -0.001 & -0.001\end{array}$

$\begin{array}{llll}0.000 & 0.000 & 0.000\end{array}$

$\begin{array}{lll}0.001 & 0.002 & 0.000\end{array}$

$\begin{array}{lll}-0.001 & 0.000 & 0.000\end{array}$

$\begin{array}{lll}0.002 & 0.001 & 0.000\end{array}$

$\begin{array}{llll}0.000 & 0.000 & 0.000\end{array}$

$\begin{array}{llll}0.002 & 0.001 & -0.001\end{array}$

$\begin{array}{llll}0.000 & 0.000 & 0.000\end{array}$

$\begin{array}{llll}-0.003 & 0.002 & -0.001\end{array}$

$0.000 \quad 0.000 \quad 0.000$

$0.000-0.002 \quad 0.000$

$\begin{array}{rrr}-0.001 & 0.000 & 0.012 \\ -0.001 & -0.001 & -0.007 \\ -0.010 & -0.025 & 0.003 \\ -0.055 & -0.160 & 0.007 \\ -0.018 & 0.033 & -0.014 \\ -0.276 & 0.325 & -0.166 \\ 0.040 & 0.008 & 0.012 \\ 0.371 & 0.108 & 0.103 \\ -0.001 & -0.018 & 0.004 \\ -0.074 & -0.271 & 0.036 \\ -0.018 & -0.004 & -0.003 \\ -0.259 & -0.113 & -0.034 \\ -0.024 & -0.013 & -0.002 \\ -0.156 & -0.083 & -0.012 \\ 0.037 & -0.015 & 0.012 \\ 0.382 & -0.241 & 0.143 \\ 0.000 & 0.043 & -0.010 \\ 0.048 & 0.406 & -0.086\end{array}$

1309.79

YES

6.281

YES

$\begin{array}{lll}X & Y & Z\end{array}$

$\begin{array}{lll}0.001 & 0.001 & 0.000\end{array}$

$\begin{array}{llll}0.047 & 0.190 & -0.035\end{array}$

$\begin{array}{llll}0.020 & -0.034 & 0.009\end{array}$

$\begin{array}{llll}-0.051 & 0.032 & -0.022\end{array}$

$\begin{array}{lll}0.166 & 0.064 & 0.044\end{array}$

$0.000-0.008 \quad 0.003$

$\begin{array}{llll}-0.004 & -0.001-0.003\end{array}$

$\begin{array}{lll}0.000 & 0.001 & 0.001\end{array}$

$0.000-0.002 \quad 0.000$

$\begin{array}{lll}-0.010 & 0.008 & 0.000\end{array}$

$0.008-0.004-0.003$

$\begin{array}{llll}0.000 & 0.000 & -0.001\end{array}$

$\begin{array}{lll}-0.002 & -0.001 & 0.008\end{array}$

$\begin{array}{rrr}0.002 & 0.001 & 0.002\end{array}$

$\begin{array}{lll}-0.074 & -0.129 & 0.001\end{array}$

$\begin{array}{llll}-0.151 & -0.354 & 0.023\end{array}$

$\begin{array}{llll}-0.063 & 0.070 & -0.039\end{array}$

$\begin{array}{llll}0.055 & -0.067 & 0.043\end{array}$

$\begin{array}{lll}0.067 & 0.036 & 0.015\end{array}$

$\begin{array}{llll}-0.080 & -0.007 & -0.027\end{array}$

$\begin{array}{llll}-0.051 & -0.094 & 0.006\end{array}$

$\begin{array}{llll}0.078 & 0.352 & -0.064\end{array}$

$\begin{array}{llll}-0.079 & -0.068 & -0.002\end{array}$

$\begin{array}{lll}0.440 & 0.164 & 0.067\end{array}$

$\begin{array}{lll}-0.115 & -0.106 & 0.000\end{array}$

$\begin{array}{lll}-0.449 & -0.281 & -0.029\end{array}$

$\begin{array}{llll}0.082 & -0.057 & 0.033\end{array}$

$\begin{array}{llll}-0.076 & 0.045 & -0.031\end{array}$

$\begin{array}{lll}0.026 & 0.068 & -0.010\end{array}$

$\begin{array}{lll}0.011 & -0.066 & 0.016\end{array}$

\subsection{5}

YES

43.945

YES

1.758

5.484

Raman Active: $\quad$ YES

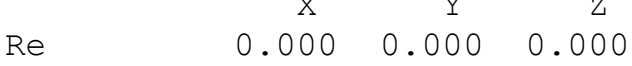

YES

$0.000 \quad 0.000$
0.000
Z
Frequency: 


$\begin{array}{lrrr}\text { N } & 0.032 & 0.012 & 0.003 \\ \mathrm{C} & -0.083 & 0.103 & -0.040 \\ \mathrm{C} & -0.102 & 0.055 & -0.045 \\ \mathrm{~N} & -0.028 & -0.043 & -0.002 \\ \mathrm{O} & 0.000 & 0.001 & 0.000 \\ \mathrm{O} & -0.001 & -0.001 & -0.001 \\ \mathrm{O} & -0.002 & 0.001 & 0.004 \\ \mathrm{C} & 0.001 & -0.001 & -0.001 \\ \mathrm{H} & 0.007 & -0.005 & -0.001 \\ \mathrm{H} & 0.006 & -0.002 & -0.004 \\ \mathrm{C} & 0.001 & 0.000 & -0.002 \\ \mathrm{H} & -0.003 & 0.001 & 0.007 \\ \mathrm{H} & -0.003 & 0.002 & 0.005 \\ \mathrm{C} & 0.055 & 0.018 & 0.013 \\ \mathrm{H} & 0.233 & 0.518 & -0.007 \\ \mathrm{C} & 0.001 & -0.007 & 0.003 \\ \mathrm{H} & 0.061 & -0.072 & 0.035 \\ \mathrm{C} & 0.024 & 0.003 & 0.008 \\ \mathrm{H} & -0.016 & -0.011 & -0.005 \\ \mathrm{C} & 0.021 & -0.039 & 0.015 \\ \mathrm{H} & -0.080 & -0.395 & 0.066 \\ \mathrm{C} & 0.054 & -0.009 & 0.014 \\ \mathrm{H} & 0.358 & 0.124 & 0.049 \\ \mathrm{C} & 0.010 & -0.044 & 0.012 \\ \mathrm{H} & -0.448 & -0.298 & -0.022 \\ \mathrm{C} & -0.004 & 0.007 & -0.002 \\ \mathrm{H} & 0.083 & -0.052 & 0.031 \\ \mathrm{C} & 0.001 & -0.037 & 0.008 \\ \mathrm{H} & 0.011 & 0.045 & -0.008\end{array}$

\begin{tabular}{lrrr} 
Frequency: & \multicolumn{3}{c}{1360.17} \\
IR Active: & \multicolumn{3}{c}{ YES } \\
IR Intens: & \multicolumn{3}{c}{ YES } \\
Raman Active: & \multicolumn{3}{c}{ Y } \\
& Z & 0.000 & 0.000 \\
Re & 0.000 & 0.000 \\
N & 0.028 & 0.195 & -0.040 \\
C & 0.208 & 0.042 & 0.043 \\
C & -0.014 & -0.209 & 0.045 \\
N & -0.185 & -0.059 & -0.052 \\
O & 0.001 & -0.002 & 0.001 \\
O & 0.000 & -0.002 & 0.001 \\
O & -0.003 & 0.002 & 0.003 \\
C & 0.004 & -0.002 & 0.001 \\
H & -0.010 & 0.004 & -0.006 \\
H & -0.007 & 0.005 & -0.006 \\
C & 0.000 & 0.000 & 0.000 \\
H & -0.003 & 0.002 & 0.001 \\
H & -0.003 & 0.000 & 0.001 \\
C & 0.113 & 0.123 & 0.015 \\
H & 0.169 & 0.264 & -0.005 \\
C & 0.054 & -0.051 & 0.031 \\
H & 0.011 & 0.008 & -0.005 \\
C & -0.092 & 0.034 & -0.040 \\
H & 0.363 & 0.181 & 0.085 \\
C & 0.011 & 0.012 & 0.000 \\
H & 0.085 & 0.280 & -0.033 \\
C & -0.007 & -0.009 & 0.000 \\
H & -0.289 & -0.131 & -0.030 \\
C & -0.099 & -0.132 & 0.010 \\
H & -0.245 & -0.212 & -0.003 \\
& & &
\end{tabular}

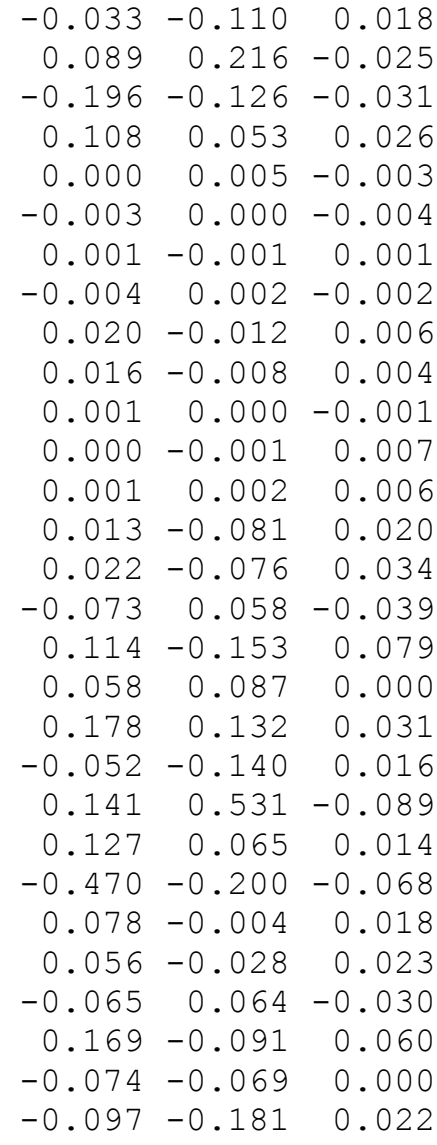

\begin{tabular}{rrrrrr} 
& 1361.57 & & & 1470.01 \\
& YES & & \multicolumn{3}{c}{ YES } \\
X & Y.511 & YES & Y & YES \\
0.000 & 0.000 & -0.001 & 0.000 & 0.000 & 0.000 \\
0.001 & 0.002 & 0.000 & 0.034 & 0.006 & 0.006 \\
0.001 & -0.003 & 0.001 & -0.053 & 0.010 & -0.014 \\
0.003 & 0.000 & 0.001 & -0.013 & 0.052 & -0.017 \\
-0.002 & -0.001 & 0.000 & -0.004 & -0.035 & 0.005 \\
-0.001 & -0.004 & 0.001 & 0.001 & 0.001 & -0.001 \\
0.003 & 0.002 & 0.003 & -0.001 & 0.000 & -0.002 \\
-0.004 & 0.004 & 0.042 & 0.003 & -0.001 & -0.003 \\
-0.112 & 0.063 & -0.093 & 0.003 & -0.005 & 0.008 \\
0.590 & -0.303 & 0.198 & -0.022 & 0.073 & 0.059 \\
0.553 & -0.345 & 0.222 & -0.072 & -0.002 & 0.058 \\
0.011 & -0.011 & 0.015 & -0.048 & 0.017 & -0.057 \\
0.061 & -0.062 & 0.005 & 0.195 & -0.395 & 0.279 \\
0.096 & 0.005 & 0.042 & 0.422 & 0.180 & 0.227 \\
0.000 & 0.002 & 0.000 & 0.042 & 0.043 & 0.006 \\
-0.002 & -0.003 & 0.000 & -0.053 & -0.238 & 0.027 \\
0.001 & -0.001 & 0.000 & -0.039 & 0.046 & -0.024 \\
-0.001 & 0.002 & -0.001 & 0.188 & -0.211 & 0.113 \\
-0.001 & -0.001 & 0.000 & -0.045 & -0.047 & -0.005 \\
0.000 & 0.000 & 0.000 & 0.213 & 0.025 & 0.067 \\
0.001 & 0.002 & 0.000 & 0.036 & -0.006 & 0.014 \\
-0.001 & -0.002 & 0.000 & 0.043 & -0.009 & 0.015 \\
-0.002 & 0.000 & 0.000 & 0.007 & -0.037 & 0.010 \\
0.002 & 0.001 & 0.000 & 0.031 & -0.033 & 0.015 \\
-0.001 & 0.000 & 0.000 & -0.040 & -0.048 & 0.003 \\
-0.002 & 0.000 & 0.001 & 0.236 & 0.096 & 0.027
\end{tabular}

$\begin{array}{rrr}-0.014 & 0.015 & -0.006 \\ 0.111 & -0.085 & 0.045 \\ -0.110 & 0.098 & -0.054 \\ 0.018 & -0.016 & 0.008 \\ 0.001 & 0.003 & -0.001 \\ 0.001 & 0.001 & 0.001 \\ 0.000 & 0.000 & 0.000 \\ 0.000 & 0.000 & 0.000 \\ 0.000 & -0.001 & -0.001 \\ 0.003 & -0.001 & 0.000 \\ 0.000 & 0.000 & 0.000 \\ 0.001 & 0.000 & -0.004 \\ -0.001 & -0.001 & -0.001 \\ 0.005 & -0.068 & 0.013 \\ 0.206 & 0.494 & -0.010 \\ -0.023 & 0.018 & -0.012 \\ -0.041 & 0.038 & -0.023 \\ 0.083 & 0.008 & 0.027 \\ -0.275 & -0.105 & -0.071 \\ 0.012 & -0.027 & 0.010 \\ -0.065 & -0.308 & 0.045 \\ -0.024 & 0.009 & -0.007 \\ -0.287 & -0.106 & -0.040 \\ -0.067 & -0.009 & -0.012 \\ 0.424 & 0.262 & 0.026 \\ 0.019 & -0.018 & 0.009 \\ 0.058 & -0.044 & 0.023 \\ -0.006 & 0.081 & -0.021 \\ -0.059 & -0.291 & 0.056\end{array}$

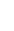

.000

14

.001 .003

59 58 
$\mathrm{C}$
$\mathrm{H}$
$\mathrm{C}$
$\mathrm{H}$

\begin{tabular}{lrrr} 
Frequency: & \multicolumn{3}{c}{1471.68} \\
IR Active: & YES \\
IR Intens: & \multicolumn{3}{c}{ 1.793 } \\
Raman Active: & YES \\
& X & Y & Z \\
Re & 0.000 & 0.000 & 0.000 \\
N & -0.029 & -0.006 & -0.005 \\
C & 0.045 & -0.008 & 0.012 \\
C & 0.011 & -0.043 & 0.014 \\
N & 0.003 & 0.028 & -0.005 \\
O & 0.000 & -0.003 & 0.001 \\
O & 0.001 & 0.001 & 0.001 \\
O & 0.005 & -0.002 & -0.005 \\
C & 0.003 & -0.005 & 0.008 \\
H & -0.025 & 0.090 & 0.073 \\
H & -0.089 & -0.004 & 0.075 \\
C & -0.054 & 0.019 & -0.063 \\
H & 0.219 & -0.441 & 0.307 \\
H & 0.468 & 0.200 & 0.253 \\
C & -0.034 & -0.034 & -0.005 \\
H & 0.043 & 0.200 & -0.019 \\
C & 0.031 & -0.038 & 0.020 \\
H & -0.152 & 0.170 & -0.092 \\
C & 0.038 & 0.038 & 0.004 \\
H & -0.175 & -0.022 & -0.055 \\
C & -0.030 & 0.005 & -0.011 \\
H & -0.036 & 0.005 & -0.012 \\
C & -0.006 & 0.032 & -0.009 \\
H & -0.026 & 0.029 & -0.013 \\
C & 0.032 & 0.041 & -0.003 \\
H & -0.194 & -0.077 & -0.023 \\
C & 0.042 & -0.027 & 0.016 \\
H & -0.201 & 0.132 & -0.078 \\
C & -0.034 & -0.043 & 0.003 \\
H & -0.010 & 0.174 & -0.043
\end{tabular}

$\begin{array}{rrr}0.001 & -0.001 & 0.000 \\ -0.003 & 0.001 & -0.001 \\ 0.000 & 0.001 & 0.000 \\ 0.000 & 0.000 & 0.000\end{array}$

1488.33

YES

68.558

YES

$\begin{array}{lll}X & Y & Z\end{array}$

$\begin{array}{llll}0.000 & 0.000 & 0.000\end{array}$

$\begin{array}{lll}-0.037-0.076 & 0.010\end{array}$

$\begin{array}{rrrr}0.109 & -0.001 & 0.026\end{array}$

$\begin{array}{lll}-0.017 & 0.102 & -0.027\end{array}$

$-0.068-0.048-0.014$

$\begin{array}{llll}-0.001 & -0.001 & 0.000\end{array}$

$0.000-0.001-0.001$

$\begin{array}{llll}0.000 & 0.000 & 0.000\end{array}$

$\begin{array}{llll}0.000 & 0.000 & 0.000\end{array}$

$\begin{array}{lll}0.001 & 0.000 & 0.000\end{array}$

$\begin{array}{lll}0.001 & 0.000 & 0.000\end{array}$

$\begin{array}{rrrr}0.001 & 0.000 & 0.001\end{array}$

$-0.005 \quad 0.009-0.005$

$-0.008-0.004-0.005$

$\begin{array}{llll}0.077 & -0.018 & 0.031\end{array}$

$\begin{array}{lll}0.098 & 0.002 & 0.035\end{array}$

$\begin{array}{lll}-0.062 & 0.120 & -0.049\end{array}$

$\begin{array}{lll}0.305 & -0.285 & 0.172\end{array}$

$-0.058-0.050-0.008$

$\begin{array}{llll}0.398 & 0.082 & 0.118\end{array}$

$\begin{array}{llll}0.039 & -0.076 & 0.030\end{array}$

$\begin{array}{lll}0.112 & 0.124 & 0.008\end{array}$

$\begin{array}{lll}-0.085 & 0.029-0.026\end{array}$

$0.1090 .135-0.002$

$-0.0320 .076-0.026$

$\begin{array}{rrrr}-0.021 & 0.099 & -0.028\end{array}$

$\begin{array}{lll}0.135 & -0.048 & 0.042\end{array}$

$\begin{array}{llll}-0.349 & 0.280 & -0.147\end{array}$

$\begin{array}{lll}-0.042 & -0.068 & 0.006\end{array}$

$\begin{array}{llll}0.016 & 0.425 & -0.096\end{array}$

$$
\begin{array}{rrr}
-0.048 & 0.033 & -0.019 \\
0.238 & -0.155 & 0.091 \\
0.040 & 0.050 & -0.003 \\
0.013 & -0.203 & 0.050
\end{array}
$$

1514.92

YES

52.753

YES

$\begin{array}{lll}X & Y\end{array}$

$\begin{array}{llll}0.000 & 0.000 & 0.000\end{array}$

$\begin{array}{llll}0.050 & -0.054 & 0.024\end{array}$

$\begin{array}{llll}-0.013 & 0.106 & -0.028\end{array}$

$\begin{array}{lll}0.091 & -0.013 & 0.032\end{array}$

$\begin{array}{llll}-0.053 & 0.040 & -0.026\end{array}$

$\begin{array}{llll}-0.001 & 0.002 & -0.001\end{array}$

$\begin{array}{lll}0.001 & -0.001 & 0.001\end{array}$

$\begin{array}{lll}-0.001 & -0.001 & 0.001\end{array}$

$\begin{array}{llll}-0.001 & 0.000 & 0.001\end{array}$

$-0.001-0.010-0.008$

$0.0110 .002-0.011$

$\begin{array}{llll}0.000 & 0.000 & 0.000\end{array}$

$\begin{array}{llll}0.002 & -0.004 & 0.003\end{array}$

$\begin{array}{lll}0.003 & 0.002 & 0.003\end{array}$

$\begin{array}{llll}-0.036 & -0.092 & 0.007\end{array}$

$\begin{array}{llll}0.155 & 0.451 & -0.029\end{array}$

$\begin{array}{lll}0.071 & -0.009 & 0.028\end{array}$

$-0.108 \quad 0.211-0.086$

$\begin{array}{llll}-0.025 & 0.057 & -0.022\end{array}$

$\begin{array}{llll}0.071 & 0.101 & 0.000\end{array}$

$\begin{array}{rrr}-0.047 & -0.099 & 0.007\end{array}$

$\begin{array}{lll}0.077 & 0.337-0.052\end{array}$

$-0.102-0.063-0.009$

$\begin{array}{llll}0.360 & 0.145 & 0.050\end{array}$

$-0.102-0.049-0.011$

$\begin{array}{rrr}0.432 & 0.240 & 0.036\end{array}$

$\begin{array}{rrrr}-0.003 & 0.067 & -0.017\end{array}$

\begin{tabular}{|c|c|c|c|c|c|}
\hline \multicolumn{3}{|c|}{1541.24} & \multicolumn{3}{|c|}{1622.45} \\
\hline & YES & & & YES & \\
\hline & 21.196 & & & 6.004 & \\
\hline & YES & & & YES & \\
\hline X & $Y$ & Z & $\mathrm{X}$ & $\mathrm{Y}$ & Z \\
\hline 0.000 & 0.000 & 0.000 & 0.000 & 0.000 & 0.000 \\
\hline 0.031 & -0.064 & 0.022 & -0.028 & -0.164 & 0.032 \\
\hline 0.090 & 0.155 & -0.013 & -0.057 & 0.124 & -0.041 \\
\hline-0.152 & -0.116 & -0.022 & -0.102 & 0.031 & -0.041 \\
\hline 0.077 & -0.025 & 0.032 & 0.126 & 0.042 & 0.034 \\
\hline 0.001 & 0.005 & -0.002 & -0.002 & -0.001 & 0.000 \\
\hline-0.003 & -0.001 & -0.003 & 0.000 & 0.001 & 0.000 \\
\hline-0.001 & 0.000 & 0.001 & 0.001 & -0.001 & -0.001 \\
\hline 0.001 & -0.001 & -0.005 & 0.000 & 0.001 & 0.001 \\
\hline 0.004 & 0.028 & 0.024 & -0.001 & -0.003 & -0.004 \\
\hline-0.021 & -0.010 & 0.030 & 0.002 & 0.002 & -0.003 \\
\hline 0.001 & 0.000 & 0.002 & -0.001 & 0.001 & -0.001 \\
\hline-0.005 & 0.009 & -0.005 & 0.005 & -0.006 & 0.001 \\
\hline-0.008 & -0.004 & -0.006 & 0.005 & 0.003 & 0.004 \\
\hline 0.011 & 0.151 & -0.028 & -0.096 & -0.051 & -0.023 \\
\hline
\end{tabular}

$\begin{array}{llll}0.205 & -0.054 & 0.059\end{array}$

$\begin{array}{llll}0.058 & -0.021 & 0.018\end{array}$

$0.0910 .120-0.009$ 


$\begin{array}{lrrr}\mathrm{H} & 0.007 & 0.013 & -0.001 \\ \mathrm{C} & 0.001 & 0.002 & 0.000 \\ \mathrm{H} & 0.003 & 0.001 & 0.001 \\ \mathrm{C} & -0.002 & 0.000 & -0.001 \\ \mathrm{H} & 0.008 & 0.004 & 0.002 \\ \mathrm{C} & 0.000 & -0.004 & 0.001 \\ \mathrm{H} & 0.004 & 0.011 & -0.001 \\ \mathrm{C} & 0.001 & -0.001 & 0.000 \\ \mathrm{H} & -0.002 & -0.003 & 0.000 \\ \mathrm{C} & 0.002 & -0.001 & 0.001 \\ \mathrm{H} & -0.001 & -0.003 & -0.001 \\ \mathrm{C} & -0.003 & 0.001 & -0.001 \\ \mathrm{H} & 0.006 & -0.005 & 0.003 \\ \mathrm{C} & 0.001 & 0.002 & 0.000 \\ \mathrm{H} & 0.000 & -0.007 & 0.002\end{array}$

$\begin{array}{lrrr}\text { Frequency: } & \text { 1632.80 } \\ \text { IR Active: } & \text { YES } \\ \text { IR Intens: } & \text { 13.592 } \\ \text { Raman Active: } & \text { YES } \\ & \text { X } & \text { Y } & \text { Z } \\ \text { Re } & 0.001 & 0.001 & 0.000 \\ \text { N } & -0.020 & -0.141 & 0.029 \\ \text { C } & -0.043 & 0.127 & -0.039 \\ \text { C } & 0.162 & -0.033 & 0.059 \\ \text { N } & -0.172 & -0.050 & -0.048 \\ \text { O } & -0.003 & -0.002 & 0.001 \\ \text { O } & -0.001 & -0.003 & -0.001 \\ \text { O } & 0.000 & 0.000 & 0.000 \\ \text { C } & 0.000 & 0.000 & 0.000 \\ \text { H } & 0.001 & 0.001 & 0.001 \\ \text { H } & 0.000 & 0.000 & 0.001 \\ \text { C } & 0.001 & 0.000 & 0.000 \\ \text { H } & -0.003 & 0.004 & 0.000 \\ \text { H } & -0.004 & -0.001 & -0.001 \\ \text { C } & 0.118 & 0.104 & 0.020 \\ \text { H } & 0.015 & -0.206 & 0.039 \\ \text { C } & -0.185 & 0.022 & -0.069 \\ \text { H } & -0.019 & -0.200 & 0.038 \\ \text { C } & 0.310 & 0.086 & 0.087 \\ \text { H } & -0.424 & -0.141 & -0.114 \\ \text { C } & -0.150 & -0.098 & -0.027 \\ \text { H } & -0.103 & 0.120 & -0.063 \\ \text { C } & -0.075 & -0.138 & 0.015 \\ \text { H } & 0.140 & -0.056 & 0.044 \\ \text { C } & 0.081 & 0.109 & -0.008 \\ \text { H } & -0.174 & -0.026 & -0.031 \\ \text { C } & 0.026 & -0.142 & 0.040 \\ \text { H } & -0.140 & -0.056 & -0.018 \\ \text { C } & 0.037 & 0.262 & -0.053 \\ \text { H } & -0.047 & -0.363 & 0.076 \\ & & & \end{array}$

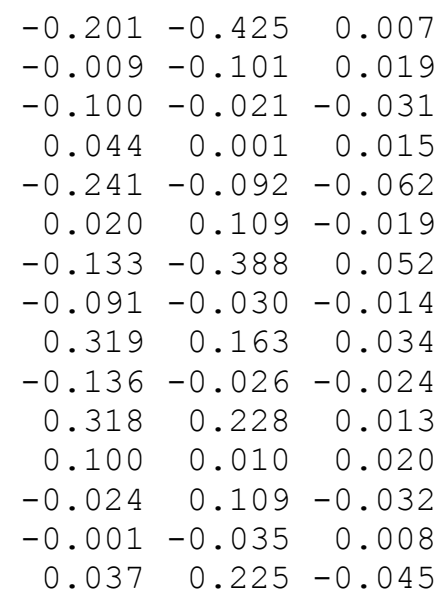

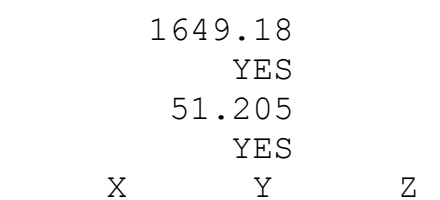

$\begin{array}{llll}0.000 & 0.000 & 0.000\end{array}$

$\begin{array}{lll}0.070 & 0.011 & 0.012\end{array}$

$\begin{array}{lll}-0.193 & -0.037 & -0.037\end{array}$

$\begin{array}{llll}0.017 & 0.181 & -0.036\end{array}$

$\begin{array}{lll}-0.003 & -0.067 & 0.012\end{array}$

$\begin{array}{lll}0.000 & 0.001 & 0.000\end{array}$

$\begin{array}{lll}-0.001 & 0.000 & 0.000\end{array}$

$\begin{array}{lll}-0.001 & 0.001 & 0.001\end{array}$

$\begin{array}{llll}0.000 & 0.000 & 0.000\end{array}$

$\begin{array}{llll}0.001 & -0.001 & 0.000\end{array}$

$0.000 \quad 0.000-0.001$

$\begin{array}{llll}0.000 & 0.000 & 0.000\end{array}$

$\begin{array}{lll}-0.001 & 0.002 & 0.001\end{array}$

$\begin{array}{lll}-0.001 & -0.001 & 0.000\end{array}$

$\begin{array}{llll}0.011 & 0.174 & -0.034\end{array}$

$\begin{array}{lll}-0.145 & -0.220 & -0.011\end{array}$

$\begin{array}{llll}0.063 & -0.156 & 0.057\end{array}$

$\begin{array}{lll}-0.199 & 0.126 & -0.101\end{array}$

$\begin{array}{lll}0.026 & 0.112 & -0.017\end{array}$

$\begin{array}{llll}-0.094 & 0.095 & -0.056\end{array}$

$\begin{array}{lll}-0.052 & -0.223 & 0.034\end{array}$

$\begin{array}{llll}0.124 & 0.360 & -0.051\end{array}$

$\begin{array}{lll}0.228 & 0.082 & 0.032\end{array}$

$-0.356-0.186-0.038$

$-0.182-0.035-0.032$

$\begin{array}{llll}0.197 & 0.188 & -0.003\end{array}$

$\begin{array}{llll}0.179 & -0.050 & 0.052\end{array}$

$\begin{array}{llll}-0.175 & 0.194 & -0.087\end{array}$

$\begin{array}{lll}-0.114 & -0.034 & -0.017\end{array}$

$\begin{array}{lll}-0.121 & 0.073 & -0.046\end{array}$

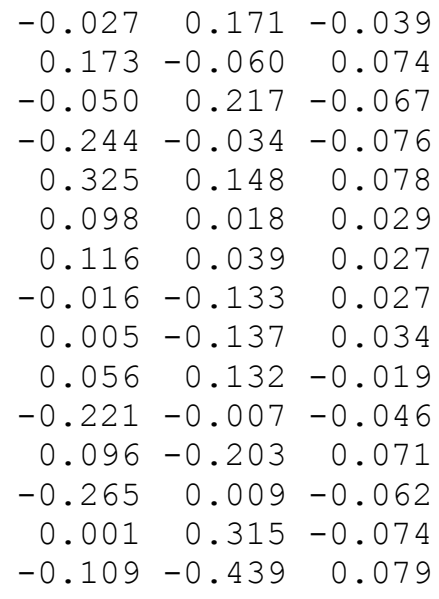

1660.61 YES

16.632 YES

$\begin{array}{ccc}X & Y & Z\end{array}$

$\begin{array}{lll}0.000 & 0.000 & 0.000\end{array}$

$\begin{array}{cccc}0.090 & -0.004 & 0.021\end{array}$

$\begin{array}{llll}-0.215 & 0.074 & -0.067\end{array}$

$\begin{array}{lll}0.103 & -0.213 & 0.079\end{array}$

$\begin{array}{llll}-0.018 & 0.094 & -0.025\end{array}$

$\begin{array}{llll}0.000 & 0.001 & -0.001\end{array}$

$\begin{array}{lll}0.001 & 0.000 & 0.001\end{array}$

$\begin{array}{lll}-0.001 & -0.001 & 0.000\end{array}$

$\begin{array}{lll}0.000 & 0.000 & 0.000\end{array}$

$\begin{array}{lll}0.000 & 0.001 & 0.002\end{array}$

$\begin{array}{llll}0.002 & -0.001 & 0.000\end{array}$

$\begin{array}{llll}0.000 & 0.000 & 0.000\end{array}$

$\begin{array}{lll}-0.002 & 0.001 & 0.002\end{array}$

$\begin{array}{rrr}0.000 & 0.000 & 0.000\end{array}$

$\begin{array}{lll}-0.013 & -0.218 & 0.043\end{array}$

$\begin{array}{lll}0.180 & 0.276 & 0.013\end{array}$

$\begin{array}{llll}-0.094 & 0.189 & -0.075\end{array}$

$\begin{array}{lll}0.209 & -0.140 & 0.107\end{array}$

$0.023-0.110 \quad 0.033$

$\begin{array}{llll}0.020 & -0.131 & 0.038\end{array}$

$\begin{array}{llll}0.010 & 0.234 & -0.050\end{array}$

$\begin{array}{lll}-0.148 & -0.264 & 0.015\end{array}$

$\begin{array}{lll}0.216 & 0.035 & 0.041\end{array}$

$-0.215-0.175-0.014$

$\begin{array}{llll}-0.201 & -0.040 & -0.035\end{array}$

$\begin{array}{llll}0.217 & 0.202 & -0.002\end{array}$

$\begin{array}{llll}0.192 & -0.067 & 0.059\end{array}$

$\begin{array}{llll}-0.166 & 0.178 & -0.082\end{array}$

$\begin{array}{llll}-0.106 & 0.014 & -0.027\end{array}$

$\begin{array}{llll}-0.127 & -0.008 & -0.028\end{array}$

$\begin{array}{rrr}3109.30 & \\ \text { YES } & \\ 13.946 & \\ \text { YES } & \\ & Y & Z \\ & 0.000 & 0.000 \\ 0 & 0.000 & 0.000 \\ 0 & 0.000 \\ 0 & 0.000 & 0.000 \\ 0 & 0.000 & 0.000\end{array}$

3135.55

YES

1.078

YES

$\begin{array}{ccc}X & Y & Z \\ 0.000 & 0.000 & 0.000 \\ 0.000 & 0.000 & 0.000 \\ 0.000 & 0.000 & 0.000 \\ 0.000 & 0.000 & 0.000\end{array}$




\begin{tabular}{|c|c|c|c|c|c|c|c|c|}
\hline 0.000 & 0.000 & 0.000 & 0.000 & 0.000 & 0.000 & 0.000 & 0.000 & 0.000 \\
\hline 0.000 & 0.000 & 0.000 & 0.000 & 0.000 & 0.000 & 0.000 & 0.000 & 0.000 \\
\hline 0.000 & 0.000 & 0.000 & 0.000 & 0.000 & 0.000 & 0.000 & 0.000 & 0.000 \\
\hline 0.002 & -0.001 & -0.001 & 0.000 & 0.000 & 0.000 & 0.000 & 0.000 & 0.000 \\
\hline 0.011 & -0.017 & -0.063 & -0.051 & -0.082 & 0.012 & 0.002 & 0.006 & -0.004 \\
\hline-0.381 & -0.387 & 0.427 & 0.387 & 0.389 & -0.458 & -0.029 & -0.032 & 0.035 \\
\hline 0.225 & 0.611 & 0.308 & 0.209 & 0.574 & 0.311 & -0.010 & -0.029 & -0.016 \\
\hline 0.000 & 0.001 & 0.001 & 0.006 & 0.006 & 0.002 & 0.056 & -0.016 & 0.032 \\
\hline 0.006 & 0.005 & 0.002 & -0.074 & -0.062 & -0.019 & -0.556 & -0.454 & -0.153 \\
\hline 0.003 & -0.015 & 0.002 & 0.001 & -0.007 & 0.001 & -0.092 & 0.635 & -0.201 \\
\hline 0.000 & 0.000 & 0.000 & 0.000 & 0.000 & 0.000 & 0.000 & 0.000 & 0.000 \\
\hline 0.000 & 0.000 & 0.000 & 0.001 & 0.000 & 0.000 & 0.001 & 0.000 & 0.000 \\
\hline 0.000 & 0.000 & 0.000 & 0.000 & 0.000 & 0.000 & 0.000 & 0.000 & 0.000 \\
\hline 0.000 & -0.001 & 0.000 & 0.000 & 0.000 & 0.000 & -0.001 & -0.001 & 0.000 \\
\hline 0.000 & 0.000 & 0.000 & 0.000 & 0.000 & 0.000 & 0.000 & 0.000 & 0.000 \\
\hline 0.000 & 0.001 & 0.000 & 0.000 & 0.000 & 0.000 & 0.000 & 0.001 & 0.000 \\
\hline 0.000 & 0.000 & 0.000 & 0.000 & 0.000 & 0.000 & 0.000 & 0.000 & 0.000 \\
\hline-0.002 & 0.000 & -0.001 & 0.000 & 0.000 & 0.000 & -0.002 & 0.000 & -0.001 \\
\hline 0.000 & 0.000 & 0.000 & 0.000 & 0.000 & 0.000 & 0.000 & 0.000 & 0.000 \\
\hline-0.001 & 0.002 & -0.001 & 0.000 & 0.000 & 0.000 & 0.000 & 0.001 & 0.000 \\
\hline 0.000 & 0.000 & 0.000 & 0.000 & 0.000 & 0.000 & 0.000 & 0.000 & 0.000 \\
\hline 0.000 & -0.001 & 0.000 & -0.001 & 0.001 & -0.001 & 0.001 & -0.001 & 0.001 \\
\hline 0.000 & 0.000 & 0.000 & 0.000 & 0.000 & 0.000 & 0.000 & 0.000 & 0.000 \\
\hline 0.000 & 0.000 & 0.000 & 0.000 & 0.000 & 0.000 & 0.000 & 0.000 & 0.000 \\
\hline 0.000 & 0.000 & 0.000 & 0.000 & 0.000 & 0.000 & 0.000 & 0.000 & 0.000 \\
\hline-0.001 & 0.000 & 0.000 & 0.000 & 0.000 & 0.000 & 0.000 & 0.000 & 0.000 \\
\hline & 3217.03 & & & 3219.75 & & & 3220.86 & \\
\hline & YES & & & YES & & & YES & \\
\hline & 0.037 & & & 1.817 & & & 0.292 & \\
\hline ive: & YES & & & YES & & & YES & \\
\hline X & $\mathrm{Y}$ & Z & $\mathrm{X}$ & $\mathrm{Y}$ & z & $\mathrm{x}$ & $\mathrm{Y}$ & Z \\
\hline 0.000 & 0.000 & 0.000 & 0.000 & 0.000 & 0.000 & 0.000 & 0.000 & 0.000 \\
\hline 0.000 & 0.000 & 0.000 & 0.000 & 0.000 & 0.000 & 0.000 & 0.000 & 0.000 \\
\hline 0.000 & 0.000 & 0.000 & -0.001 & 0.000 & 0.000 & 0.000 & 0.000 & 0.000 \\
\hline 0.000 & 0.000 & 0.000 & 0.000 & 0.000 & 0.000 & 0.000 & 0.000 & 0.000 \\
\hline 0.000 & 0.000 & 0.000 & 0.000 & 0.000 & 0.000 & 0.000 & 0.000 & 0.000 \\
\hline 0.000 & 0.000 & 0.000 & 0.000 & 0.000 & 0.000 & 0.000 & 0.000 & 0.000 \\
\hline 0.000 & 0.000 & 0.000 & 0.000 & 0.000 & 0.000 & 0.000 & 0.000 & 0.000 \\
\hline 0.000 & 0.000 & 0.000 & 0.000 & 0.000 & 0.000 & 0.000 & 0.000 & 0.000 \\
\hline-0.004 & -0.006 & 0.000 & 0.000 & 0.000 & 0.000 & 0.000 & 0.000 & 0.000 \\
\hline 0.024 & 0.026 & -0.032 & 0.000 & 0.000 & 0.000 & 0.001 & 0.001 & -0.001 \\
\hline 0.018 & 0.044 & 0.027 & 0.000 & 0.001 & 0.000 & 0.000 & 0.000 & 0.000 \\
\hline-0.033 & -0.092 & 0.007 & 0.000 & -0.001 & 0.000 & -0.001 & -0.001 & 0.000 \\
\hline 0.513 & 0.398 & 0.143 & 0.006 & 0.005 & 0.002 & 0.007 & 0.006 & 0.002 \\
\hline-0.121 & 0.691 & -0.224 & -0.001 & 0.008 & -0.003 & -0.002 & 0.008 & -0.003 \\
\hline 0.001 & 0.000 & 0.000 & -0.004 & 0.001 & -0.002 & -0.008 & 0.002 & -0.003 \\
\hline-0.010 & 0.003 & -0.004 & 0.044 & -0.014 & 0.019 & 0.088 & -0.028 & 0.039 \\
\hline-0.001 & -0.001 & 0.000 & 0.009 & 0.010 & 0.001 & 0.017 & 0.019 & 0.002 \\
\hline 0.006 & 0.006 & 0.001 & -0.113 & -0.109 & -0.016 & -0.219 & -0.212 & -0.030 \\
\hline 0.000 & 0.001 & 0.000 & 0.008 & -0.036 & 0.011 & 0.014 & -0.063 & 0.019 \\
\hline 0.003 & -0.014 & 0.004 & -0.092 & 0.418 & -0.128 & -0.159 & 0.718 & -0.220 \\
\hline 0.000 & 0.000 & 0.000 & -0.014 & 0.005 & -0.006 & -0.014 & 0.006 & -0.006 \\
\hline-0.002 & 0.000 & -0.001 & 0.169 & -0.042 & 0.066 & 0.174 & -0.044 & 0.068 \\
\hline 0.000 & 0.000 & 0.000 & 0.010 & -0.017 & 0.006 & -0.003 & 0.004 & -0.002 \\
\hline 0.000 & 0.001 & 0.000 & -0.094 & 0.220 & -0.069 & 0.027 & -0.062 & 0.020 \\
\hline 0.000 & 0.000 & 0.000 & 0.003 & -0.006 & 0.002 & -0.002 & 0.004 & -0.002 \\
\hline 0.002 & -0.003 & 0.001 & -0.036 & 0.071 & -0.026 & 0.026 & -0.051 & 0.018 \\
\hline 0.000 & 0.000 & 0.000 & 0.014 & 0.017 & -0.001 & -0.010 & -0.012 & 0.001 \\
\hline 0.001 & 0.001 & 0.000 & -0.153 & -0.219 & 0.019 & 0.103 & 0.147 & -0.013 \\
\hline 0.000 & 0.000 & 0.000 & -0.065 & 0.005 & -0.016 & 0.040 & -0.003 & 0.010 \\
\hline
\end{tabular}


3233.67

IR Active:

YES

IR Intens:

1.188

Raman Active: $\mathrm{X}$

0.000

YES

0.000

$Y$

Z

0.000

0.000

$-0.001$

0.000

0.000

0.000

$-0.001-0.001$

$0.000 \quad 0.000$

$0.000 \quad 0.000$

$0.000 \quad 0.000$

0.000

0.000

0.000

0.000

0.000

0.000

0.000

0.000

0.000

0.000

0.000

0.000

0.000

0.000

0.000

0.000

0.001

0.000

$-0.005-0.004$

0.000

$0.001-0.006$

0.001

$0.034-0.010$

0.002

$\begin{array}{lll}-0.394 & 0.127 & -0.172\end{array}$

$-0.039-0.034-0.006$

$\begin{array}{llll}0.428 & 0.406 & 0.061\end{array}$

$0.006-0.015 \quad 0.005$

$\begin{array}{llll}-0.040 & 0.170 & -0.053\end{array}$

$-0.020 \quad 0.006-0.008$

$\begin{array}{llll}0.234 & -0.057 & 0.092\end{array}$

$\begin{array}{llll}0.008 & -0.017 & 0.005\end{array}$

$\begin{array}{llll}-0.083 & 0.198 & -0.062\end{array}$

$\begin{array}{llll}-0.011 & 0.024 & -0.009\end{array}$

$0.140-0.278 \quad 0.101$

$\begin{array}{llll}-0.020 & -0.031 & 0.003\end{array}$

$\begin{array}{llll}0.235 & 0.344 & -0.031\end{array}$

$\begin{array}{lll}-0.008 & 0.003 & -0.002\end{array}$

$0.086-0.009 \quad 0.021$

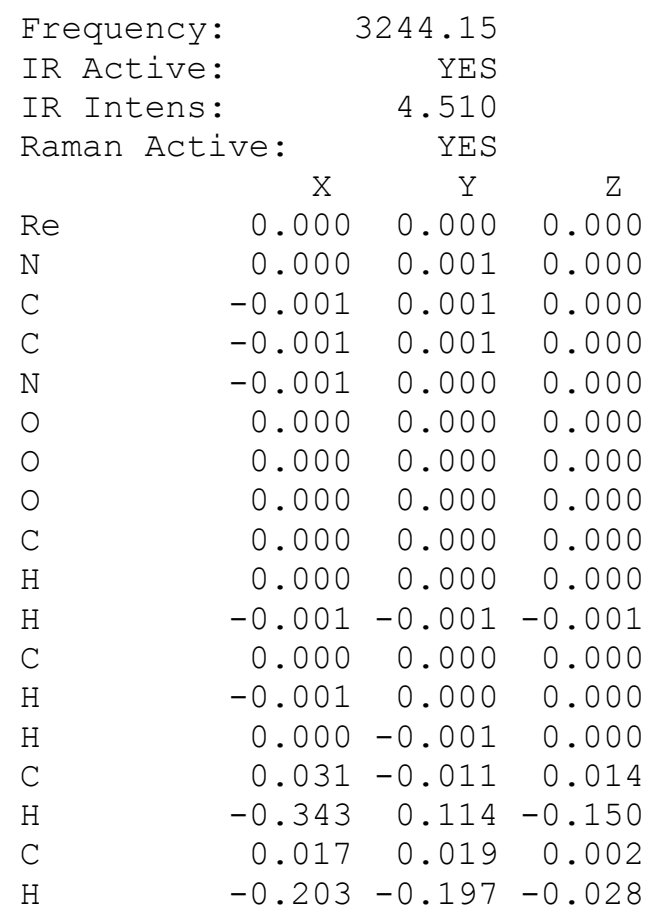

3234.80

YES

5.029

YES

$\begin{array}{cc}X & Y \\ 0.000 & 0.000\end{array}$

$0.000 \quad 0.000$

$0.000-0.001$

0.0010 .000

0.000

0.000

0.000

0.000

0.000

0.000

0.000

0.000

0.000

0.000

$0.000 \quad 0.000$

0.000

0.000

0

0.000

0.000

0.002

0.002

0.000

0.000

$-0.028$

0.008

0.317

$-0.102$

$\begin{array}{ll}0.027 & 0.023\end{array}$

$-0.293-0.277$

$-0.005$

0.018

$0.045-0.197$

$0.003-0.001$

0.010

$-0.009$

0.005

$-0.046$

0.107

$-0.018$

0.038

$0.220-0.435$

$-0.026-0.042$

$\begin{array}{llll}0.317 & 0.464 & -0.042\end{array}$

$\begin{array}{rrrr}-0.021 & 0.004 & -0.006\end{array}$

$\begin{array}{llll}0.230 & -0.021 & 0.056\end{array}$

3244.85

YES

0.268

YES

$\mathrm{X}$

0.000

$Y$

0.000

0.000

0.000

0.001

0.000

0.001

0.000

0.001

0.000

0.000

0.000

0.000

0.000

0.000

0.000

0.000

0.000

$-0.001-0.001$

0.000

0.000

0.000

0.003

$-0.001$

0.002

$-0.001$

0.005

$-0.050$

0.019

$0.558-0.185$

0.000

0.000

0.000

0.000

0.000

0.000

0.000

0.000

0.000

0.001

0.000

0.000

0.001

$-0.002$

$-0.031$

0.354
3238.09

YES

1.433

YES

$\begin{array}{rrr}X & Y & Z \\ 0.000 & 0.000 & 0.000 \\ 0.000 & 0.001 & 0.000 \\ -0.003 & 0.001 & -0.001\end{array}$

$\begin{array}{lll}0.001 & -0.002 & 0.001\end{array}$

$\begin{array}{lll}0.001 & 0.000 & 0.000\end{array}$

$\begin{array}{llll}0.000 & 0.000 & 0.000\end{array}$

$\begin{array}{lll}0.000 & 0.000 & 0.000\end{array}$

$\begin{array}{llll}0.000 & 0.000 & 0.000\end{array}$

$\begin{array}{llll}0.000 & 0.000 & 0.000\end{array}$

$0.000 \quad 0.000 \quad 0.000$

$\begin{array}{llll}0.000 & 0.000 & 0.000\end{array}$

$\begin{array}{lll}0.000 & 0.000 & 0.000\end{array}$

$\begin{array}{llll}0.000 & 0.000 & 0.000\end{array}$

$\begin{array}{llll}0.000 & 0.001 & 0.000\end{array}$

$-0.0190 .006-0.008$

$\begin{array}{llll}0.213 & -0.069 & 0.093\end{array}$

$\begin{array}{rrrr}0.003 & 0.001 & 0.001\end{array}$

$-0.023-0.019-0.004$

$\begin{array}{llll}-0.004 & 0.023 & -0.007\end{array}$

$\begin{array}{lll}0.053 & -0.248 & 0.075\end{array}$

$\begin{array}{rrr}-0.049 & 0.011 & -0.019\end{array}$

$\begin{array}{llll}0.553 & -0.131 & 0.215\end{array}$

$\begin{array}{rrrr}0.019 & -0.048 & 0.015\end{array}$

$-0.2220 .538-0.168$

$\begin{array}{llll}0.010 & -0.019 & 0.007\end{array}$

$-0.110 \quad 0.216-0.079$

$0.000 \quad 0.003-0.001$

$-0.013-0.023 \quad 0.003$

$\begin{array}{llll}0.022 & 0.000 & 0.005\end{array}$

$\begin{array}{lll}-0.239 & 0.019 & -0.057\end{array}$

\begin{tabular}{rrr}
\multicolumn{3}{c}{3249.00} \\
YES \\
X & YES \\
0.000 & 0.000 & 0.000 \\
0.000 & 0.000 & 0.000 \\
0.002 & 0.000 & 0.000 \\
0.000 & -0.002 & 0.000 \\
0.000 & 0.000 & 0.000 \\
0.000 & 0.000 & 0.000 \\
0.000 & 0.000 & 0.000 \\
0.000 & 0.000 & 0.000 \\
0.000 & 0.000 & 0.000 \\
-0.001 & -0.001 & 0.001 \\
0.001 & 0.002 & 0.001 \\
0.000 & 0.000 & 0.000 \\
-0.001 & -0.001 & 0.000 \\
0.000 & 0.001 & 0.000 \\
0.014 & -0.006 & 0.006 \\
-0.157 & 0.052 & -0.069 \\
0.012 & 0.011 & 0.002 \\
-0.125 & -0.120 & -0.018
\end{tabular}




$\begin{array}{lrrrrrr}\mathrm{C} & -0.002 & -0.001 & 0.000 & 0.004 & -0.013 & 0.004 \\ \mathrm{H} & 0.001 & 0.003 & 0.000 & -0.031 & 0.134 & -0.042 \\ \mathrm{C} & 0.022 & -0.006 & 0.009 & 0.006 & -0.001 & 0.002 \\ \mathrm{H} & -0.259 & 0.061 & -0.101 & -0.061 & 0.014 & -0.024 \\ \mathrm{C} & 0.008 & -0.017 & 0.005 & -0.006 & 0.016 & -0.005 \\ \mathrm{H} & -0.082 & 0.198 & -0.062 & 0.071 & -0.172 & 0.054 \\ \mathrm{C} & 0.026 & -0.045 & 0.017 & 0.017 & -0.029 & 0.011 \\ \mathrm{H} & -0.266 & 0.515 & -0.188 & -0.169 & 0.327 & -0.119 \\ \mathrm{C} & -0.028 & -0.036 & 0.003 & -0.018 & -0.025 & 0.002 \\ \mathrm{H} & 0.286 & 0.410 & -0.036 & 0.191 & 0.275 & -0.025 \\ \mathrm{C} & -0.006 & 0.003 & -0.002 & -0.012 & 0.002 & -0.003 \\ \mathrm{H} & 0.067 & -0.008 & 0.017 & 0.125 & -0.011 & 0.030\end{array}$

STANDARD THERMODYNAMIC QUANTITIES AT $298.18 \mathrm{~K}$ AND

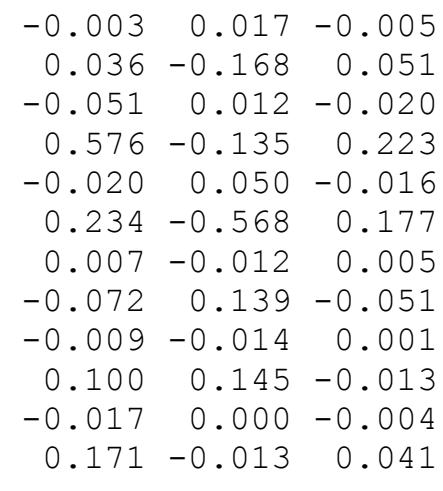

$1.00 \mathrm{ATM}$

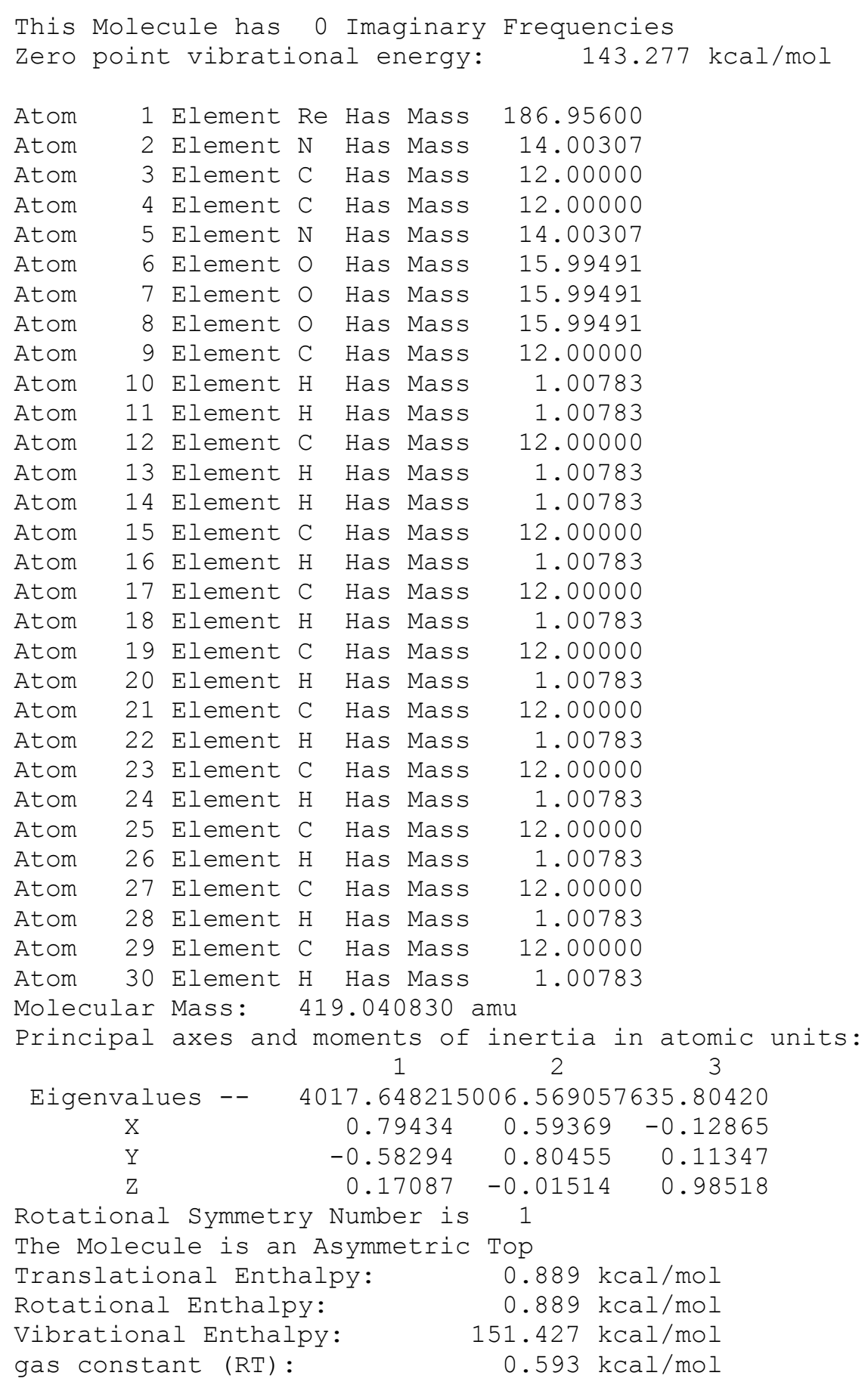

This Molecule has 0 Imaginary Frequencies 
Translational Entropy:

Rotational Entropy:

Vibrational Entropy:

Total Enthalpy:

Total Entropy:
$43.989 \mathrm{cal} / \mathrm{mol} . \mathrm{K}$

$33.396 \mathrm{cal} / \mathrm{mol} . \mathrm{K}$

$51.277 \mathrm{cal} / \mathrm{mol} . \mathrm{K}$

$153.797 \mathrm{kcal} / \mathrm{mol}$

$128.662 \mathrm{cal} / \mathrm{mol} . \mathrm{K}$

Spartan '02 Properties Program: (PC/x86)

Release

Reason for exit: Successful completion

Properties Program CPU Time : 000:00:09.1

Properties Program Wall Time: 000:00:09.2 


\author{
Spartan '02 \\ build 116 (May 14 2002)
}

Wavefunction Developers:

B.J. Deppmeier, A.J. Driessen, T.S. Hehre, W.J. Hehre,

J.A. Johnson, P.E. Klunzinger, J.M. Leonard, I.N. Pham

W.J. Pietro, Jianguo Yu

Q-Chem Developers:

J. Kong, C.A. White, A.I. Krylov, C.D. Sherrill,

R.D. Adamson, T.R. Furlani, M.S. Lee, A.M. Lee,

S.R. Gwaltney, T.R. Adams, C. Ochsenfeld, A.T.B. Gilbert,

G.S. Kedziora, V.A. Rassolov, D. R. Maurice, N. Nair,

Y. Shao, N.A. Besley, P.E. Maslen, J.P. Dombroski,

H. Dachsel, W.M. Zhang, P.P. Korambath, J. Baker,

E.F. C. Byrd, T. Van Voorhis, M. Oumi, S. Hirata,

C.P. Hsu, N. Ishikawa, J. Florian, A. Warshel,

B.G. Johnson, P.M.W. Gill, M. Head-Gordon, J.A. Pople

$\begin{array}{lll}\text { Wavefunction Inc. } & \text { Sales: } & \text { sales@wavefun.com } \\ \text { Irvine CA } & \text { Support: } & \text { support@wavefun.com } \\ & \text { Web: } & \text { www.wavefun.com }\end{array}$

Copyright () $1995-2002$

Spartan '02 Mechanics Program: (PC/x86)

Release 116

Reason for exit: Successful completion

Mechanics CPU Time : 000:00:00.2

Mechanics Wall Time: 000:00:00.6

Spartan '02 Quantum Mechanics Program: (PC/x86)

Release 116

Job type: Geometry optimization.

Method: RB3LYP

Basis set: LACVP**

Number of shells: 79

Number of basis functions: 234

SCF model:

A restricted hybrid HF-DFT SCF calculation will be performed using Pulay DIIS extrapolation

Optimization:

$\begin{array}{rc}\text { Step } & \text { Energy } \\ 1 & -631.5069222 \\ 2 & -631.5038839 \\ 3 & -631.5022668 \\ 4 & -631.5023759 \\ 5 & -631.5063230 \\ 6 & -631.5066469 \\ 7 & -631.5068441 \\ 8 & -631.5071417 \\ 9 & -631.5072780 \\ 10 & -631.5072669 \\ 11 & -631.5073518 \\ 12 & -631.5073021\end{array}$

$\begin{array}{rr}\text { Max Grad. } & \text { Max Dist. } \\ 0.002888 & 0.123325 \\ 0.011506 & 0.146545 \\ 0.014345 & 0.140963 \\ 0.012624 & 0.112753 \\ 0.003779 & 0.146747 \\ 0.003342 & 0.062556 \\ 0.005314 & 0.052977 \\ 0.003677 & 0.057909 \\ 0.001307 & 0.024687 \\ 0.000687 & 0.042155 \\ 0.000642 & 0.010975 \\ 0.000602 & 0.019069\end{array}$




$\begin{array}{llll}13 & -631.5073129 & 0.000376 & 0.046487 \\ 14 & -631.5073315 & 0.000336 & 0.080745 \\ 15 & -631.5074108 & 0.000449 & 0.036963 \\ 16 & -631.5073871 & 0.000322 & 0.009473 \\ 17 & -631.5073709 & 0.000212 & 0.002395 \\ 18 & -631.5075101 & 0.000052 & 0.002900 \\ 19 & -631.5074438 & 0.000083 & 0.001538 \\ 20 & -631.5073854 & 0.000076 & 0.000698\end{array}$

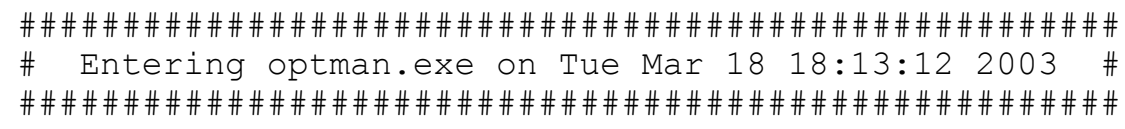

Cartesian Hessian Update

Hessian Updated using BFGS Update

** GEOMETRY OPTIMIZATION IN DELOCALIZED INTERNAL COORDINATES ** Searching for a Minimum

Optimization Cycle: 20

\begin{tabular}{|c|c|c|c|c|}
\hline \multirow{2}{*}{\multicolumn{2}{|c|}{ АTOM }} & \multicolumn{3}{|c|}{ Coordinates (Angstroms) } \\
\hline & & $\mathrm{X}$ & Y & Z \\
\hline 1 & $\mathrm{Re}$ & -1.010108 & 1.083462 & -0.632839 \\
\hline 2 & 0 & -0.996068 & 1.581524 & -2.236299 \\
\hline 3 & O & -2.463849 & 1.416433 & 0.480067 \\
\hline 4 & 0 & -1.596057 & -0.711945 & -0.475169 \\
\hline 5 & $\mathrm{C}$ & -3.324183 & 0.291143 & 0.825991 \\
\hline 6 & $\mathrm{H}$ & -3.059965 & -0.048328 & 1.831783 \\
\hline 7 & $\mathrm{C}$ & -3.031518 & -0.769110 & -0.243919 \\
\hline 8 & $\mathrm{H}$ & -3.275696 & -1.787856 & 0.067355 \\
\hline 9 & $\mathrm{H}$ & -3.554948 & -0.537264 & -1.177397 \\
\hline 10 & $\mathrm{~N}$ & 0.924865 & 0.380373 & -0.166892 \\
\hline 11 & $\mathrm{C}$ & 3.541513 & -0.468492 & 0.239260 \\
\hline 12 & $\mathrm{C}$ & 1.913762 & 1.279820 & 0.083077 \\
\hline 13 & $\mathrm{C}$ & 1.231054 & -0.943338 & -0.207251 \\
\hline 14 & $\mathrm{C}$ & 2.526425 & -1.394074 & -0.007565 \\
\hline 15 & $\mathrm{C}$ & 3.227117 & 0.891436 & 0.286206 \\
\hline 16 & $\mathrm{H}$ & 1.625782 & 2.326222 & 0.105890 \\
\hline 17 & $\mathrm{H}$ & 0.403353 & -1.613419 & -0.402002 \\
\hline 18 & $\mathrm{H}$ & 2.731080 & -2.457589 & -0.052158 \\
\hline 19 & $\mathrm{H}$ & 3.983997 & 1.643591 & 0.475444 \\
\hline 20 & $\mathrm{H}$ & 4.563062 & -0.800927 & 0.393081 \\
\hline 21 & $\mathrm{H}$ & -4.359615 & 0.638336 & 0.813337 \\
\hline
\end{tabular}

Energy is $\quad-631.507385427$

Hessian Updated using BFGS Update

internal optimization with constraints (0)

57 Hessian modes will be used to form the next step Hessian Eigenvalues:
0.001363
0.004924
0.007982
0.014532
0.020243
0.022500
0.023033
0.025547
0.027611
0.028763
0.030120
0.032107
0.040289
0.042859
0.049821
0.055903
0.064311
0.127335
0.128429
0.129094
0.133581
0.099821
0.125638
0.147548 


$\begin{array}{llllll}0.156167 & 0.173834 & 0.214130 & 0.220815 & 0.231469 & 0.236950 \\ 0.241847 & 0.253774 & 0.276472 & 0.288023 & 0.302523 & 0.305687 \\ 0.308656 & 0.309002 & 0.312200 & 0.332250 & 0.337995 & 0.339424 \\ 0.342201 & 0.343148 & 0.351575 & 0.356242 & 0.362670 & 0.379083 \\ 0.392848 & 0.468407 & 1.033618 & & \end{array}$

Minimum Search - Taking Simple RFO Step

Searching for Lamda that Minimizes Along All modes

Value Taken Lamda $=-0.00000005$

Step Taken. Stepsize is 0.001635

$\begin{array}{lccc} & \text { Maximum } & \text { Tolerance } & \text { Cnvgd? } \\ \text { Gradient } & 0.000076 & 0.000300 & \text { YES } \\ \text { Displacement } & 0.000698 & 0.001200 & \text { YES } \\ \text { Energy change } & 0.000058 & 0.000001 & \text { NO }\end{array}$

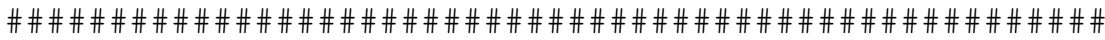 \\ \# Entering anlman.exe on Tue Mar 18 18:13:13 2003 \#

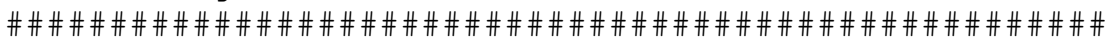

Analysis of SCF Wavefunction

Cartesian Multipole Moments

\begin{tabular}{|c|c|c|c|c|c|}
\hline \multicolumn{6}{|c|}{$\begin{array}{r}\text { Charge }\left(\mathrm{ESU} \times 10^{\wedge} 10\right) \\
4.8032\end{array}$} \\
\hline \multicolumn{6}{|c|}{ Dipole Moment (Debye) } \\
\hline $\mathrm{X}$ & 2.0207 & $\mathrm{Y}$ & 0.3317 & Z & 1.7805 \\
\hline Tot & 2.7136 & & & & \\
\hline \multicolumn{6}{|c|}{ Quadrupole Moments (Debye-Ang) } \\
\hline $\mathrm{XX}$ & -36.1451 & XY & 2.9028 & YY & -71.1532 \\
\hline $\mathrm{XZ}$ & -0.3405 & YZ & 1.6927 & ZZ & -87.3991 \\
\hline \multicolumn{6}{|c|}{ Octapole Moments (Debye-Ang^2) } \\
\hline $\mathrm{XXX}$ & 64.4391 & XXY & -46.3075 & XYY & 36.9348 \\
\hline YYY & -90.3109 & XXz & 37.9134 & XYZ & 1.8283 \\
\hline YYZ & 22.6202 & XZZ & 7.8411 & YZZ & -37.9192 \\
\hline ZZZ & 73.5945 & & & & \\
\hline \multicolumn{6}{|c|}{ Hexadecapole Moments (Debye-Ang^3) } \\
\hline XXXX & -1795.6817 & XXXY & 137.6255 & XXYY & -468.7261 \\
\hline XYYY & 127.5594 & YYYY & -593.7932 & XXXZ & -39.4629 \\
\hline XXYZ & 28.7347 & XYYZ & -15.9023 & YYYZ & 72.4022 \\
\hline XXZZ & -478.1302 & XYZZ & 47.1869 & YYZZ & -188.4767 \\
\hline XZZZ & -77.4447 & YZZZ & 85.7328 & ZZZZ & -387.5276 \\
\hline
\end{tabular}

Reason for exit: Sucessful completion

Quantum Mechanics Program CPU Time : 004:44:34.5

Quantum Mechanics Program Wall Time: 009:30:36.3

Spartan '02 Properties Program: (PC/x86)

Reason for exit: Successful completion

Properties Program CPU Time : 000:00:03.4

Properties Program Wall Time: 000:00:36.8 
Basis set: LACVP**

Number of shells: 79

Number of basis functions: 234

SCF model:

A restricted hybrid HF-DFT SCF calculation will be performed using Pulay DIIS extrapolation

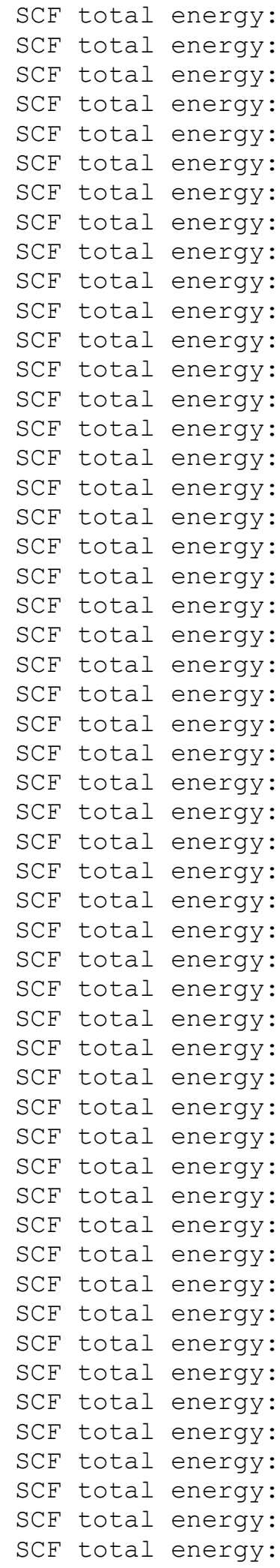

-631.5073654 hartrees -631.5075848 hartrees -631.5073634 hartrees -631.5073627 hartrees -631.5073577 hartrees -631.5073604 hartrees -631.5073635 hartrees -631.5073651 hartrees -631.5075192 hartrees -631.5073624 hartrees -631.5073636 hartrees -631.5073567 hartrees -631.5073630 hartrees -631.5073598 hartrees -631.5073669 hartrees -631.5073669 hartrees -631.5073557 hartrees -631.5073656 hartrees -631.5073621 hartrees -631.5073608 hartrees -631.5074215 hartrees -631.5073625 hartrees -631.5073652 hartrees -631.5073648 hartrees -631.5073629 hartrees -631.5073607 hartrees -631.5073554 hartrees -631.5073601 hartrees -631.5073603 hartrees -631.5073631 hartrees -631.5073652 hartrees -631.5073627 hartrees -631.5073669 hartrees -631.5073626 hartrees -631.5073645 hartrees -631.5073614 hartrees -631.5073570 hartrees -631.5073606 hartrees -631.5073573 hartrees -631.5073633 hartrees -631.5073612 hartrees -631.5073642 hartrees -631.5073609 hartrees -631.5073646 hartrees -631.5073573 hartrees -631.5073607 hartrees -631.5073665 hartrees -631.5073643 hartrees -631.5073606 hartrees -631.5073674 hartrees -631.5073615 hartrees -631.5073655 hartrees -631.5073629 hartrees 


\begin{tabular}{|c|c|c|c|c|}
\hline $\mathrm{SCF}$ & total & & & \\
\hline $\mathrm{SCF}$ & total & energy: & -631.5073621 & hartrees \\
\hline $\mathrm{SCF}$ & total & energy: & -631.5073664 & hartrees \\
\hline $\mathrm{SCF}$ & total & energy: & -631.5073614 & hartrees \\
\hline $\mathrm{SCF}$ & total & energy: & -631.5073783 & hartrees \\
\hline $\mathrm{SCF}$ & total & energy: & -631.5073622 & hartrees \\
\hline $\mathrm{SCF}$ & total & energy: & -631.5073852 & hartrees \\
\hline $\mathrm{SCF}$ & total & energy: & -631.5073628 & hartrees \\
\hline $\mathrm{SCF}$ & total & energy: & -631.5073648 & hartrees \\
\hline $\mathrm{SCF}$ & total & energy: & -631.5073590 & hartrees \\
\hline $\mathrm{SCF}$ & total & energy: & -631.5073604 & hartrees \\
\hline$S C F$ & total & energy: & -631.5073635 & hartrees \\
\hline $\mathrm{SCF}$ & total & energy: & -631.5073611 & hartrees \\
\hline $\mathrm{SCF}$ & total & energy: & -631.5073646 & hartrees \\
\hline $\mathrm{SCF}$ & total & energy: & -631.5073609 & hartrees \\
\hline $\mathrm{SCF}$ & total & energy: & -631.5073649 & hartrees \\
\hline $\mathrm{SCF}$ & total & energy: & -631.5073646 & hartrees \\
\hline$S C F$ & total & energy: & -631.5073616 & hartrees \\
\hline $\mathrm{SCF}$ & total & energy: & -631.5073610 & hartrees \\
\hline $\mathrm{SCF}$ & total & energy: & -631.5073640 & hartrees \\
\hline SCE & total & energy: & -631.5073627 & hartrees \\
\hline $\mathrm{SCF}$ & total & energy: & -631.5073623 & hartrees \\
\hline $\mathrm{SCF}$ & total & energy: & -631.5073624 & hartrees \\
\hline$S C F$ & total & energy: & -631.5073624 & hartrees \\
\hline $\mathrm{SCF}$ & total & energy: & -631.5073640 & hartrees \\
\hline SCE & total & energy: & -631.5073566 & hartrees \\
\hline $\mathrm{SCF}$ & total & energy: & -631.5073621 & hartrees \\
\hline $\mathrm{SCF}$ & total & energy: & -631.5073647 & hartrees \\
\hline $\mathrm{SCF}$ & total & energy: & -631.5073600 & hartrees \\
\hline $\mathrm{SCF}$ & total & energy: & -631.5073613 & hartrees \\
\hline $\mathrm{SCF}$ & total & energy: & -631.5073643 & hartrees \\
\hline SCF & total & energy: & -631.5073643 & hartrees \\
\hline $\mathrm{SCF}$ & total & energy: & -631.5073574 & hartrees \\
\hline $\mathrm{SCF}$ & total & energy: & -631.5073638 & hartrees \\
\hline $\mathrm{SCF}$ & total & energy: & -631.5073661 & hartrees \\
\hline $\mathrm{SCF}$ & total & energy: & -631.5073595 & hartrees \\
\hline $\mathrm{SCF}$ & total & energy: & -631.5073590 & hartrees \\
\hline $\mathrm{SCF}$ & total & energy: & -631.5073631 & hartrees \\
\hline $\mathrm{SCF}$ & total & energy: & -631.5073624 & hartrees \\
\hline $\mathrm{SCF}$ & total & energy: & -631.5073651 & hartrees \\
\hline $\mathrm{SCF}$ & total & energy: & -631.5073621 & hartrees \\
\hline $\mathrm{SCF}$ & total & energy: & -631.5073630 & hartrees \\
\hline $\mathrm{SCF}$ & total & energy: & -631.5073641 & hartrees \\
\hline SCE & total & energy: & -631.5073605 & hartrees \\
\hline$S C F$ & total & energy: & -631.5073630 & hartrees \\
\hline $\mathrm{SCF}$ & total & energy: & -631.5073670 & hartrees \\
\hline SCE & total & energy: & -631.5073623 & hartrees \\
\hline$S C F$ & total & energy: & -631.5073644 & hartrees \\
\hline $\mathrm{SCF}$ & total & energy: & -631.5073611 & hartrees \\
\hline $\mathrm{SCE}$ & total & energy: & -631.5073651 & hartrees \\
\hline $\mathrm{SCF}$ & total & energy: & -631.5073638 & hartrees \\
\hline $\mathrm{SCF}$ & total & energy: & -631.5073634 & hartrees \\
\hline $\mathrm{SCF}$ & total & energy: & -631.5073621 & hartrees \\
\hline $\mathrm{SCF}$ & total & energy: & -631.5073596 & hartrees \\
\hline $\mathrm{SCF}$ & total & energy: & -631.5073632 & hartrees \\
\hline $\mathrm{SCF}$ & total & energy: & -631.5073669 & hartrees \\
\hline $\mathrm{SCF}$ & total & energy: & -631.5073645 & hartrees \\
\hline $\mathrm{SCF}$ & total & energy: & -631.5073599 & hartrees \\
\hline$S C E$ & & energy: & -631.5073634 & hartrees \\
\hline $\mathrm{SCF}$ & tota & energy: & -631.5073636 & hartrees \\
\hline $\mathrm{SCF}$ & & energy: & -631.5073651 & hartrees \\
\hline
\end{tabular}




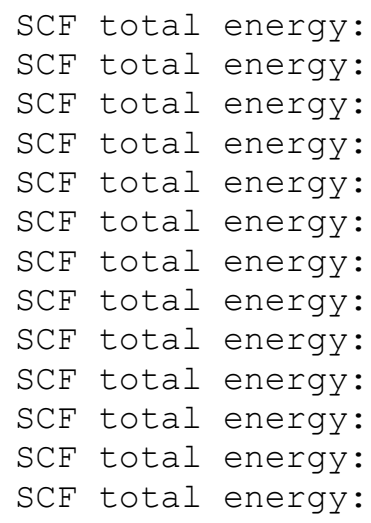

-631.5073602 hartrees

-631.5073624 hartrees

-631.5073632 hartrees

-631.5073655 hartrees

-631.5073611 hartrees

-631.5073631 hartrees

-631.5073643 hartrees

-631.5073601 hartrees

-631.5073669 hartrees

-631.5073628 hartrees

-631.5073663 hartrees

-631.5073638 hartrees

-631.5073612 hartrees

Reason for exit: Sucessful completion

Quantum Mechanics Program CPU Time : 022:06:55.7

Quantum Mechanics Program Wall Time: 022:40:34.8

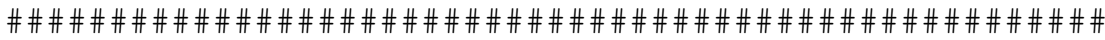 \\ \# Entering vibman.exe on Sat Jan 10 13:10:00 2004 \#

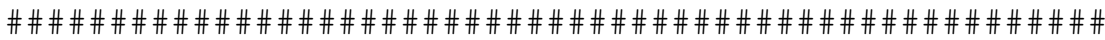

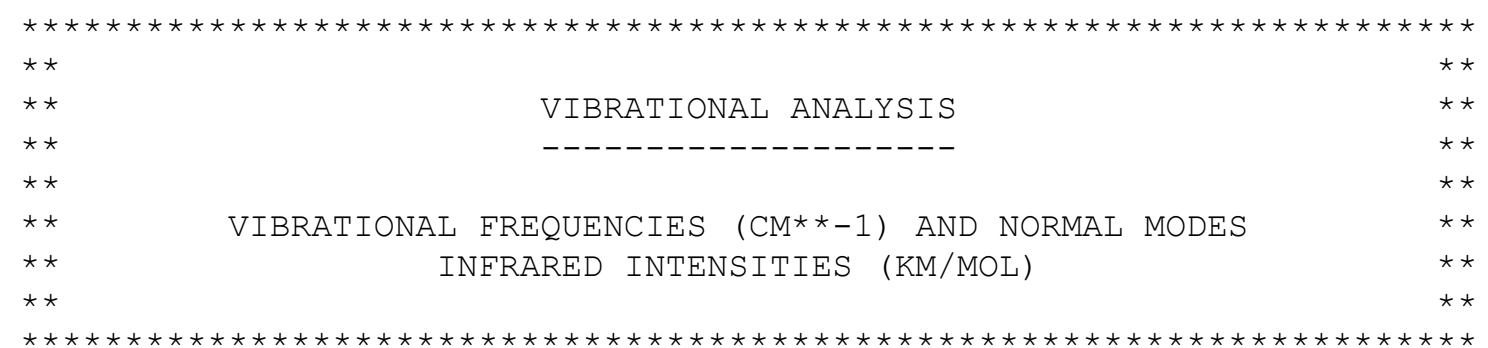

\begin{tabular}{|c|c|c|c|c|c|c|c|c|c|}
\hline Frequency: & & 35.45 & & & 54.39 & & & 118.72 & \\
\hline IR Active: & & YES & & & YES & & & YES & \\
\hline IR Intens: & & 0.411 & & & 4.278 & & & 4.877 & \\
\hline Raman Acti & ve: & YES & & & YES & & & YES & \\
\hline & X & $Y$ & Z & $\mathrm{X}$ & $Y$ & Z & $\mathrm{X}$ & $\mathrm{Y}$ & Z \\
\hline $\operatorname{Re}$ & -0.001 & -0.007 & -0.020 & -0.002 & 0.010 & -0.045 & -0.019 & 0.047 & -0.003 \\
\hline O & -0.011 & -0.106 & -0.051 & -0.165 & 0.101 & -0.023 & -0.003 & 0.007 & -0.012 \\
\hline 0 & 0.012 & 0.064 & -0.027 & 0.143 & -0.065 & 0.166 & -0.110 & -0.043 & -0.096 \\
\hline O & 0.003 & 0.003 & 0.106 & -0.004 & -0.001 & -0.127 & 0.184 & -0.004 & 0.124 \\
\hline $\mathrm{C}$ & 0.019 & 0.087 & 0.063 & 0.205 & -0.101 & 0.212 & 0.001 & -0.110 & -0.036 \\
\hline $\mathrm{H}$ & 0.033 & 0.157 & 0.082 & 0.386 & -0.197 & 0.132 & -0.034 & 0.046 & 0.025 \\
\hline $\mathrm{C}$ & 0.007 & 0.015 & 0.131 & 0.027 & 0.004 & 0.062 & 0.200 & -0.210 & 0.113 \\
\hline $\mathrm{H}$ & 0.015 & 0.036 & 0.205 & 0.047 & -0.023 & -0.010 & 0.371 & -0.207 & 0.257 \\
\hline $\mathrm{H}$ & -0.009 & -0.050 & 0.124 & -0.101 & 0.116 & 0.161 & 0.173 & -0.438 & 0.073 \\
\hline $\mathrm{N}$ & -0.003 & 0.003 & -0.002 & -0.005 & 0.002 & -0.085 & 0.024 & 0.048 & -0.053 \\
\hline C & -0.004 & 0.012 & 0.014 & -0.062 & -0.031 & 0.207 & -0.040 & -0.139 & 0.052 \\
\hline $\mathrm{C}$ & -0.046 & -0.010 & 0.214 & -0.012 & -0.006 & -0.024 & 0.083 & -0.020 & -0.042 \\
\hline C & 0.038 & 0.018 & -0.209 & -0.023 & -0.004 & -0.024 & -0.052 & 0.028 & -0.039 \\
\hline C & 0.041 & 0.024 & -0.218 & -0.051 & -0.020 & 0.123 & -0.095 & -0.066 & 0.013 \\
\hline C & -0.049 & -0.005 & 0.241 & -0.041 & -0.023 & 0.124 & 0.049 & -0.117 & 0.010 \\
\hline $\mathrm{H}$ & -0.078 & -0.022 & 0.369 & 0.004 & 0.000 & -0.088 & 0.158 & -0.001 & -0.065 \\
\hline $\mathrm{H}$ & 0.067 & 0.024 & -0.356 & -0.017 & 0.005 & -0.083 & -0.083 & 0.074 & -0.051 \\
\hline $\mathrm{H}$ & 0.078 & 0.038 & -0.397 & -0.065 & -0.025 & 0.177 & -0.176 & -0.082 & 0.031 \\
\hline $\mathrm{H}$ & -0.084 & -0.016 & 0.429 & -0.046 & -0.030 & 0.177 & 0.094 & -0.168 & 0.027 \\
\hline $\mathrm{H}$ & -0.004 & 0.016 & 0.019 & -0.085 & -0.044 & 0.333 & -0.071 & -0.207 & 0.110 \\
\hline $\mathrm{H}$ & 0.018 & 0.084 & 0.053 & 0.203 & -0.101 & 0.427 & -0.042 & -0.240 & -0.153 \\
\hline Frequency: & & 138.18 & & & 162.04 & & & 207.37 & \\
\hline IR Active: & & YES & & & YES & & & YES & \\
\hline
\end{tabular}




\begin{tabular}{|c|c|c|c|c|c|c|c|c|c|}
\hline IR Intens: & & 0.886 & & & 7.306 & & & 1.121 & \\
\hline Raman Active & ve: & YES & & & YES & & & YES & \\
\hline & $\mathrm{X}$ & $Y$ & Z & $\mathrm{X}$ & $\mathrm{Y}$ & Z & $\mathrm{X}$ & $Y$ & Z \\
\hline $\mathrm{Re}$ & 0.001 & 0.011 & -0.030 & -0.015 & -0.022 & -0.003 & 0.004 & -0.002 & -0.025 \\
\hline 0 & 0.173 & 0.023 & -0.030 & 0.405 & 0.304 & 0.098 & -0.211 & 0.235 & 0.048 \\
\hline O & 0.002 & -0.021 & -0.022 & -0.072 & 0.033 & -0.103 & 0.054 & 0.013 & 0.008 \\
\hline 0 & 0.024 & -0.006 & -0.045 & -0.064 & -0.007 & 0.029 & 0.020 & -0.003 & -0.001 \\
\hline $\mathrm{C}$ & 0.137 & -0.086 & 0.120 & 0.014 & 0.043 & 0.107 & -0.084 & 0.099 & -0.079 \\
\hline $\mathrm{H}$ & 0.347 & -0.080 & 0.067 & 0.140 & 0.149 & 0.110 & -0.351 & 0.223 & 0.032 \\
\hline $\mathrm{C}$ & 0.048 & -0.046 & 0.062 & -0.042 & -0.050 & 0.174 & 0.043 & -0.079 & 0.112 \\
\hline $\mathrm{H}$ & 0.096 & -0.061 & 0.053 & 0.042 & -0.031 & 0.302 & 0.173 & -0.041 & 0.339 \\
\hline $\mathrm{H}$ & -0.047 & -0.024 & 0.121 & -0.158 & -0.167 & 0.212 & -0.026 & -0.352 & 0.085 \\
\hline $\mathrm{N}$ & -0.100 & -0.002 & 0.241 & -0.019 & -0.098 & -0.159 & -0.010 & -0.111 & 0.079 \\
\hline C & -0.041 & 0.005 & -0.201 & -0.014 & 0.071 & 0.107 & 0.073 & 0.026 & -0.057 \\
\hline C & -0.086 & -0.004 & 0.184 & -0.088 & -0.033 & -0.143 & -0.045 & -0.076 & 0.084 \\
\hline $\mathrm{C}$ & -0.110 & -0.004 & 0.191 & 0.053 & -0.079 & -0.121 & 0.057 & -0.102 & 0.102 \\
\hline $\mathrm{C}$ & -0.078 & 0.000 & -0.031 & 0.063 & 0.005 & 0.024 & 0.099 & -0.029 & 0.032 \\
\hline$-c$ & -0.051 & -0.002 & -0.046 & -0.083 & 0.057 & -0.018 & -0.010 & 0.003 & 0.009 \\
\hline $\mathrm{H}$ & -0.097 & -0.009 & 0.288 & -0.150 & -0.049 & -0.208 & -0.097 & -0.090 & 0.125 \\
\hline $\mathrm{H}$ & -0.135 & 0.005 & 0.275 & 0.102 & -0.129 & -0.165 & 0.082 & -0.145 & 0.140 \\
\hline$-c$ & -0.075 & 0.003 & -0.100 & 0.132 & 0.016 & 0.089 & 0.155 & -0.018 & 0.026 \\
\hline $\mathrm{H}$ & -0.028 & -0.007 & -0.124 & -0.143 & 0.110 & 0.008 & -0.049 & 0.048 & -0.013 \\
\hline $\mathrm{H}$ & -0.005 & 0.012 & -0.433 & -0.017 & 0.129 & 0.255 & 0.107 & 0.083 & -0.158 \\
\hline $\mathrm{H}$ & 0.110 & -0.162 & 0.308 & -0.005 & -0.009 & 0.188 & -0.058 & 0.170 & -0.365 \\
\hline Frequency: & & 228.38 & & & 260.26 & & & 294.70 & \\
\hline IR Active: & & YES & & & YES & & & YES & \\
\hline IR Intens: & & 2.083 & & & 1.848 & & & 8.684 & \\
\hline Raman Active & ve: & YES & & & YES & & & YES & \\
\hline & $\mathrm{X}$ & $Y$ & Z & $\mathrm{X}$ & $Y$ & Z & $\mathrm{X}$ & $\mathrm{Y}$ & Z \\
\hline $\mathrm{Re}$ & -0.051 & -0.007 & -0.014 & 0.031 & -0.047 & -0.006 & -0.051 & 0.034 & -0.031 \\
\hline 0 & -0.026 & 0.075 & 0.013 & 0.013 & 0.126 & 0.045 & 0.253 & -0.106 & -0.079 \\
\hline$-c$ & -0.134 & 0.040 & -0.140 & 0.059 & -0.052 & 0.028 & 0.172 & -0.161 & 0.332 \\
\hline O & -0.112 & 0.006 & -0.069 & -0.091 & -0.037 & -0.059 & -0.043 & 0.026 & 0.006 \\
\hline $\mathrm{C}$ & 0.039 & -0.024 & 0.099 & -0.095 & 0.071 & -0.018 & -0.161 & -0.011 & -0.004 \\
\hline $\mathrm{H}$ & 0.347 & -0.014 & 0.022 & -0.310 & 0.134 & 0.060 & -0.608 & -0.060 & 0.096 \\
\hline $\mathrm{C}$ & -0.116 & 0.028 & 0.025 & -0.074 & -0.061 & 0.101 & -0.021 & -0.053 & 0.040 \\
\hline$-c$ & -0.114 & 0.016 & -0.016 & 0.031 & -0.034 & 0.271 & 0.061 & -0.057 & 0.094 \\
\hline$-c$ & -0.187 & 0.109 & 0.086 & -0.175 & -0.247 & 0.113 & -0.048 & -0.163 & 0.026 \\
\hline $\mathrm{N}$ & 0.153 & 0.072 & 0.069 & 0.025 & 0.219 & 0.014 & 0.045 & -0.008 & 0.013 \\
\hline $\mathrm{C}$ & 0.205 & -0.090 & -0.002 & -0.101 & -0.038 & -0.018 & 0.107 & -0.030 & 0.003 \\
\hline $\mathrm{C}$ & 0.240 & -0.004 & 0.085 & 0.094 & 0.157 & 0.019 & 0.074 & -0.031 & 0.022 \\
\hline $\mathrm{C}$ & 0.143 & 0.065 & 0.068 & -0.117 & 0.202 & -0.014 & 0.076 & -0.005 & 0.031 \\
\hline $\mathrm{C}$ & 0.134 & -0.015 & 0.020 & -0.180 & 0.061 & -0.038 & 0.089 & -0.010 & 0.014 \\
\hline $\mathrm{C}$ & 0.239 & -0.083 & 0.051 & 0.050 & 0.002 & 0.015 & 0.083 & -0.037 & 0.023 \\
\hline $\mathrm{H}$ & 0.320 & 0.017 & 0.114 & 0.183 & 0.182 & 0.014 & 0.100 & -0.025 & 0.026 \\
\hline $\mathrm{H}$ & 0.127 & 0.079 & 0.079 & -0.192 & 0.296 & -0.020 & 0.086 & -0.022 & 0.045 \\
\hline $\mathrm{H}$ & 0.055 & -0.029 & -0.011 & -0.287 & 0.043 & -0.071 & 0.070 & -0.014 & 0.008 \\
\hline $\mathrm{H}$ & 0.269 & -0.112 & 0.053 & 0.145 & -0.096 & 0.026 & 0.070 & -0.025 & 0.028 \\
\hline $\mathrm{H}$ & 0.197 & -0.142 & -0.058 & -0.133 & -0.143 & -0.031 & 0.111 & -0.030 & -0.019 \\
\hline $\mathrm{H}$ & 0.009 & -0.106 & 0.382 & -0.056 & 0.186 & -0.220 & -0.090 & 0.193 & -0.387 \\
\hline Frequency: & & 312.52 & & & 399.70 & & & 406.84 & \\
\hline IR Active: & & YES & & & YES & & & YES & \\
\hline IR Intens: & & 17.935 & & & 0.483 & & & 2.015 & \\
\hline Raman Active & ve : & YES & & & YES & & & YES & \\
\hline & $\mathrm{X}$ & $\mathrm{Y}$ & Z & $\mathrm{X}$ & Y & Z & $\mathrm{X}$ & $\mathrm{Y}$ & Z \\
\hline $\mathrm{Re}$ & -0.024 & -0.029 & -0.027 & 0.021 & 0.011 & -0.008 & -0.060 & -0.033 & 0.019 \\
\hline 0 & 0.018 & 0.186 & 0.033 & -0.026 & 0.001 & -0.014 & 0.068 & -0.013 & 0.032 \\
\hline O & 0.065 & -0.017 & 0.053 & -0.036 & -0.087 & -0.016 & 0.111 & 0.228 & 0.052 \\
\hline 0 & 0.122 & -0.047 & 0.320 & -0.060 & 0.026 & 0.082 & 0.167 & -0.074 & -0.213 \\
\hline
\end{tabular}




$\mathrm{C}$
$\mathrm{H}$
$\mathrm{C}$
$\mathrm{H}$
$\mathrm{H}$
$\mathrm{N}$
$\mathrm{C}$
$\mathrm{C}$
$\mathrm{C}$
$\mathrm{C}$
$\mathrm{C}$
$\mathrm{H}$
$\mathrm{H}$
$\mathrm{H}$
$\mathrm{H}$
$\mathrm{H}$
$\mathrm{H}$

$\begin{array}{rrr}0.019 & 0.005 & 0.000 \\ 0.020 & -0.108 & -0.039 \\ 0.055 & 0.141 & -0.121 \\ -0.299 & 0.132 & -0.433 \\ 0.388 & 0.504 & -0.221 \\ 0.010 & 0.045 & 0.004 \\ 0.000 & -0.020 & -0.025 \\ 0.028 & 0.025 & 0.025 \\ -0.014 & 0.042 & 0.026 \\ -0.026 & 0.007 & 0.002 \\ 0.021 & -0.016 & 0.018 \\ 0.048 & 0.029 & 0.037 \\ -0.023 & 0.047 & 0.058 \\ -0.061 & 0.000 & -0.001 \\ 0.039 & -0.037 & 0.031 \\ -0.001 & -0.041 & -0.061 \\ 0.028 & 0.032 & 0.022\end{array}$

\begin{tabular}{lrrr} 
Frequency: & \multicolumn{3}{c}{439.63} \\
IR Active: & YES \\
IR Intens: & \multicolumn{3}{c}{1.901} \\
Raman Active: & YES \\
& Y & Z \\
Re & 0.003 & 0.003 & -0.009 \\
O & -0.021 & 0.021 & -0.006 \\
O & 0.006 & -0.026 & -0.001 \\
O & -0.012 & 0.001 & 0.033 \\
C & -0.014 & -0.014 & 0.007 \\
H & -0.008 & -0.016 & 0.005 \\
C & -0.017 & -0.002 & 0.003 \\
H & -0.027 & -0.006 & -0.018 \\
H & -0.003 & 0.019 & 0.001 \\
N & -0.058 & -0.023 & 0.301 \\
C & -0.032 & -0.020 & 0.249 \\
C & 0.011 & 0.000 & -0.053 \\
C & 0.020 & 0.000 & -0.066 \\
C & 0.040 & 0.009 & -0.168 \\
C & 0.035 & 0.008 & -0.181 \\
H & 0.032 & 0.007 & -0.150 \\
H & 0.044 & 0.005 & -0.191 \\
H & 0.077 & 0.024 & -0.380 \\
H & 0.070 & 0.028 & -0.403 \\
H & -0.093 & -0.042 & 0.611 \\
H & -0.007 & 0.006 & 0.012
\end{tabular}

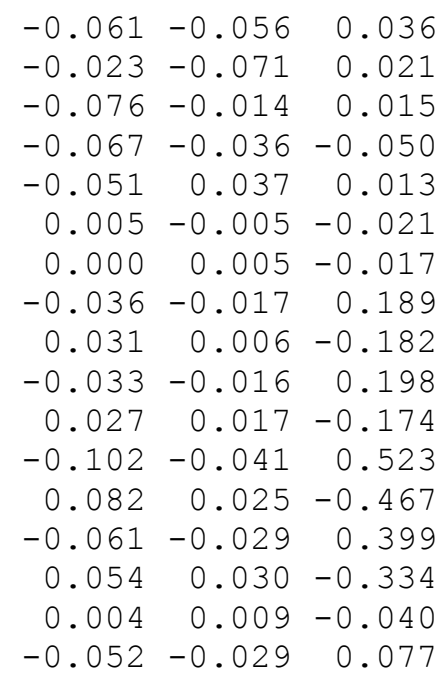

\begin{tabular}{rrr}
\multicolumn{4}{c}{603.04} \\
\multicolumn{3}{c}{1.874} \\
YES & YES & $Z$ \\
-0.008 & 0.016 & 0.008 \\
0.004 & 0.003 & -0.004 \\
0.157 & 0.068 & -0.011 \\
-0.116 & -0.094 & 0.124 \\
0.152 & -0.014 & -0.175 \\
0.413 & -0.205 & -0.310 \\
-0.133 & -0.167 & -0.092 \\
-0.102 & -0.063 & 0.298 \\
-0.138 & -0.518 & -0.177 \\
-0.004 & 0.000 & -0.004 \\
0.009 & 0.002 & -0.002 \\
0.005 & -0.007 & -0.001 \\
-0.004 & 0.004 & -0.006 \\
-0.001 & 0.010 & 0.005 \\
0.008 & 0.001 & 0.004 \\
0.008 & -0.006 & -0.002 \\
-0.004 & 0.002 & -0.008 \\
-0.004 & 0.010 & 0.010 \\
0.003 & 0.006 & 0.005 \\
0.007 & -0.007 & -0.008 \\
0.201 & 0.122 & 0.195
\end{tabular}

\begin{tabular}{rrr}
\multicolumn{4}{c}{676.15} \\
YES \\
45.045 \\
X & YES \\
0.016 & -0.029 & -0.005 \\
-0.003 & 0.020 & -0.005 \\
-0.072 & 0.111 & 0.107 \\
-0.023 & 0.233 & 0.037 \\
-0.012 & -0.046 & -0.031 \\
0.093 & -0.215 & -0.114 \\
0.024 & -0.009 & -0.045 \\
0.315 & -0.037 & 0.092 \\
-0.028 & -0.272 & -0.080 \\
-0.244 & 0.078 & -0.025 \\
0.271 & -0.084 & 0.033
\end{tabular}

$\begin{array}{rrr}0.157 & 0.168 & -0.091 \\ 0.025 & 0.210 & -0.043 \\ 0.209 & 0.051 & -0.036 \\ 0.171 & 0.111 & 0.130 \\ 0.144 & -0.072 & -0.031 \\ 0.000 & 0.032 & 0.033 \\ 0.005 & -0.023 & 0.031 \\ 0.006 & 0.010 & 0.128 \\ 0.029 & 0.050 & -0.148 \\ -0.030 & -0.001 & 0.115 \\ 0.051 & -0.004 & -0.149 \\ -0.011 & 0.000 & 0.346 \\ 0.074 & 0.064 & -0.379 \\ -0.082 & -0.015 & 0.220 \\ 0.090 & -0.006 & -0.297 \\ -0.007 & -0.043 & 0.072 \\ 0.139 & 0.108 & -0.235\end{array}$

657.41

YES

0.853

YES

\begin{tabular}{rrr}
$X$ & \multicolumn{1}{c}{$Y$} & $Z$ \\
-0.002 & -0.001 & 0.000 \\
0.001 & 0.001 & -0.002 \\
-0.001 & -0.008 & -0.006 \\
0.008 & -0.017 & -0.006 \\
-0.003 & 0.003 & 0.008 \\
-0.028 & 0.026 & 0.023 \\
0.011 & 0.007 & 0.007 \\
-0.019 & 0.005 & -0.024 \\
0.019 & 0.046 & 0.012 \\
-0.038 & -0.130 & -0.017 \\
0.041 & 0.144 & 0.022 \\
0.157 & -0.273 & 0.010 \\
-0.280 & -0.134 & -0.056 \\
-0.171 & 0.304 & -0.012 \\
0.310 & 0.149 & 0.062 \\
0.046 & -0.307 & -0.006 \\
-0.221 & -0.216 & -0.049 \\
0.014 & 0.343 & 0.019 \\
0.184 & 0.286 & 0.046 \\
-0.085 & -0.265 & -0.026 \\
-0.004 & -0.002 & -0.027
\end{tabular}

699.22

YES

39.827

YES

\begin{tabular}{rrr} 
X & \multicolumn{1}{c}{$Y$} & $Z$ \\
0.000 & -0.001 & 0.000 \\
0.001 & -0.002 & 0.002 \\
-0.001 & 0.003 & 0.003 \\
-0.001 & 0.008 & -0.001 \\
0.002 & 0.000 & -0.002 \\
0.007 & -0.010 & -0.007 \\
-0.001 & -0.001 & -0.003 \\
0.011 & -0.001 & 0.008 \\
-0.003 & -0.017 & -0.005 \\
0.016 & 0.014 & -0.137 \\
0.001 & -0.009 & 0.058
\end{tabular}




$\mathrm{C}$
$\mathrm{C}$
$\mathrm{C}$
$\mathrm{C}$
$\mathrm{H}$
$\mathrm{H}$
$\mathrm{H}$
$\mathrm{H}$
$\mathrm{H}$
$\mathrm{H}$

$\begin{array}{rrr}0.073 & 0.093 & 0.030 \\ -0.007 & -0.107 & -0.003 \\ -0.035 & -0.118 & -0.019 \\ 0.067 & 0.127 & 0.014 \\ -0.071 & 0.058 & -0.007 \\ -0.108 & 0.019 & -0.025 \\ 0.147 & -0.085 & -0.004 \\ 0.204 & -0.007 & 0.004 \\ -0.183 & 0.041 & -0.025 \\ -0.050 & -0.191 & 0.177\end{array}$

Frequi
IR
IR
Raman
Re
O
O
O
C
H
C
H
H
N
C
C
C
C
C
H
H
H
H
H
H

717.56

YES

60.514

YES

\begin{tabular}{rrr}
$X$ & \multicolumn{1}{c}{$Y$} & $Z$ \\
-0.038 & -0.019 & 0.030 \\
-0.003 & -0.017 & -0.012 \\
0.260 & -0.381 & -0.297 \\
-0.022 & 0.394 & 0.012 \\
0.164 & 0.119 & -0.071 \\
0.130 & 0.199 & -0.034 \\
0.077 & 0.110 & -0.003 \\
0.397 & 0.068 & 0.075 \\
-0.065 & -0.026 & 0.046 \\
0.002 & -0.001 & 0.000 \\
0.000 & 0.002 & -0.003 \\
-0.005 & -0.007 & -0.008 \\
-0.001 & 0.012 & 0.003 \\
-0.006 & 0.007 & 0.001 \\
-0.004 & -0.002 & 0.004 \\
-0.011 & -0.010 & -0.008 \\
0.001 & 0.012 & -0.010 \\
-0.009 & 0.007 & -0.007 \\
-0.009 & 0.001 & 0.012 \\
-0.001 & -0.002 & -0.005 \\
0.265 & 0.405 & -0.089
\end{tabular}

Frequency:

IR Active:

IR Intens:

Raman Active:
888.14

YES

0.716

YES
Z

$$
\begin{array}{lll}
0.000 & 0.000 & 0.000
\end{array}
$$

$\begin{array}{llll}0.000 & 0.000 & -0.001\end{array}$

$\begin{array}{lll}-0.003 & -0.003 & 0.001\end{array}$

$\begin{array}{rrr}-0.004 & 0.000 & 0.000\end{array}$

$\begin{array}{llll}0.005 & 0.000 & -0.004\end{array}$

$0.001-0.001-0.003$

$\begin{array}{llll}0.003 & 0.004 & 0.003\end{array}$

$\begin{array}{ccc}-0.003 & 0.008 & 0.008\end{array}$

$\begin{array}{llll}0.002 & 0.001 & 0.003\end{array}$

$\begin{array}{rrrr}-0.001 & 0.000 & 0.003\end{array}$

$\begin{array}{llll}0.004 & 0.002 & -0.015\end{array}$

$\begin{array}{llll}-0.016 & -0.007 & 0.092\end{array}$

$\begin{array}{llll}0.012 & 0.004 & -0.074\end{array}$

$0.013 \quad 0.004-0.068$

$\begin{array}{llll}-0.009 & -0.003 & 0.053\end{array}$

$\begin{array}{llll}0.093 & 0.036 & -0.514\end{array}$

$\begin{array}{lll}-0.080 & -0.023 & 0.411\end{array}$

$\begin{array}{lll}-0.095 & -0.042 & 0.566\end{array}$

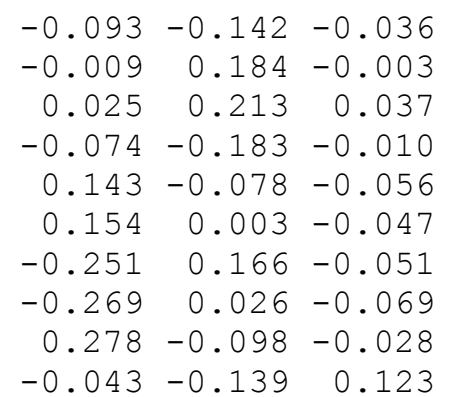

\begin{tabular}{rrr}
\multicolumn{3}{c}{783.18} \\
Y & YES \\
X & YES \\
0.000 & 0.000 & -0.001 \\
-0.002 & 0.002 & -0.001 \\
0.000 & 0.002 & 0.002 \\
-0.001 & 0.003 & 0.000 \\
-0.002 & -0.002 & 0.001 \\
-0.001 & -0.002 & 0.001 \\
0.001 & 0.000 & 0.000 \\
0.006 & -0.001 & -0.001 \\
-0.001 & -0.002 & 0.000 \\
-0.023 & -0.008 & 0.124 \\
0.018 & 0.009 & -0.110 \\
0.022 & 0.008 & -0.115 \\
0.016 & 0.005 & -0.094 \\
-0.003 & -0.002 & 0.014 \\
0.001 & 0.001 & 0.011 \\
-0.038 & -0.015 & 0.221 \\
-0.025 & -0.007 & 0.124 \\
-0.095 & -0.042 & 0.564 \\
-0.105 & -0.044 & 0.628 \\
-0.066 & -0.027 & 0.376 \\
-0.002 & -0.002 & 0.001
\end{tabular}

$\begin{array}{lll}-0.043 & -0.139 & 0.123\end{array}$

$\begin{array}{rrr}-0.021 & -0.010 & 0.090 \\ -0.018 & 0.003 & 0.104 \\ 0.023 & 0.018 & -0.130 \\ 0.018 & 0.001 & -0.131 \\ -0.093 & -0.040 & 0.549 \\ -0.087 & -0.026 & 0.505 \\ -0.044 & -0.006 & 0.158 \\ -0.044 & -0.014 & 0.182 \\ -0.079 & -0.041 & 0.526 \\ 0.001 & -0.002 & 0.008\end{array}$

861.00

YES

9. 044

YES

$\begin{array}{ccc}X & Y & Z \\ 0.002 & 0.001 & -0.004\end{array}$

$0.001-0.005-0.010$

$\begin{array}{llll}-0.080 & -0.113 & 0.052\end{array}$

$0.147-0.037-0.027$

$\begin{array}{rrrr}-0.041 & 0.118-0.040\end{array}$

$0.281-0.272-0.246$

$\begin{array}{llll}-0.092 & 0.111 & 0.000\end{array}$

$\begin{array}{rrrr}0.028 & 0.239 & 0.491\end{array}$

$-0.006-0.371-0.156$

$\begin{array}{llll}-0.001 & 0.000 & 0.003\end{array}$

$\begin{array}{llll}-0.002 & 0.001 & -0.001\end{array}$

$0.001-0.002-0.002$

$\begin{array}{llll}-0.001 & -0.005 & 0.000\end{array}$

$\begin{array}{rrr}-0.001 & 0.000 & 0.001\end{array}$

$\begin{array}{lll}0.001 & 0.000 & 0.000\end{array}$

$\begin{array}{lll}0.001 & -0.002 & 0.002\end{array}$

$0.005-0.010-0.003$

$0.003 \quad 0.001-0.002$

$\begin{array}{lll}0.000 & 0.000 & 0.003\end{array}$

$\begin{array}{llll}-0.002 & 0.000 & -0.001\end{array}$

$\begin{array}{llll}-0.062 & 0.091 & 0.479\end{array}$

123. 248

YES

$\begin{array}{lll}X & Y\end{array}$

$\begin{array}{lll}-0.004 & -0.002 & 0.004\end{array}$

$-0.002-0.002-0.002$

$\begin{array}{llll}0.126 & 0.081 & -0.074\end{array}$

$\begin{array}{llll}0.172 & 0.049 & -0.028\end{array}$

$\begin{array}{lll}-0.192 & 0.033 & 0.189\end{array}$

$\begin{array}{lll}0.038 & 0.074 & 0.139\end{array}$

$-0.141-0.180-0.110$

$0.162-0.334-0.385$

$-0.007-0.099-0.158$

$0.008-0.003 \quad 0.001$

$\begin{array}{rrr}-0.004 & 0.001-0.001\end{array}$

$\begin{array}{llll}-0.004 & -0.009 & 0.003\end{array}$

$0.0020 .006-0.004$

$\begin{array}{llll}-0.005 & 0.008 & -0.004\end{array}$

$-0.006-0.004 \quad 0.004$

$-0.008-0.010-0.033$

$\begin{array}{llll}-0.003 & 0.007 & 0.026\end{array}$

$\begin{array}{lll}-0.016 & 0.005 & 0.031\end{array}$
972.69

YES

74.370

\begin{tabular}{rrr}
\multicolumn{4}{c}{972.69} \\
\multicolumn{3}{c}{ YES } \\
X & YES \\
0.006 & 0.003 & -0.002 \\
-0.007 & 0.000 & -0.006 \\
-0.121 & -0.098 & 0.061 \\
-0.201 & -0.075 & 0.032 \\
0.002 & 0.297 & 0.118 \\
0.182 & 0.167 & 0.046 \\
0.271 & -0.121 & -0.198 \\
0.394 & -0.187 & -0.281 \\
0.166 & -0.208 & -0.182 \\
-0.023 & 0.009 & -0.001 \\
0.006 & -0.001 & 0.005 \\
0.005 & 0.014 & -0.006 \\
-0.005 & -0.014 & -0.005 \\
0.009 & -0.018 & 0.000 \\
0.012 & 0.010 & 0.008 \\
0.003 & 0.014 & 0.044 \\
-0.005 & -0.025 & 0.022 \\
0.025 & -0.017 & 0.001
\end{tabular}




$\mathrm{H}$
$\mathrm{H}$
$\mathrm{H}$
Freque
IR A
IR I
Rama

$\mathrm{Re}$
$\mathrm{O}$
$\mathrm{O}$
$\mathrm{O}$
$\mathrm{C}$
$\mathrm{H}$
$\mathrm{C}$
$\mathrm{H}$
$\mathrm{H}$
$\mathrm{N}$
$\mathrm{C}$
$\mathrm{C}$
$\mathrm{C}$
$\mathrm{C}$
$\mathrm{C}$
$\mathrm{H}$
$\mathrm{H}$
$\mathrm{H}$
$\mathrm{H}$
$\mathrm{H}$
$\mathrm{H}$

$$
\begin{array}{rrr}
0.073 & 0.032 & -0.426 \\
-0.014 & -0.004 & 0.089 \\
0.001 & -0.010 & -0.008
\end{array}
$$$$
\begin{array}{rrr}
-0.003 & 0.002 & -0.036 \\
-0.006 & -0.003 & 0.000 \\
-0.040 & 0.485 & 0.461
\end{array}
$$

0.025

0.013

$-0.011$
$0.005-0.020$
$0.005-0.020$

$\begin{array}{lll}-0.040 & 0.485 & 0.461\end{array}$

\begin{tabular}{rrr}
\multicolumn{3}{c}{987.30} \\
Y & YES \\
X & YES \\
0.003 & -0.003 & -0.002 \\
0.001 & 0.004 & -0.010 \\
-0.162 & -0.059 & 0.100 \\
0.110 & 0.083 & -0.001 \\
0.253 & 0.165 & -0.051 \\
-0.162 & 0.310 & 0.114 \\
-0.208 & -0.187 & 0.017 \\
-0.328 & -0.275 & -0.379 \\
-0.040 & 0.295 & 0.042 \\
-0.005 & 0.001 & 0.005 \\
0.005 & -0.001 & 0.006 \\
0.002 & 0.006 & -0.013 \\
-0.004 & -0.007 & -0.006 \\
0.002 & -0.004 & -0.002 \\
0.000 & 0.001 & 0.009 \\
-0.006 & 0.002 & 0.077 \\
-0.023 & 0.005 & 0.029 \\
0.006 & -0.005 & 0.012 \\
0.009 & 0.007 & -0.047 \\
0.013 & 0.005 & -0.029 \\
0.231 & 0.114 & -0.373
\end{tabular}

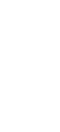

1013.72

78.49

0.825

ve: $\quad \begin{array}{r}\mathrm{X} \\ \mathrm{X}\end{array}$

$\begin{array}{lll}0.000 & 0.000 & 0.000\end{array}$

$\begin{array}{llll}0.000 & -0.001 & 0.004\end{array}$

$\begin{array}{llll}0.008 & 0.004 & -0.004\end{array}$

$-0.001-0.001-0.001$

$-0.008-0.011-0.001$

$0.003-0.015-0.005$

$\begin{array}{llll}0.003 & 0.009 & 0.003\end{array}$

$\begin{array}{llll}0.006 & 0.014 & 0.020\end{array}$

$\begin{array}{lll}-0.002 & -0.008 & 0.002\end{array}$

$\begin{array}{llll}-0.004 & 0.000 & 0.019\end{array}$

$\begin{array}{rrrr}-0.010 & -0.004 & 0.056\end{array}$

$0.0210 .007-0.119$

$\begin{array}{llll}0.010 & 0.004 & -0.051\end{array}$

$\begin{array}{llll}0.003 & 0.000 & -0.018\end{array}$

$\begin{array}{lll}-0.014 & -0.006 & 0.087\end{array}$

$\begin{array}{llll}-0.120 & -0.048 & 0.674\end{array}$

$\begin{array}{lll}-0.067-0.016 & 0.353\end{array}$

$\begin{array}{llll}-0.015 & -0.007 & 0.088\end{array}$

$0.083 \quad 0.037-0.485$

$\begin{array}{llll}0.058 & 0.024 & -0.336\end{array}$

$\begin{array}{llll}-0.008 & -0.011 & 0.005\end{array}$

\begin{tabular}{lrrr} 
Frequency: & \multicolumn{3}{c}{ 1036.16 } \\
IR Active: & \multicolumn{3}{c}{39.559} \\
IR Intens: & \multicolumn{3}{c}{ YES } \\
Raman Active: & Y \\
& Z \\
Re & 0.002 & -0.006 & 0.016 \\
O & 0.001 & 0.052 & -0.170 \\
O & 0.013 & 0.013 & -0.015 \\
O & 0.023 & 0.001 & -0.004 \\
C & -0.013 & -0.024 & 0.007 \\
H & -0.033 & -0.037 & 0.007 \\
C & -0.016 & 0.014 & 0.004 \\
H & 0.000 & 0.026 & 0.052 \\
H & -0.004 & -0.020 & -0.008 \\
N & -0.228 & 0.076 & -0.048 \\
C & 0.300 & -0.093 & 0.017 \\
C & -0.064 & 0.272 & 0.004 \\
C & -0.206 & -0.188 & -0.040 \\
C & 0.029 & -0.242 & -0.002 \\
C & 0.157 & 0.175 & 0.061 \\
H & -0.177 & 0.267 & 0.025 \\
H & -0.293 & -0.104 & -0.102 \\
H & -0.005 & -0.274 & -0.080 \\
H & 0.160 & 0.244 & -0.063 \\
H & 0.298 & -0.110 & 0.204 \\
H & -0.002 & 0.006 & -0.015 \\
& & &
\end{tabular}

1045.04

YES

0.614

YES

$\begin{array}{ccc}X & Y & Z\end{array}$

$\begin{array}{llr}0.000 & 0.000 & 0.001 \\ 0.000 & 0.004 & -0.012\end{array}$

$\begin{array}{rrr}0.000 & 0.004 & -0.012 \\ -0.001 & 0.000 & 0.001\end{array}$

$\begin{array}{lll}-0.001 & -0.001 & 0.000\end{array}$

$\begin{array}{lll}0.000 & 0.001 & 0.000\end{array}$

$\begin{array}{llll}0.001 & 0.001 & 0.000\end{array}$

$\begin{array}{llll}0.001 & 0.000 & 0.000\end{array}$

$\begin{array}{rrrr}-0.002 & 0.001 & 0.001\end{array}$

$0.003-0.001-0.001$

$0.015-0.003-0.007$

$0.0090 .015-0.132$

$0.004-0.014-0.006$

$0.0140 .011-0.019$

$\begin{array}{rrr}-0.018 & 0.005 & 0.089\end{array}$

$\begin{array}{lll}-0.021 & -0.014 & 0.070\end{array}$

$\begin{array}{lll}-0.005 & -0.020 & 0.076\end{array}$

$\begin{array}{llll}-0.018 & -0.001 & 0.166\end{array}$

$\begin{array}{llll}0.081 & 0.048 & -0.478\end{array}$

$\begin{array}{llll}0.058 & 0.020 & -0.396\end{array}$

$\begin{array}{lll}-0.138 & -0.041 & 0.718\end{array}$

$-0.001-0.0020 .000$
0.442

YES

$\begin{array}{rrr}X & Y & Z \\ 0.000 & 0.000 & 0.000 \\ 0.000 & 0.001 & -0.003 \\ 0.001 & 0.000 & 0.000 \\ 0.001 & 0.002 & -0.002 \\ 0.000 & -0.001 & -0.001 \\ 0.001 & 0.000 & -0.001 \\ -0.001 & 0.000 & 0.001 \\ -0.003 & 0.000 & -0.001 \\ 0.004 & 0.000 & -0.001 \\ -0.006 & 0.002 & 0.013 \\ 0.000 & -0.003 & 0.044 \\ -0.008 & 0.000 & 0.037 \\ 0.018 & 0.005 & -0.132 \\ -0.013 & -0.013 & 0.087 \\ 0.014 & 0.008 & -0.068 \\ 0.028 & 0.014 & -0.156 \\ -0.152 & -0.034 & 0.729 \\ 0.085 & 0.026 & -0.440 \\ -0.062 & -0.016 & 0.343 \\ 0.051 & 0.021 & -0.244 \\ 0.000 & -0.001 & 0.001\end{array}$

1057.62

YES

101.677

YES

$\begin{array}{rrr}X & Y & Z \\ 0.000 & 0.018 & -0.067 \\ 0.001 & -0.239 & 0.789 \\ 0.039 & -0.011 & -0.012 \\ -0.004 & 0.041 & 0.014 \\ 0.007 & -0.006 & -0.019 \\ 0.025 & -0.010 & -0.026 \\ -0.007 & -0.013 & 0.015 \\ 0.056 & -0.077 & -0.141 \\ -0.065 & 0.056 & 0.061 \\ -0.189 & 0.066 & -0.030 \\ 0.085 & -0.023 & -0.022 \\ -0.016 & 0.033 & 0.005 \\ -0.056 & -0.020 & -0.013 \\ 0.038 & -0.171 & 0.019 \\ 0.112 & 0.122 & 0.043 \\ -0.013 & 0.044 & -0.021 \\ -0.109 & 0.028 & -0.006 \\ 0.022 & -0.183 & -0.120 \\ 0.077 & 0.199 & -0.043 \\ 0.063 & -0.032 & 0.194 \\ 0.020 & 0.024 & 0.009\end{array}$

1113.64

YES

3.798 


Raman
Re
O
O
C
H
C
H
H
N
C
C
C
C
C
H
H
H
H
H
H

$$
\begin{array}{rrr}
\text { ive: } \begin{array}{c}
\text { YES } \\
\text { X }
\end{array} & \multicolumn{1}{c}{Z} \\
-0.004 & 0.004 & -0.010 \\
-0.001 & -0.030 & 0.099 \\
0.002 & -0.005 & 0.002 \\
-0.010 & 0.002 & 0.001 \\
-0.003 & 0.008 & -0.004 \\
0.031 & 0.013 & -0.011 \\
0.011 & -0.006 & 0.002 \\
-0.007 & -0.011 & -0.027 \\
0.022 & 0.013 & 0.000 \\
0.310 & -0.097 & 0.056 \\
0.225 & -0.065 & 0.030 \\
-0.057 & 0.283 & 0.009 \\
-0.221 & -0.204 & -0.056 \\
-0.039 & 0.256 & 0.015 \\
-0.190 & -0.193 & -0.044 \\
-0.149 & 0.267 & 0.004 \\
-0.368 & -0.051 & -0.068 \\
0.134 & 0.296 & 0.036 \\
-0.105 & -0.289 & -0.053 \\
0.236 & -0.037 & 0.055 \\
-0.009 & -0.008 & 0.028
\end{array}
$$

\begin{tabular}{rrr}
\multicolumn{3}{c}{ YES } \\
X & \multicolumn{1}{c}{$Y$} & \multicolumn{1}{c}{$Z$} \\
0.001 & -0.001 & 0.001 \\
0.000 & 0.000 & 0.001 \\
0.003 & 0.004 & -0.006 \\
0.005 & 0.002 & 0.002 \\
0.004 & -0.008 & 0.007 \\
-0.034 & -0.016 & 0.014 \\
-0.007 & 0.006 & -0.006 \\
0.025 & 0.004 & 0.013 \\
-0.032 & -0.017 & 0.003 \\
-0.135 & 0.051 & -0.024 \\
-0.096 & 0.040 & -0.015 \\
0.047 & 0.066 & 0.014 \\
-0.008 & -0.091 & -0.009 \\
0.071 & 0.065 & 0.018 \\
0.015 & -0.103 & -0.005 \\
0.312 & 0.143 & 0.069 \\
0.122 & -0.265 & 0.012 \\
0.578 & 0.163 & 0.106 \\
0.350 & -0.457 & 0.025 \\
-0.100 & 0.066 & -0.007 \\
0.014 & 0.018 & -0.026
\end{tabular}

1.397

YES Y Z

Raman Active

$\begin{array}{rrr}X & Y & Z \\ 0.000 & -0.001 & 0.002 \\ 0.002 & 0.003 & -0.012 \\ 0.037 & -0.009 & 0.026 \\ -0.016 & 0.034 & -0.036 \\ -0.094 & 0.045 & -0.134 \\ 0.450 & 0.059 & -0.277 \\ 0.047 & -0.077 & 0.148 \\ -0.305 & -0.128 & -0.287 \\ 0.356 & 0.346 & 0.079 \\ -0.012 & 0.010 & -0.002 \\ -0.003 & 0.010 & 0.000 \\ 0.009 & -0.005 & 0.001 \\ -0.009 & -0.003 & -0.001 \\ 0.007 & -0.008 & 0.000 \\ -0.002 & -0.006 & -0.001 \\ 0.060 & 0.009 & 0.016 \\ -0.026 & 0.019 & -0.012 \\ 0.057 & 0.000 & 0.014 \\ 0.001 & -0.009 & -0.002 \\ 0.010 & 0.052 & 0.005 \\ -0.168 & -0.152 & 0.382\end{array}$

\begin{tabular}{lrrr} 
Frequency: & \multicolumn{3}{c}{1242.56} \\
IR Active: & YES \\
IR Intens: & \multicolumn{3}{c}{14.875} \\
Raman Active: & YES \\
& $\mathrm{X}$ & Y & Z \\
Re & -0.001 & 0.000 & 0.000 \\
O & 0.001 & 0.003 & -0.007 \\
O & -0.013 & 0.004 & 0.011 \\
O & 0.012 & -0.041 & 0.020 \\
C & -0.004 & -0.005 & -0.070
\end{tabular}

1195.74

YES

2.870

YES

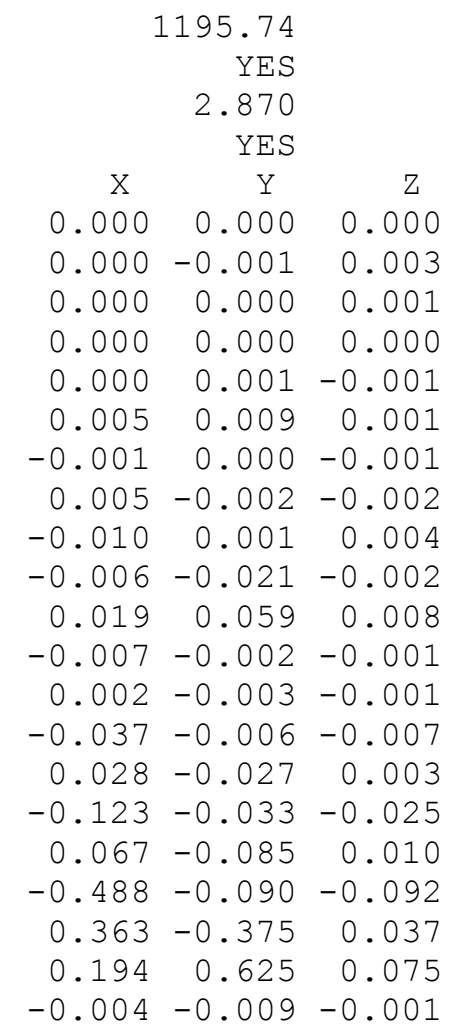

$\begin{array}{rrr} & 1248.85 \\ \text { YES } \\ \text { 15.014 } \\ \text { X } & \text { YES } \\ 0.000 & 0.000 & 0.000 \\ 0.000 & -0.003 & 0.008 \\ 0.009 & -0.002 & -0.006 \\ -0.005 & 0.021 & -0.009 \\ 0.000 & 0.001 & 0.029\end{array}$

\begin{tabular}{rrr}
\multicolumn{1}{c}{ YES } & \multicolumn{1}{c}{ Y } & \multicolumn{1}{c}{$Z$} \\
0.000 & -0.001 & 0.000 \\
0.001 & 0.004 & -0.006 \\
-0.002 & 0.004 & -0.002 \\
0.001 & -0.002 & 0.003 \\
0.007 & -0.005 & 0.012 \\
-0.045 & -0.020 & 0.021 \\
-0.002 & 0.008 & -0.012 \\
0.025 & 0.014 & 0.029 \\
-0.014 & -0.034 & -0.015 \\
0.033 & 0.096 & 0.012 \\
0.029 & 0.096 & 0.012 \\
0.071 & -0.107 & 0.004 \\
-0.105 & -0.027 & -0.016 \\
0.023 & -0.093 & -0.004 \\
-0.070 & -0.045 & -0.016 \\
0.408 & -0.025 & 0.075 \\
-0.336 & 0.258 & -0.068 \\
0.270 & -0.058 & 0.053 \\
-0.315 & 0.194 & -0.039 \\
0.173 & 0.563 & 0.069 \\
0.017 & 0.020 & -0.032
\end{tabular}

1214.45

YES

10.198 YES

$\begin{array}{rrr}\text { X } & \text { Y r } & \text { Z } \\ 0.000 & 0.000 & 0.000 \\ 0.001 & -0.001 & 0.002 \\ -0.034 & 0.017 & -0.021 \\ -0.022 & 0.010 & -0.020 \\ 0.023 & -0.028 & 0.006 \\ -0.267 & -0.565 & -0.089 \\ 0.034 & -0.015 & 0.062 \\ -0.171 & 0.014 & -0.001 \\ 0.330 & 0.027 & -0.086 \\ 0.002 & -0.001 & 0.001 \\ 0.000 & 0.000 & 0.000 \\ -0.002 & 0.000 & 0.000 \\ 0.003 & -0.001 & 0.001 \\ -0.001 & 0.002 & 0.000 \\ 0.000 & 0.000 & 0.000 \\ -0.006 & -0.001 & -0.001 \\ 0.006 & -0.005 & 0.000 \\ -0.020 & -0.001 & -0.003 \\ 0.007 & -0.007 & 0.001 \\ 0.000 & 0.001 & 0.000 \\ 0.250 & 0.618 & 0.041\end{array}$

1324.14 YES 2. 391 YES

$\begin{array}{ccc}X & Y & Z \\ 0.000 & -0.001 & -0.001 \\ 0.001 & 0.000 & 0.007 \\ 0.006 & 0.003 & 0.001 \\ -0.006 & 0.007 & -0.002 \\ -0.002 & 0.010 & 0.002\end{array}$




H
C
H
H
N
C
C
C
C
C
H
H
H
H
H
H

$\begin{array}{rrr}0.042 & -0.148 & -0.135 \\ -0.010 & 0.041 & 0.039 \\ 0.543 & -0.187 & -0.306 \\ -0.424 & 0.182 & 0.303 \\ 0.000 & 0.006 & 0.000 \\ -0.003 & 0.004 & 0.000 \\ 0.024 & 0.015 & 0.006 \\ 0.015 & -0.034 & -0.001 \\ -0.020 & 0.006 & -0.003 \\ -0.016 & 0.003 & -0.003 \\ 0.188 & 0.060 & 0.038 \\ 0.130 & -0.180 & 0.019 \\ -0.168 & -0.021 & -0.033 \\ -0.128 & 0.118 & -0.014 \\ -0.004 & -0.001 & -0.001 \\ 0.081 & 0.234 & 0.067\end{array}$

\begin{tabular}{lrrr} 
Frequency: & \multicolumn{3}{c}{1351.40} \\
IR Active: & YES \\
IR Intens: & 2.965 \\
Raman Active: & YES \\
& X & Y & Z \\
Re & -0.001 & 0.001 & 0.001 \\
O & 0.001 & 0.000 & 0.000 \\
O & 0.024 & 0.001 & -0.020 \\
O & -0.022 & -0.026 & -0.009 \\
C & 0.036 & 0.050 & 0.028 \\
H & -0.087 & -0.231 & -0.035 \\
C & -0.085 & 0.050 & 0.071 \\
H & 0.424 & -0.197 & -0.342 \\
H & 0.482 & -0.254 & -0.321 \\
N & -0.001 & -0.002 & 0.000 \\
C & 0.000 & 0.003 & 0.000 \\
C & -0.002 & 0.001 & 0.000 \\
C & 0.005 & -0.005 & 0.001 \\
C & 0.000 & 0.002 & 0.000 \\
C & 0.000 & 0.000 & 0.000 \\
H & 0.026 & 0.008 & 0.006 \\
H & -0.009 & 0.014 & -0.003 \\
H & -0.025 & -0.003 & -0.004 \\
H & 0.004 & -0.004 & 0.000 \\
H & -0.006 & -0.016 & -0.002 \\
H & -0.107 & -0.379 & -0.154
\end{tabular}
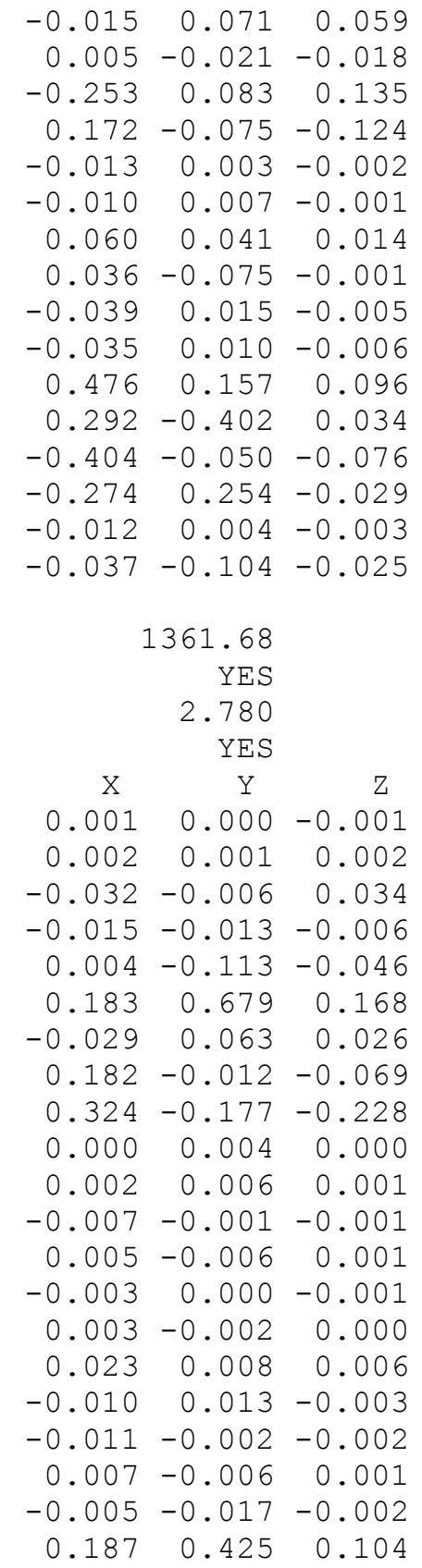

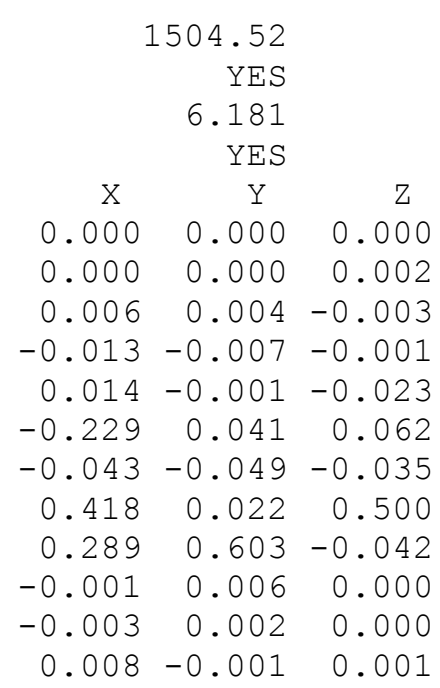

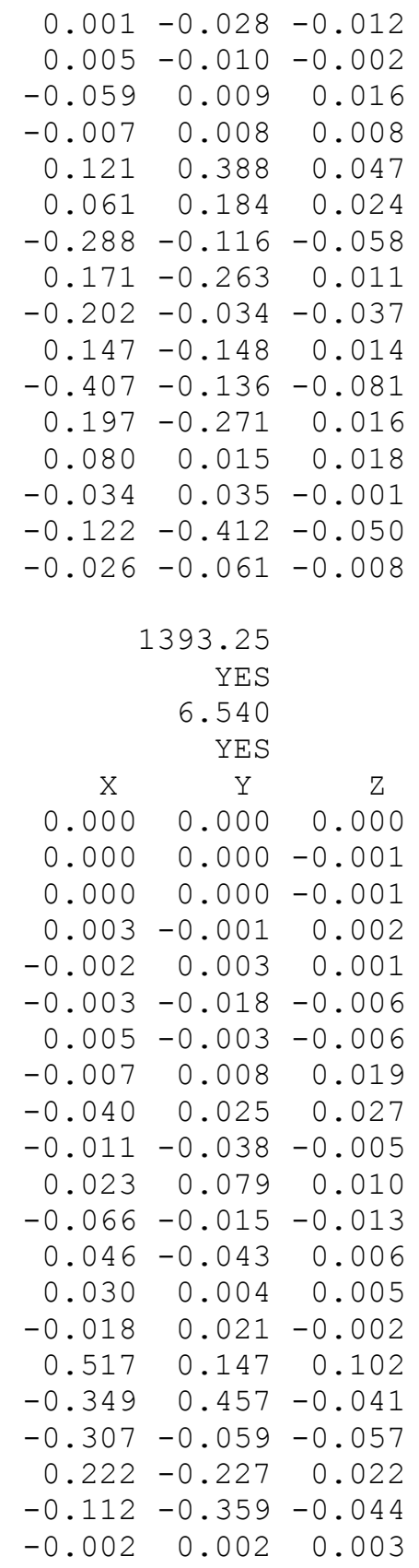

1512.06

YES

0.584

YES

$\begin{array}{rrr}X & Y & Z \\ 0.000 & 0.000 & 0.000 \\ 0.000 & 0.000 & -0.001 \\ 0.010 & 0.004 & -0.009 \\ 0.003 & 0.002 & 0.002 \\ 0.047 & -0.004 & -0.062 \\ -0.628 & 0.107 & 0.174 \\ 0.018 & 0.020 & 0.013 \\ -0.135 & -0.010 & -0.195 \\ -0.117 & -0.208 & 0.025 \\ 0.002 & -0.003 & 0.000 \\ 0.003 & -0.001 & 0.000 \\ -0.005 & -0.001 & -0.001\end{array}$




$\begin{array}{lrrr}\mathrm{C} & -0.126 & -0.026 & -0.024 \\ \mathrm{C} & 0.180 & -0.022 & 0.030 \\ \mathrm{C} & -0.157 & 0.071 & -0.022 \\ \mathrm{H} & -0.334 & -0.229 & -0.074 \\ \mathrm{H} & 0.091 & -0.337 & -0.002 \\ \mathrm{H} & -0.406 & -0.145 & -0.082 \\ \mathrm{H} & 0.200 & -0.323 & 0.010 \\ \mathrm{H} & -0.152 & -0.467 & -0.058 \\ \mathrm{H} & 0.000 & -0.001 & 0.004\end{array}$

\begin{tabular}{lrrr} 
Frequency: & \multicolumn{3}{c}{1521.19} \\
IR Active: & YES \\
IR Intens: & 4.581 \\
Raman Active: & YES \\
& X & Y & Z \\
Re & 0.000 & 0.000 & 0.000 \\
O & 0.000 & 0.000 & 0.002 \\
O & -0.001 & 0.000 & 0.001 \\
O & -0.002 & -0.002 & -0.001 \\
C & -0.001 & 0.001 & 0.003 \\
H & 0.016 & -0.007 & -0.005 \\
C & -0.005 & -0.004 & -0.002 \\
H & 0.034 & -0.001 & 0.037 \\
H & 0.029 & 0.044 & -0.008 \\
N & 0.076 & -0.017 & 0.014 \\
C & 0.089 & -0.018 & 0.014 \\
C & -0.092 & -0.067 & -0.021 \\
C & -0.056 & 0.109 & -0.003 \\
C & -0.103 & -0.071 & -0.023 \\
C & -0.064 & 0.122 & -0.002 \\
H & 0.476 & 0.081 & 0.091 \\
H & 0.356 & -0.398 & 0.045 \\
H & 0.404 & 0.011 & 0.070 \\
H & 0.346 & -0.290 & 0.038 \\
H & 0.092 & -0.077 & 0.011 \\
H & 0.000 & 0.001 & -0.021
\end{tabular}

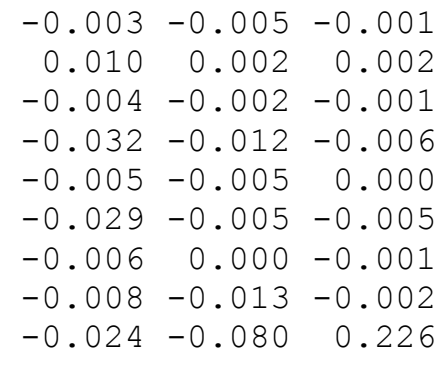

\begin{tabular}{rrr}
\multicolumn{4}{c}{1616.93} \\
\multicolumn{4}{c}{0.172} \\
YES & YES \\
0.000 & 0.001 & 0.000 \\
-0.001 & -0.002 & 0.002 \\
-0.001 & -0.001 & 0.000 \\
0.001 & -0.001 & 0.000 \\
0.001 & 0.000 & 0.001 \\
-0.003 & -0.004 & 0.000 \\
-0.001 & 0.000 & 0.001 \\
0.001 & -0.001 & 0.001 \\
0.005 & 0.002 & -0.002 \\
-0.044 & -0.162 & -0.019 \\
0.103 & 0.386 & 0.046 \\
0.109 & 0.118 & 0.028 \\
-0.059 & 0.174 & 0.001 \\
-0.093 & -0.181 & -0.029 \\
0.018 & -0.235 & -0.013 \\
-0.276 & 0.015 & -0.048 \\
0.231 & -0.184 & 0.035 \\
0.108 & -0.173 & 0.007 \\
-0.238 & -0.007 & -0.042 \\
-0.198 & -0.583 & -0.076 \\
0.001 & 0.000 & -0.002
\end{tabular}

\begin{tabular}{rrr}
\multicolumn{3}{c}{3087.26} \\
\multicolumn{3}{c}{13.446} \\
YES \\
YES & Y & $Z$ \\
0.000 & 0.000 & 0.000 \\
0.000 & 0.000 & 0.000 \\
0.001 & 0.001 & 0.000 \\
0.000 & 0.000 & 0.000 \\
0.026 & 0.004 & -0.063 \\
0.216 & -0.255 & 0.753 \\
-0.003 & -0.008 & 0.002 \\
0.025 & 0.096 & -0.034 \\
0.006 & -0.004 & 0.017 \\
0.000 & 0.000 & 0.000 \\
0.000 & 0.000 & 0.000 \\
0.000 & 0.000 & 0.000 \\
0.000 & 0.000 & 0.000 \\
0.000 & 0.000 & 0.000 \\
0.000 & 0.000 & 0.000 \\
-0.001 & 0.003 & 0.000 \\
0.000 & 0.000 & 0.000 \\
0.000 & 0.000 & 0.000 \\
0.000 & 0.000 & 0.000
\end{tabular}

$\begin{array}{rrr}0.000 & 0.004 & 0.000 \\ -0.006 & -0.002 & -0.001 \\ 0.001 & 0.002 & 0.000 \\ 0.020 & 0.006 & 0.004 \\ 0.007 & -0.003 & 0.000 \\ 0.019 & 0.003 & 0.003 \\ 0.008 & -0.005 & 0.001 \\ 0.005 & 0.004 & 0.001 \\ -0.032 & -0.173 & 0.641\end{array}$

1660.80

YES

58.496 YES

\begin{tabular}{rrr}
$X$ & \multicolumn{1}{c}{$Y$} & $Z$ \\
0.000 & 0.000 & 0.000 \\
-0.001 & 0.002 & -0.005 \\
-0.001 & 0.000 & 0.000 \\
0.000 & -0.001 & -0.001 \\
0.001 & 0.001 & 0.000 \\
-0.002 & -0.004 & 0.000 \\
0.000 & -0.001 & 0.000 \\
0.002 & -0.001 & -0.001 \\
0.001 & 0.001 & 0.000 \\
0.114 & -0.017 & 0.021 \\
-0.142 & 0.015 & -0.024 \\
-0.283 & 0.009 & -0.050 \\
-0.234 & 0.130 & -0.033 \\
0.290 & -0.010 & 0.050 \\
0.242 & -0.128 & 0.033 \\
0.373 & 0.212 & 0.083 \\
0.154 & -0.397 & 0.007 \\
-0.322 & -0.137 & -0.066 \\
-0.154 & 0.308 & -0.005 \\
-0.146 & 0.091 & -0.016 \\
0.000 & -0.001 & -0.001
\end{tabular}

3139.85 YES

2.489 YES

$\begin{array}{cc}X & Y \\ 0.000 & 0.000\end{array}$ Z

0.000

0.000

0.000

0.000

0.000

0.000

0.000

0.000

0.000

$-0.031$

0.000

0.033

$-0.042$

$-0.010$

$0.002-0$.

$-0.072$

0.125

0.173

$0.762-0.231$

$-0.202$

$0.085-0.367$

0.000

0.000

0.000

0.000

0.000

0.000

0.000

0.000

0.000

0.000

0.000

0.000

0.000

0.000

0.000

0.000

0.000

0.000

$-0.001$

0.002

0.000

0.000

0.000

0.000

0.000

0.000

0.000

0.000

0.000

0.000 


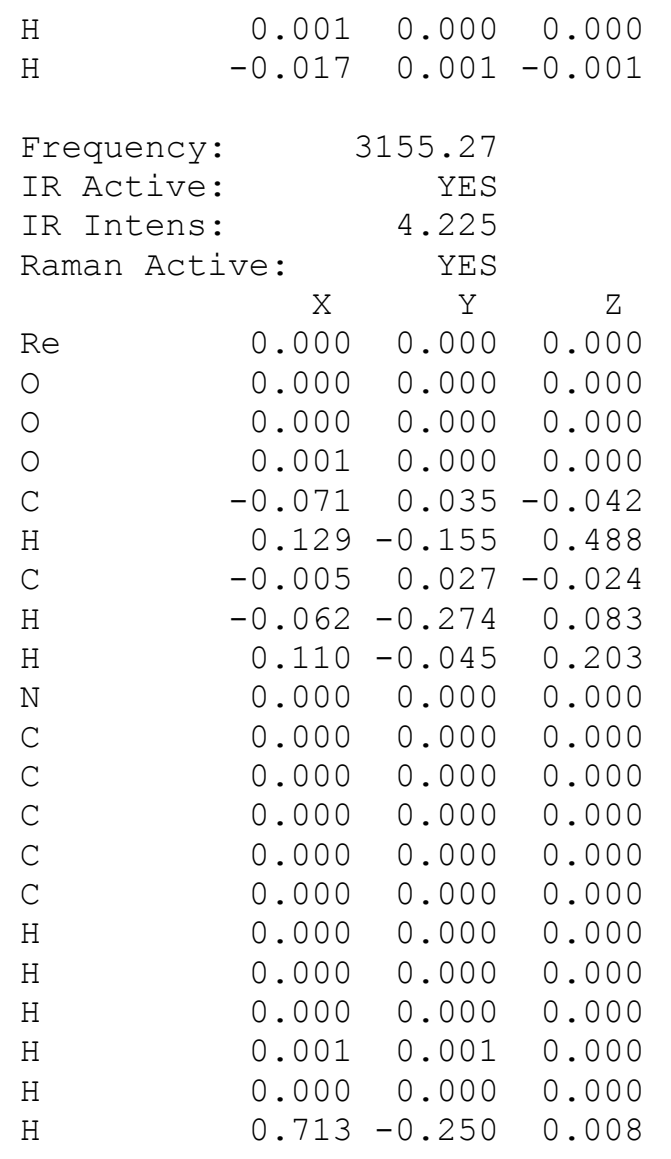

$$
\begin{array}{rrr}
-0.001 & 0.000 & 0.000 \\
-0.521 & 0.181 & -0.023
\end{array}
$$$$
0.000 \quad 0.000
$$$$
0.335-0.118
$$$$
0.000
$$$$
3206.71
$$$$
\text { YES }
$$$$
0.737
$$$$
\text { YES }
$$$$
\begin{array}{cc}
X & Y \\
0.000 & 0.000
\end{array}
$$$$
0.000 \quad 0.000
$$$$
0.000 \quad 0.000
$$$$
0.000
$$$$
\begin{aligned}
& 0.000 \\
& 0.000
\end{aligned}
$$$$
0.000 \quad 0.000
$$$$
-0.001
$$$$
0.000
$$$$
0.000-0.000-0.002
$$$$
0.000-0.000 \quad 0.000
$$$$
0.000-0.002 \quad 0.000
$$$$
\begin{array}{llll}
0.000 & 0.000 & 0.001
\end{array}
$$$$
\begin{array}{llll}
0.001 & 0.001 & 0.000
\end{array}
$$$$
\begin{array}{rrr}
-0.007 & 0.002-0.001
\end{array}
$$$$
0.024-0.082-0.001
$$$$
\begin{array}{rrrr}
-0.001 & 0.001 & 0.000
\end{array}
$$$$
0.001-0.003 \quad 0.000
$$$$
\begin{array}{lll}
0.010 & 0.014 & 0.003
\end{array}
$$$$
\begin{array}{llll}
-0.260 & 0.931 & 0.018
\end{array}
$$$$
\begin{array}{llll}
0.012 & 0.009 & 0.003
\end{array}
$$$$
\begin{array}{llll}
-0.007 & 0.038 & 0.001
\end{array}
$$$$
-0.152-0.154-0.038
$$$$
0.086-0.028 \quad 0.013
$$$$
\begin{array}{lll}
0.001 & 0.000 & 0.000
\end{array}
$$

Frequency:
IR Active:
IR Intens:

IR Intens:

3234.10

YES

2. 720

YES

\begin{tabular}{lrrr} 
& \multicolumn{1}{c}{ X } & Y \\
Re & 0.000 & 0.000 & 0.000 \\
O & 0.000 & 0.000 & 0.000 \\
O & 0.000 & 0.000 & 0.000 \\
O & 0.000 & 0.000 & 0.000 \\
$\mathrm{C}$ & 0.000 & 0.000 & 0.000 \\
$\mathrm{H}$ & 0.000 & 0.000 & -0.001 \\
$\mathrm{C}$ & 0.000 & 0.000 & 0.000 \\
$\mathrm{H}$ & 0.000 & 0.000 & 0.000 \\
$\mathrm{H}$ & 0.000 & 0.000 & 0.000 \\
$\mathrm{~N}$ & 0.000 & 0.001 & 0.000 \\
$\mathrm{C}$ & 0.025 & -0.004 & 0.004 \\
$\mathrm{C}$ & 0.002 & -0.006 & 0.000 \\
$\mathrm{C}$ & 0.017 & 0.017 & 0.004 \\
$\mathrm{C}$ & 0.013 & -0.075 & -0.003 \\
$\mathrm{C}$ & -0.020 & -0.017 & -0.005 \\
$\mathrm{H}$ & -0.018 & 0.062 & 0.001 \\
$\mathrm{H}$ & -0.218 & -0.181 & -0.051 \\
$\mathrm{H}$ & -0.160 & 0.842 & 0.033 \\
$\mathrm{H}$ & 0.216 & 0.212 & 0.053 \\
$\mathrm{H}$ & -0.260 & 0.081 & -0.039 \\
$\mathrm{H}$ & 0.000 & 0.000 & 0.000
\end{tabular}

$$
\begin{array}{r}
3239.49 \\
\text { YES } \\
5.076 \\
\text { YES }
\end{array}
$$

$\mathrm{X}$

0.000

0.000

0.000

0.000

0.000

0.000

0.000

0.000

0.000

0.001

$-0.029$

0.008

$-0.012$

$-0.001$

$-0.056$

$-0.050$

0.137

$0.029-0.161-0.007$

$\begin{array}{llll}0.623 & 0.617 & 0.153\end{array}$

$\begin{array}{llll}0.313 & -0.105 & 0.047\end{array}$

$\begin{array}{lll}-0.001 & 0.000 & 0.000\end{array}$

\begin{tabular}{rrr}
\multicolumn{3}{c}{3220.31} \\
Y 0.330 \\
Y & YES \\
0.000 & 0.000 & 0.000 \\
0.000 & 0.000 & 0.000 \\
0.000 & 0.000 & 0.000 \\
0.000 & 0.000 & 0.000 \\
0.000 & 0.000 & 0.000 \\
0.000 & 0.000 & 0.001 \\
0.000 & 0.000 & 0.000 \\
0.000 & 0.000 & 0.000 \\
0.000 & 0.000 & -0.001 \\
0.000 & 0.000 & 0.000 \\
-0.073 & 0.024 & -0.011 \\
-0.004 & 0.012 & 0.000 \\
0.005 & 0.005 & 0.001 \\
0.008 & -0.029 & -0.001 \\
0.016 & 0.011 & 0.003 \\
0.040 & -0.141 & -0.003 \\
-0.061 & -0.051 & -0.014 \\
-0.069 & 0.345 & 0.013 \\
-0.160 & -0.154 & -0.039 \\
0.836 & -0.273 & 0.125 \\
-0.001 & 0.000 & 0.000
\end{tabular}

3250.60 YES

13.476 YES

\begin{tabular}{rrr} 
X & \multicolumn{1}{c}{$Y$} & $Z$ \\
0.000 & 0.000 & 0.000 \\
0.000 & 0.000 & 0.000 \\
0.000 & 0.000 & 0.000 \\
0.000 & 0.000 & 0.000 \\
0.000 & 0.000 & 0.000 \\
0.000 & 0.000 & 0.000 \\
0.000 & 0.000 & 0.000 \\
0.000 & 0.000 & 0.000 \\
0.000 & 0.000 & 0.000 \\
0.000 & 0.001 & 0.000 \\
0.007 & -0.001 & 0.001 \\
-0.001 & 0.005 & 0.000 \\
-0.066 & -0.050 & -0.015 \\
0.010 & -0.029 & 0.000 \\
0.006 & 0.006 & 0.001 \\
0.011 & -0.038 & -0.001 \\
0.713 & 0.579 & 0.166 \\
-0.065 & 0.317 & 0.012 \\
-0.065 & -0.064 & -0.016 \\
-0.069 & 0.022 & -0.010 \\
0.000 & 0.000 & 0.000
\end{tabular}

STANDARD THERMODYNAMIC QUANTITIES AT $298.18 \mathrm{~K}$ AND 1.00 ATM

This Molecule has 0 Imaginary Frequencies 


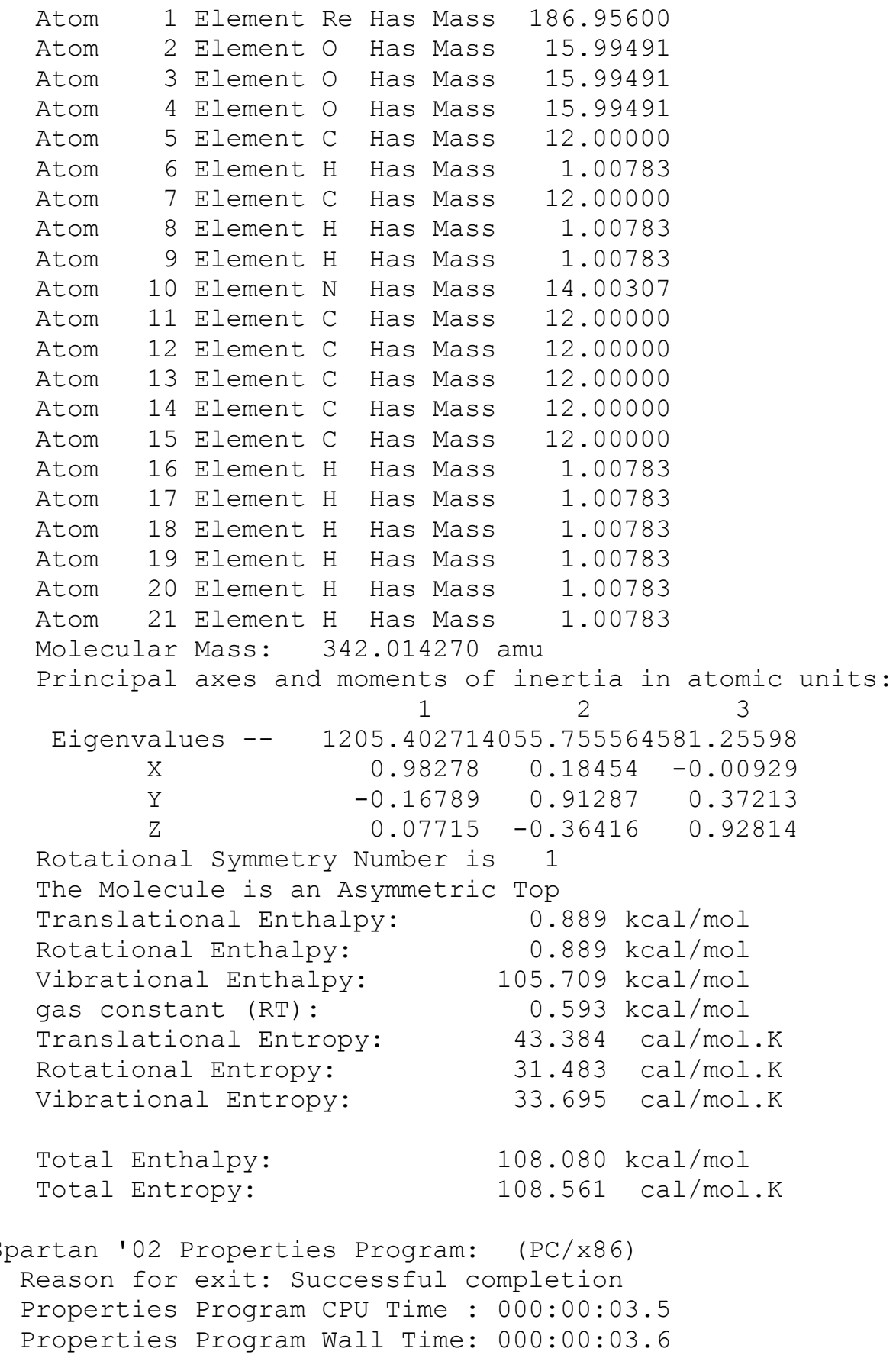

Rotational symmetry Number is 1

The Molecule is an Asymmetric Top

Translational Enthalpy: $\quad 0.889 \mathrm{kcal} / \mathrm{mol}$

Rotational Enthalpy: $\quad 0.889 \mathrm{kcal} / \mathrm{mol}$

Vibrational Enthalpy: $\quad 105.709 \mathrm{kcal} / \mathrm{mol}$

gas constant (RT):

Translational Entropy:

Rotational Entropy:

Vibrational Entropy:

$0.593 \mathrm{kcal} / \mathrm{mol}$

$43.384 \mathrm{cal} / \mathrm{mol} . \mathrm{K}$

$31.483 \mathrm{cal} / \mathrm{mol} . \mathrm{K}$

$33.695 \mathrm{cal} / \mathrm{mol} . \mathrm{K}$

Total Enthalpy:

$108.080 \mathrm{kcal} / \mathrm{mol}$

Total Entropy:

$108.561 \mathrm{cal} / \mathrm{mol} . \mathrm{K}$

15.99491

15.99491

15.99491

12.00000

12.00000

12.00000

1.00783

1.00783

.00783

1.00783

1.00783

partan '02 Properties Program: (PC/x86)

Reason for exit: Successful completion

Properties Program CPU Time : 000:00:03.5

Properties Program Wall Time: 000:00:03.6 


\title{
5-coordinate metallaoxetane in Figure 5
}

\author{
Spartan '02 \\ build 116 (May 14 2002)
}

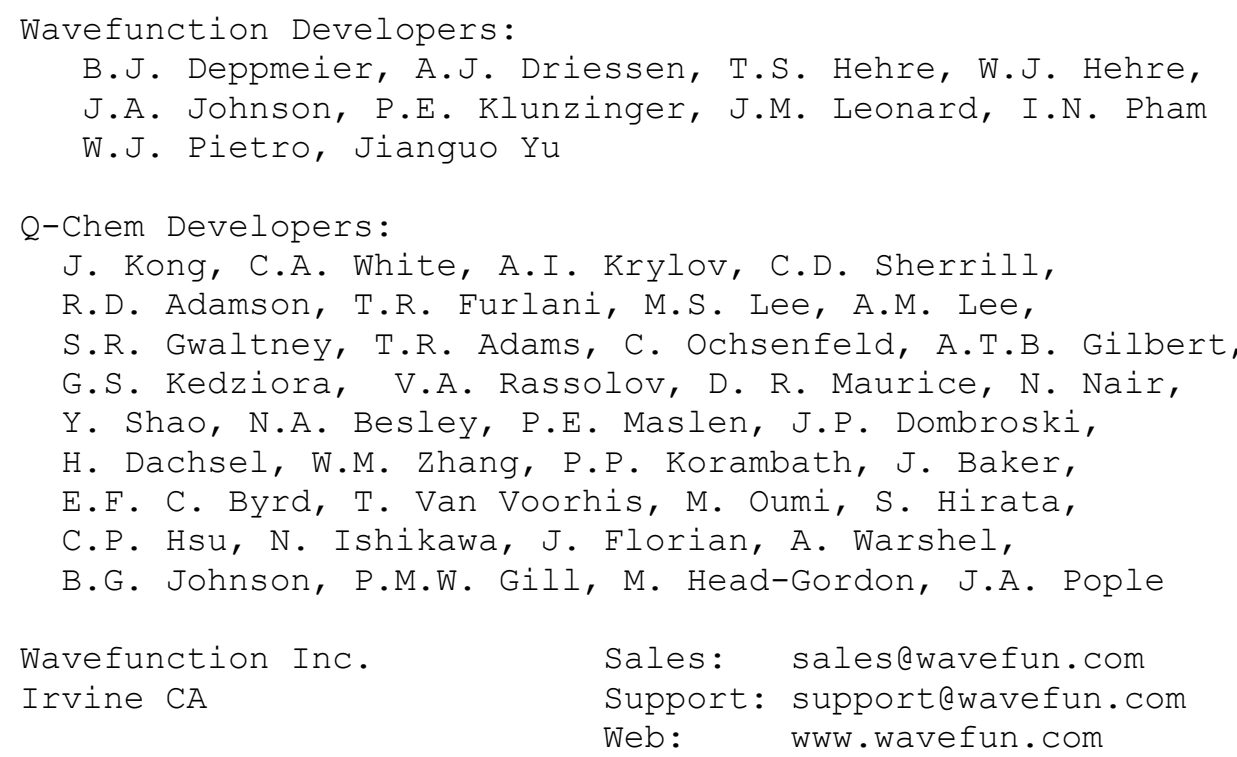

Copyright (c) $1995-2002$

Spartan '02 Mechanics Program: (PC/x86)

Release 116

Reason for exit: Successful completion

Mechanics CPU Time : 000:00:00.2

Mechanics Wall Time: 000:00:00.8

Spartan '02 Quantum Mechanics Program: (PC/x86) Release 116

Job type: Geometry optimization.

Method: RB3LYP

Basis set: LACVP**

Number of shells: 79

Number of basis functions: 234

SCF model:

A restricted hybrid HF-DFT SCF calculation will be performed using Pulay DIIS extrapolation

$\begin{array}{rcrrr}\text { Optimization: } & \text { Max Grad. } & \text { Max Dist. } & \\ \text { Step } & \text { Energy } & 0.005460 & 0.139659 & 6 \\ 1 & -631.5081138 & 0.011826 & 0.146992 & 5 \\ 2 & -631.5062851 & 0.019201 & 0.175312 & 4 \\ 3 & -631.5033750 & 0.020011 & 0.190043 & 3 \\ 4 & -631.5023952 & 0.021148 & 0.139739 & 2 \\ 5 & -631.4989986 & 0.017682 & 0.111635 & 1 \\ 6 & -631.5002473 & 0.006346 & 0.102266 & 1 \\ 7 & -631.5070284 & 0.005434 & 0.093956 & 1 \\ 8 & -631.5083713 & 0.001806 & 0.146006 & 1 \\ 9 & -631.5086083 & 0.006648 & 0.077316 & \\ 10 & -631.5083084 & 0.000783 & 0.028352 & \\ 11 & -631.5089249 & 0.000601 & 0.090706 & \end{array}$




$\begin{array}{llll}13 & -631.5088656 & 0.000791 & 0.068997 \\ 14 & -631.5088985 & 0.000896 & 0.072544 \\ 15 & -631.5089090 & 0.000927 & 0.032338 \\ 16 & -631.5089661 & 0.000783 & 0.023423 \\ 17 & -631.5090735 & 0.000531 & 0.031718 \\ 18 & -631.5090689 & 0.000162 & 0.049473 \\ 19 & -631.5089275 & 0.000251 & 0.037856 \\ 20 & -631.5089342 & 0.000174 & 0.018171 \\ 21 & -631.5089495 & 0.000051 & 0.004601 \\ 22 & -631.5089419 & 0.000021 & 0.002118 \\ 23 & -631.5089501 & 0.000026 & 0.001009\end{array}$

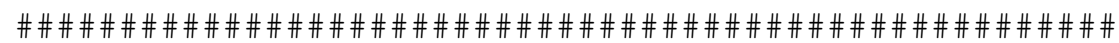

\# Entering optman.exe on Tue Mar 18 18:54:22 2003 \#

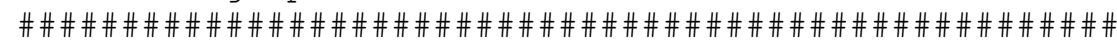

\begin{tabular}{|c|c|c|c|c|c|c|}
\hline \multicolumn{7}{|c|}{$\begin{array}{l}\text { Cartesian Hessian Update } \\
\text { Hessian Updated using BFGS Update }\end{array}$} \\
\hline \multirow[t]{2}{*}{$\star \star$} & \multicolumn{2}{|c|}{$\begin{array}{l}\text { GEOMETRY OPTIMIZATION IN } \\
\text { Searching for a Minimum }\end{array}$} & DELOCALIZED & \multicolumn{2}{|c|}{ INTERNAL COORDINATES } & $\star \star$ \\
\hline & \multicolumn{6}{|c|}{ Optimization Cycle: 23} \\
\hline \multicolumn{7}{|c|}{ Coordinates (Angstroms) } \\
\hline \multicolumn{2}{|c|}{ ATOM } & $\mathrm{X}$ & $\mathrm{Y}$ & \multicolumn{2}{|l|}{ Z } & \\
\hline 1 & $\mathrm{Re}$ & -1.226849 & 0.976181 & \multicolumn{2}{|l|}{-0.140083} & \\
\hline 2 & 0 & -1.362048 & 1.686228 & \multicolumn{2}{|l|}{-1.674044} & \\
\hline 3 & 0 & -1.250314 & 2.099539 & \multicolumn{2}{|l|}{1.133994} & \\
\hline 4 & 0 & -1.506897 & -0.929777 & \multicolumn{2}{|l|}{0.045419} & \\
\hline 5 & $\mathrm{C}$ & -2.942393 & -1.078326 & \multicolumn{2}{|l|}{0.104952} & \\
\hline 6 & $\mathrm{H}$ & -3.224290 & -1.595673 & \multicolumn{2}{|l|}{1.025676} & \\
\hline 7 & $\mathrm{H}$ & -3.287895 & -1.650384 & \multicolumn{2}{|l|}{-0.760239} & \\
\hline 8 & $\mathrm{C}$ & -3.312688 & 0.399782 & \multicolumn{2}{|l|}{0.071336} & \\
\hline 9 & $\mathrm{H}$ & -3.691319 & 0.825218 & \multicolumn{2}{|l|}{1.000372} & \\
\hline 10 & $\mathrm{~N}$ & 0.797372 & 0.240854 & \multicolumn{2}{|l|}{-0.029847} & \\
\hline 11 & $\mathrm{C}$ & 3.484947 & -0.423744 & \multicolumn{2}{|l|}{0.017688} & \\
\hline 12 & $\mathrm{C}$ & 1.741783 & 1.220419 & \multicolumn{2}{|l|}{-0.010458} & \\
\hline 13 & $\mathrm{C}$ & 1.168987 & -1.059328 & \multicolumn{2}{|l|}{-0.025151} & \\
\hline 14 & $\mathrm{C}$ & 2.508625 & -1.419146 & \multicolumn{2}{|l|}{-0.001097} & \\
\hline 15 & $\mathrm{C}$ & 3.091848 & 0.917029 & \multicolumn{2}{|l|}{0.013229} & \\
\hline 16 & $\mathrm{H}$ & 2.772647 & -2.470375 & \multicolumn{2}{|l|}{0.000382} & \\
\hline 17 & $\mathrm{H}$ & 3.816124 & 1.723113 & \multicolumn{2}{|l|}{0.031650} & \\
\hline 18 & $\mathrm{H}$ & 4.537793 & -0.686320 & \multicolumn{2}{|l|}{0.036332} & \\
\hline 19 & $\mathrm{H}$ & 1.393328 & 2.247497 & \multicolumn{2}{|l|}{-0.007342} & \\
\hline 20 & $\mathrm{H}$ & 0.363703 & -1.782290 & \multicolumn{2}{|l|}{-0.040732} & \\
\hline 21 & & -3.872464 & 0.759501 & \multicolumn{2}{|l|}{-0.792038} & \\
\hline $\mathrm{Po}$ & nt Group: & : c1 Number & of degrees & of freedom: & 57 & \\
\hline En & rgy is & -631.50895014 & & & & \\
\hline $\begin{array}{l}\text { Hess } \\
\text { int }\end{array}$ & $\begin{array}{l}\text { an Update } \\
\text { rnal opti }\end{array}$ & $\begin{array}{l}\text { ed using BFGS } \\
\text { imization with }\end{array}$ & $\begin{array}{l}\text { Update } \\
\text { constraints }\end{array}$ & $(0)$ & & \\
\hline $\begin{array}{l}57 \mathrm{H} \\
\text { Hes }\end{array}$ & $\begin{array}{l}\text { ssian moc } \\
\text { ian Eigen }\end{array}$ & $\begin{array}{l}\text { des will be us } \\
\text { nvalues: }\end{array}$ & sed to form $t$ & the next step & & \\
\hline & .000159 & 0.001889 & 0.003631 & 0.013278 & 0.020799 & 0.022071 \\
\hline & .022892 & 0.024639 & 0.025408 & 0.025704 & 0.026972 & 0.029754 \\
\hline
\end{tabular}




$\begin{array}{llllll}0.030064 & 0.034984 & 0.039984 & 0.042552 & 0.045813 & 0.051770 \\ 0.063306 & 0.071461 & 0.077378 & 0.080252 & 0.085176 & 0.106259 \\ 0.107965 & 0.127875 & 0.129394 & 0.129537 & 0.132499 & 0.143579 \\ 0.151675 & 0.174727 & 0.184363 & 0.203310 & 0.214484 & 0.232246 \\ 0.253812 & 0.260586 & 0.306725 & 0.313434 & 0.314782 & 0.316680 \\ 0.317505 & 0.330980 & 0.333640 & 0.337939 & 0.343517 & 0.344142 \\ 0.345231 & 0.362302 & 0.364401 & 0.374851 & 0.395602 & 0.467647\end{array}$

Minimum Search - Taking Simple RFO Step

Searching for Lamda that Minimizes Along All modes

Value Taken Lamda $=-0.00000002$

Step Taken. Stepsize is 0.001708

$\begin{array}{lrrr} & \text { Maximum } & \text { Tolerance } & \text { Cnvgd? } \\ \text { Gradient } & 0.000026 & 0.000300 & \text { YES } \\ \text { Displacement } & 0.001009 & 0.001200 & \text { YES } \\ \text { Energy change } & -0.000008 & 0.000001 & \text { NO }\end{array}$

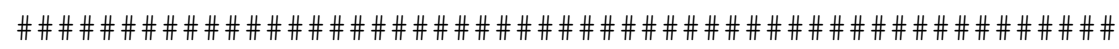

\# Entering anlman.exe on Tue Mar 18 18:54:22 2003 \#

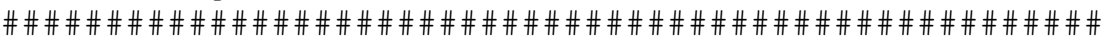

Analysis of SCF Wavefunction

Cartesian Multipole Moments

\begin{tabular}{|c|c|c|c|c|c|}
\hline \multicolumn{6}{|c|}{ Charge (ESU x 10^10) } \\
\hline \multicolumn{6}{|c|}{ Dipole Moment (Debye) } \\
\hline $\mathrm{X}$ & 1.9243 & Y & -0.7211 & Z & 0.1851 \\
\hline Tot & 2.0633 & & & & \\
\hline \multicolumn{6}{|c|}{ Quadrupole Moments (Debye-Ang) } \\
\hline $\mathrm{XX}$ & -35.7872 & $X Y$ & 1.8861 & YY & -74.8255 \\
\hline $\mathrm{XZ}$ & -0.6159 & $\mathrm{YZ}$ & -0.1041 & $\mathrm{ZZ}$ & -87.9628 \\
\hline \multicolumn{6}{|c|}{ Octapole Moments (Debye-Ang^2) } \\
\hline $\mathrm{XXX}$ & 79.8572 & XXY & -36.8543 & XYY & 38.1818 \\
\hline YYY & -100.4014 & XXZ & 7.5934 & $\mathrm{XYZ}$ & 0.0615 \\
\hline YYZ & 3.7326 & $\mathrm{XZZ}$ & 11.9111 & YZZ & -36.9402 \\
\hline ZZZ & 17.9298 & & & & \\
\hline \multicolumn{6}{|c|}{ Hexadecapole Moments (Debye-Ang^3) } \\
\hline XXXX & -1712.9537 & XXXY & 118.9672 & XXYY & -437.7029 \\
\hline$X Y Y Y$ & 135.9115 & YYYY & -731.3907 & XXXZ & -12.7734 \\
\hline XXYZ & 5.9932 & XYYZ & -4.2819 & YYYZ & 13.8331 \\
\hline XXZZ & -437.5590 & XYZZ & 50.9530 & YYZZ & -182.4169 \\
\hline XZZZ & -21.0066 & YZZZ & 22.4372 & ZZZZ & -242.1595 \\
\hline
\end{tabular}

Reason for exit: Sucessful completion

Quantum Mechanics Program CPU Time : 005:25:17.9

Quantum Mechanics Program Wall Time: 010:11:04.9

Spartan '02 Properties Program: (PC/x86)

Release 116

Reason for exit: Successful completion

Properties Program CPU Time : 000:00:03.4

Properties Program Wall Time: 000:00:03.4 
Job type: Frequency calculation.

Method: RB3LYP

Basis set: LACVP**

Number of shells: 79

Number of basis functions: 234

SCF model:

A restricted hybrid HF-DFT SCF calculation will be performed using Pulay DIIS extrapolation

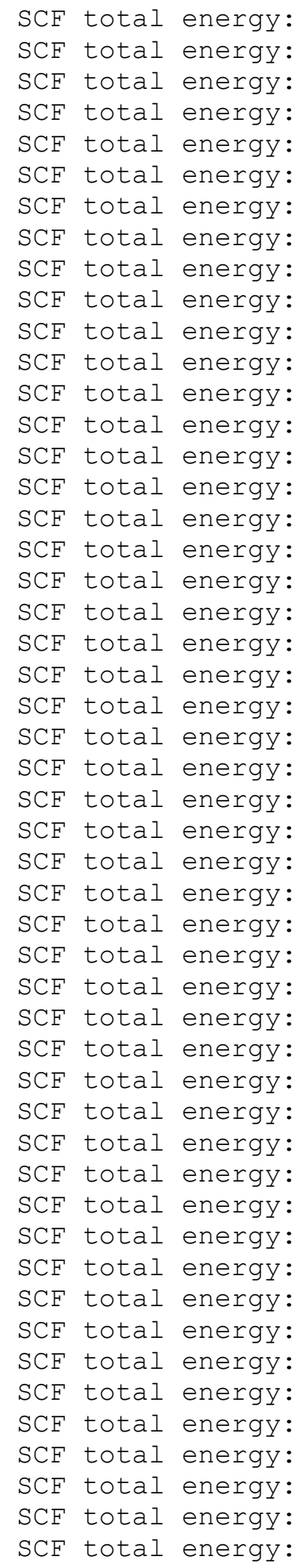

-631.5090888 hartrees -631.5089748 hartrees -631.5090662 hartrees -631.5089839 hartrees -631.5089407 hartrees -631.5089708 hartrees -631.5089751 hartrees -631.5090920 hartrees -631.5090907 hartrees -631.5090902 hartrees -631.5090916 hartrees -631.5090883 hartrees -631.5090900 hartrees -631.5092347 hartrees -631.5090904 hartrees -631.5090933 hartrees -631.5090881 hartrees -631.5090925 hartrees -631.5090941 hartrees -631.5092803 hartrees -631.5092768 hartrees -631.5092346 hartrees -631.5092771 hartrees -631.5092851 hartrees -631.5092825 hartrees -631.5092293 hartrees -631.5092296 hartrees -631.5092377 hartrees -631.5092276 hartrees -631.5092357 hartrees -631.5092304 hartrees -631.5092344 hartrees -631.5092274 hartrees -631.5092331 hartrees -631.5092327 hartrees -631.5092313 hartrees -631.5092350 hartrees -631.5092335 hartrees -631.5092280 hartrees -631.5092345 hartrees -631.5092307 hartrees -631.5092343 hartrees -631.5092313 hartrees -631.5092208 hartrees -631.5092238 hartrees -631.5092172 hartrees -631.5092278 hartrees -631.5092201 hartrees -631.5092193 hartrees -631.5092335 hartrees 


\begin{tabular}{|c|c|c|c|c|}
\hline $\mathrm{SCF}$ & total & & & \\
\hline $\mathrm{SCF}$ & total & energy: & -631.5092293 & hartrees \\
\hline $\mathrm{SCF}$ & total & energy: & -631.5092326 & hartrees \\
\hline $\mathrm{SCF}$ & total & energy: & -631.5092335 & hartrees \\
\hline $\mathrm{SCF}$ & total & energy: & -631.5092290 & hartrees \\
\hline $\mathrm{SCF}$ & total & energy: & -631.5092173 & hartrees \\
\hline $\mathrm{SCF}$ & total & energy: & -631.5092172 & hartrees \\
\hline $\mathrm{SCF}$ & total & energy: & -631.5092334 & hartrees \\
\hline $\mathrm{SCF}$ & total & energy: & -631.5092354 & hartrees \\
\hline $\mathrm{SCF}$ & total & energy: & -631.5092173 & hartrees \\
\hline $\mathrm{SCF}$ & total & energy: & -631.5092184 & hartrees \\
\hline$S C F$ & total & energy: & -631.5092317 & hartrees \\
\hline $\mathrm{SCF}$ & total & energy: & -631.5092312 & hartrees \\
\hline $\mathrm{SCF}$ & total & energy: & -631.5092291 & hartrees \\
\hline SCE & total & energy: & -631.5092328 & hartrees \\
\hline $\mathrm{SCF}$ & total & energy: & -631.5092338 & hartrees \\
\hline $\mathrm{SCF}$ & total & energy: & -631.5092350 & hartrees \\
\hline$S C F$ & total & energy: & -631.5092313 & hartrees \\
\hline $\mathrm{SCF}$ & total & energy: & -631.5092349 & hartrees \\
\hline $\mathrm{SCF}$ & total & energy: & -631.5092307 & hartrees \\
\hline SCE & total & energy: & -631.5092328 & hartrees \\
\hline $\mathrm{SCF}$ & total & energy: & -631.5092329 & hartrees \\
\hline$S C F$ & total & energy: & -631.5092338 & hartrees \\
\hline$S C F$ & total & energy: & -631.5092311 & hartrees \\
\hline$S C F$ & total & energy: & -631.5092313 & hartrees \\
\hline SCE & total & energy: & -631.5092335 & hartrees \\
\hline $\mathrm{SCE}$ & total & energy: & -631.5092294 & hartrees \\
\hline $\mathrm{SCF}$ & total & energy: & -631.5092337 & hartrees \\
\hline $\mathrm{SCF}$ & total & energy: & -631.5092304 & hartrees \\
\hline $\mathrm{SCE}$ & total & energy: & -631.5092326 & hartrees \\
\hline$S C E$ & total & energy: & -631.5092307 & hartrees \\
\hline SCF & total & energy: & -631.5092365 & hartrees \\
\hline $\mathrm{SCF}$ & total & energy: & -631.5092316 & hartrees \\
\hline SCE & total & energy: & -631.5092335 & hartrees \\
\hline $\mathrm{SCF}$ & total & energy: & -631.5092323 & hartrees \\
\hline $\mathrm{SCF}$ & total & energy: & -631.5092259 & hartrees \\
\hline$S C F$ & total & energy: & -631.5092354 & hartrees \\
\hline $\mathrm{SCF}$ & total & energy: & -631.5092304 & hartrees \\
\hline $\mathrm{SCF}$ & total & energy: & -631.5092302 & hartrees \\
\hline$S C F$ & total & energy: & -631.5092308 & hartrees \\
\hline $\mathrm{SCF}$ & total & energy: & -631.5092353 & hartrees \\
\hline $\mathrm{SCE}$ & total & energy: & -631.5092321 & hartrees \\
\hline $\mathrm{SCF}$ & total & energy: & -631.5092336 & hartrees \\
\hline SCE & total & energy: & -631.5092342 & hartrees \\
\hline $\mathrm{SCF}$ & total & energy: & -631.5092310 & hartrees \\
\hline $\mathrm{SCF}$ & total & energy: & -631.5092344 & hartrees \\
\hline SCE & total & energy: & -631.5092345 & hartrees \\
\hline$S C F$ & total & energy: & -631.5092337 & hartrees \\
\hline $\mathrm{SCF}$ & total & energy: & -631.5092342 & hartrees \\
\hline $\mathrm{SCE}$ & total & energy: & -631.5092306 & hartrees \\
\hline $\mathrm{SCF}$ & total & energy: & -631.5092374 & hartrees \\
\hline $\mathrm{SCF}$ & total & energy: & -631.5092316 & hartrees \\
\hline $\mathrm{SCE}$ & total & energy: & -631.5092319 & hartrees \\
\hline $\mathrm{SCF}$ & total & energy: & -631.5092311 & hartrees \\
\hline $\mathrm{SCF}$ & total & energy: & -631.5092342 & hartrees \\
\hline SCE & total & energy: & -631.5092331 & hartrees \\
\hline $\mathrm{SCF}$ & total & energy: & -631.5092302 & hartrees \\
\hline $\mathrm{SCF}$ & total & energy: & -631.5092341 & hartrees \\
\hline$S C E$ & total & energy: & -631.5092345 & hartrees \\
\hline $\mathrm{SCF}$ & tota & energy: & -631.5092320 & hartrees \\
\hline $\mathrm{SCF}$ & & energy: & -631.5092362 & hartrees \\
\hline
\end{tabular}




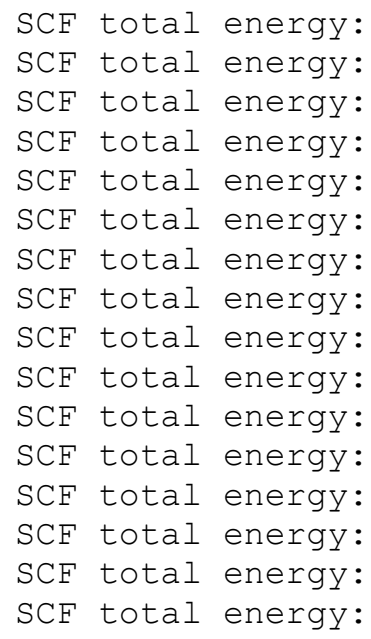

-631.5092326 hartrees

-631.5092341 hartrees

-631.5092329 hartrees

-631.5092342 hartrees

-631.5092334 hartrees

-631.5092305 hartrees

-631.5092356 hartrees

-631.5092338 hartrees

-631.5092339 hartrees

-631.5092329 hartrees

-631.5092416 hartrees

-631.5092255 hartrees

-631.5092325 hartrees

-631.5092344 hartrees

-631.5092358 hartrees

-631.5092353 hartrees

Reason for exit: Sucessful completion

Quantum Mechanics Program CPU Time : 022:30:34.9

Quantum Mechanics Program Wall Time: 023:03:05.3

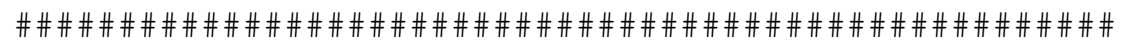

\# Entering vibman.exe on Fri Jan 09 14:16:22 2004 \#

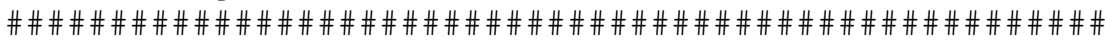

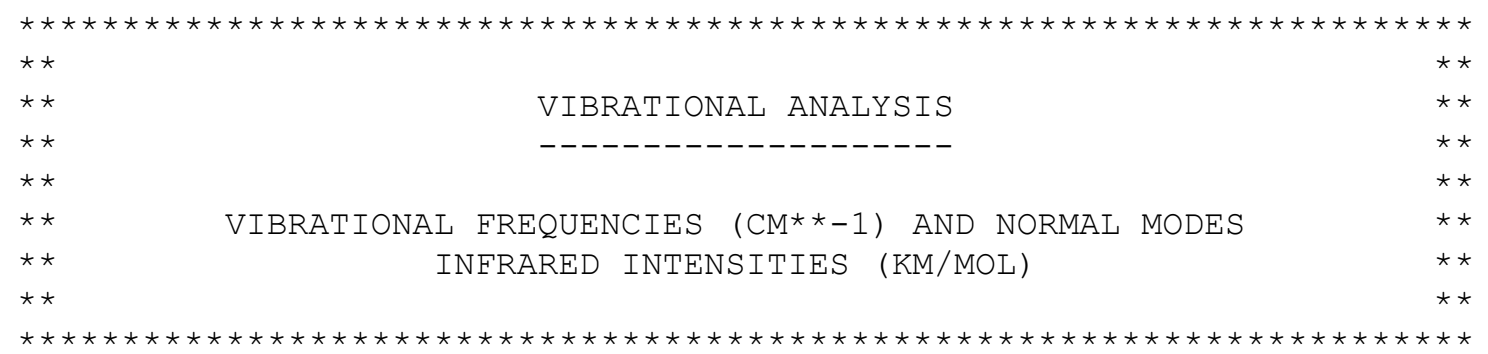

\begin{tabular}{|c|c|c|c|c|c|c|c|c|c|}
\hline \multicolumn{2}{|c|}{ Frequency: } & \multicolumn{2}{|l|}{19.81} & \multicolumn{3}{|c|}{42.67} & \multicolumn{3}{|c|}{95.38} \\
\hline IR $A C$ & & YES & & & YES & & & YES & \\
\hline IR In & & 3.194 & & & 0.795 & & & 1.026 & \\
\hline Raman & ive: & YES & & & YES & & & YES & \\
\hline & X & $\mathrm{Y}$ & Z & X & $\mathrm{Y}$ & Z & X & $\mathrm{Y}$ & Z \\
\hline $\mathrm{Re}$ & -0.001 & -0.004 & -0.022 & -0.001 & 0.002 & 0.000 & -0.003 & 0.006 & -0.027 \\
\hline 0 & -0.108 & -0.101 & -0.058 & 0.070 & -0.040 & -0.029 & -0.128 & 0.162 & 0.058 \\
\hline 0 & 0.105 & 0.090 & -0.094 & -0.060 & 0.028 & -0.027 & 0.071 & -0.132 & 0.087 \\
\hline 0 & -0.005 & -0.005 & -0.075 & 0.012 & 0.013 & 0.205 & 0.028 & 0.001 & -0.035 \\
\hline $\mathrm{C}$ & -0.005 & 0.011 & 0.028 & 0.013 & -0.002 & 0.148 & 0.040 & -0.019 & 0.214 \\
\hline $\mathrm{H}$ & 0.062 & -0.113 & -0.024 & -0.029 & 0.178 & 0.242 & 0.201 & 0.126 & 0.351 \\
\hline $\mathrm{H}$ & -0.086 & 0.141 & -0.022 & 0.072 & -0.188 & 0.242 & -0.092 & -0.173 & 0.362 \\
\hline C & 0.023 & 0.018 & 0.268 & -0.016 & -0.009 & -0.175 & 0.005 & -0.027 & 0.042 \\
\hline $\mathrm{H}$ & 0.214 & -0.099 & 0.397 & -0.174 & 0.167 & -0.316 & -0.040 & 0.095 & -0.030 \\
\hline $\mathrm{N}$ & 0.001 & 0.011 & 0.042 & -0.001 & 0.002 & -0.011 & 0.010 & -0.002 & -0.202 \\
\hline $\mathrm{C}$ & 0.001 & 0.002 & 0.044 & -0.003 & -0.007 & -0.038 & -0.001 & -0.022 & 0.232 \\
\hline $\mathrm{C}$ & 0.002 & 0.005 & $0.1 \xi$ & -0.001 & -0.005 & 0.129 & 0.011 & -0.008 & -0.119 \\
\hline C & 0.002 & 0.013 & -0.102 & -0.002 & 0.004 & -0.163 & 0.005 & -0.005 & -0.129 \\
\hline $\mathrm{C}$ & 0.001 & 0.009 & -0.103 & -0.003 & 0.000 & -0.188 & -0.001 & -0.015 & 0.083 \\
\hline $\mathrm{C}$ & 0.001 & -0.001 & 0.191 & -0.002 & -0.010 & 0.126 & 0.005 & -0.018 & 0.105 \\
\hline $\mathrm{H}$ & -0.001 & 0.011 & -0.221 & -0.004 & 0.002 & -0.317 & -0.0 & -0.017 & 0.149 \\
\hline $\mathrm{H}$ & 0.000 & -0.007 & 0.313 & -0.002 & -0.016 & 0.248 & 0.005 & -0.021 & 0.187 \\
\hline $\mathrm{H}$ & 0.000 & -0.002 & 0.044 & -0.004 & -0.011 & -0.047 & -0.007 & -0.030 & 0.436 \\
\hline $\mathrm{H}$ & 0.003 & 0.001 & 0.3 & & -0.007 & 0.244 & 0.016 & -0.004 & -0.214 \\
\hline $\mathrm{H}$ & 0.003 & 0.017 & -0.213 & -0.002 & 0.009 & -0.248 & 0.006 & -0.002 & -0.205 \\
\hline $\mathrm{H}$ & -0.137 & 0.144 & 0.427 & 0.111 & -0.191 & -0.337 & 0.028 & -0.164 & -0.033 \\
\hline
\end{tabular}




\begin{tabular}{|c|c|c|c|c|c|c|c|c|}
\hline Frequency: & \multicolumn{2}{|l|}{139.77} & \multicolumn{3}{|c|}{149.05} & \multicolumn{3}{|c|}{198.28} \\
\hline IR Active: & YES & & & YES & & & YES & \\
\hline IR Intens: & 1.984 & & & 2.991 & & & 2.176 & \\
\hline Raman Active: & YES & & & YES & & & YES & \\
\hline$x$ & $\mathrm{Y}$ & Z & $\mathrm{X}$ & $\mathrm{Y}$ & Z & $\mathrm{X}$ & $\mathrm{Y}$ & Z \\
\hline 0.009 & -0.035 & -0.018 & 0.003 & -0.023 & 0.023 & 0.023 & 0.015 & 0.027 \\
\hline 0.150 & 0.061 & 0.015 & 0.044 & -0.076 & -0.009 & 0.407 & -0.091 & -0.054 \\
\hline 0.120 & -0.099 & 0.040 & 0.188 & 0.023 & -0.011 & -0.151 & 0.093 & -0.030 \\
\hline-0.126 & -0.018 & -0.134 & -0.075 & -0.001 & 0.180 & 0.075 & -0.037 & -0.171 \\
\hline-0.140 & 0.121 & 0.168 & -0.106 & 0.081 & -0.173 & 0.105 & -0.084 & 0.072 \\
\hline-0.002 & 0.307 & 0.322 & -0.354 & -0.059 & -0.335 & 0.264 & -0.084 & 0.124 \\
\hline-0.361 & -0.008 & 0.336 & 0.069 & 0.255 & -0.350 & -0.031 & -0.101 & 0.134 \\
\hline-0.020 & 0.153 & 0.006 & -0.012 & 0.105 & -0.038 & 0.074 & -0.095 & 0.146 \\
\hline-0.066 & 0.304 & -0.078 & 0.054 & 0.013 & 0.031 & 0.140 & -0.127 & 0.187 \\
\hline-0.032 & -0.039 & 0.096 & -0.023 & -0.035 & -0.166 & -0.129 & -0.020 & -0.119 \\
\hline-0.003 & 0.130 & -0.073 & -0.009 & 0.097 & 0.127 & -0.169 & 0.026 & 0.088 \\
\hline-0.093 & 0.020 & 0.081 & -0.075 & 0.013 & -0.129 & -0.166 & 0.008 & -0.128 \\
\hline 0.027 & -0.017 & 0.075 & 0.025 & -0.018 & -0.138 & -0.137 & -0.021 & -0.126 \\
\hline 0.054 & 0.068 & -0.009 & 0.043 & 0.049 & 0.002 & -0.140 & 0.005 & -0.028 \\
\hline-0.076 & 0.107 & -0.009 & -0.066 & 0.081 & 0.027 & -0.174 & 0.026 & -0.009 \\
\hline 0.124 & 0.087 & -0.035 & 0.102 & 0.063 & 0.039 & -0.115 & 0.012 & -0.013 \\
\hline-0.122 & 0.150 & -0.033 & -0.108 & 0.116 & 0.082 & -0.182 & 0.031 & 0.015 \\
\hline 0.014 & 0.192 & -0.164 & -0.001 & 0.142 & 0.287 & -0.168 & 0.041 & 0.225 \\
\hline-0.156 & -0.003 & 0.126 & -0.125 & -0.003 & -0.199 & -0.191 & 0.001 & -0.207 \\
\hline 0.057 & -0.056 & 0.099 & 0.051 & -0.048 & -0.183 & -0.132 & -0.019 & -0.175 \\
\hline 0.063 & 0.078 & -0.083 & -0.036 & 0.242 & 0.038 & -0.008 & -0.080 & 0.202 \\
\hline Frequency: & 202.45 & & & 217.81 & & & 253.50 & \\
\hline IR Active: & YES & & & YES & & & YES & \\
\hline IR Intens: & 1.790 & & & 12.990 & & & 0.376 & \\
\hline Raman Active: & YES & & & YES & & & YES & \\
\hline X & $\mathrm{Y}$ & Z & $\mathrm{X}$ & $\mathrm{Y}$ & Z & $\mathrm{X}$ & $\mathrm{Y}$ & Z \\
\hline-0.016 & 0.018 & -0.023 & 0.126 & 0.009 & -0.019 & -0.012 & -0.002 & -0.050 \\
\hline 0.032 & 0.095 & 0.003 & -0.389 & 0.006 & 0.015 & 0.180 & 0.165 & 0.003 \\
\hline 0.469 & -0.042 & 0.039 & -0.242 & -0.086 & 0.048 & -0.129 & -0.170 & 0.072 \\
\hline 0.146 & -0.017 & 0.115 & 0.005 & 0.001 & 0.115 & 0.007 & 0.020 & 0.267 \\
\hline 0.169 & -0.149 & -0.034 & 0.018 & 0.046 & -0.027 & -0.004 & 0.007 & -0.001 \\
\hline 0.122 & -0.179 & -0.067 & -0.098 & 0.016 & -0.080 & -0.164 & -0.186 & -0.166 \\
\hline 0.297 & -0.170 & -0.071 & 0.074 & 0.099 & -0.084 & 0.148 & 0.197 & -0.179 \\
\hline 0.067 & -0.174 & -0.077 & 0.043 & 0.044 & -0.011 & 0.012 & 0.008 & 0.212 \\
\hline-0.016 & -0.194 & -0.099 & 0.122 & 0.057 & 0.012 & 0.329 & -0.121 & 0.397 \\
\hline-0.112 & 0.020 & 0.106 & -0.173 & -0.135 & 0.042 & 0.012 & 0.003 & 0.019 \\
\hline-0.150 & -0.004 & -0.082 & -0.182 & 0.103 & -0.044 & 0.018 & -0.005 & -0.032 \\
\hline-0.119 & 0.022 & 0.102 & -0.275 & -0.051 & 0.051 & 0.016 & -0.002 & 0.031 \\
\hline-0.145 & 0.015 & 0.111 & -0.115 & -0.124 & 0.045 & 0.012 & 0.003 & 0.036 \\
\hline-0.156 & 0.001 & 0.031 & -0.089 & 0.004 & 0.000 & 0.013 & -0.001 & 0.001 \\
\hline-0.129 & 0.001 & -0.011 & -0.266 & 0.075 & 0.009 & 0.018 & -0.005 & 0.012 \\
\hline-0.169 & -0.002 & 0.025 & 0.024 & 0.033 & -0.013 & 0.007 & -0.002 & -0.005 \\
\hline-0.113 & -0.012 & -0.042 & -0.324 & 0.126 & 0.005 & 0.019 & -0.007 & 0.015 \\
\hline-0.150 & -0.011 & -0.202 & -0.159 & 0.190 & -0.109 & 0.018 & -0.006 & -0.079 \\
\hline-0.117 & 0.019 & 0.169 & -0.369 & -0.082 & 0.080 & 0.020 & 0.000 & 0.038 \\
\hline-0.153 & 0.028 & 0.145 & -0.079 & -0.162 & 0.061 & 0.011 & 0.003 & 0.064 \\
\hline 0.060 & -0.235 & -0.102 & 0.069 & 0.095 & 0.001 & -0.260 & 0.145 & 0.447 \\
\hline Frequency: & 268.15 & & & 323.54 & & & 398.89 & \\
\hline IR Active: & YES & & & YES & & & YES & \\
\hline IR Intens: & 3.317 & & & 2.493 & & & 0.048 & \\
\hline Raman Active: & YES & & & YES & & & YES & \\
\hline X & $Y$ & Z & $\mathrm{X}$ & Y & Z & $\mathrm{X}$ & $\mathrm{Y}$ & Z \\
\hline 0.039 & -0.044 & -0.001 & 0.027 & -0.077 & 0.006 & 0.001 & 0.001 & 0.000 \\
\hline
\end{tabular}




$\begin{array}{lrrr}\mathrm{O} & -0.021 & -0.068 & -0.006 \\ \mathrm{O} & -0.013 & -0.101 & 0.041 \\ \mathrm{O} & -0.061 & -0.031 & 0.012 \\ \mathrm{C} & -0.080 & 0.039 & -0.004 \\ \mathrm{H} & -0.110 & 0.049 & -0.009 \\ \mathrm{H} & -0.090 & 0.054 & -0.010 \\ \mathrm{C} & -0.009 & 0.051 & -0.010 \\ \mathrm{H} & -0.003 & 0.072 & -0.017 \\ \mathrm{~N} & 0.011 & 0.287 & -0.005 \\ \mathrm{C} & -0.127 & -0.061 & 0.013 \\ \mathrm{C} & 0.115 & 0.213 & -0.013 \\ \mathrm{C} & -0.171 & 0.258 & -0.017 \\ \mathrm{C} & -0.245 & 0.066 & -0.002 \\ \mathrm{C} & 0.070 & 0.006 & -0.009 \\ \mathrm{H} & -0.393 & 0.029 & 0.001 \\ \mathrm{H} & 0.204 & -0.112 & -0.012 \\ \mathrm{H} & -0.163 & -0.207 & 0.037 \\ \mathrm{H} & 0.219 & 0.249 & -0.014 \\ \mathrm{H} & -0.268 & 0.369 & -0.024 \\ \mathrm{H} & 0.025 & 0.078 & -0.019\end{array}$

\begin{tabular}{lrrr} 
Frequency: & \multicolumn{3}{c}{425.02} \\
IR Active: & \multicolumn{3}{c}{ YES } \\
IR Intens: & \multicolumn{3}{c}{ I.461 } \\
Raman Active: & YES \\
& Y & Y \\
Re & -0.049 & -0.042 & 0.005 \\
O & -0.034 & 0.016 & 0.032 \\
O & -0.050 & -0.001 & -0.029 \\
O & 0.261 & 0.024 & -0.014 \\
C & 0.220 & 0.246 & -0.018 \\
H & 0.169 & 0.297 & -0.010 \\
H & 0.170 & 0.275 & -0.012 \\
C & 0.355 & 0.285 & -0.040 \\
H & 0.284 & 0.281 & -0.067 \\
N & -0.007 & 0.006 & 0.020 \\
C & -0.031 & -0.015 & 0.013 \\
C & -0.017 & 0.008 & -0.014 \\
C & -0.013 & 0.007 & 0.007 \\
C & -0.026 & -0.012 & -0.020 \\
C & -0.022 & -0.011 & -0.002 \\
H & -0.038 & -0.014 & -0.043 \\
H & -0.010 & -0.022 & -0.006 \\
H & -0.031 & -0.014 & 0.033 \\
H & -0.013 & 0.011 & -0.038 \\
H & 0.000 & 0.001 & 0.011 \\
H & 0.351 & 0.282 & -0.039
\end{tabular}

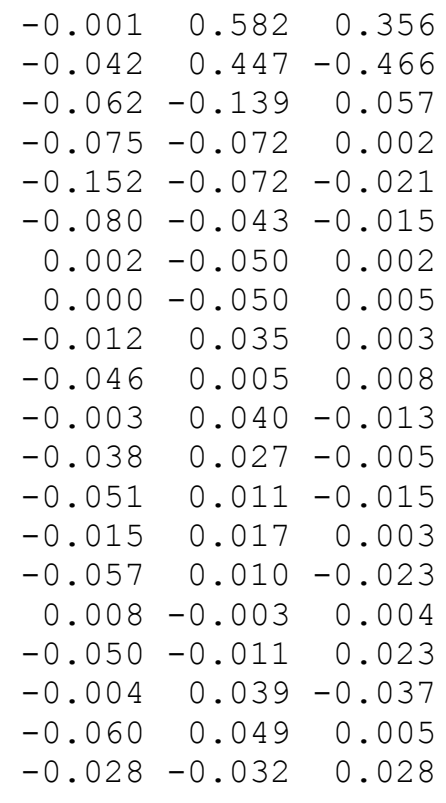

\begin{tabular}{rrr}
\multicolumn{4}{c}{437.12} \\
\multicolumn{3}{c}{1.892} \\
YES & YES & $Z$ \\
0.001 & 0.003 & -0.007 \\
-0.013 & 0.016 & -0.004 \\
0.012 & -0.019 & 0.008 \\
-0.005 & 0.007 & 0.014 \\
-0.002 & -0.010 & 0.002 \\
-0.002 & -0.012 & 0.001 \\
0.008 & -0.014 & 0.000 \\
-0.015 & -0.012 & 0.001 \\
-0.012 & -0.012 & 0.001 \\
-0.009 & -0.011 & 0.313 \\
-0.001 & -0.006 & 0.249 \\
0.001 & -0.004 & -0.085 \\
0.004 & -0.003 & -0.051 \\
0.010 & 0.004 & -0.191 \\
0.004 & 0.002 & -0.161 \\
0.017 & 0.010 & -0.429 \\
0.005 & 0.012 & -0.369 \\
-0.008 & -0.013 & 0.606 \\
0.005 & 0.001 & -0.232 \\
0.010 & -0.004 & -0.151 \\
-0.013 & -0.015 & -0.001
\end{tabular}

$-0.004-0.005-0.003$

$\begin{array}{rrr}0.009 & -0.002 & 0.002\end{array}$

$-0.005-0.001-0.008$

$-0.002-0.006-0.002$

$0.000-0.006-0.001$

$-0.003-0.007-0.001$

$-0.006-0.007-0.001$

$-0.005-0.007-0.001$

$0.002 \quad 0.003-0.012$

$\begin{array}{rrr}-0.001 & 0.000-0.020\end{array}$

$0.006 \quad 0.006-0.182$

$\begin{array}{rrrr}-0.006 & -0.003 & 0.209\end{array}$

$0.002 \quad 0.005-0.190$

$\begin{array}{llll}-0.004 & -0.005 & 0.213\end{array}$

$\begin{array}{rrrr}0.004 & 0.010 & -0.378\end{array}$

$\begin{array}{lll}-0.007 & -0.011 & 0.392\end{array}$

$0.000-0.001-0.060$

$0.0130 .018-0.519$

$\begin{array}{lll}-0.016 & -0.008 & 0.519\end{array}$

$-0.005-0.006-0.002$

\begin{tabular}{rrr}
\multicolumn{3}{c}{554.88} \\
Y & YES \\
X & YES \\
-0.009 & 0.039 & -0.002 \\
-0.020 & -0.012 & -0.027 \\
-0.010 & -0.004 & 0.035 \\
-0.107 & -0.321 & 0.027 \\
-0.165 & -0.135 & 0.014 \\
-0.349 & 0.003 & 0.041 \\
-0.325 & -0.072 & 0.033 \\
0.378 & -0.043 & -0.036 \\
0.471 & 0.064 & -0.043 \\
-0.027 & 0.006 & 0.003 \\
0.050 & -0.006 & 0.004 \\
0.015 & -0.017 & 0.001 \\
0.001 & 0.009 & 0.001 \\
0.017 & 0.026 & -0.001 \\
0.025 & -0.014 & -0.002 \\
-0.006 & 0.020 & -0.006 \\
0.002 & 0.006 & -0.005 \\
0.047 & -0.019 & 0.006 \\
0.045 & -0.007 & 0.004 \\
0.011 & -0.005 & 0.001 \\
0.445 & 0.130 & -0.005
\end{tabular}

\section{(2)}

$\begin{array}{lll}-0.009 & 0.039 & -0.002\end{array}$

$-0.020-0.012-0.027$

$\begin{array}{llll}-0.010 & -0.004 & 0.035\end{array}$

$\begin{array}{lll}-0.107 & -0.321 & 0.027\end{array}$

$\begin{array}{lll}-0.165 & -0.135 & 0.014\end{array}$

$\begin{array}{llll}-0.349 & 0.003 & 0.041\end{array}$

$\begin{array}{lll}-0.325 & -0.072 & 0.033\end{array}$

$0.378-0.043-0.036$

$\begin{array}{llll}0.471 & 0.064 & -0.043\end{array}$

$\begin{array}{rrr}-0.027 & 0.006 & 0.003\end{array}$

$\begin{array}{lll}0.050 & -0.006 & 0.004\end{array}$

$\begin{array}{llll}0.015 & -0.017 & 0.001\end{array}$

$\begin{array}{lll}0.001 & 0.009 & 0.001\end{array}$

$\begin{array}{llll}0.017 & 0.026 & -0.001\end{array}$

$0.025-0.014-0.002$

$\begin{array}{llll}-0.006 & 0.020 & -0.006\end{array}$

$0.0020 .006-0.005$

$0.047-0.0190 .006$

$\begin{array}{lll}0.011 & -0.005 & 0.001\end{array}$

$0.445-0.130-0.005$

$\begin{array}{ccc} & 664.81 & \\ & \text { YES } \\ & 9.648 \\ & \text { YES } & \\ \text { X } & \text { Y } & \text { Z }\end{array}$

$\begin{array}{rrr}-0.010 & 0.006 & 0.000\end{array}$

$-0.004-0.003-0.004$

$\begin{array}{llll}-0.003 & 0.001 & 0.004\end{array}$

$\begin{array}{lll}-0.024 & 0.018 & -0.001\end{array}$

$\begin{array}{lll}-0.031 & -0.035 & 0.002\end{array}$

$\begin{array}{lll}-0.022 & -0.009 & 0.020\end{array}$

$\begin{array}{llll}-0.024 & -0.054 & 0.012\end{array}$

$0.061-0.018-0.013$

\begin{tabular}{rrr} 
& 693.19 \\
\multicolumn{4}{c}{ YES } \\
37.056 \\
Y & YES & $Z$ \\
0.000 & 0.000 & 0.000 \\
-0.002 & 0.002 & -0.001 \\
0.002 & -0.002 & 0.000 \\
0.000 & 0.000 & 0.003 \\
-0.002 & -0.001 & 0.000 \\
-0.001 & -0.003 & -0.001 \\
-0.003 & 0.002 & -0.002 \\
0.001 & -0.001 & 0.002
\end{tabular}




$\mathrm{H}$
$\mathrm{N}$
$\mathrm{C}$
$\mathrm{C}$
$\mathrm{C}$
$\mathrm{C}$
$\mathrm{C}$
$\mathrm{H}$
$\mathrm{H}$
$\mathrm{H}$
$\mathrm{H}$
$\mathrm{H}$
$\mathrm{H}$

$$
\begin{array}{rrr}
-0.024 & 0.000 & -0.001 \\
-0.088 & -0.112 & -0.005 \\
0.087 & 0.132 & 0.005 \\
0.153 & -0.291 & -0.003 \\
-0.272 & -0.119 & -0.008 \\
-0.185 & 0.330 & 0.005 \\
0.290 & 0.128 & 0.012 \\
-0.060 & 0.366 & 0.001 \\
0.114 & 0.291 & 0.002 \\
-0.015 & -0.284 & -0.017 \\
0.100 & -0.314 & -0.015 \\
-0.163 & -0.239 & -0.017 \\
-0.005 & -0.007 & -0.005
\end{array}
$$

\begin{tabular}{lrrr} 
Frequency: & \multicolumn{3}{c}{732.36} \\
IR Active: & \multicolumn{3}{c}{5.880} \\
IR Intens: & \multicolumn{3}{c}{ YES } \\
Raman Active: & \multicolumn{3}{c}{ Y } \\
& X \\
Re & 0.000 & -0.004 & 0.004 \\
O & -0.007 & -0.004 & 0.007 \\
O & 0.006 & 0.007 & 0.002 \\
O & -0.030 & 0.071 & -0.008 \\
C & -0.005 & -0.019 & -0.009 \\
H & 0.026 & 0.244 & 0.162 \\
H & 0.029 & -0.303 & 0.160 \\
C & 0.033 & -0.013 & -0.120 \\
H & 0.603 & -0.181 & 0.188 \\
N & -0.006 & 0.002 & 0.003 \\
C & 0.009 & -0.002 & -0.001 \\
C & -0.004 & -0.004 & -0.002 \\
C & 0.000 & 0.009 & -0.003 \\
C & 0.000 & 0.007 & 0.002 \\
C & -0.004 & -0.006 & 0.001 \\
H & -0.010 & 0.005 & 0.004 \\
H & -0.011 & 0.000 & 0.001 \\
H & 0.009 & -0.002 & -0.003 \\
H & 0.002 & -0.002 & -0.003 \\
H & 0.001 & 0.007 & -0.001 \\
H & -0.475 & 0.169 & 0.294
\end{tabular}

$$
\begin{array}{rrr}
0.106 & -0.023 & 0.008 \\
0.330 & -0.109 & -0.001 \\
-0.354 & 0.114 & 0.002 \\
0.133 & 0.138 & 0.019 \\
-0.036 & -0.251 & 0.001 \\
-0.076 & -0.205 & -0.016 \\
0.125 & 0.270 & -0.001 \\
0.306 & -0.110 & 0.000 \\
0.366 & 0.058 & -0.002 \\
-0.374 & 0.048 & 0.023 \\
-0.167 & 0.039 & 0.022 \\
-0.249 & -0.028 & 0.028 \\
0.019 & 0.007 & 0.027
\end{array}
$$

758.47

YES

44.081

YES

$\begin{array}{ccc}X & Y & Z \\ 0.000 & -0.020 & 0.000\end{array}$

$-0.0010 .016-0.006$

$\begin{array}{lll}-0.008 & 0.009-0.001\end{array}$

$-0.193 \quad 0.385-0.005$

$\begin{array}{llll}-0.048 & -0.139 & 0.011\end{array}$

$0.098-0.367-0.079$

$0.070-0.028-0.111$

$\begin{array}{lll}0.281 & -0.072 & 0.065\end{array}$

$\begin{array}{llll}-0.133 & 0.062 & -0.167\end{array}$

$\begin{array}{llll}-0.011 & 0.003 & 0.004\end{array}$

$0.026-0.004-0.008$

$-0.013-0.013-0.010$

$\begin{array}{llll}0.001 & 0.024 & -0.008\end{array}$

$\begin{array}{rrr}0.004 & 0.016 & 0.001\end{array}$

$\begin{array}{llll}-0.014 & -0.017 & 0.001\end{array}$

$\begin{array}{lll}-0.020 & 0.010 & 0.046\end{array}$

$\begin{array}{llll}-0.038 & 0.000 & 0.049\end{array}$

$\begin{array}{llll}0.027 & 0.003 & 0.029\end{array}$

$\begin{array}{llll}-0.004 & -0.010 & 0.024\end{array}$

$\begin{array}{lll}-0.012 & 0.032 & 0.017\end{array}$

$0.650-0.117-0.192$

\begin{tabular}{rrr} 
& \multicolumn{3}{c}{907.88} \\
& YES \\
X & YES \\
0.000 & 0.000 & 0.000 \\
0.002 & 0.009 & -0.015 \\
-0.002 & -0.010 & -0.010 \\
0.006 & -0.001 & 0.025 \\
-0.003 & -0.010 & -0.069 \\
0.067 & 0.353 & 0.169 \\
-0.037 & -0.376 & 0.175 \\
-0.008 & 0.010 & 0.039 \\
-0.353 & -0.440 & 0.093 \\
0.000 & 0.000 & -0.002 \\
-0.001 & 0.001 & 0.000 \\
0.000 & 0.000 & -0.001 \\
0.000 & -0.001 & 0.002 \\
0.000 & -0.001 & 0.001 \\
0.000 & 0.000 & -0.001
\end{tabular}

$\begin{array}{rrr}-0.009 & 0.003 & -0.004 \\ 0.003 & -0.005 & 0.150 \\ -0.005 & 0.004 & -0.072 \\ 0.005 & 0.005 & -0.108 \\ 0.003 & -0.001 & -0.121 \\ -0.003 & -0.009 & 0.145 \\ -0.001 & 0.002 & 0.142 \\ 0.010 & 0.000 & -0.120 \\ 0.011 & 0.005 & -0.132 \\ 0.006 & 0.015 & -0.545 \\ 0.010 & 0.018 & -0.551 \\ 0.011 & 0.011 & -0.520 \\ 0.013 & 0.000 & -0.007\end{array}$

782.44

YES

34.527

YES

$\begin{array}{rrr}X & Y & Z \\ 0.000 & 0.001 & -0.001 \\ -0.001 & 0.001 & 0.000 \\ 0.000 & -0.001 & 0.001 \\ 0.005 & -0.009 & 0.001 \\ 0.001 & 0.003 & 0.000 \\ -0.002 & 0.007 & 0.001 \\ -0.001 & 0.001 & 0.002 \\ -0.006 & 0.001 & 0.000 \\ -0.004 & -0.001 & 0.002 \\ -0.002 & -0.004 & 0.109 \\ 0.001 & 0.003 & -0.103 \\ 0.004 & 0.003 & -0.113 \\ 0.001 & -0.001 & -0.080 \\ 0.000 & -0.001 & 0.005 \\ 0.002 & 0.002 & 0.003 \\ -0.008 & -0.014 & 0.550 \\ -0.013 & -0.016 & 0.636 \\ -0.011 & -0.011 & 0.393 \\ -0.007 & -0.010 & 0.272 \\ -0.006 & -0.006 & 0.146 \\ -0.009 & 0.002 & 0.001\end{array}$

983.15

YES

0.048

YES

$\begin{array}{rrr}X & Y & Z \\ 0.000 & 0.000 & -0.001 \\ 0.001 & -0.003 & 0.007 \\ -0.001 & 0.006 & 0.008 \\ -0.002 & -0.001 & 0.000 \\ 0.004 & 0.002 & -0.001 \\ 0.001 & 0.005 & 0.000 \\ 0.003 & -0.001 & 0.001 \\ 0.000 & 0.000 & 0.001 \\ -0.005 & -0.004 & 0.001 \\ -0.004 & 0.000 & 0.017 \\ 0.001 & -0.002 & 0.060 \\ 0.003 & 0.007 & -0.117 \\ -0.001 & -0.002 & -0.049 \\ 0.002 & -0.001 & -0.023 \\ 0.000 & -0.001 & 0.087\end{array}$




$\begin{array}{rrrr}\mathrm{H} & -0.014 & -0.015 & 0.586 \\ \mathrm{H} & 0.008 & 0.012 & -0.412 \\ \mathrm{H} & -0.002 & -0.001 & 0.106 \\ \mathrm{H} & 0.013 & 0.017 & -0.528 \\ \mathrm{H} & -0.013 & -0.007 & 0.415 \\ \mathrm{H} & 0.007 & 0.007 & 0.000\end{array}$

\begin{tabular}{lrrr} 
Frequency: & \multicolumn{3}{c}{1003.89} \\
IR Active: & \multicolumn{3}{c}{82.247} \\
IR Intens: & \multicolumn{3}{c}{ YES } \\
Raman Active: & \multicolumn{3}{c}{ Y } \\
& 0.000 & 0.004 & 0.007 \\
Re & 0.001 & 0.002 & -0.010 \\
O & 0.005 & -0.059 & -0.068 \\
O & -0.192 & -0.038 & 0.007 \\
O & 0.190 & 0.268 & -0.019 \\
C & -0.044 & 0.403 & -0.011 \\
H & -0.033 & 0.308 & 0.040 \\
H & 0.083 & -0.191 & 0.004 \\
C & -0.146 & -0.492 & 0.032 \\
H & -0.068 & 0.014 & -0.002 \\
N & 0.034 & -0.011 & 0.001 \\
C & 0.002 & 0.054 & 0.004 \\
C & -0.023 & -0.036 & -0.005 \\
C & 0.023 & -0.051 & 0.003 \\
C & 0.039 & 0.038 & -0.001 \\
C & 0.044 & -0.051 & -0.021 \\
H & 0.067 & 0.019 & 0.022 \\
H & 0.039 & -0.007 & 0.000 \\
H & 0.008 & 0.061 & -0.009 \\
H & -0.019 & -0.048 & 0.012 \\
H & -0.103 & -0.478 & 0.009 \\
H & & &
\end{tabular}

$$
\begin{array}{rrr}
0.002 & 0.000 & -0.009 \\
0.000 & 0.000 & 0.008 \\
0.000 & 0.001 & -0.001 \\
0.000 & -0.001 & 0.007 \\
0.000 & -0.001 & -0.006 \\
0.340 & 0.467 & 0.014
\end{array}
$$

\begin{tabular}{rrr}
\multicolumn{3}{c}{1015.84} \\
Y & YES \\
X & YES \\
-0.002 & -0.013 & -0.077 \\
0.038 & -0.200 & 0.436 \\
-0.013 & 0.351 & 0.436 \\
-0.049 & -0.012 & 0.033 \\
0.052 & 0.070 & -0.033 \\
0.046 & 0.189 & 0.034 \\
-0.045 & -0.009 & 0.052 \\
0.015 & -0.049 & 0.022 \\
-0.126 & -0.183 & 0.018 \\
-0.054 & 0.008 & -0.005 \\
0.023 & -0.010 & -0.027 \\
-0.002 & 0.039 & 0.004 \\
-0.015 & -0.025 & 0.075 \\
0.013 & -0.035 & -0.051 \\
0.034 & 0.031 & 0.026 \\
0.011 & -0.045 & 0.256 \\
0.063 & 0.018 & -0.130 \\
0.018 & -0.030 & 0.159 \\
-0.017 & 0.039 & -0.060 \\
0.016 & -0.037 & -0.437 \\
0.077 & -0.039 & -0.012
\end{tabular}

1037.10 YES

52.084

YES

\section{Raman Active}

64.852

$$
\begin{array}{cc} 
& Y E S \\
X & Y
\end{array}
$$

$\mathrm{Re}$

O

0

O

C

$\mathrm{H}$

C

$\mathrm{H}$

$\mathrm{N}$

C

C

C

C

$\mathrm{H}$

$\mathrm{H}$

$\mathrm{H}$

$\mathrm{H}$

$$
\begin{array}{rrr}
-0.001 & 0.001 & 0.000 \\
0.001 & -0.003 & 0.005 \\
0.002 & -0.003 & -0.001 \\
-0.162 & -0.055 & 0.006 \\
0.238 & -0.141 & -0.001 \\
0.264 & -0.241 & -0.038 \\
0.258 & -0.178 & -0.001 \\
-0.080 & 0.159 & -0.003 \\
0.192 & 0.408 & 0.007 \\
-0.134 & 0.023 & 0.001 \\
0.055 & -0.022 & 0.017 \\
-0.002 & 0.085 & 0.019 \\
-0.034 & -0.050 & -0.048 \\
0.034 & -0.100 & 0.029 \\
0.082 & 0.082 & -0.024 \\
0.052 & -0.101 & -0.156 \\
0.123 & 0.050 & 0.146 \\
0.061 & -0.035 & -0.092 \\
-0.010 & 0.092 & -0.072 \\
-0.028 & -0.081 & 0.258 \\
0.146 & 0.410 & -0.046
\end{array}
$$

$$
\begin{array}{rrr}
0.001 & -0.005 & 0.112 \\
0.016 & 0.015 & -0.502 \\
0.011 & 0.008 & -0.351 \\
-0.017 & -0.019 & 0.676 \\
-0.015 & -0.009 & 0.354 \\
0.003 & 0.001 & -0.001
\end{array}
$$

$$
1019.77
$$$$
\text { YES }
$$

6.729

YES

$\begin{array}{rrr}X & Y & Z \\ 0.000 & -0.001 & -0.006 \\ 0.003 & -0.017 & 0.037 \\ -0.001 & 0.024 & 0.030 \\ 0.020 & 0.006 & 0.000 \\ -0.030 & 0.024 & -0.002 \\ -0.037 & 0.047 & 0.007 \\ -0.036 & 0.024 & 0.003 \\ 0.012 & -0.025 & 0.003 \\ -0.039 & -0.070 & 0.000 \\ 0.028 & -0.005 & 0.015 \\ -0.007 & 0.003 & 0.047 \\ -0.002 & -0.016 & 0.036 \\ 0.007 & 0.012 & -0.128 \\ -0.009 & 0.017 & 0.082 \\ -0.015 & -0.014 & -0.072 \\ 0.000 & 0.031 & -0.416 \\ -0.039 & -0.017 & 0.357 \\ 0.000 & 0.016 & -0.270 \\ 0.004 & -0.011 & -0.154 \\ -0.030 & 0.007 & 0.737 \\ -0.012 & -0.058 & 0.005\end{array}$

\begin{tabular}{rrr} 
X & \multicolumn{1}{c}{$Y$ Y } & \multicolumn{1}{c}{$Z$} \\
-0.006 & 0.030 & -0.010 \\
0.026 & -0.154 & 0.295 \\
0.006 & -0.166 & -0.183 \\
-0.193 & -0.059 & 0.011 \\
0.236 & -0.046 & -0.012 \\
0.173 & -0.058 & -0.027 \\
0.150 & -0.065 & 0.020 \\
-0.022 & 0.075 & 0.004 \\
0.047 & 0.139 & 0.007 \\
0.256 & -0.041 & 0.006 \\
-0.197 & 0.068 & -0.014 \\
0.047 & -0.213 & -0.013 \\
0.125 & 0.146 & 0.020 \\
-0.047 & 0.207 & 0.000 \\
-0.159 & -0.180 & 0.004 \\
-0.006 & 0.239 & 0.023 \\
-0.191 & -0.181 & -0.073 \\
-0.208 & 0.123 & 0.060 \\
0.132 & -0.206 & 0.047 \\
0.109 & 0.188 & -0.077 \\
0.076 & 0.155 & -0.026
\end{tabular}

1046.46 YES

0.171 YES

\begin{tabular}{rrr}
\multicolumn{3}{c}{1046.46} \\
\multicolumn{3}{c}{ Y.171 } \\
Y & YES \\
0.000 & -0.001 & 0.000 \\
-0.001 & 0.004 & -0.007 \\
0.000 & 0.009 & 0.010 \\
-0.002 & -0.002 & 0.000 \\
0.003 & 0.001 & 0.000 \\
0.002 & 0.001 & 0.000 \\
0.003 & 0.001 & 0.000 \\
-0.001 & 0.000 & 0.000 \\
0.000 & -0.001 & 0.001 \\
0.004 & -0.001 & -0.007 \\
0.006 & 0.003 & -0.130 \\
-0.001 & 0.004 & -0.007 \\
-0.001 & -0.002 & -0.027 \\
-0.003 & 0.001 & 0.093 \\
-0.004 & -0.005 & 0.072 \\
0.011 & 0.017 & -0.500 \\
0.009 & 0.007 & -0.396 \\
-0.014 & -0.019 & 0.718 \\
-0.006 & 0.000 & 0.086 \\
-0.010 & -0.004 & 0.194 \\
0.002 & 0.002 & -0.001
\end{tabular}




\begin{tabular}{|c|c|c|c|c|c|c|c|c|}
\hline Frequency: & \multicolumn{2}{|l|}{1049.33} & \multicolumn{3}{|c|}{1054.22} & \multicolumn{3}{|c|}{1089.63} \\
\hline IR Active: & YES & & \multicolumn{3}{|c|}{ YES } & \multicolumn{3}{|c|}{ YES } \\
\hline IR Intens: & \multicolumn{2}{|l|}{$\begin{array}{r}\text { YES } \\
9.810\end{array}$} & \multicolumn{3}{|c|}{0.858} & \multicolumn{3}{|c|}{24.037} \\
\hline Raman Active: & \multicolumn{2}{|l|}{ YES } & \multicolumn{3}{|c|}{ YES } & \multicolumn{3}{|c|}{ YES } \\
\hline$x$ & $\mathrm{Y}$ & Z & $\mathrm{X}$ & Y & Z & $X$ & Y & Z \\
\hline-0.002 & 0.033 & -0.007 & -0.002 & -0.011 & 0.002 & 0.000 & 0.001 & 0.000 \\
\hline 0.029 & -0.179 & 0.342 & -0.014 & 0.068 & -0.128 & 0.002 & -0.009 & 0.017 \\
\hline 0.007 & -0.231 & -0.259 & -0.006 & 0.089 & 0.099 & 0.001 & -0.013 & -0.014 \\
\hline 0.143 & 0.069 & -0.007 & -0.087 & -0.045 & 0.005 & 0.020 & 0.015 & -0.002 \\
\hline-0.172 & -0.052 & 0.005 & 0.115 & 0.009 & -0.004 & -0.026 & -0.006 & 0.002 \\
\hline-0.101 & -0.055 & 0.021 & 0.094 & 0.001 & -0.012 & -0.019 & -0.010 & 0.001 \\
\hline-0.119 & -0.056 & -0.008 & 0.090 & 0.003 & 0.005 & -0.017 & -0.006 & -0.002 \\
\hline 0.035 & 0.020 & 0.000 & -0.027 & 0.013 & 0.000 & 0.005 & 0.001 & -0.001 \\
\hline-0.120 & -0.058 & -0.028 & 0.064 & 0.067 & 0.014 & -0.011 & -0.007 & -0.004 \\
\hline-0.016 & 0.006 & 0.000 & 0.330 & -0.082 & 0.007 & -0.107 & 0.018 & -0.002 \\
\hline 0.284 & -0.063 & -0.008 & 0.236 & -0.055 & 0.014 & -0.099 & 0.021 & -0.001 \\
\hline-0.082 & 0.226 & 0.004 & -0.080 & 0.255 & 0.005 & 0.041 & 0.095 & 0.004 \\
\hline-0.206 & -0.179 & -0.007 & -0.198 & -0.192 & 0.000 & 0.004 & -0.094 & -0.005 \\
\hline 0.005 & -0.081 & 0.005 & -0.067 & 0.248 & -0.008 & 0.057 & 0.103 & 0.005 \\
\hline 0.015 & 0.063 & 0.008 & -0.177 & -0.190 & -0.013 & 0.008 & -0.114 & -0.004 \\
\hline-0.036 & -0.108 & -0.042 & 0.005 & 0.271 & 0.078 & 0.512 & 0.227 & 0.009 \\
\hline-0.060 & 0.153 & -0.033 & -0.149 & -0.226 & 0.016 & 0.402 & -0.476 & 0.001 \\
\hline 0.315 & -0.024 & 0.076 & 0.245 & -0.051 & -0.050 & -0.112 & 0.013 & -0.004 \\
\hline-0.190 & 0.208 & 0.000 & -0.209 & 0.222 & -0.007 & 0.235 & 0.168 & 0.002 \\
\hline-0.387 & -0.011 & -0.013 & -0.359 & -0.036 & -0.079 & 0.192 & -0.305 & 0.014 \\
\hline-0.088 & -0.061 & 0.042 & 0.051 & 0.071 & -0.024 & -0.012 & -0.007 & 0.006 \\
\hline Frequency: & 1120.85 & & & 148.23 & & & 1178.89 & \\
\hline IR Active: & YES & & & YES & & & YES & \\
\hline IR Intens: & 1.718 & & & 26.829 & & & 2.080 & \\
\hline Raman Active: & YES & & & YES & & & YES & \\
\hline 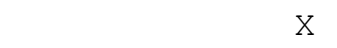 & $Y$ & Z & $\mathrm{X}$ & $\mathrm{Y}$ & Z & $\mathrm{X}$ & $\mathrm{Y}$ & Z \\
\hline 0.000 & -0.003 & 0.000 & 0.002 & 0.000 & 0.000 & 0.000 & 0.000 & -0.001 \\
\hline-0.001 & 0.006 & -0.012 & -0.003 & 0.004 & -0.014 & 0.001 & -0.002 & 0.006 \\
\hline 0.000 & 0.010 & 0.012 & -0.003 & 0.007 & 0.014 & 0.000 & 0.002 & 0.004 \\
\hline-0.001 & -0.004 & 0.000 & -0.027 & -0.022 & 0.002 & -0.002 & -0.001 & -0.053 \\
\hline 0.003 & -0.001 & 0.001 & 0.003 & -0.068 & 0.000 & 0.007 & 0.008 & 0.156 \\
\hline 0.016 & -0.011 & -0.001 & 0.052 & -0.114 & -0.006 & -0.510 & -0.197 & -0.118 \\
\hline 0.017 & -0.005 & -0.002 & 0.083 & -0.127 & 0.000 & 0.512 & 0.198 & -0.148 \\
\hline 0.002 & 0.009 & -0.002 & 0.093 & 0.164 & -0.008 & -0.004 & -0.005 & -0.065 \\
\hline-0.017 & -0.011 & -0.001 & -0.548 & -0.368 & -0.027 & -0.093 & -0.401 & 0.068 \\
\hline-0.001 & 0.098 & 0.001 & 0.000 & -0.006 & 0.000 & 0.001 & 0.000 & 0.001 \\
\hline 0.016 & 0.100 & 0.002 & -0.001 & -0.004 & 0.000 & 0.000 & 0.000 & 0.000 \\
\hline 0.083 & -0.082 & -0.001 & -0.002 & 0.002 & 0.000 & 0.000 & 0.000 & 0.000 \\
\hline-0.112 & -0.043 & -0.001 & 0.006 & 0.002 & 0.000 & 0.000 & -0.001 & 0.001 \\
\hline 0.039 & -0.087 & -0.002 & -0.002 & 0.005 & 0.000 & -0.001 & 0.000 & 0.000 \\
\hline-0.056 & -0.061 & -0.003 & 0.000 & 0.002 & 0.000 & 0.000 & 0.000 & 0.000 \\
\hline 0.340 & -0.021 & 0.010 & -0.018 & 0.001 & 0.000 & -0.006 & -0.001 & 0.002 \\
\hline-0.235 & 0.086 & -0.004 & 0.001 & 0.002 & 0.001 & -0.001 & 0.001 & -0.001 \\
\hline 0.144 & 0.622 & 0.020 & -0.008 & -0.029 & -0.003 & 0.000 & 0.000 & 0.000 \\
\hline 0.415 & 0.023 & 0.012 & -0.004 & 0.002 & -0.002 & -0.002 & -0.001 & -0.001 \\
\hline-0.358 & 0.215 & -0.015 & 0.012 & -0.004 & -0.001 & 0.004 & -0.004 & -0.007 \\
\hline-0.024 & -0.009 & 0.006 & -0.544 & -0.408 & 0.137 & 0.084 & 0.387 & 0.049 \\
\hline Frequency: & 1197.18 & & & 219.66 & & & 1246.76 & \\
\hline IR Active: & YES & & & YES & & & YES & \\
\hline IR Intens: & 2.883 & & & 0.326 & & & 30.124 & \\
\hline Raman Active: & YES & & & YES & & & YES & \\
\hline $\mathrm{X}$ & $\mathrm{Y}$ & Z & $\mathrm{X}$ & $\mathrm{Y}$ & Z & X & $\mathrm{Y}$ & Z \\
\hline 0.000 & 0.000 & 0.000 & 0.000 & 0.000 & 0.000 & 0.000 & 0.000 & 0.000 \\
\hline 0.000 & 0.000 & 0.001 & 0.000 & 0.000 & 0.000 & 0.001 & -0.002 & 0.003 \\
\hline
\end{tabular}




O
$\mathrm{O}$
$\mathrm{C}$
$\mathrm{H}$
$\mathrm{H}$
$\mathrm{C}$
$\mathrm{H}$
$\mathrm{N}$
$\mathrm{C}$
$\mathrm{C}$
$\mathrm{C}$
$\mathrm{C}$
$\mathrm{C}$
$\mathrm{H}$
$\mathrm{H}$
$\mathrm{H}$
$\mathrm{H}$
$\mathrm{H}$
$\mathrm{H}$

$\begin{array}{rrr}0.000 & -0.001 & -0.001 \\ -0.001 & 0.000 & 0.000 \\ 0.000 & -0.001 & -0.001 \\ -0.003 & 0.002 & 0.000 \\ -0.002 & -0.003 & 0.001 \\ 0.001 & 0.001 & 0.001 \\ -0.004 & 0.000 & -0.001 \\ 0.000 & -0.022 & 0.000 \\ 0.018 & 0.056 & 0.002 \\ -0.011 & 0.000 & 0.000 \\ 0.008 & -0.005 & -0.001 \\ -0.043 & -0.007 & -0.001 \\ 0.030 & -0.021 & 0.000 \\ -0.530 & -0.131 & -0.015 \\ 0.376 & -0.330 & 0.002 \\ 0.156 & 0.615 & 0.018 \\ -0.124 & -0.038 & -0.003 \\ 0.107 & -0.110 & 0.002 \\ -0.003 & -0.005 & 0.001\end{array}$

Freque
IR A t IR I t
Raman
Re
O
O
O
C
H
H
C
H
N
C
C
C
C
C
H
H
H
H
H
H

$$
\begin{array}{rrr}
X & Y & Z \\
0.001 & -0.001 & 0.000 \\
0.001 & 0.000 & 0.000 \\
0.002 & -0.001 & 0.000 \\
-0.013 & -0.003 & 0.001 \\
0.004 & 0.000 & -0.001 \\
0.006 & -0.006 & -0.003 \\
-0.001 & -0.008 & 0.004 \\
0.003 & 0.002 & -0.001 \\
-0.001 & 0.009 & -0.005 \\
0.095 & 0.404 & 0.011 \\
0.060 & 0.178 & 0.006 \\
-0.296 & -0.131 & -0.012 \\
0.159 & -0.236 & -0.002 \\
-0.197 & -0.069 & -0.005 \\
0.160 & -0.131 & 0.000 \\
0.214 & 0.030 & 0.004 \\
-0.019 & 0.028 & 0.002 \\
-0.076 & -0.385 & -0.013 \\
-0.386 & -0.149 & -0.004 \\
0.242 & -0.295 & -0.002 \\
-0.010 & -0.001 & 0.005
\end{array}
$$

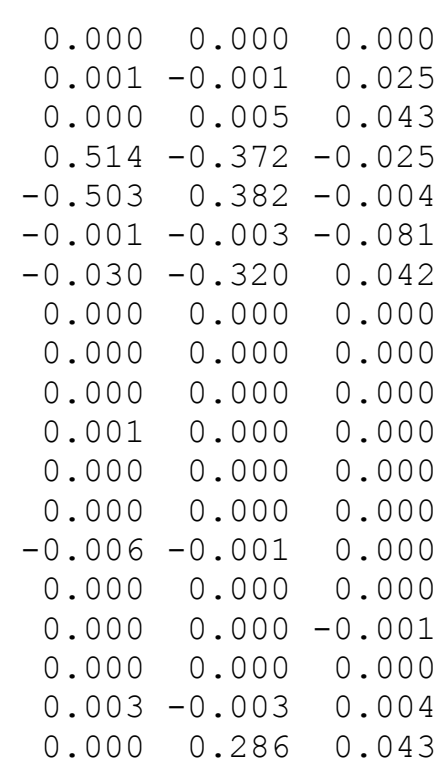

1366.74

YES

1.390

4.058

YES

$\begin{array}{rrr}0.000 & -0.002 & -0.002 \\ -0.001 & 0.005 & 0.000 \\ -0.001 & -0.003 & -0.001 \\ -0.013 & 0.001 & -0.001 \\ -0.014 & -0.003 & 0.003 \\ 0.000 & -0.001 & 0.001 \\ 0.003 & 0.010 & -0.003 \\ -0.003 & 0.016 & 0.000 \\ -0.011 & 0.005 & 0.000 \\ 0.074 & 0.043 & 0.003 \\ 0.037 & -0.077 & -0.002 \\ -0.046 & 0.008 & 0.000 \\ -0.046 & 0.003 & -0.001 \\ -0.390 & -0.077 & -0.013 \\ -0.385 & 0.303 & -0.001 \\ -0.012 & 0.008 & 0.000 \\ 0.464 & 0.179 & 0.016 \\ 0.372 & -0.442 & 0.005 \\ 0.002 & 0.003 & 0.001\end{array}$

$\begin{array}{ccc}X & Y & Z \\ 0.000 & 0.002 & 0.000\end{array}$

$\begin{array}{lll}0.000 & -0.002 & 0.004\end{array}$

$0.000-0.003-0.004$

$\begin{array}{llll}-0.017 & -0.040 & 0.002\end{array}$

$\begin{array}{lll}-0.105 & 0.114 & 0.001\end{array}$

$\begin{array}{llll}0.604 & -0.324 & -0.042\end{array}$

$\begin{array}{llll}0.608 & -0.331 & 0.010\end{array}$

$\begin{array}{lll}0.011 & -0.017 & 0.002\end{array}$

$\begin{array}{lll}0.086 & -0.033 & 0.033\end{array}$

$\begin{array}{lll}-0.002 & -0.002 & 0.000\end{array}$

$\begin{array}{lll}-0.001 & 0.003 & 0.000\end{array}$

$\begin{array}{lll}-0.002 & 0.000 & 0.000\end{array}$

$\begin{array}{lll}0.005 & -0.004 & 0.000\end{array}$

$\begin{array}{lll}0.002 & 0.002 & 0.000\end{array}$

$\begin{array}{llll}-0.001 & 0.001 & 0.000\end{array}$

$-0.029-0.006-0.001$

$\begin{array}{lll}0.007 & -0.007 & 0.000\end{array}$

$\begin{array}{llll}-0.007 & -0.019 & -0.001\end{array}$

$\begin{array}{lll}0.029 & 0.011 & 0.001\end{array}$

$\begin{array}{rrr}-0.007 & 0.008 & 0.000\end{array}$

$\begin{array}{llll}0.078 & -0.049 & -0.048\end{array}$

1495.29

YES

46.433

YES

$\begin{array}{ccc}X & Y & Z \\ 0.000 & 0.001 & 0.000\end{array}$

$\begin{array}{lll}0.000 & 0.000 & 0.001\end{array}$

$\begin{array}{llll}0.000 & -0.001 & -0.001\end{array}$

$0.002 \quad 0.000 \quad 0.000$

$\begin{array}{lll}-0.002 & -0.001 & 0.000\end{array}$

$\begin{array}{lll}0.014 & 0.017 & 0.015\end{array}$

$\begin{array}{llll}0.015 & 0.018 & -0.016\end{array}$

$\begin{array}{rrrr}0.001 & -0.004 & 0.000\end{array}$

$\begin{array}{llll}-0.015 & 0.013 & -0.014\end{array}$

$$
\begin{aligned}
& \begin{array}{ccc} 
& 1395.68 \\
& \text { YES } & \\
& 5.409 & \\
& \text { YES } & \\
\mathrm{X} & \mathrm{Y} & \mathrm{Z}
\end{array} \\
& 0.000 \quad 0.000 \quad 0.000 \\
& \begin{array}{llll}
0.000 & 0.001 & -0.001
\end{array} \\
& \begin{array}{lll}
0.000 & 0.001 & 0.001
\end{array} \\
& \begin{array}{lll}
0.003 & 0.002 & 0.000
\end{array} \\
& \begin{array}{llll}
0.005 & -0.004 & 0.000
\end{array} \\
& \begin{array}{lll}
-0.026 & 0.020 & 0.005
\end{array} \\
& \begin{array}{llll}
-0.024 & 0.021 & -0.004
\end{array} \\
& \begin{array}{lll}
-0.002 & 0.000 & 0.000
\end{array} \\
& \begin{array}{lll}
0.002 & 0.001 & 0.002
\end{array} \\
& -0.018-0.028-0.001 \\
& \begin{array}{lll}
0.014 & 0.088 & 0.002
\end{array} \\
& -0.054-0.013-0.002 \\
& \begin{array}{llll}
0.060 & -0.060 & 0.001
\end{array} \\
& \begin{array}{lll}
0.028 & 0.010 & 0.001
\end{array} \\
& \begin{array}{llll}
-0.020 & 0.011 & 0.000
\end{array} \\
& -0.361-0.089-0.010 \\
& \begin{array}{llll}
0.199 & -0.187 & 0.000
\end{array} \\
& \begin{array}{llll}
-0.100 & -0.372 & -0.011
\end{array} \\
& \begin{array}{lll}
0.529 & 0.192 & 0.019
\end{array} \\
& \begin{array}{lll}
-0.367 & 0.405 & -0.004
\end{array} \\
& 0.002 \quad 0.002-0.002 \\
& 1523.90 \\
& \text { YES } \\
& 8.482 \\
& \text { YES } \\
& \begin{array}{ccc}
X & Y & Z \\
0.000 & 0.000 & 0.000
\end{array} \\
& 0.000 \quad 0.000 \quad 0.000 \\
& \begin{array}{ccc}
0.000 & 0.001 & 0.000
\end{array} \\
& \begin{array}{lll}
-0.002 & -0.002 & 0.000
\end{array} \\
& \begin{array}{lll}
0.002 & 0.003 & 0.000
\end{array} \\
& -0.009-0.020-0.016 \\
& \begin{array}{lll}
-0.009 & -0.019 & 0.017
\end{array} \\
& 0.000-0.001 \quad 0.000 \\
& \begin{array}{llll}
-0.001 & 0.000 & -0.001
\end{array}
\end{aligned}
$$




N
C
C
C
C
C
H
H
H
H
H
H

$\begin{array}{rr}0.001 & 0.001 \\ 0.001 & 0.001 \\ 0.001 & -0.002 \\ -0.003 & 0.000 \\ 0.004 & 0.000 \\ -0.004 & 0.002 \\ -0.006 & -0.003 \\ 0.009 & -0.009 \\ -0.002 & -0.011 \\ 0.000 & -0.003 \\ 0.002 & -0.005 \\ -0.315 & 0.426\end{array}$

0.000
0.000
0.000
0.000
0.000
0.000
0.000
0.000
0.000
0.000
0.000
0.441

Frequency:

1532.67

IR Active:

YES

IR Intens:

0.913

Raman Active:

YES

$\begin{array}{lrrr} & \text { X } & \text { Y } & \text { Z } \\ \text { Re } & 0.000 & 0.000 & 0.000 \\ \text { O } & 0.000 & 0.000 & 0.001 \\ \text { O } & 0.000 & 0.000 & -0.001 \\ \text { O } & -0.009 & -0.007 & 0.000 \\ \text { C } & -0.038 & -0.077 & 0.002 \\ \text { H } & 0.308 & 0.467 & 0.411 \\ \text { H } & 0.291 & 0.463 & -0.440 \\ \text { C } & -0.008 & 0.013 & 0.001 \\ \text { H } & 0.046 & -0.057 & 0.051 \\ \text { N } & 0.001 & 0.002 & 0.000 \\ \text { C } & 0.002 & -0.001 & 0.000 \\ \text { C } & -0.001 & -0.002 & 0.000 \\ \text { C } & -0.002 & 0.002 & 0.000 \\ \text { C } & -0.001 & -0.002 & 0.000 \\ \text { C } & -0.003 & 0.004 & 0.000 \\ \text { H } & 0.007 & 0.000 & 0.000 \\ \text { H } & 0.009 & -0.007 & 0.000 \\ \text { H } & 0.002 & -0.003 & 0.000 \\ \text { H } & 0.010 & 0.001 & 0.000 \\ \text { H } & 0.016 & -0.019 & 0.000 \\ \text { H } & 0.036 & -0.062 & -0.058\end{array}$

Frequency:

IR Active:

IR Intens:

Raman Active:

Re
O
O
O
C
H
H
C
H
N
C
C
C
C
C
H

$\begin{array}{cc}X & Y \\ 0.000 & 0.000 \\ 0.000 & 0.000 \\ 0.000 & 0.000 \\ -0.002 & 0.000 \\ -0.031 & -0.059 \\ 0.172 & 0.329 \\ 0.214 & 0.350 \\ 0.000 & 0.001 \\ -0.002 & 0.001 \\ 0.000 & 0.000 \\ 0.000 & 0.000 \\ 0.000 & 0.000 \\ 0.000 & 0.000 \\ 0.000 & 0.000 \\ 0.000 & 0.000 \\ 0.000 & 0.000\end{array}$

3083.10

YES

27.450

YES

Z

0.000

0.000

0.000

0.000

0.000

$-0.586$

0.588

0.000

0.004

0.000

0.000

0.000

0.000

0.000

0.000

0.000

$\begin{array}{rrr}-0.051 & -0.118 & -0.003 \\ -0.032 & -0.044 & -0.002 \\ -0.079 & 0.096 & 0.001 \\ 0.151 & -0.006 & 0.004 \\ -0.154 & 0.037 & -0.003 \\ 0.176 & -0.091 & 0.002 \\ 0.255 & 0.160 & 0.010 \\ -0.325 & 0.376 & 0.002 \\ 0.094 & 0.486 & 0.015 \\ 0.172 & 0.208 & 0.009 \\ -0.214 & 0.417 & 0.001 \\ -0.010 & 0.016 & 0.016\end{array}$

1616.89

YES

0.024

YES

\begin{tabular}{rrr}
$X$ & \multicolumn{1}{c}{$Y$} & $Z$ \\
0.000 & 0.001 & 0.000 \\
0.000 & 0.000 & 0.001 \\
-0.001 & -0.001 & -0.002 \\
0.002 & -0.001 & 0.000 \\
-0.002 & 0.000 & 0.000 \\
0.006 & -0.001 & 0.002 \\
0.005 & 0.000 & -0.003 \\
0.001 & -0.001 & 0.000 \\
-0.004 & -0.003 & -0.001 \\
-0.029 & -0.176 & -0.004 \\
0.089 & 0.396 & 0.012 \\
0.096 & 0.124 & 0.006 \\
-0.069 & 0.181 & 0.002 \\
-0.096 & -0.193 & -0.007 \\
0.035 & -0.231 & -0.005 \\
0.148 & -0.160 & -0.001 \\
-0.231 & -0.028 & -0.006 \\
-0.155 & -0.587 & -0.019 \\
-0.277 & -0.001 & -0.009 \\
0.254 & -0.166 & 0.006 \\
-0.001 & -0.002 & 0.001
\end{tabular}

3125.12

YES

4.688

YES

$\mathrm{x}$

0.000

0.000

0.000

0.000

0.000

0.005

$-0.006$

$0.050-0$.

$-0.233$

0.000

0.000

$Y$

0.000

0.000

0.000

0.001

0.003

0.008

$-0.011$

-0.044
0.253

0.253

0.000

0.000

0.000

0.000

0.000

0.000

0.000

0.000

0.000

0.000

0.000
$0.062-0.050$

$\begin{array}{llll}0.086 & -0.027 & 0.001\end{array}$

$\begin{array}{llll}-0.110 & -0.051 & -0.004\end{array}$

$\begin{array}{lll}-0.026 & 0.105 & 0.002\end{array}$

$-0.133-0.066-0.005$

$\begin{array}{lll}-0.038 & 0.106 & 0.002\end{array}$

$\begin{array}{lll}0.459 & 0.078 & 0.012\end{array}$

$\begin{array}{lll}0.315 & -0.194 & 0.002\end{array}$

$\begin{array}{lll}0.123 & 0.038 & 0.004\end{array}$

$\begin{array}{lll}0.608 & 0.197 & 0.023\end{array}$

$\begin{array}{lll}0.298 & -0.233 & 0.005\end{array}$

$\begin{array}{lll}0.002 & 0.001 & 0.000\end{array}$

1656.25

YES

37.806

YES

\begin{tabular}{rrr}
$X$ & \multicolumn{1}{c}{$Y$} & $Z$ \\
-0.001 & 0.000 & 0.000 \\
0.001 & -0.001 & 0.000 \\
0.001 & -0.002 & 0.000 \\
0.001 & 0.001 & 0.000 \\
-0.001 & 0.001 & 0.000 \\
0.001 & 0.000 & 0.000 \\
0.002 & 0.000 & 0.000 \\
0.001 & 0.000 & 0.000 \\
0.001 & 0.001 & -0.001 \\
-0.107 & 0.021 & -0.002 \\
0.142 & -0.003 & 0.003 \\
0.285 & 0.004 & 0.007 \\
0.237 & -0.115 & 0.003 \\
-0.285 & -0.012 & -0.007 \\
-0.251 & 0.110 & -0.003 \\
0.307 & 0.150 & 0.010 \\
0.172 & -0.293 & -0.003 \\
0.147 & -0.088 & 0.001 \\
-0.418 & -0.265 & -0.020 \\
-0.164 & 0.356 & 0.002 \\
0.000 & 0.001 & 0.000
\end{tabular}

3140.86

YES

6.035

YES

$\begin{array}{crr}X & Y & Z \\ 0.000 & 0.000 & 0.000 \\ 0.000 & 0.000 & 0.000 \\ 0.000 & 0.000 & 0.000 \\ 0.000 & 0.000 & 0.001 \\ -0.002 & 0.000 & -0.097 \\ -0.178 & -0.352 & 0.591 \\ 0.207 & 0.349 & 0.554 \\ 0.003 & -0.001 & 0.012 \\ 0.021 & -0.021 & -0.055 \\ 0.000 & 0.000 & 0.000 \\ 0.000 & 0.000 & 0.000 \\ 0.000 & 0.000 & 0.000 \\ 0.000 & 0.000 & 0.000 \\ 0.000 & 0.000 & 0.000 \\ 0.000 & 0.000 & 0.000 \\ 0.000 & 0.000 & 0.000\end{array}$




$\mathrm{H}$
$\mathrm{H}$
$\mathrm{H}$
$\mathrm{H}$
$\mathrm{H}$

Fre
IR
IR
Ram
Re
O
O
O
C
H
H
C
H
N
C
C
C
C
C
H
H
H
H
H
H

$\mathrm{H}$

$$
\begin{array}{rrr}
0.000 & 0.000 & 0.000 \\
0.001 & 0.000 & 0.000 \\
-0.001 & 0.002 & 0.000 \\
-0.001 & -0.003 & 0.000 \\
-0.005 & 0.002 & -0.006
\end{array}
$$

Frequency:

IR Active:

R Intens:

Raman Active:

$$
\begin{array}{rr}
\multicolumn{1}{c}{\text { X }} & \multicolumn{1}{c}{Y} \\
0.000 & 0.000 \\
0.000 & 0.000 \\
0.000 & 0.000 \\
0.000 & 0.000 \\
0.000 & 0.000 \\
-0.019 & -0.042 \\
0.023 & 0.044 \\
-0.009 & -0.002 \\
-0.250 & 0.261 \\
0.000 & 0.000 \\
0.000 & 0.000 \\
0.000 & 0.000 \\
0.000 & 0.000 \\
0.000 & 0.000 \\
0.000 & 0.000 \\
0.000 & 0.001 \\
-0.001 & -0.001 \\
-0.001 & 0.000 \\
-0.001 & 0.004 \\
-0.002 & -0.002 \\
0.355 & -0.239
\end{array}
$$

3205.44

YES

1.161

YES

$$
\begin{array}{rrr}
0.000 & 0.000 & 0.000 \\
-0.001 & 0.000 & 0.000 \\
0.000 & -0.001 & 0.000 \\
0.001 & 0.001 & 0.000 \\
-0.350 & 0.240 & -0.569
\end{array}
$$

3220.27

YES

0.235

YES

Z

0.000

0.000

0.000

0.000

$-0.011$

0.067

0.066

$-0.096$

0.606

0.000

0.000

0.000

0.000

0.000

0.000

0.000

0.000

0.000

0.000

0.000

0.543
Z

0.000

0.000

0.000

0.000

0.001

$-0.001$

0.000

0.000

0.000

$-0.001$

$-0.001$

0.000

0.000

0.001

0.001
0.006

$-0.013$

0.013

0.009

$-0.001$

$-0.001$
3236.23

YES

3.777

YES

Raman Active:

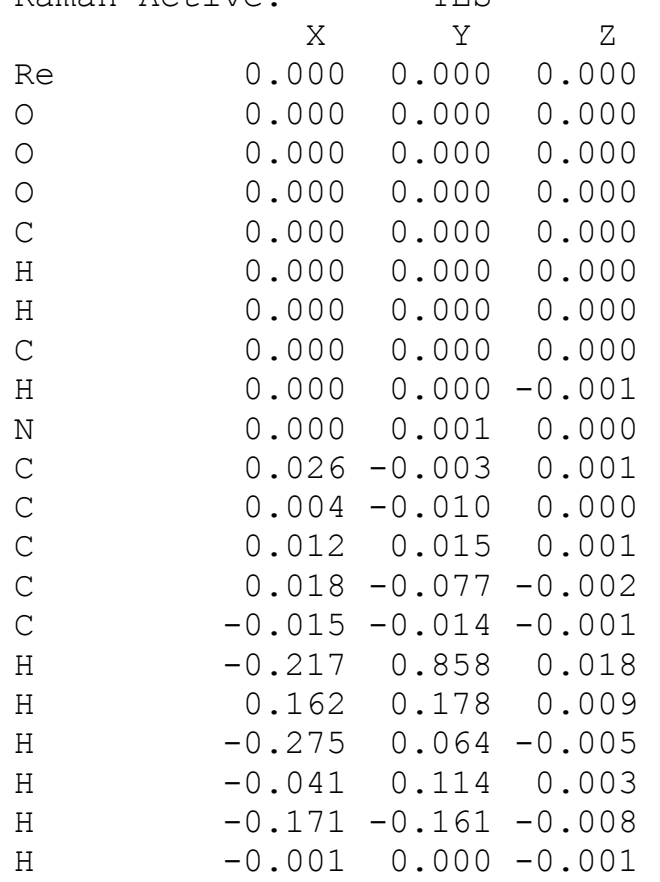

$$
\begin{array}{rrr}
0.000 & 0.000 & 0.000 \\
0.000 & 0.000 & 0.000 \\
0.000 & 0.000 & 0.000 \\
0.000 & 0.000 & 0.000 \\
-0.050 & 0.032 & -0.081
\end{array}
$$

\begin{tabular}{rrr}
\multicolumn{3}{c}{3225.28} \\
\multicolumn{3}{c}{ 2.005 } \\
YES & YES & Z \\
0.000 & 0.000 & 0.000 \\
0.000 & 0.000 & 0.000 \\
0.000 & 0.000 & 0.000 \\
0.000 & 0.000 & 0.000 \\
0.000 & 0.000 & 0.000 \\
0.000 & 0.000 & 0.001 \\
0.000 & 0.000 & -0.001 \\
0.000 & 0.000 & 0.000 \\
0.001 & -0.001 & -0.002 \\
0.000 & 0.001 & 0.000 \\
0.036 & -0.010 & 0.001 \\
0.026 & -0.071 & -0.002 \\
-0.003 & -0.002 & 0.000 \\
-0.005 & 0.014 & 0.000 \\
0.010 & 0.018 & 0.001 \\
0.045 & -0.170 & -0.004 \\
-0.151 & -0.175 & -0.009 \\
-0.405 & 0.101 & -0.007 \\
-0.282 & 0.807 & 0.020 \\
0.036 & 0.033 & 0.002 \\
-0.001 & 0.001 & -0.002
\end{tabular}

\begin{tabular}{rrr}
\multicolumn{3}{c}{3256.02} \\
YES \\
X & YES \\
0.000 & 0.000 & 0.000 \\
0.000 & 0.000 & 0.000 \\
0.000 & 0.000 & 0.000 \\
-0.001 & 0.000 & 0.000 \\
-0.001 & 0.000 & 0.000 \\
0.001 & 0.001 & -0.002 \\
0.000 & 0.001 & 0.001 \\
0.000 & 0.000 & 0.000 \\
0.000 & 0.000 & 0.000 \\
0.000 & 0.000 & 0.000 \\
0.006 & -0.001 & 0.000 \\
-0.001 & 0.005 & 0.000 \\
-0.064 & -0.056 & -0.003 \\
0.010 & -0.022 & 0.000 \\
0.004 & 0.005 & 0.000 \\
-0.067 & 0.249 & 0.005 \\
-0.045 & -0.049 & -0.003 \\
-0.050 & 0.012 & -0.001 \\
0.015 & -0.041 & -0.001 \\
0.705 & 0.647 & 0.034 \\
0.001 & -0.001 & 0.002
\end{tabular}

3242.06

YES

6.744

YES

$x$

0.000

0.000

0.000

0.000

0.000

0.000

0.000

0.000

0.000

0.001

$-0.025$

$\begin{array}{rr}0.014 & -0.028 \\ -0.008 & -0.008\end{array}$

$-0.002$

$-0.053-0.058$

$0.037-0.152$

$\begin{array}{lll}0.582 & 0.649\end{array}$

$0.272-0.070$

$\begin{array}{rr}-0.113 & 0.312\end{array}$

0.099

0.000
0.092

0.000 
This Molecule has 0 Imaginary Frequencies

Zero point vibrational energy:

$99.140 \mathrm{kcal} / \mathrm{mol}$

\begin{tabular}{|c|c|c|c|c|c|c|}
\hline Atom & 1 & Element & $\mathrm{Re}$ & Has & Mass & 186.9560 \\
\hline Atom & 2 & Element & 0 & Has & Mass & 15.9949 \\
\hline tom & 3 & Element & O & Has & Mass & 15.9 \\
\hline tom & 4 & Element & 0 & Has & Mass & \\
\hline tom & 5 & Element & $\mathrm{C}$ & Has & Mass & 12 \\
\hline com & 6 & Element & $\mathrm{H}$ & Has & Mass & \\
\hline tom & 7 & Element & $\mathrm{H}$ & Has & Mass & \\
\hline tom & 8 & Element & $\mathrm{C}$ & Has & Mass & 12 . \\
\hline om & 9 & Element & $\mathrm{H}$ & Has & Mass & \\
\hline tom & 10 & Element & $\mathrm{N}$ & Has & Mass & 14.0 \\
\hline tom & 11 & Element & $\mathrm{C}$ & Has & Mass & 12 . \\
\hline om & 12 & Element & $\mathrm{C}$ & Has & Mass & 12 \\
\hline tom & 13 & Element & $\mathrm{C}$ & Has & Mass & 12. \\
\hline Lom & 14 & Element & $\mathrm{C}$ & Has & Mass & 12. \\
\hline & 15 & Element & C & Has & Mass & 12 . \\
\hline & 16 & Element & $\mathrm{H}$ & Has & Mass & 1.0 \\
\hline & 17 & Element & $\mathrm{H}$ & Has & Mass & \\
\hline & 18 & Element & $\mathrm{H}$ & Has & Mass & 1.0 \\
\hline & 19 & Element & $\mathrm{H}$ & & Mass & \\
\hline & 20 & ement & $\mathrm{H}$ & & Mass & \\
\hline & 21 & Element & $\mathrm{H}$ & Has & Mass & 1. \\
\hline
\end{tabular}

Molecular Mass: $\quad 342.014270$ amu

Principal axes and moments of inertia in atomic units:

Eigenvalues -- $\quad 1177.914673780 .142034468 .35477$

$\begin{array}{rrrr}X & 0.98257 & 0.18529 & -0.01520 \\ Y & -0.18464 & 0.98211 & 0.03698 \\ Z & 0.02178 & -0.03353 & 0.99920\end{array}$

Rotational symmetry Number is 1

The Molecule is an Asymmetric Top

Translational Enthalpy: $\quad 0.889 \mathrm{kcal} / \mathrm{mol}$

Rotational Enthalpy:

$0.889 \mathrm{kcal} / \mathrm{mol}$

Vibrational Enthalpy:

gas constant (RT):

Translational Entropy:

Rotational Entropy:

Vibrational Entropy:

$104.846 \mathrm{kcal} / \mathrm{mol}$

$0.593 \mathrm{kcal} / \mathrm{mol}$

$43.384 \mathrm{cal} / \mathrm{mol} . \mathrm{K}$

$31.365 \mathrm{cal} / \mathrm{mol} . \mathrm{K}$

$38.146 \mathrm{cal} / \mathrm{mol} . \mathrm{K}$

Total Enthalpy:

$107.216 \mathrm{kcal} / \mathrm{mol}$

Total Entropy:

$112.895 \mathrm{cal} / \mathrm{mol} . \mathrm{K}$

Spartan '02 Properties Program: (PC/x86)

Release 116

Reason for exit: Successful completion

Properties Program CPU Time : 000:00:03.5

Properties Program Wall Time: 000:00:03.6 